INL/EXT-16-38755

Revision: 0

\title{
Transient Testing Reactor Physics Workshop - May 2016
}

May 2016

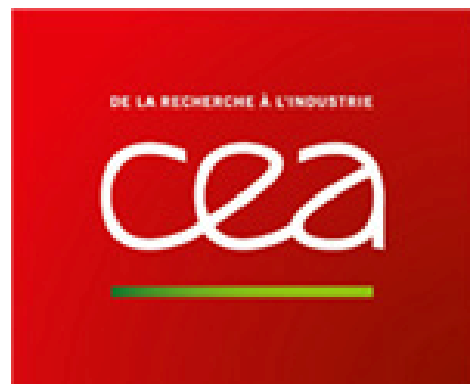




\section{DISCLAIMER}

This information was prepared as an account of work sponsored by an agency of the U.S. Government. Neither the U.S. Government nor any agency thereof, nor any of their employees, makes any warranty, expressed or implied, or assumes any legal liability or responsibility for the accuracy, completeness, or usefulness, of any information, apparatus, product, or process disclosed, or represents that its use would not infringe privately owned rights. References herein to any specific commercial product, process, or service by trade name, trade mark, manufacturer, or otherwise, does not necessarily constitute or imply its endorsement, recommendation, or favoring by the U.S. Government or any agency thereof. The views and opinions of authors expressed herein do not necessarily state or reflect those of the U.S. Government or any agency thereof. 


\title{
Transient Testing Reactor Physics Workshop - May 2016
}

\author{
Daniel Wachs
}

May 2016

Idaho National Laboratory Idaho Falls, Idaho 83415

http://www.inl.gov

\author{
Prepared for the \\ U.S. Department of Energy \\ Under DOE Idaho Operations Office \\ Contract DE-AC07-05ID14517
}


INTENTIONALLY BLANK 


\begin{abstract}
This Workshop was conducted in conjunction with the PHYSOR Conference being held in Sun Valley, Idaho, USA from May $1 \mathrm{st}-5^{\text {th }}, 2016$. The intent was to conduct a technical exchange on transient test reactor physics and modeling techniques used to support the Idaho National Laboratory TREAT facility and CABRI (CEA/IRSN) of France. Currently, several parallel programs are independently supporting critical aspects of TREAT physics analysis. Successful implementation will require active collaboration across programmatic boundaries to ensure the transfer of critical information as well as coordination and prioritization of activities.
\end{abstract}


INTENTIONALLY BLANK 
WORKSHOP PARTICIPANTS

\begin{tabular}{|c|c|c|}
\hline CABRI Experiments & & \\
\hline Jean-Pascal Hudelot & CEA & Jean-pascal.hudelot@cea.fr \\
\hline Bruno Biard & IRSN & Bruno.biard@irsn.fr \\
\hline \multicolumn{3}{|l|}{ TREAT Experiments } \\
\hline Dan Wachs & INL & Daniel.wachs@inl.gov \\
\hline Colby Jensen & INL & Colby.jensen@inl.gov \\
\hline Nick Woolstenhulme & INL & Nicolas.woolstenhulme@inl.gov \\
\hline Andy Beasley & INL & a.beasley@inl.gov \\
\hline John Darrell Bess & INL & John.bess@inl.gov \\
\hline David Chichester & INL & David.chichester@inl.gov \\
\hline Rob O'Brien & INL & Robert.obrien@inl.gov \\
\hline Connie Hill & INL & Connie.hill@inl.gov \\
\hline Charlie Folsom & INL & Charles.folsom@inl.gov \\
\hline Mary Lou Dunzik-Gougar & Idaho State Univ. & mldg@isu.edu \\
\hline \multicolumn{3}{|l|}{ TREAT Operations } \\
\hline Jim Parry & INL & James.parry@inl.gov \\
\hline Ben Chase & INL & Benjamin.chase@inl.gov \\
\hline \multicolumn{3}{|l|}{ NEAMS - TREAT } \\
\hline Mark DeHart & INL & Mark.dehart@inl.gov \\
\hline Javier Ortensi & INL & Javier.ortensi@inl.gov \\
\hline \multicolumn{3}{|l|}{ TREAT Benchmark IRP } \\
\hline Tom Downar & Univ. of Michigan & downer@umich.edu \\
\hline Bill Martin & Univ. of Michigan & wrm@umich.edu \\
\hline George Imel & Idaho State Univ. & gimel@isu.edu \\
\hline \multicolumn{3}{|l|}{ TREAT LEU Conversion } \\
\hline Dimitris Kontogeorgakos & ANL & dikontog@anl.gov \\
\hline \multicolumn{3}{|l|}{ DOE } \\
\hline Bradley Heath & DOE-ID & heathbk@id.doe.gov \\
\hline
\end{tabular}


INTENTIONALLY BLANK 


\section{CONTENTS}

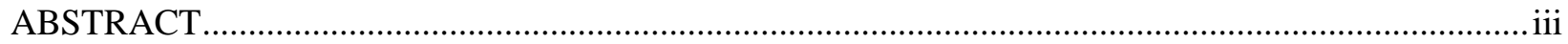

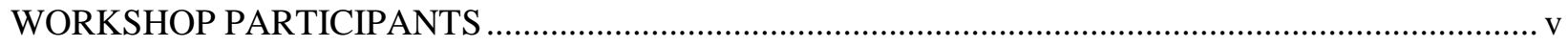

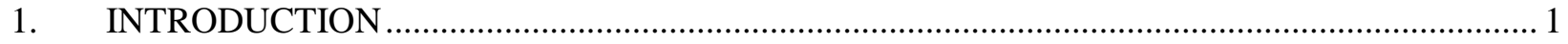

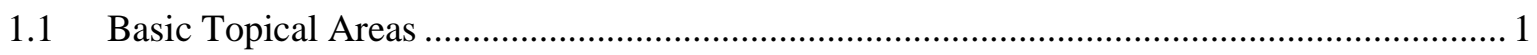

2. TOPIC 1 - TRANSIENT TESTING FACILITY OVERVIEWS ................................................ 1

3. TOPIC 2 - REACTOR STARTUP REQUIREMENTS …................................................... 1

4. TOPIC 3 - ADVANCED MODELING AND SIMULATION TOOL DEVELOPMENT ............... 3

5. TOPIC 4 - FUEL MOTION MONITORING SYSTEM RECOVERY ......................................... 4

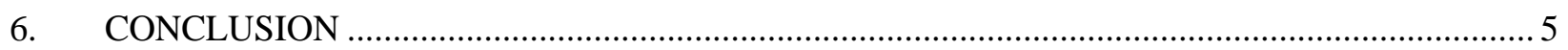

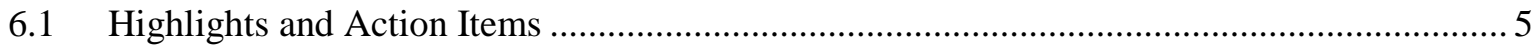

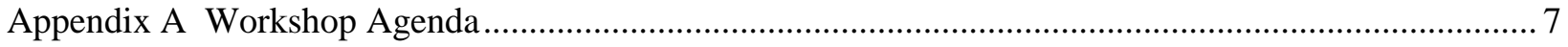

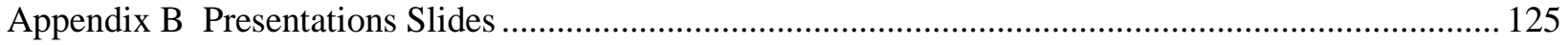


INTENTIONALLY BLANK 


\section{Transient Testing Reactor Physics Workshop - May 2016}

\section{INTRODUCTION}

The Workshop was conducted in conjunction with the PHYSOR Conference being held in Sun Valley, Idaho, USA from May $1 \mathrm{st}-5^{\text {th }}, 2016$. The Transient Testing Physics Workshop had two primary objectives. The first was to open a dialogue between U.S. and French technical experts related to reactor physics at the Idaho National Laboratory (INL) Transient Testing Reactor (TREAT) facility and the French CABRI Research Reactor project (CEA/IRSN). This dialogue was to inform the development of action plans within both the DOE/CEA and INL/IRSN bilateral cooperative agreements. The second objective was to help integrate the diverse domestic efforts in this area related to the TREAT project. Currently, several parallel programs are independently supporting critical aspects of TREAT physics analysis. Successful implementation will require active collaboration across programmatic boundaries to ensure the transfer of critical information as well as coordination and prioritization of activities.

\subsection{Basic Topical Areas}

The workshop consisted of four basic topical areas (as shown in the attached agenda) including:

- Transient testing facility overviews (TREAT and CABRI descriptions and programs);

- Reactor startup requirements (relationship between operating requirement, reactor analysis codes, and physics testing);

- Advanced modeling and simulation tool development (code development and benchmark cases for validation), and

- Fuel motion monitoring system recovery.

This workshop was constrained to these areas in order to focus discussion. However, the scope of follow-up workshops will expand to other critical areas outlined in the bilateral agreements.

\section{TOPIC 1 - TRANSIENT TESTING FACILITY OVERVIEWS}

Presentations were provided on both TREAT and CABRI. Nick Woolstenhulme presented the history of transient testing at INL (emphasizing TREAT). A joint presentation was provided by Jean-Pascal Hudelot (CEA) and Bruno Biard (IRSN) on CABRI. Both presentations are included in the appendix.

\section{TOPIC 2 - REACTOR STARTUP REQUIREMENTS}

Presentations were provided by Jim Parry (TREAT Chief Reactor Scientist) on the TREAT startup plan and Jean-Pascal Hudelot (CEA) on the CABRI commissioning progress and plan. Both presentations are included in the appendix.

Parry summarized some key aspects of the TREAT physics plan. TREAT operational limits are based on experimentally obtained reactivity insertion limits. Physics codes are used to design sub-maximal transients of interest to experimenters and do not serve any safety function. As such, conservative pointkinetics codes have traditionally been used for first-order core and transient design for experiments. Detailed reactor operation parameters were then refined through operational testing (e.g. calibration tests).

The approach to physics testing for new core configurations was reviewed. Fundamentally, reactor engineering will characterize a new core design by first conducting rod worth tests, heat balance tests (to calibrate power instruments), and, finally, a series of sub-maximal temperature-limited transients to estimate the limiting reactivity insertion. Subsequent testing is then conducted by the experiment program to determine coupling between the experiment and the reactor for the specific test device being used. 
While computational analysis is used to inform these tests, the data required for experiments is supplied by empirical results collected during these calibration tests.

The existing instrumentation was reviewed (power measurements are based on instruments located in biological shielding, far from the experiment). It is also important to note that the thermocouples in many of the existing instrumented fuel elements have failed during service and only a limited number are still functional. New devices will need to be developed and qualified to measure local power. Potential areas for insertion of new instruments were described and include the 'coolant channels' between elements, unused control rod drive positions, and specially designed replacement elements. The use of more sophisticated codes to reliably design experiments without calibration runs may require improved material property data for TREAT driver fuel and the availability of advanced instrumentation in order to validate the codes.

TREAT reactor engineering is working to maintain a suite of codes that can be validated (benchmark studies or future physics testing) and made available to experimenters. These codes will include both steady-state and transient codes. The steady-state codes are used to estimate the power coupling factor (PCF) between the reactor and experiment.

Steady-state models are being developed on multiple platforms by various TREAT stakeholders and include

- SCALE (TREAT Reactor Engineering -INL),

- MCNP (TREAT Experiments - INL, Hodoscope modeling - Texas A\&M University/Idaho State University, and LEU Conversion - ANL),

- SERPENT (Advanced TREAT M\&S - INL), and

- PARCS (TREAT Benchmark studies - University of Michigan).

Transient codes include the

- ARCS simulator (TREAT Reactor Engineering),

- S-TREK (a code being adapted by TREAT Reactor Engineering from the ARCS simulator for use on a more universal platform),

- a RELAP-5 model (being developed by the TREAT Experiments program to support experiment design),

- TREKIN (being updated by ANL for LEU Conversion core design), and

- MAMMOTH/RATLESNAKE (NEAMS-TREAT)

Jean-Pascal Hudelot provided an overview of the CABRI physics modeling and commissioning effort. CABRI steady-state core neutronics are described using TRIPOLI4 (also possible with MCNP). These calculations were initially validated against historic critical tests that were conducted while configured with the sodium loop. Further validation for the water-loop configuration is based on updates and improvement to representation of the core configuration. This includes both the core geometry and the material compositions within the core.

In addition to the core neutronics, tools to describe test pins are also required. The calculations performed at commercial power plants to estimate fuel pin composition are not always accurate enough to support determination of the power coupling factor during transient testing. CEA and IRSN both maintain a suite of codes to perform this function (CEA - APOLLO2/REL2005, APOLLO3, CESAR5.3 and IRSN- MORET4, MCNP, and VESTA).

CABRI transient behavior is treated using the point kinetics code DULCINEE. This code uses a pseudo steady-state method where the feedback parameters are calculated after each time step using 
preliminary TRIPOLI4 calculated results (this includes Doppler effect and delayed phenomena like clad expansion and coolant density). Other critical parameters (delayed neutron fraction and generation lifetime) are determined either computationally, using MCNP or the latest version of the TRIPOLI4 code, or measured during the commissioning tests. DULCINEE also embeds simplified thermal and thermal hydraulics models for processing single- or two-phase flows in natural or forced convection. Heat transfer in the fuel rods is modeled from the inside to the outside. Several types of regions are described (fuel/gap/clad). It allows the user to specify the control system operational parameters and predict the resulting transient or to input the desired transient and calculate the necessary control strategy to achieve it. Validation of DULCINEE is based on 19 past transient tests.

CABRI driver fuel performance during each transient is analyzed using SCANAIR to ensure compliance with safety criteria including fuel temperature, clad temperature, and clad strain. The use of multi-physics codes that couple phenomena to predict driver fuel performance is being pursued using the ALCYONE code (fuel performance) coupled with APOLLO3 (neutronics).

CABRI primary cooling system thermal hydraulics are being treated using CATHARE, TRIO-U and the commercial CFD code STAR-CCM+.

A variety of tests are being conducted during the commission phase of the reactor to provide critical inputs to these models. Examples include the following areas.

- Prior to nuclear operations - He-3 system depressurization rate as a function of valve positions and impacts of He-3 purity.

- Low power operations (Commission \#1) - neutronic characterization of the core. The results were compared with uncertainty targets laid out in advance of the testing program. First criticality was achieved October 20, 2015.

- Full power operations (Commission \#7) - heat balances for up to $25 \mathrm{MW}$ steady-state power and neutron detector calibration for transient power from $100 \mathrm{~kW}$ to $\sim 25 \mathrm{GW}$.

Discussion revealed a few common physics issues related to uncertainties in the use of point kinetics methods. Most significantly, the neutron spectrum is known to change during the transient. In the case of TREAT, the spectrum hardens during the transient due to the increase in graphite temperature. In CABRI the spectrum softens as the He-3 is removed from the reactor. In both TREAT and CABRI, the radial power distribution also moves during the transient. Advanced codes may allow for explicit treatment of these phenomena.

\section{TOPIC 3 - ADVANCED MODELING AND SIMULATION TOOL DEVELOPMENT}

The development and validation of new codes requires detailed comparison of calculated results with relevant measured parameters. Benchmark documentation of historic TREAT tests is being developed (by TREAT Reactor Engineering, TREAT Experiments, NE IRP projects, and NEUP projects) to enable the new MOOSE based codes (to be developed under NEAMS).

A presentation was prepared by John Bess (attached) to describe the objective, status, and early findings of the benchmark efforts. Dr. Bess is leading an effort within INL to develop the benchmark cases required to understand uncertainties in the TREAT core that could impact reactor operations (criticality, control rod worth, excess reactivity, shutdown margin) and to support validation of nuclear data utilized in the existing TREAT operation codes. Three core configurations are being assessed including the minimal critical core, a mid-size core to be determined, and the M8CAL configuration. At the end of this process, results will be packaged and documented for the International Reactor Physics Experiments Benchmark (IRPhEP) handbook. These cases will include the required core geometry, 
component compositions, and experimental results. A Baseline Assessment of TREAT for Modeling and Analysis Needs (BATMAN) report (INL/EXT-15-35372) outlining the core component geometry and material compositions is complete and is being widely used for model development. The experimental data for the selected transients is being collected but users are encountering difficulty finding all the desired data as well as defining the quality level/pedigree on the data that is located. It is anticipated that additional testing will be required during startup physics testing to 'fill in the blanks'.

However, a few dominant dimensional and material property unknowns have already been identified that substantially impact the uncertainty in reactor modeling. In particular, the actual position of the poison section of the control rods in the core (to be measured by TREAT Operations), boron content in the TREAT driver fuel, extent of graphitization in the TREAT fuel blocks, and the specific heat of the TREAT graphite. Strategies to measure or estimate these properties are being developed.

Additional benchmark cases are being developed under NE-4 sponsored projects to support complex experiment analysis. Tom Downar and Bill Martin (University of Michigan) are working within an NE IRP (led by Oregon State University) to develop benchmark cases for the minimum critical, M8CAL, and a third core to be determined. The UM team is using PARCS to assess sensitivity and uncertainty in the measured parameters. Ayman Hawari (North Carolina State University) is working within an NE NEUP to develop similar benchmark cases for the M2 and M3 experiments. The NCSU team is using SERPENT for sensitivity and uncertainty calculations.

Progress in the development of modeling tools to perform coupled kinetics calculations was presented by Mark DeHart. This team is working to use MAMMOTH to couple nuclear time-dependent neutron transport (RattleSnake) in the TREAT core with thermal mechanical behavior (BISON) of the fuel elements. This tool will allow for prediction of the full time dependent response of TREAT and its experiments to transient tests. A simplified model of TREAT was constructed from an infinite lattice of fuel elements to explore general physical response of the system. The analysis showed the appropriate qualitative pulse response as well as the fuel element temperature and flux distribution. However, difficulty was encountered due to the streaming effects of the coolant channels located at each elements corner. These openings allow for axial streaming of neutrons that required a modification to the computational methods used. This feature is expected to be even more significant in treatment of the hodoscope slot.

A MAMMOTH model of the minimum critical core was developed by Tony Alberti (Oregon State University). This was followed by 'small core' configuration that allowed for simulation of TREAT transient \#15. Initial results suggest excellent agreement after analysis/reduction of the available historic measured reactor power data. MAMMOTH predicts as wide array of additional reactor parameters that were not historically measured that could be used for more comprehensive validation in the future.

M8CAL analysis is underway and poses some new challenges. Most notably, the presence of the hodoscope slot, multiple control rod types, complex geometry of the test section, and the use of dysprosium flux shaping collars in the test. Currently, the steady state calculations and measurements don't match and must be resolved prior to meaningful transient analysis.

Developers plan to follow analysis of these tests with work on the Multi-SERTTA device to be used in the ATF-3 campaign. Exchange of device descriptions and modeling needs was initiated between device designer (Nick Woolstehnulme) and MAMMOTH analysts (Javier Ortensi).

\section{TOPIC 4 - FUEL MOTION MONITORING SYSTEM RECOVERY}

The status of hodoscope recovery at both CABRI and TREAT was presented (see attached).

David Chichester described TREAT hodoscope recovery goals and specific activities completed to date. Although the TREAT hodoscope includes two detector banks (proton recoil scintillator detectors 
and proton recoil proportional counters), only one set is currently being addressed to support startup operations. The scintillators were selected for initial use due to their relative simplicity, to mitigate technical risks. All of the scintillator detectors were extracted from the hodocopse, an evaluation process was developed and implemented, and a refurbishment technique was developed. 99 of 327 detectors are considered candidates for refurbishment and 20 have been refurbished to date. The photomultiplier tubes attached to the scintillator were found to have substantially degraded and must be replaced. Candidates were tested and a preferred commercial product was selected. A data acquisition system (DAS) was designed and a prototype constructed. The 16-channel system will be replicated as many times as necessary to support deployment of a limited view system (64-96 channels) in the near term and eventually to support the full system. Advanced DAS capabilities may result in collection of neutron and gamma ray data. This could substantially enhance data gathered from the hodoscope during future test. Further analysis and testing is required.

The hodoscope was suggested as a tool that could be used to support physics testing. The device provides unique time and space-dependent fast neutron flux distribution data that is being predicted using the advanced codes. The device could be enhanced with additional detectors to simultaneously collect thermal neutron data.

Bruno Biard presented the status of the CABRI hodoscope. The device consists of 51 rows and 3 columns of collimated neutron impinging on 153 fission chambers (Np-237) and 153 proton recoil counters (methane). The system is capable of collecting data every $1 \mathrm{~ms}$. The fission chambers are designed to provide measurements during full-power transient mode and the proton recoil counters provide measurements at low power. A few operating concerns were identified. Some electronic components may not be readily available and there are only a limited number of spares. Also, the system had to be moved during the facility refurbishment and confirmation of alignment is a critical first step in commissioning.

The process for calibrating and converting the hodoscope signal to a local mass distribution was described. The background neutron signal was processed during first critical tests and showed excellent agreement with the power profile measured via dosimetry.

A follow-up workshop on hodoscope technology to be hosted by IRSN in Cadarache in October was proposed.

\section{CONCLUSION}

\subsection{Highlights and Action Items}

- Significant synergy exists between the TREAT and CABRI programs. Formal collaboration in the future is empowered by bilateral agreements between DOE/CEA and DOE/IRSN.

- $\quad$ TREAT startup physics lead (Jim Parry) should engage CABRI commissioning lead (Jean-Pascal Hudelot) to discuss methodologies used and lessons learned during early physics testing at CABRI.

- TREAT reactor engineering should be formally tasked with reviewing all TREAT core models being developed, act as a hub for storing and distributing the models to users, and provide revision control functions to the validated versions of these models. Active participation of TREAT engineering in advanced code development is also recommended to accelerate implementation.

- Advanced codes should be used to explore time dependent reactor behavior (spectrum shifts in particular) that currently cannot be described using point kinetics.

- A strategy to reduce uncertainty in key TREAT material properties (boron content, graphitization, and specific heat) should be developed for consideration. 
- Integration of the MAMMOTH team with the ATF-3 Experiments team should be expanded to provide 'qualitative' analysis of phenomena of interest that cannot be determined using existing codes (to later become 'quantitative' after validation of the codes during physics testing).

- Expanded technical exchange between CABRI and TREAT experts in the areas of experiment design, safety basis development, instrumentation, irradiation test device, modeling and simulation, and fuel motion monitoring would substantially improve the programs in both countries.

An expanded workshop to be hosted by CEA and IRSN is proposed for October 2016. 


\section{Appendix A}

\section{Workshop Agenda}


INTENTIONALLY BLANK 


\section{Transient Test Reactor Physics Workshop Thursday, May 5, 2016}

Objective: Conduct technical exchange on transient test reactor physics and modeling techniques used to support TREAT (INL) and CABRI (CEA/IRSN). The workshop will be conducted in conjunction with the PHYSOR conference being held in Sun Valley, Idaho, USA from May 1-5.

Attire: Business Casual 


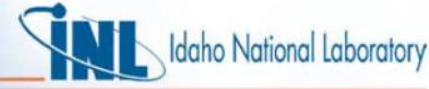

\section{Transient Test Reactor Physics Workshop Thursday, May 5, 2016}

Sun Valley Lodge, Larkspur Room, Sun Valley, Idaho, USA

08:00 Brief overview of TREAT and CABRI Reactors

Dan Wachs/Bruno Biard

09:30 BREAK

All

09:45 TREAT physics modeling and startup testing plan

Jim Parry

TREAT Reactor Chief Scientist, (208) 533-8872

10:30 CABRI physics modeling and startup testing results and plans Jean-Pascal Hudelot

11:30 IRHP Benchmark process and results for TREAT John Bess (Tom Downar) Reactor Physics Design \& Analysis, (208) 526-4375

12:00 Working Lunch: Discussion of dosimetry and radiation measurement techniques. All

1:00 Advanced modeling and simulation for TREAT. Mark DeHart Reactor Physics Design \& Analysis, (208) 526-1279

2:00 TREAT Hodoscope recovery and performance.

David Chichester Global Security/International Safeguards, (208) 526-8920

2:45 BREAK. All

3:00 CABRI Hodoscope recovery and initial testing results Bruno Biard

3:45 Discussion of development and validation of advanced analysis methods IRSN

5:00 Adjourn for Dinner. All

INL Facility tours (including TREAT) could be accommodated on Friday May 6 upon request. 

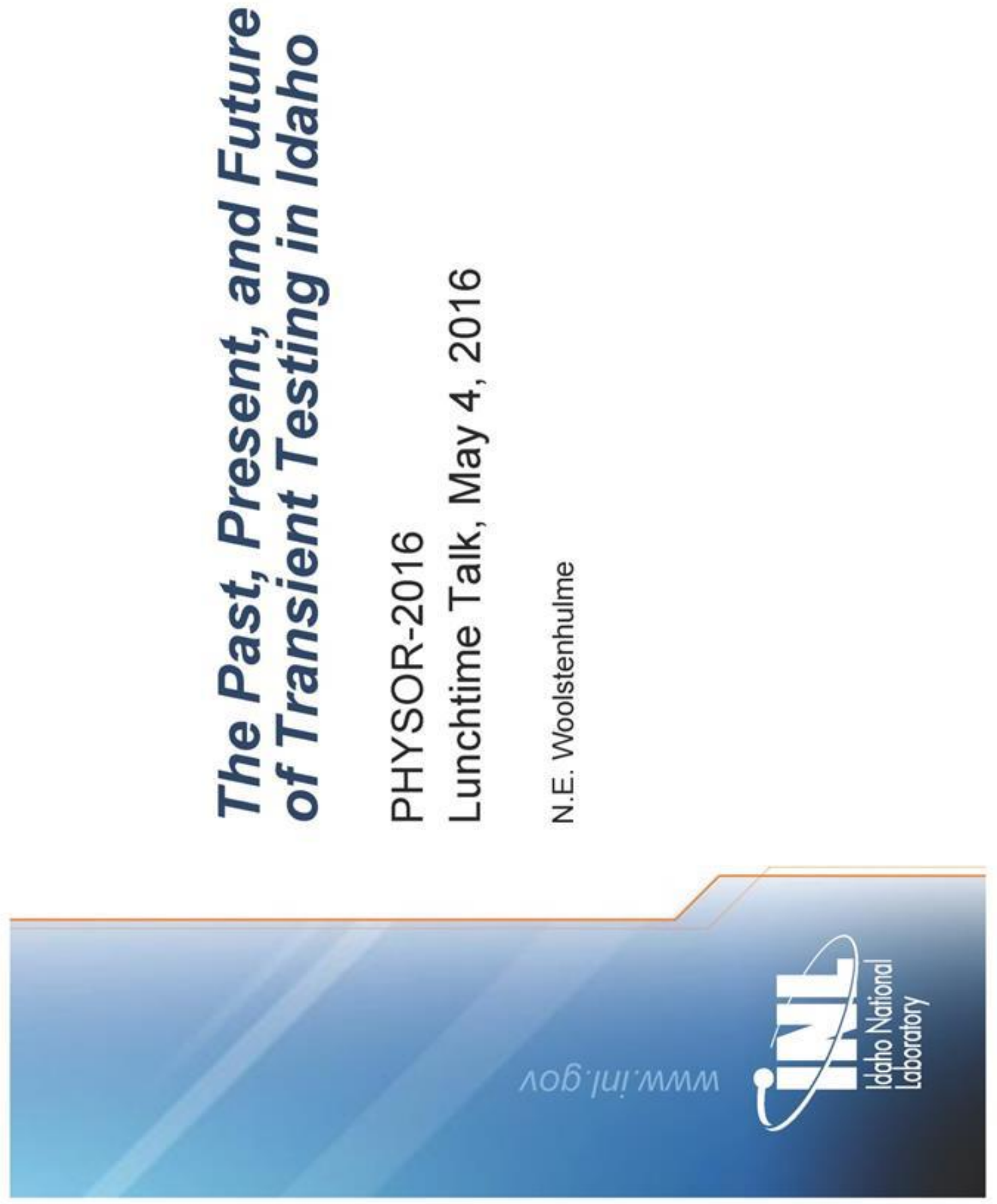

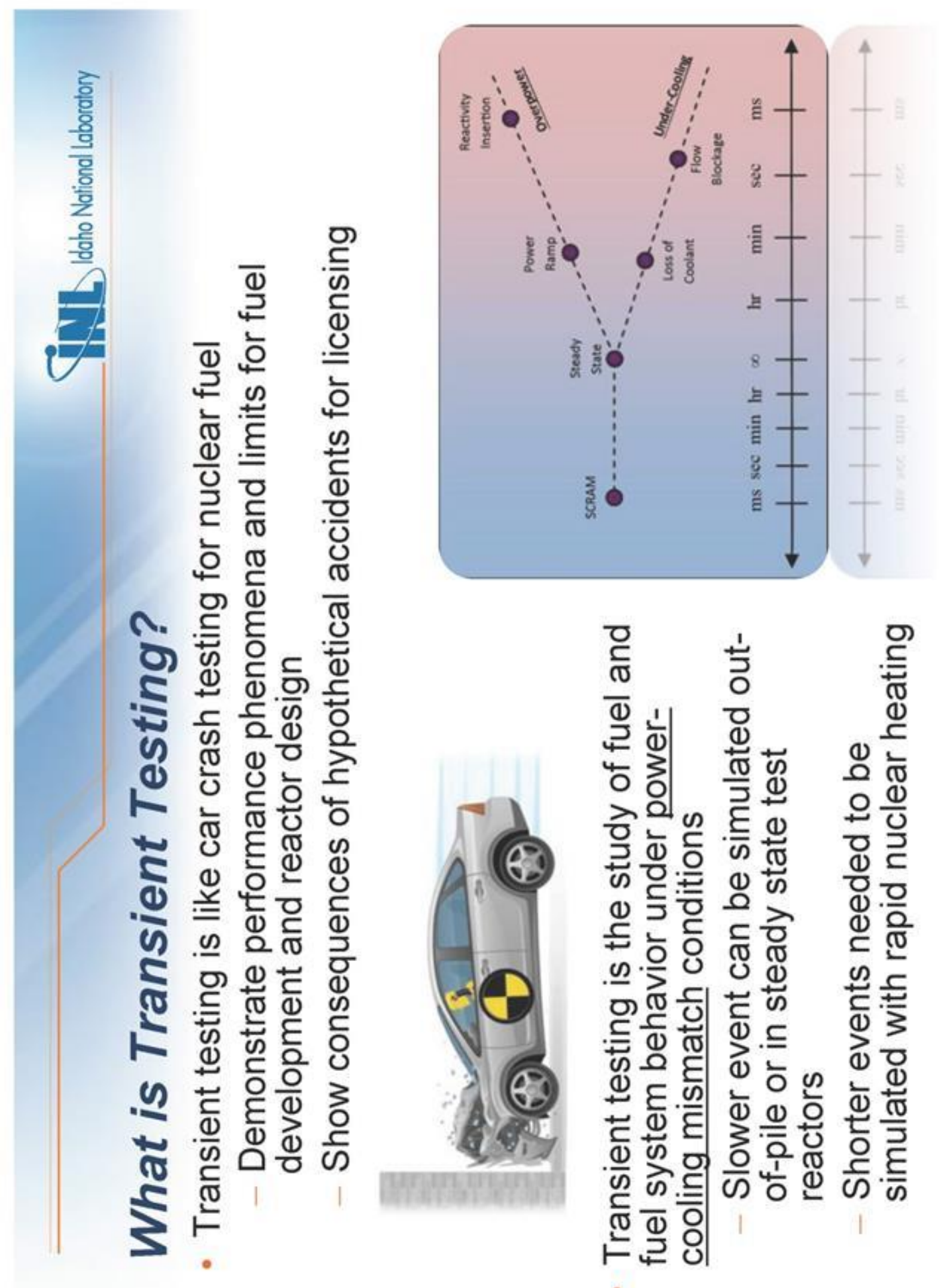

음 मे 㐫岕 离 김

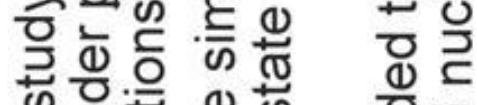

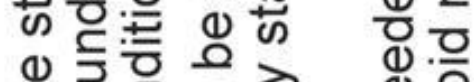

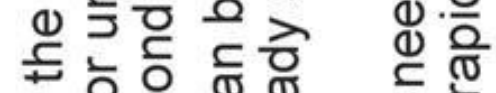

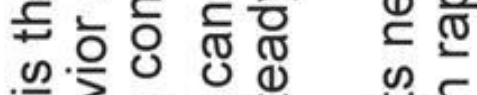

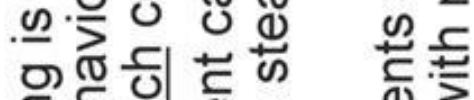

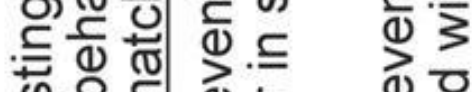

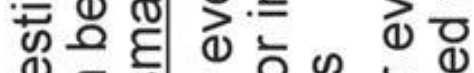

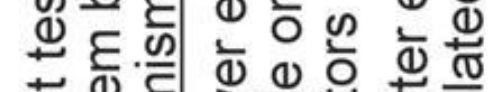
든

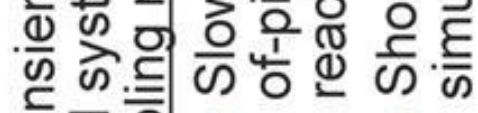
흔 


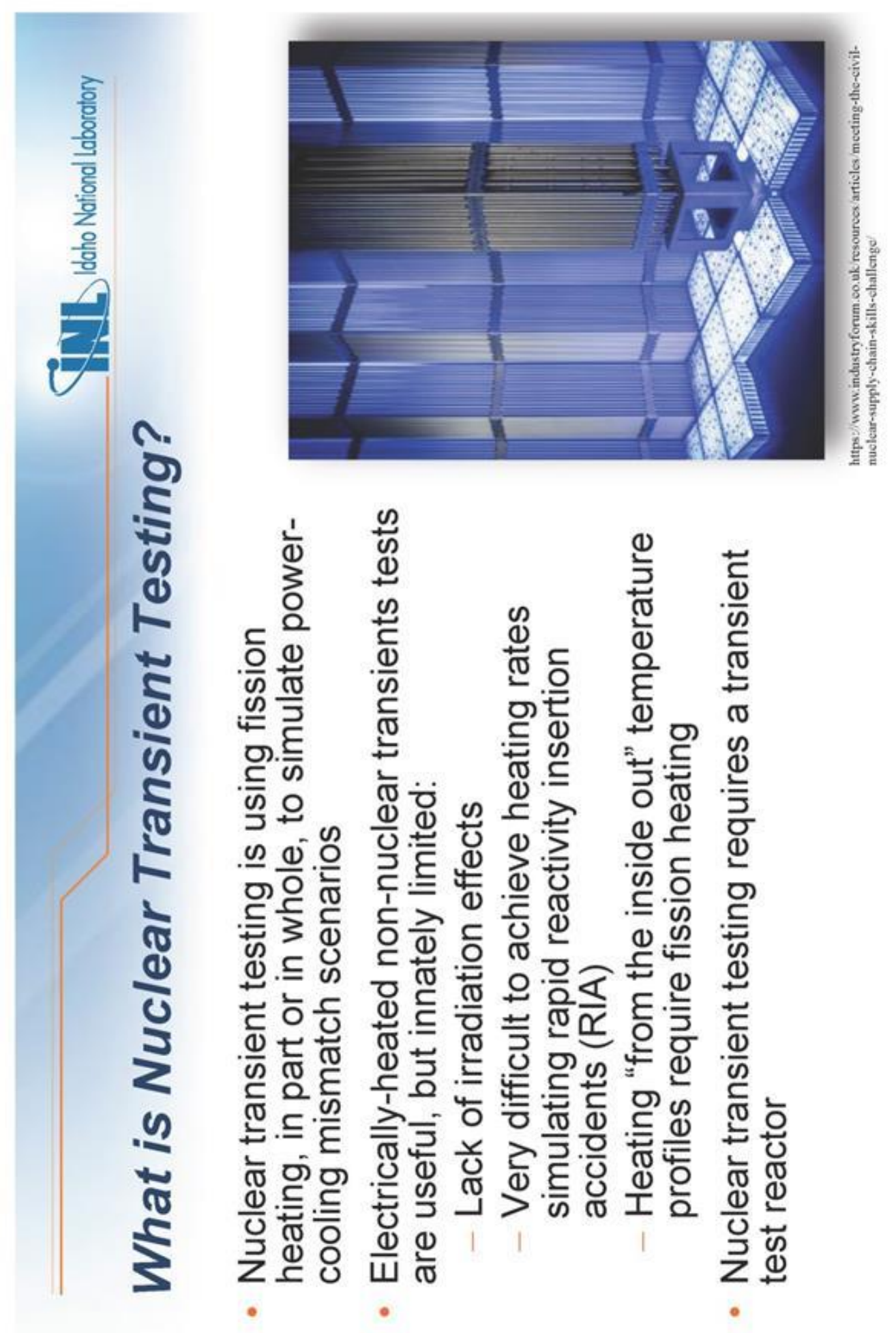




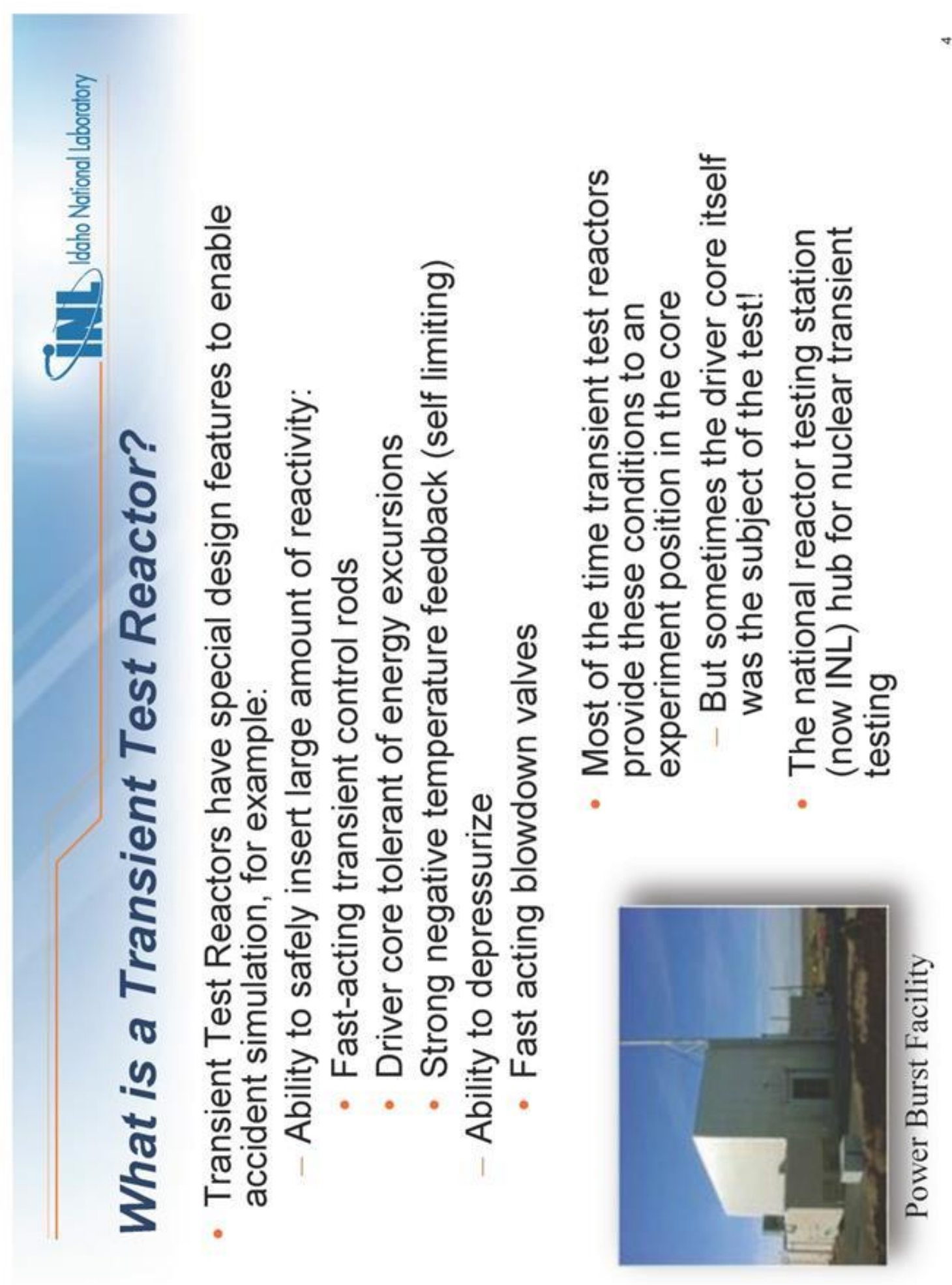



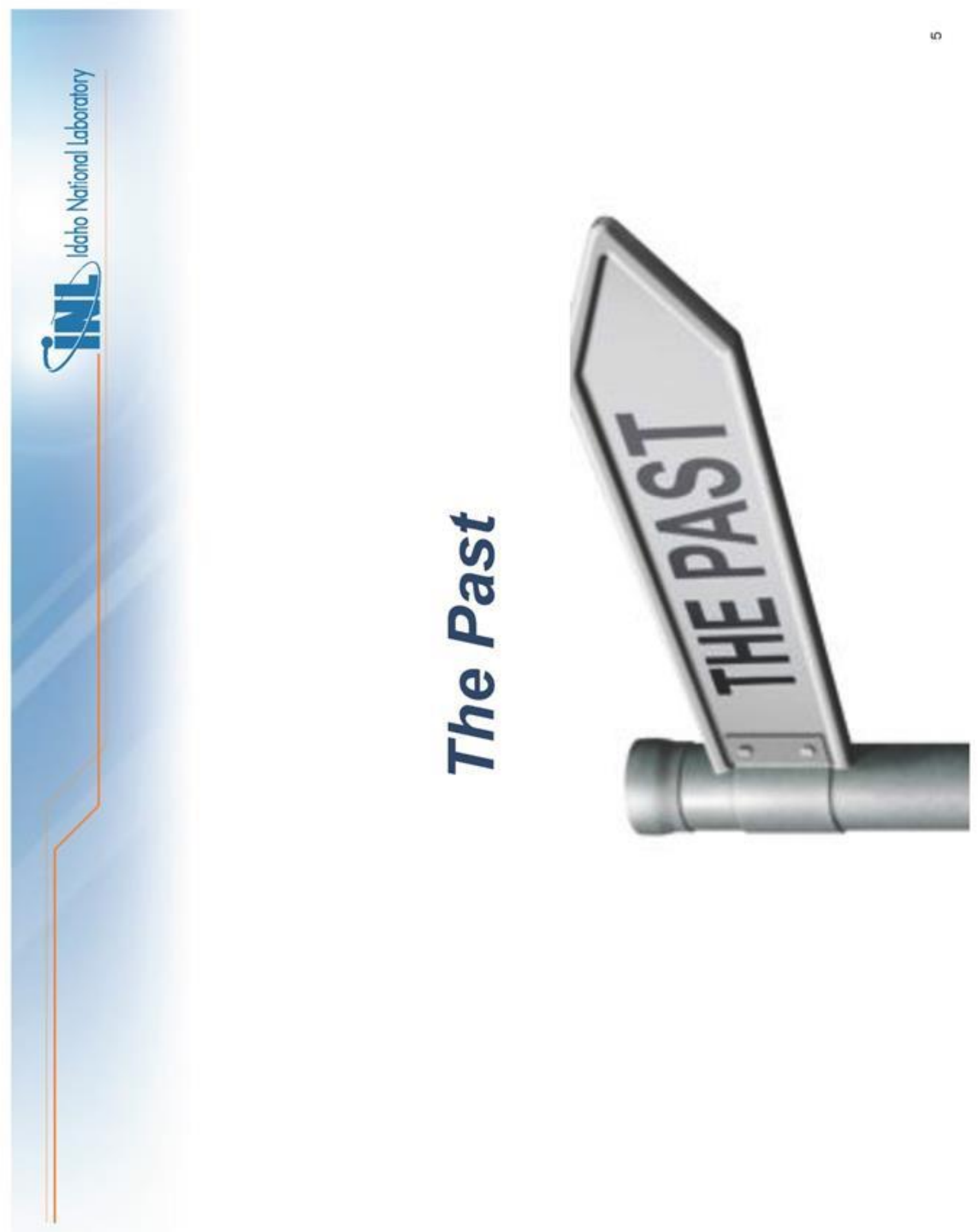


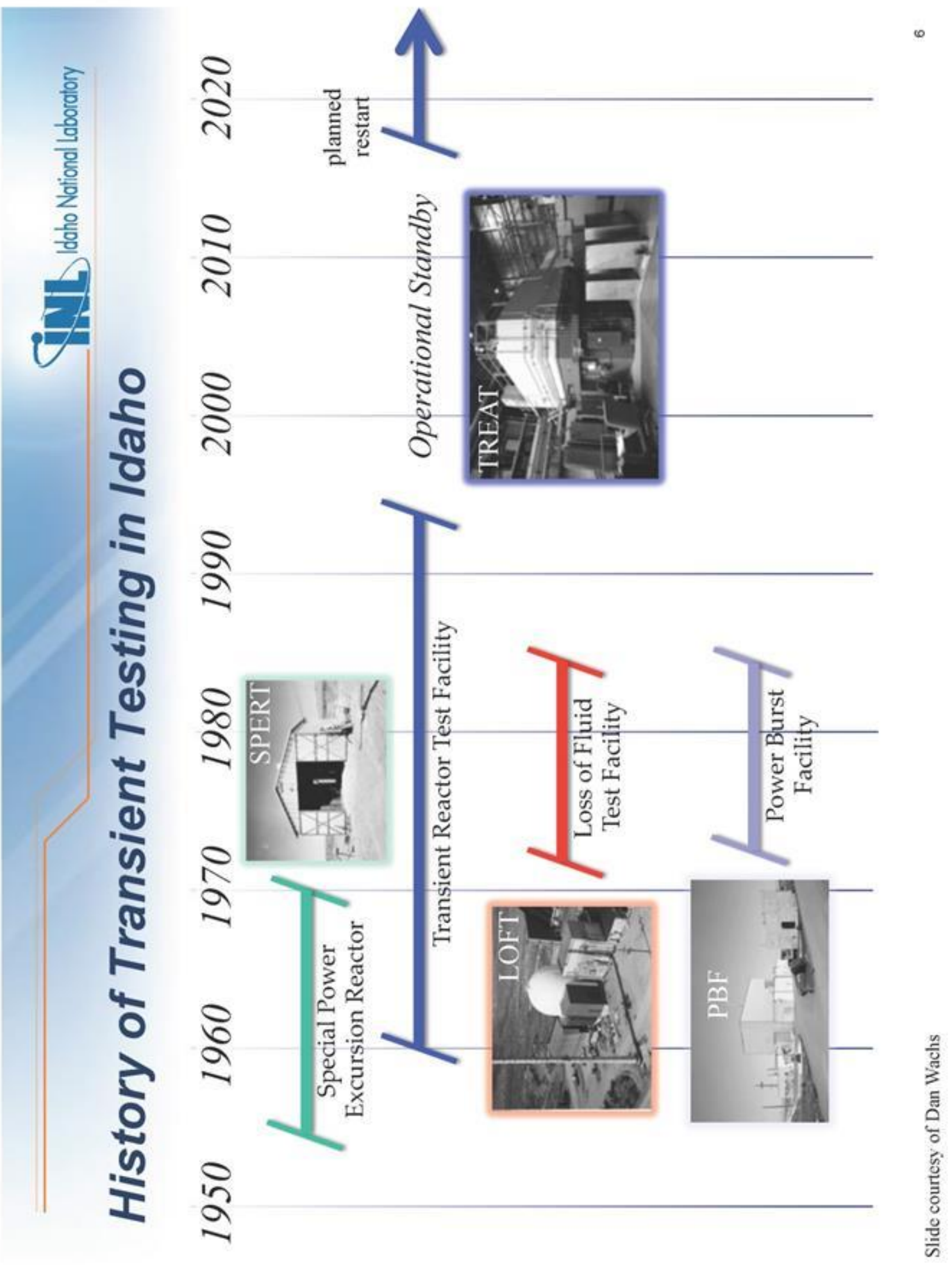




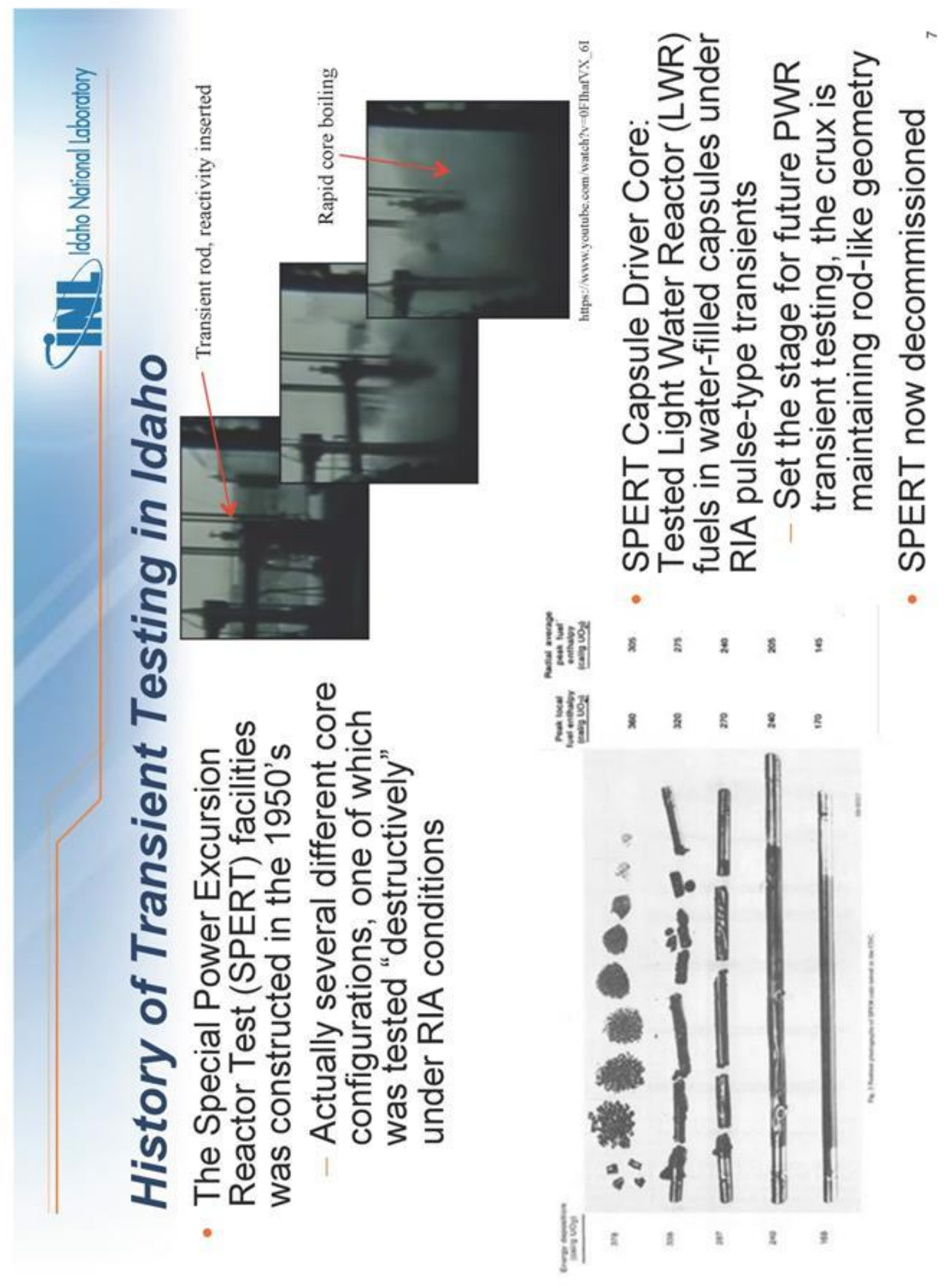



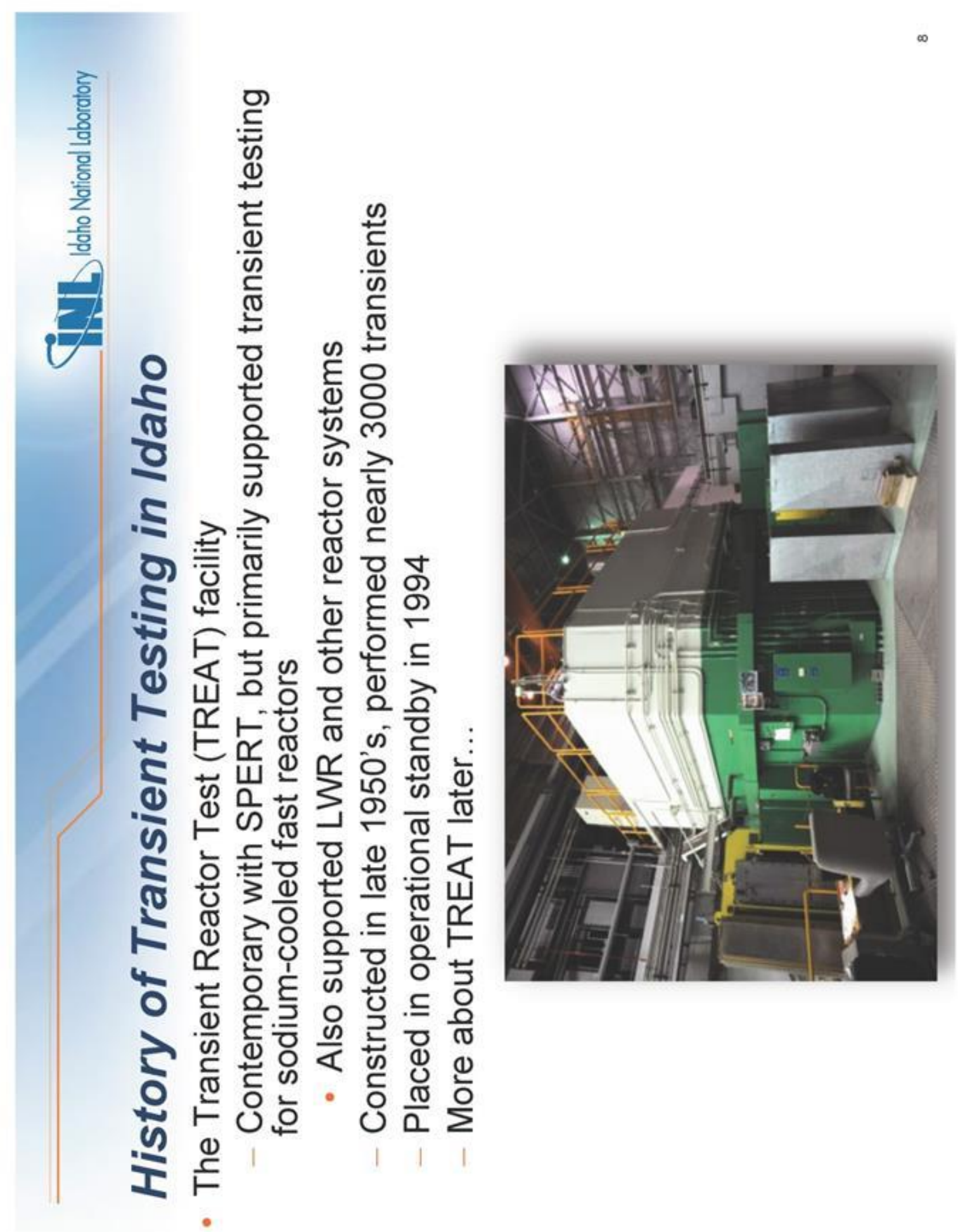


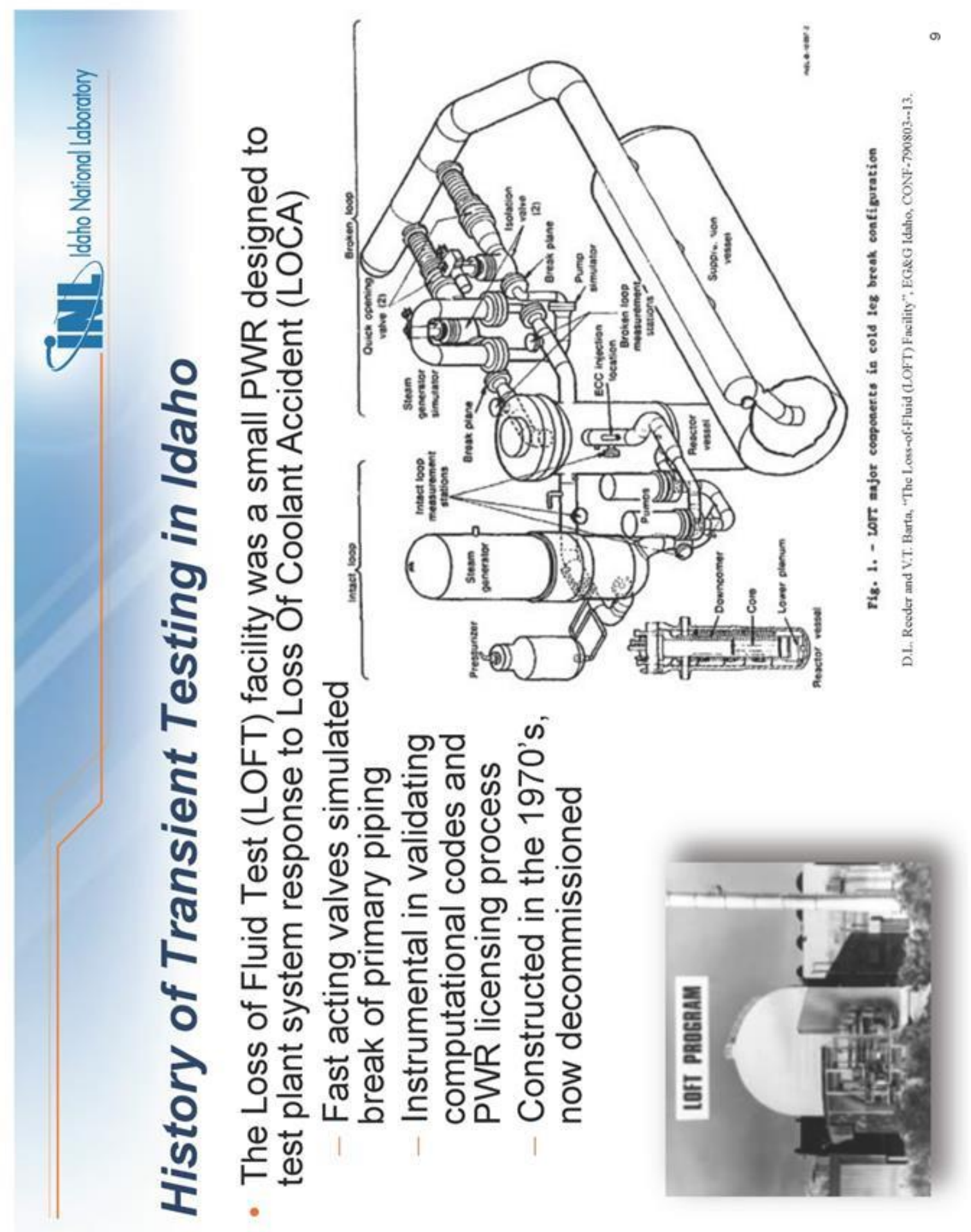




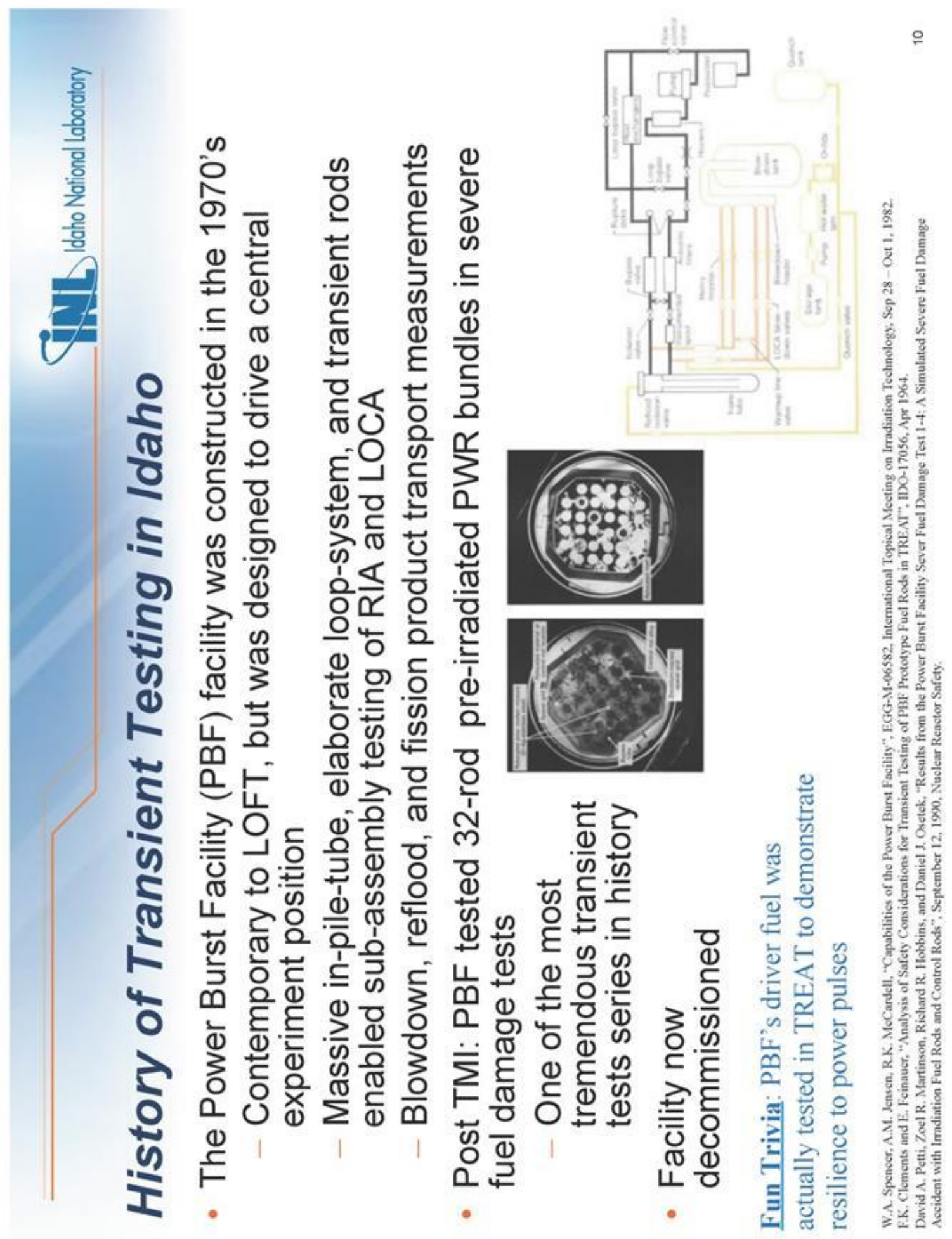




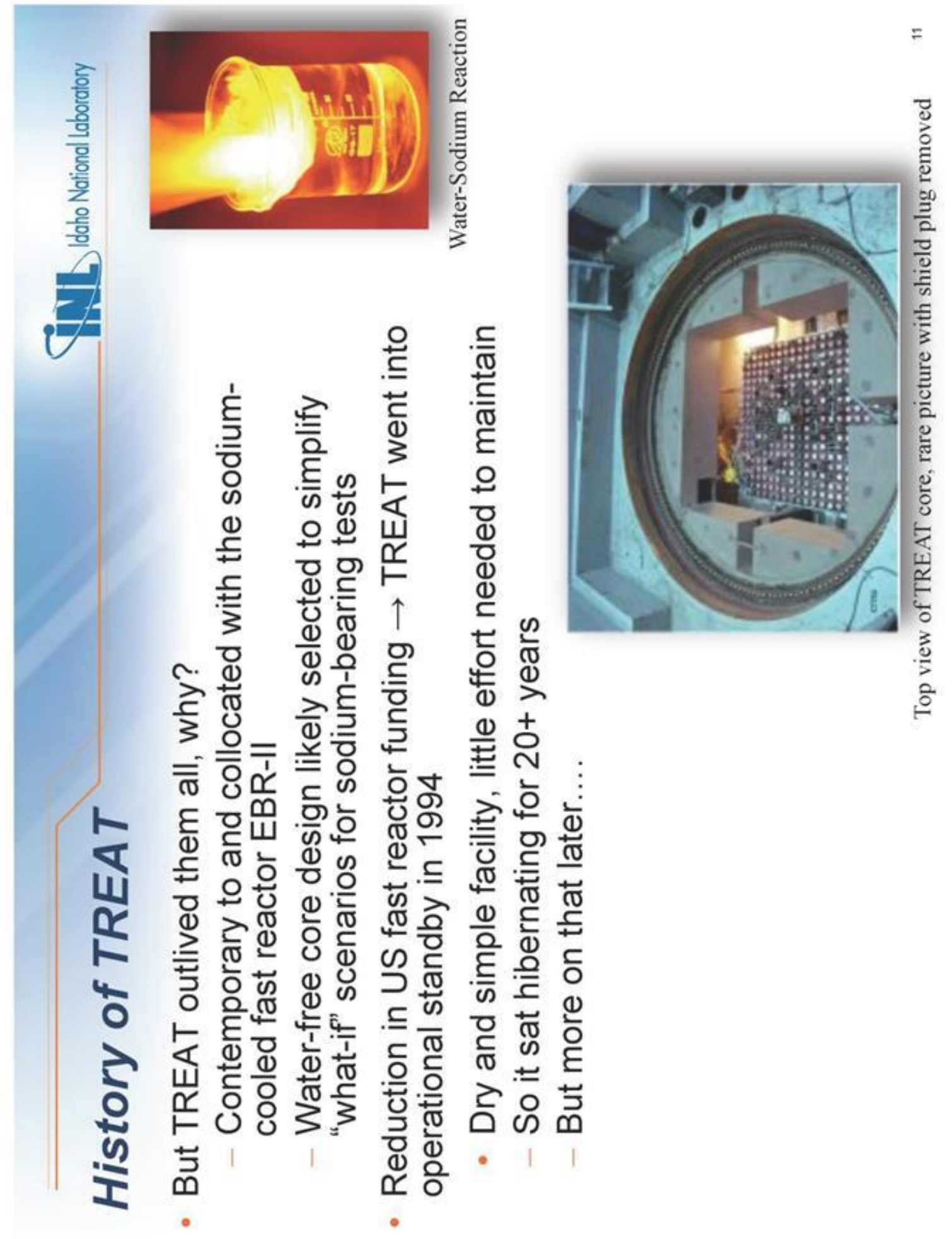




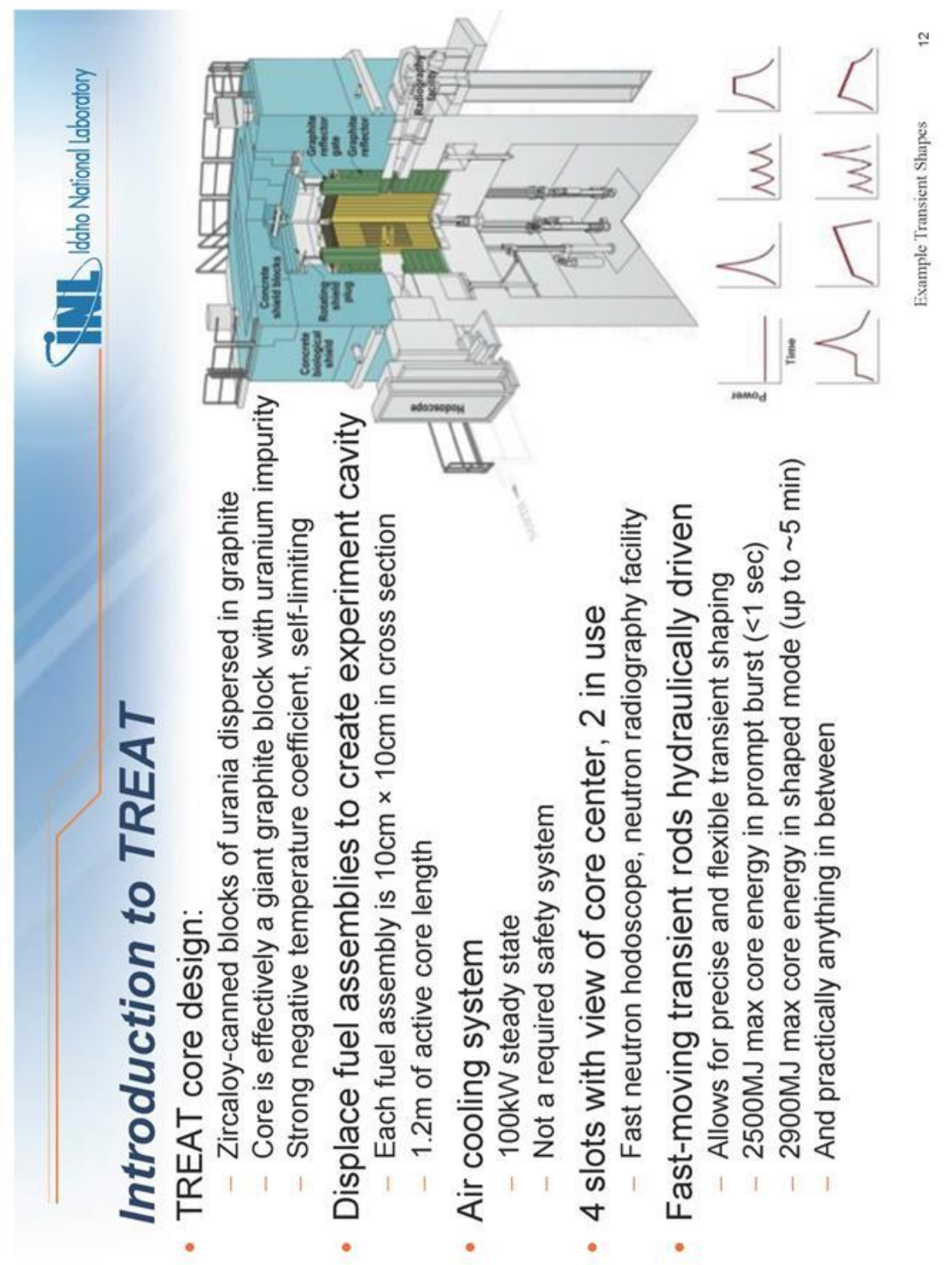




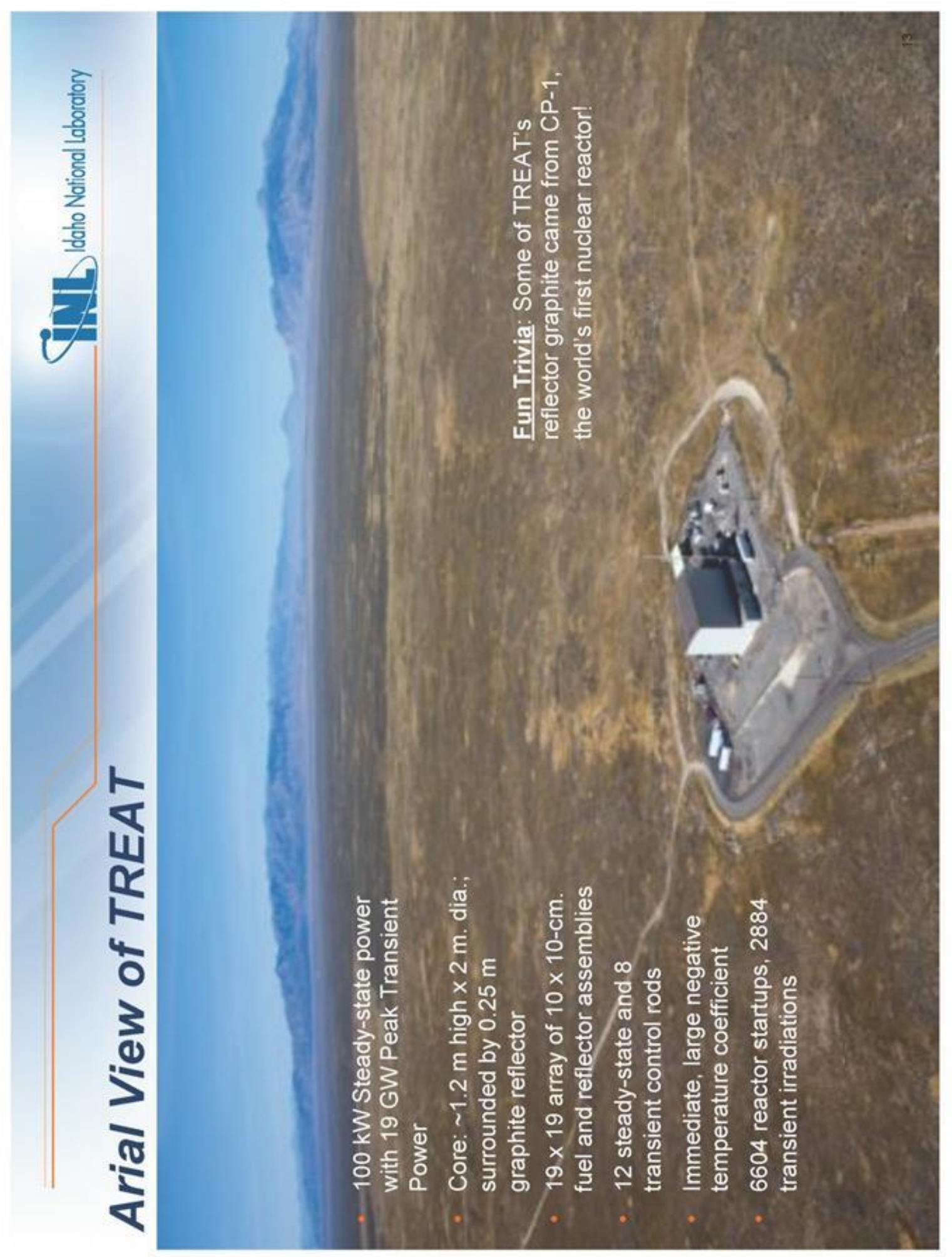




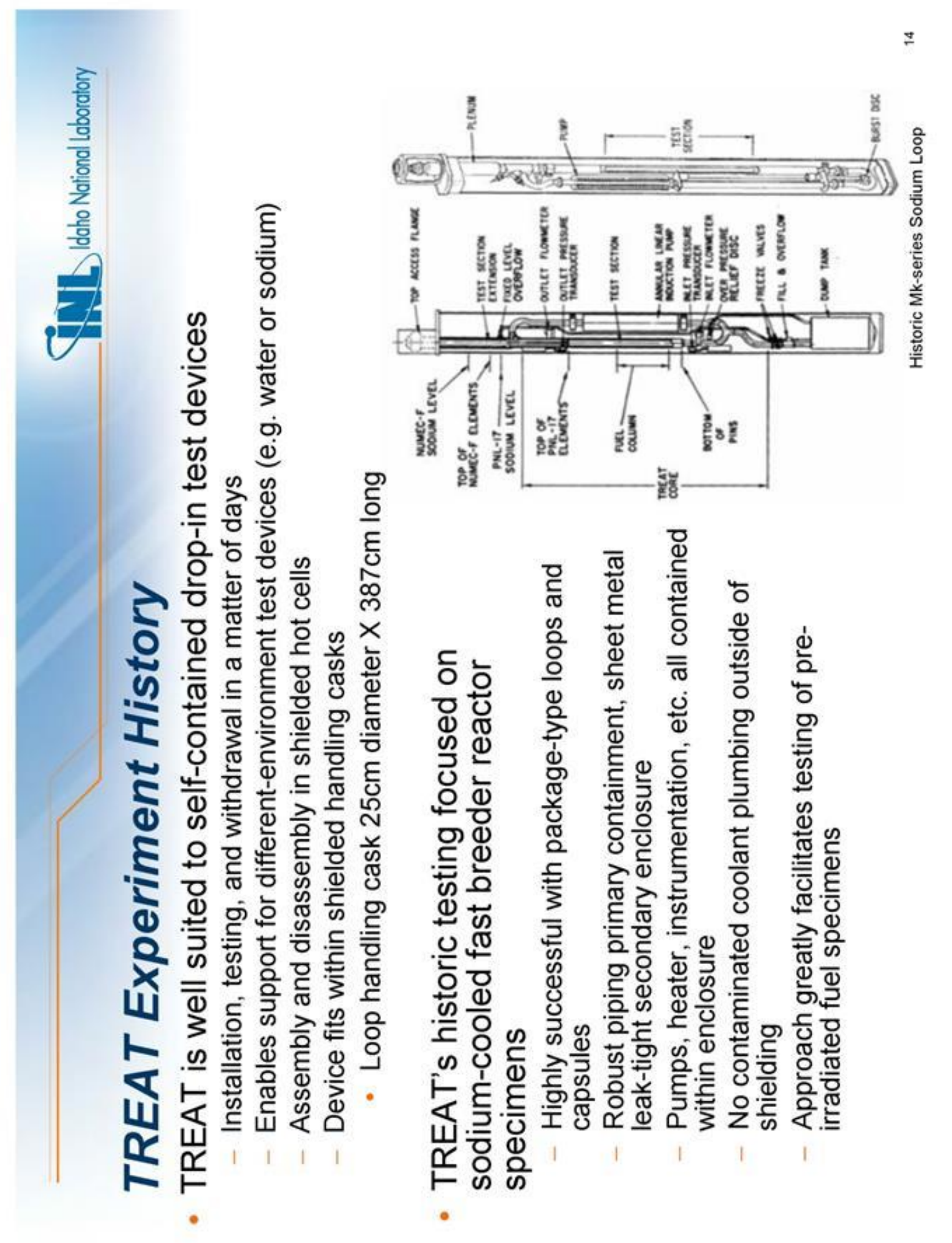




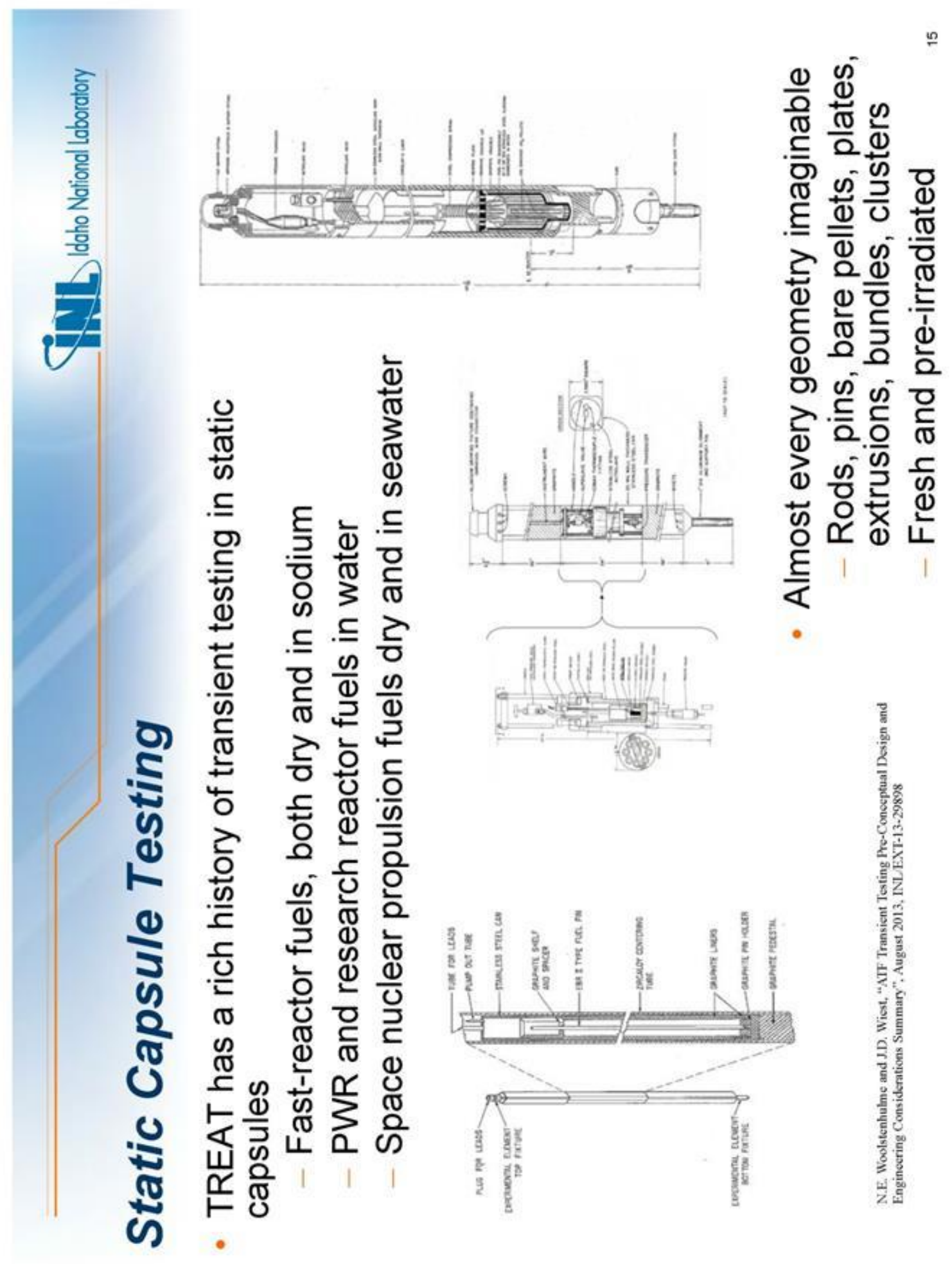




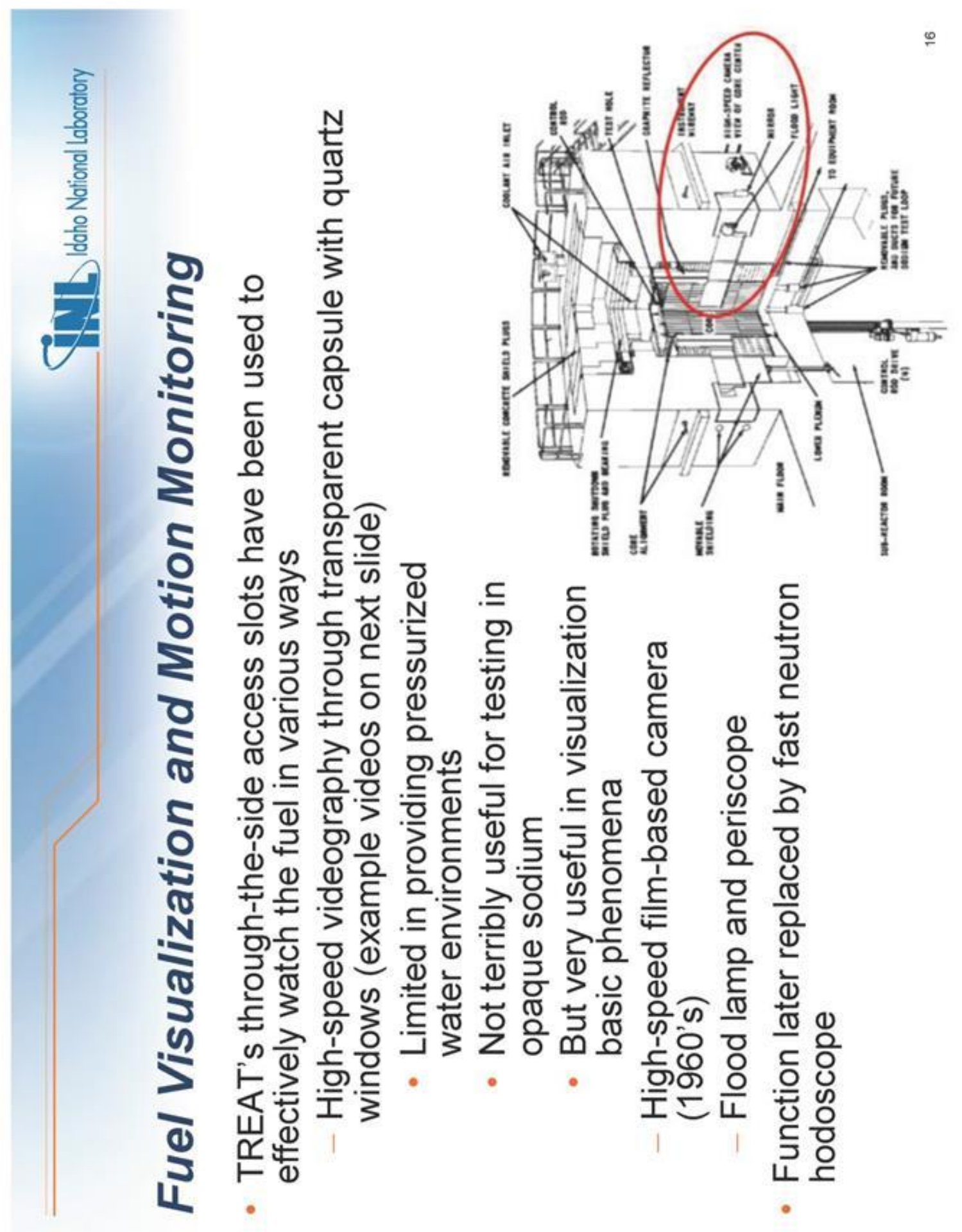




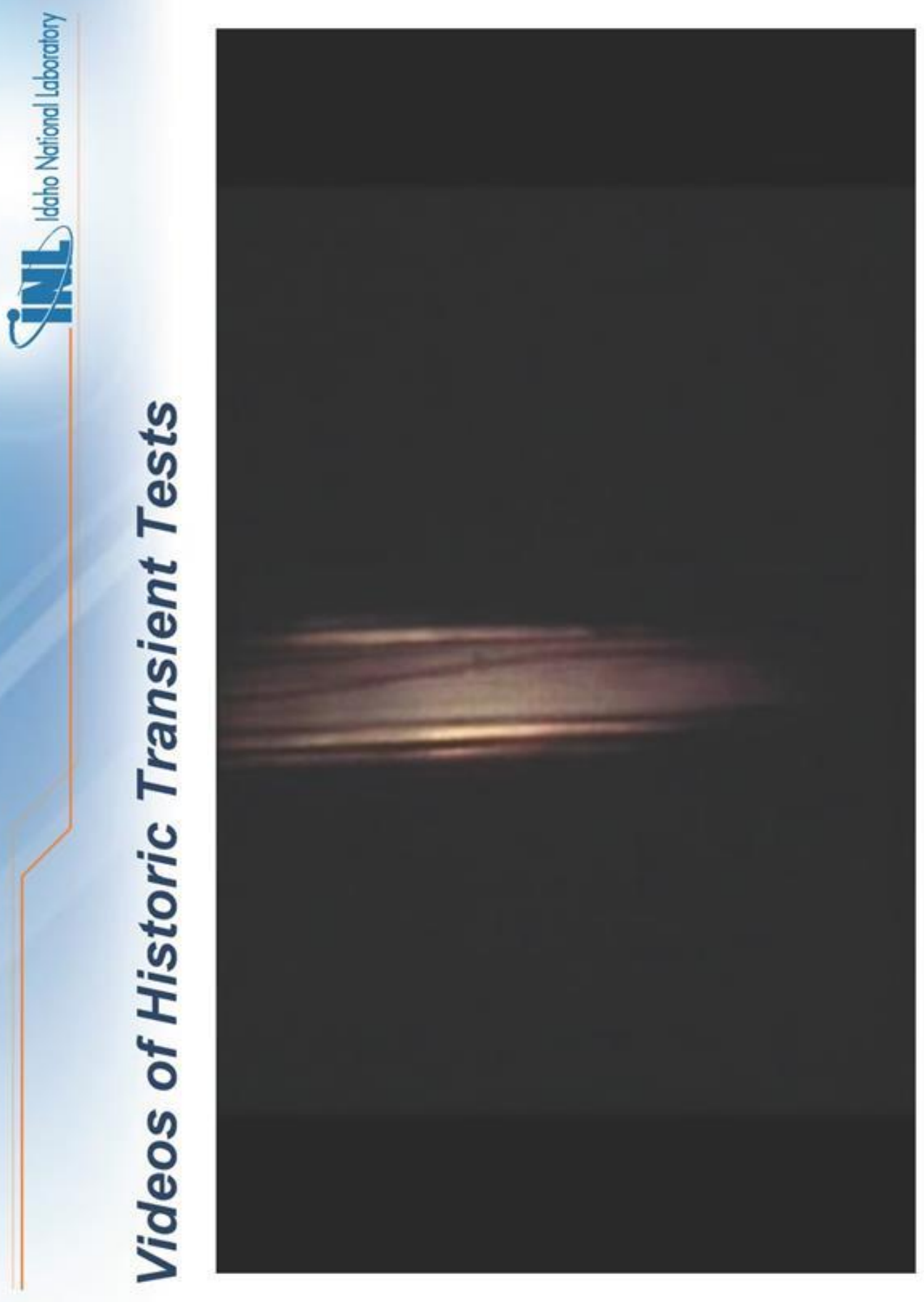




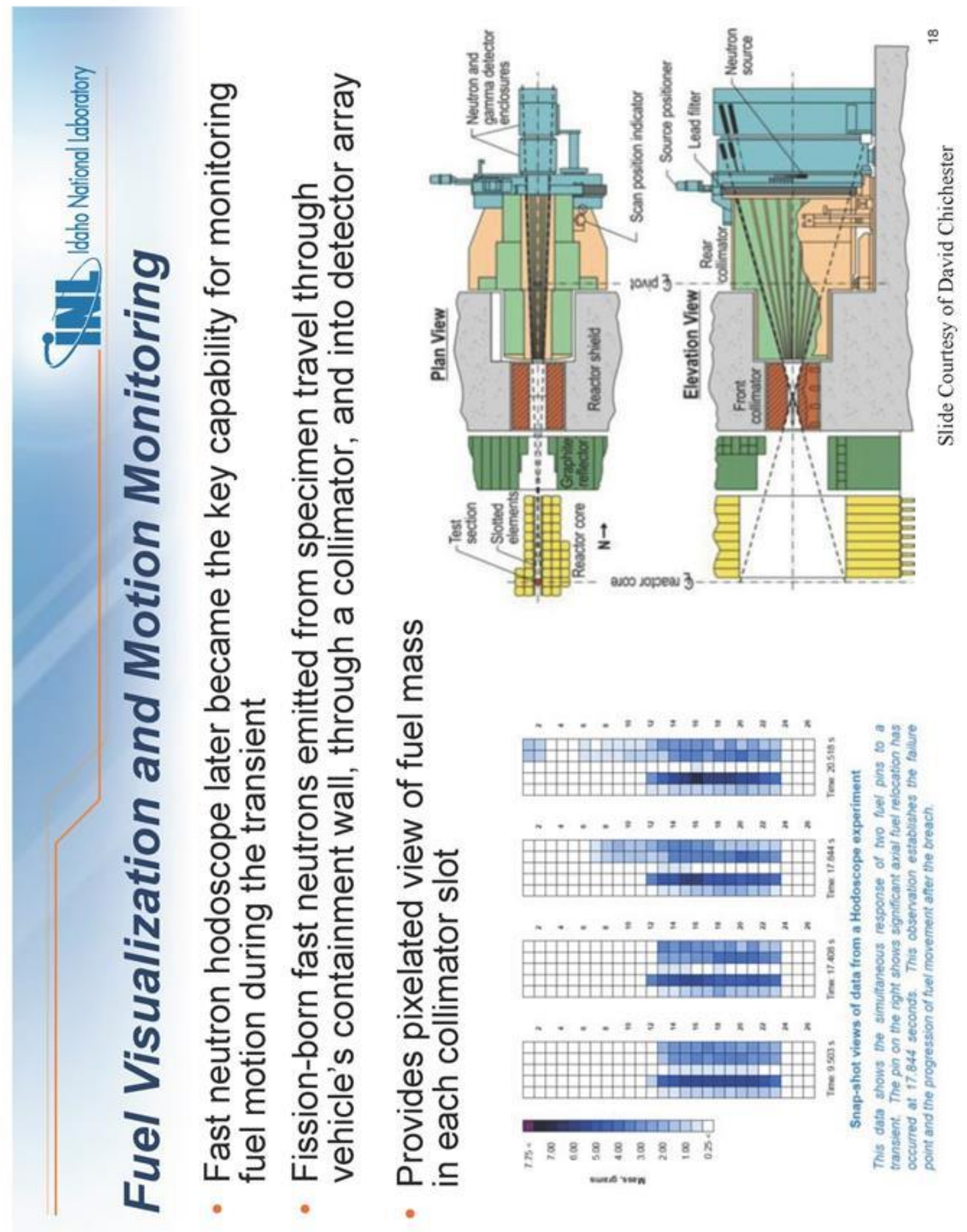




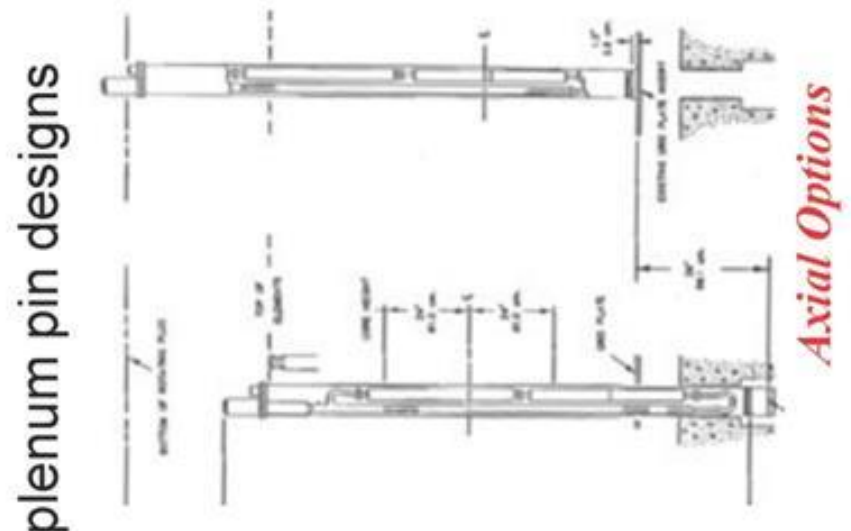

○ N

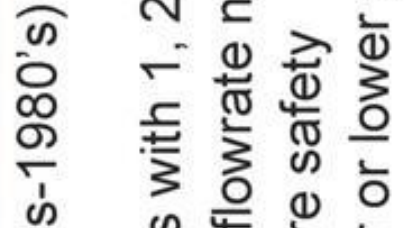

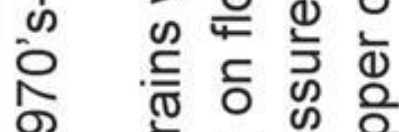

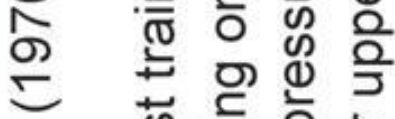

क क ष

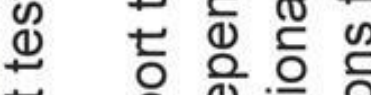

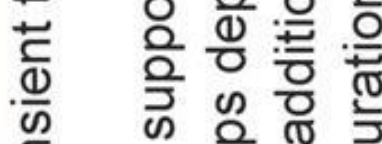

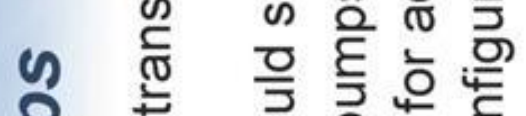

ว

-

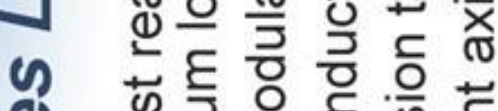

(1) 世

(1) 을윤ำ 뉸

()

$\frac{1}{\mathbb{1}} \frac{\pi}{\square}$
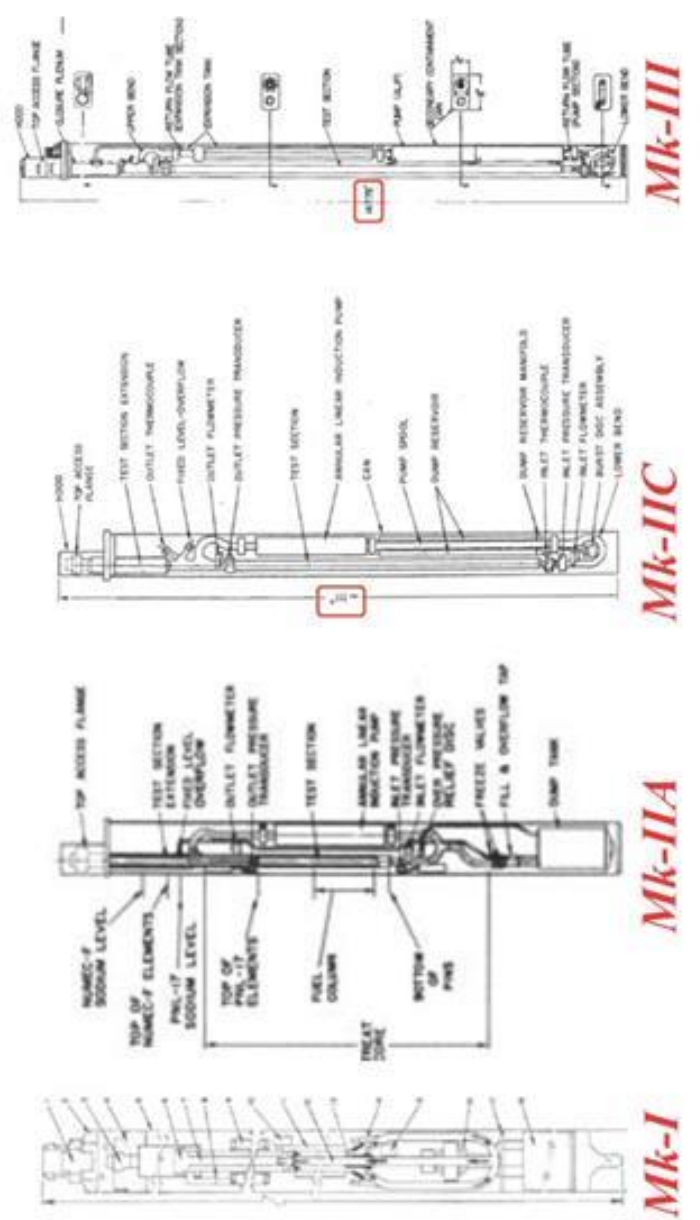

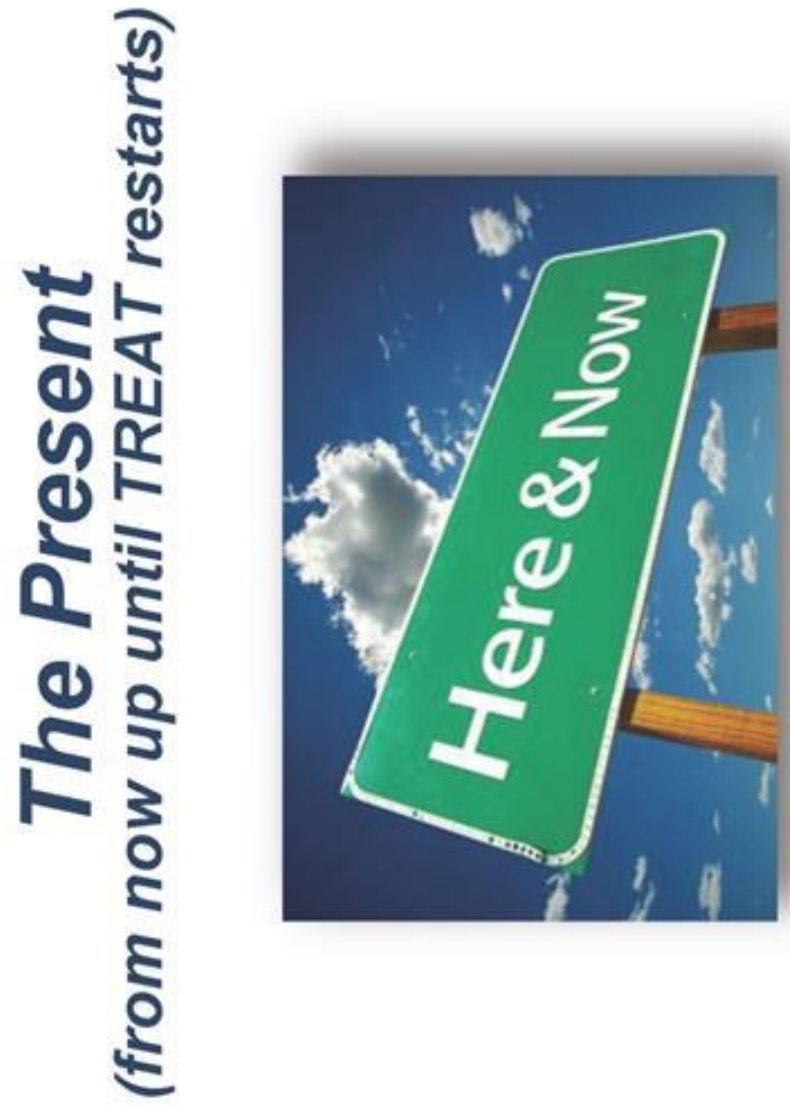


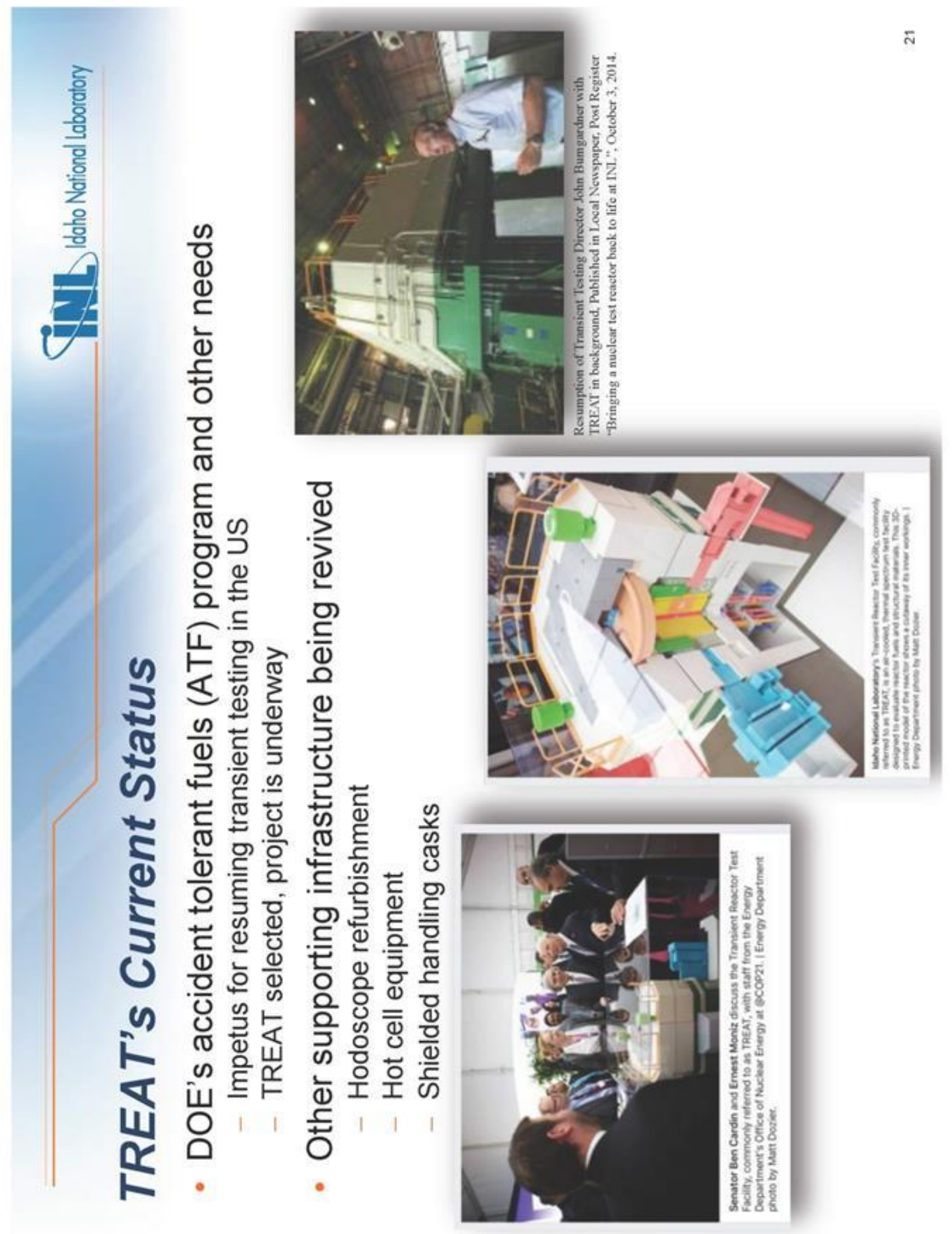




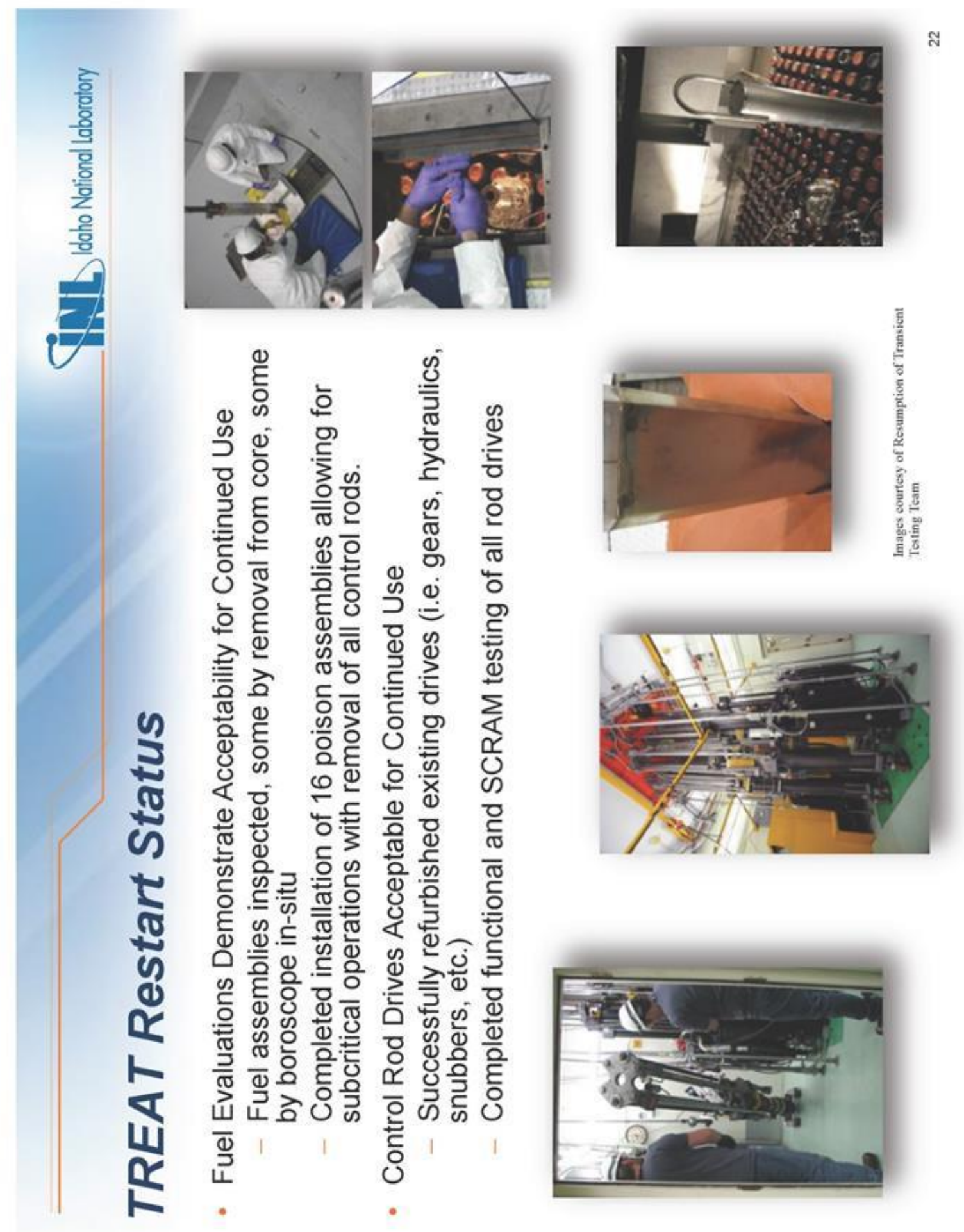




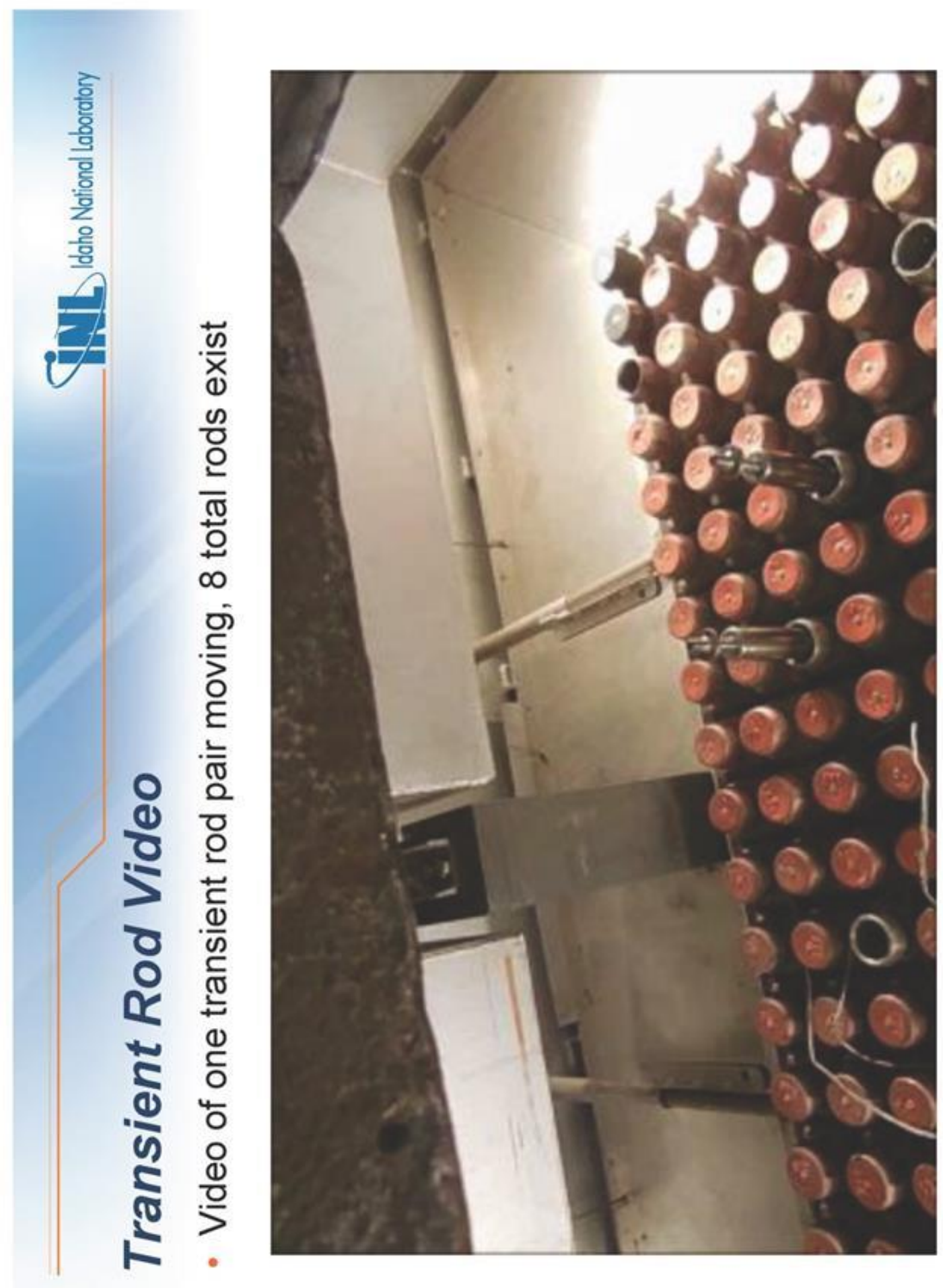

ก 


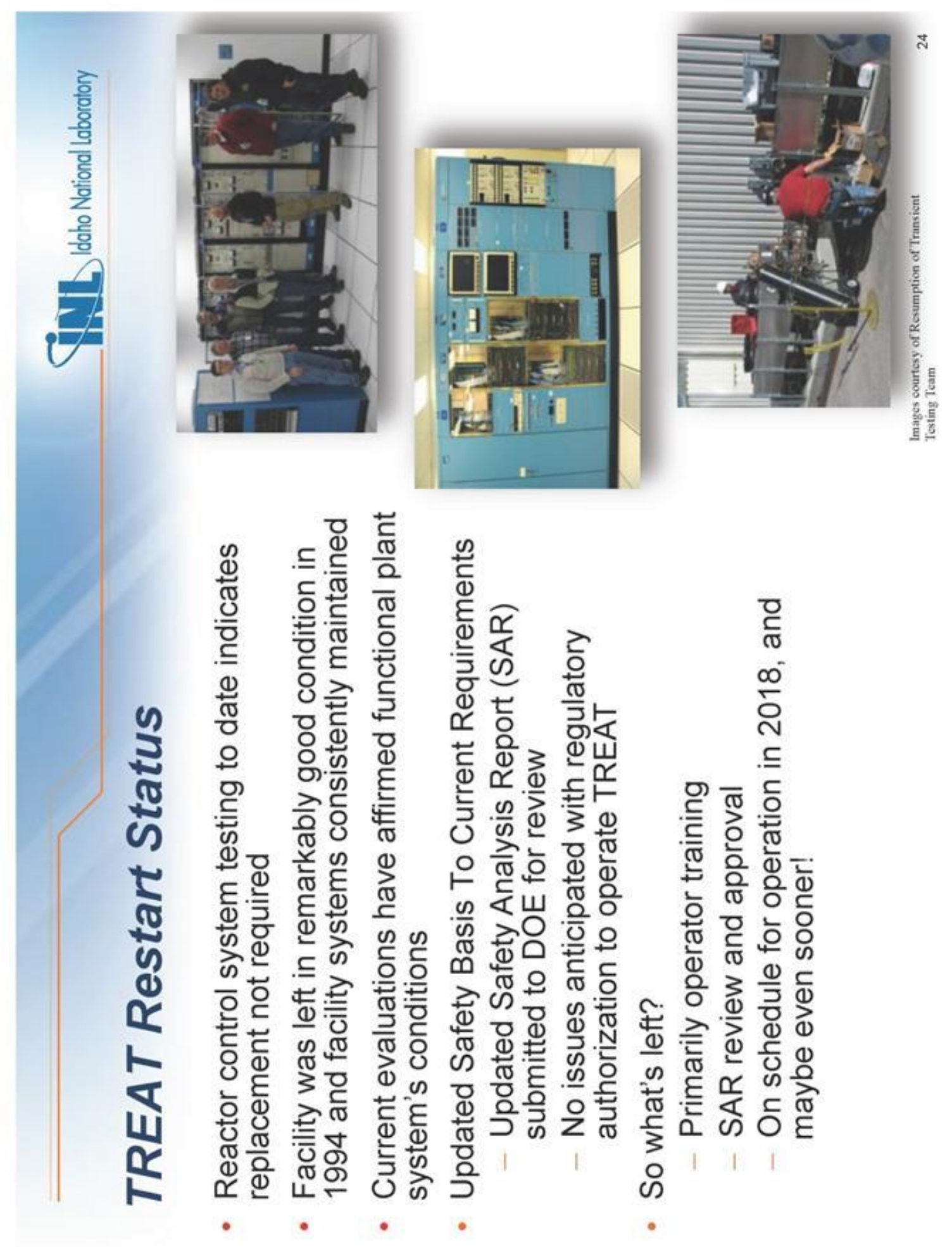




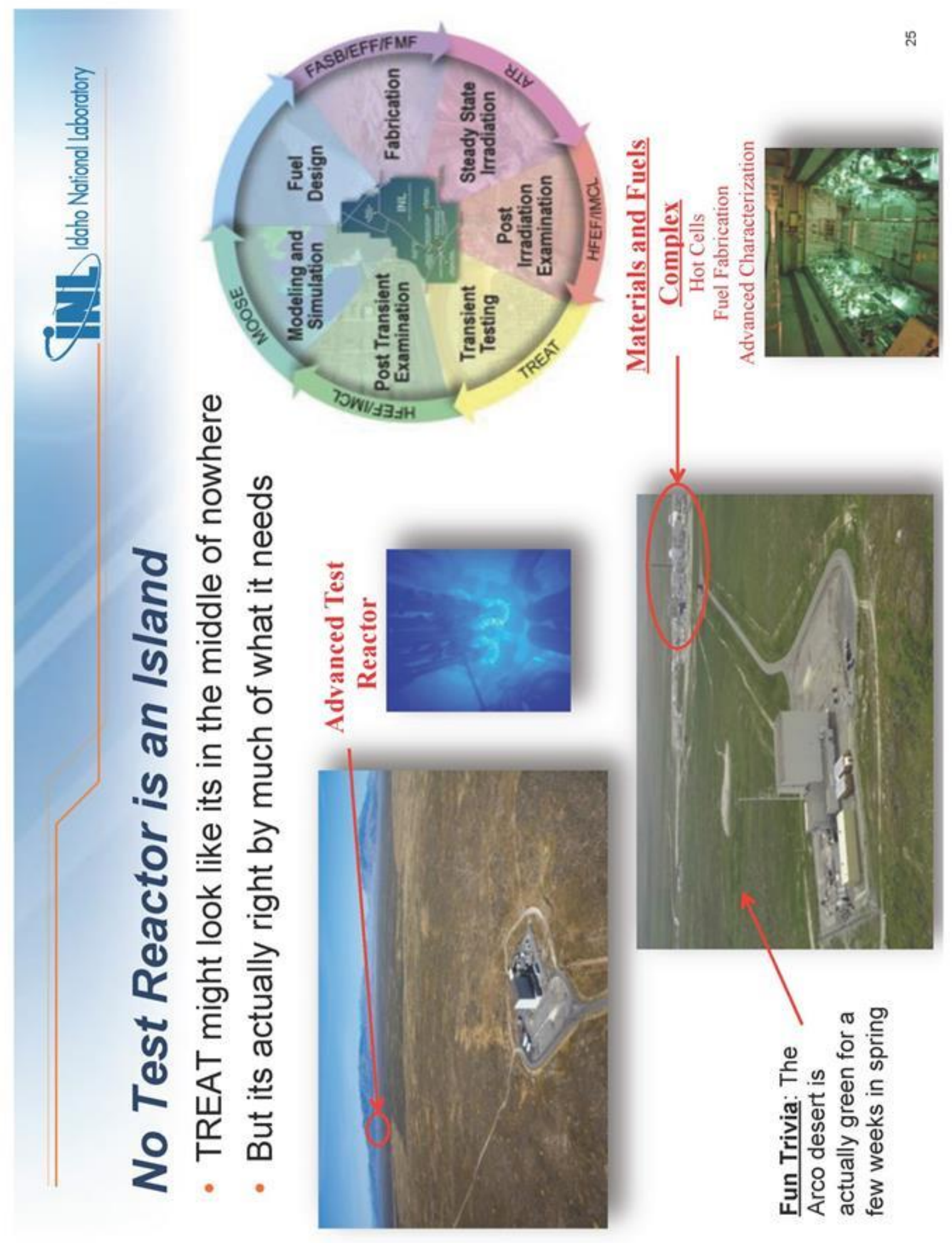




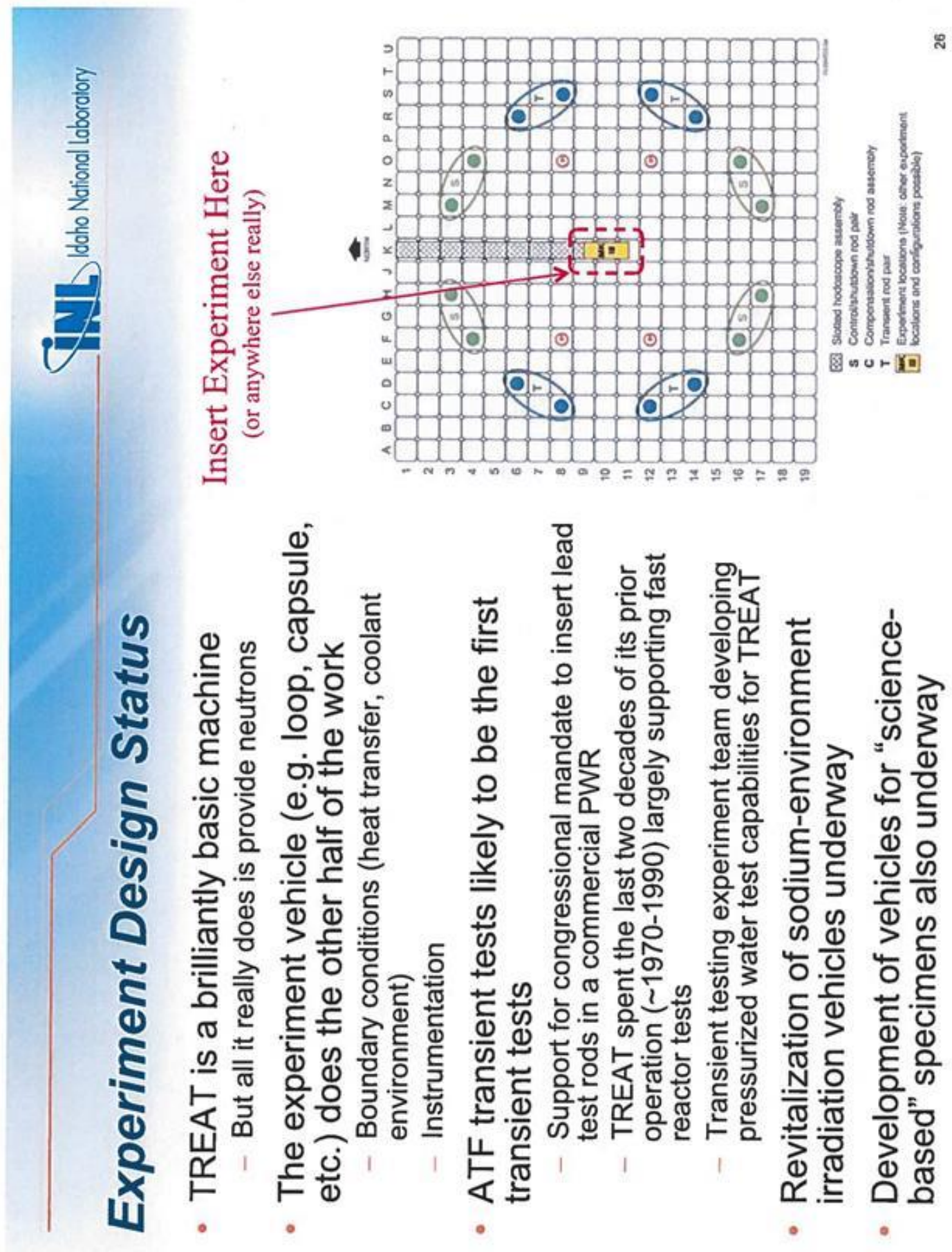




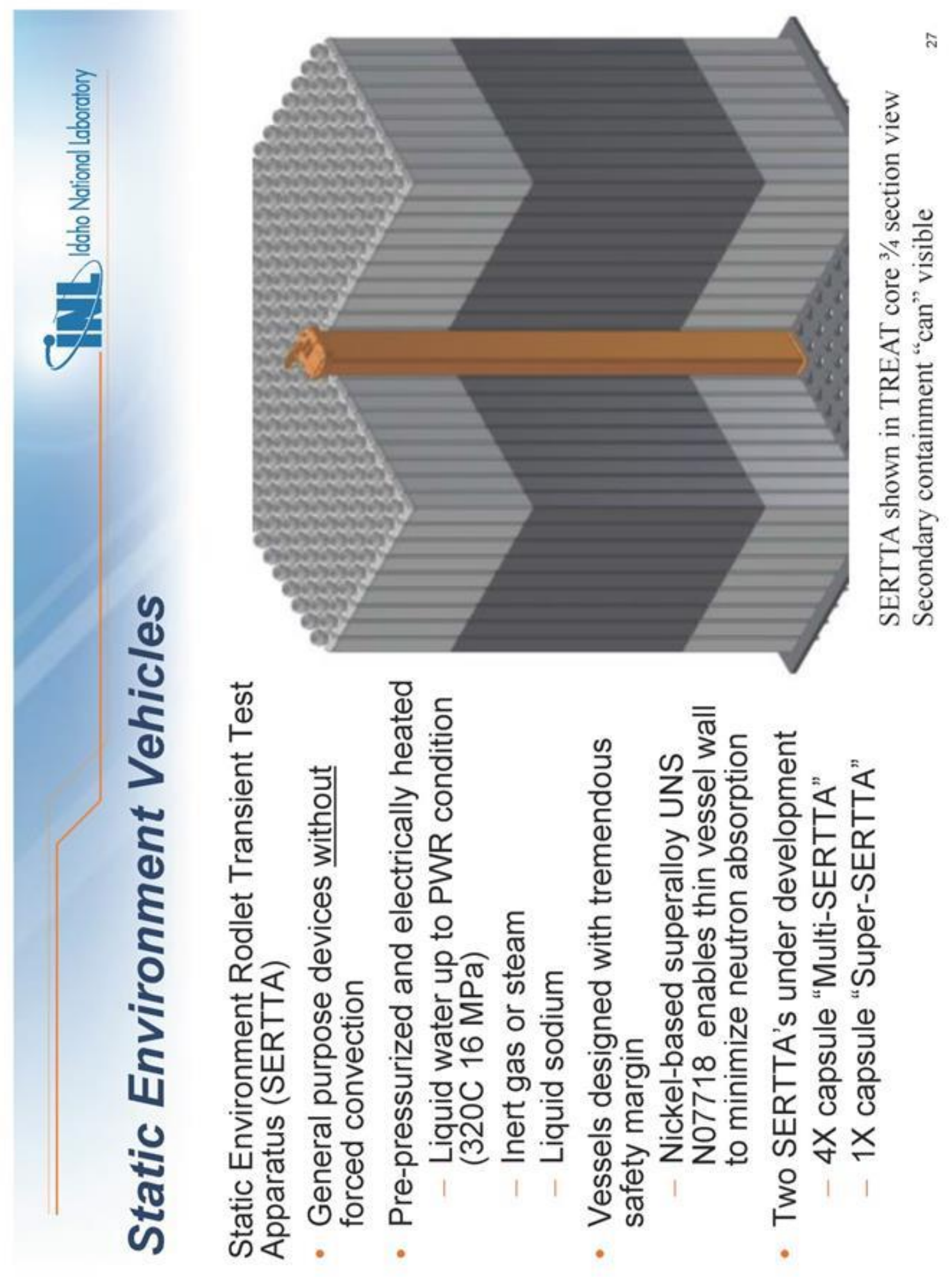




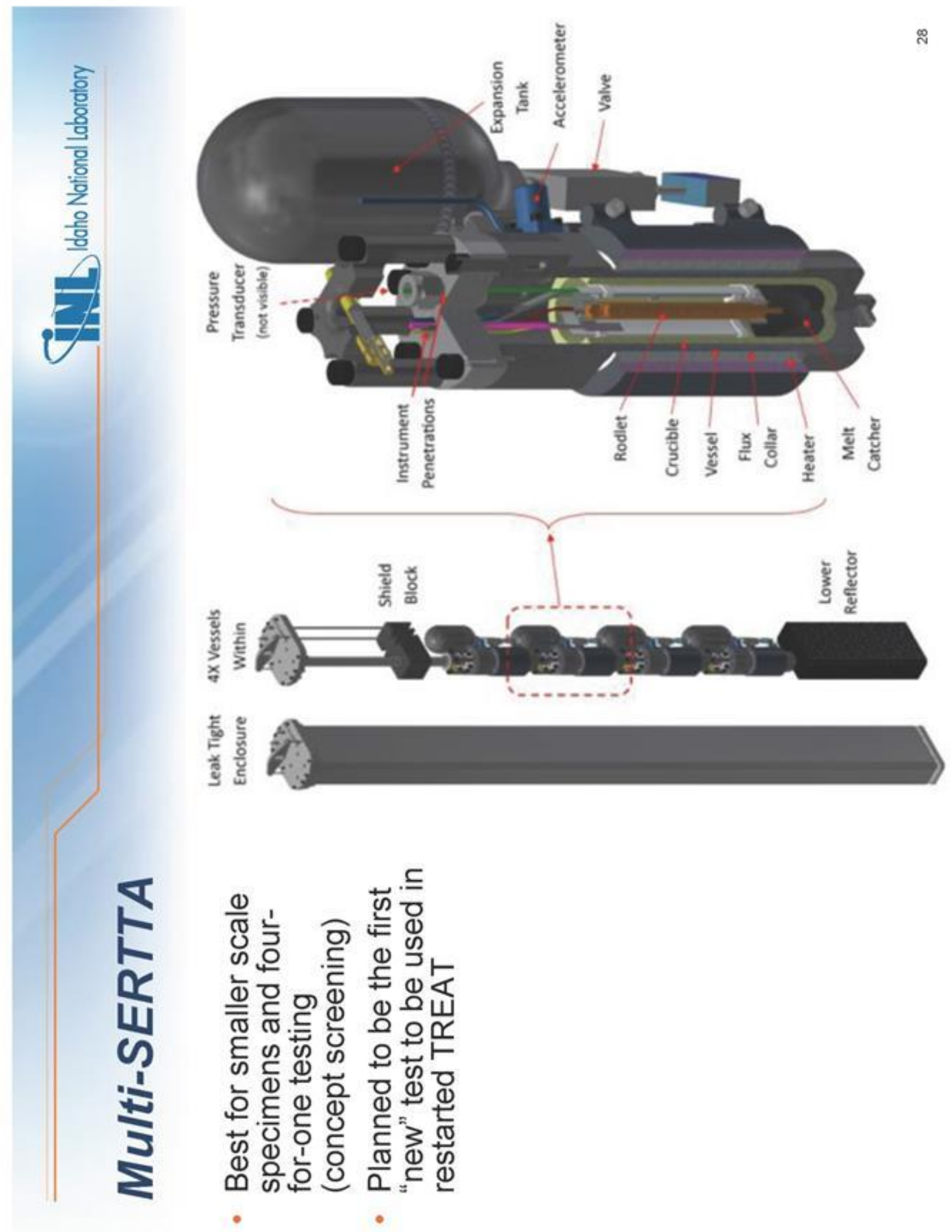




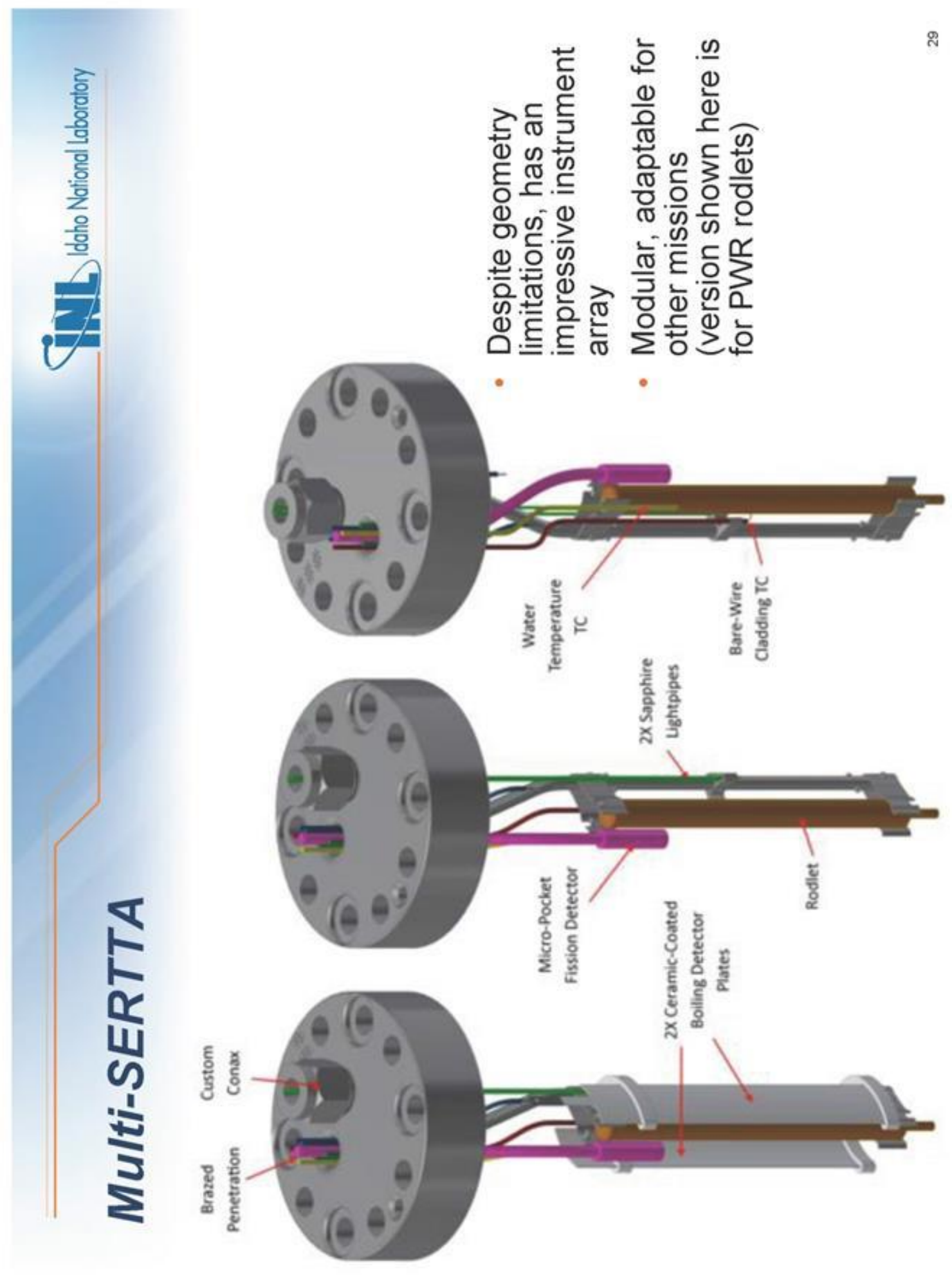



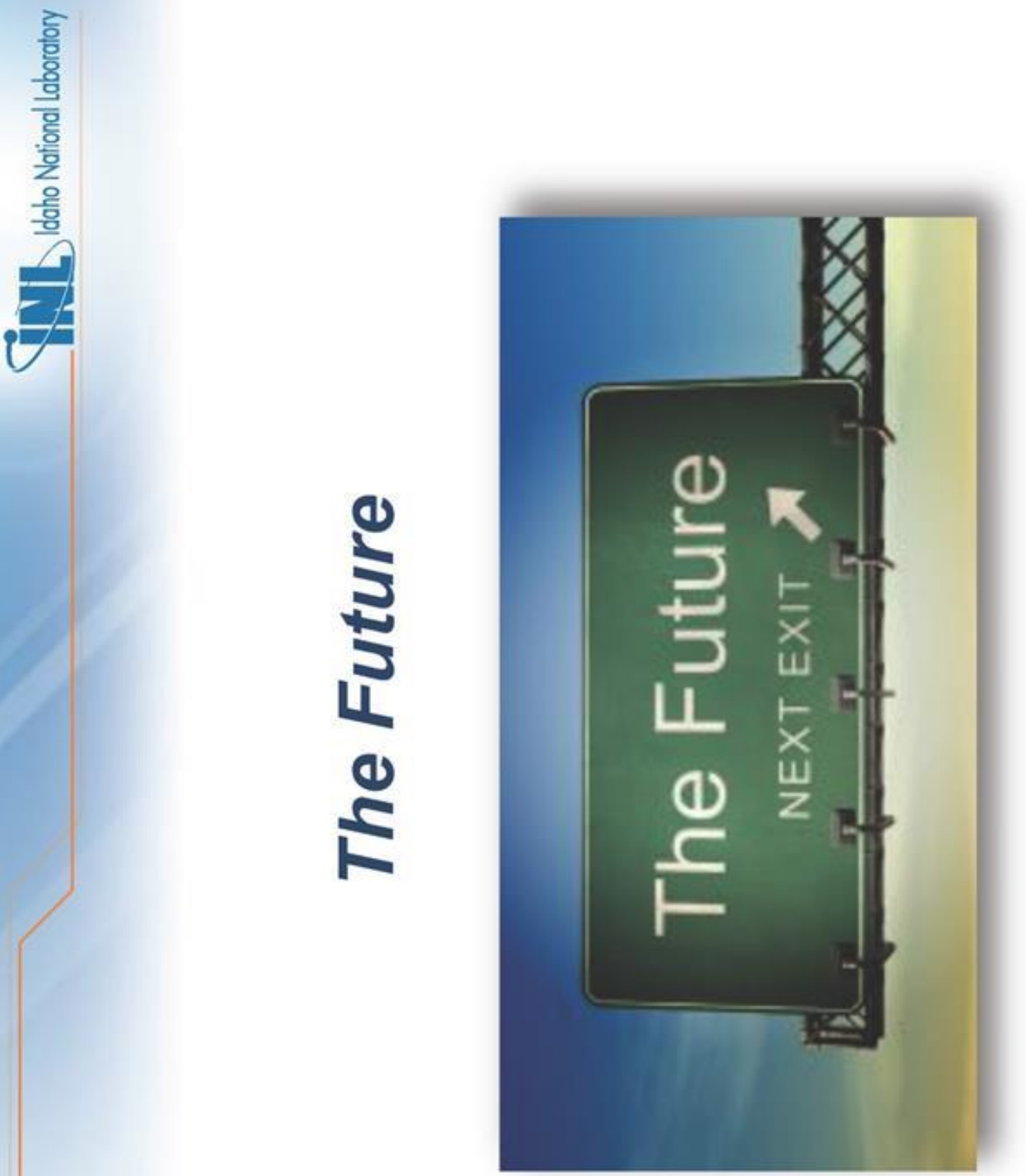

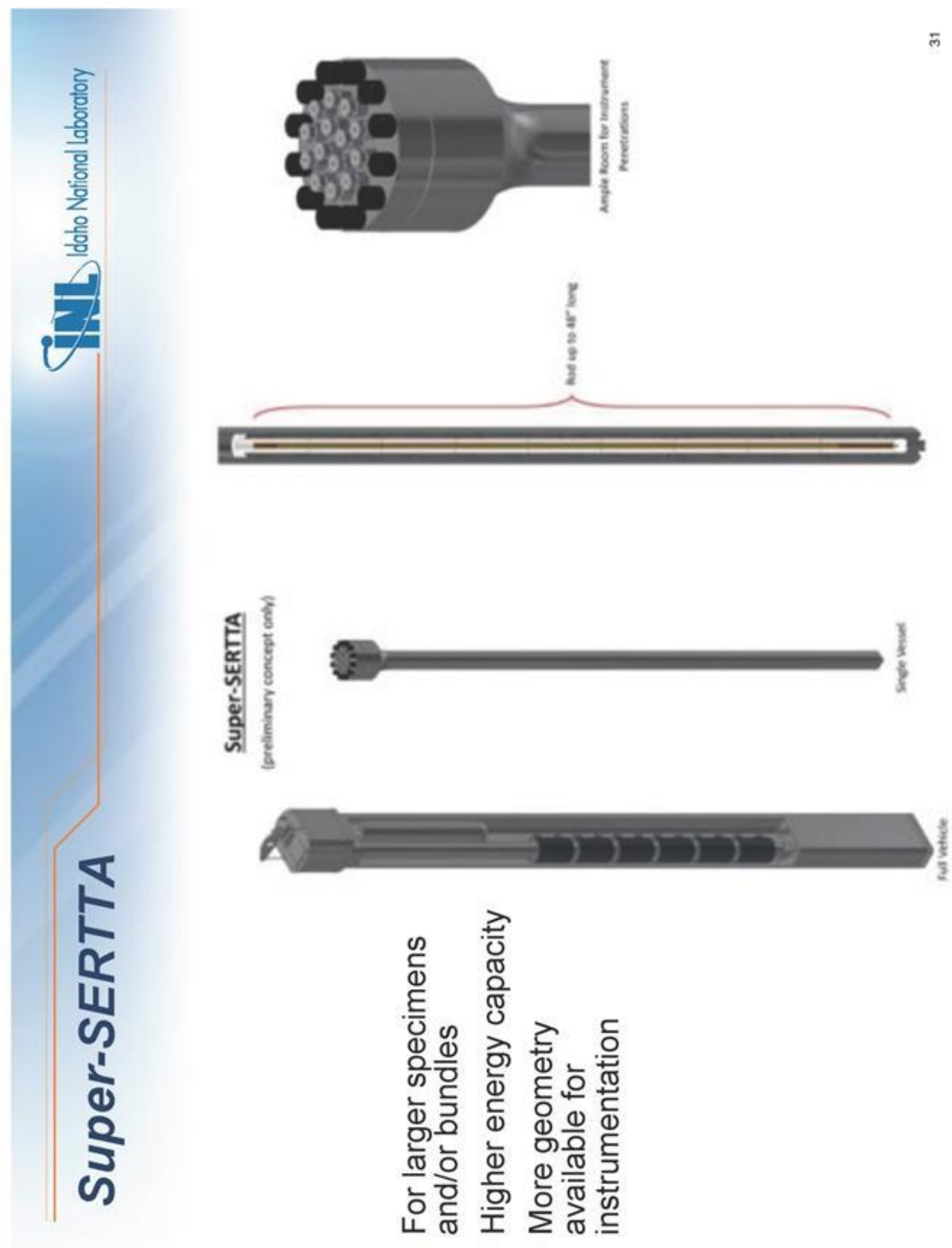

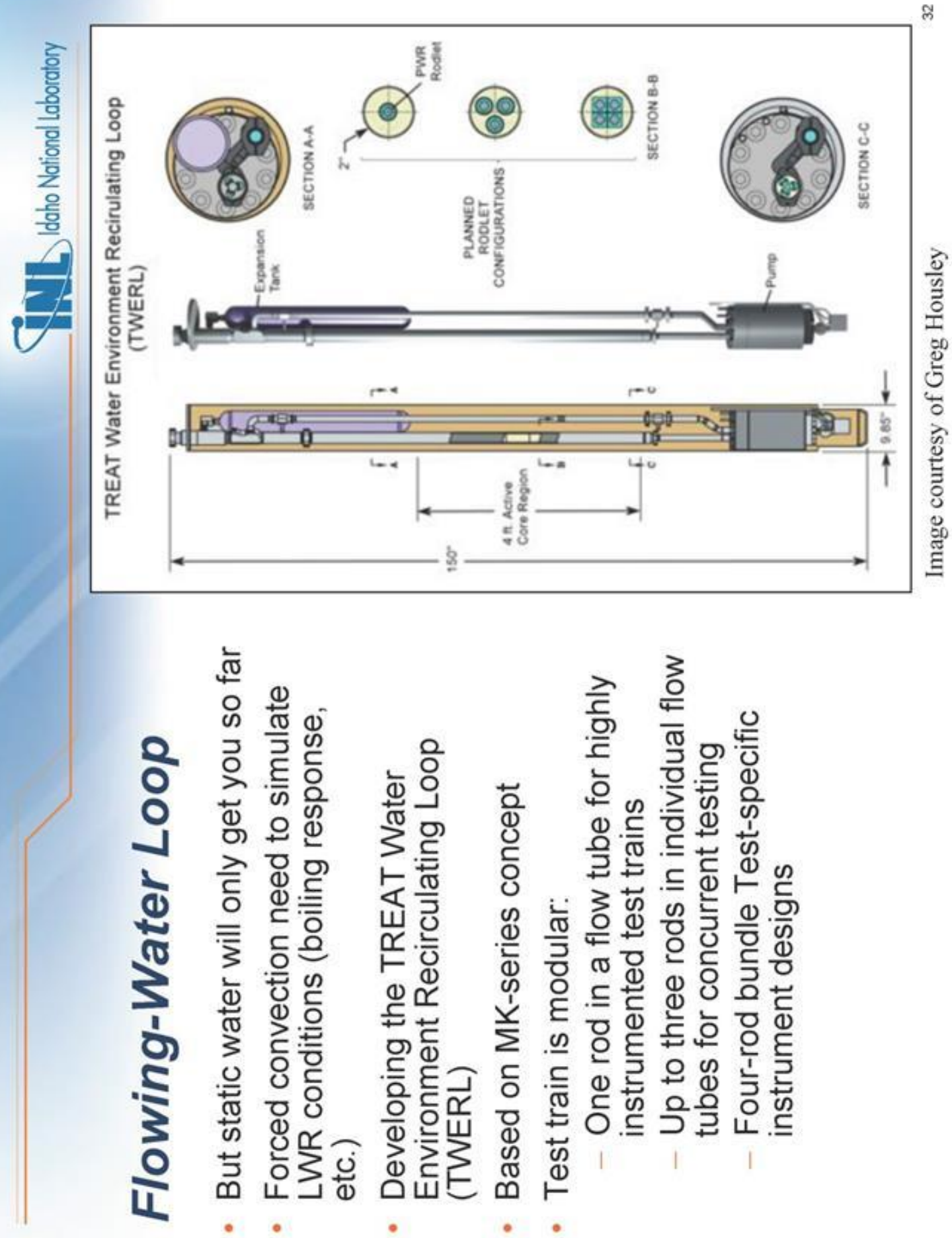

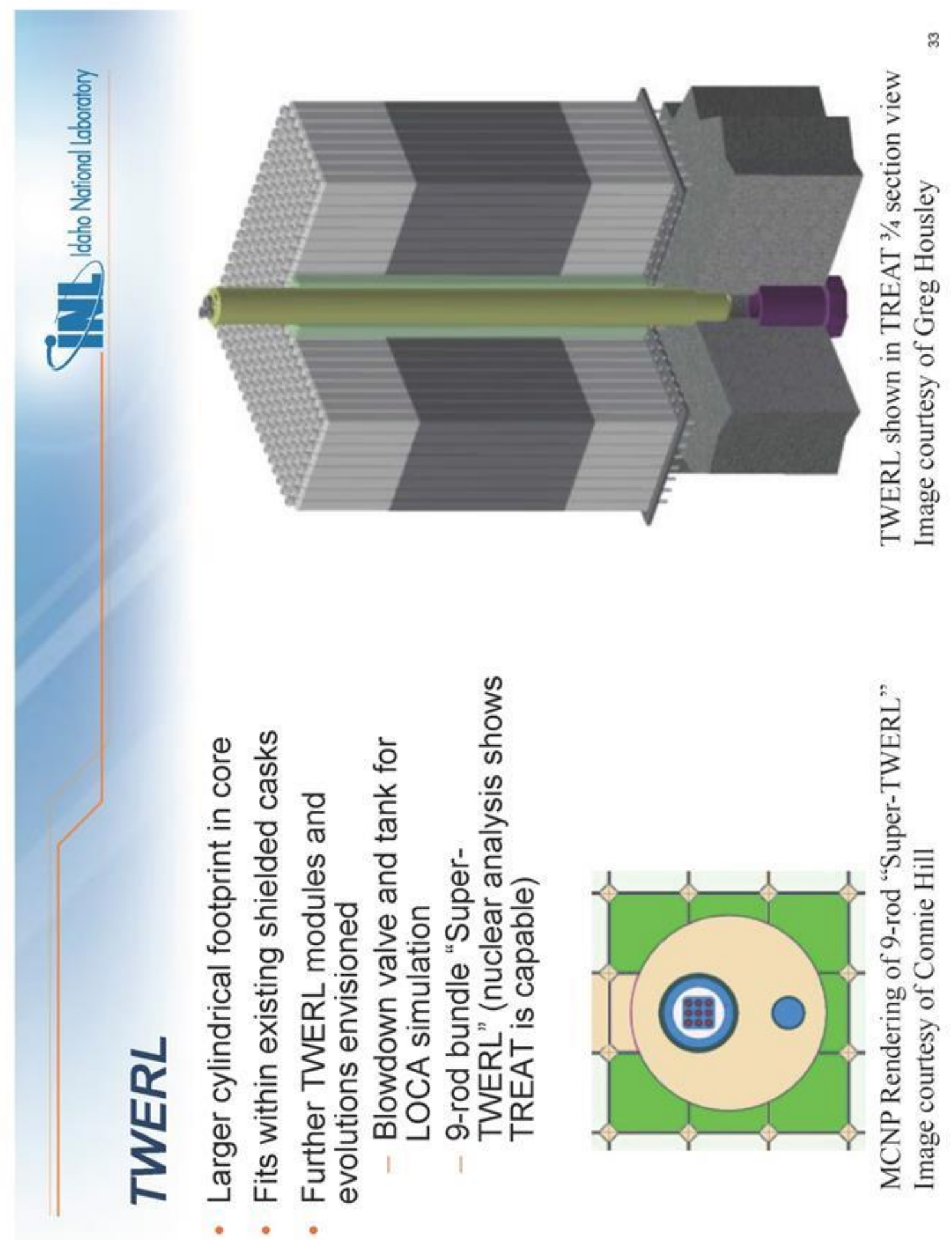


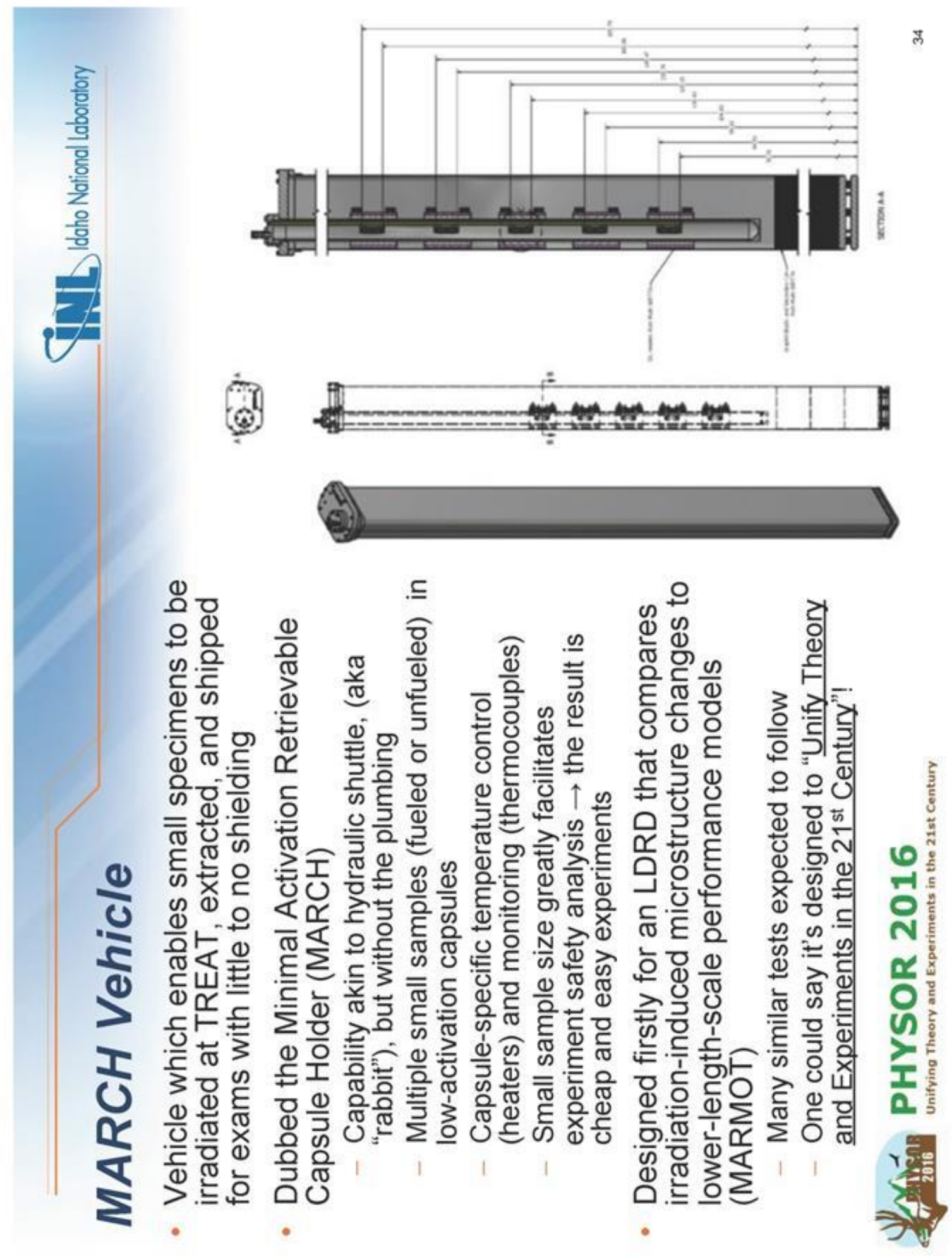




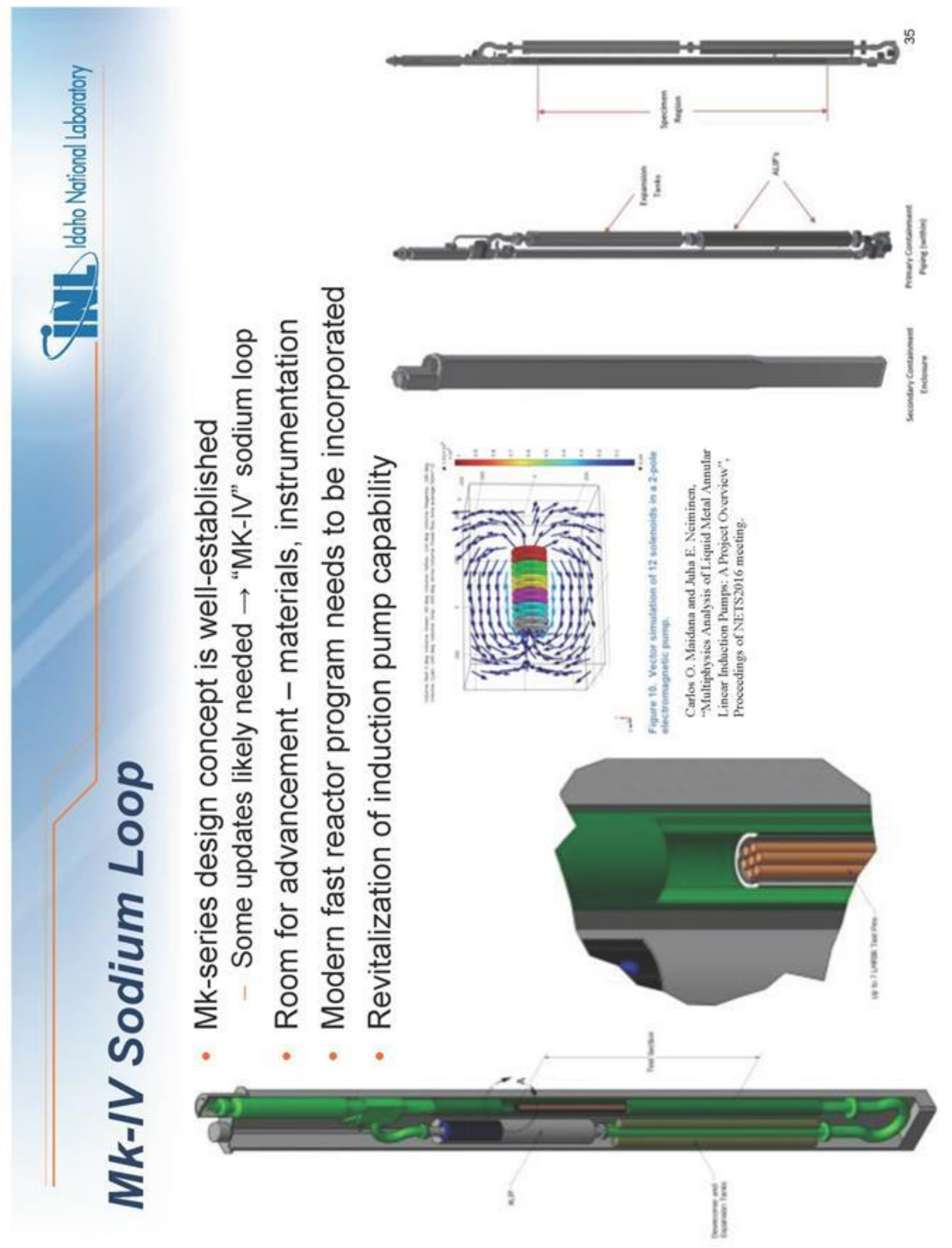




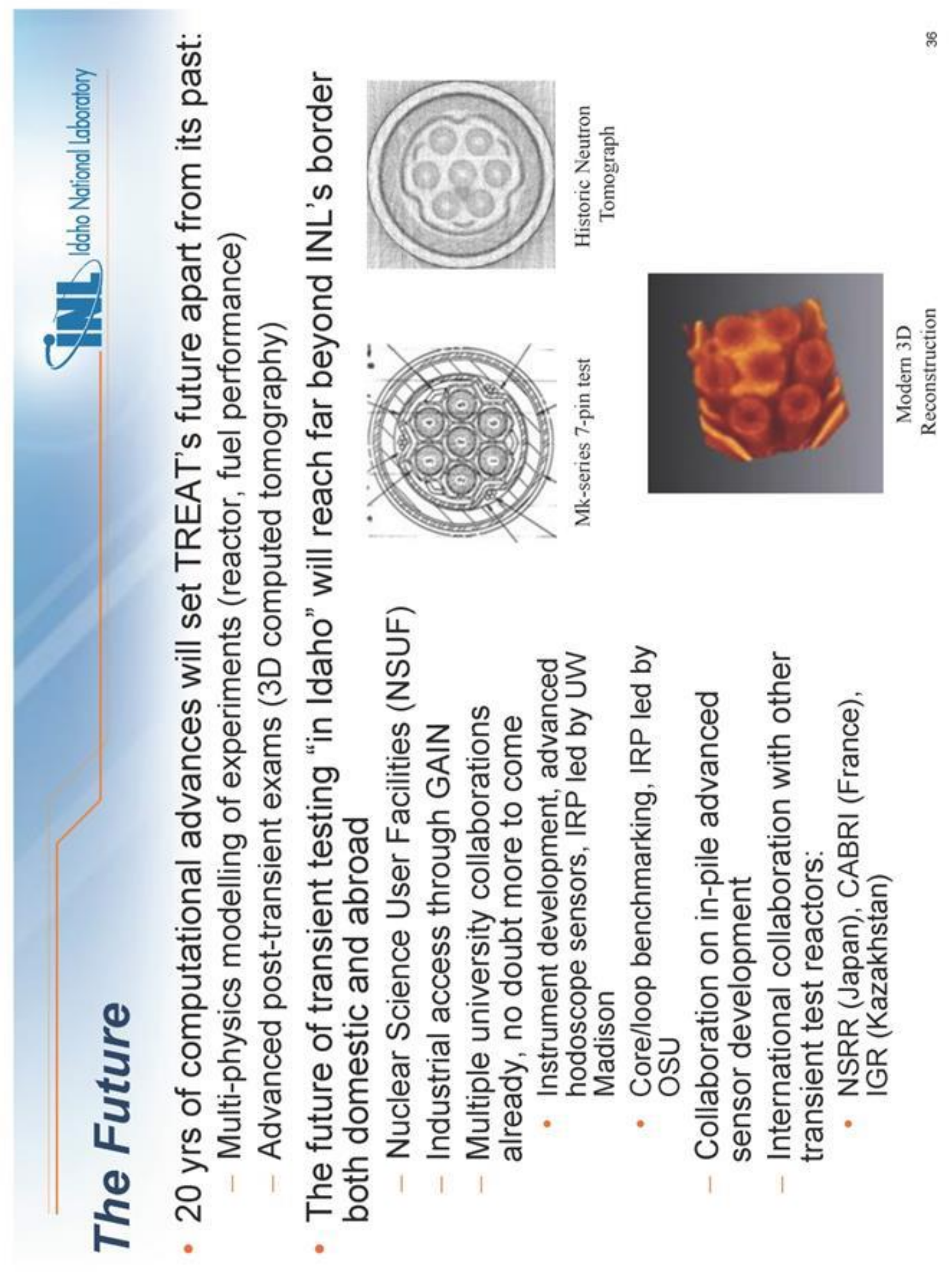




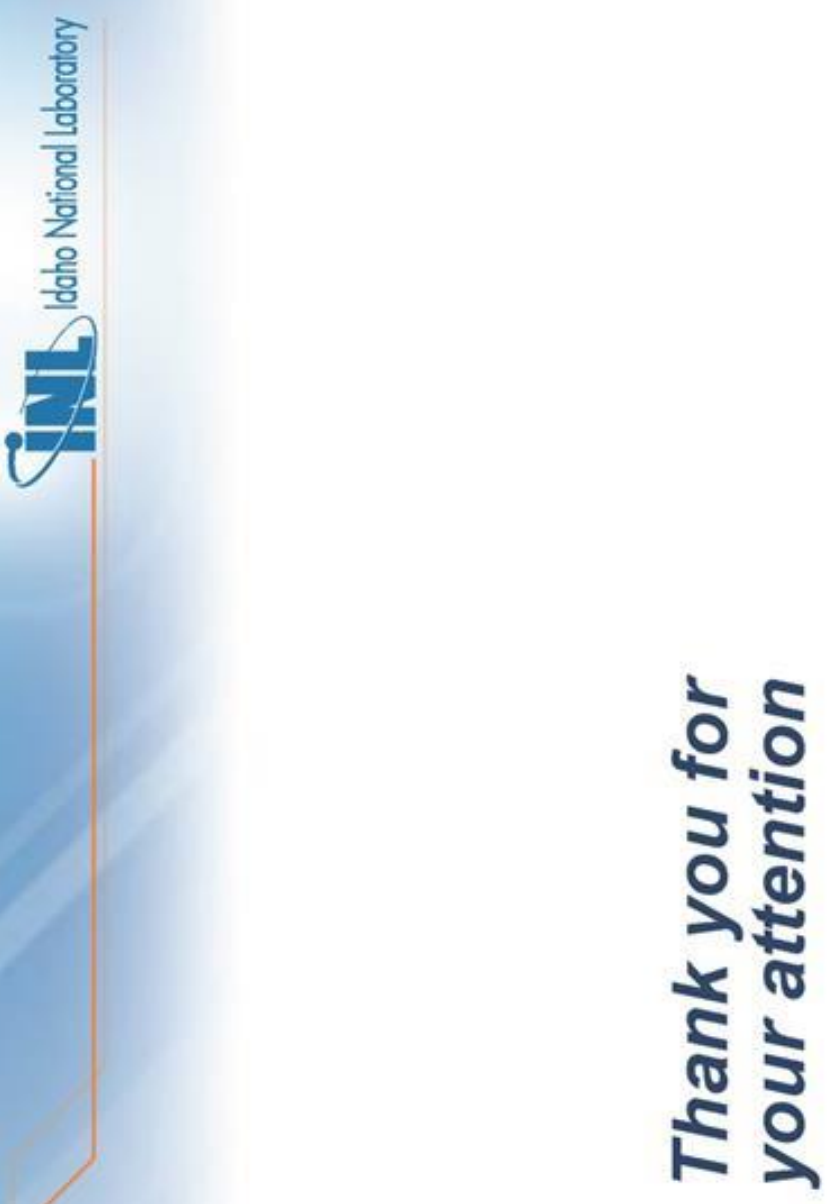




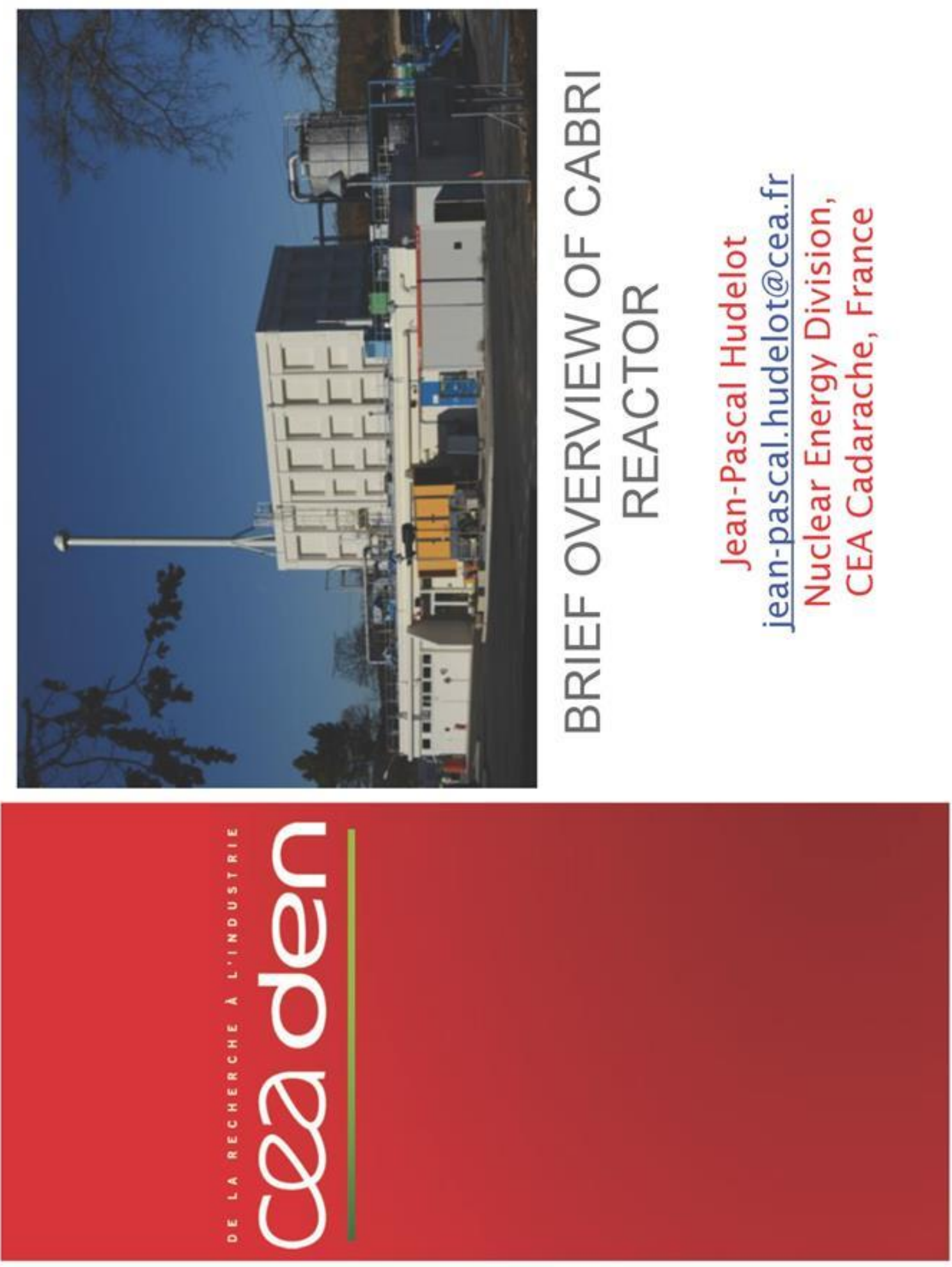



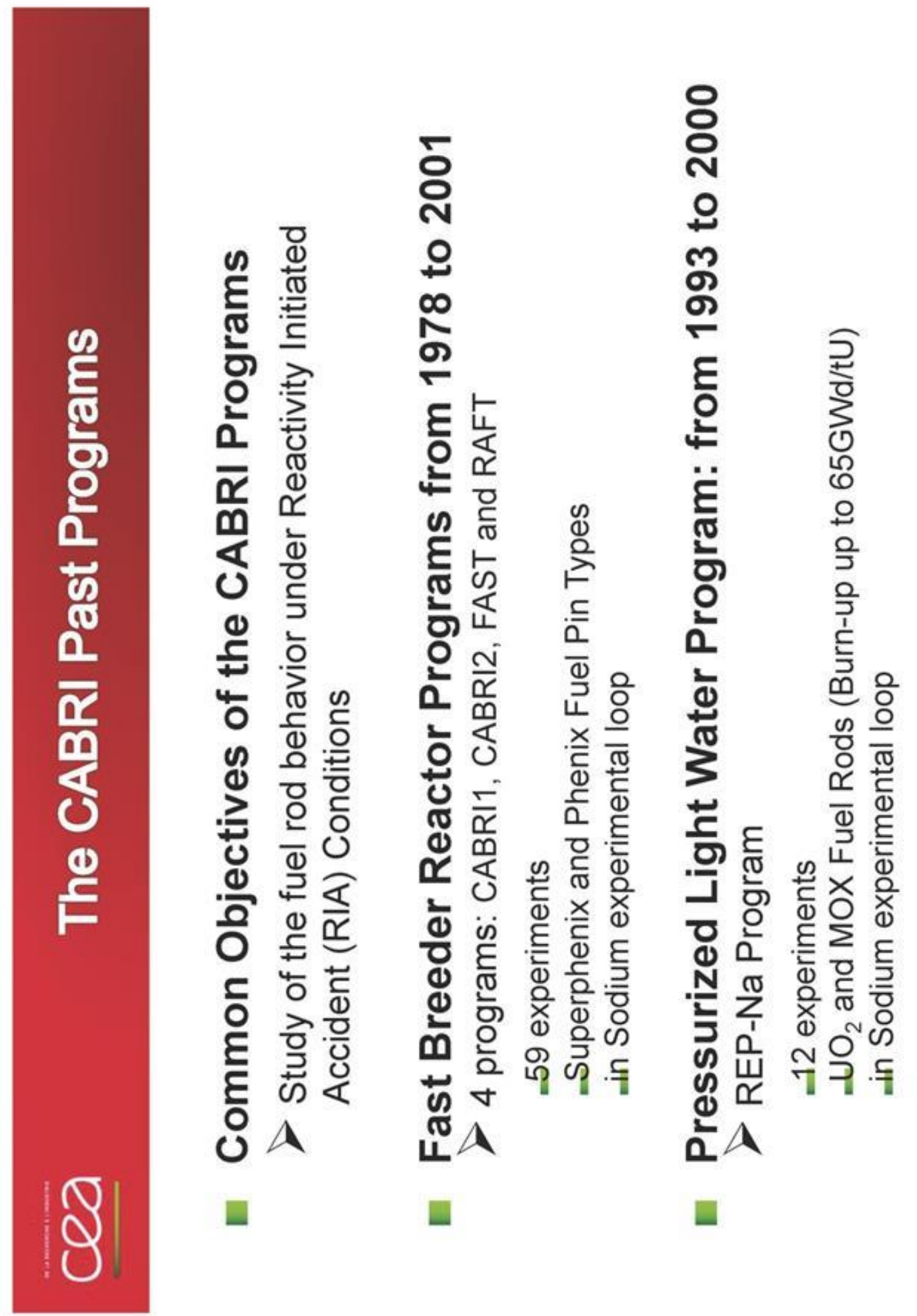


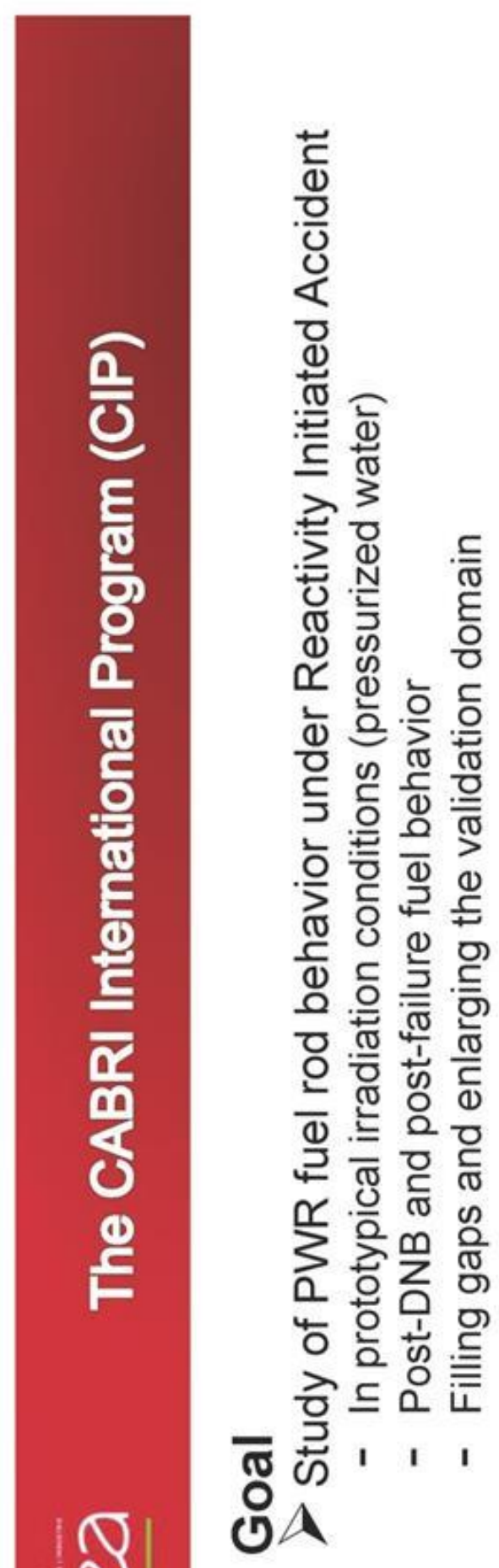

兵

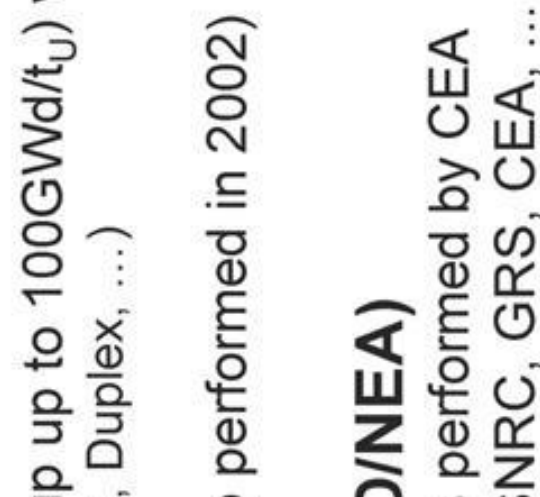

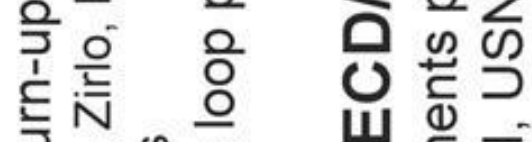

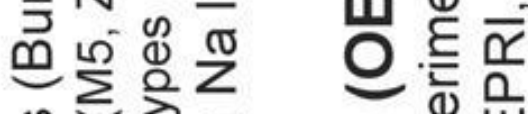

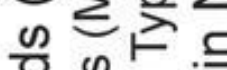

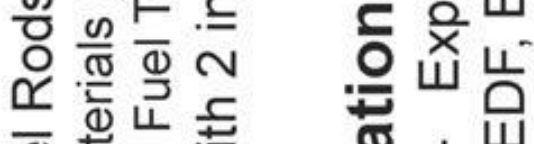

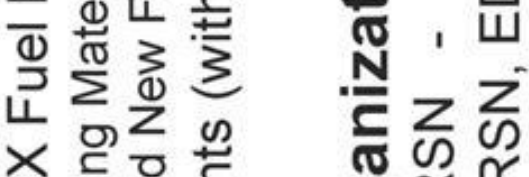

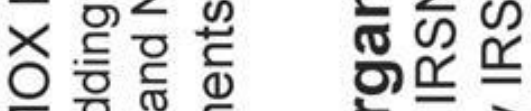

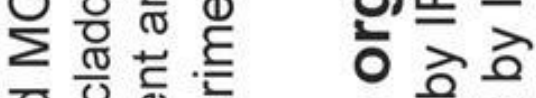

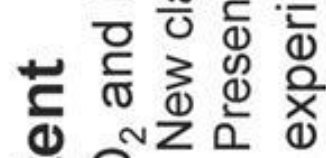

등 웡 임

过 곤

음 누

는

0

0

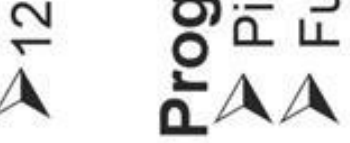



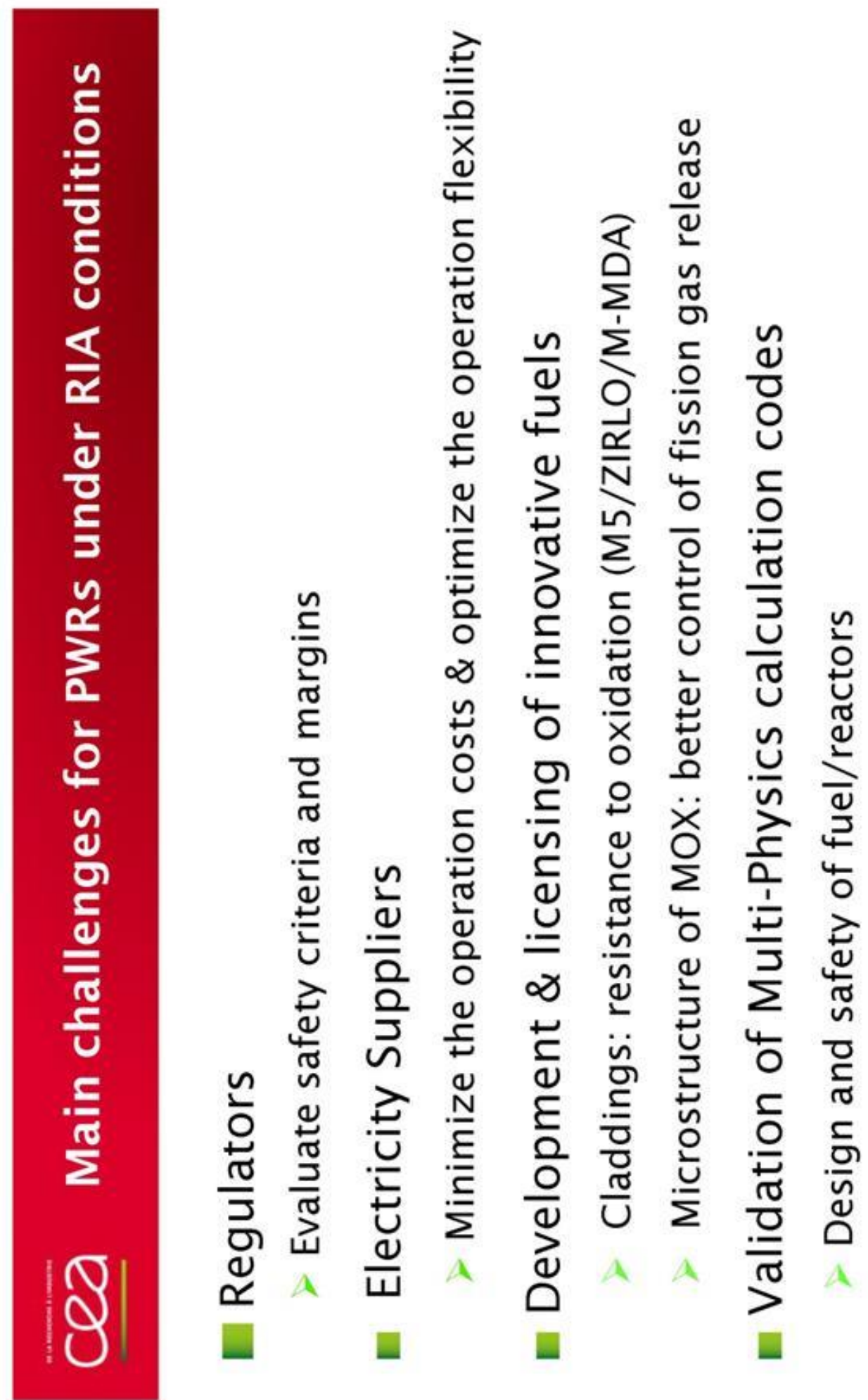


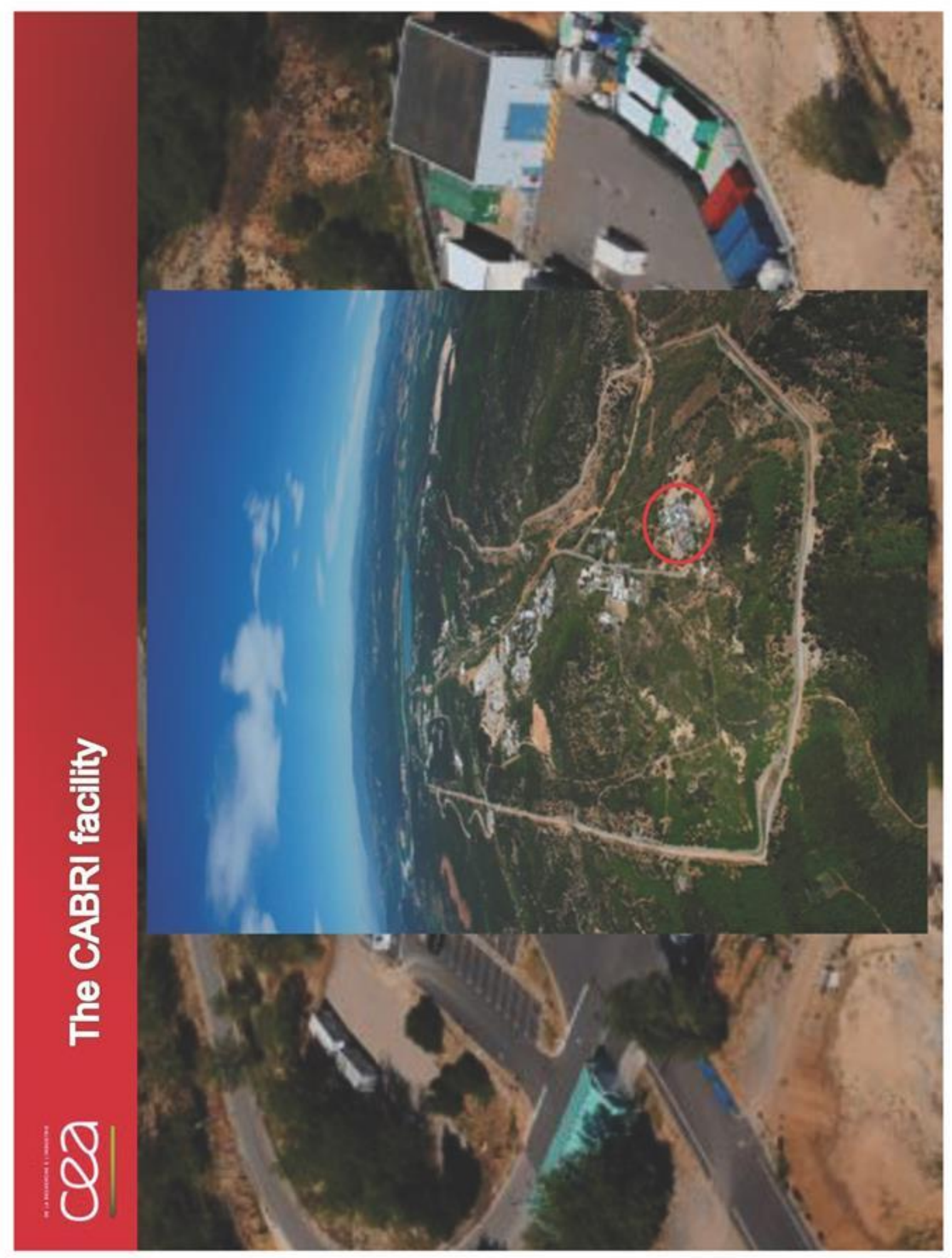



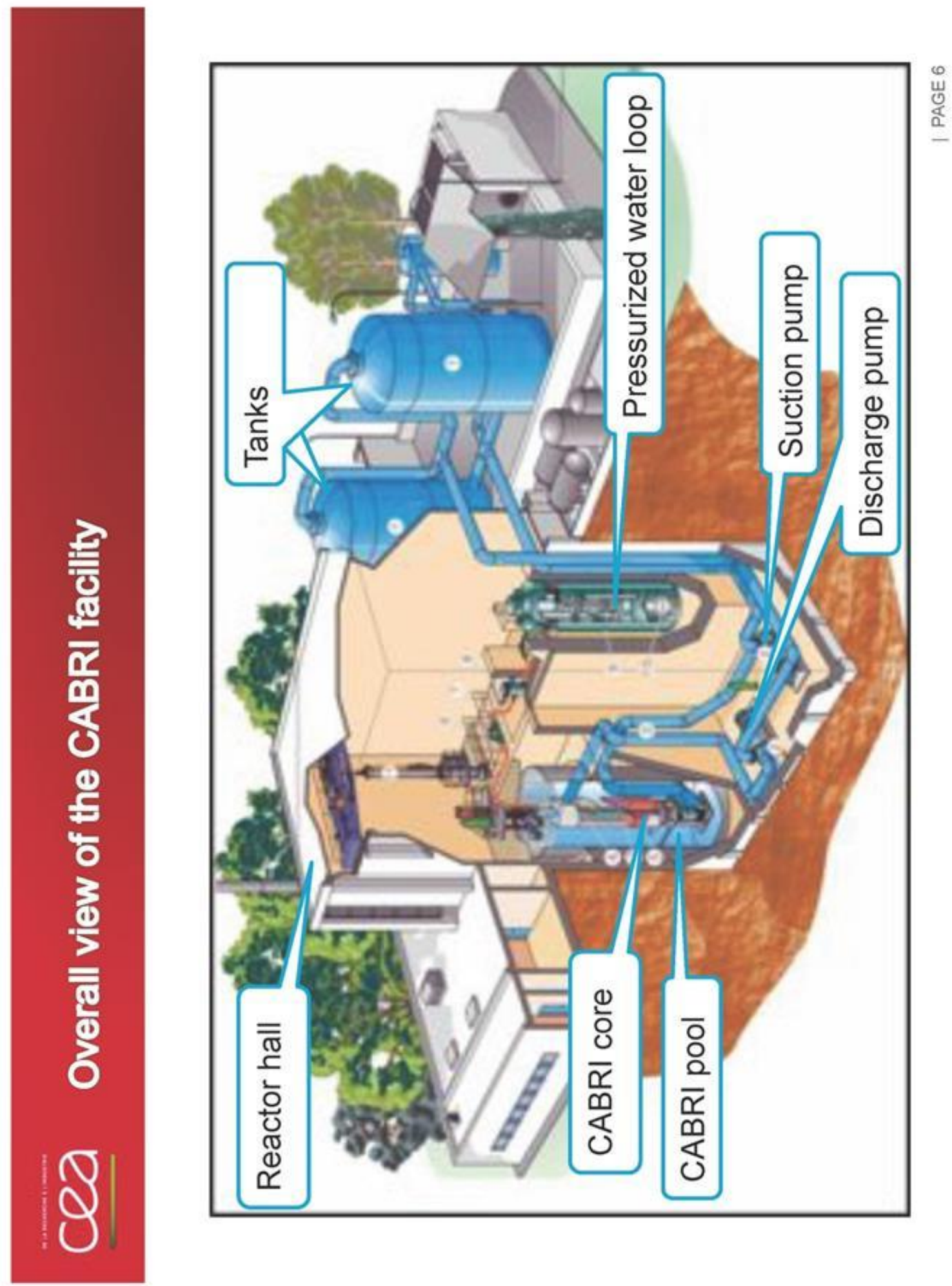

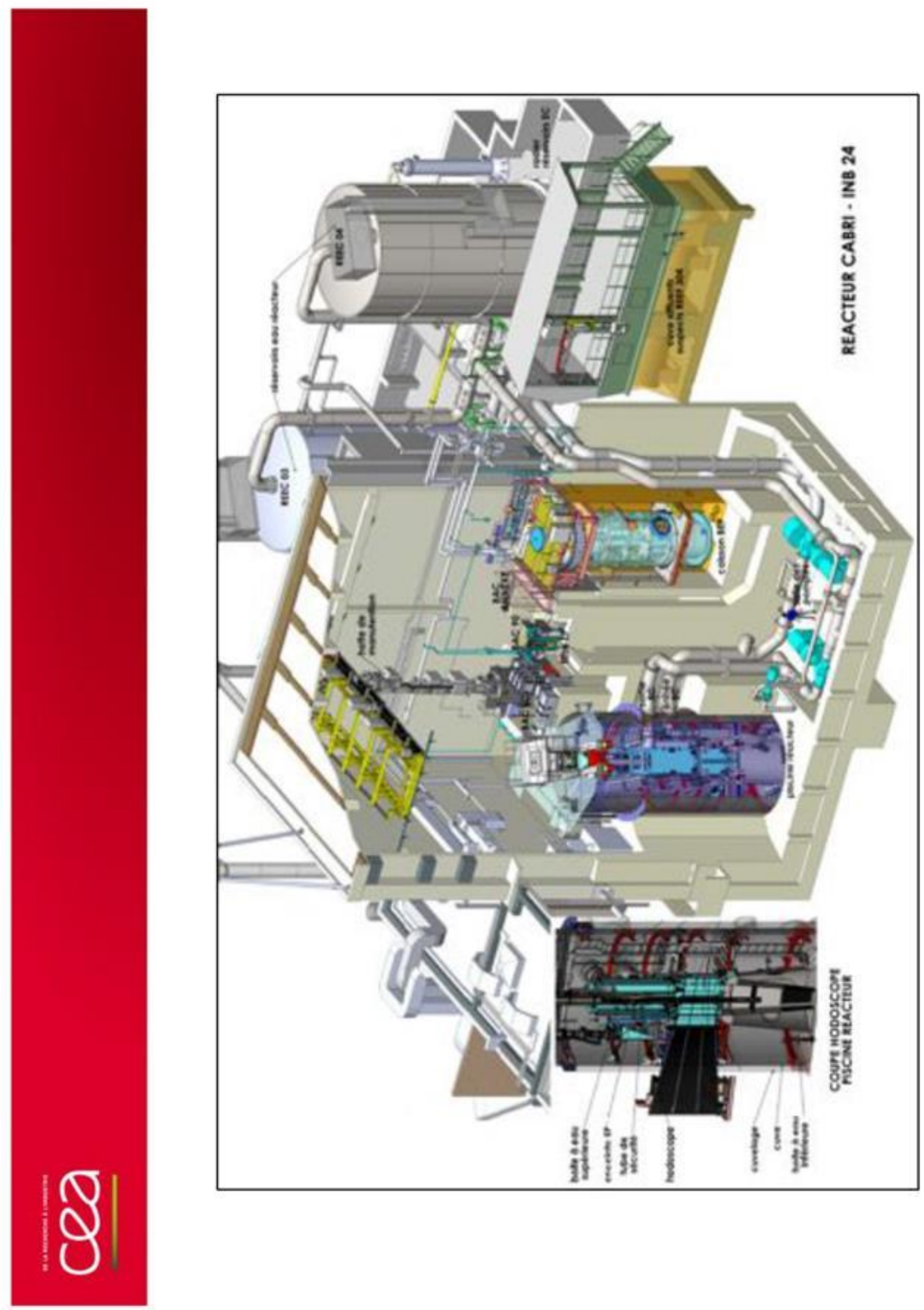

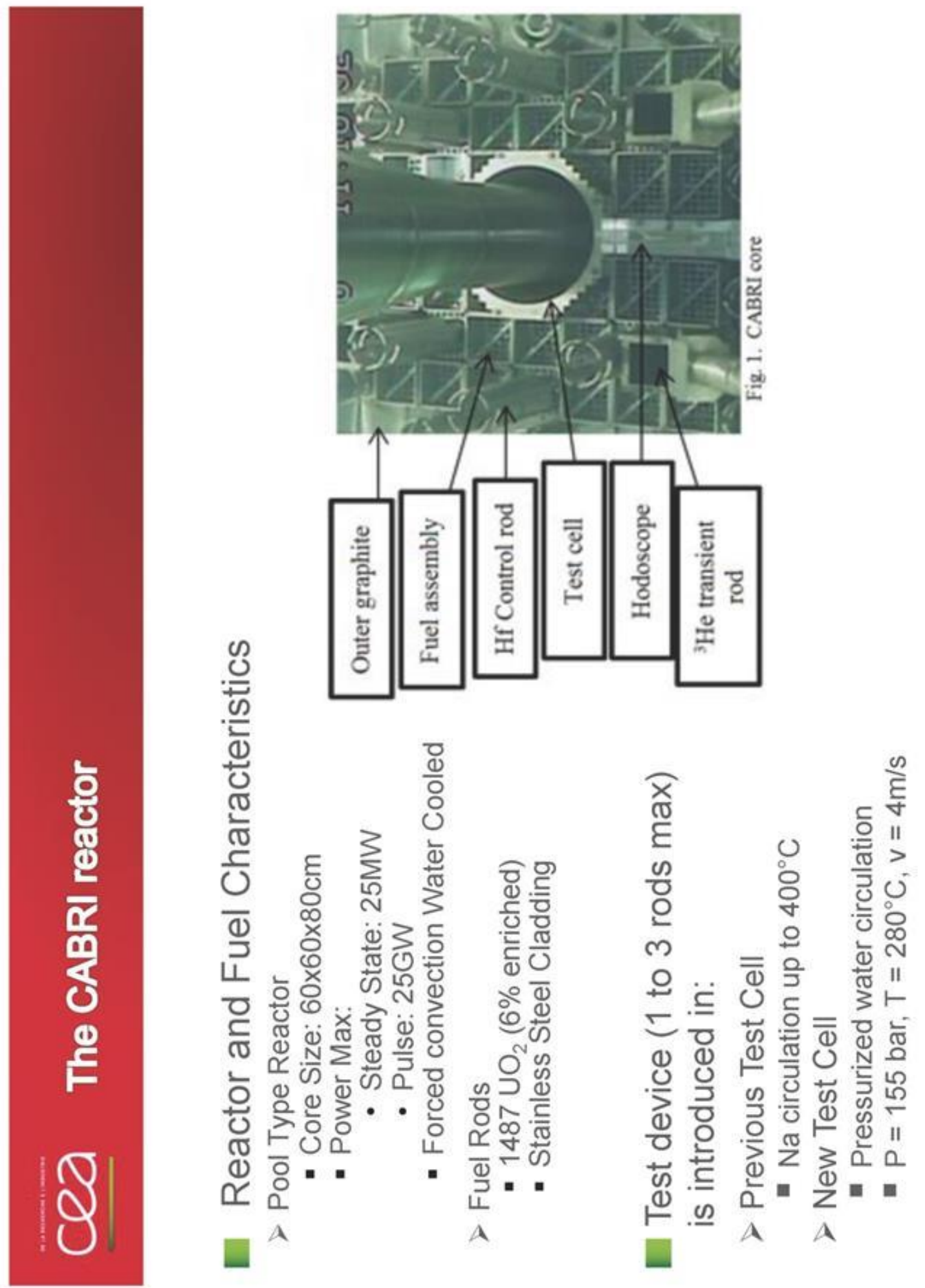


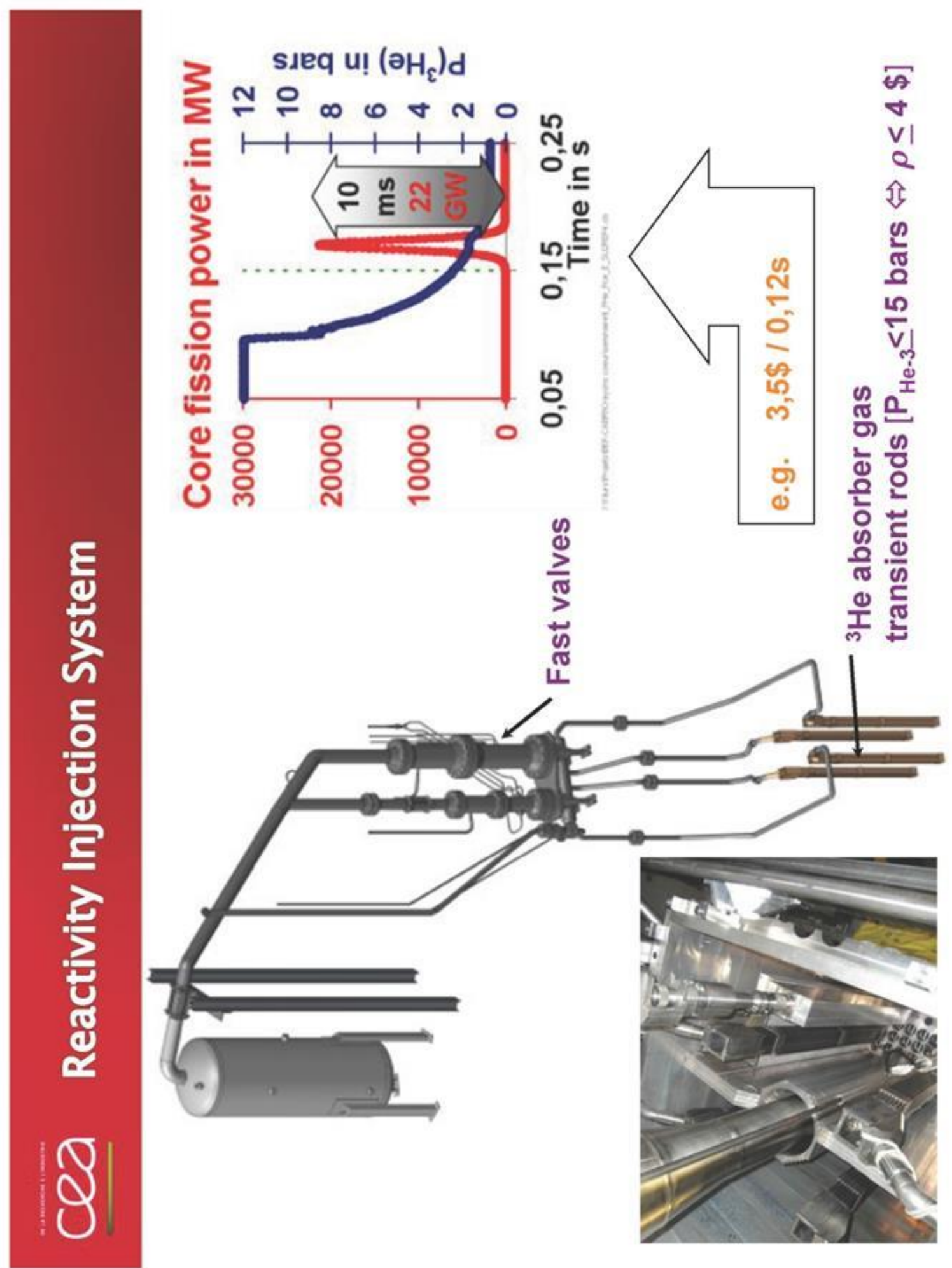




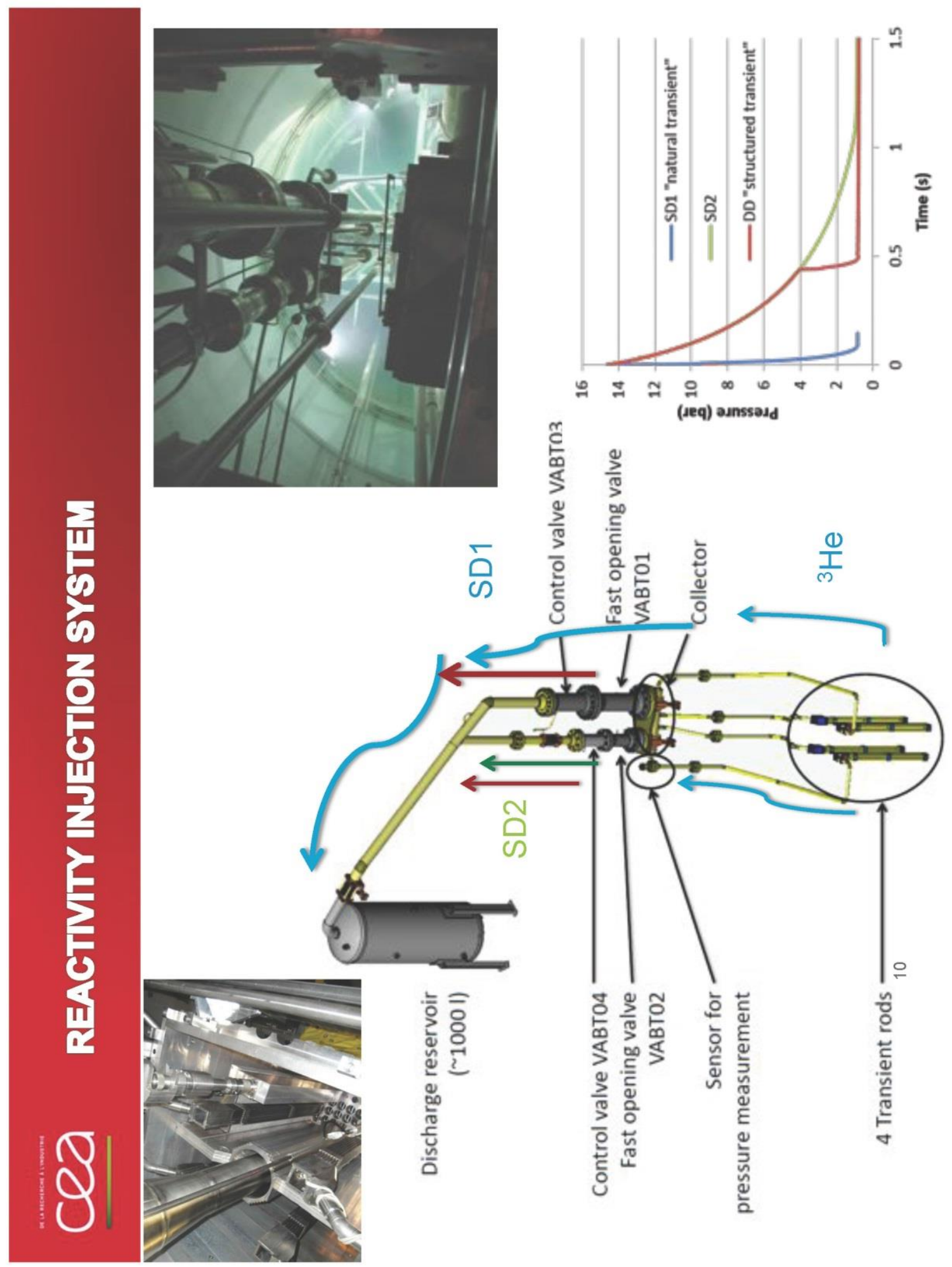



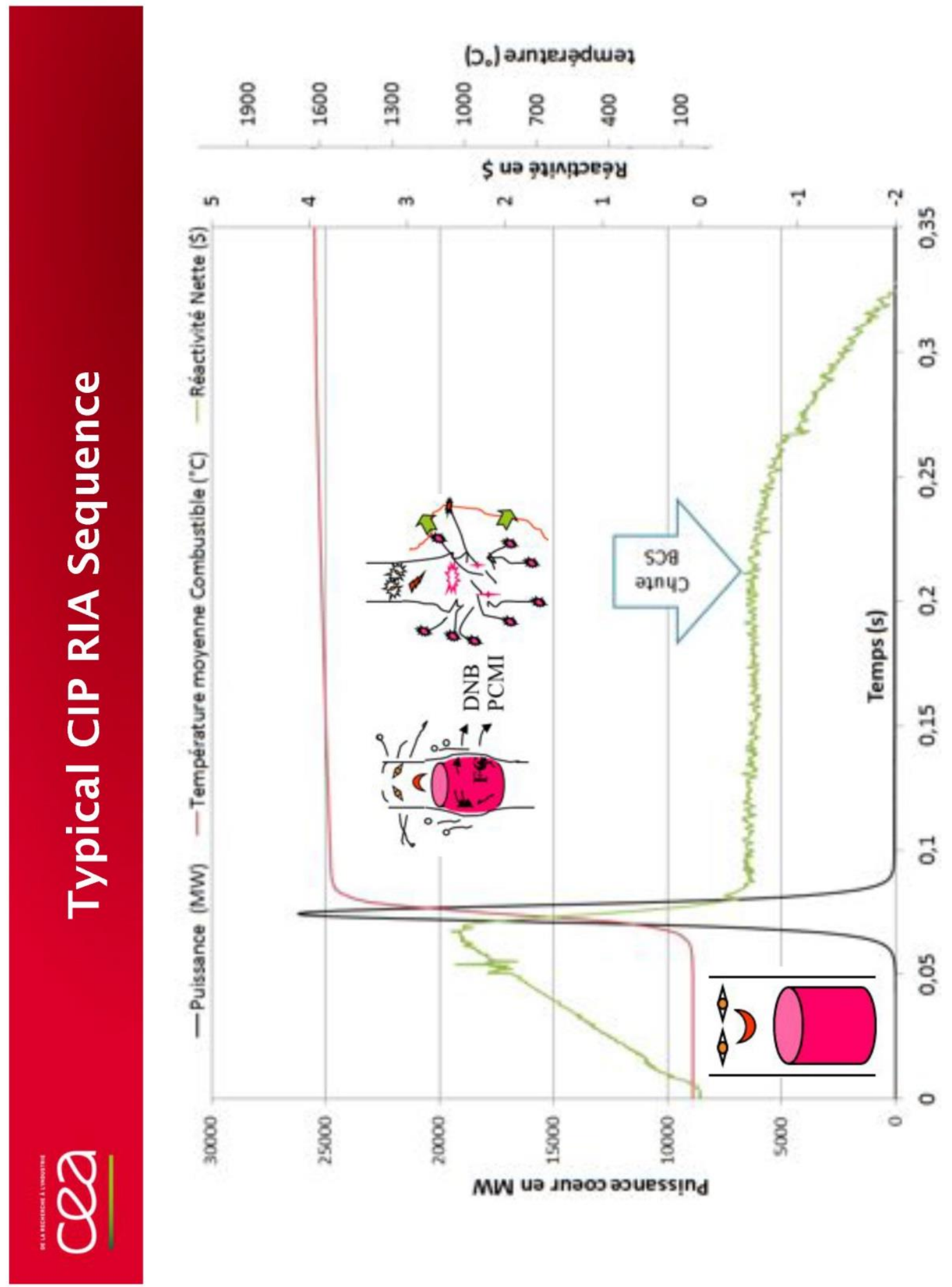


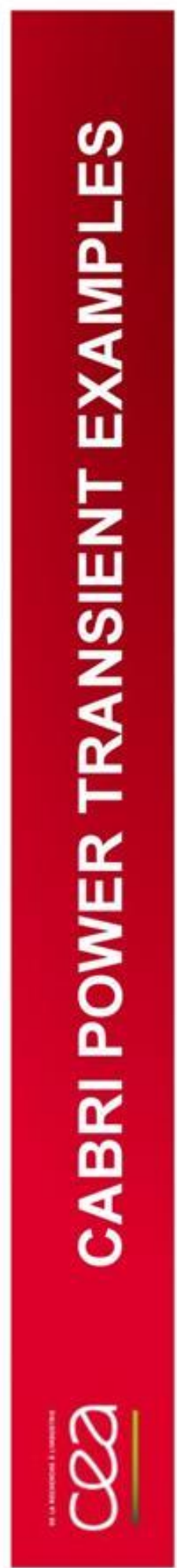

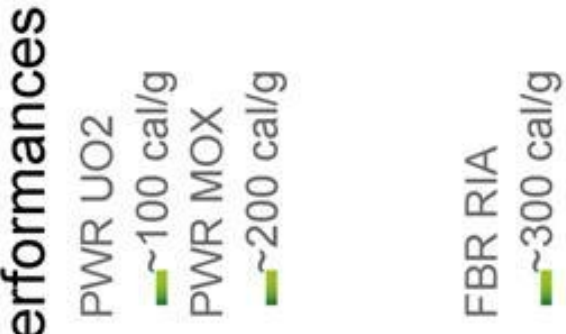
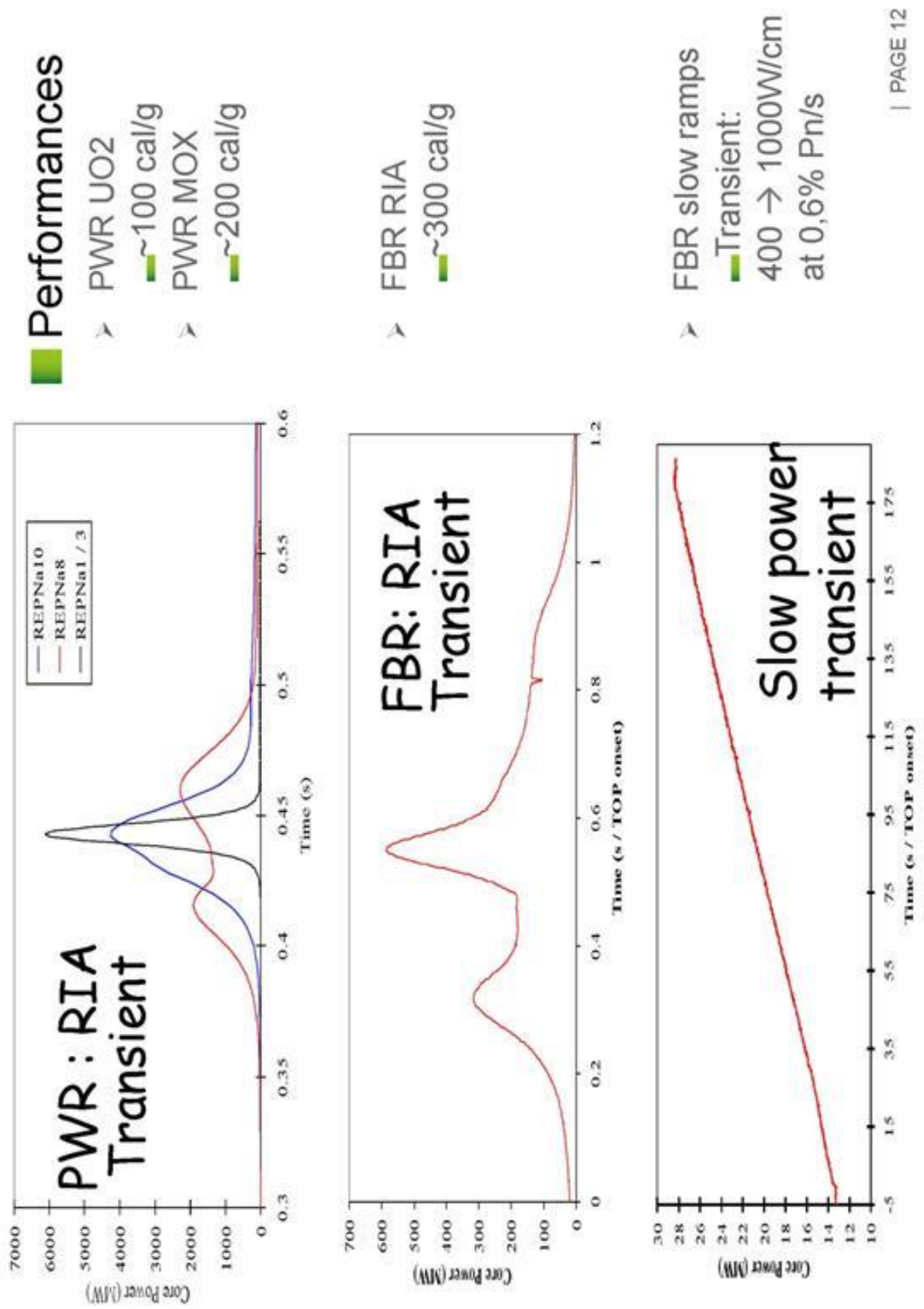


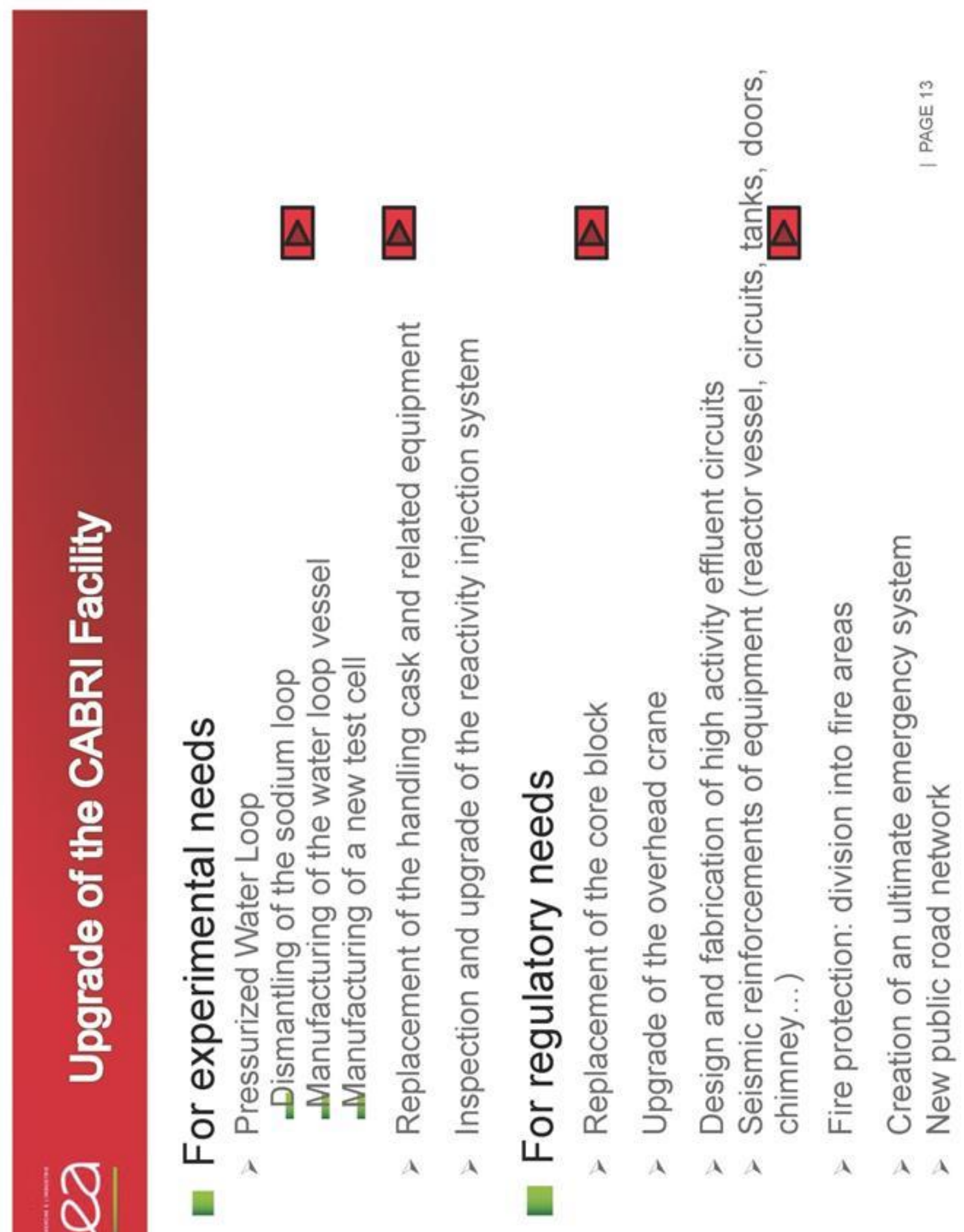



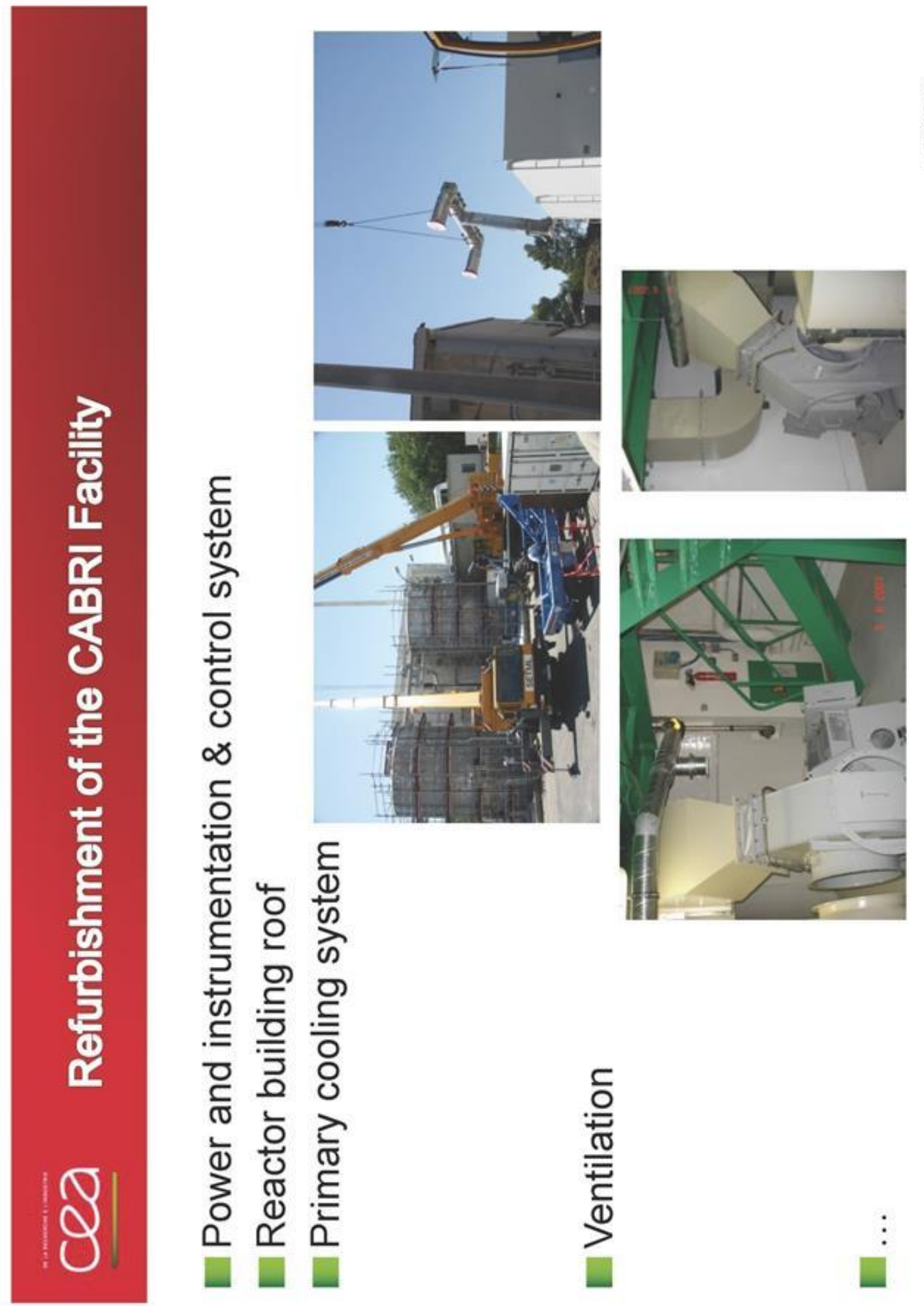


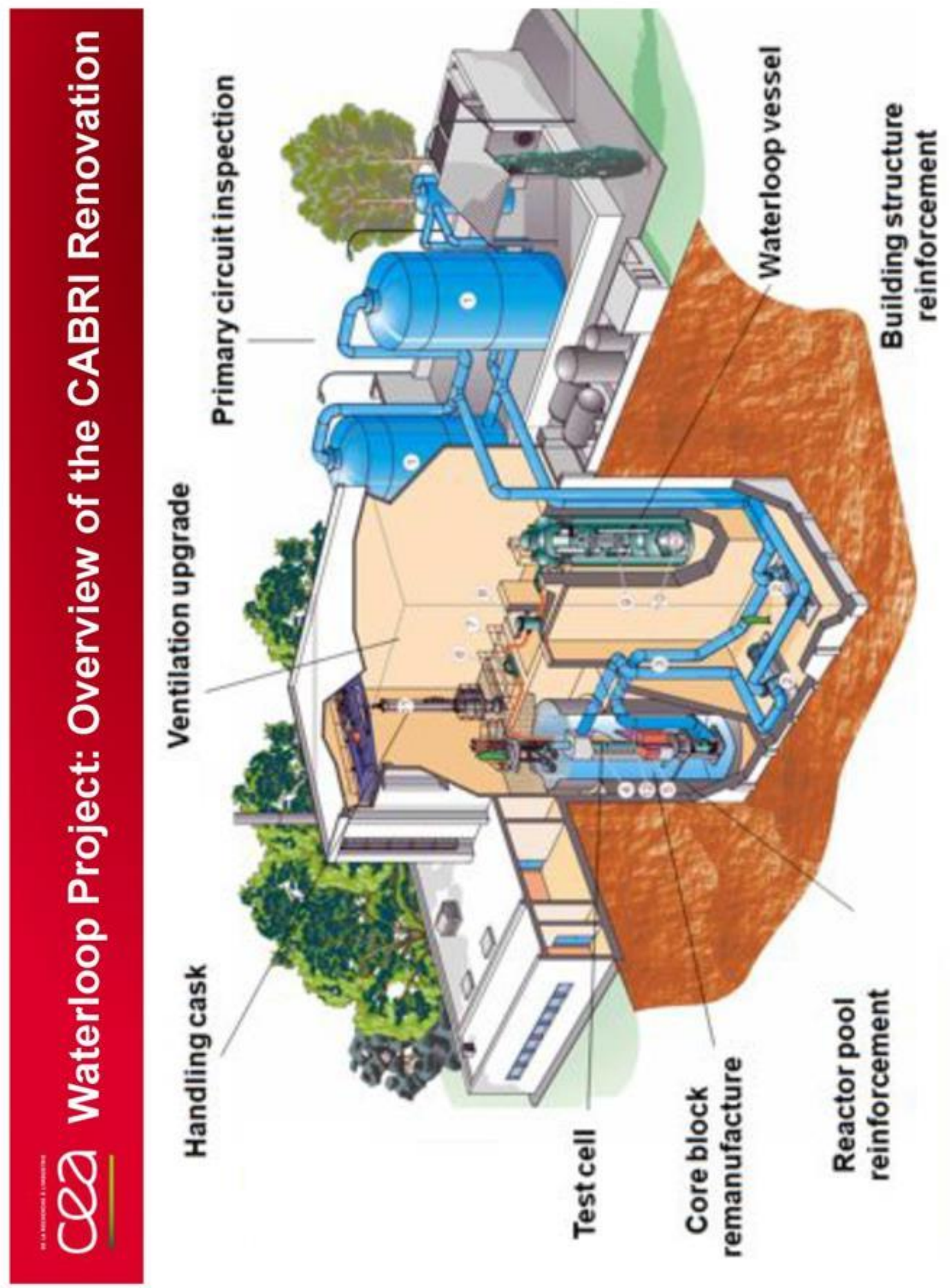




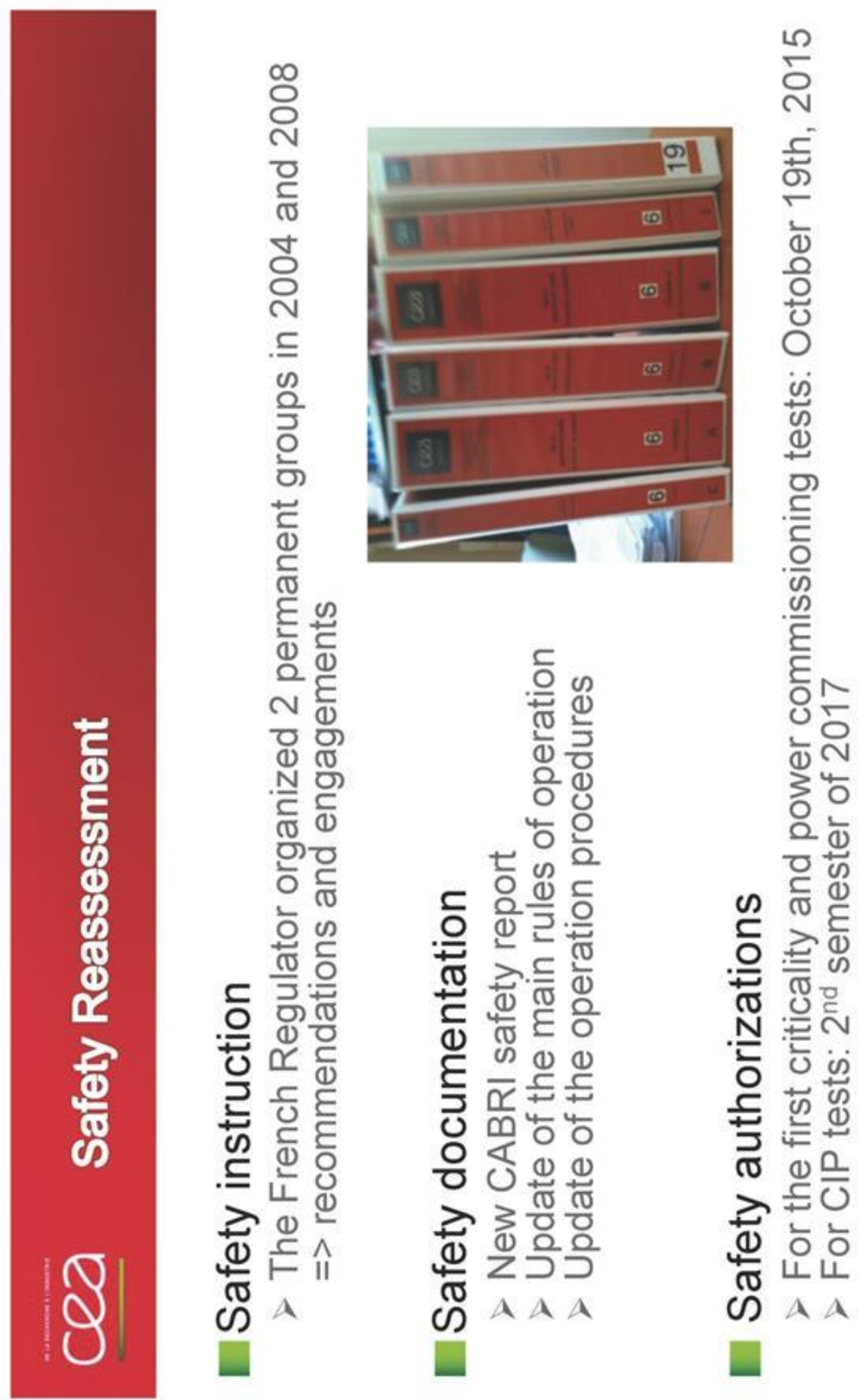




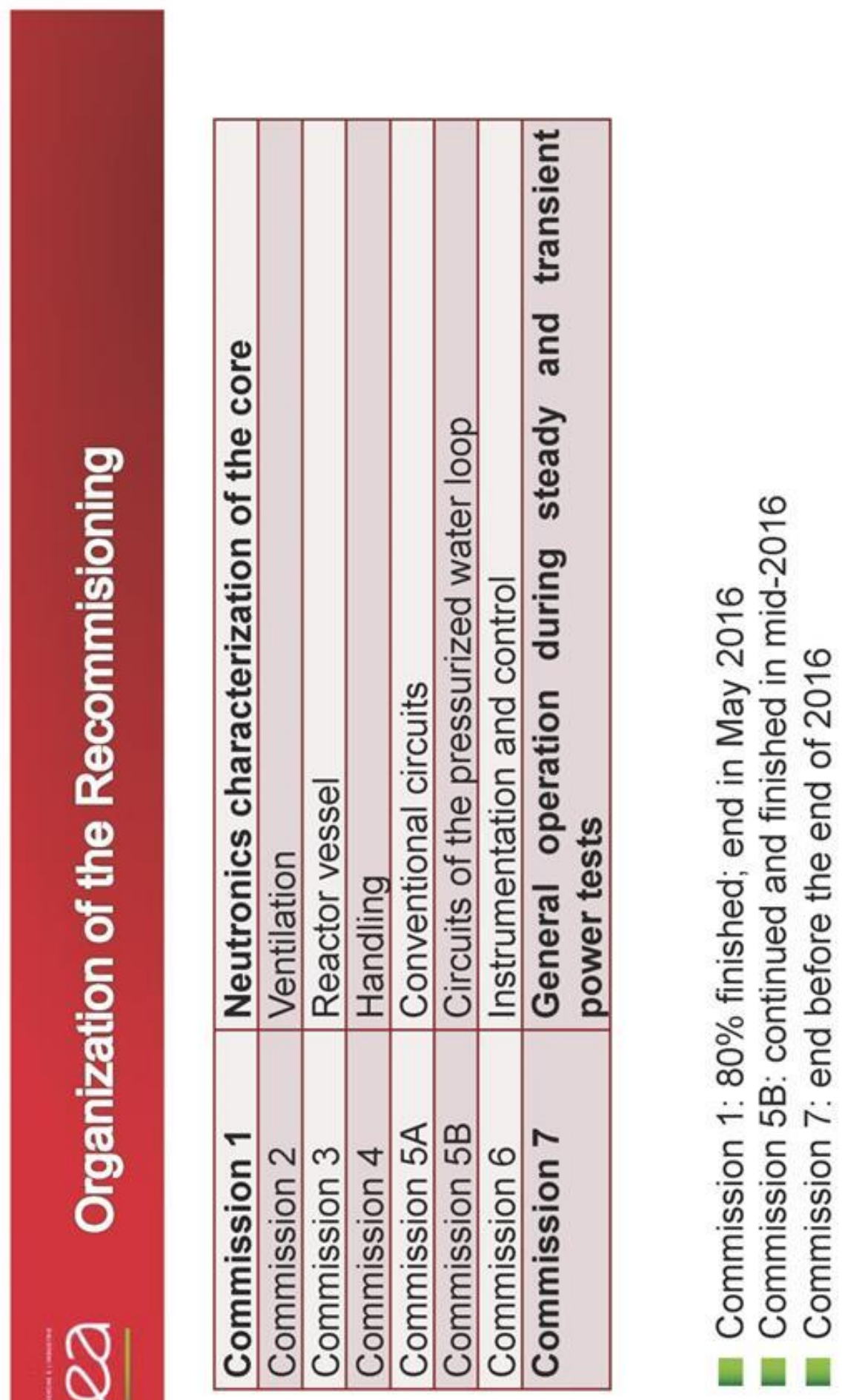




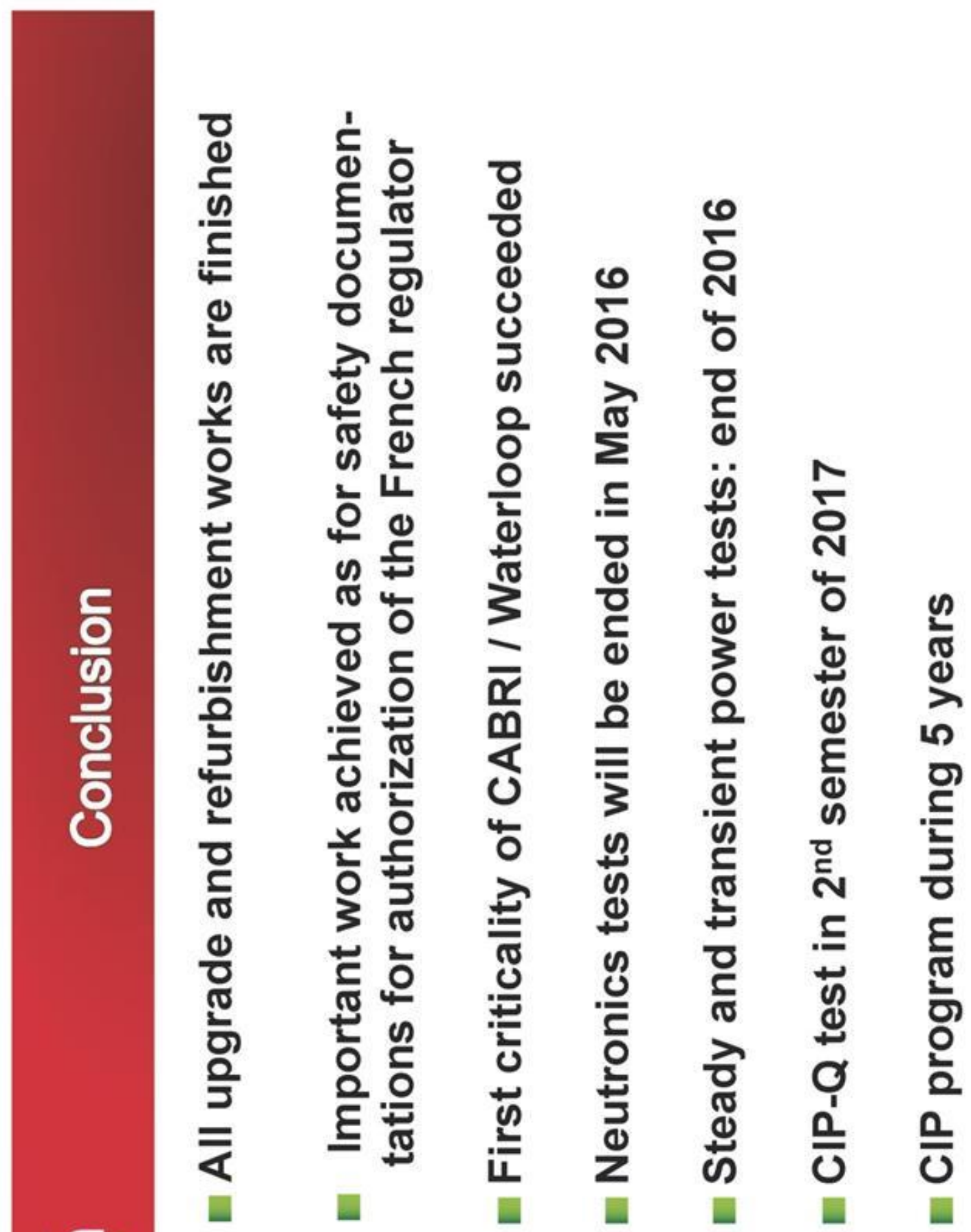




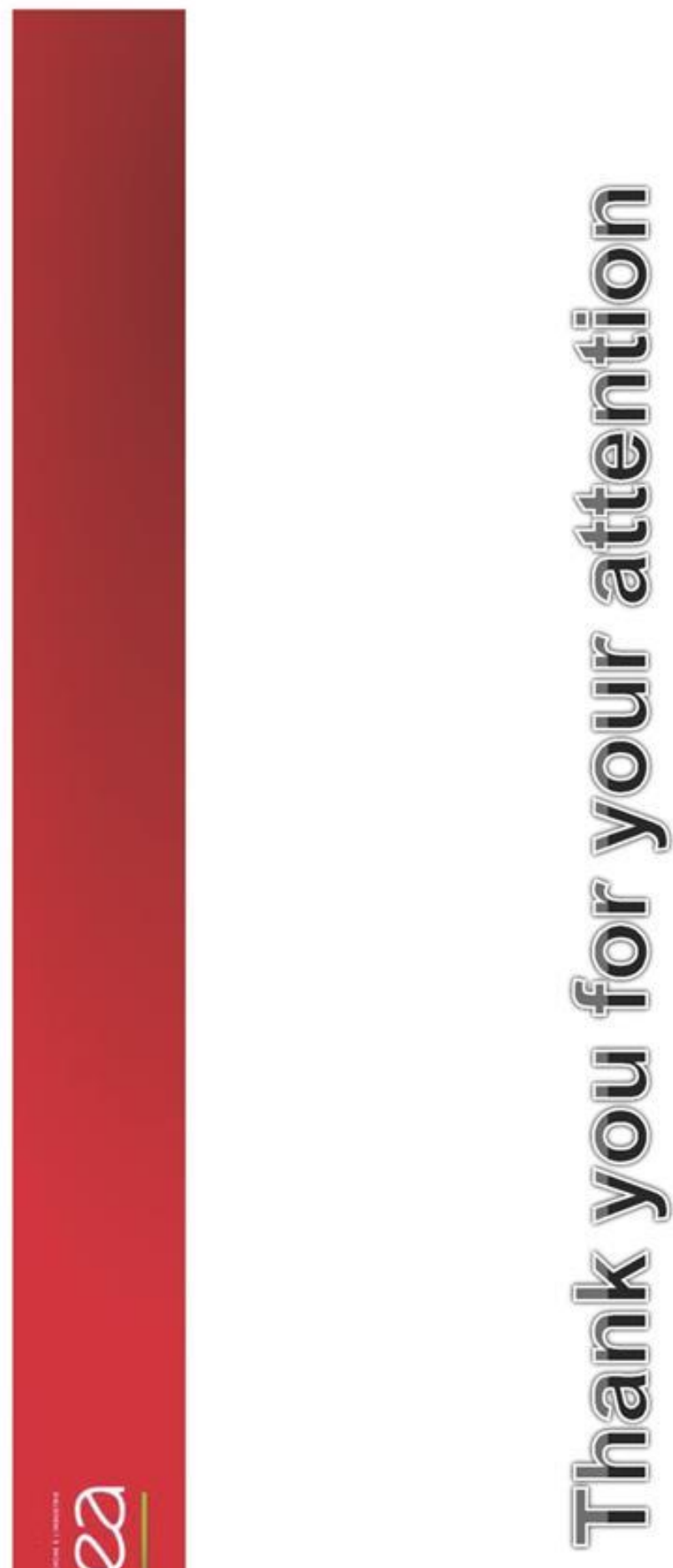




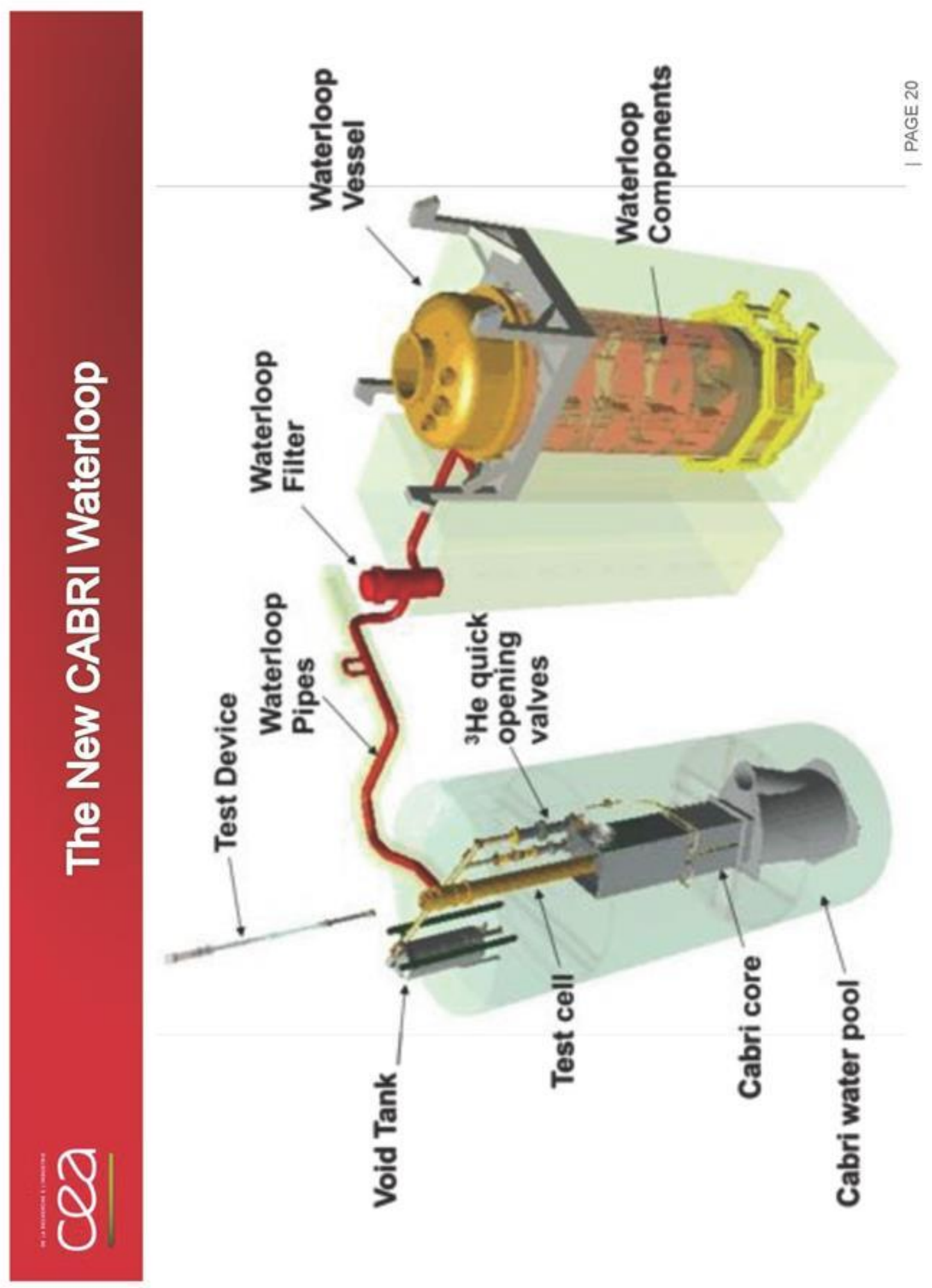




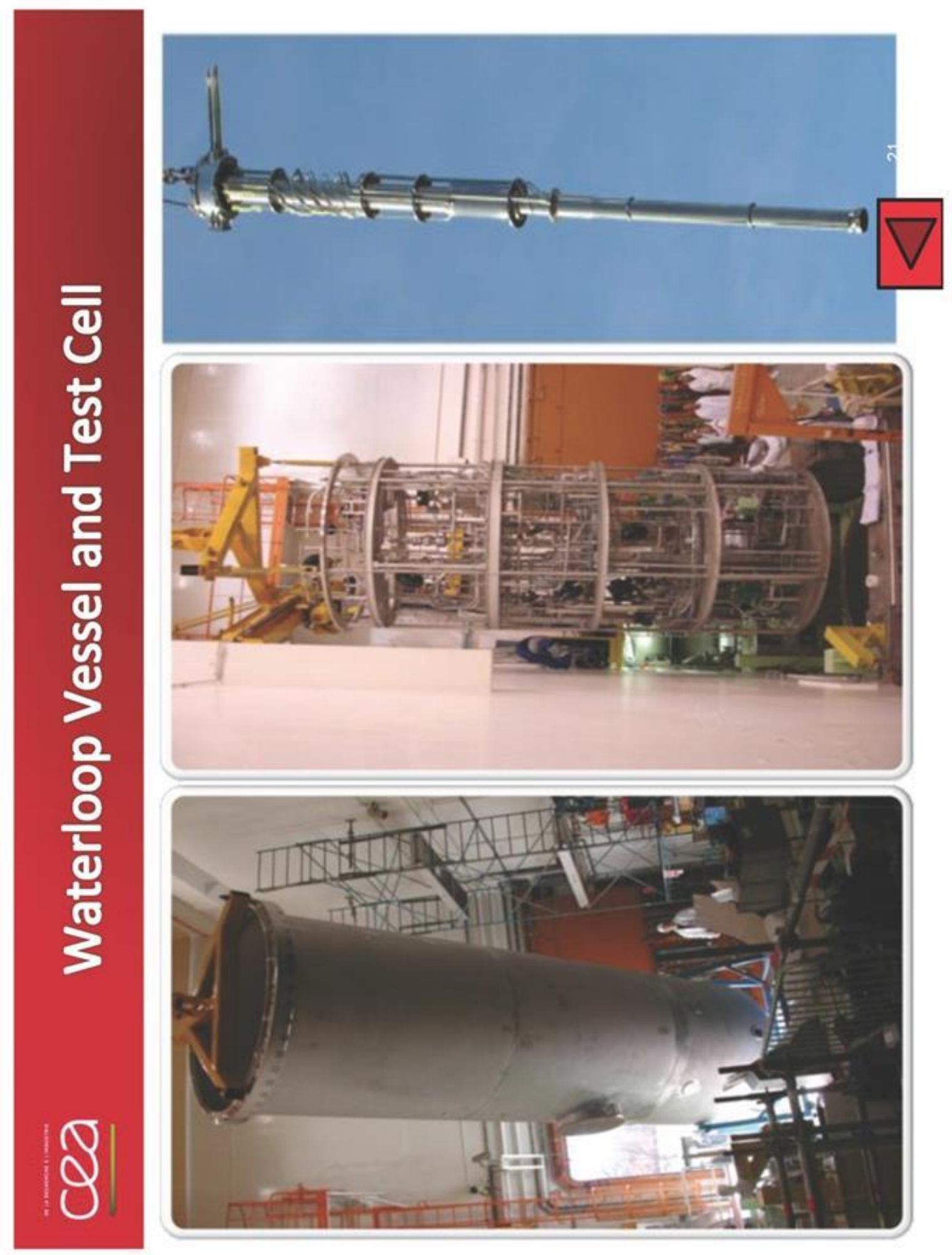




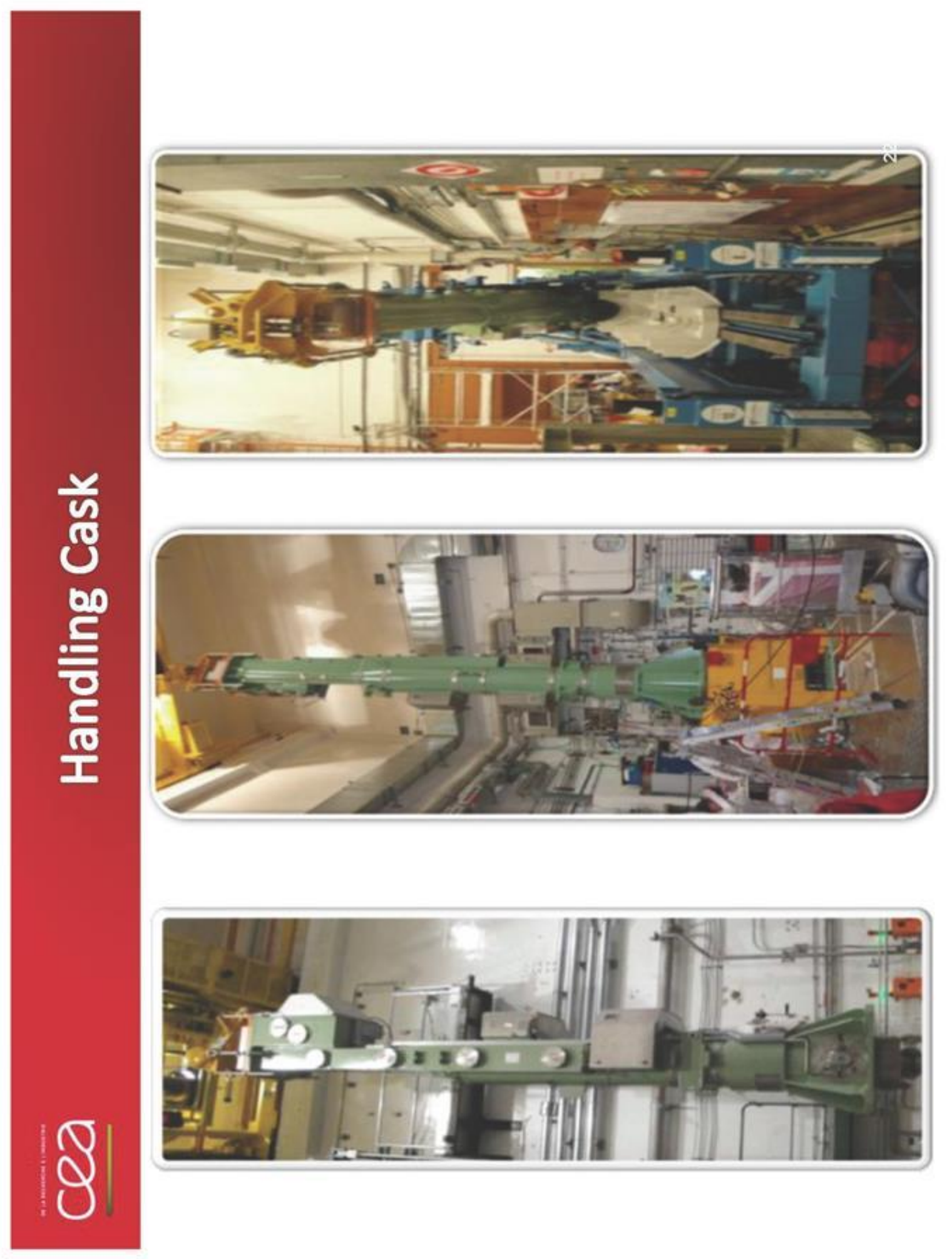



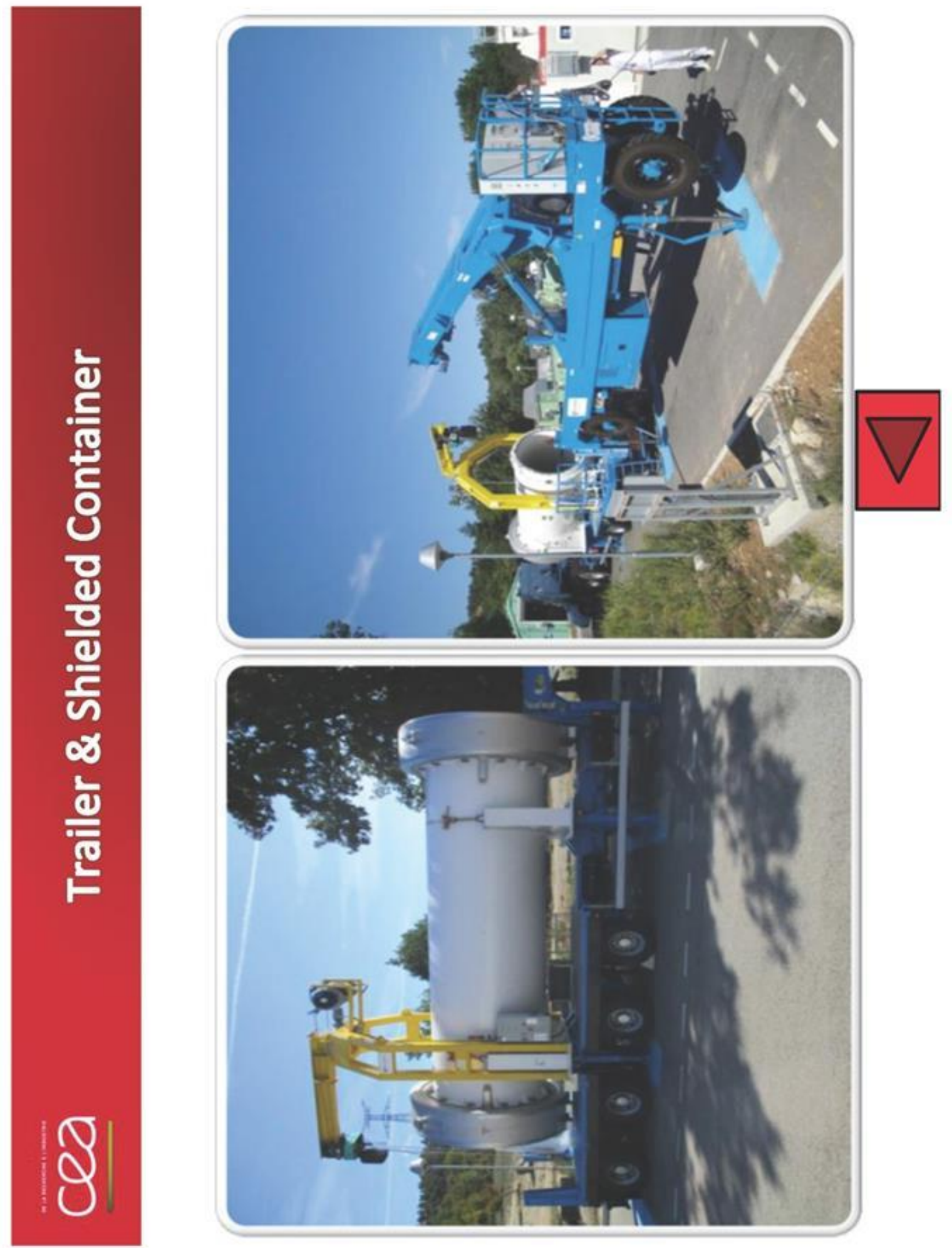


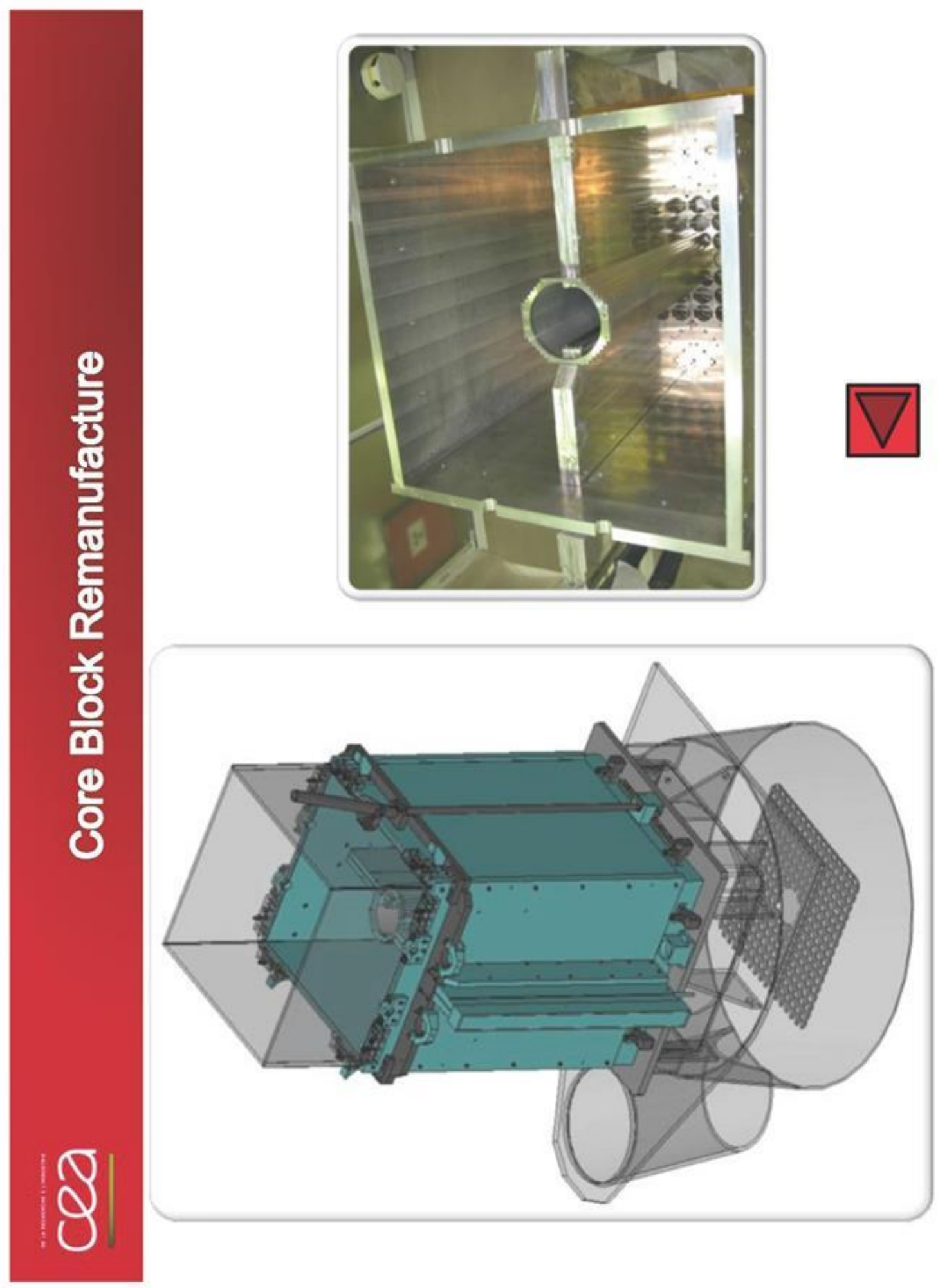



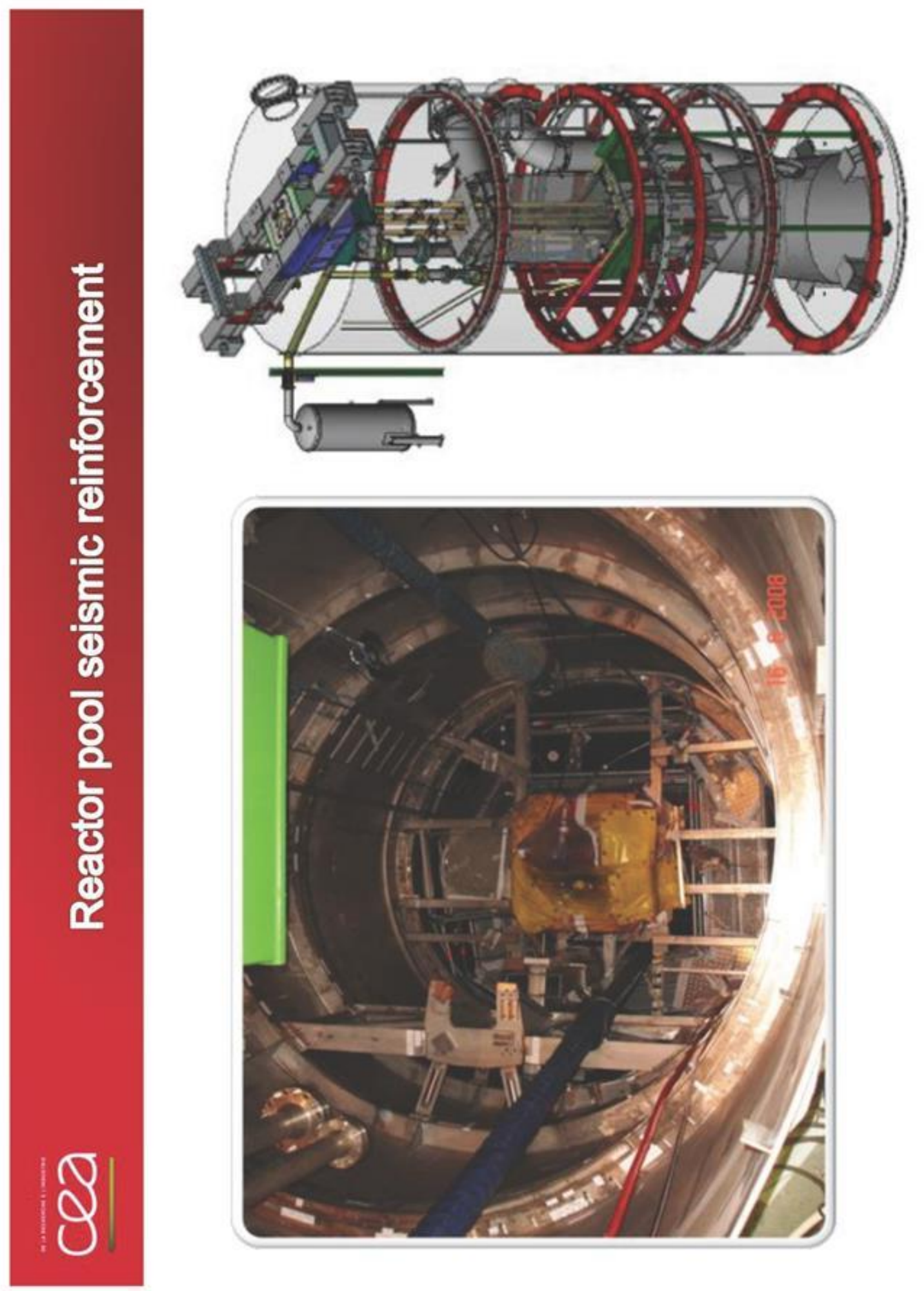


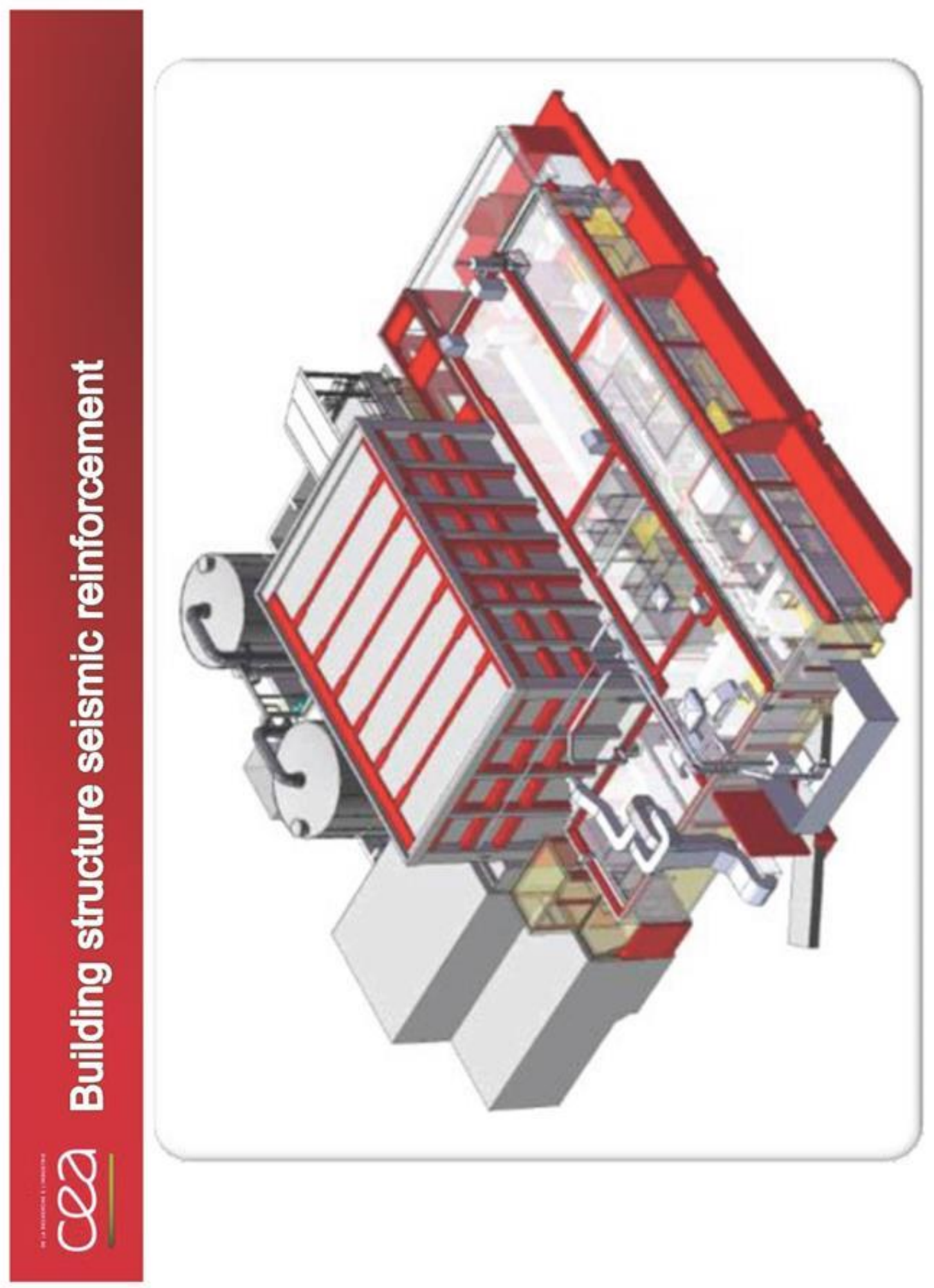



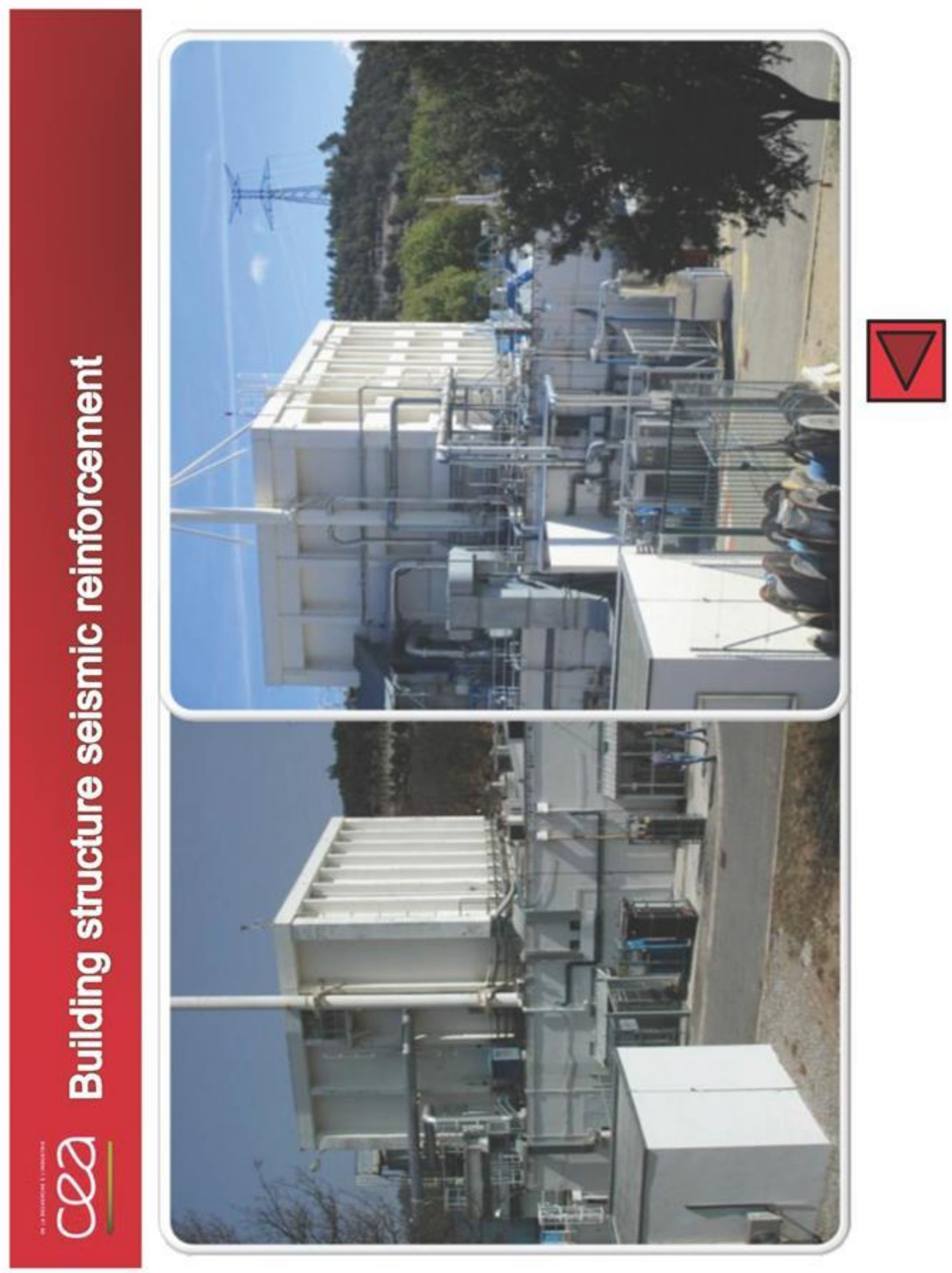

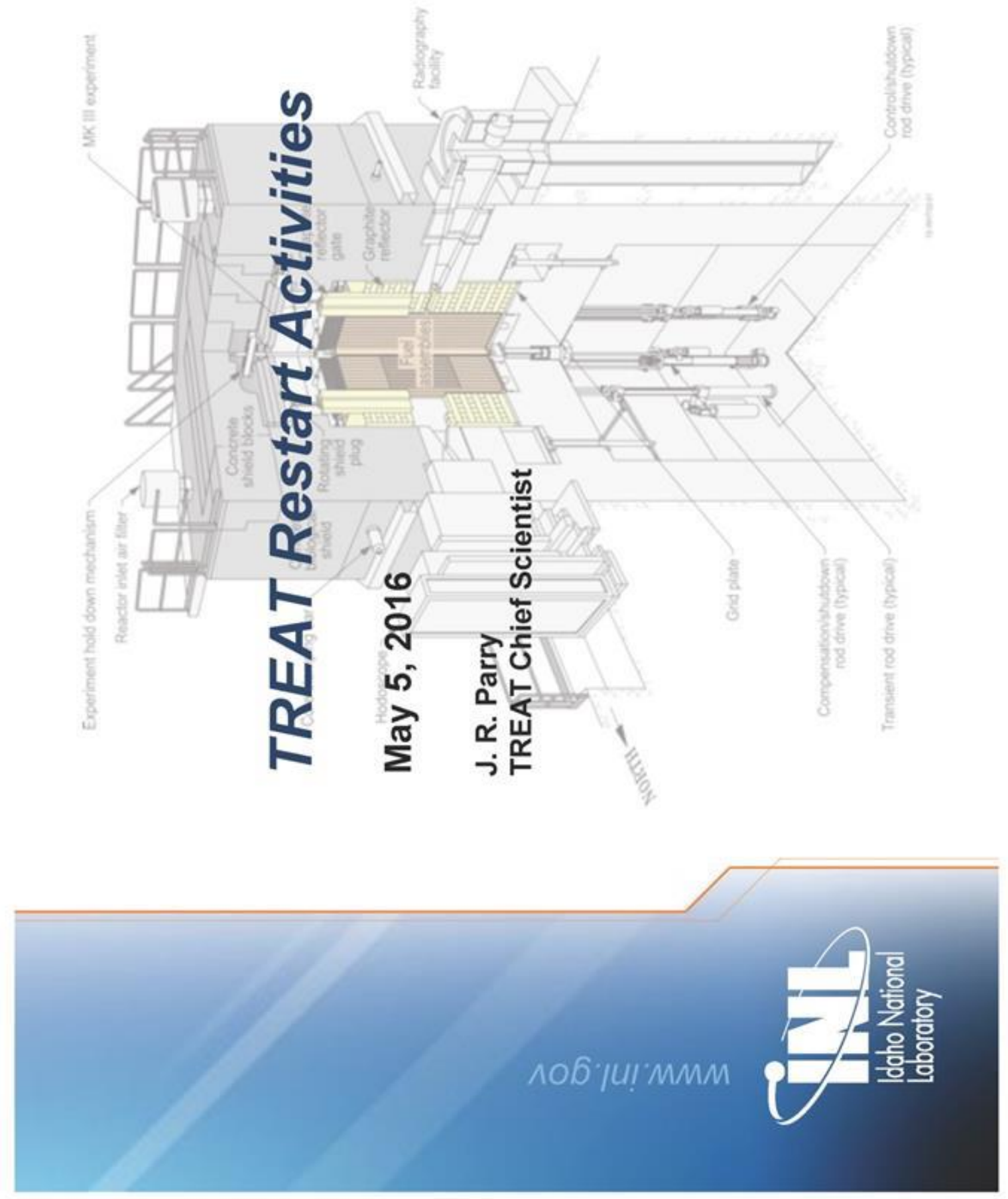


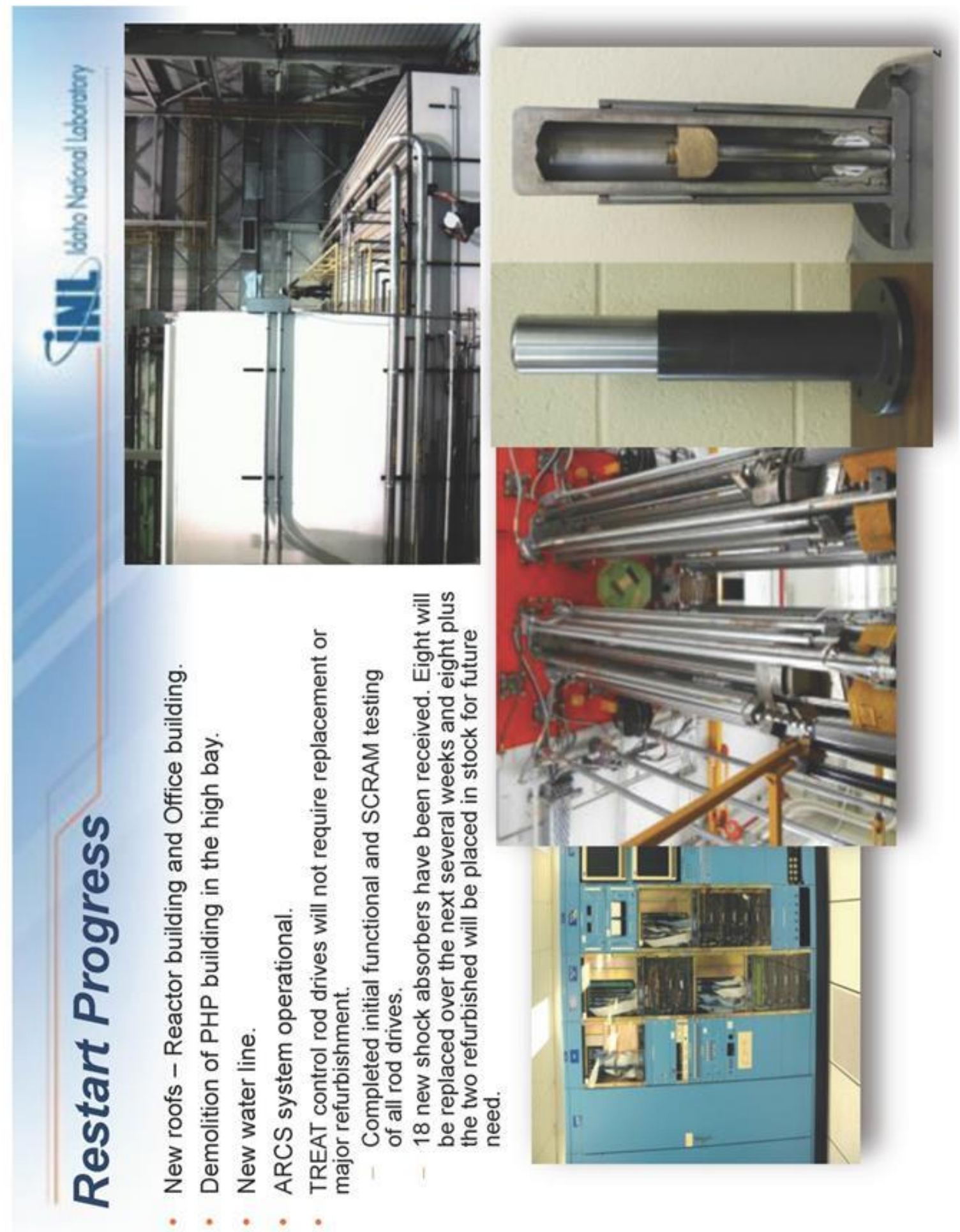




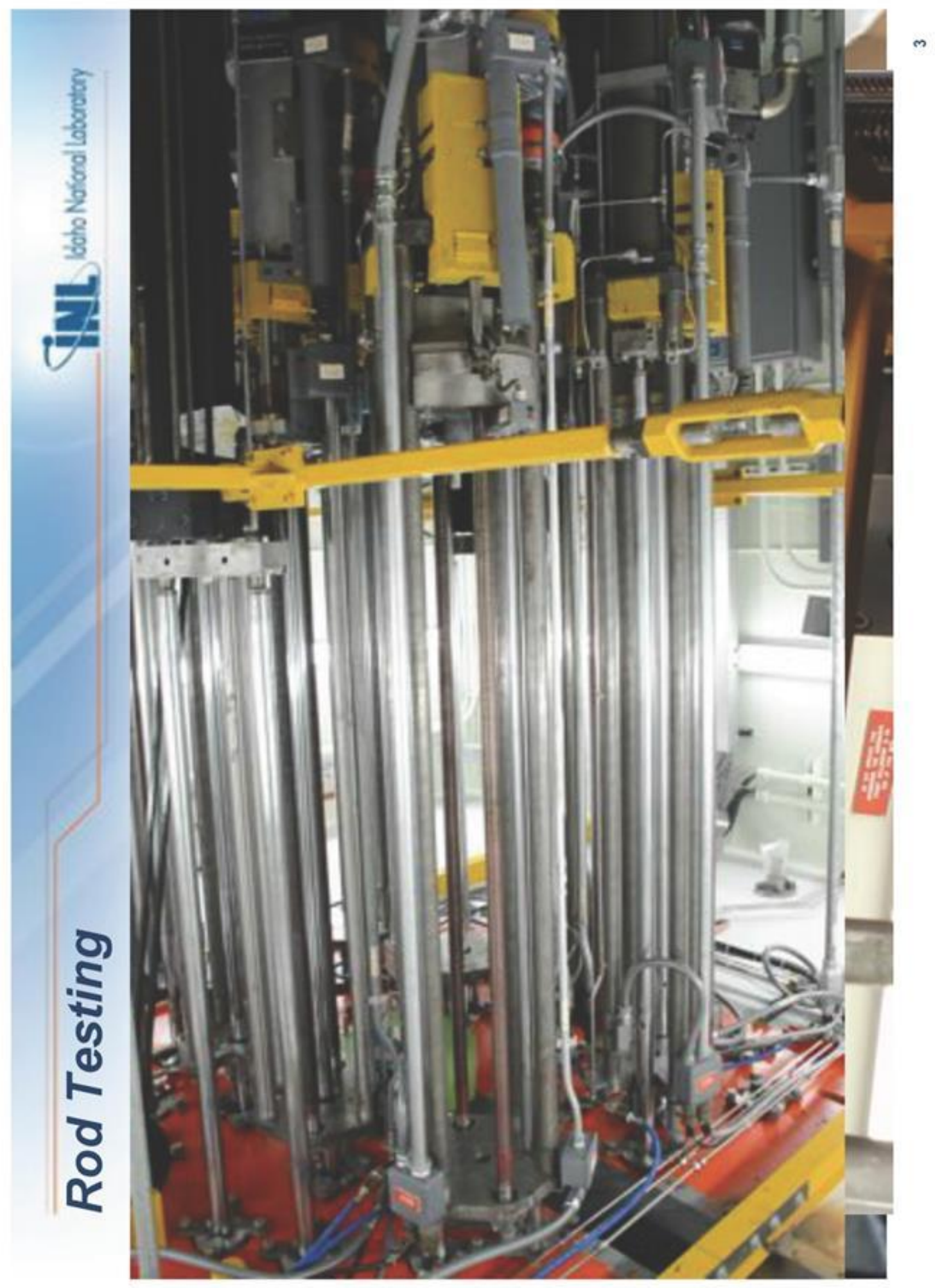




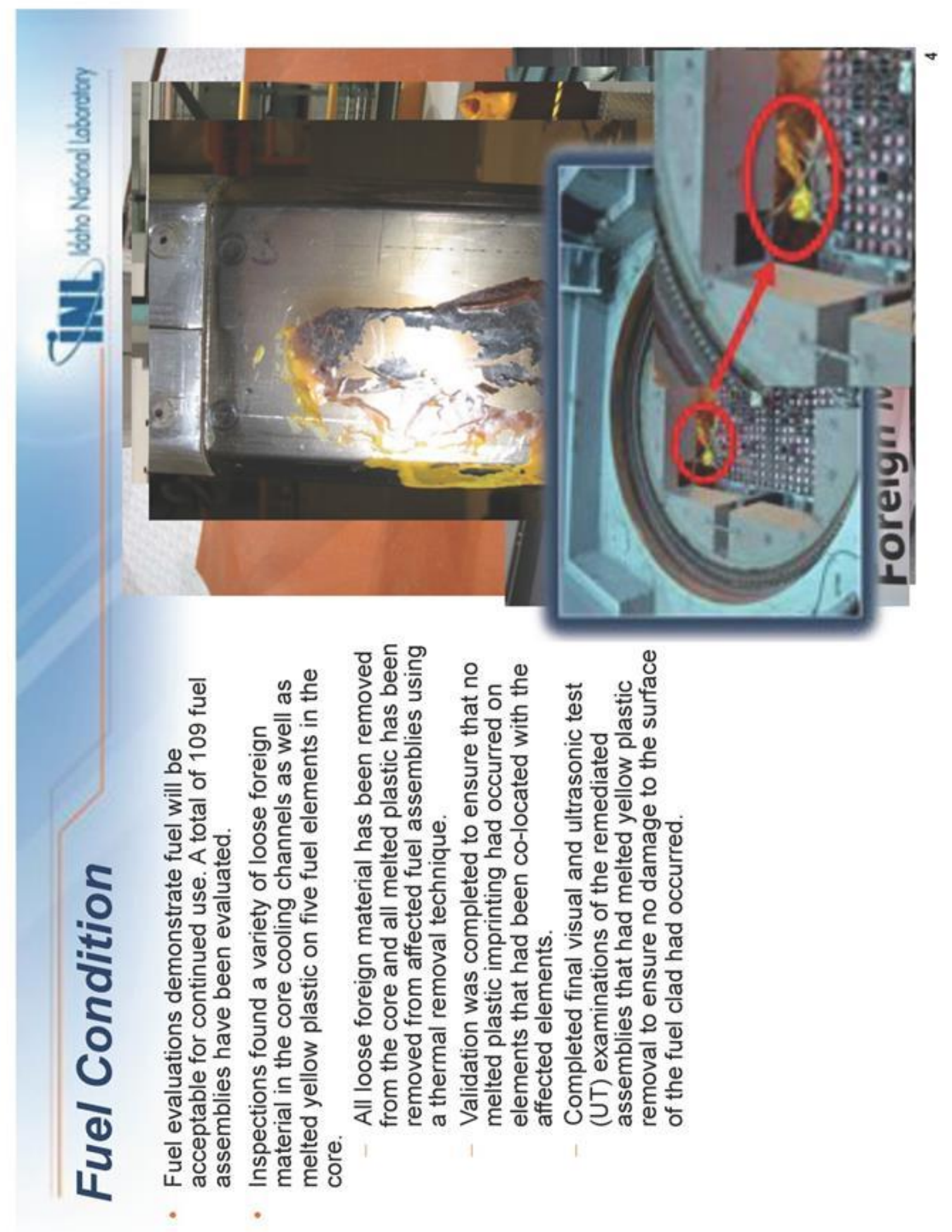




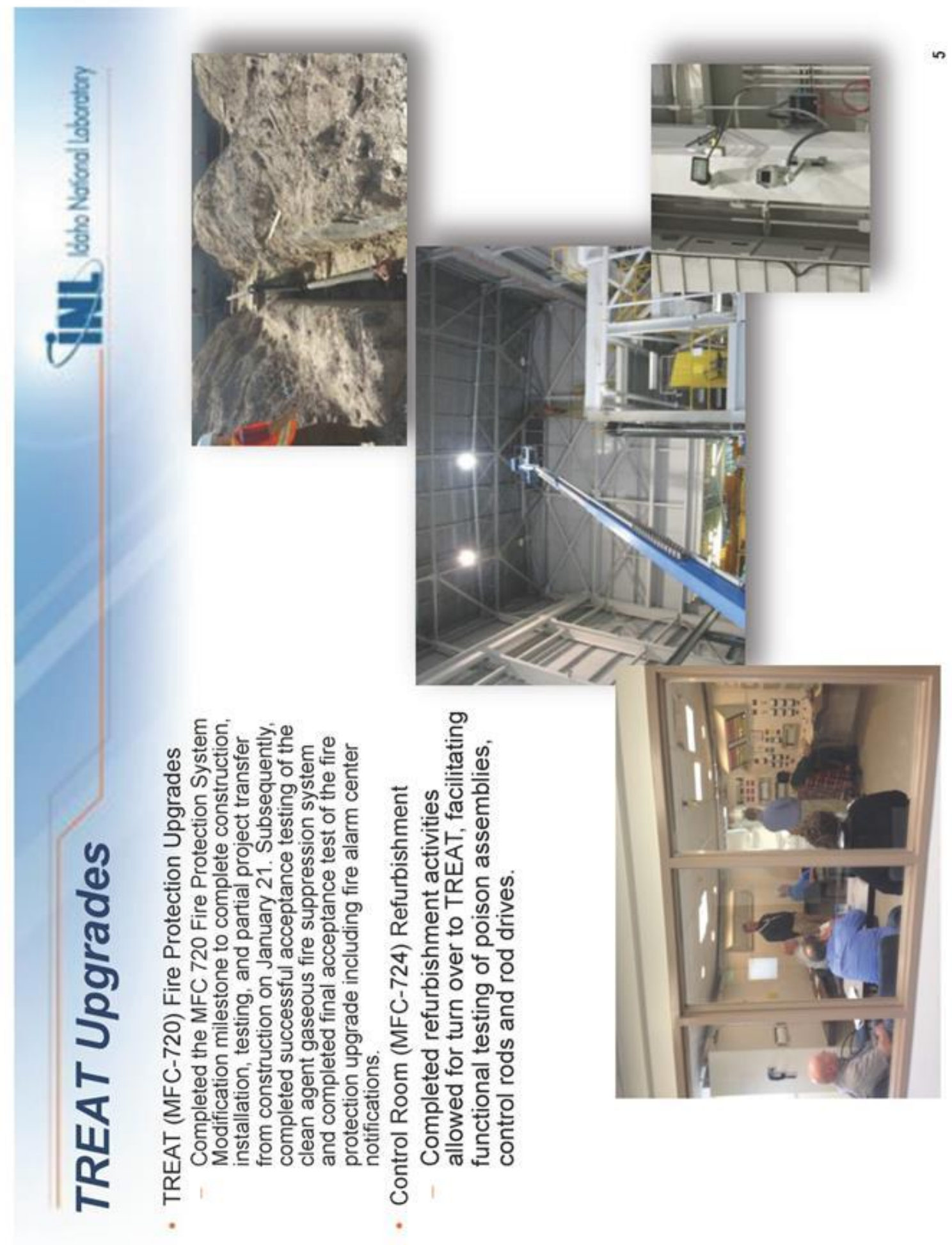



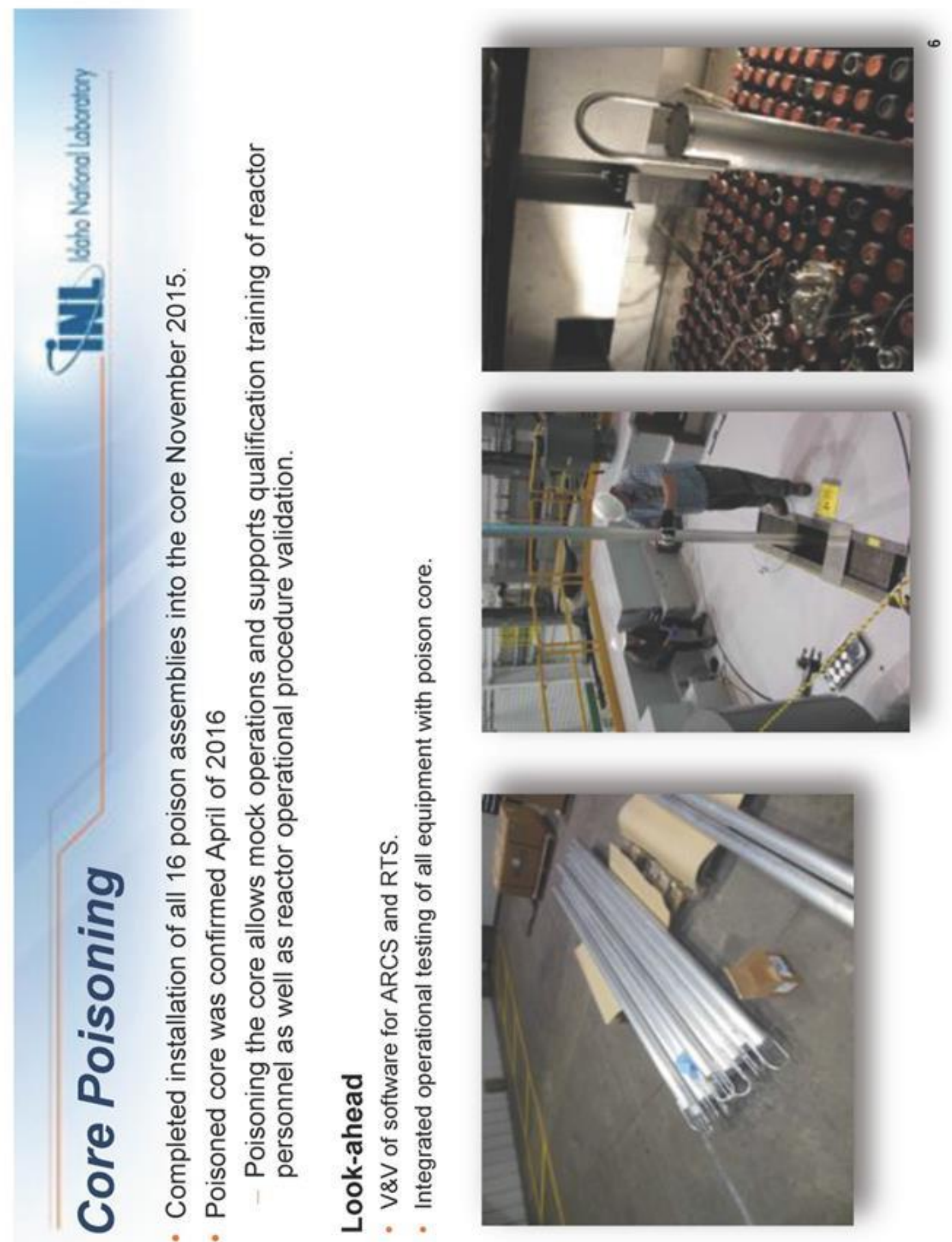


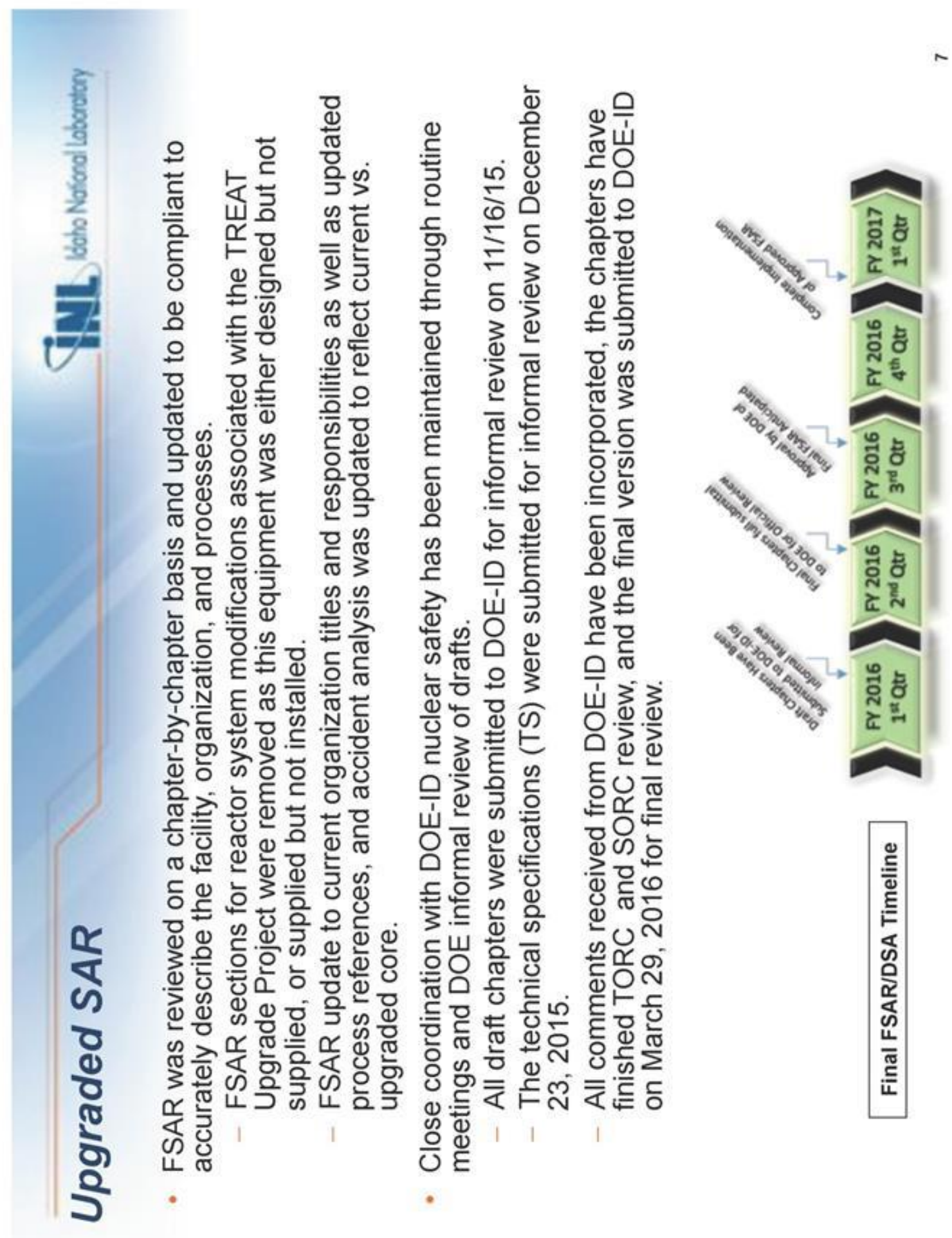




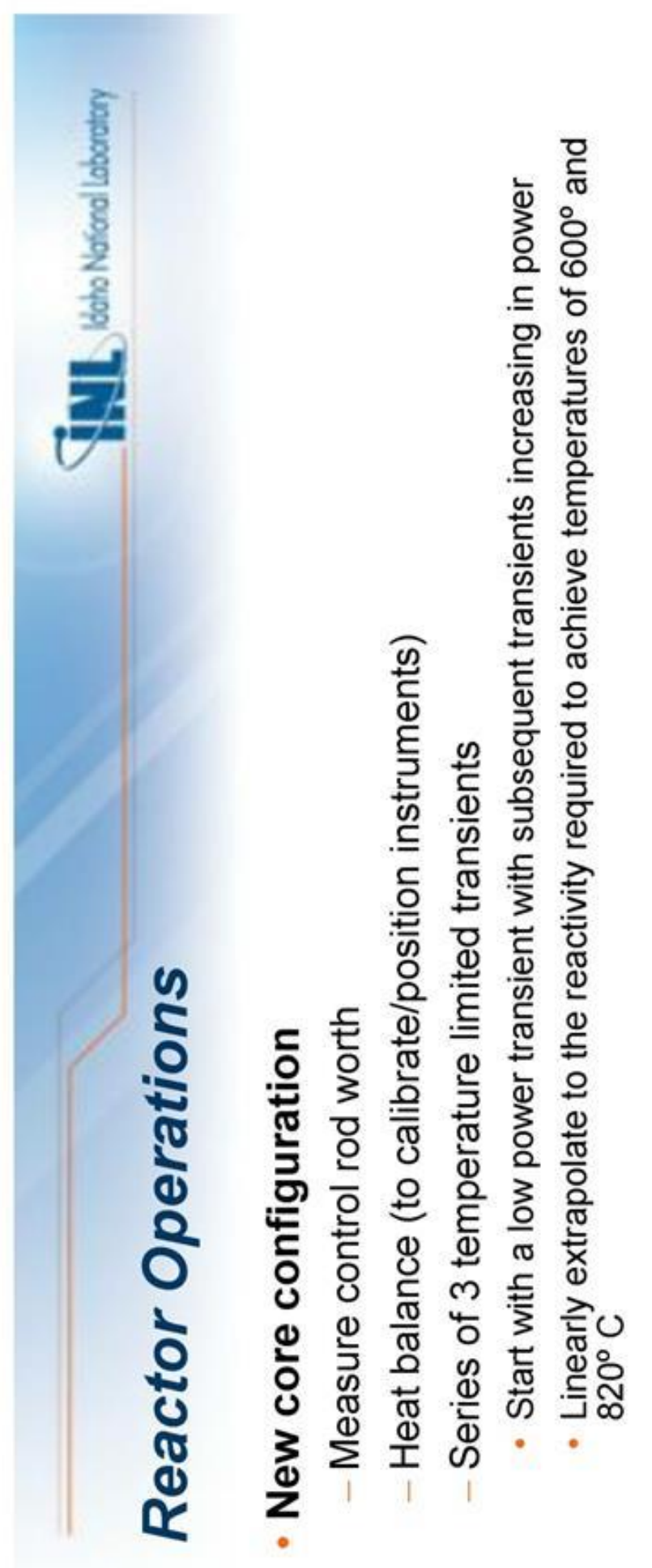




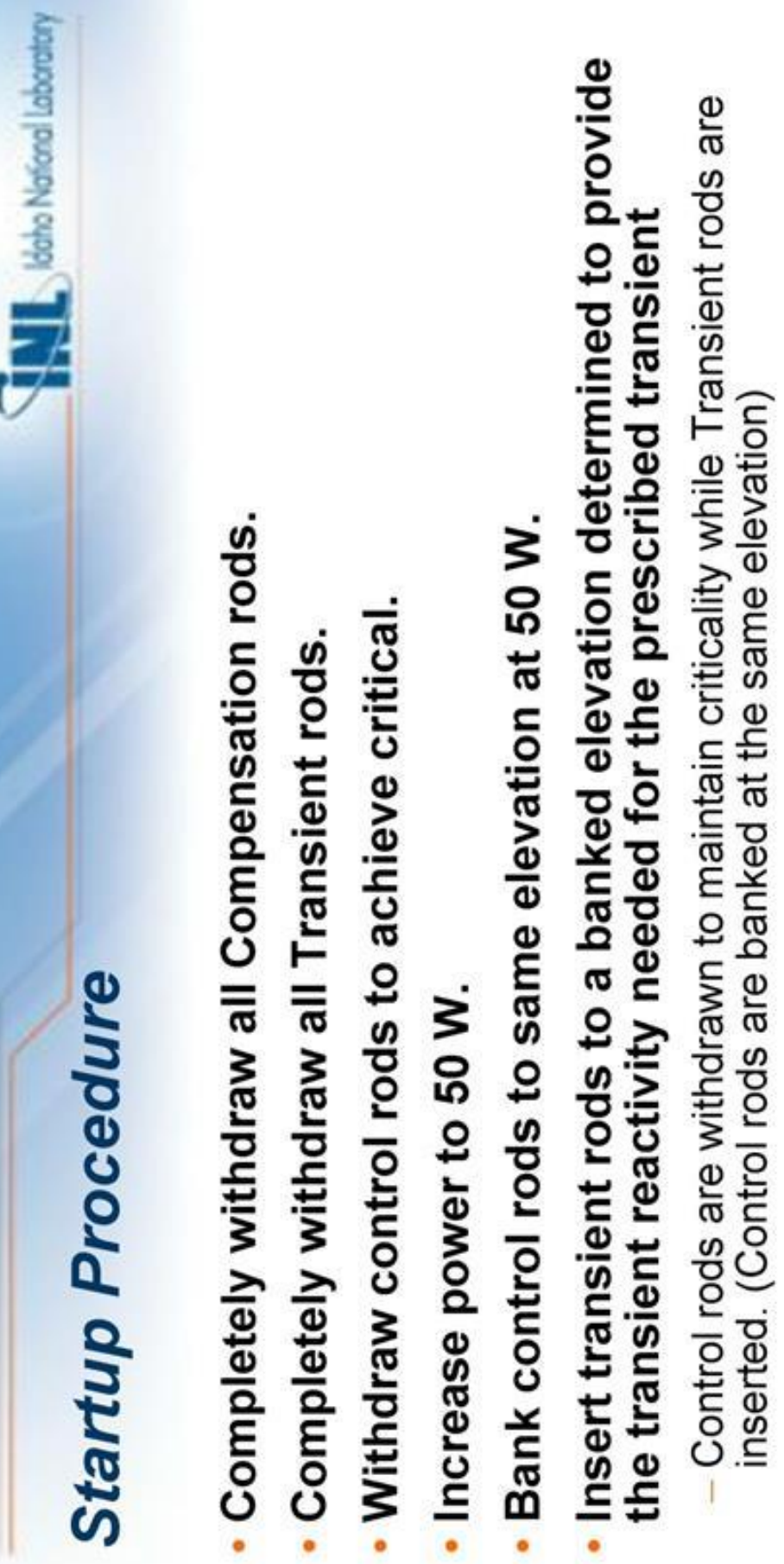




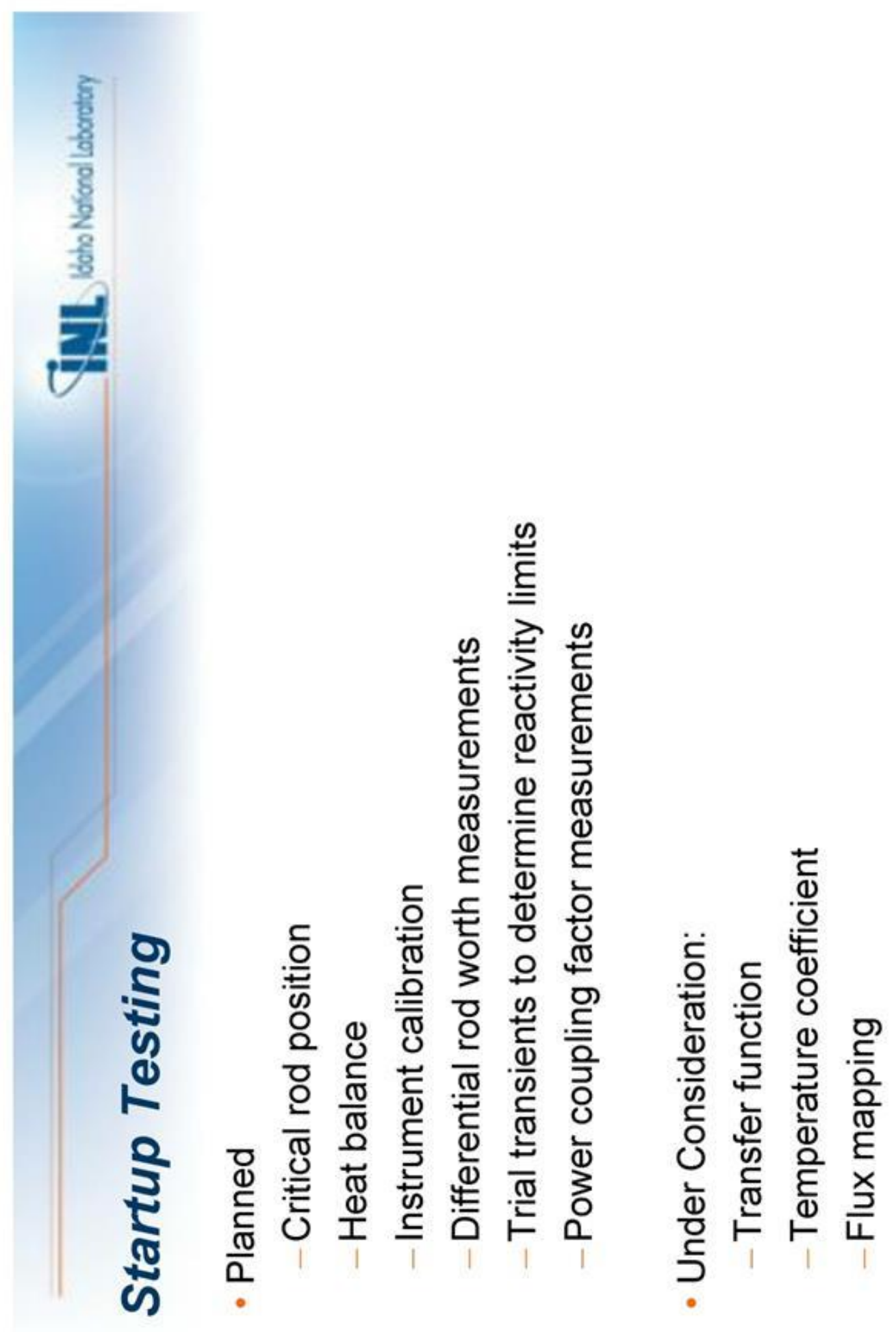




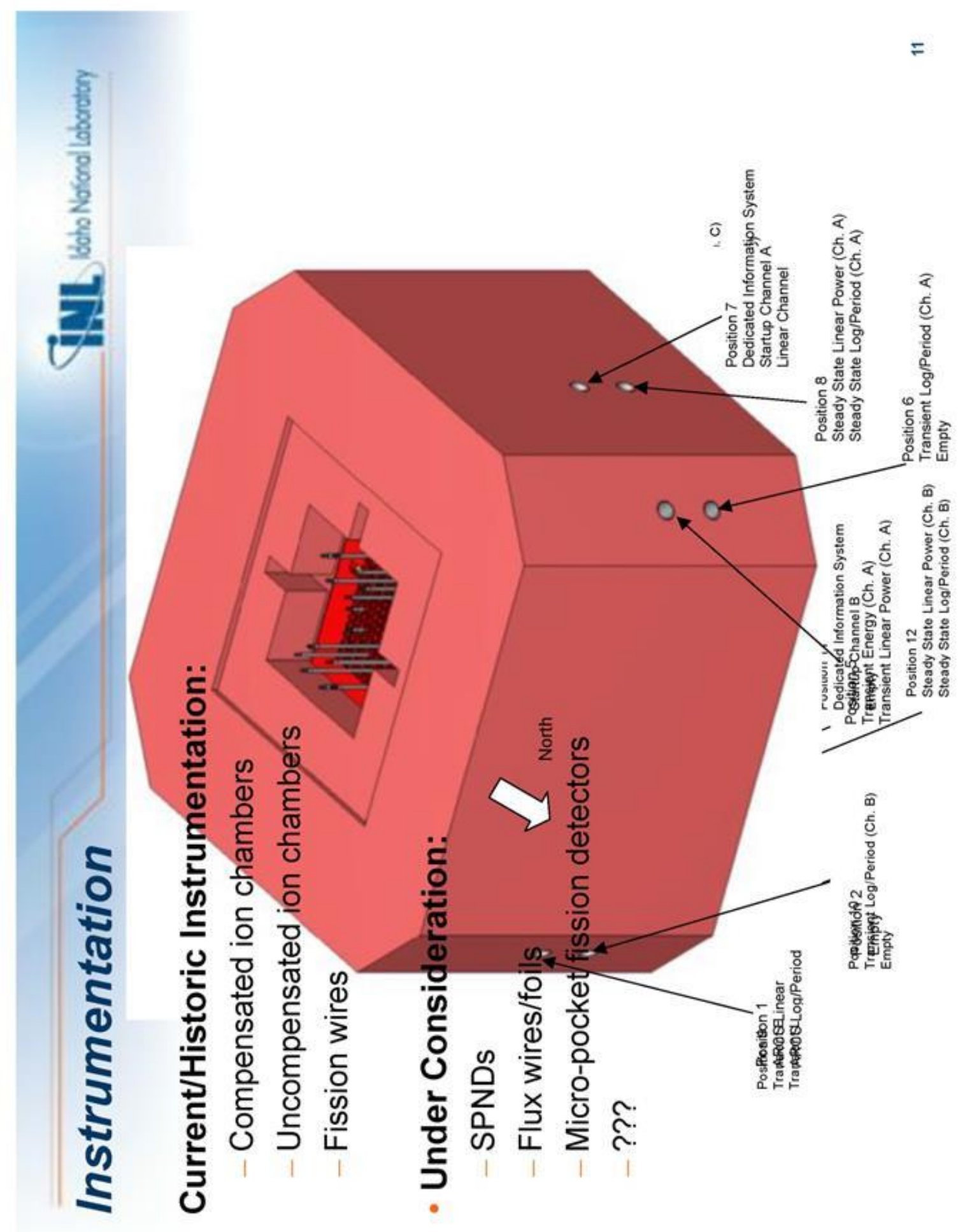




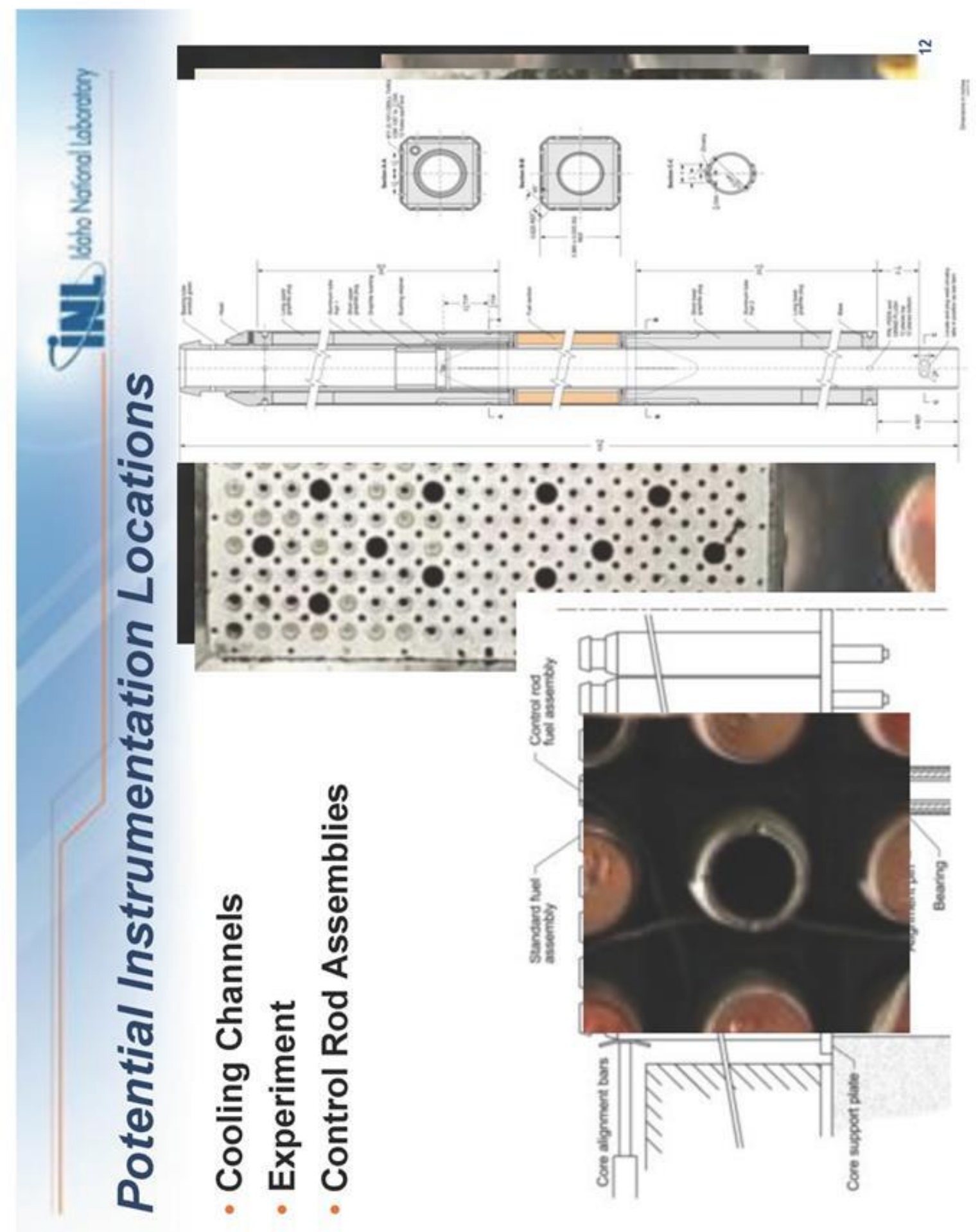




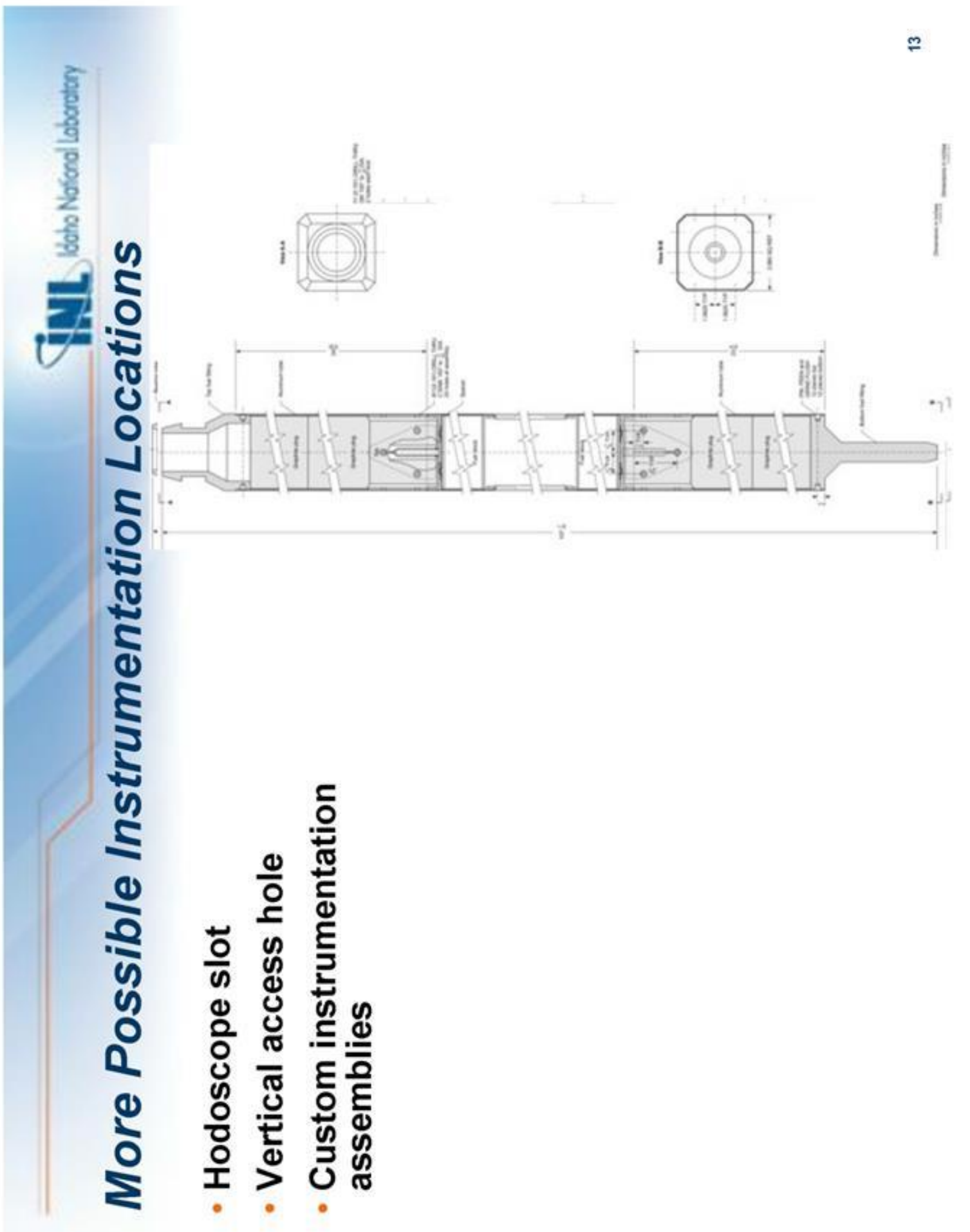




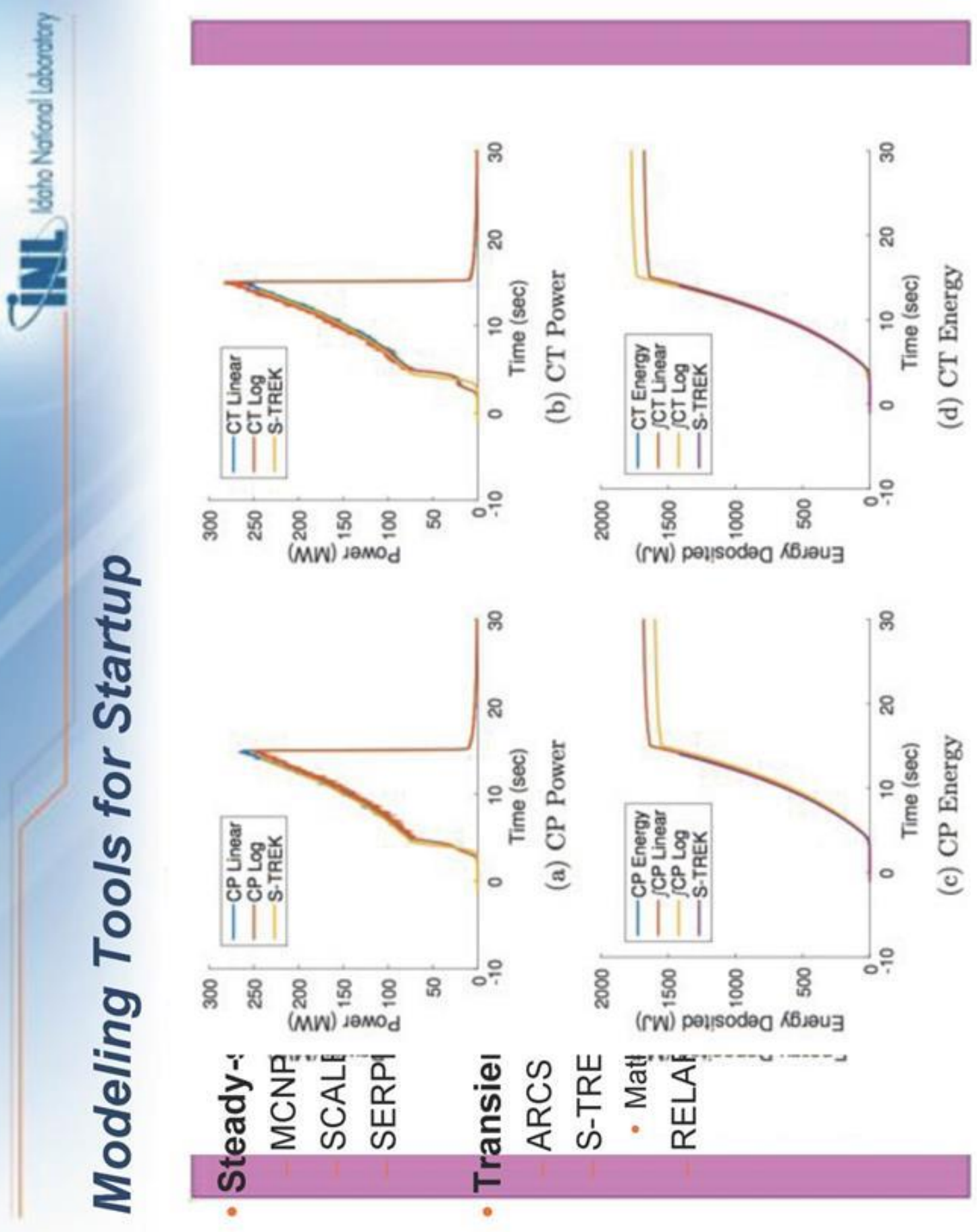


N.

()

E

(1)

a)

ช 

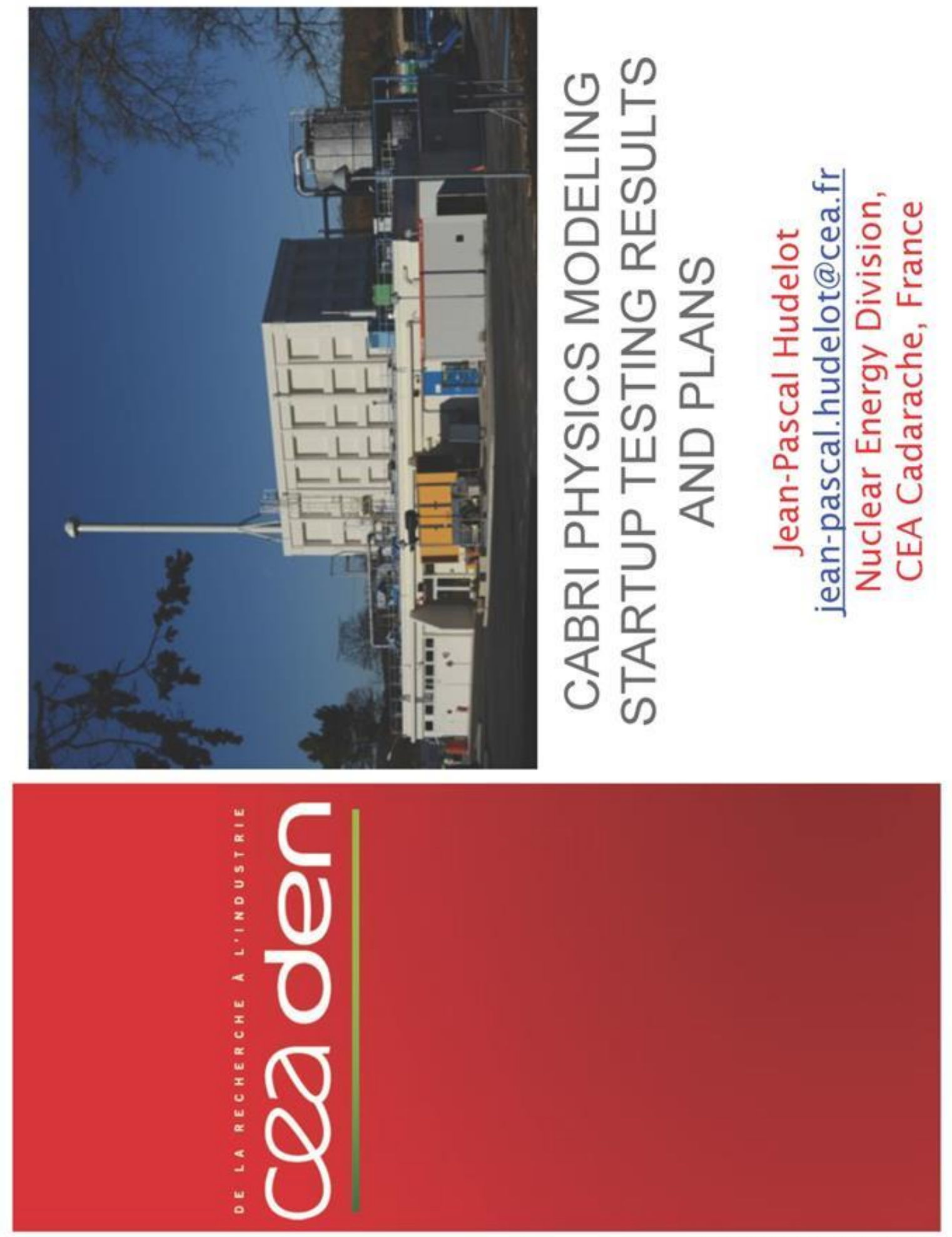


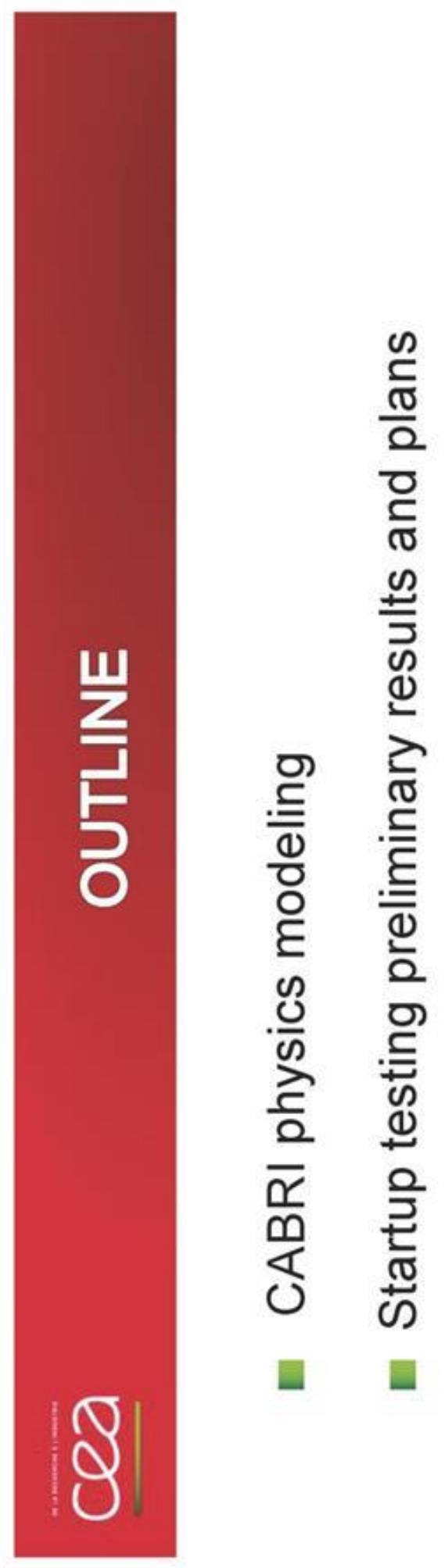




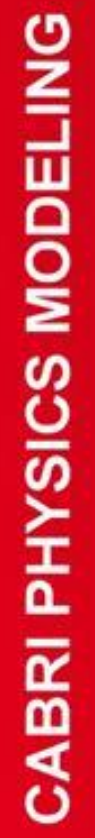



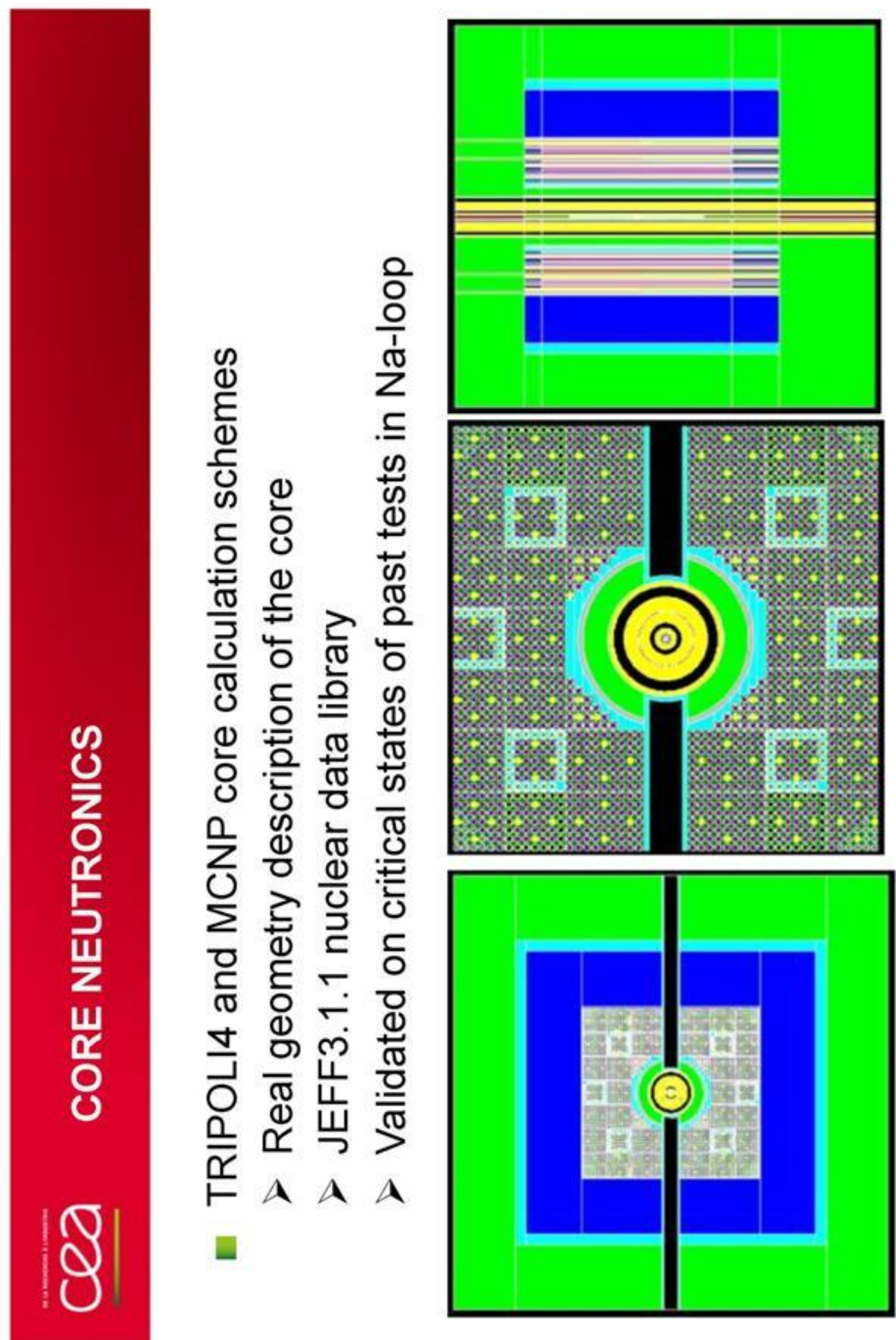

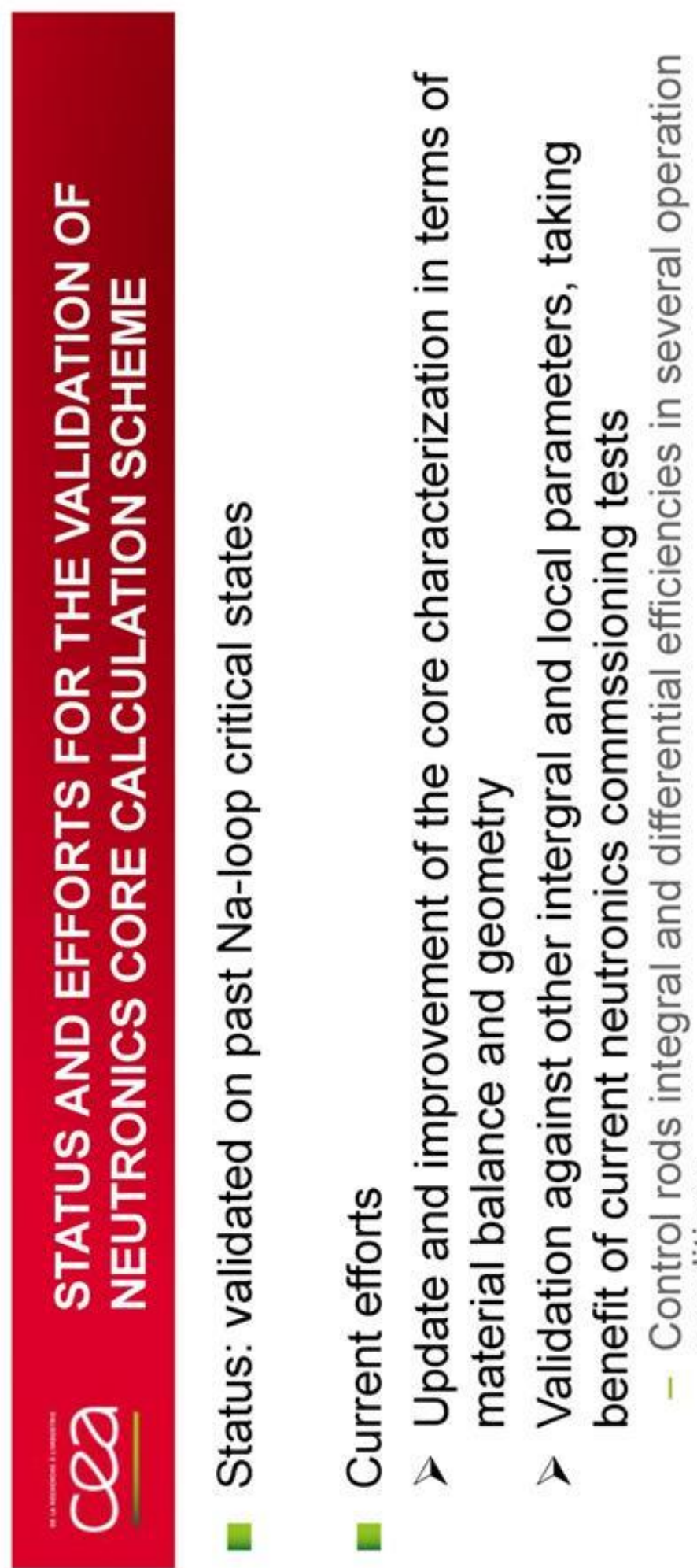

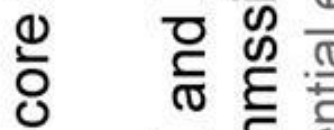

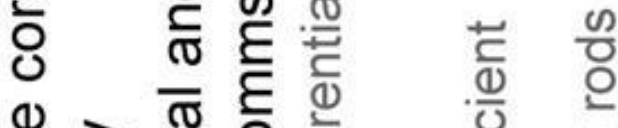

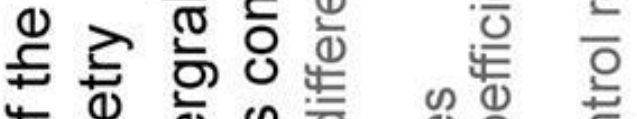

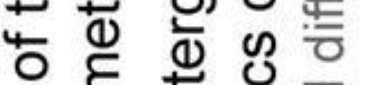

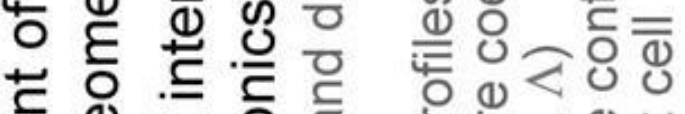

ฮั ฮั่ 은

을 ๓

×

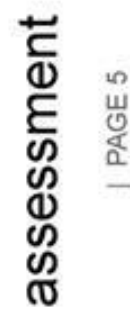

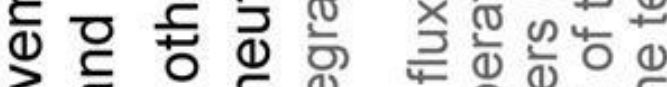

은 ญ

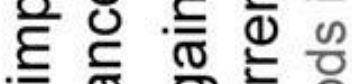

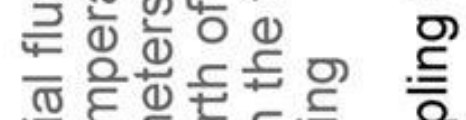

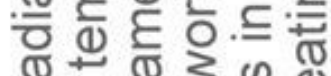

진중

$\frac{\pi}{\frac{\pi}{0}}$

त)

으

芒

产

응

훙

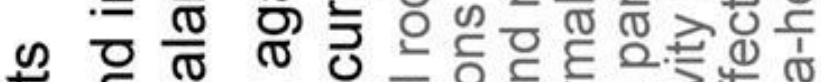

는

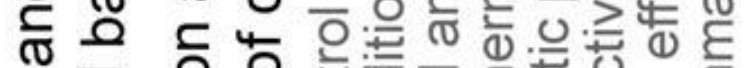

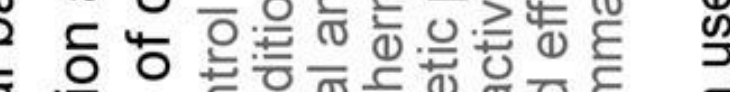

ळ

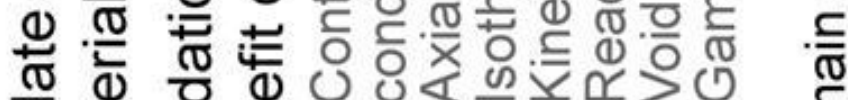

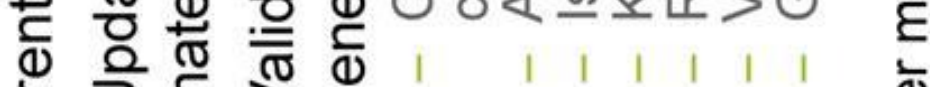

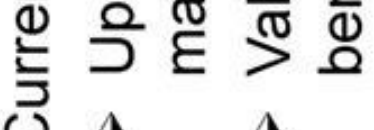

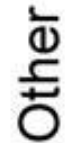




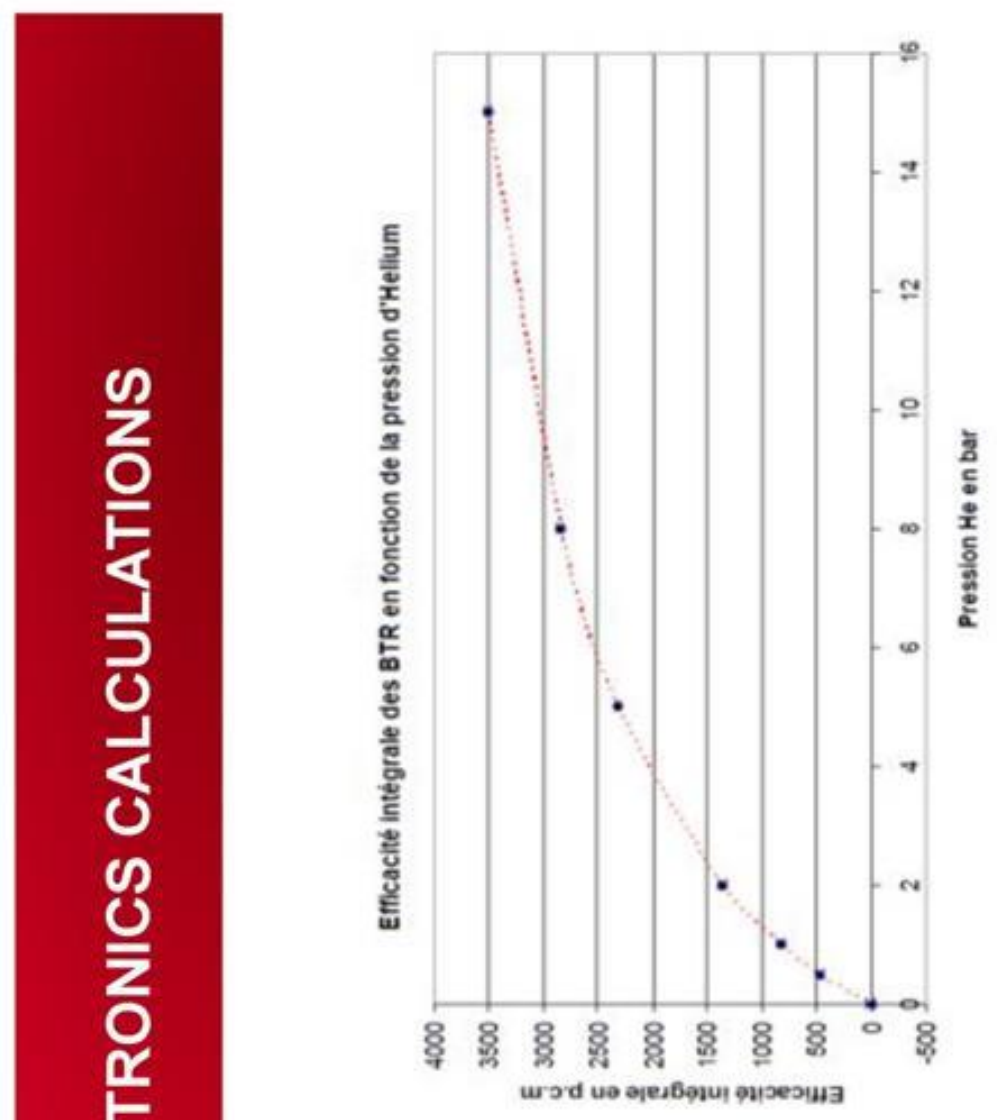

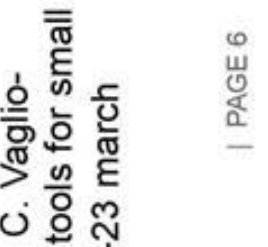

థ๐ 의

心 $\frac{\pi}{5}$

口 $\frac{0}{\pi}$ ฮ

त उ

닌 은

은 는

ㄷํㄹㅇํํ

. 눙

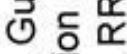

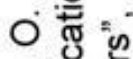

넨 논

공 ฮ

() 읃

-

울을

幽 믄 믄

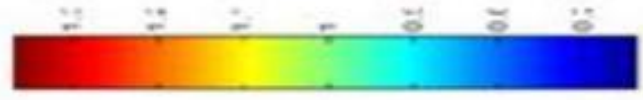

II.

ด $\frac{\pi}{7}$

ن 芒

ธัฒ

○
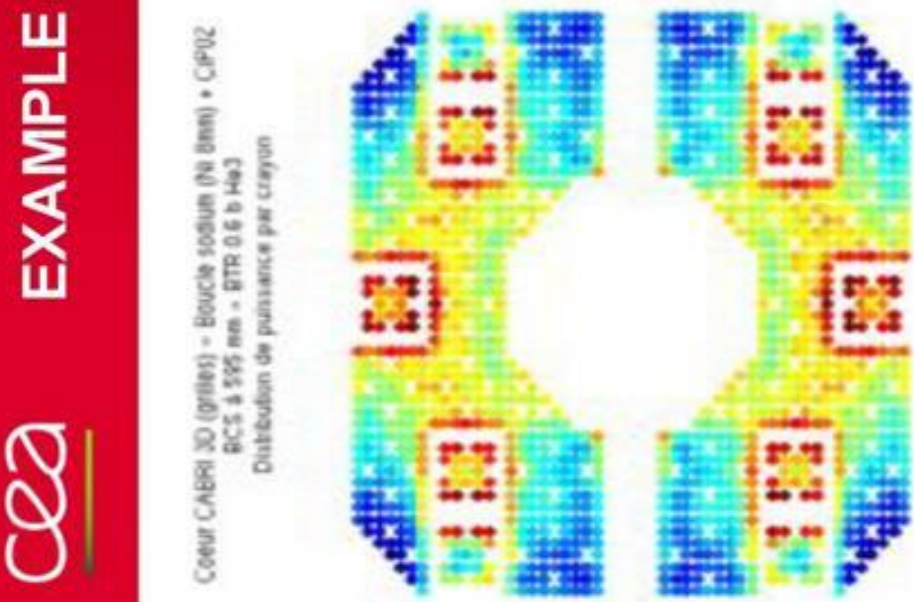

을음 뚜

응

บัด

눙ㅇ

흐는든

잉

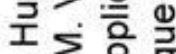

ํํㅇ ซ

$\because$ क 응

0

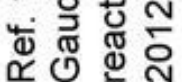




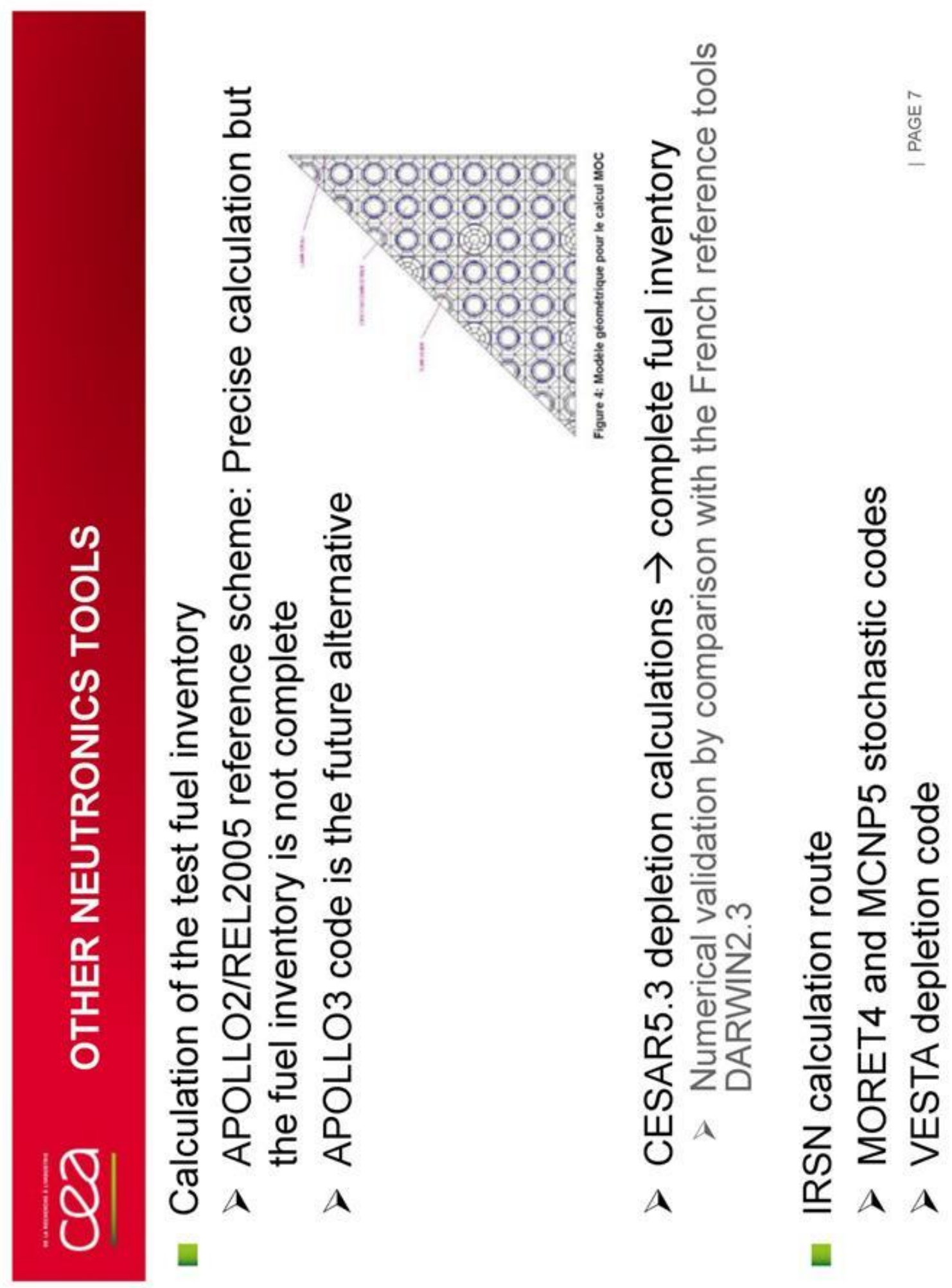




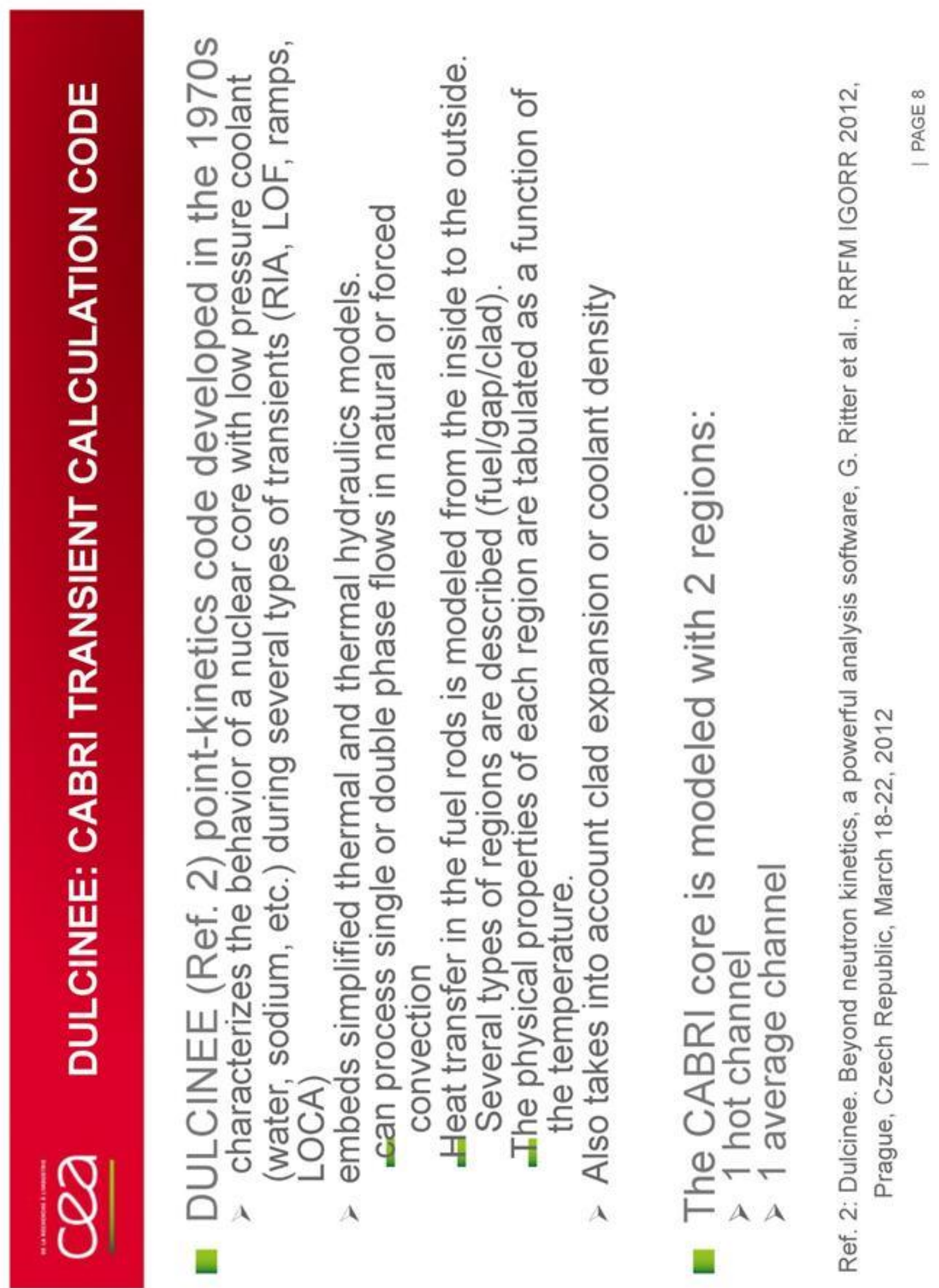




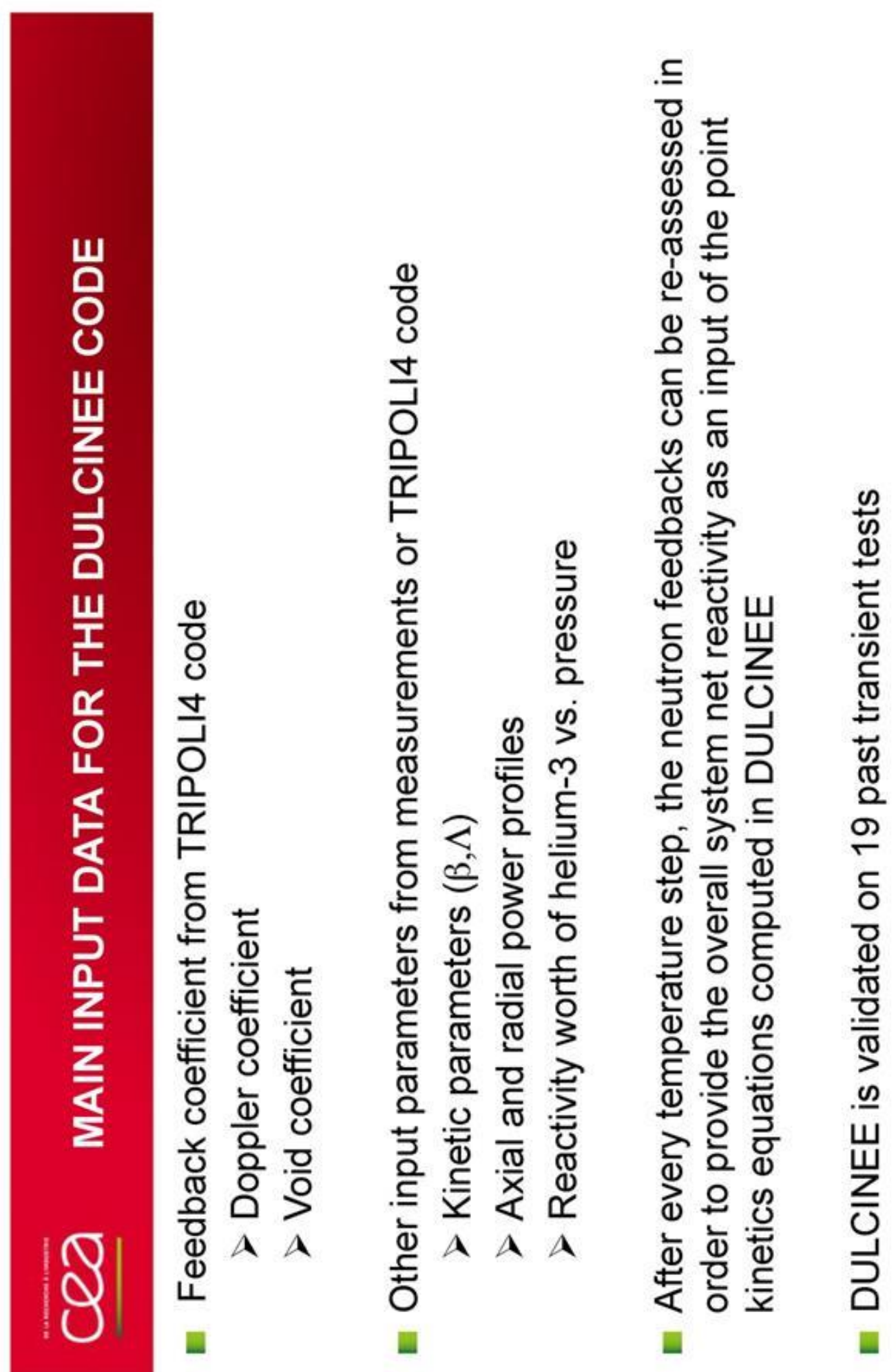




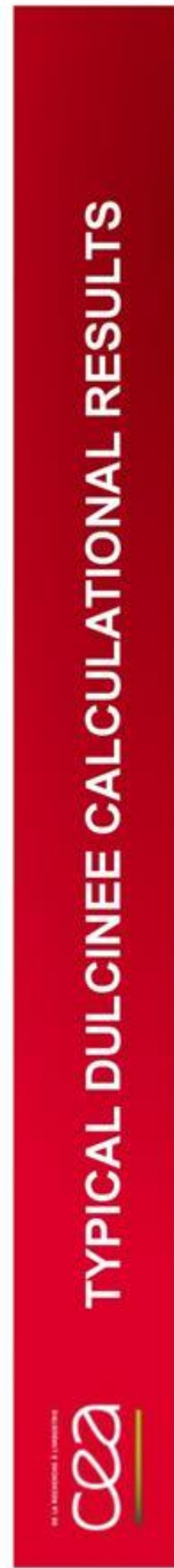

$$
\begin{aligned}
& \text { b.| aunzespduas } \\
& \begin{array}{lllllll}
8 & 8 & 8 & 8 & 8 & 8 & 8
\end{array} \\
& \text { 음 }
\end{aligned}
$$
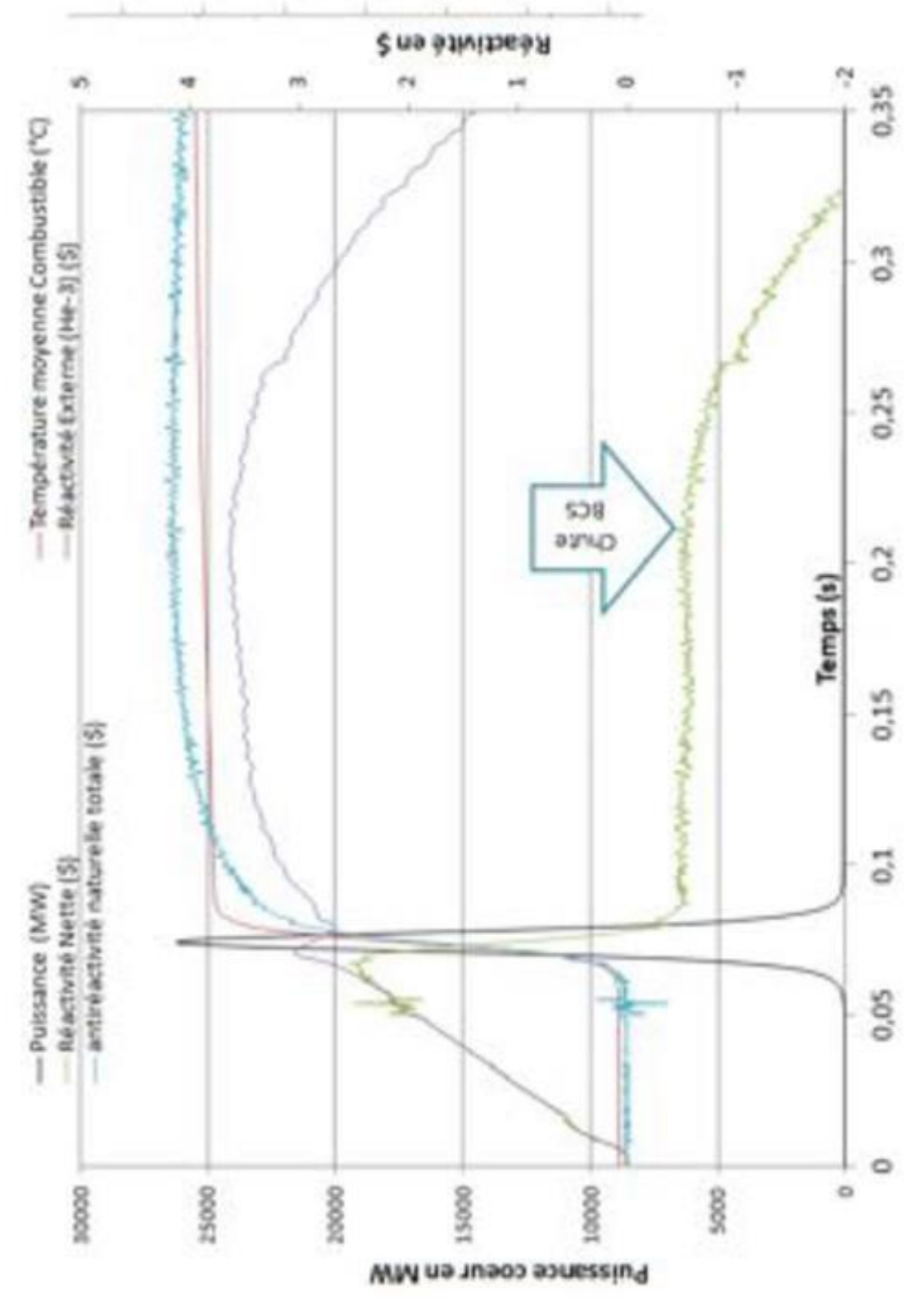


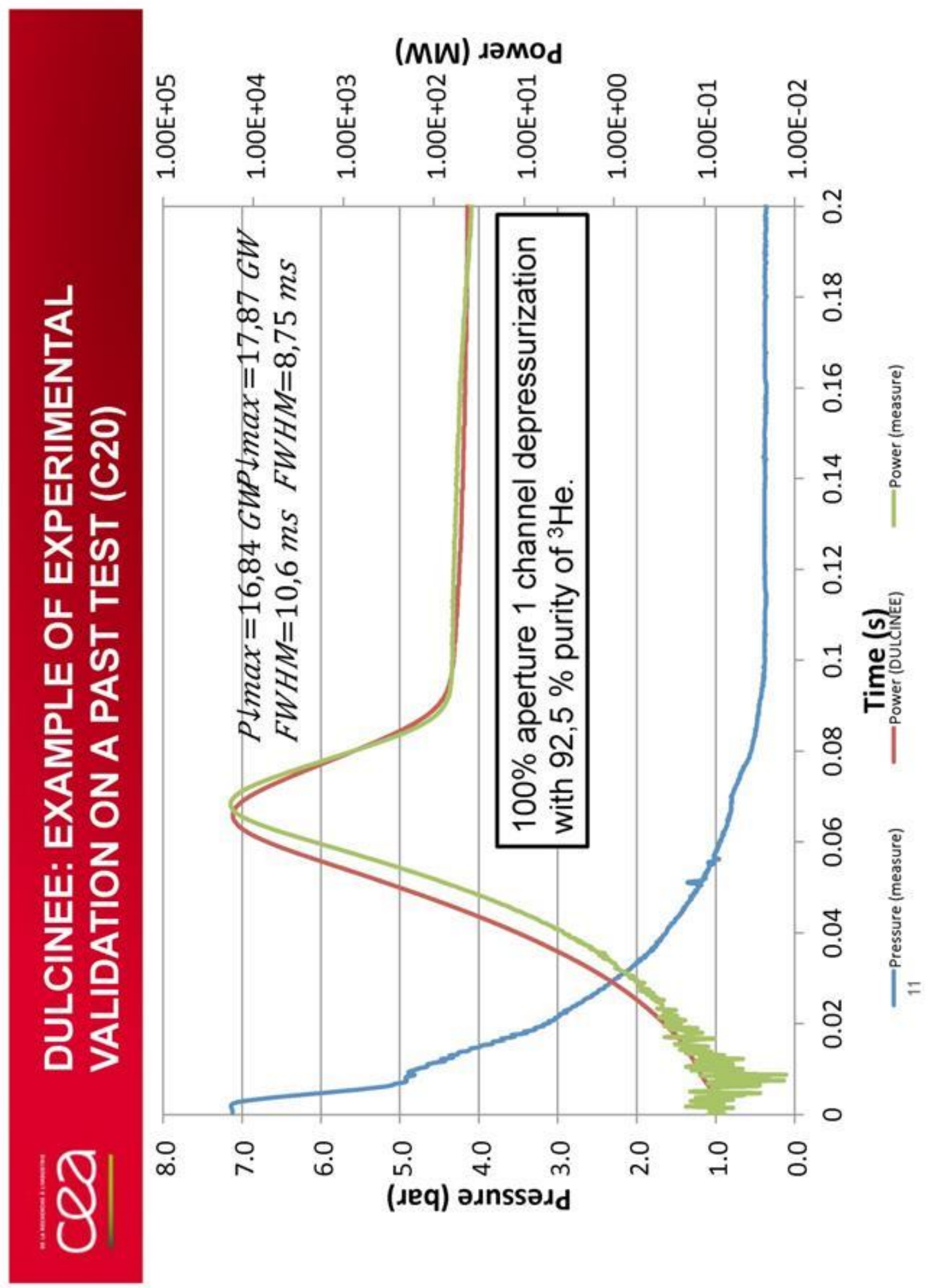



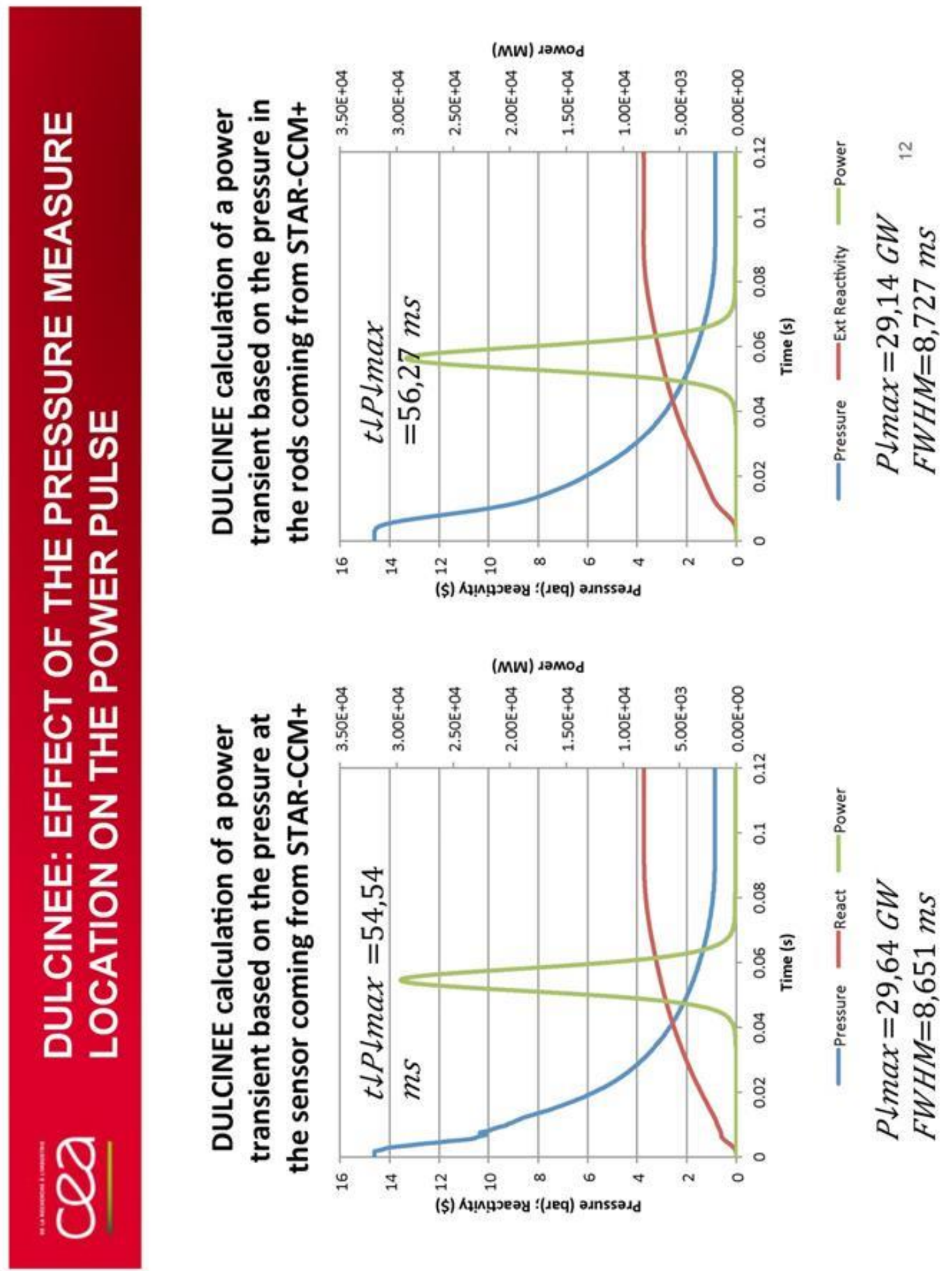


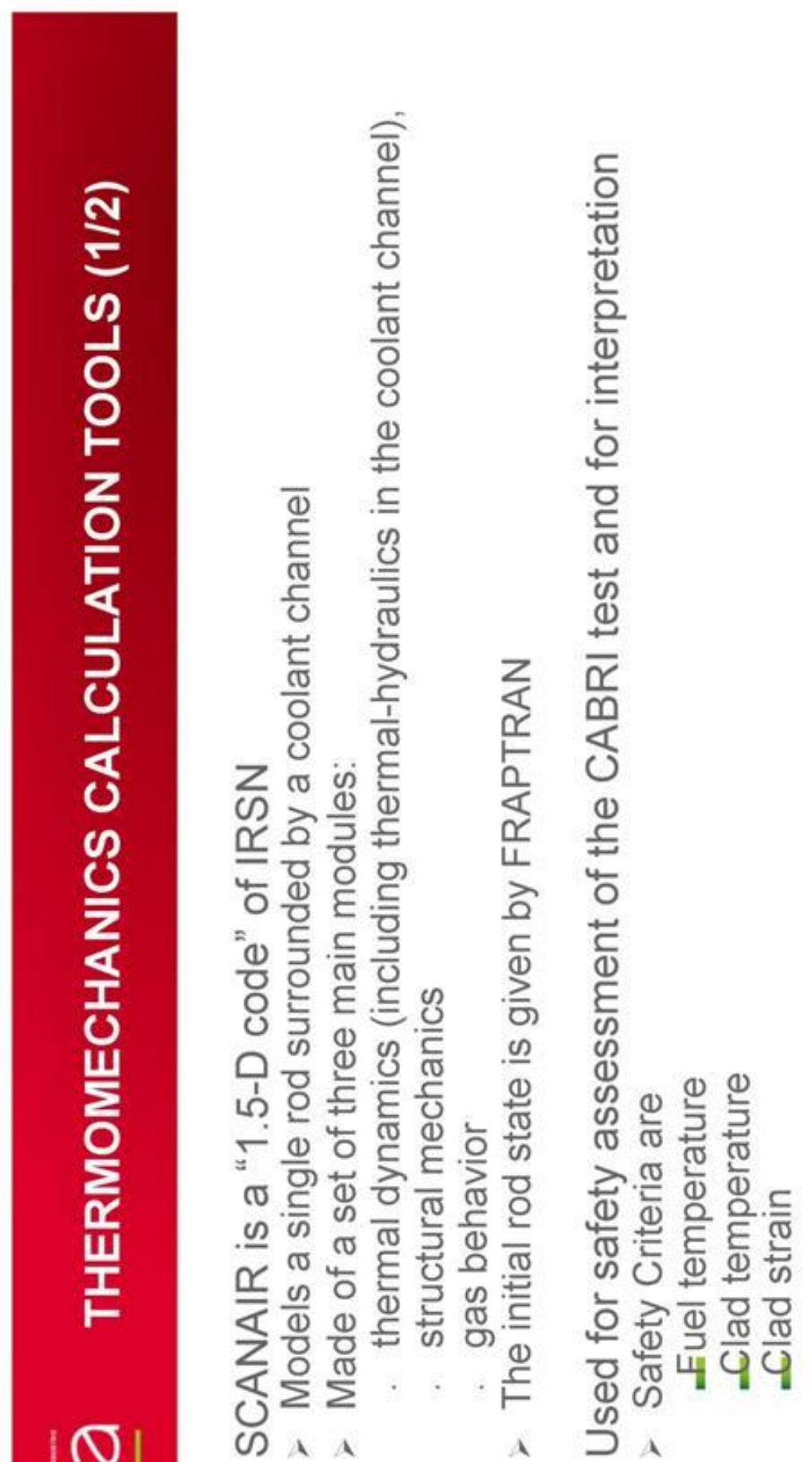

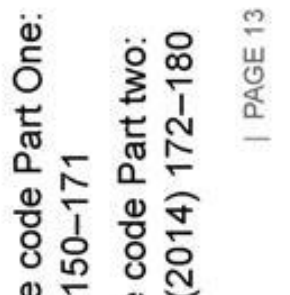

Ð

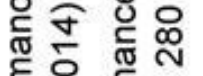

ํํㅇ 으 ह등

농요원

ฮ등 흐

는 흔

동 능 이

뜬

๙

ช 등 뚜

뻥 뜬

之绻

ธิ

फ़

등 के

ชั้

은 $=$ 은

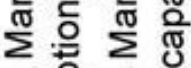

0 은 0 은

हี ญ

일응

产

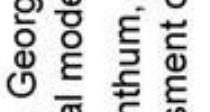

$>$ त्ष

远芯

市

范 范 

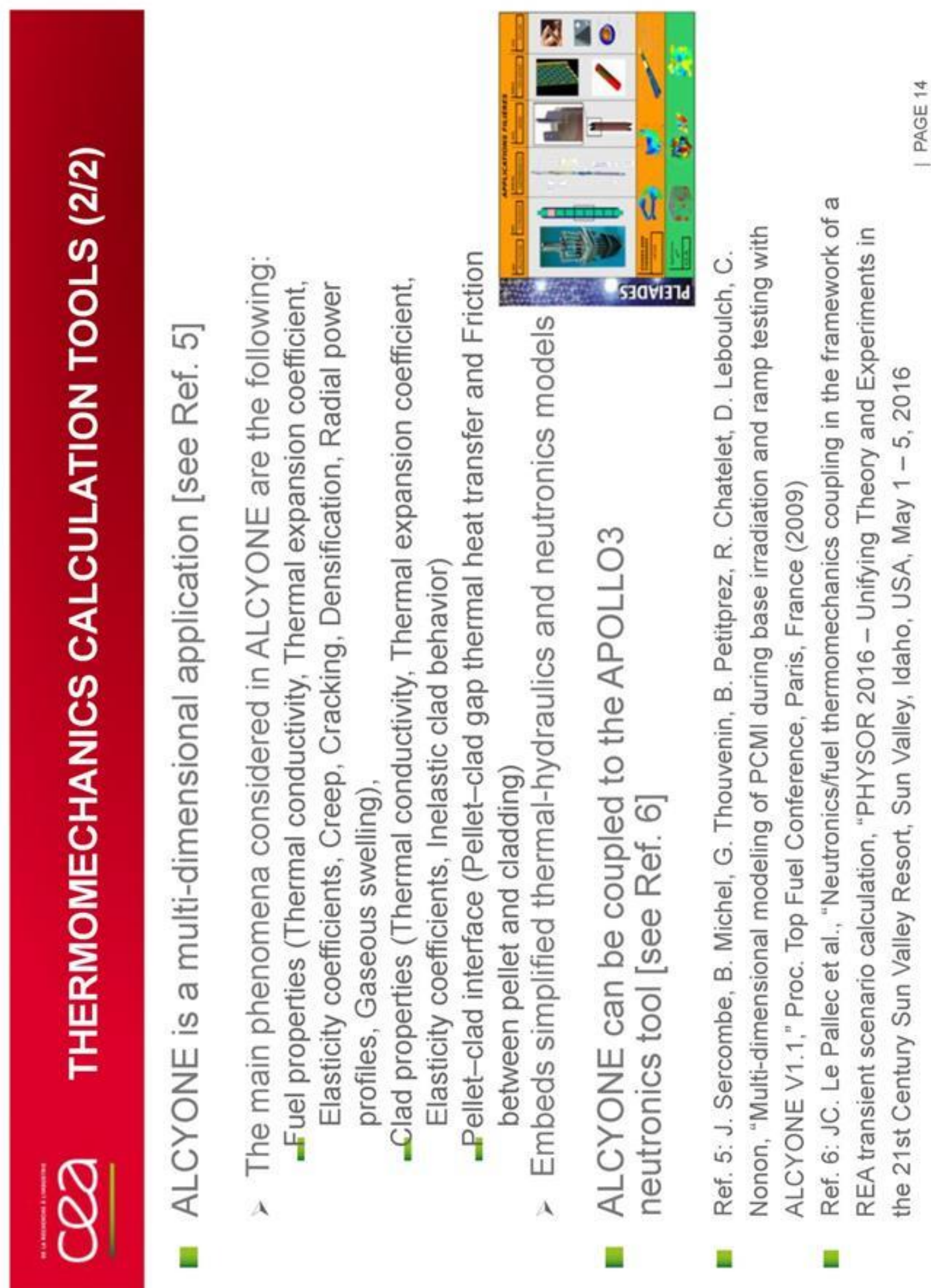

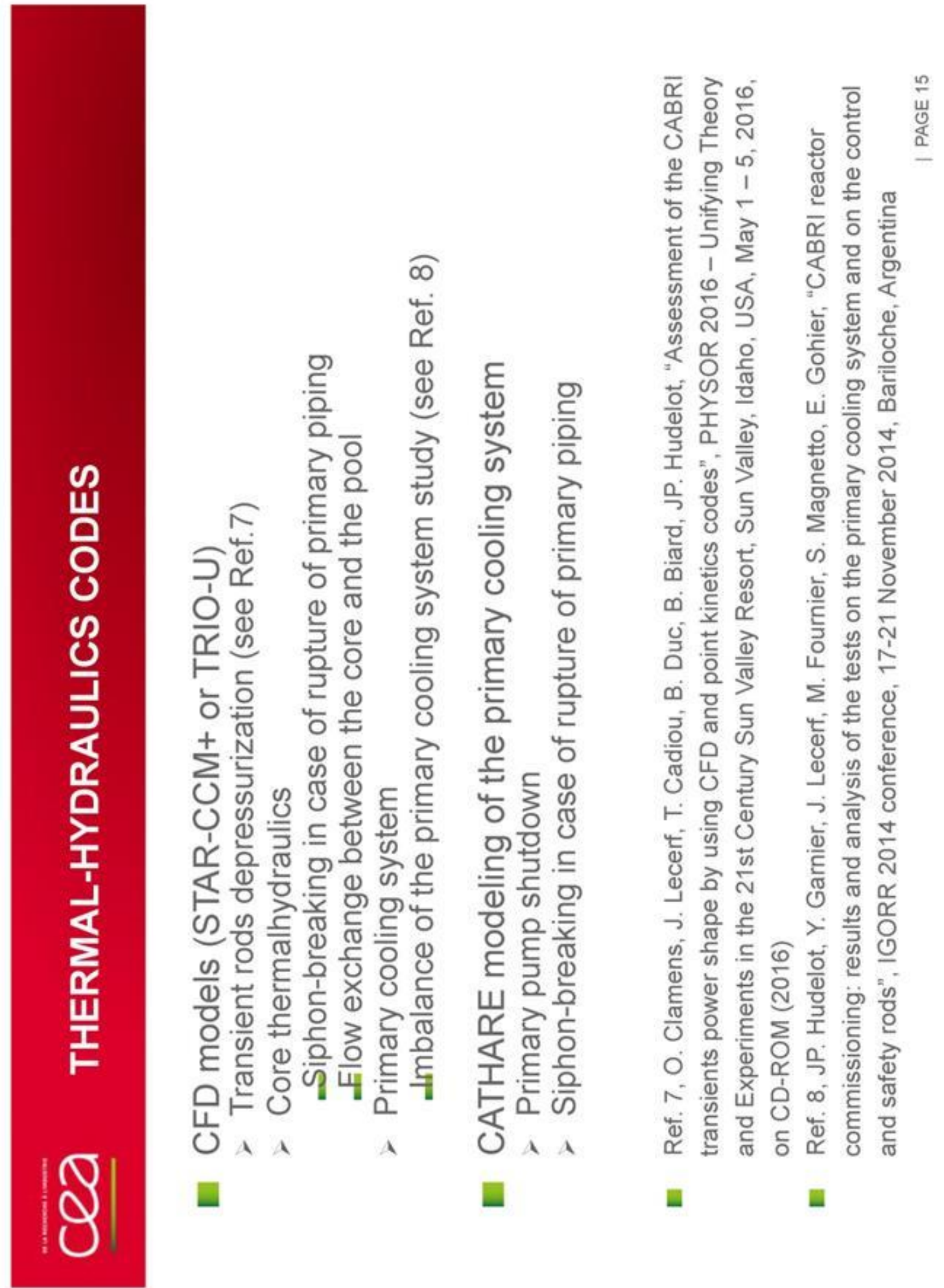

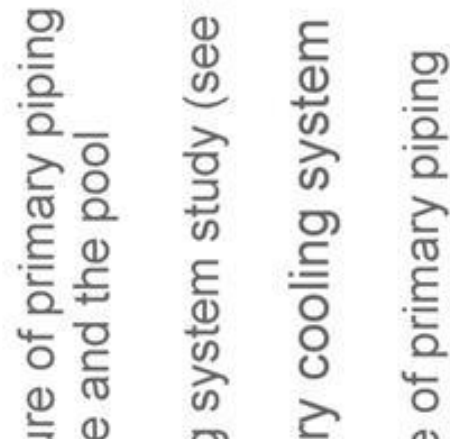

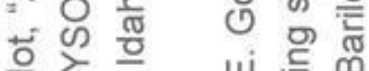

응

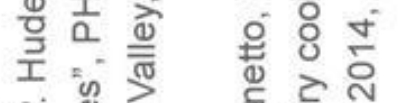

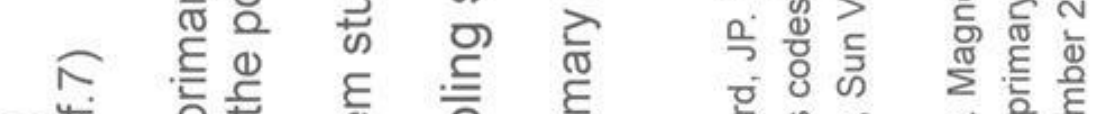

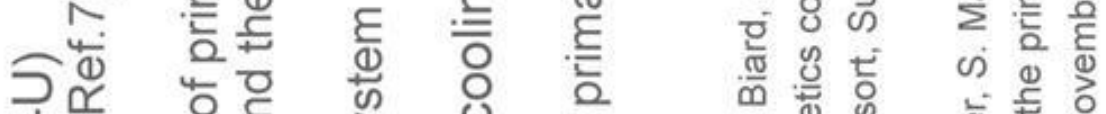

仓่

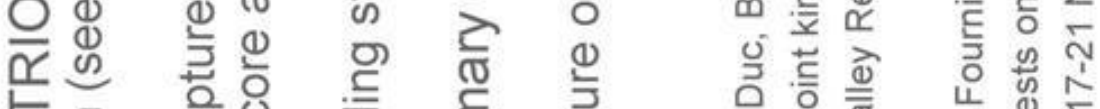

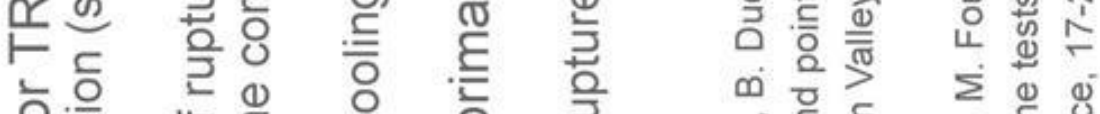
ㅎํ유 늉 茎督

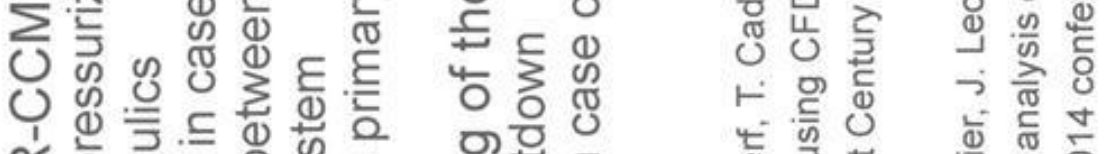

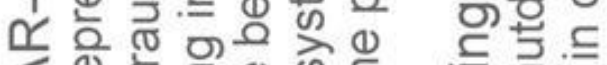

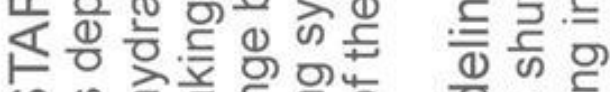

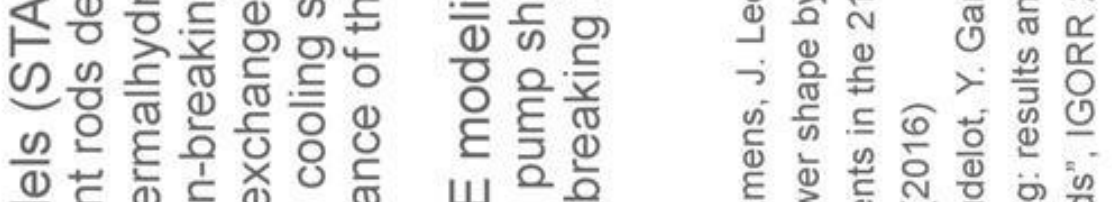

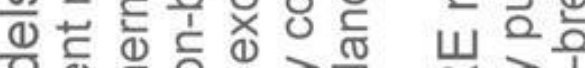
응.

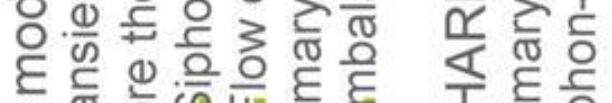

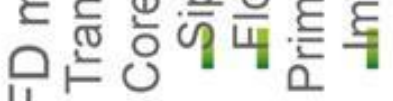
U । 도든 은 है

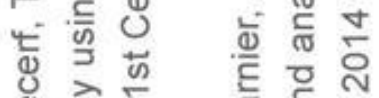
ฮั ล $\checkmark$ (1)

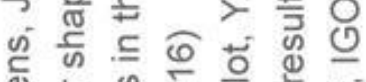

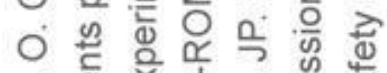
‥ 뜬

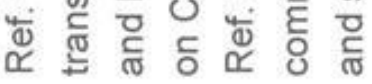




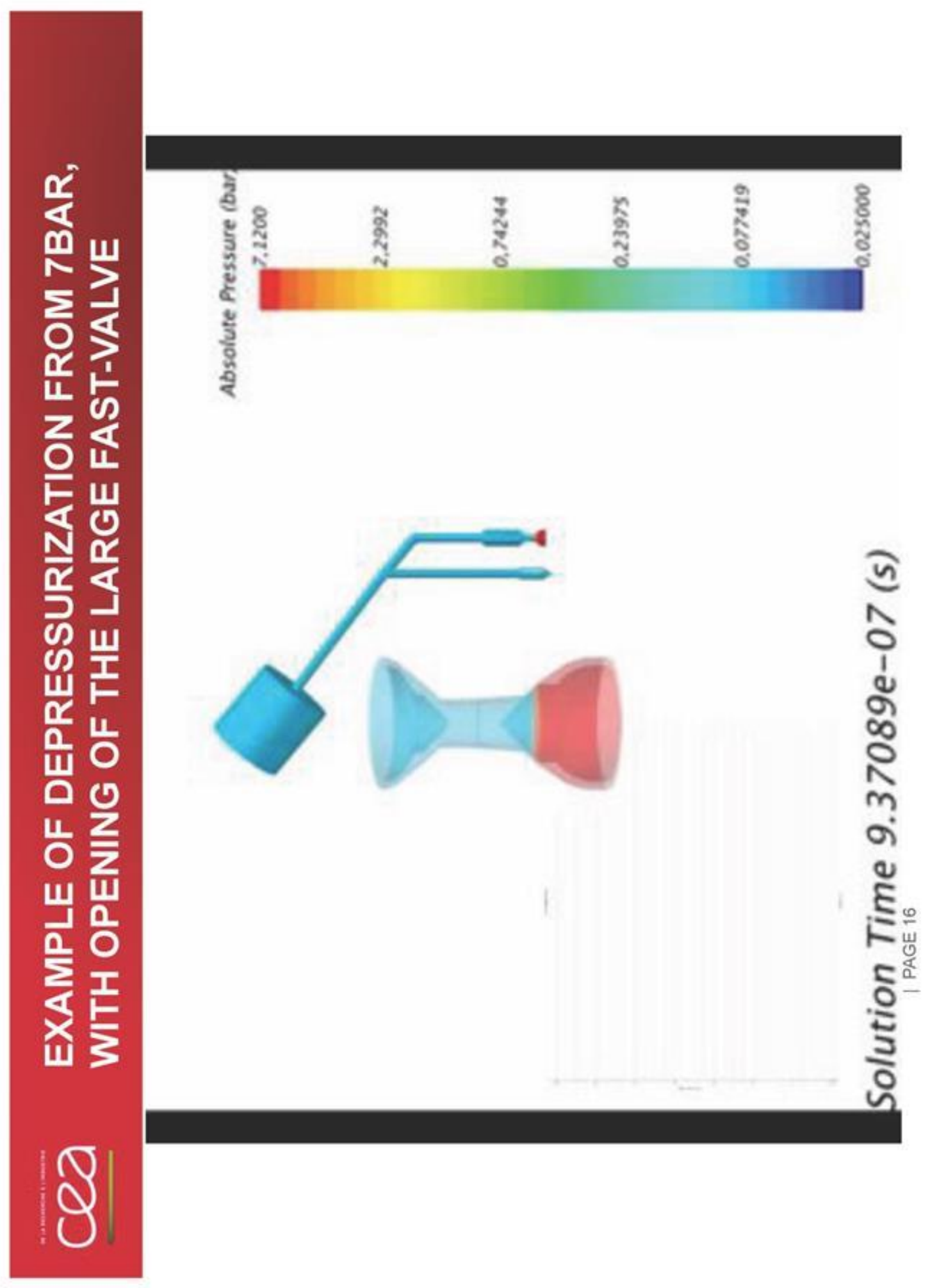



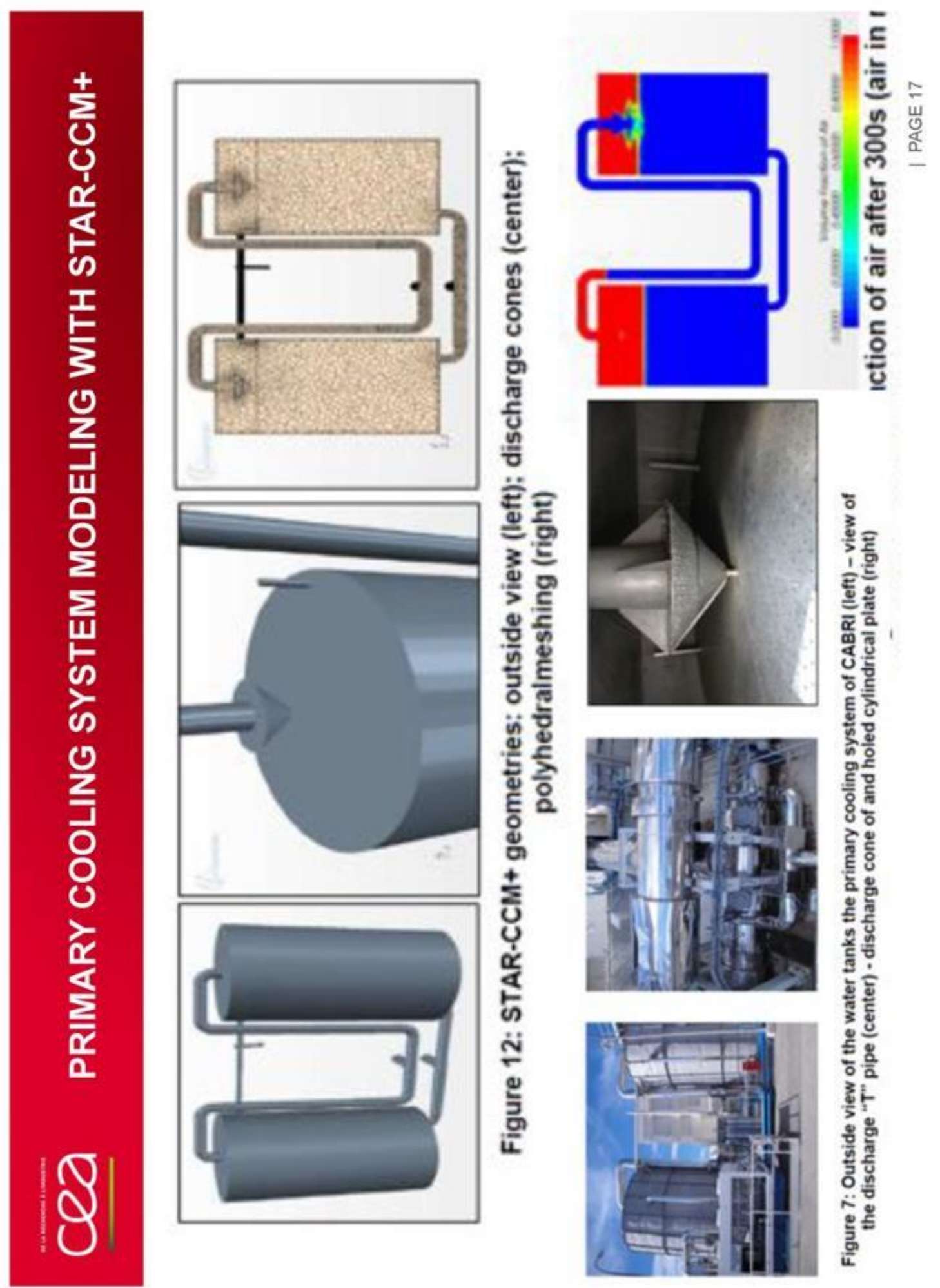

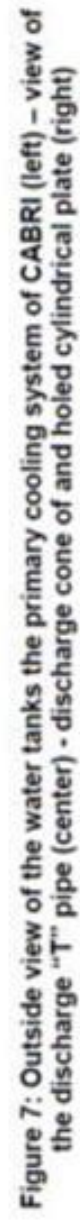



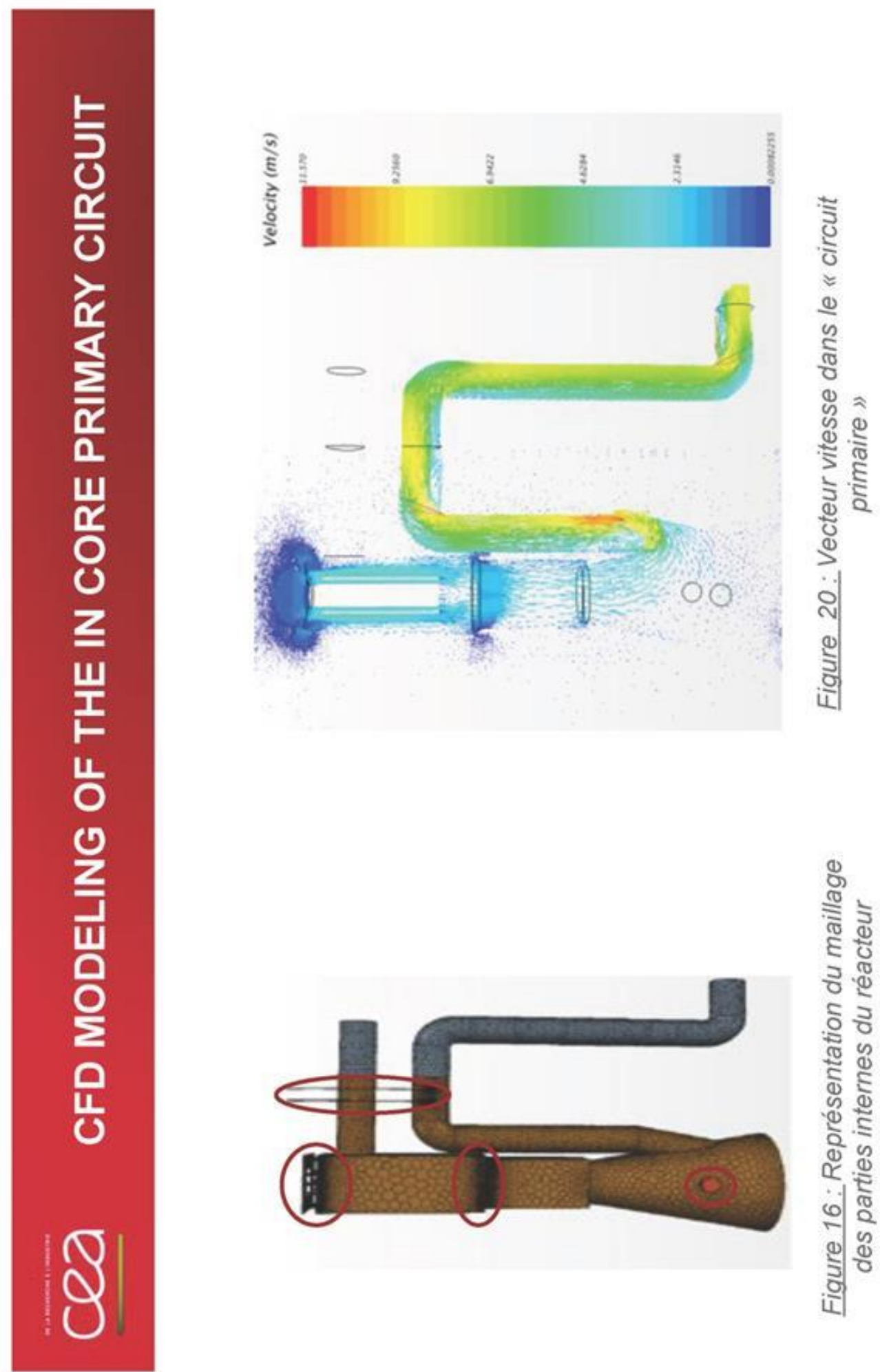

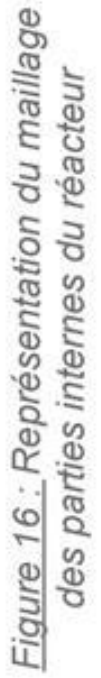



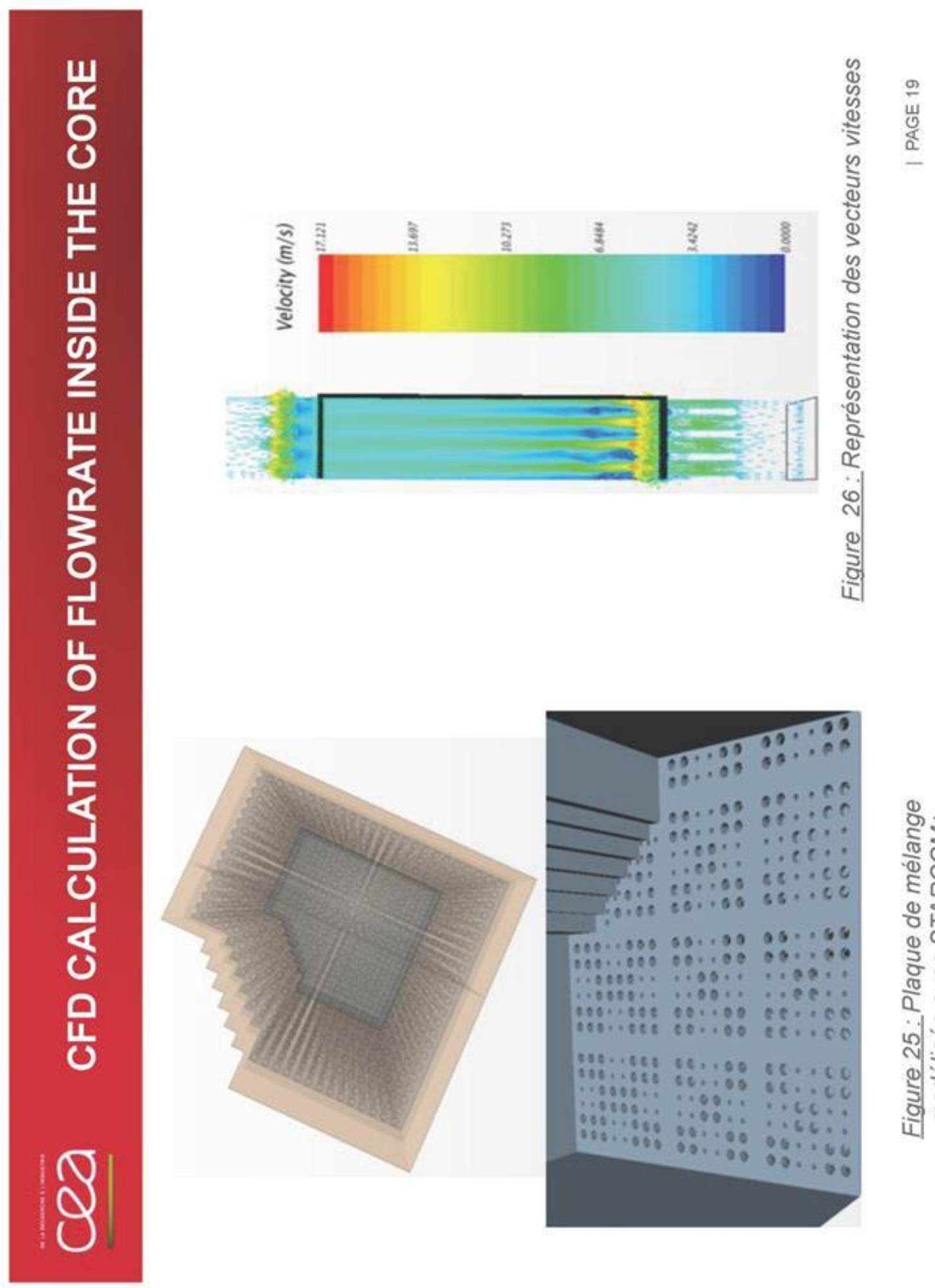

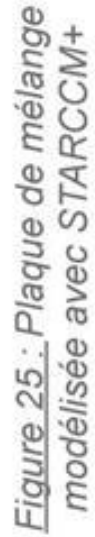



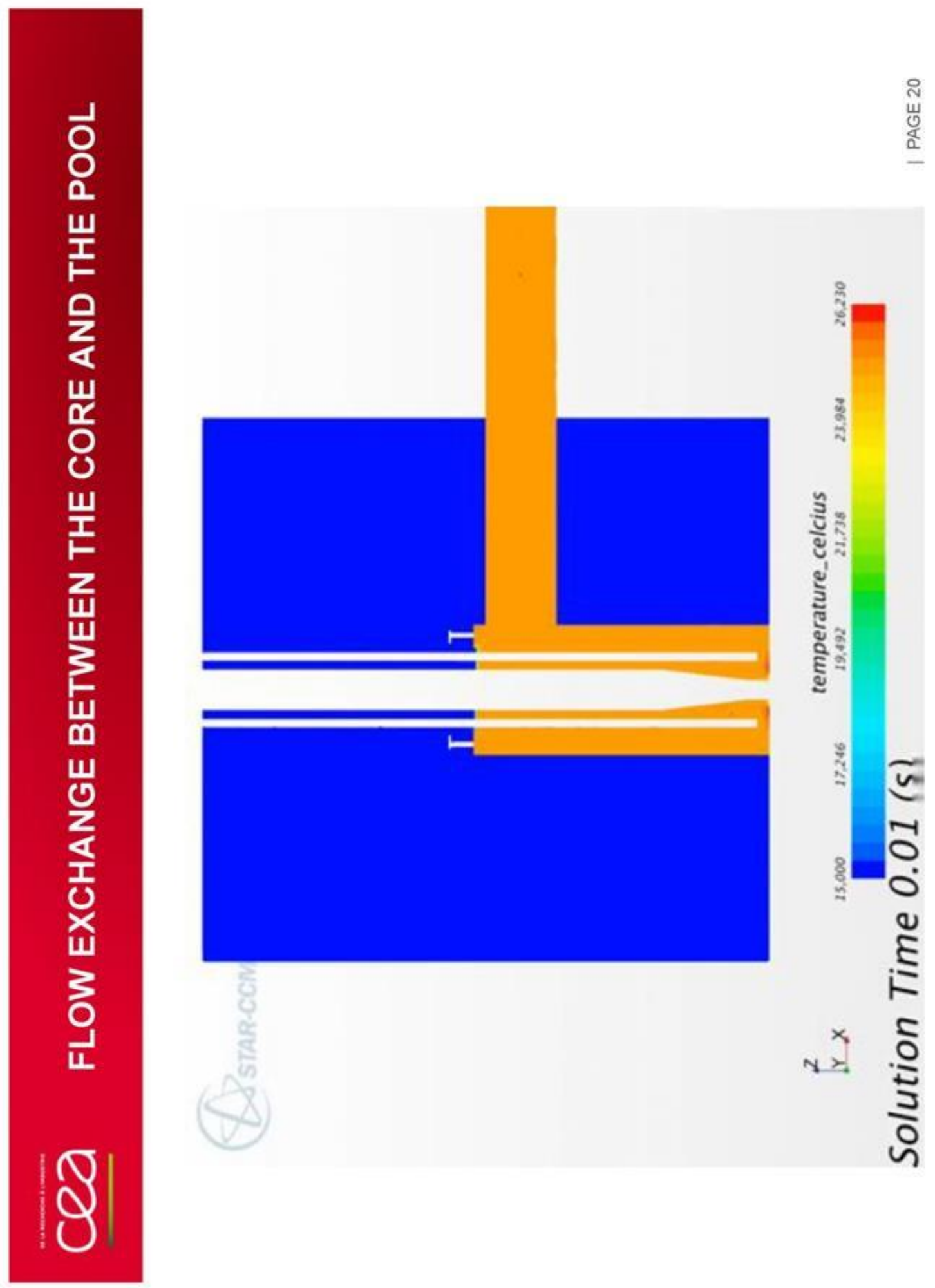


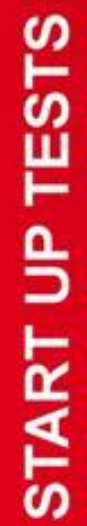




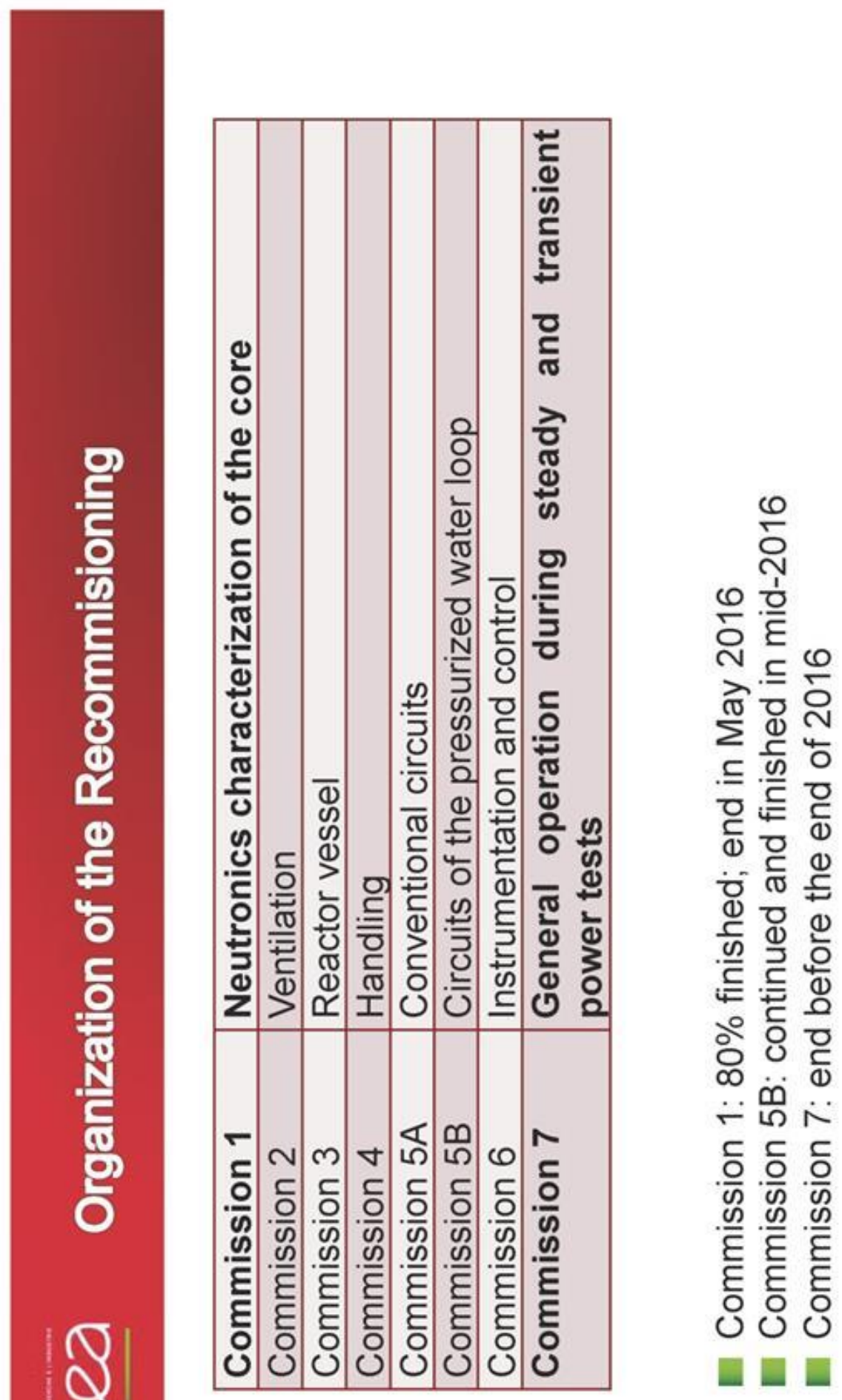



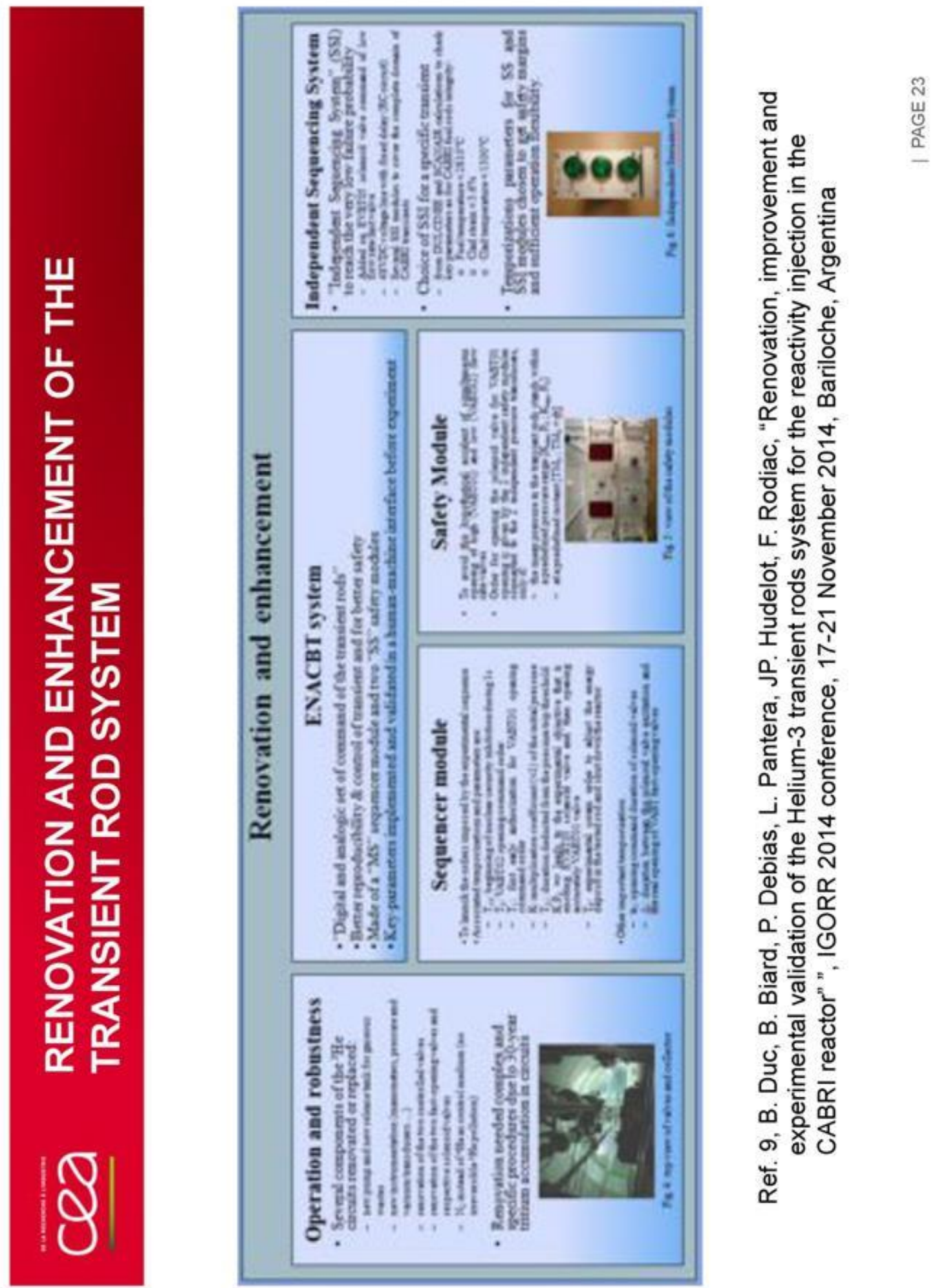

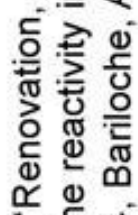
똥 뜬휴 흥 $\propto \Phi$ แ 命 흥 음 은 흥든 픈도 문 $\frac{0}{5}$ ธี่ केष ส ‥ 응 工 ஸั 능 뭉 도음 응 휴음 믈 =

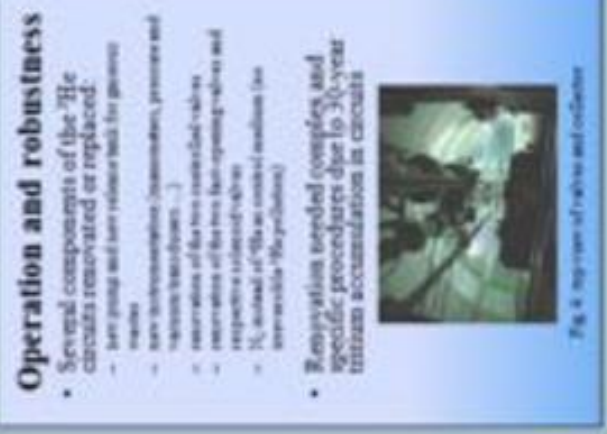
뜬 ๓ $\overline{ }$ บํํำ ํㅡㄴ 즌 ๓ क ख ष्் 


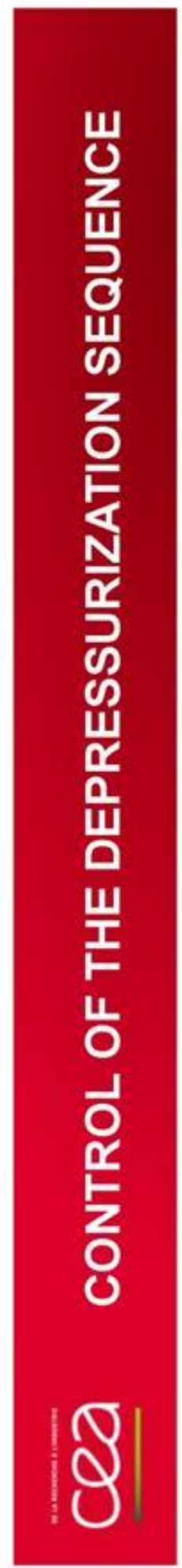

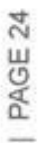

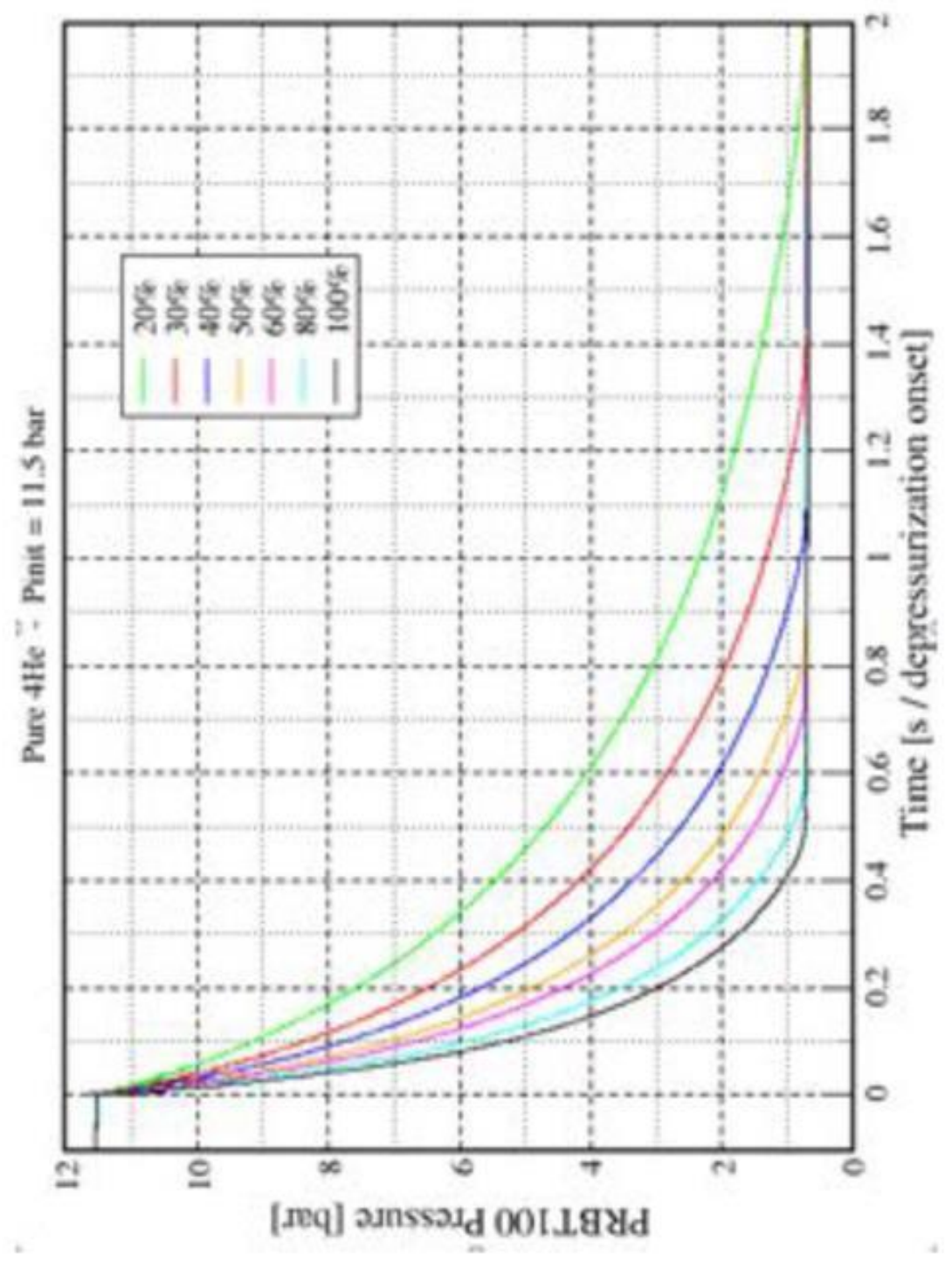



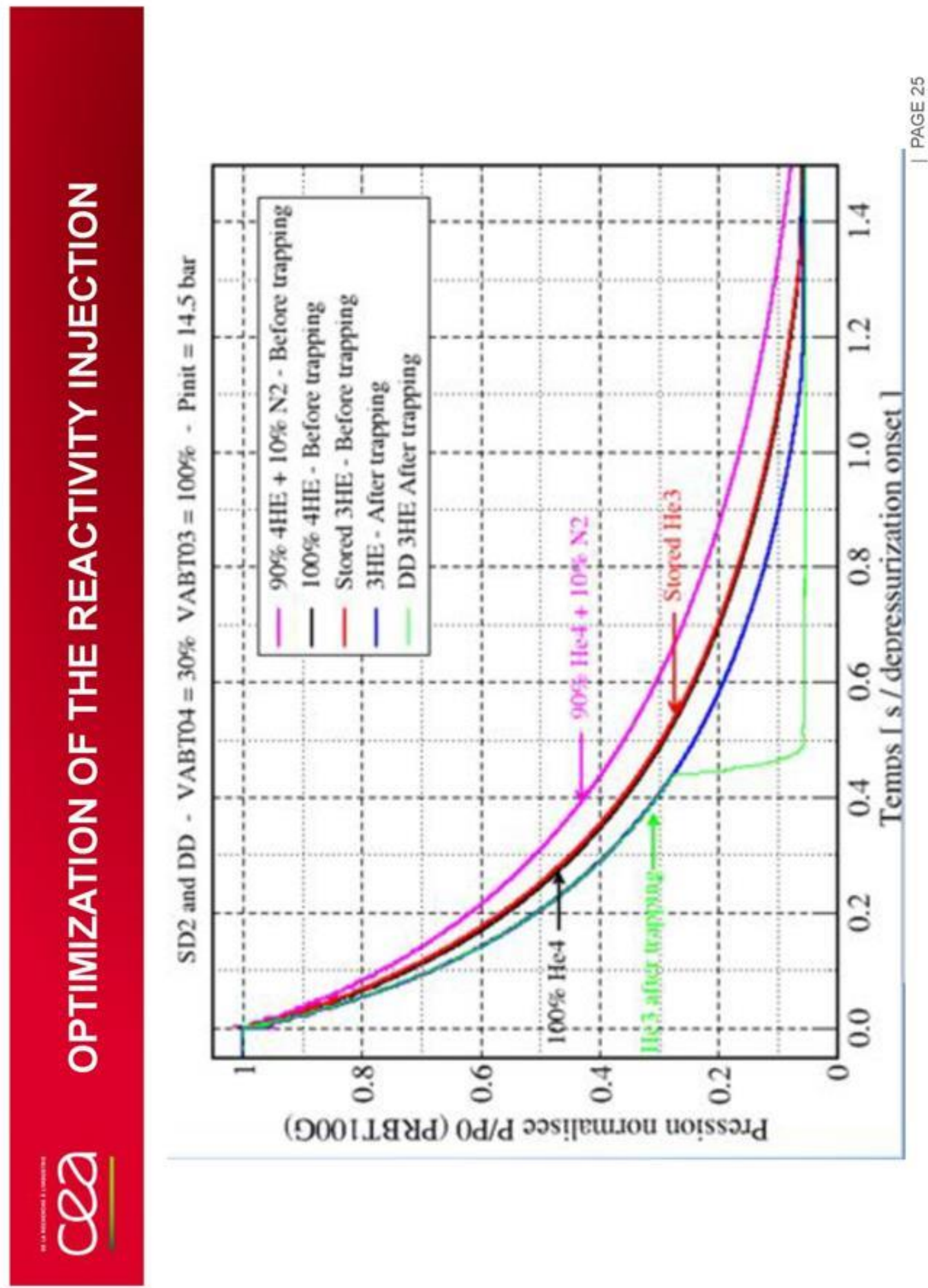


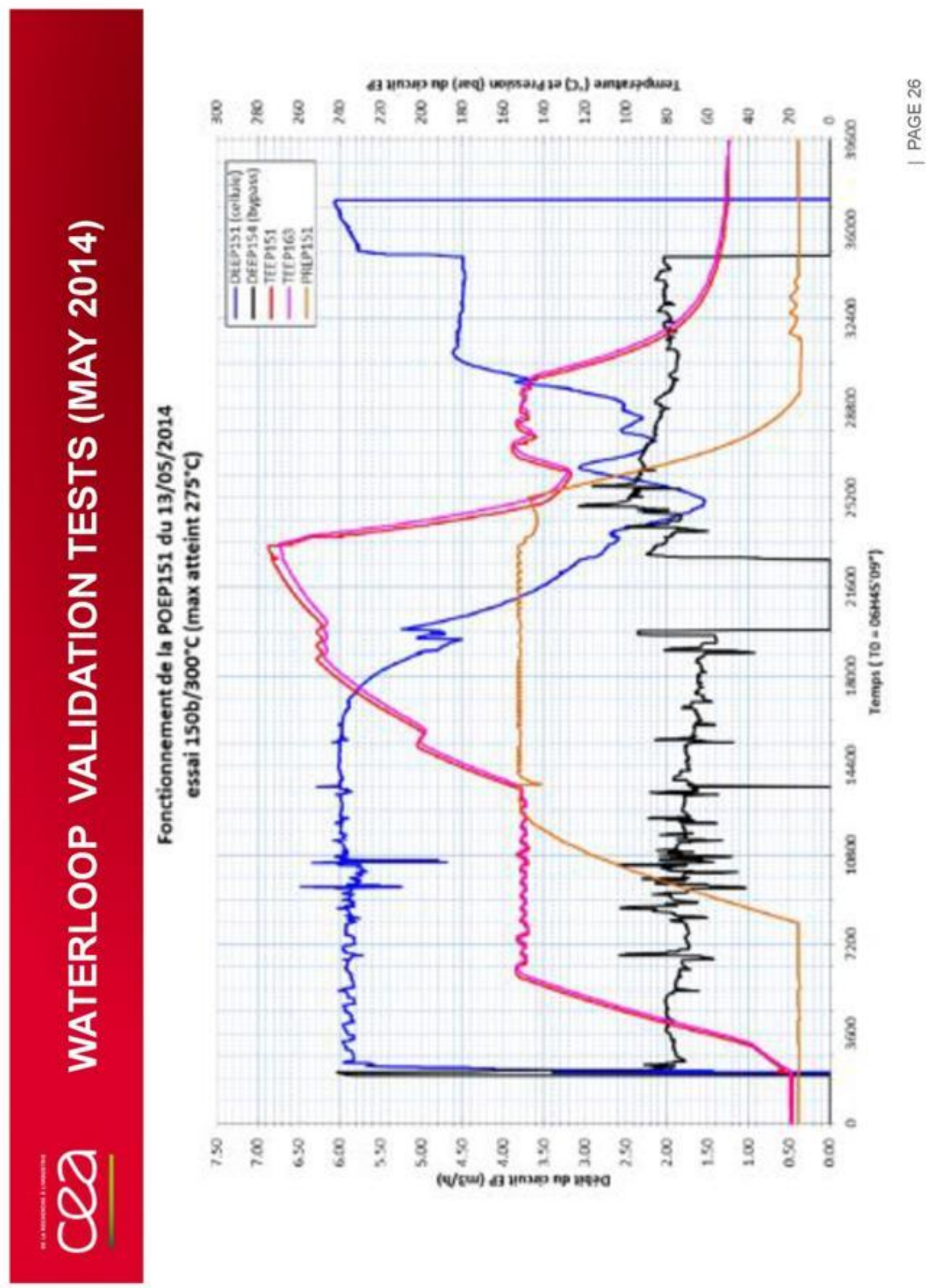




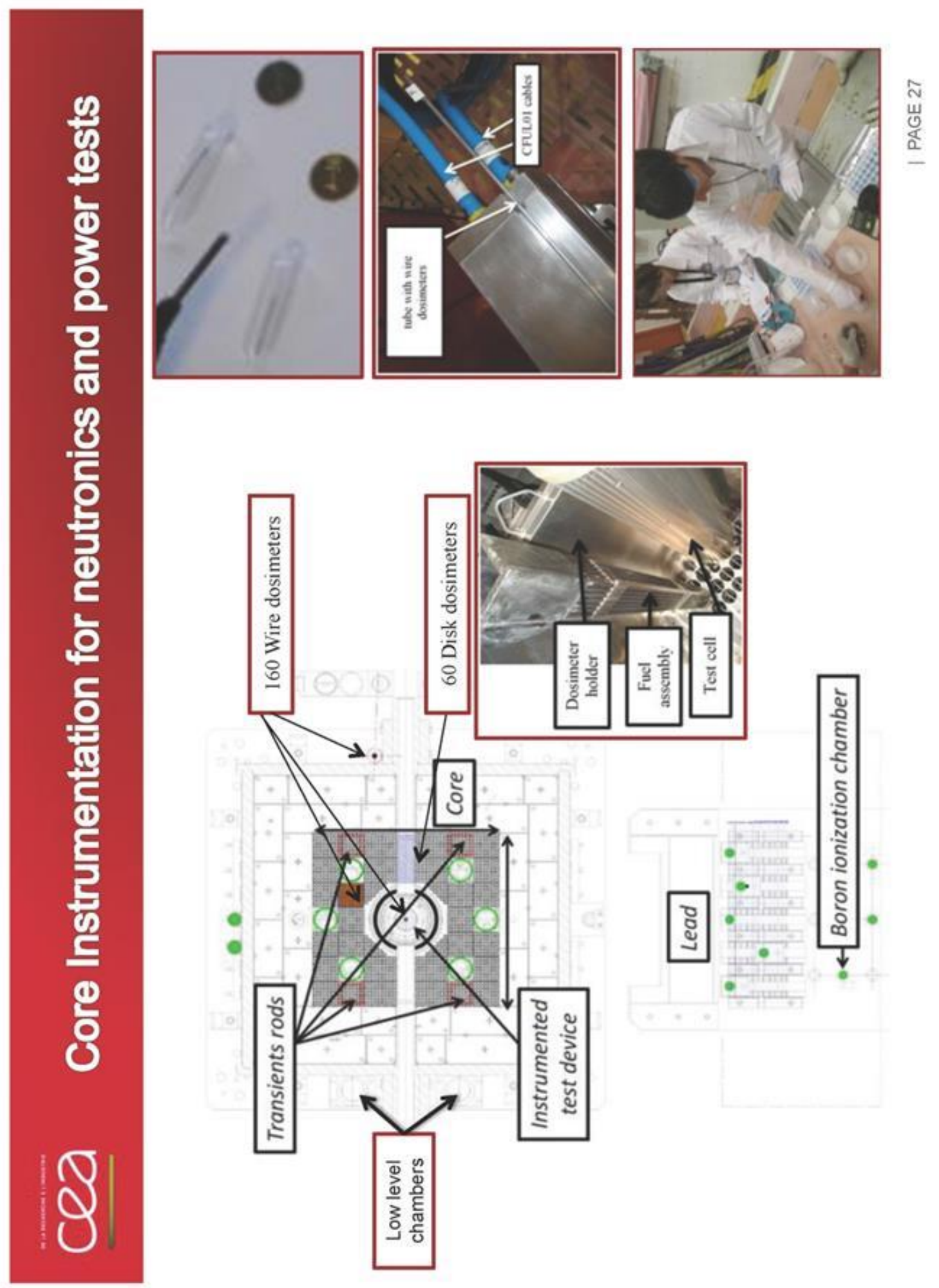




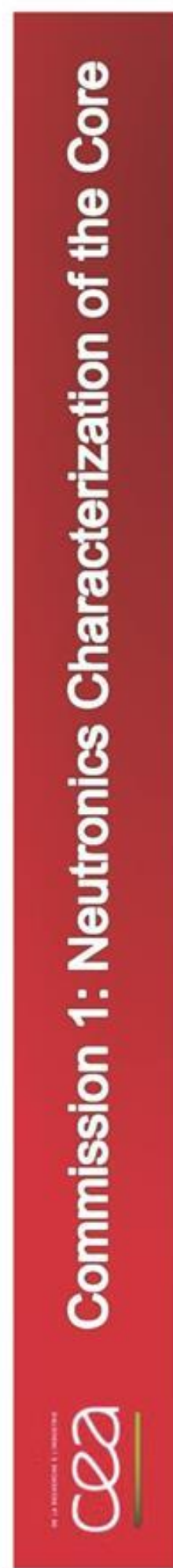

号

$\Delta$

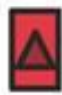

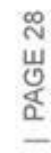

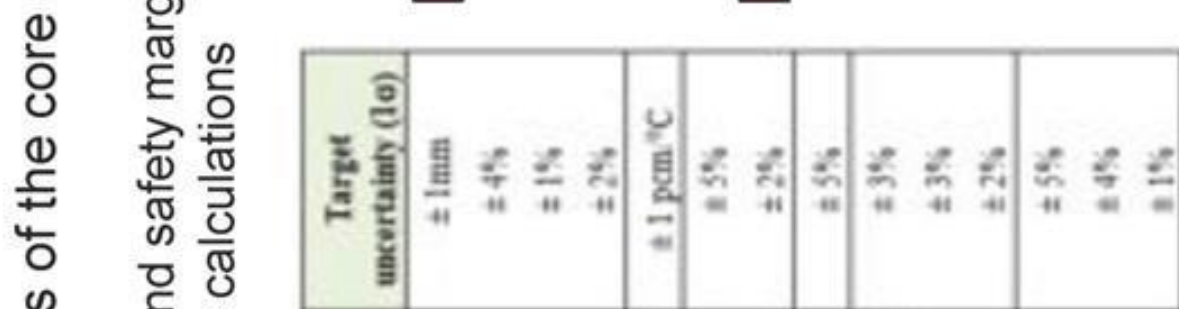

느 음

峁

어

है है क

은

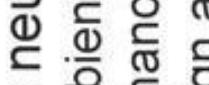

() है ह 은

든ํํ

行 范

드욯

흥 은

닌

U 넌

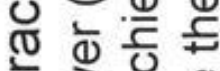

ฮั

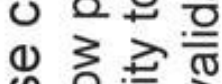

뜨으 흥

넌요은

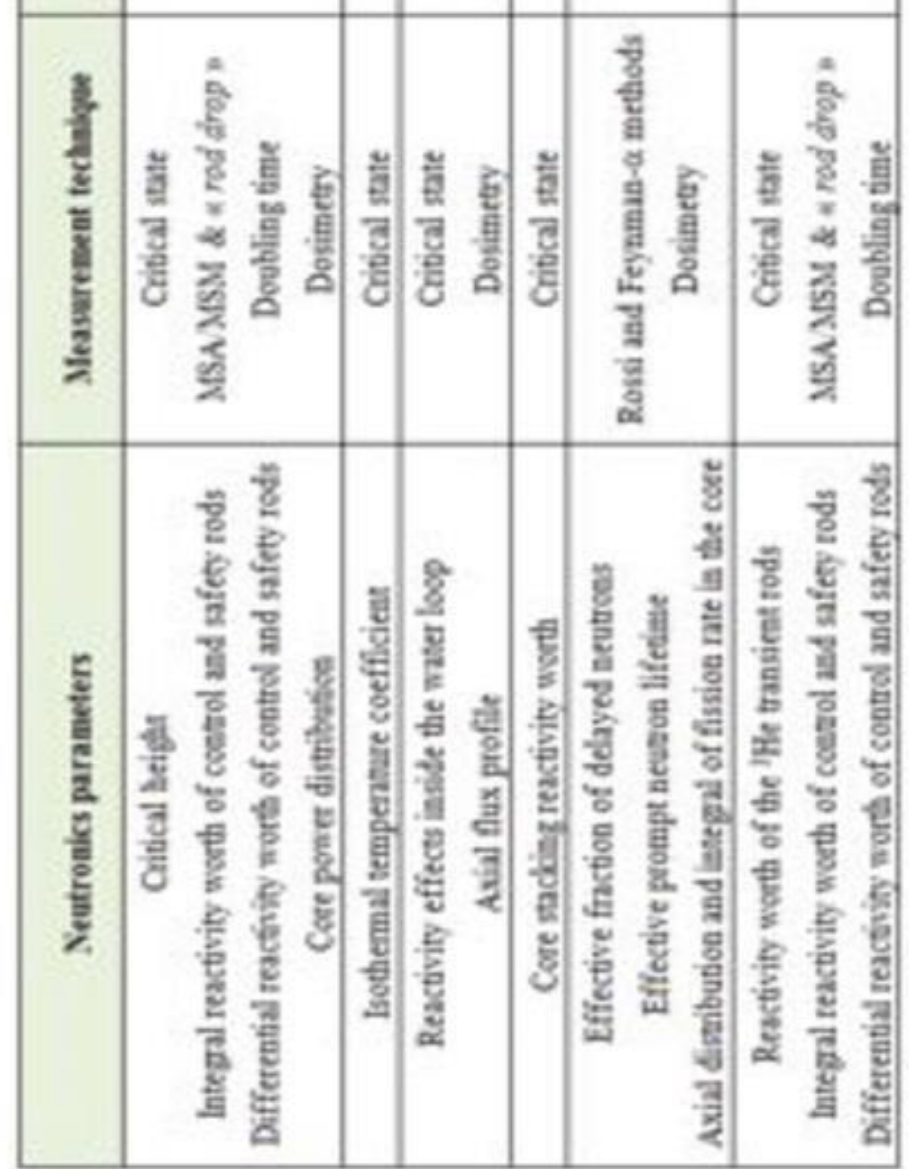

느 A 


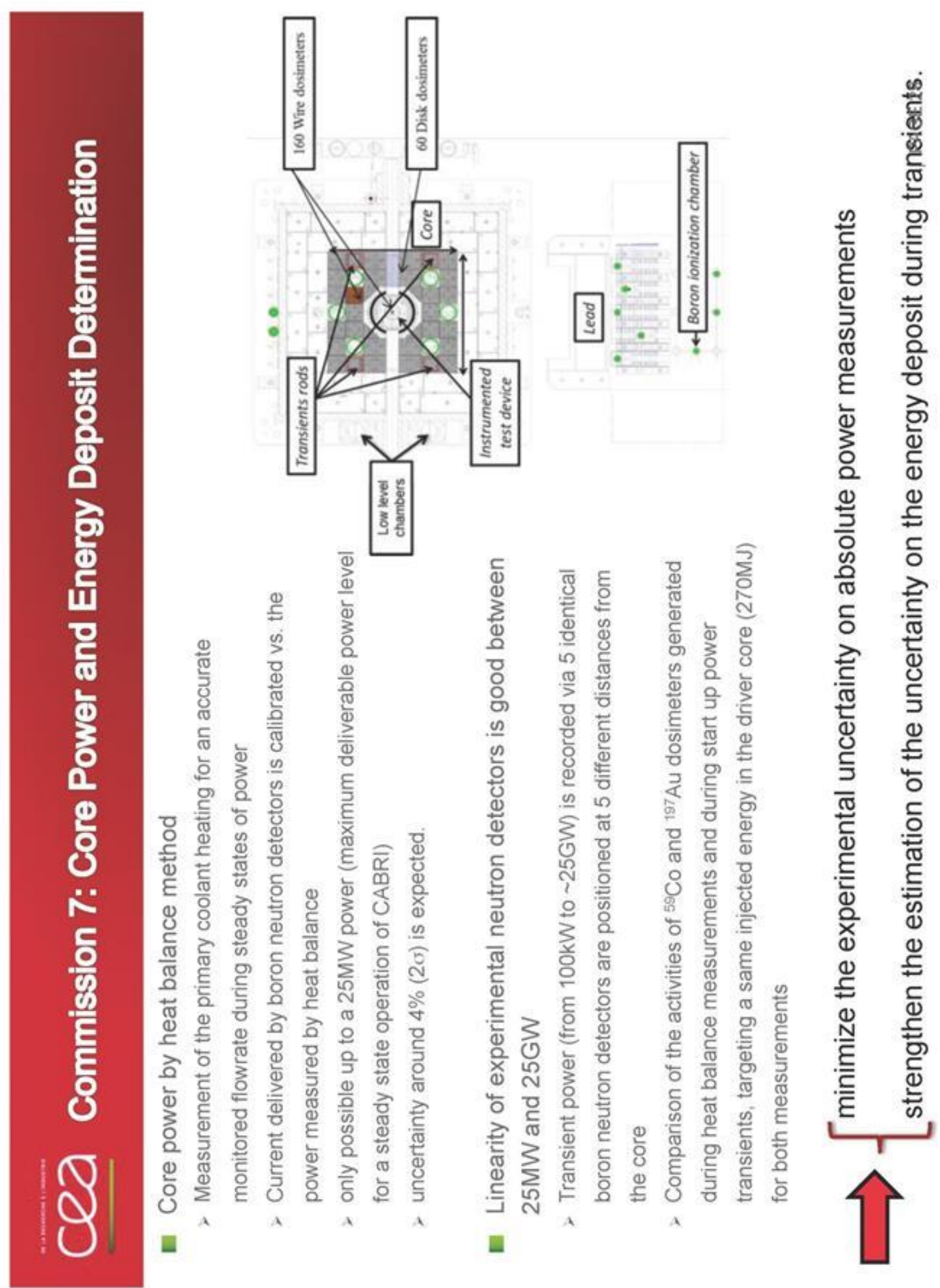




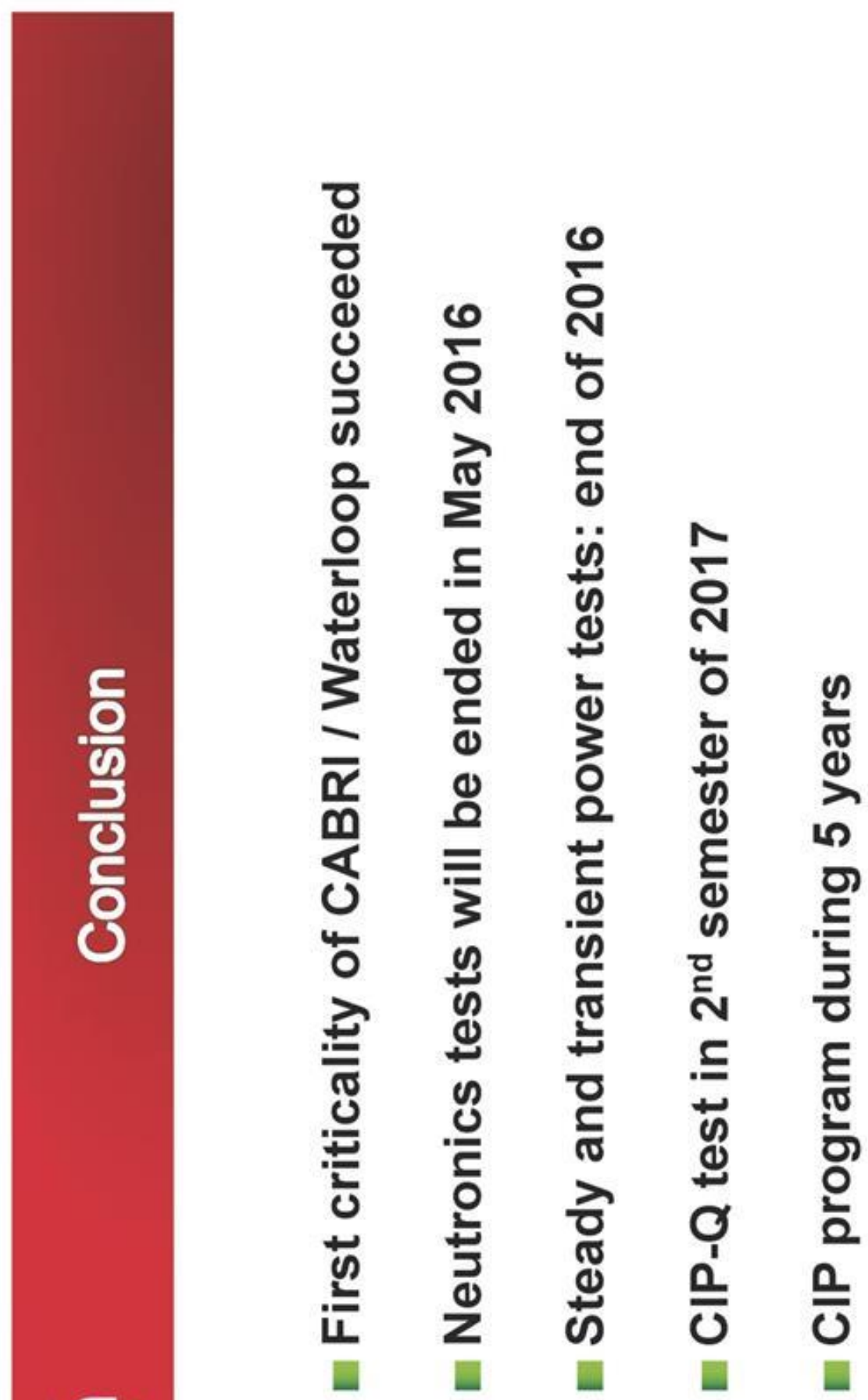




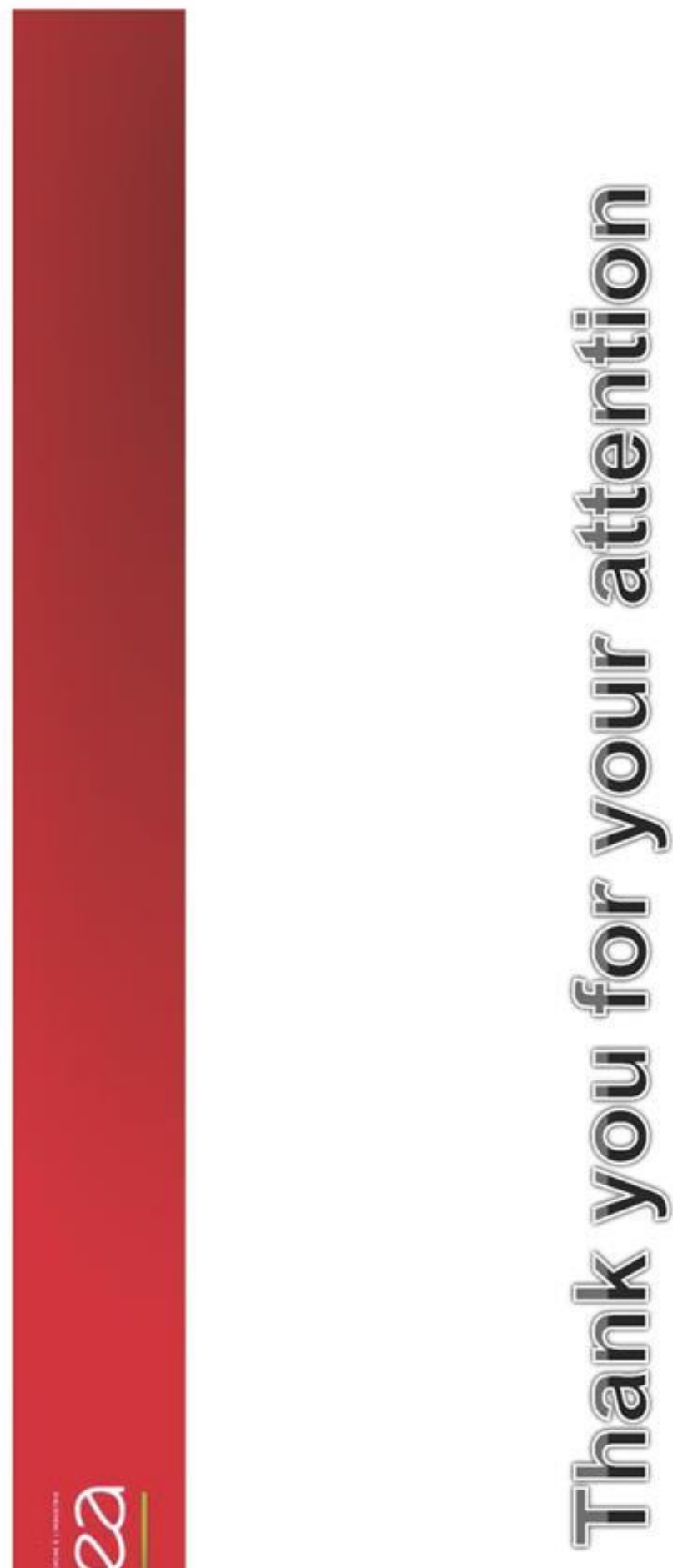



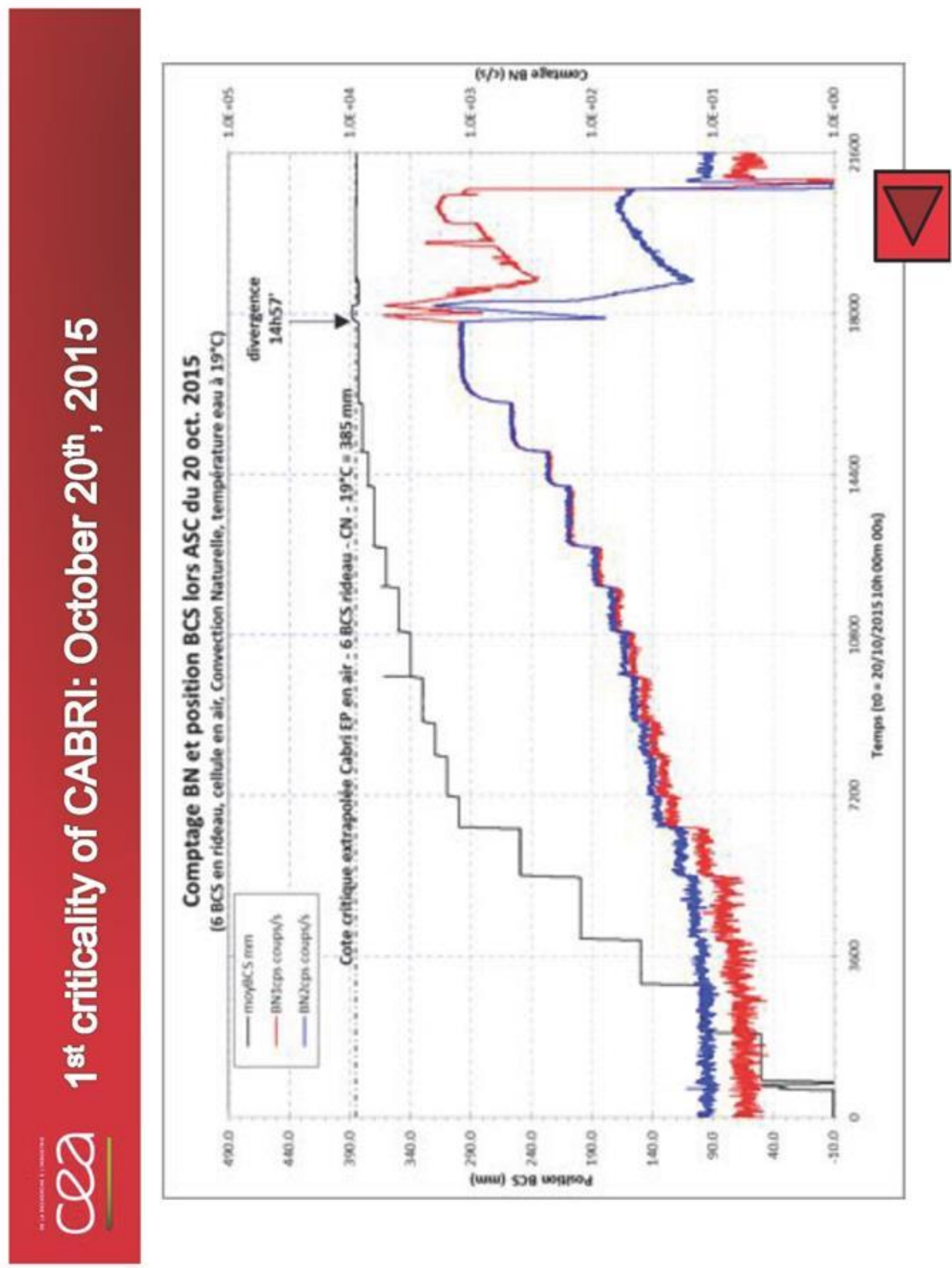


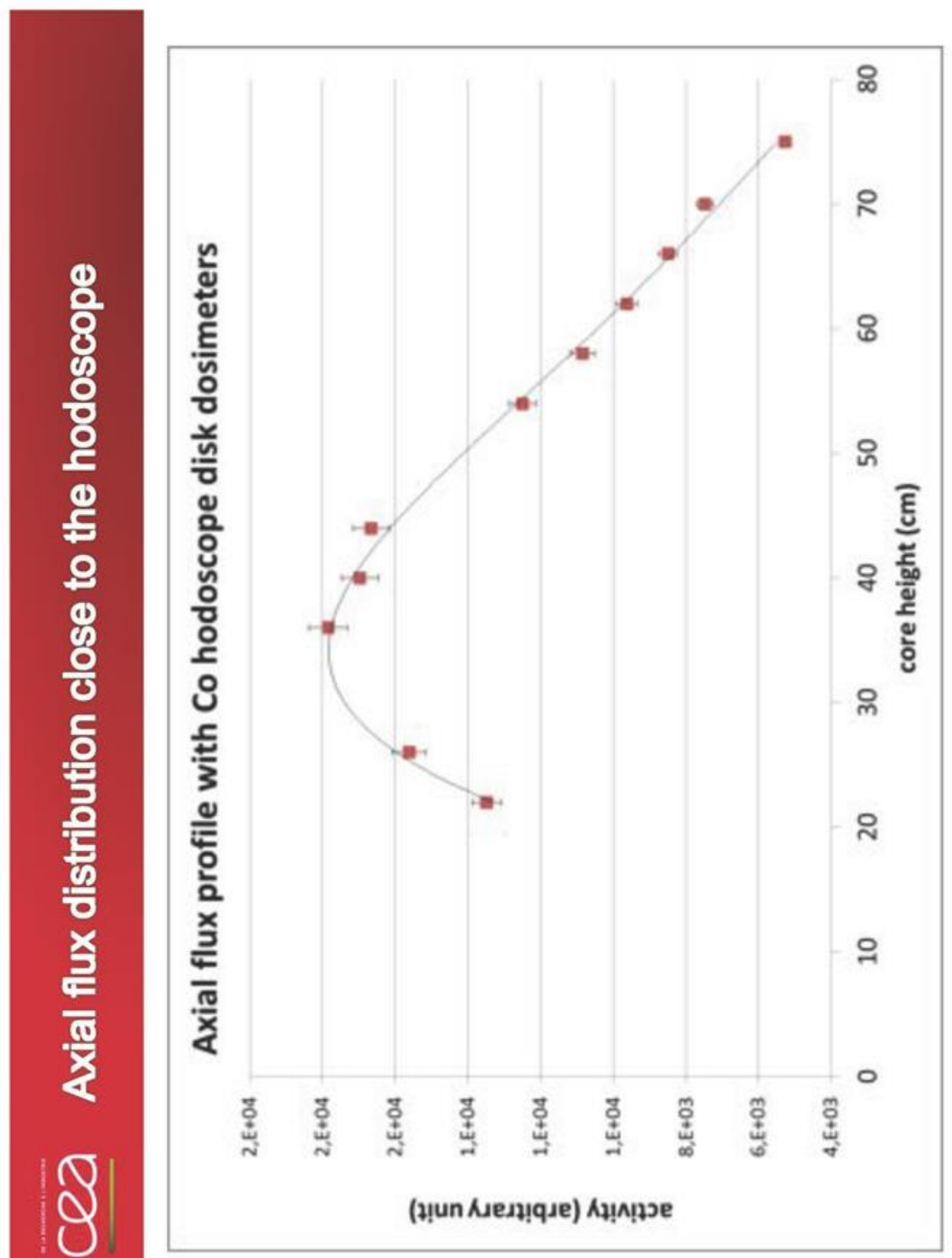




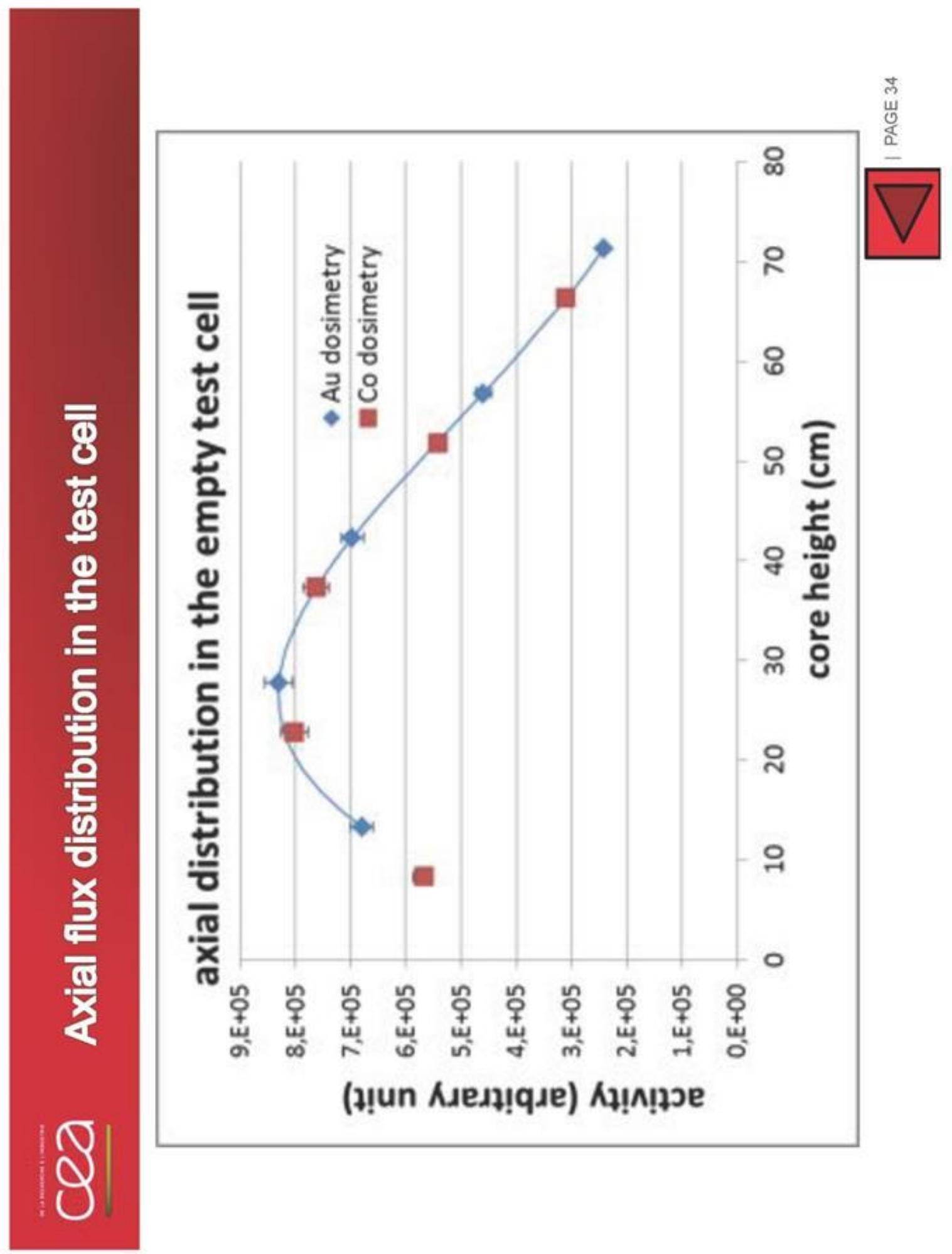


INTENTIONALLY BLANK 
Appendix B

Presentations Slides 
INTENTIONALLY BLANK 


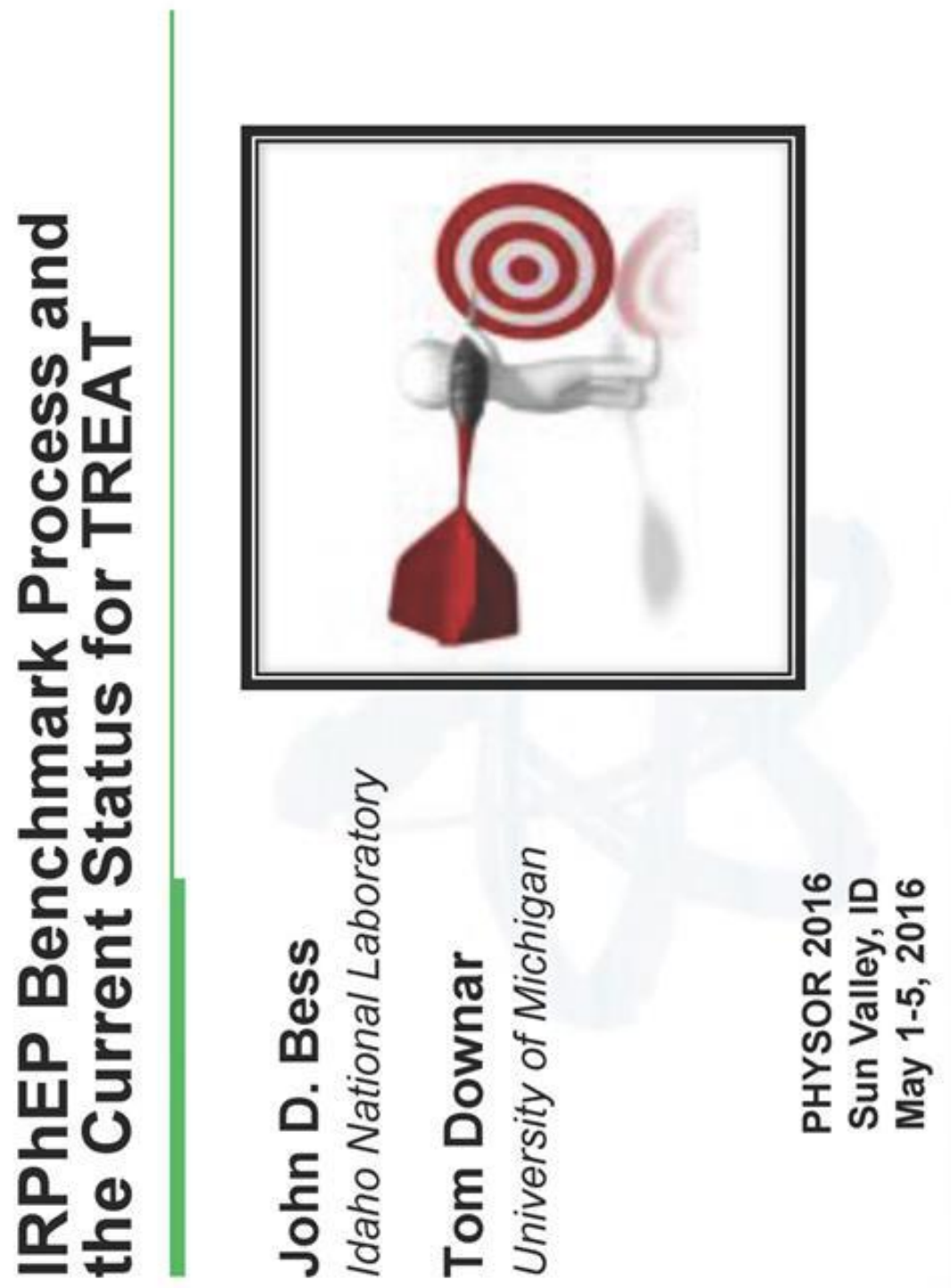

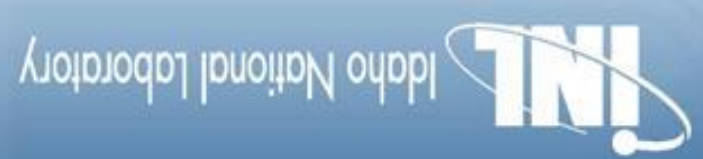

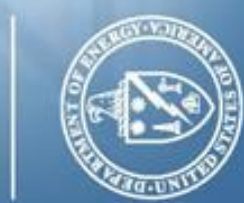



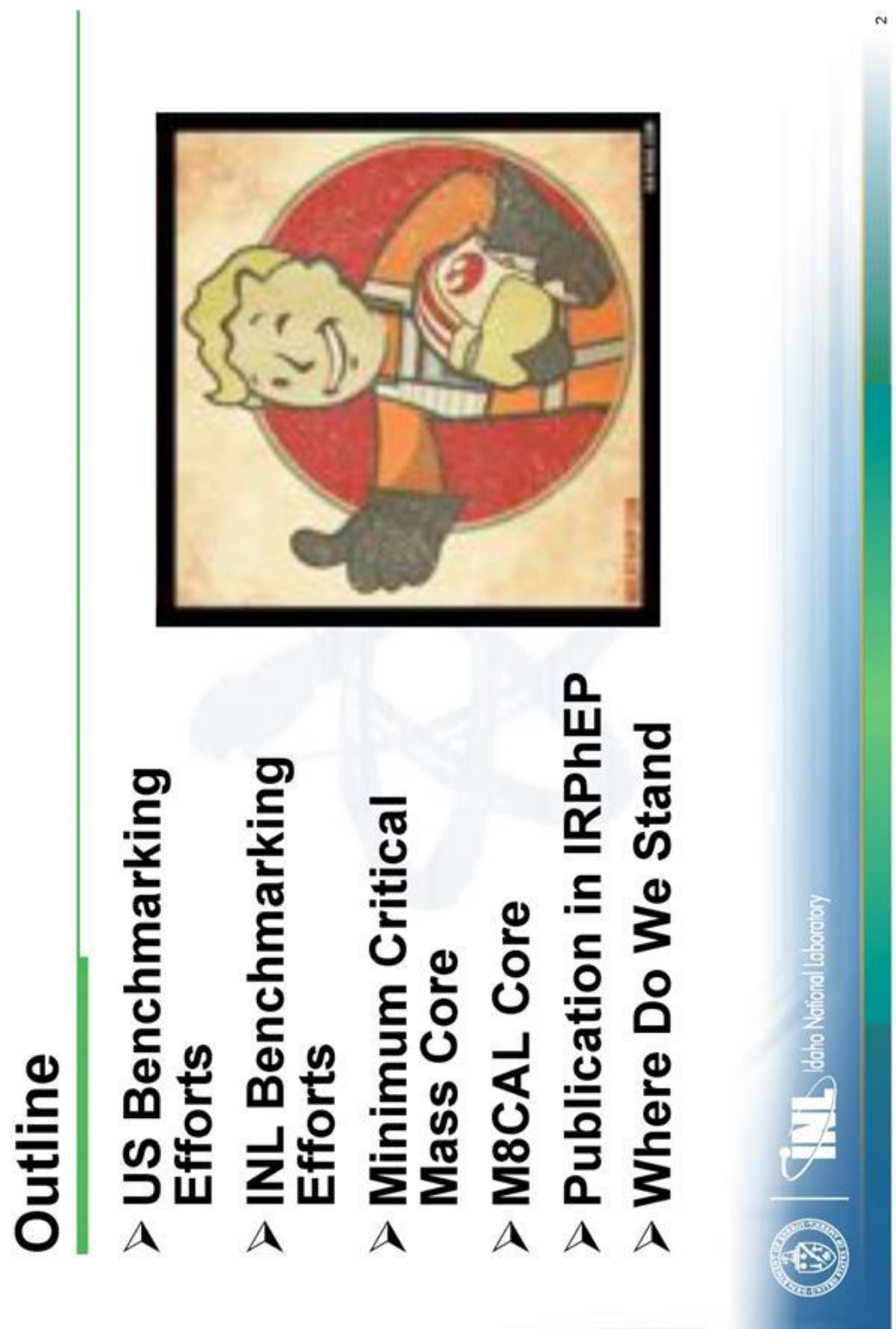


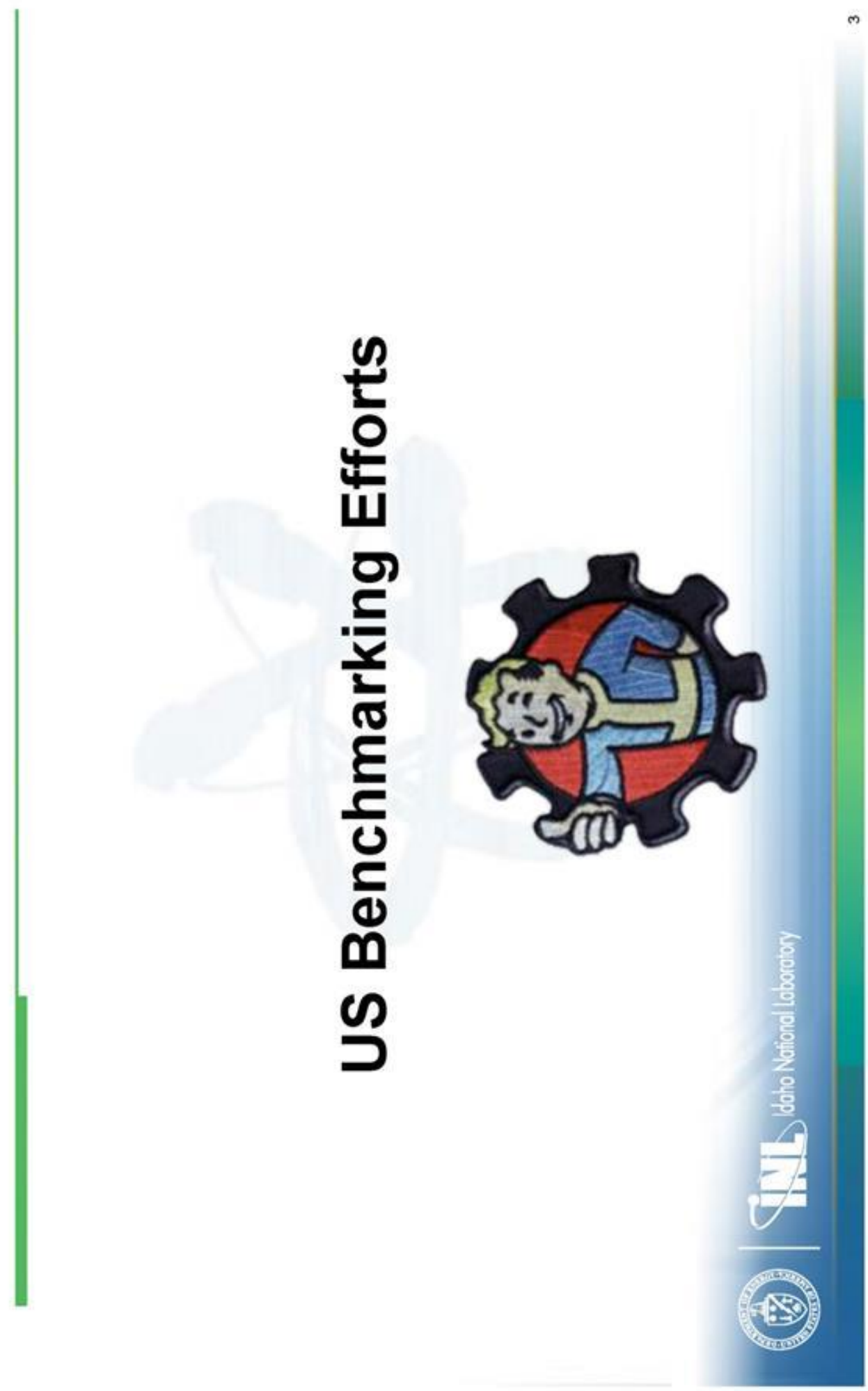




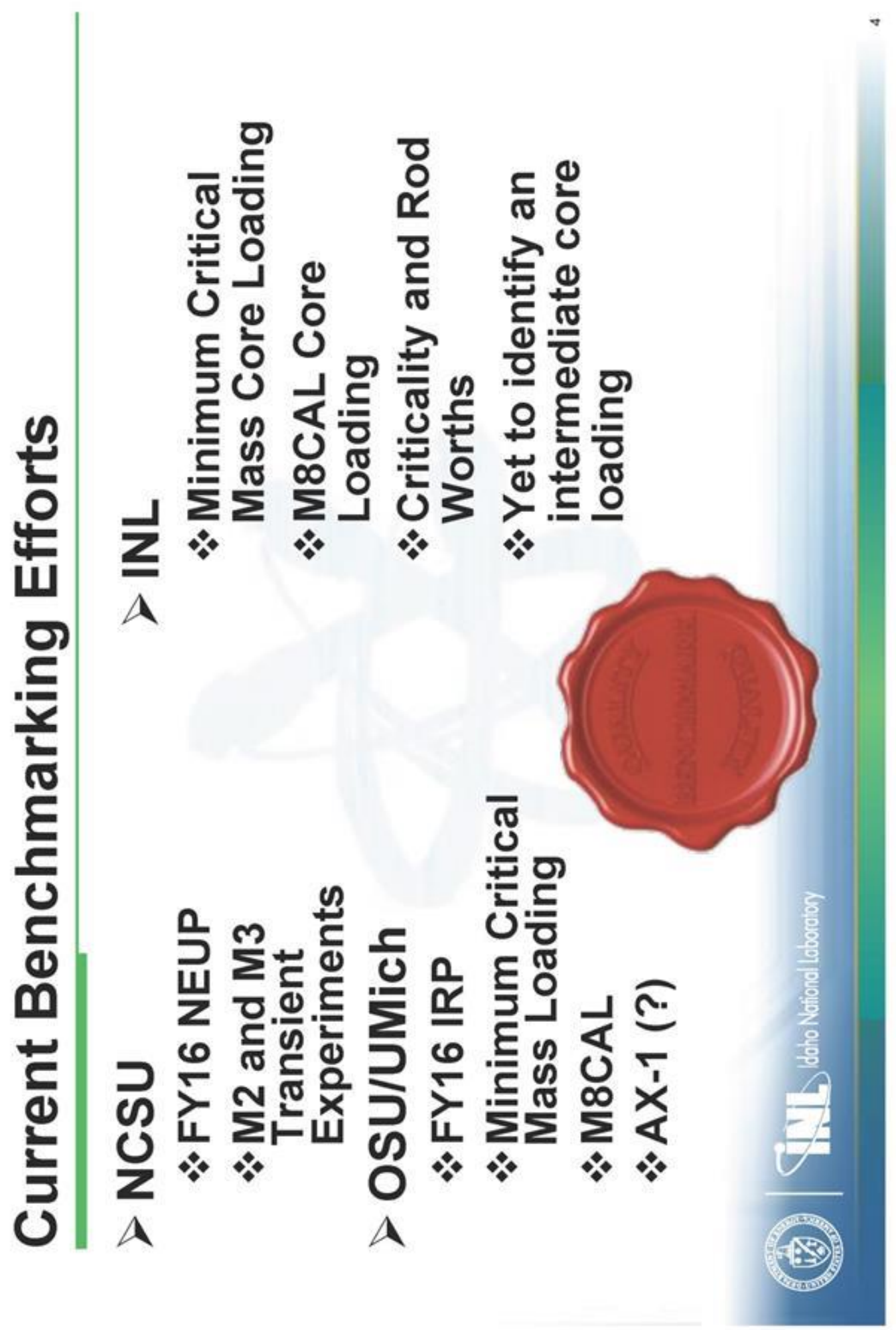




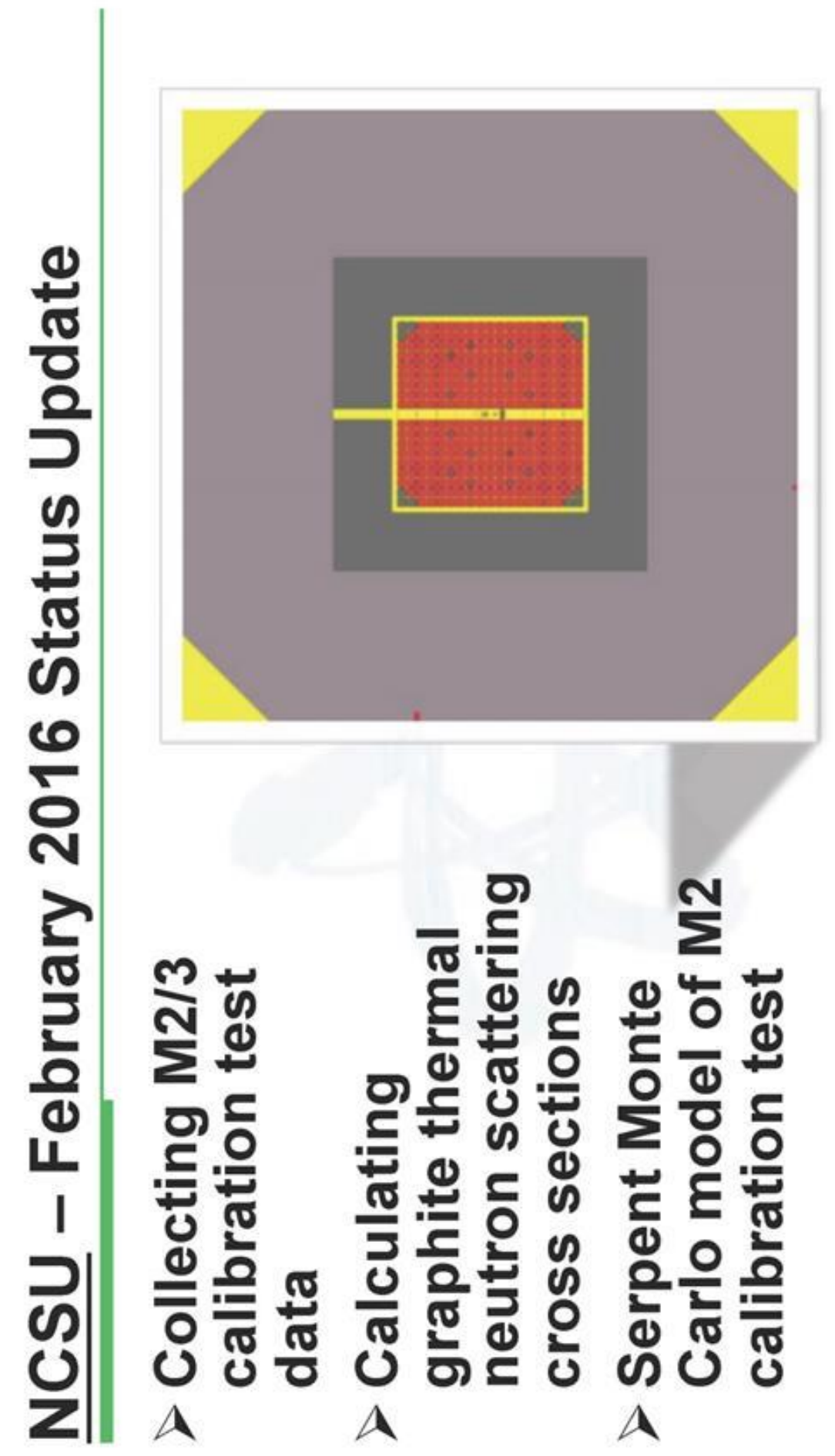




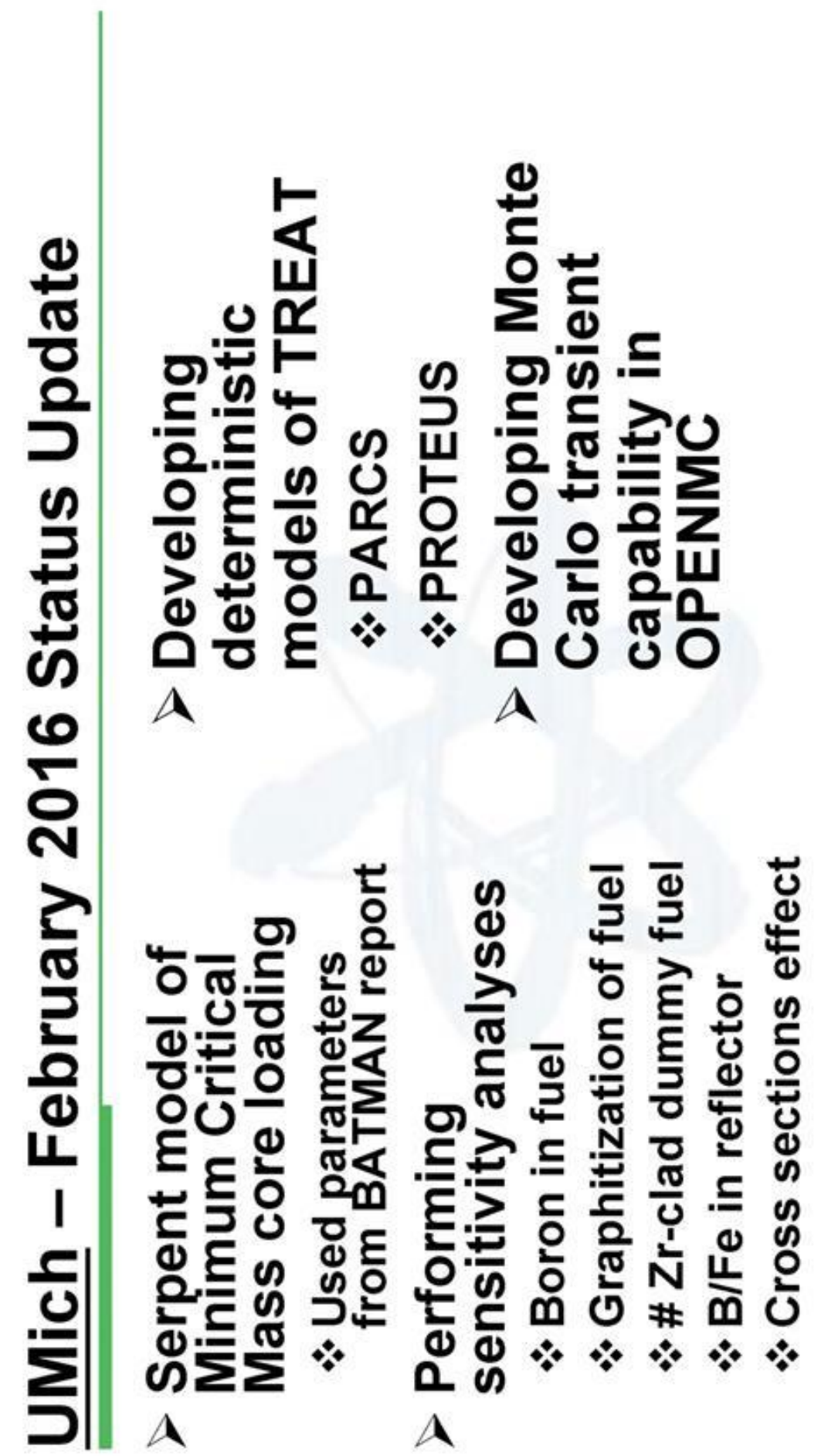




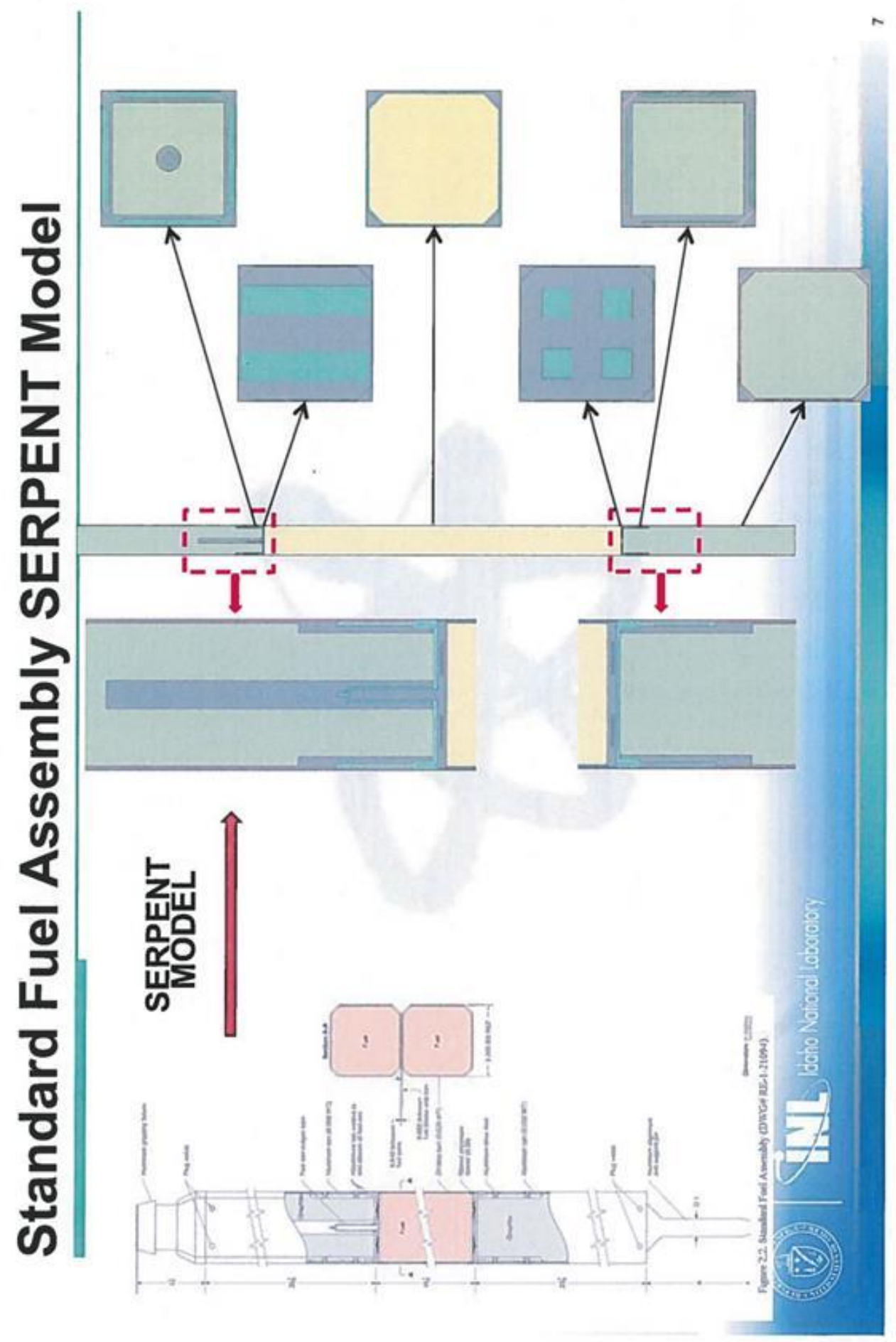




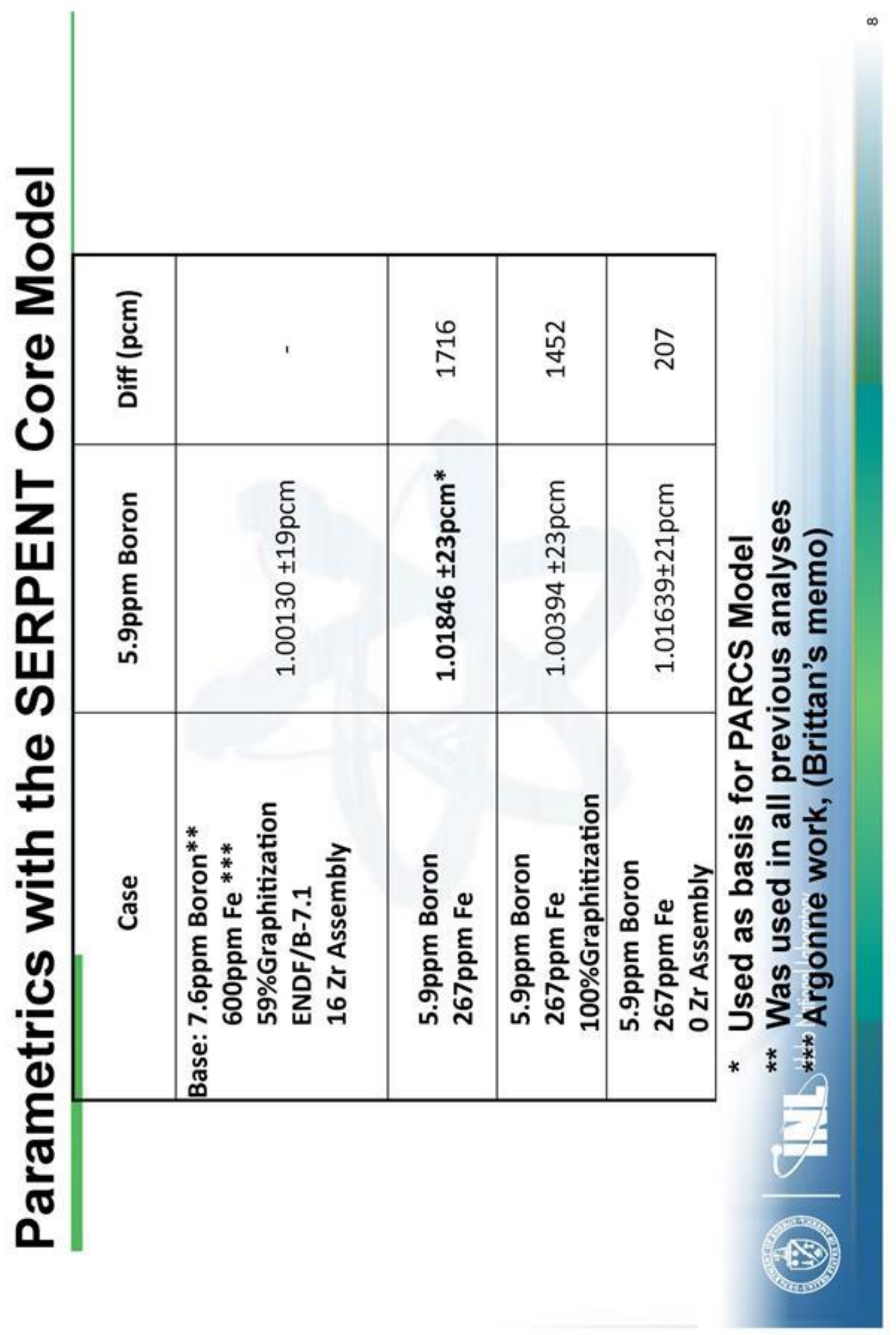




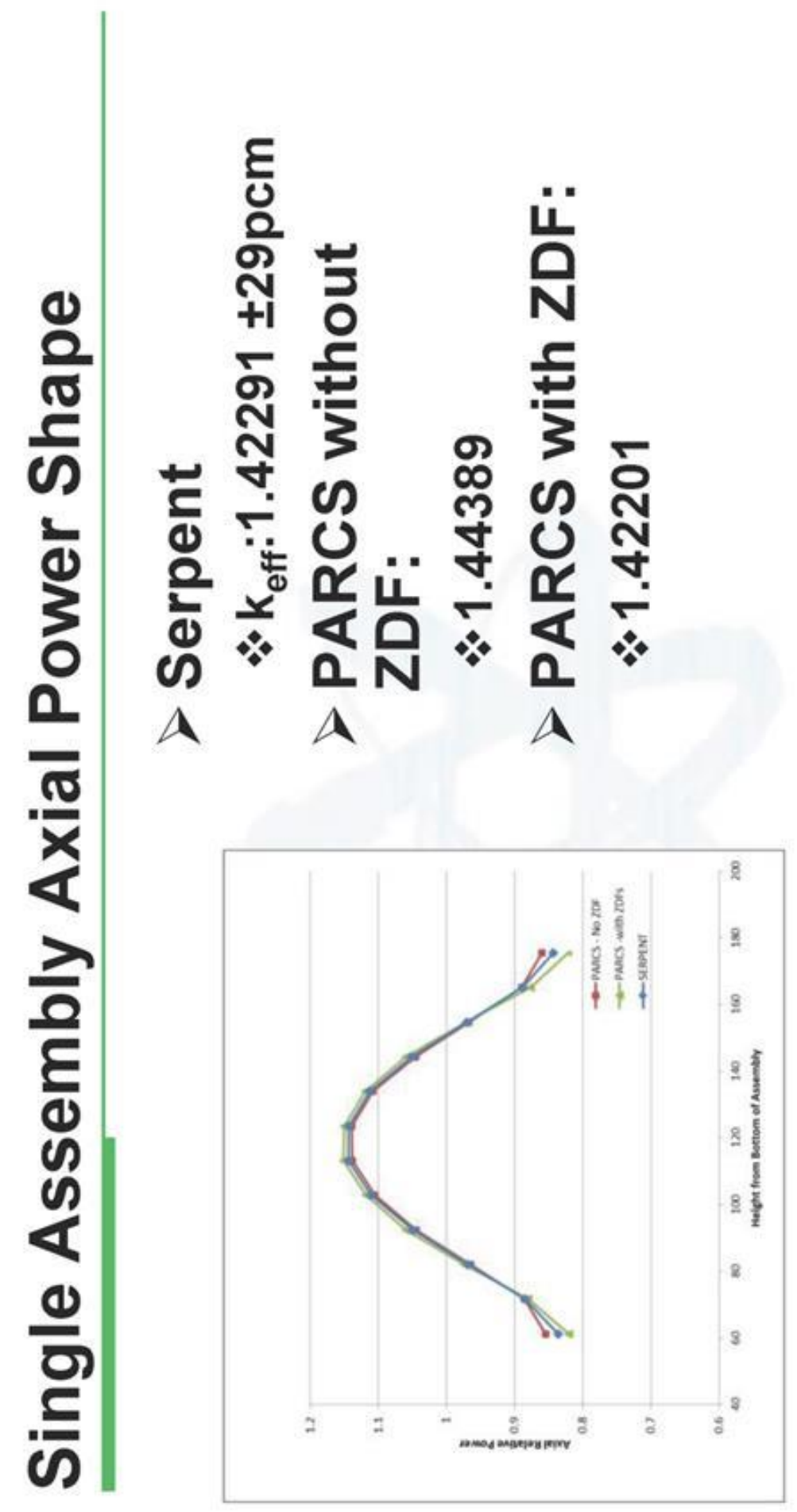




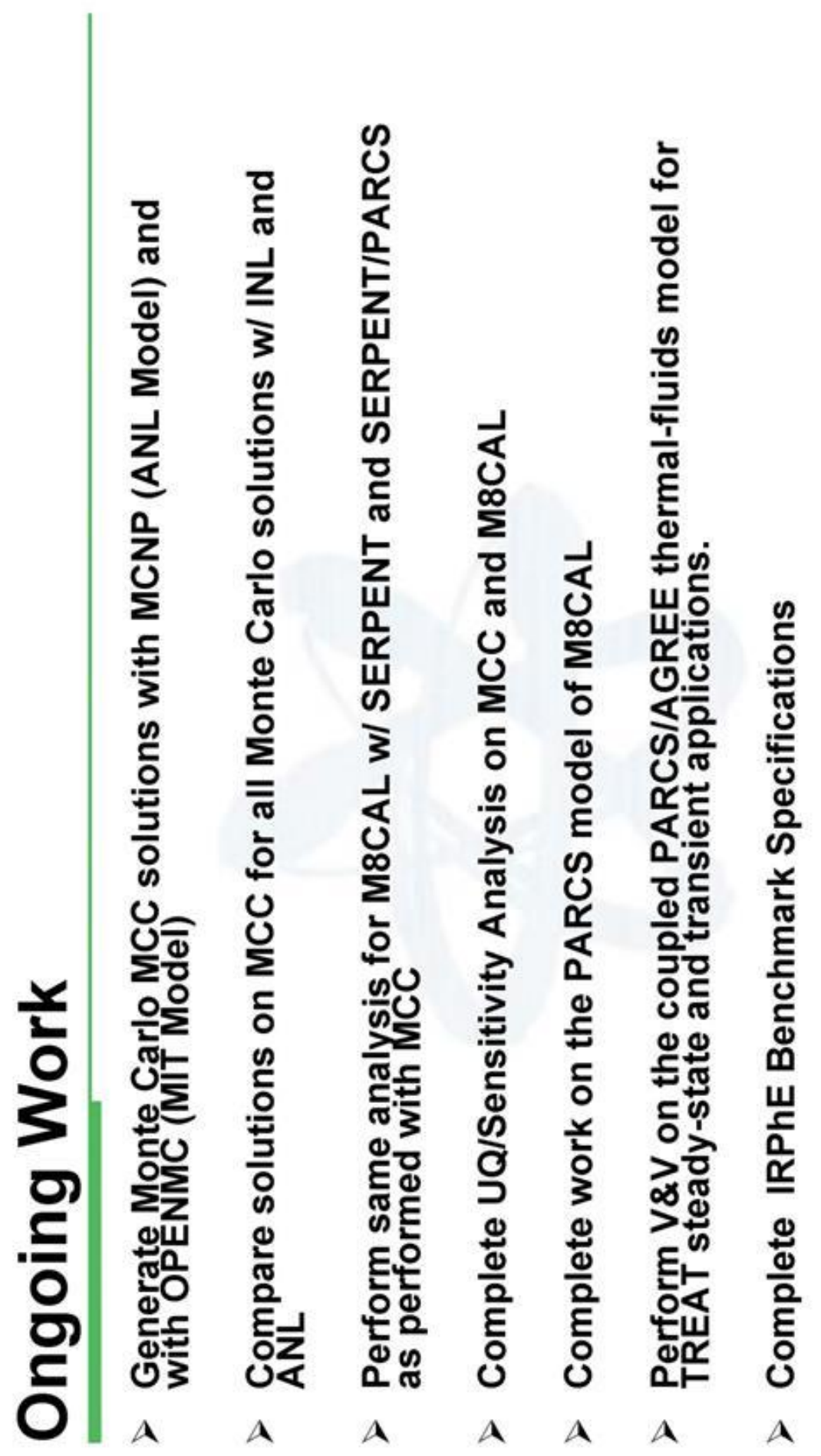


은

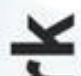

ชิ

E

ט

들

m

$\underline{Z}$
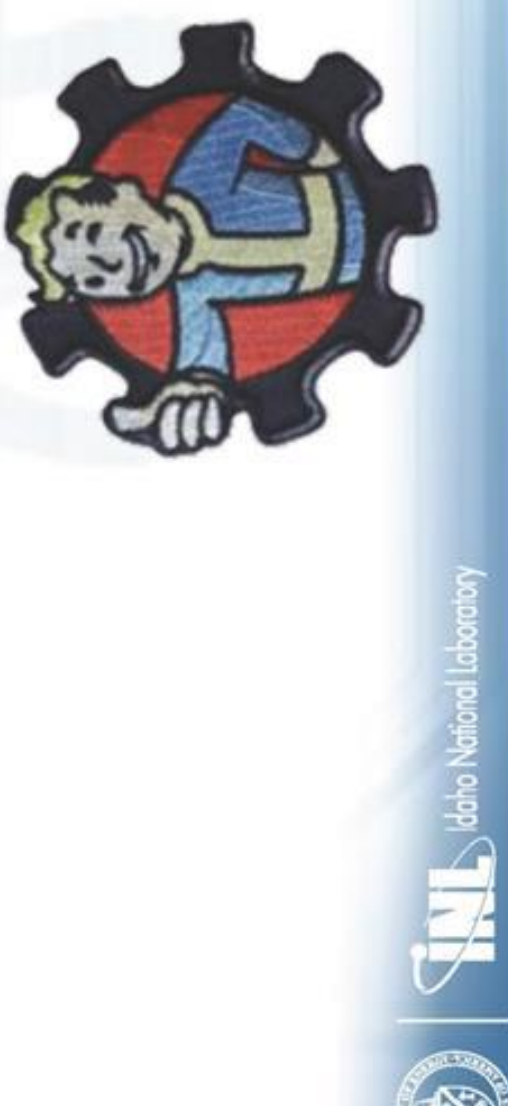

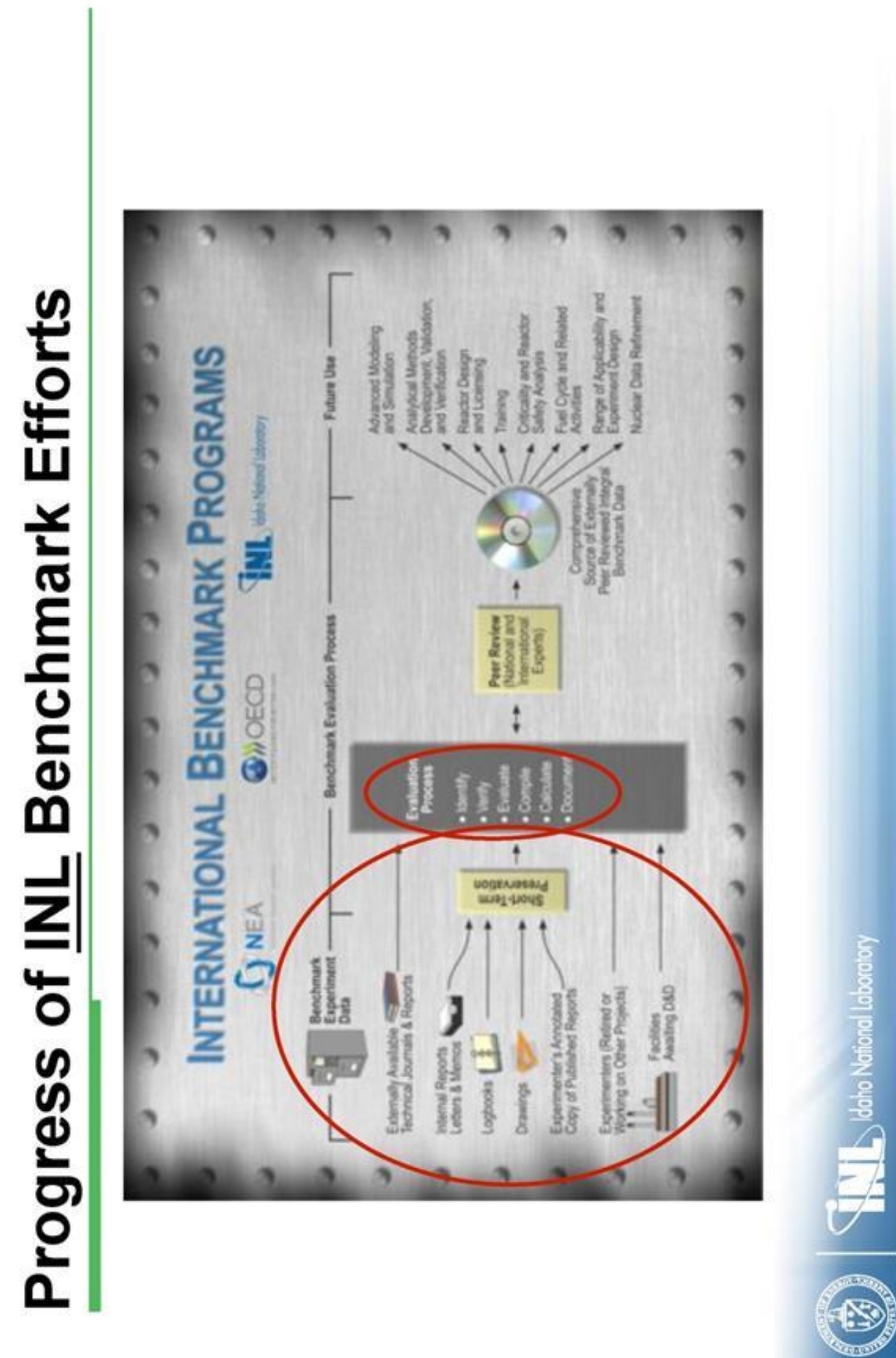


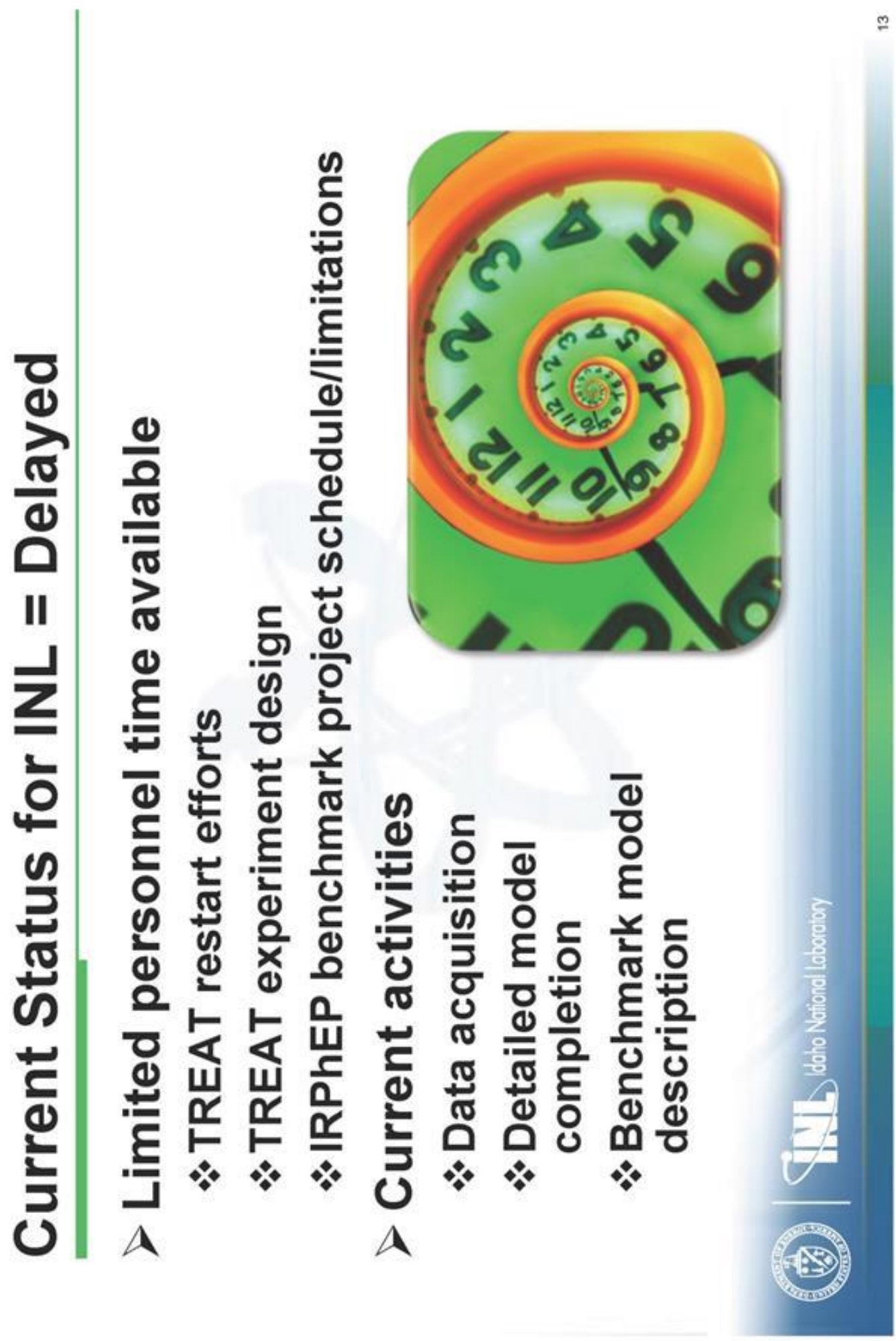




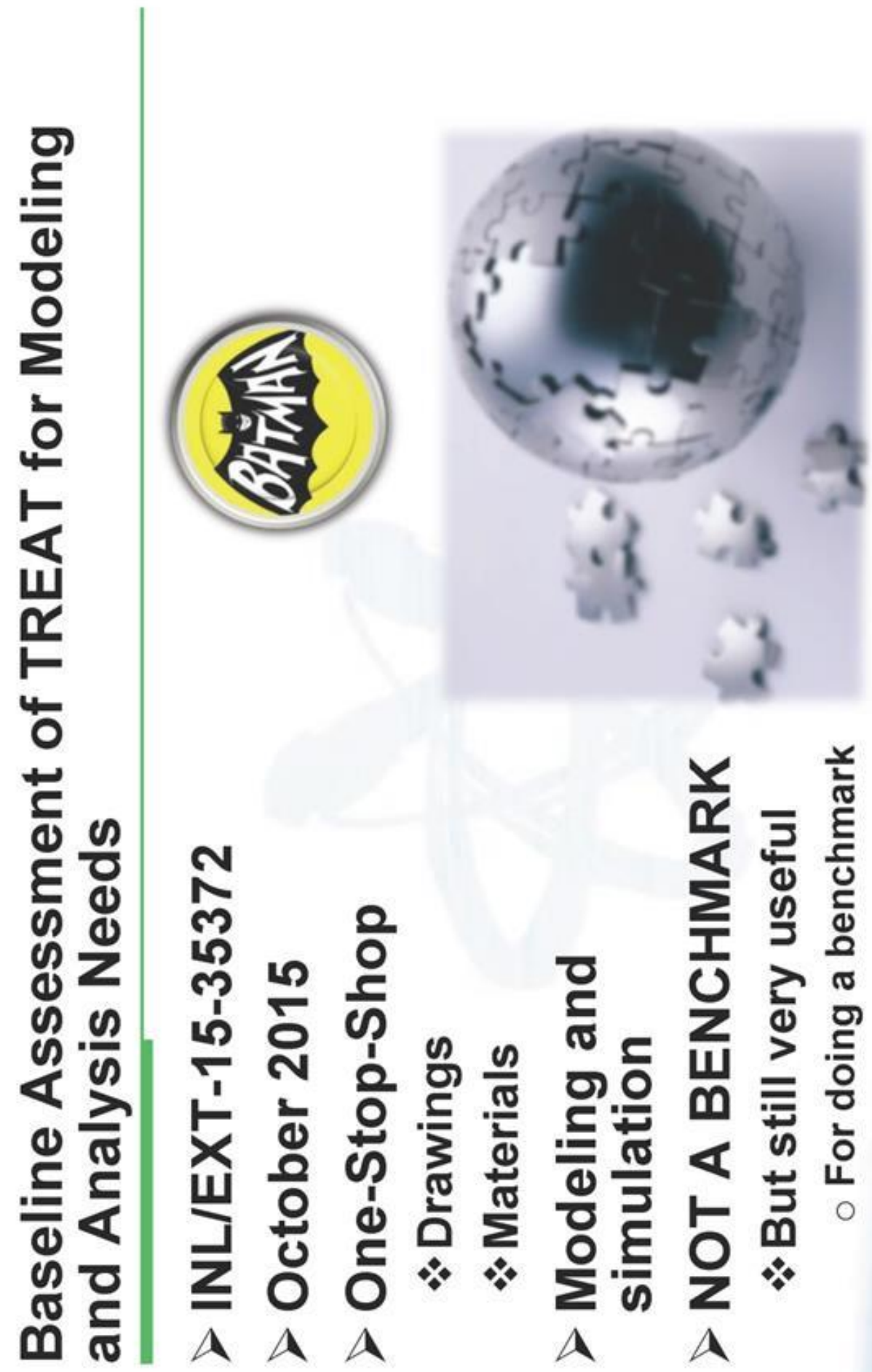




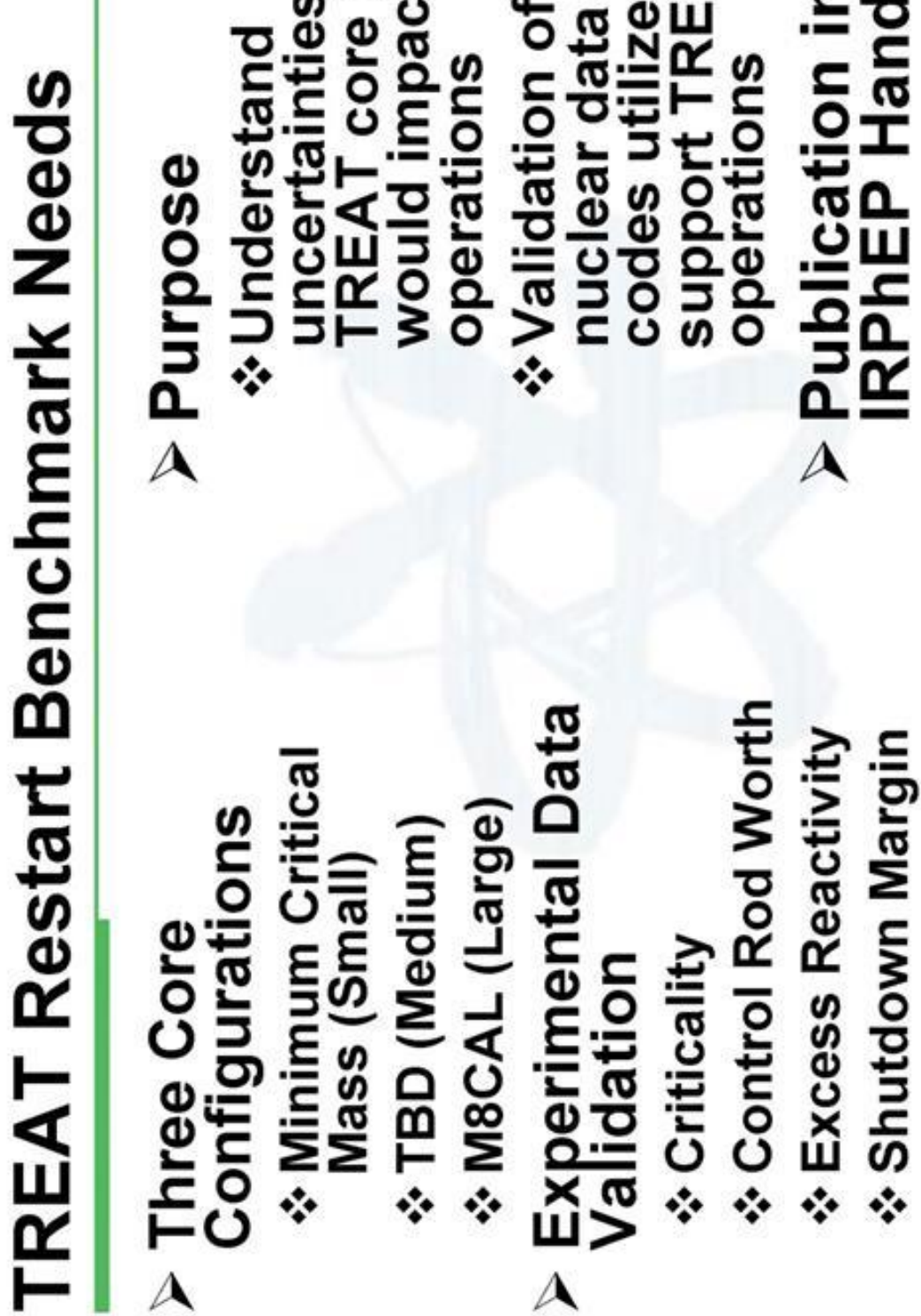


(1)

E
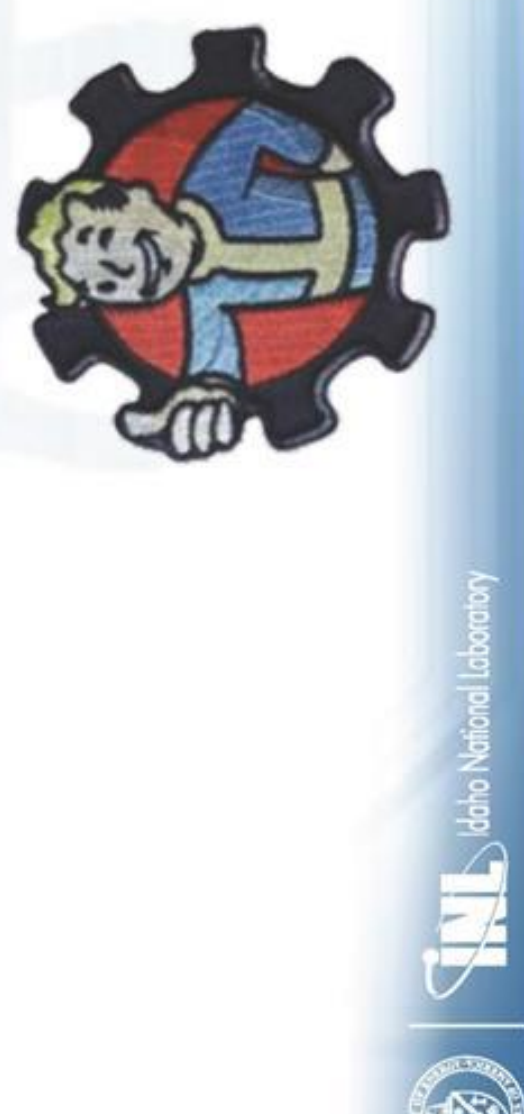


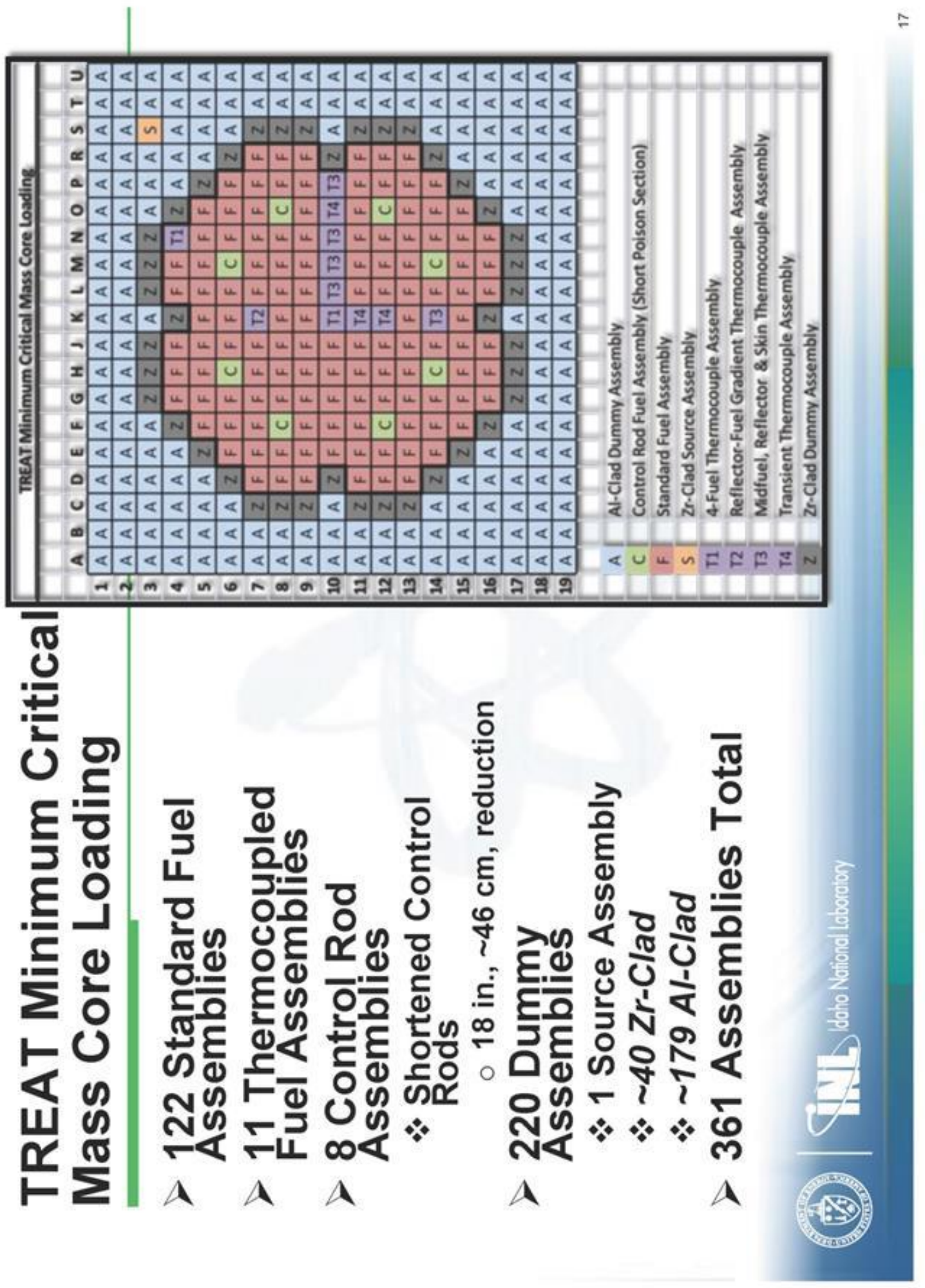



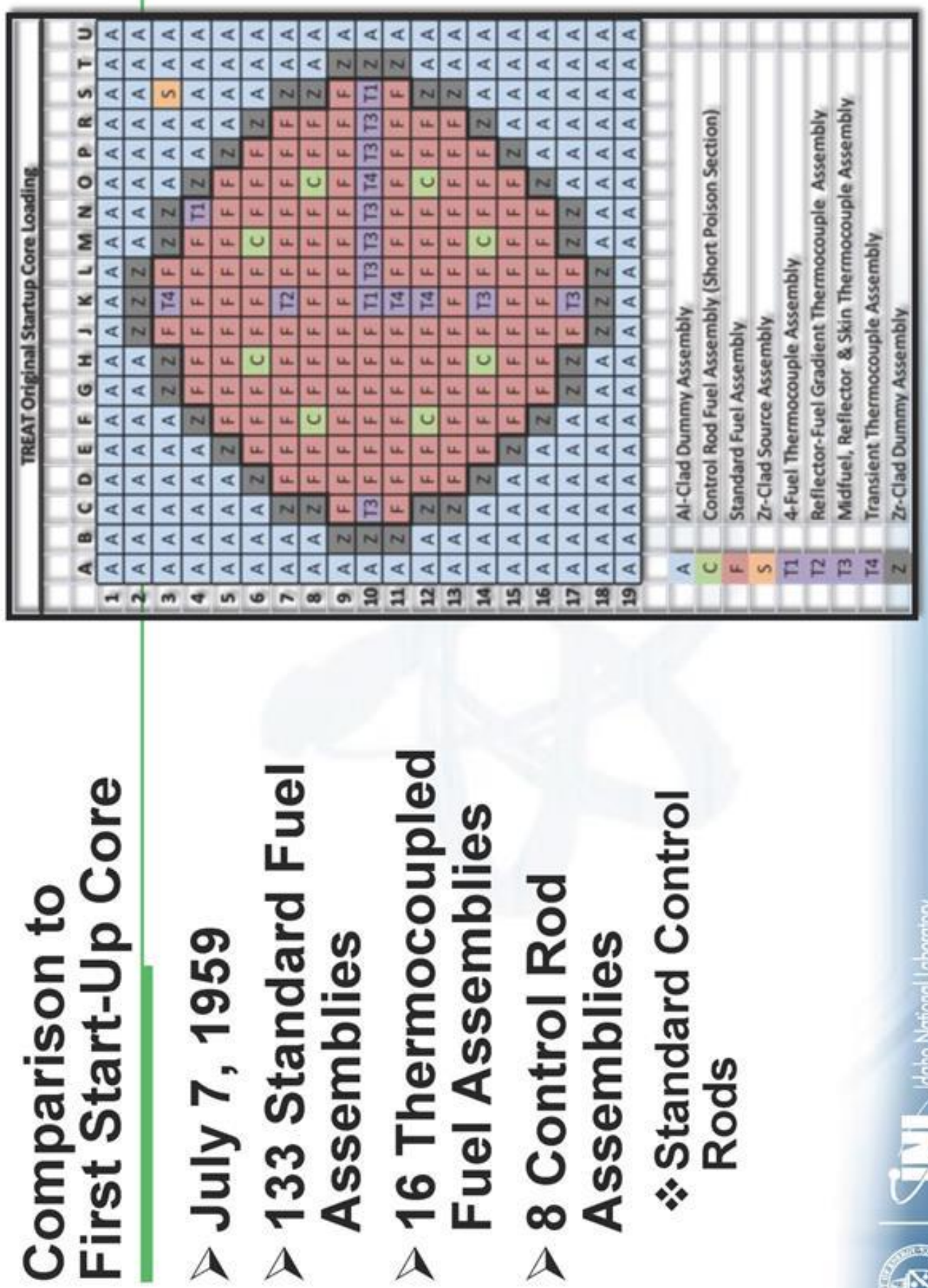


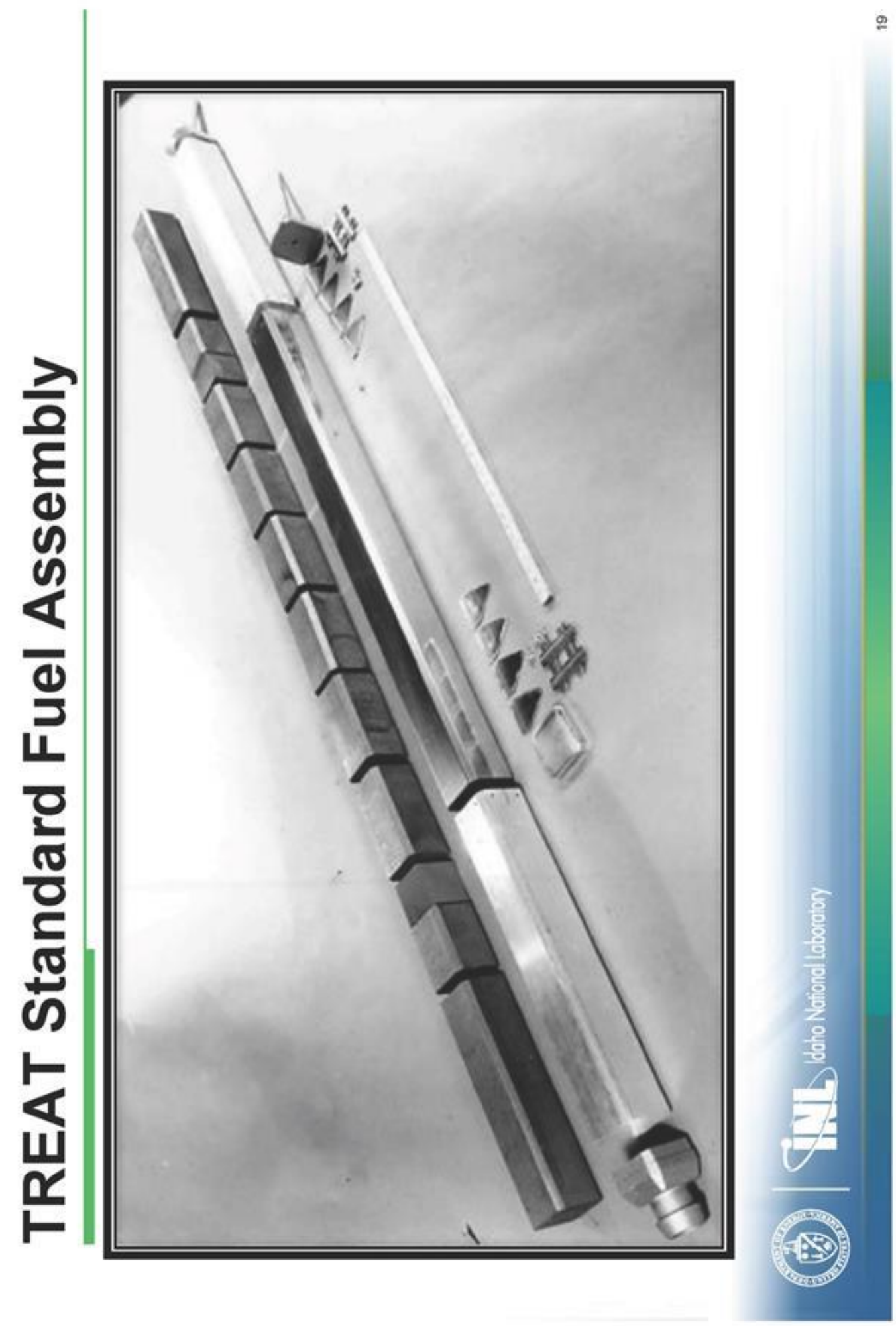




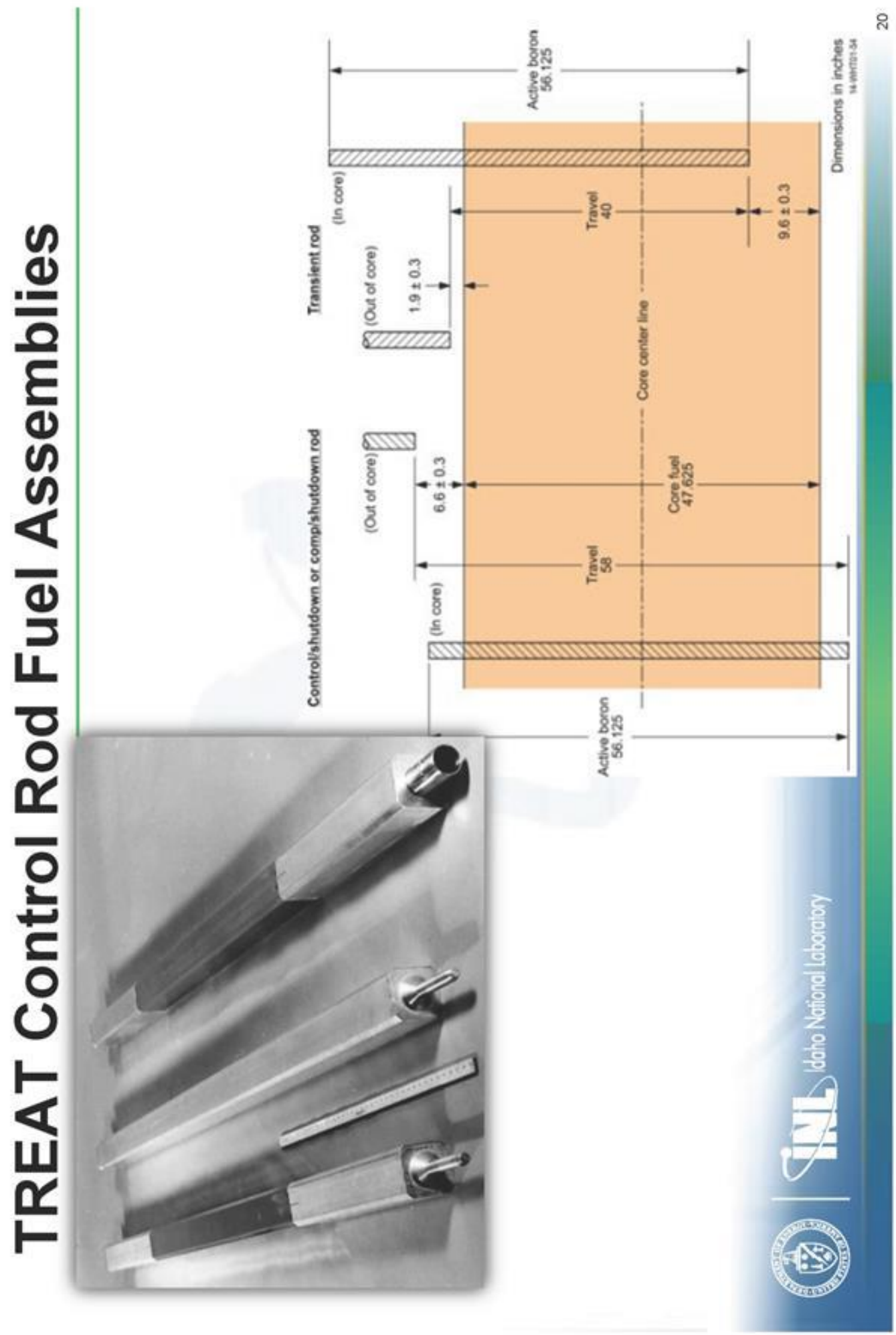




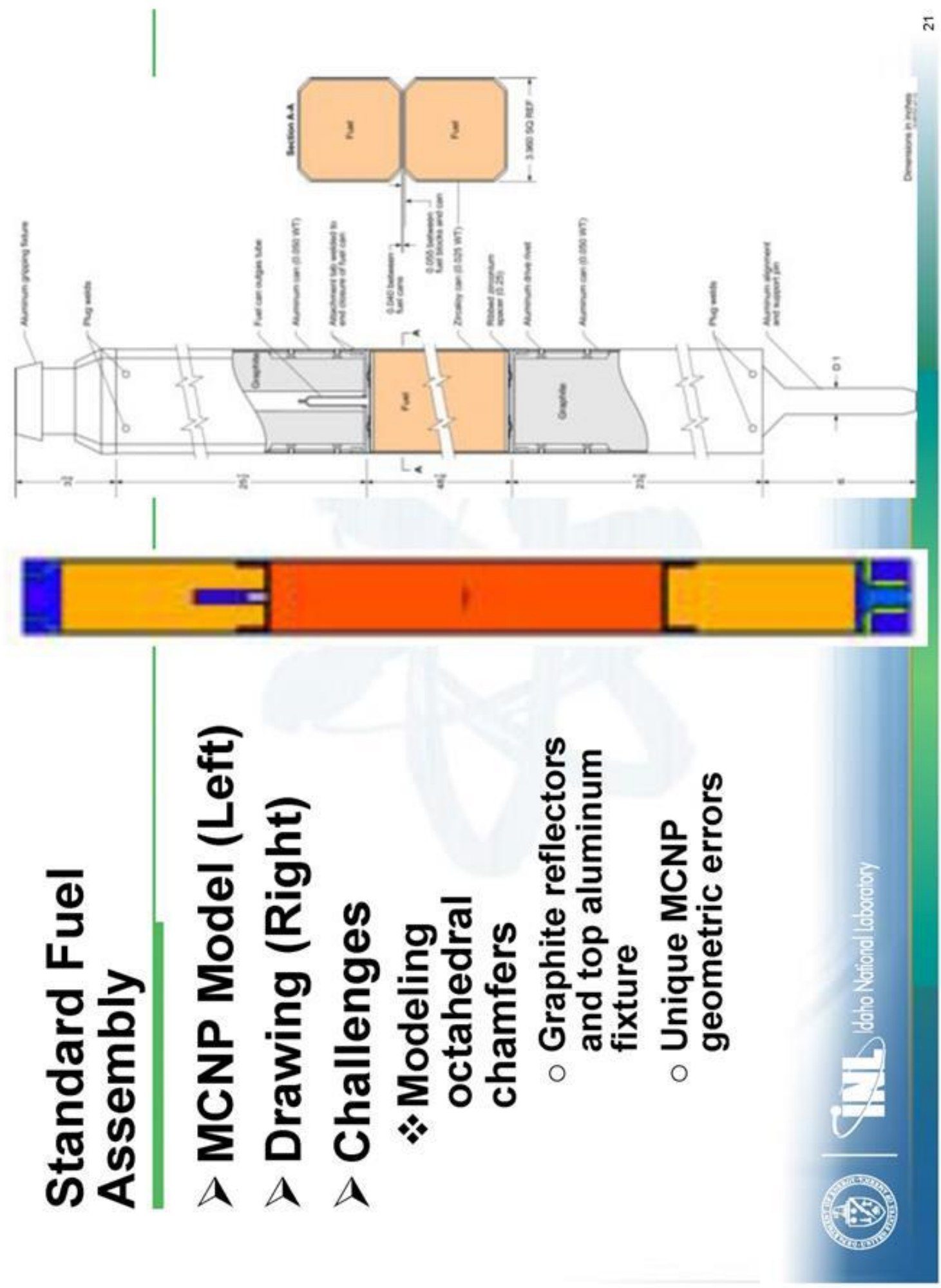




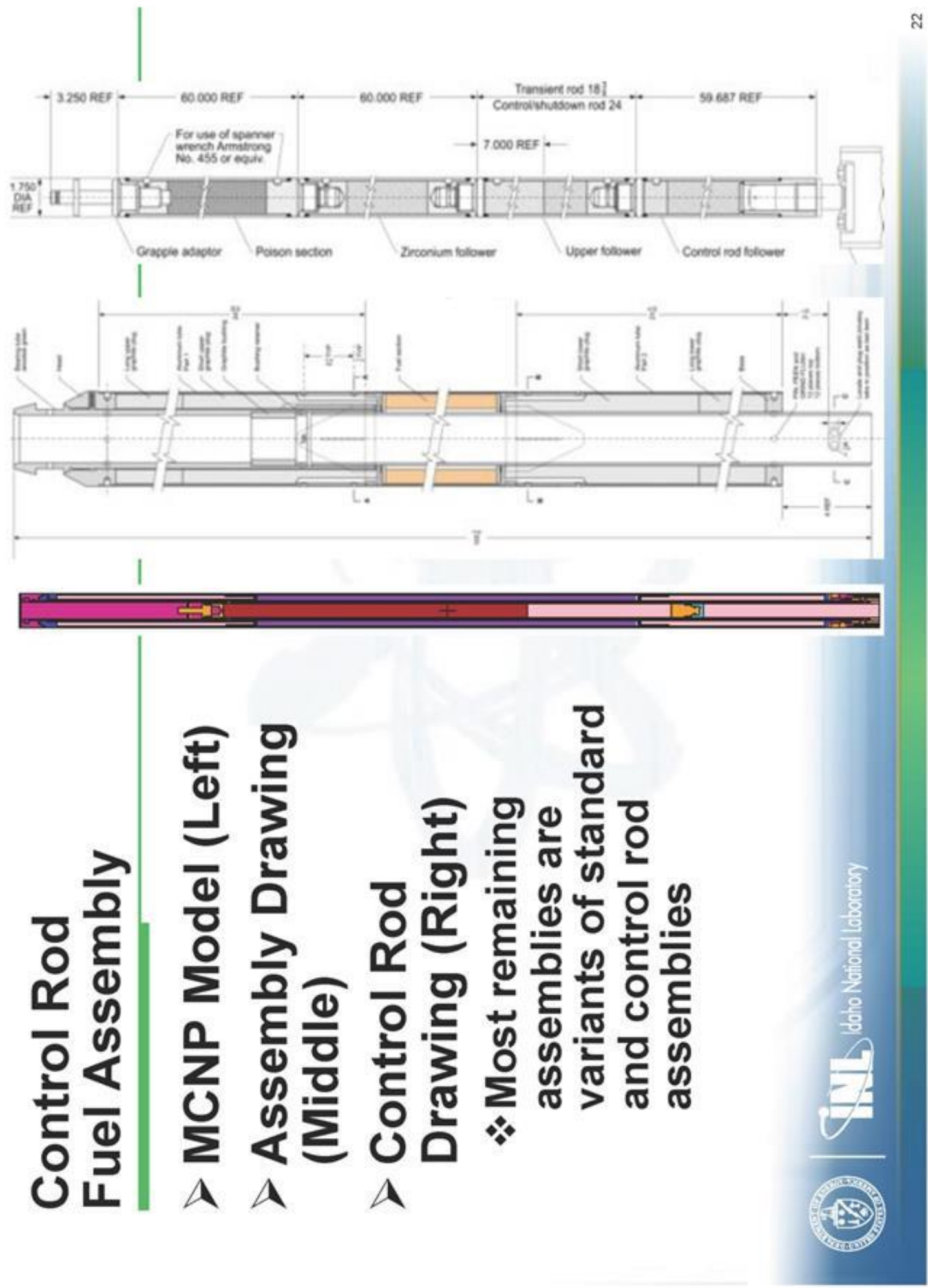




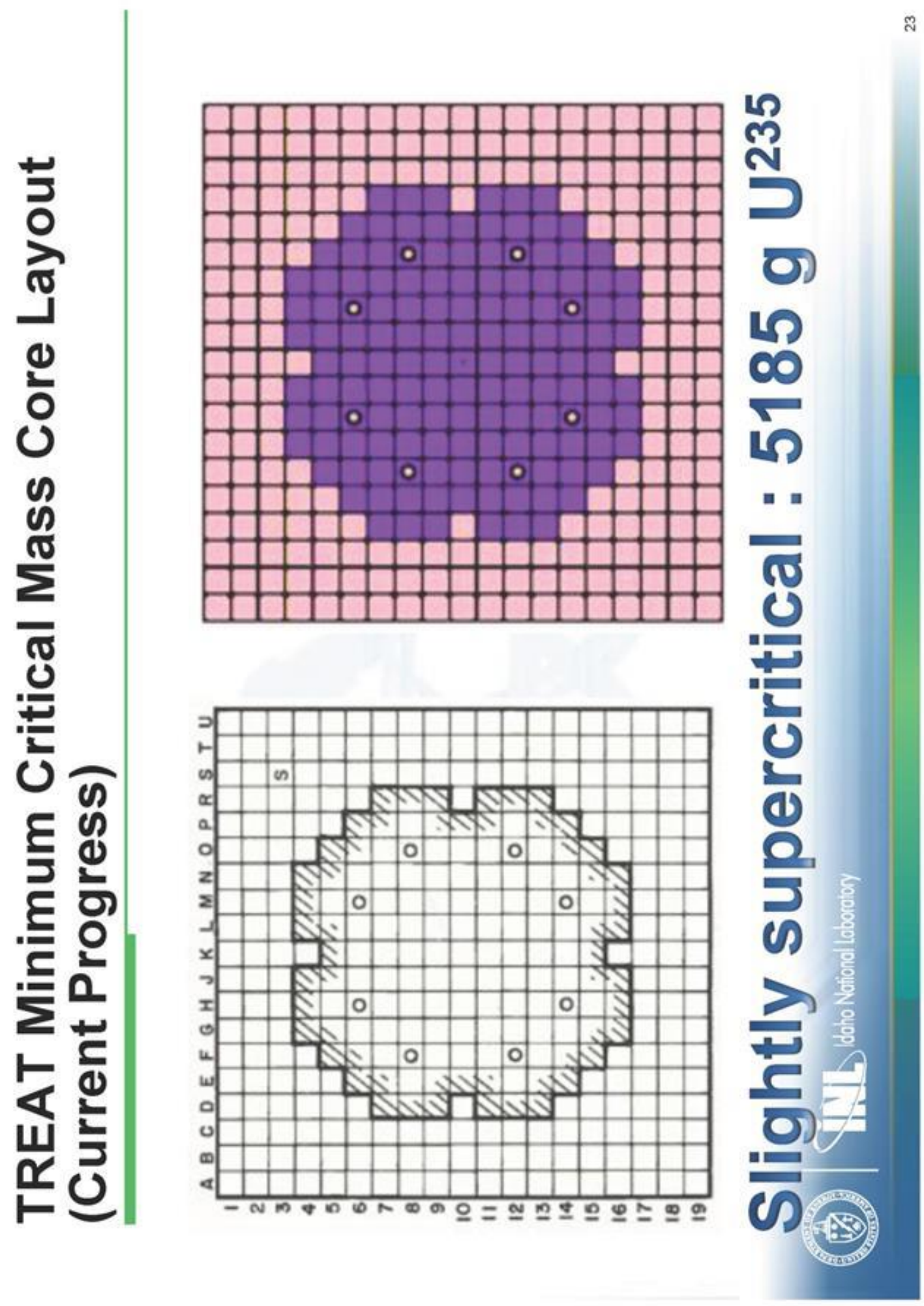




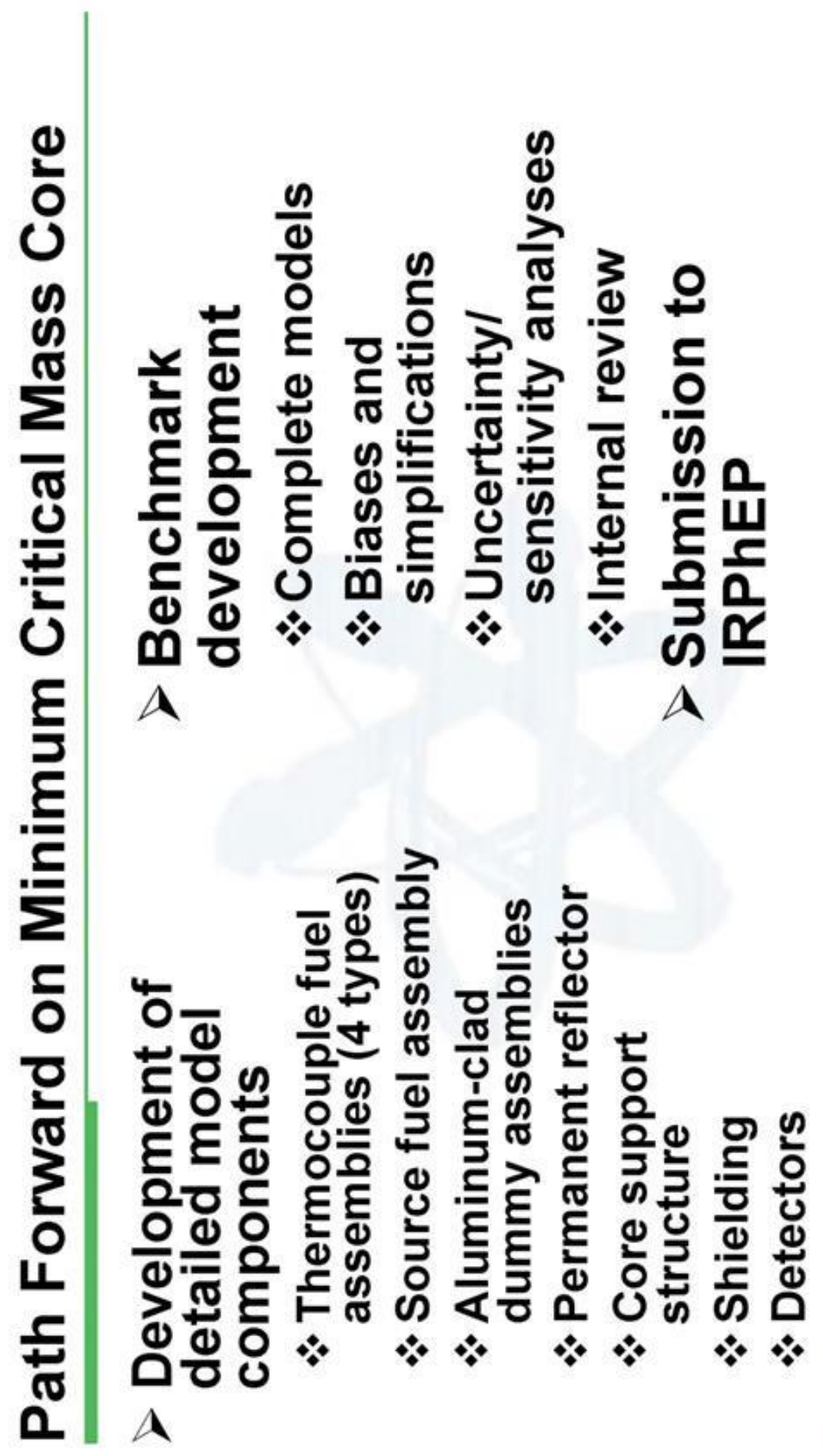




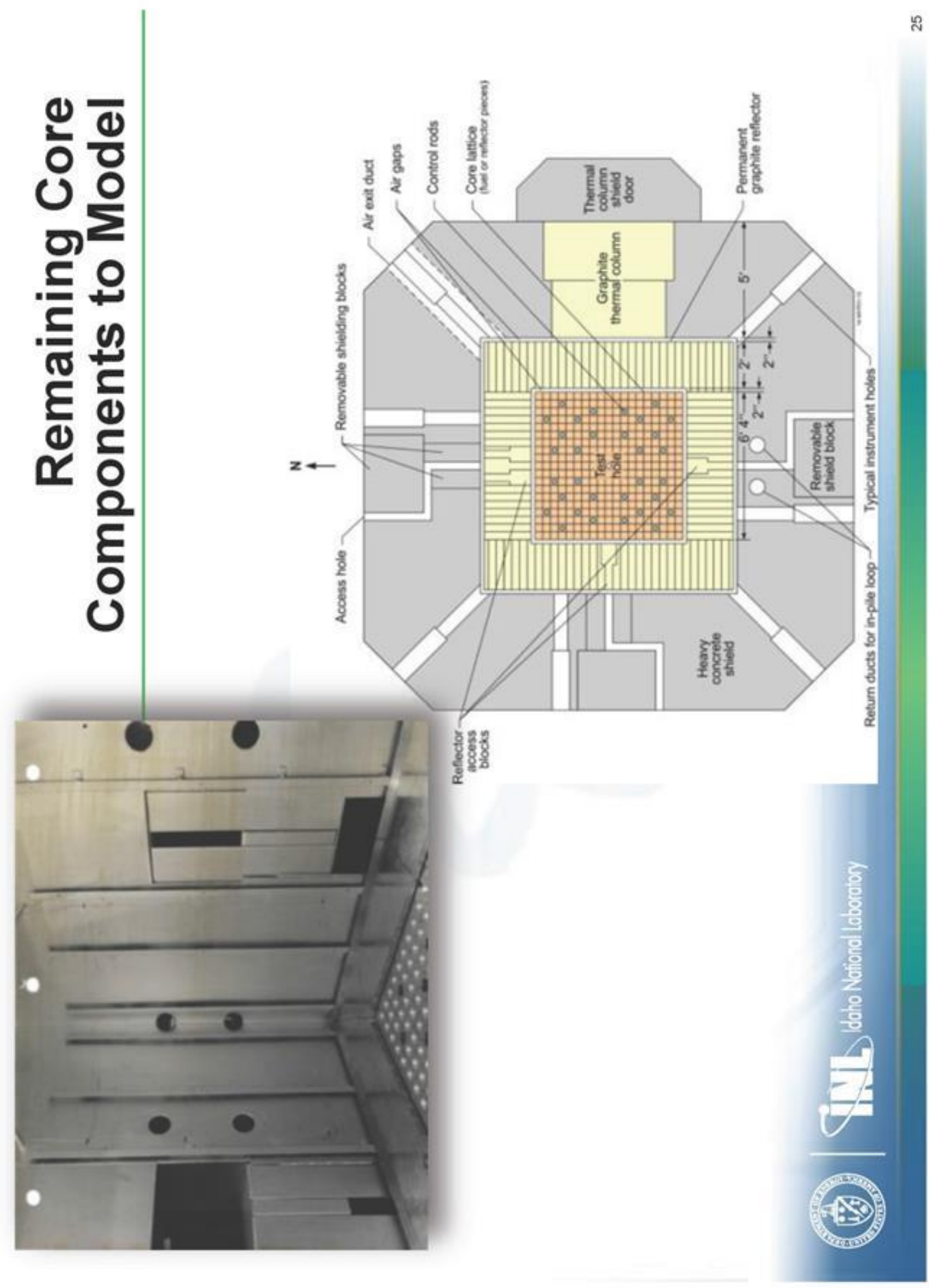




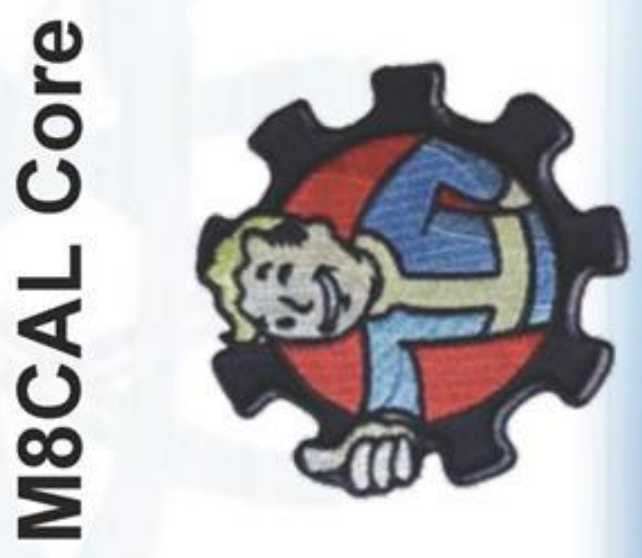




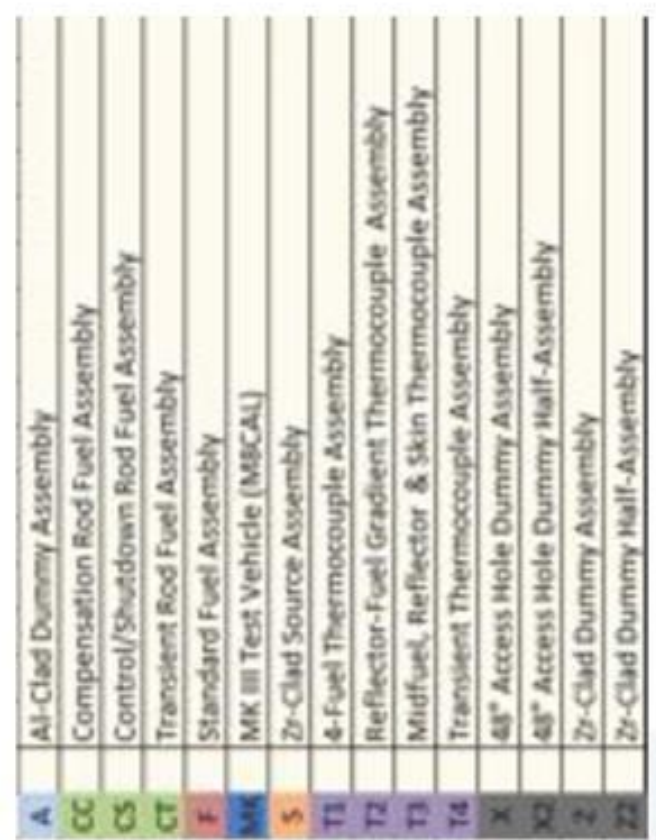

$\frac{1}{0}$

जi

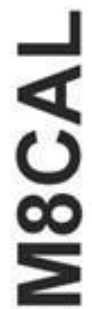

$\frac{1}{11}$

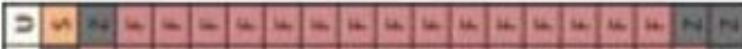

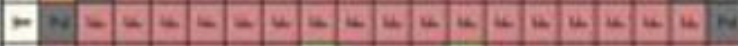

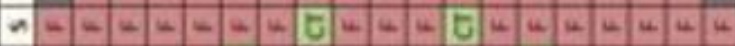

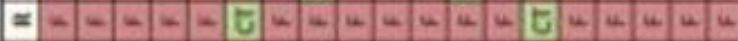

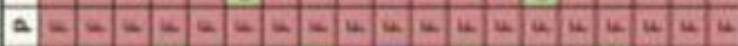

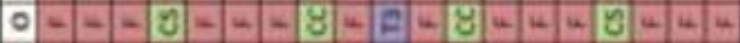

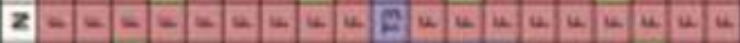

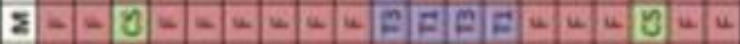

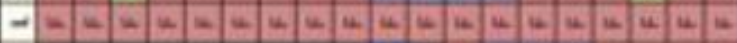

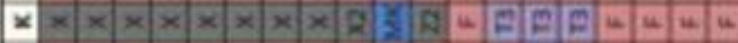

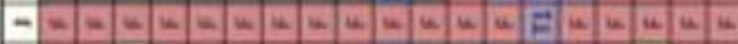

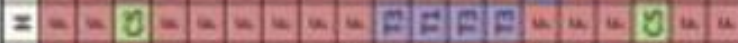

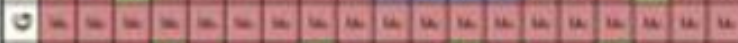

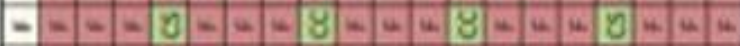

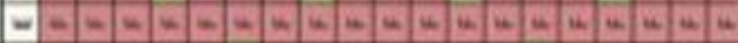

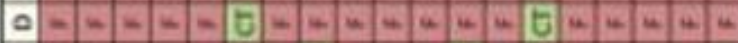

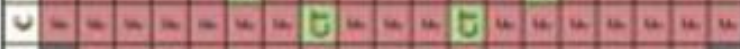

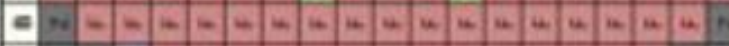

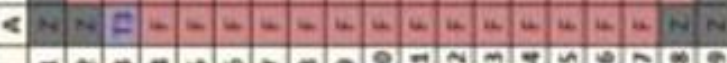




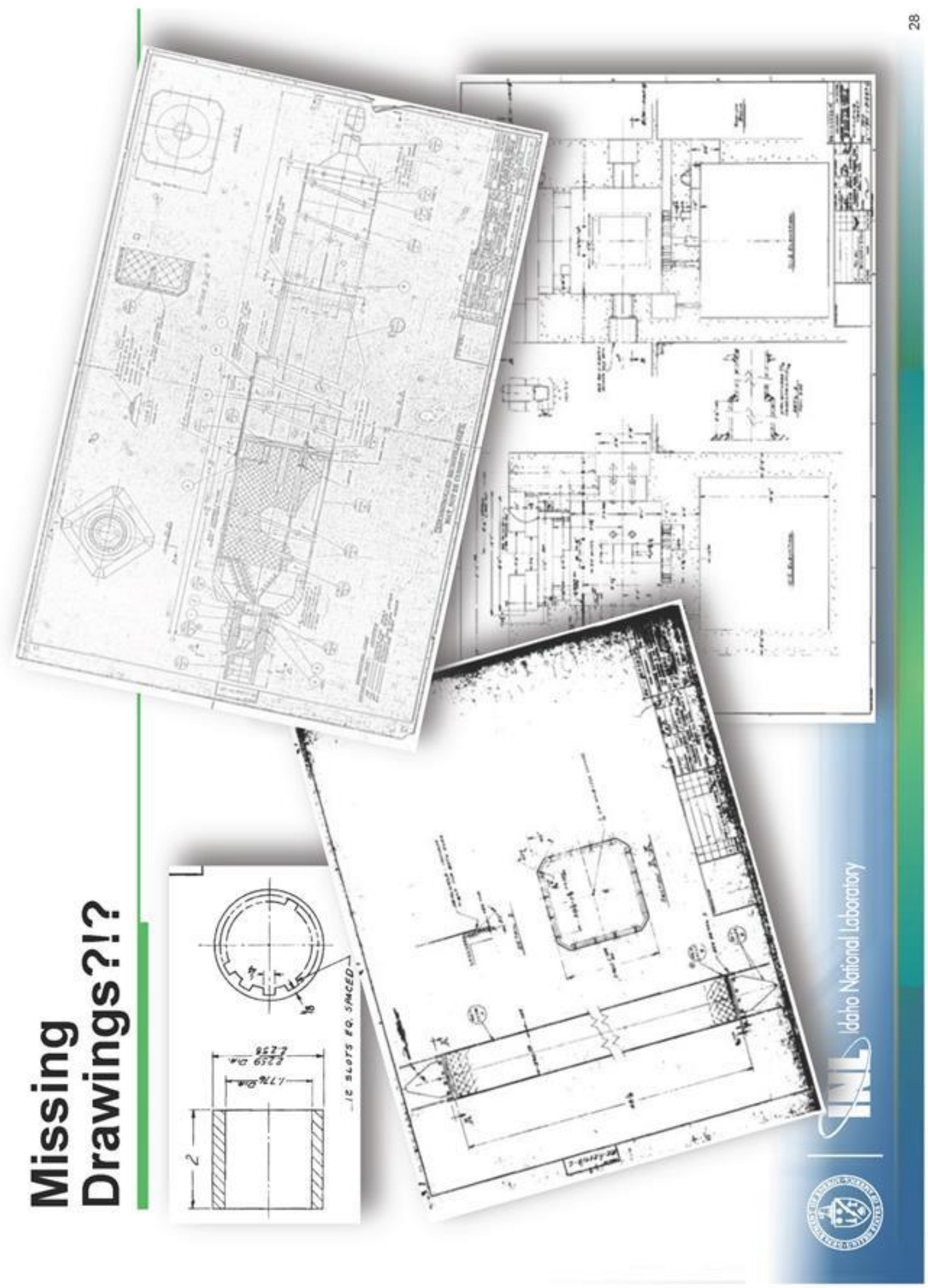




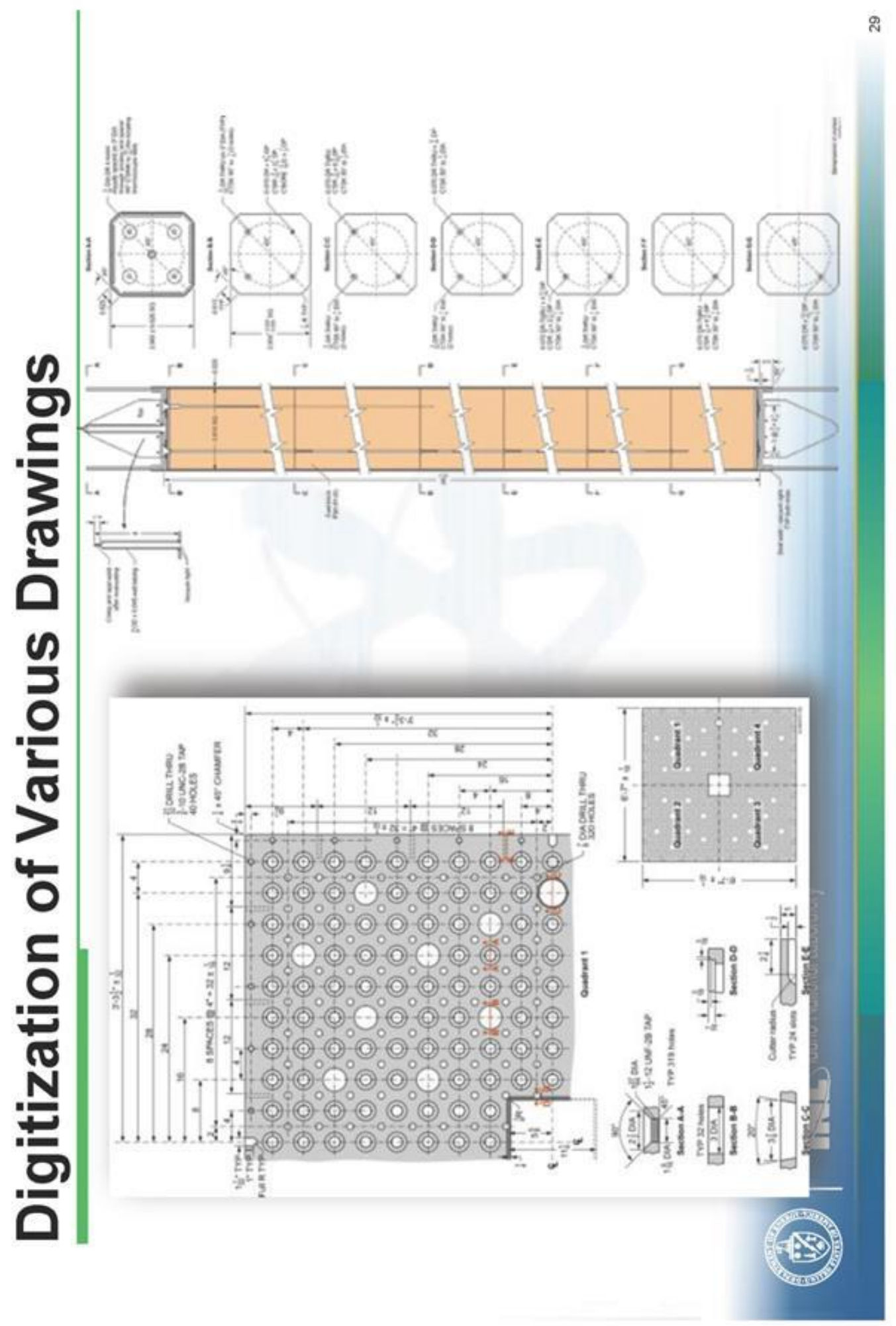




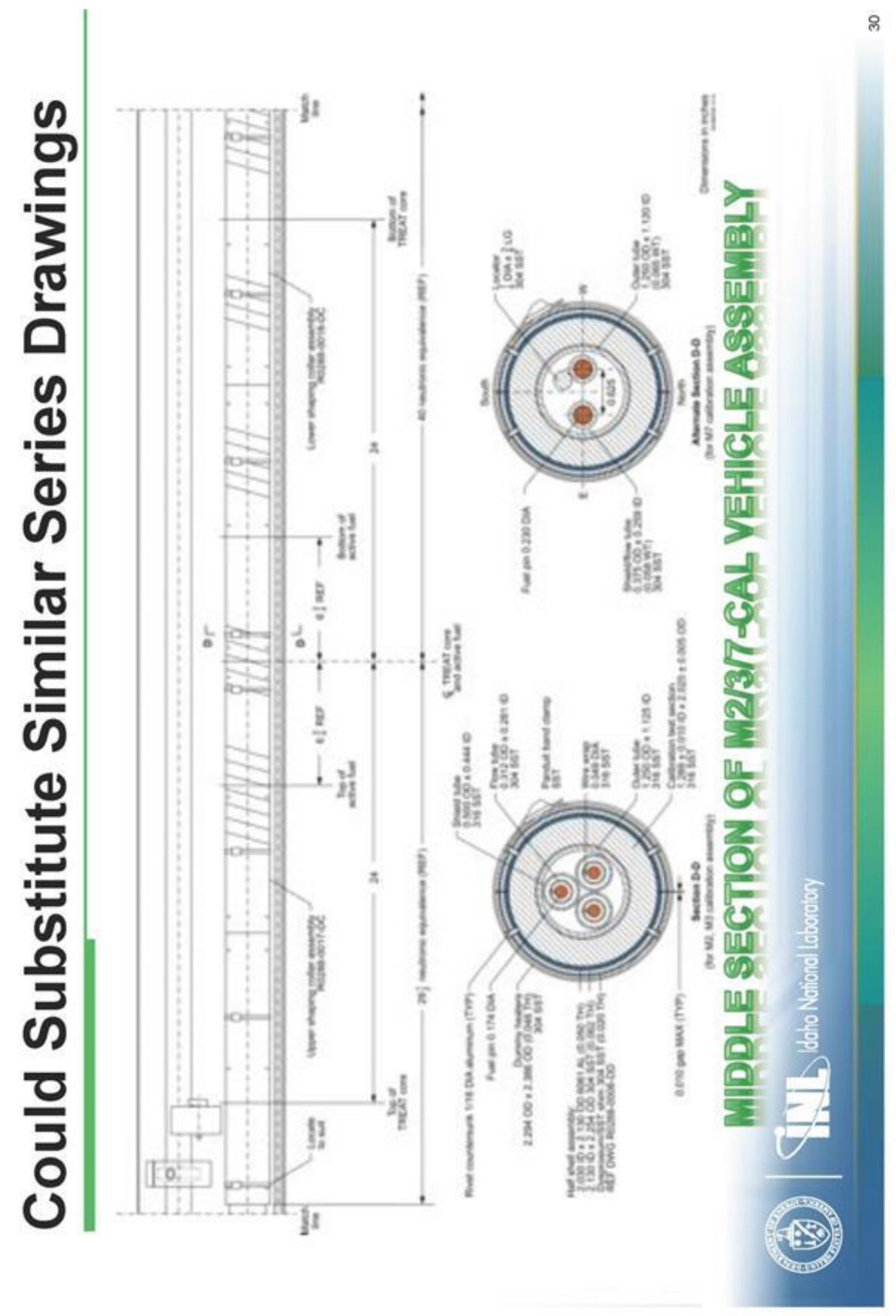




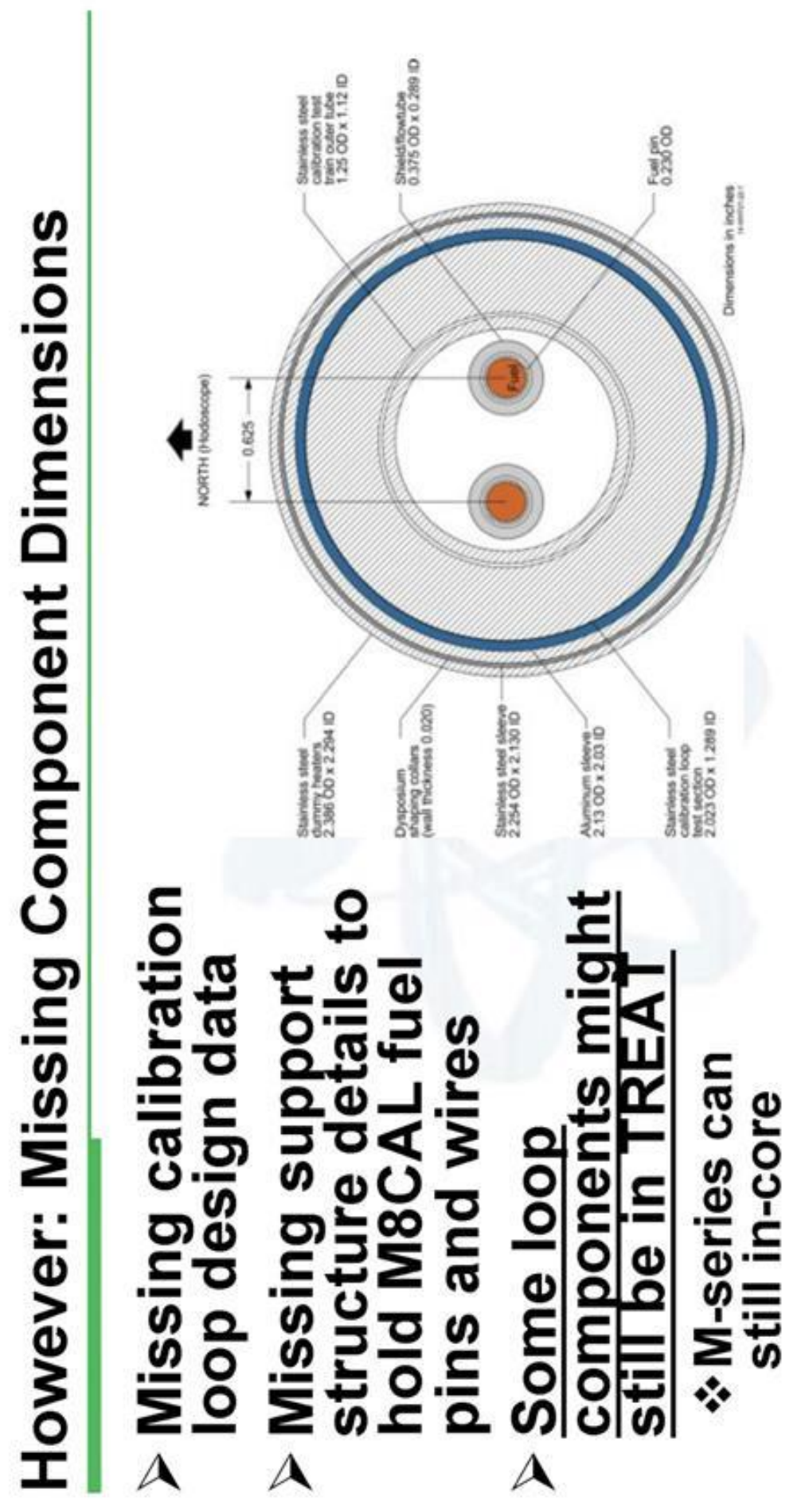




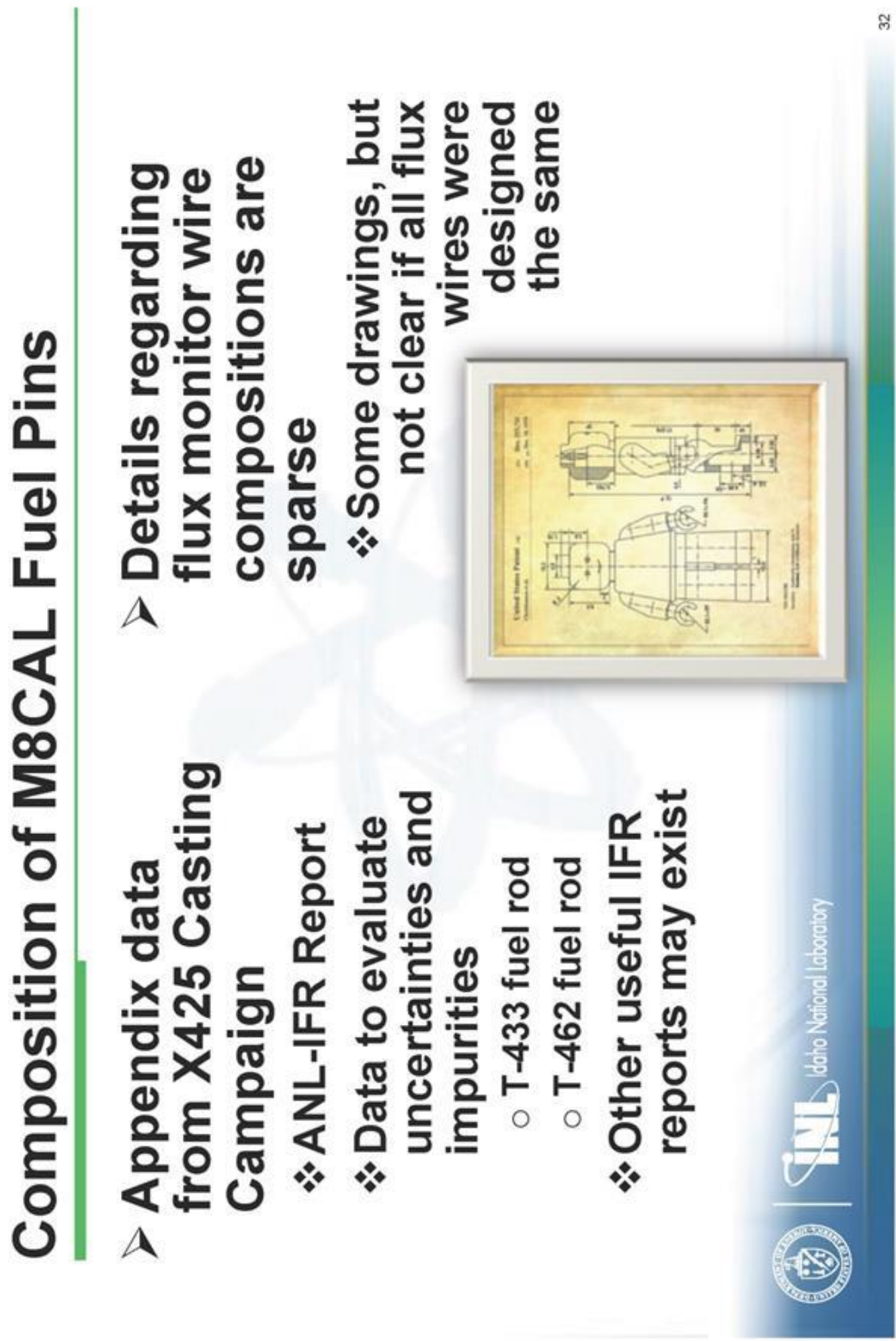




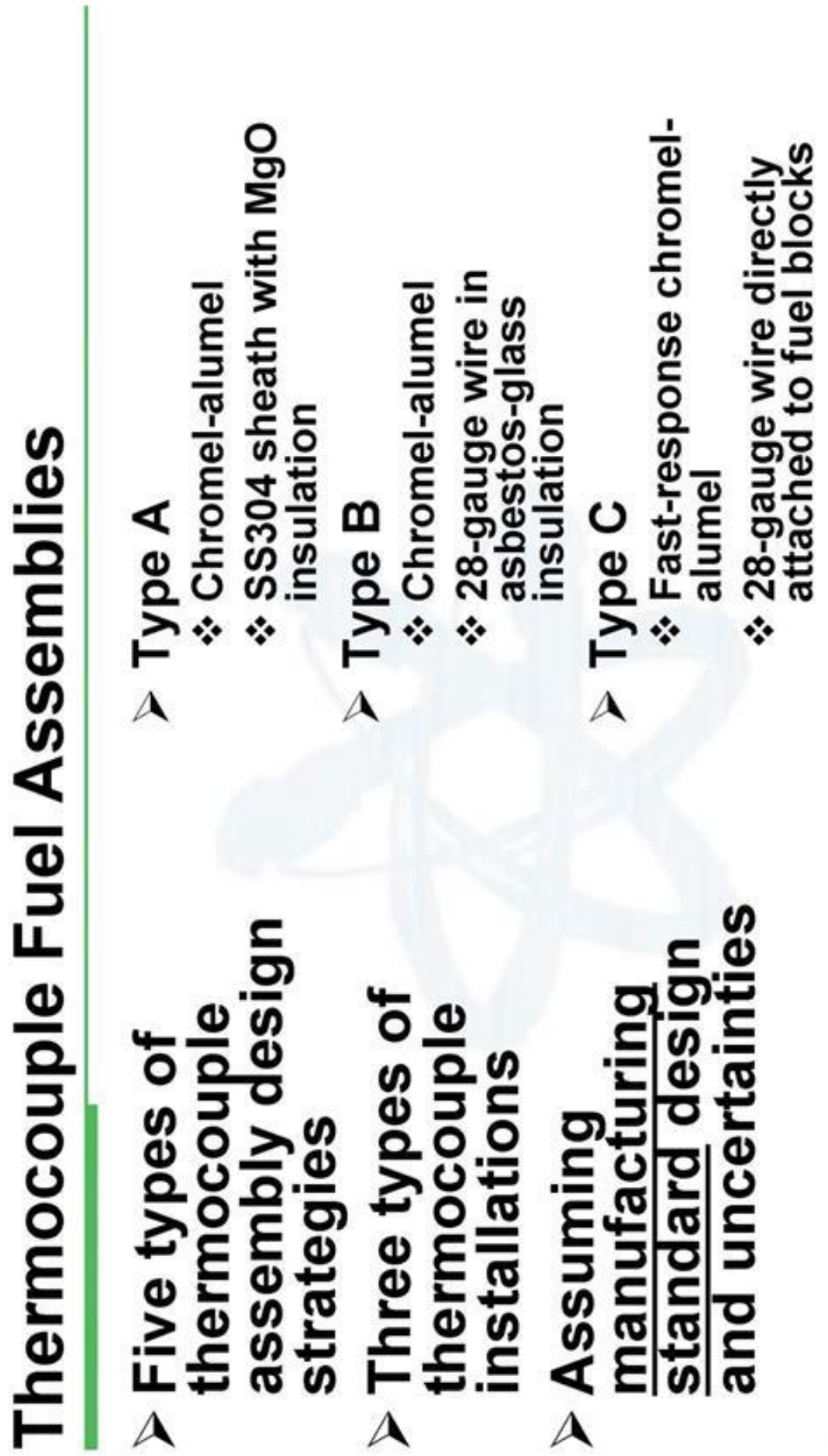




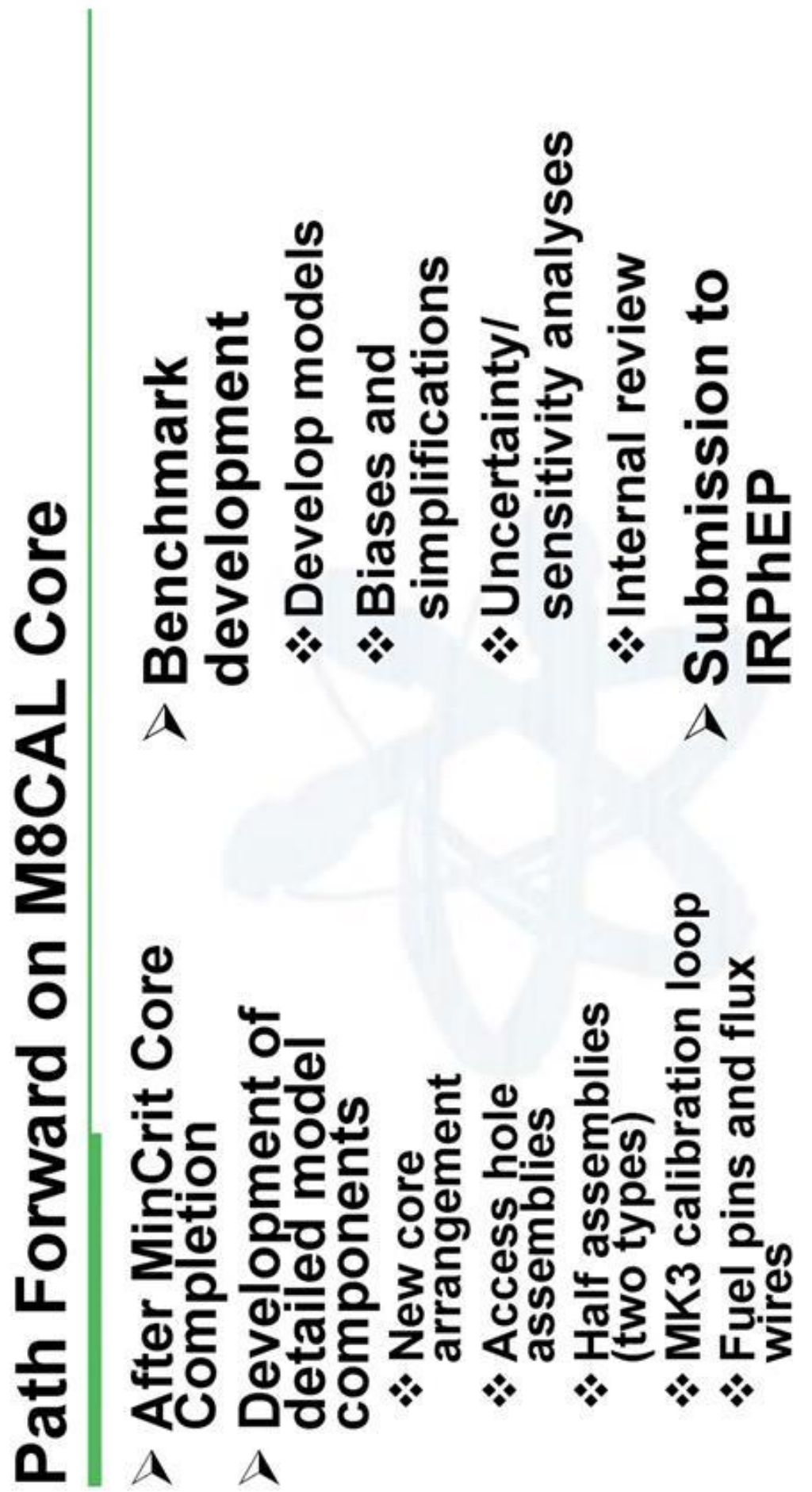




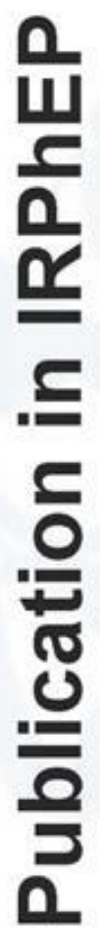
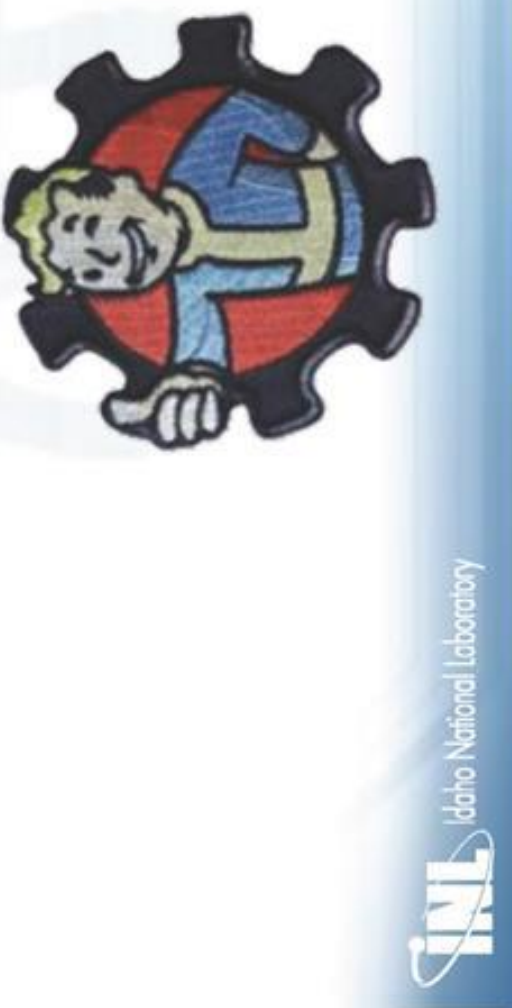

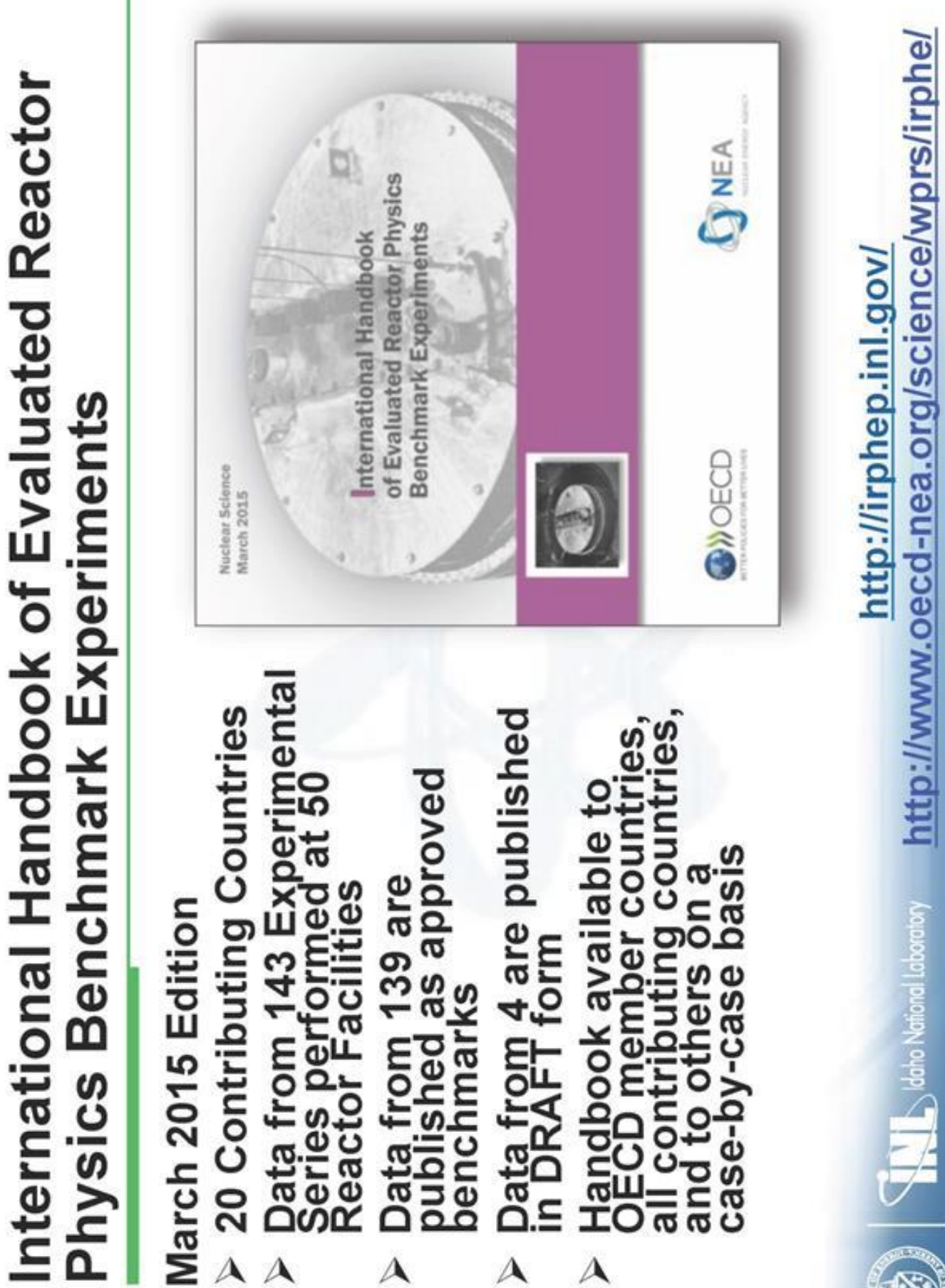

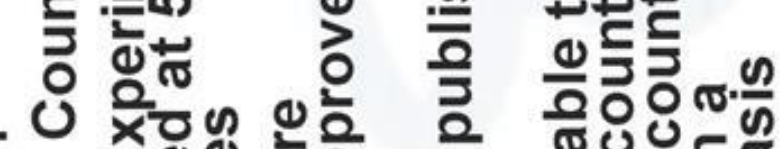

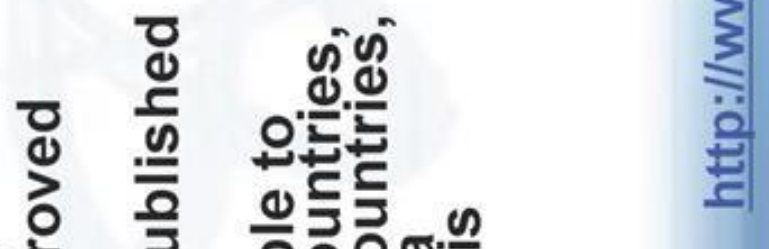

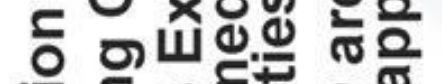

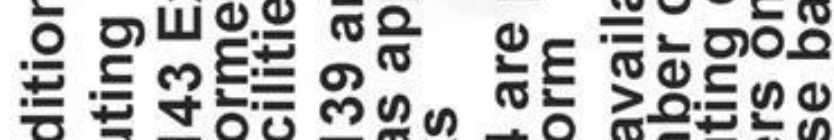

ய

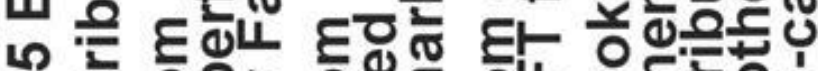

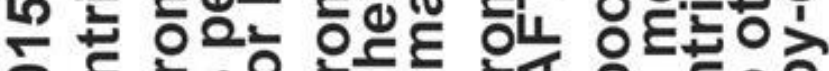

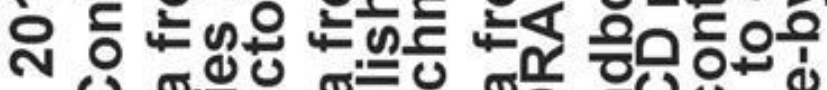

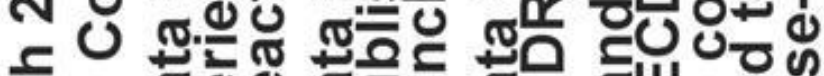

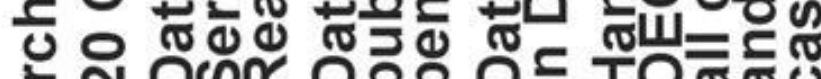

ส

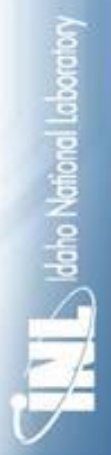

$\sum^{\mathbb{N}} \mathrm{A} \quad \mathrm{A} \quad \mathrm{A} \quad \mathrm{A}$ 

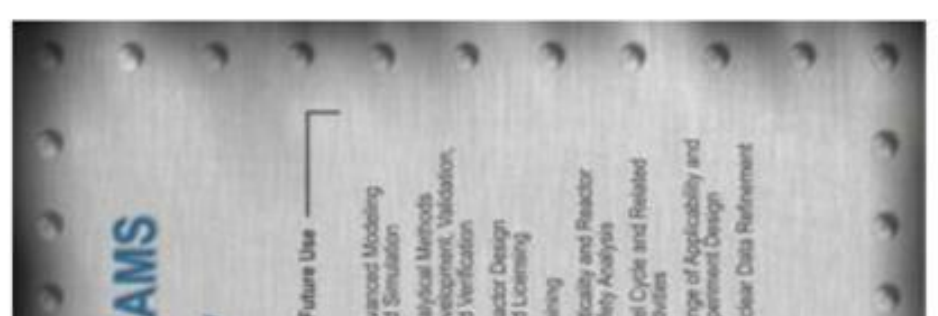

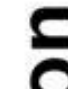

1

10

를

10

Uు

12

ช

ह

들

ఏ)

m

으

동

(2)

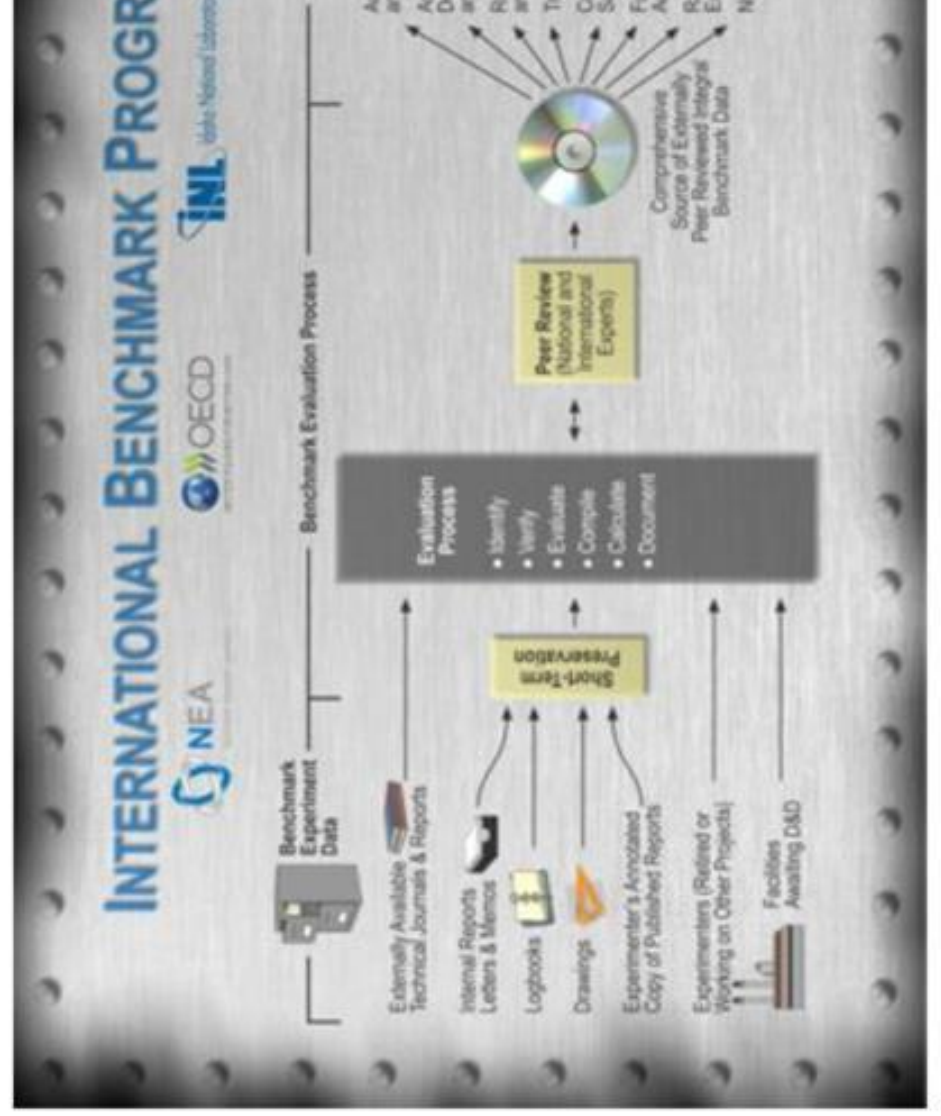




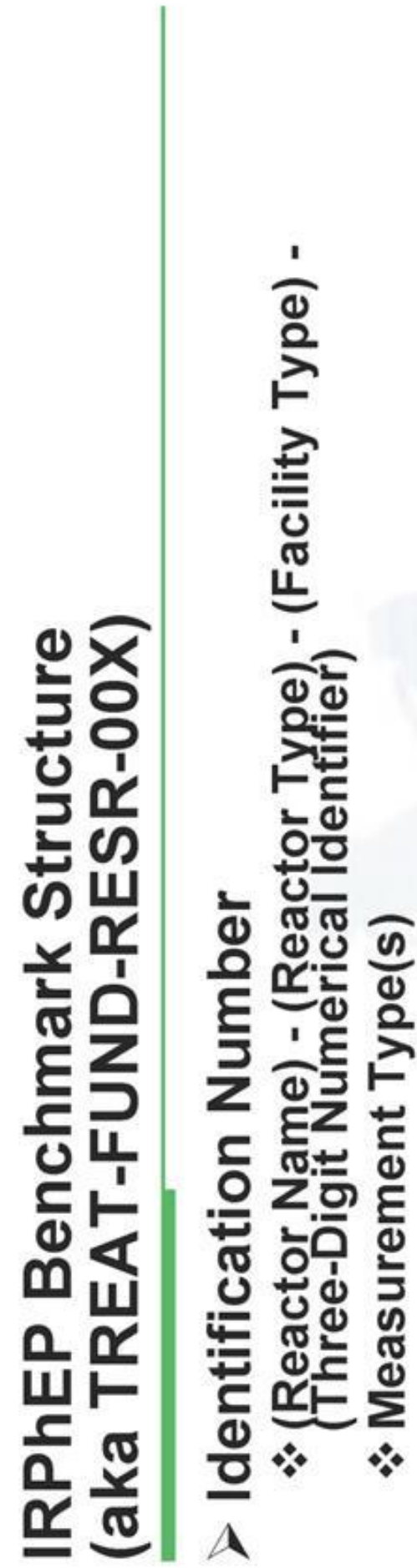

塄

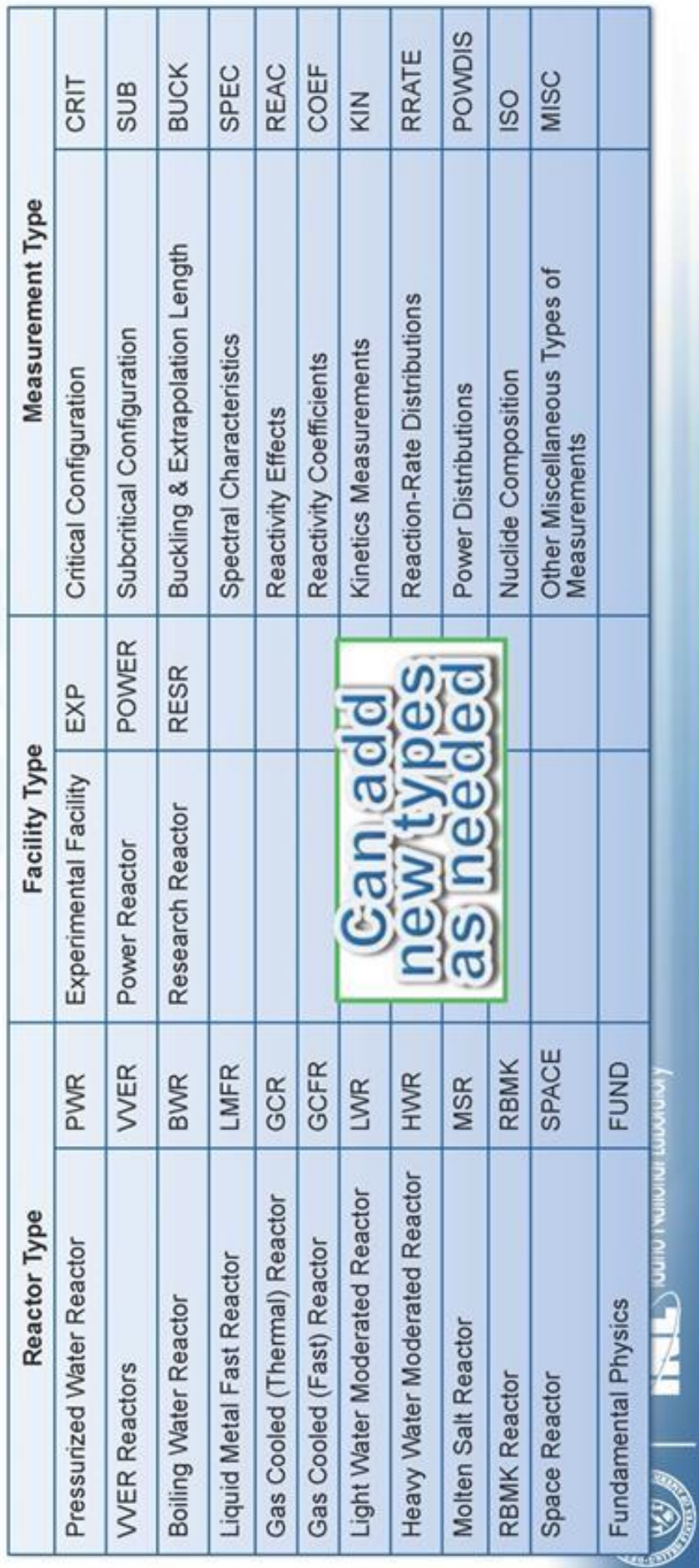


$\frac{\text { ㄴ }}{\text { 들 }}$

(1)

$\approx$

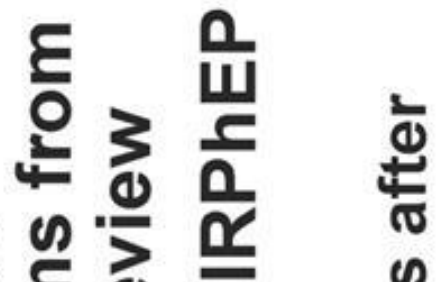

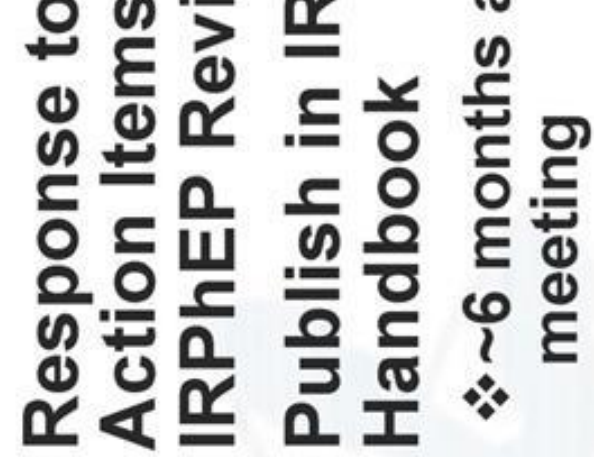
A 4

$>$

2

(1)

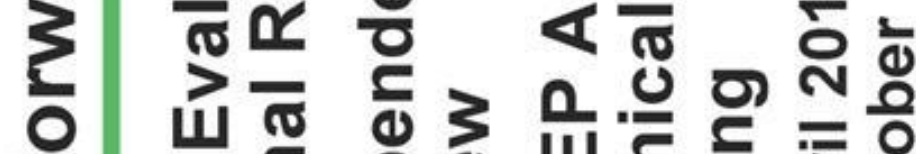

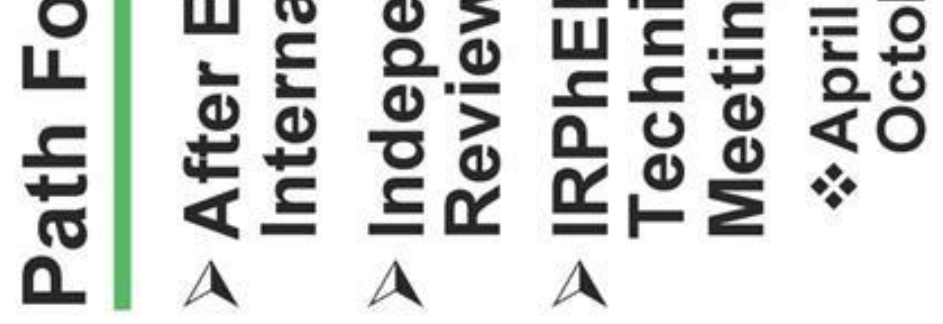



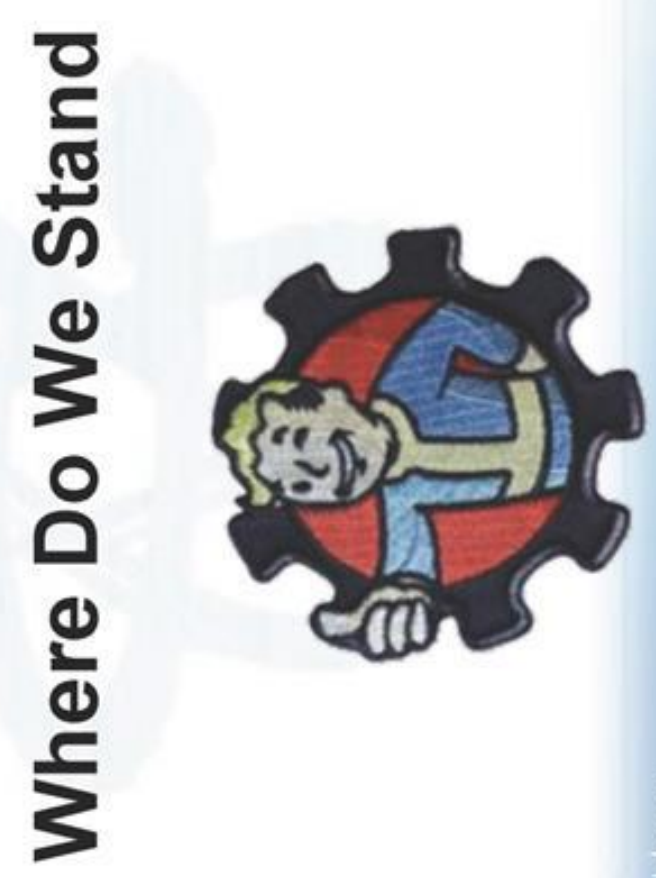


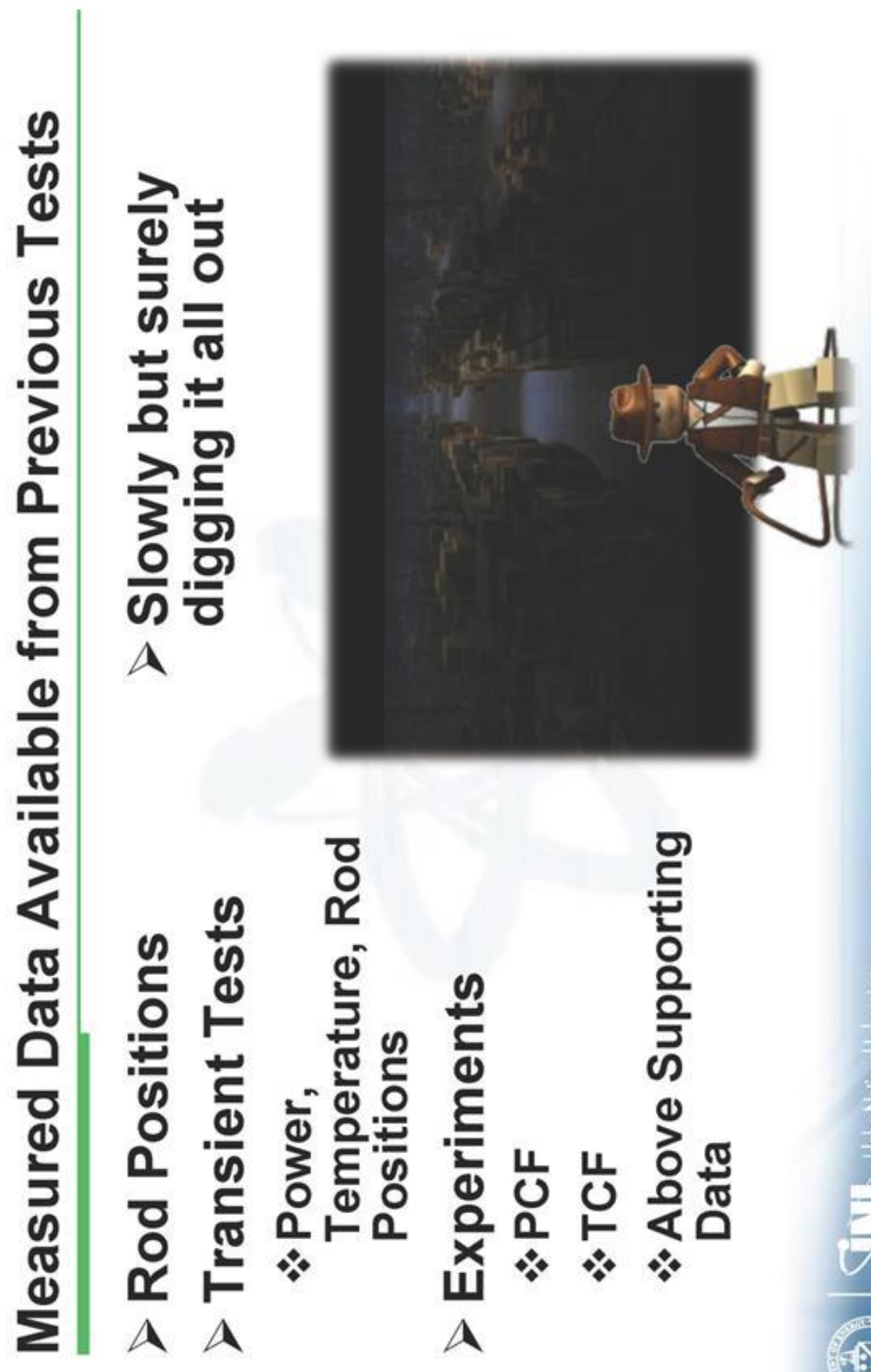




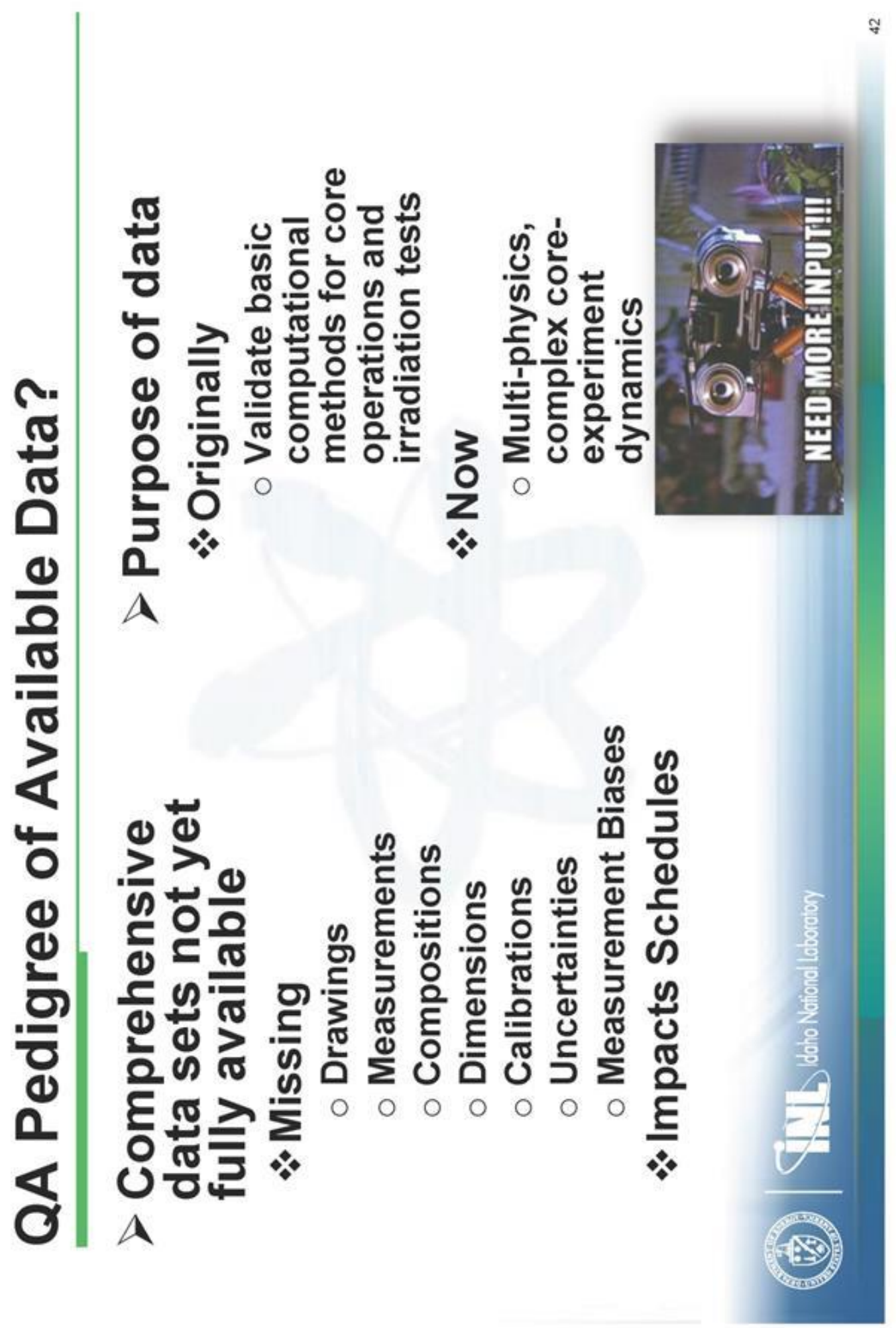




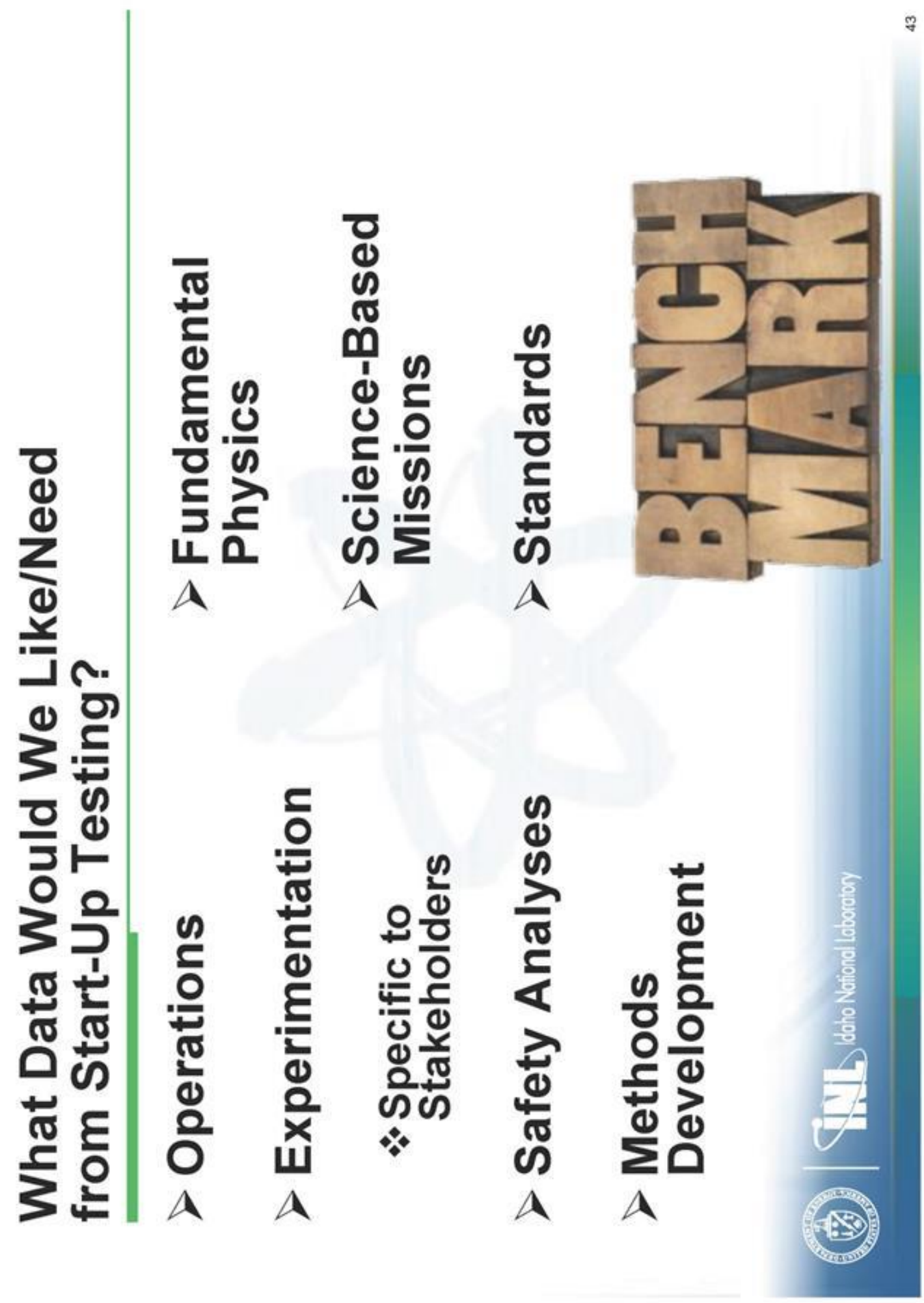




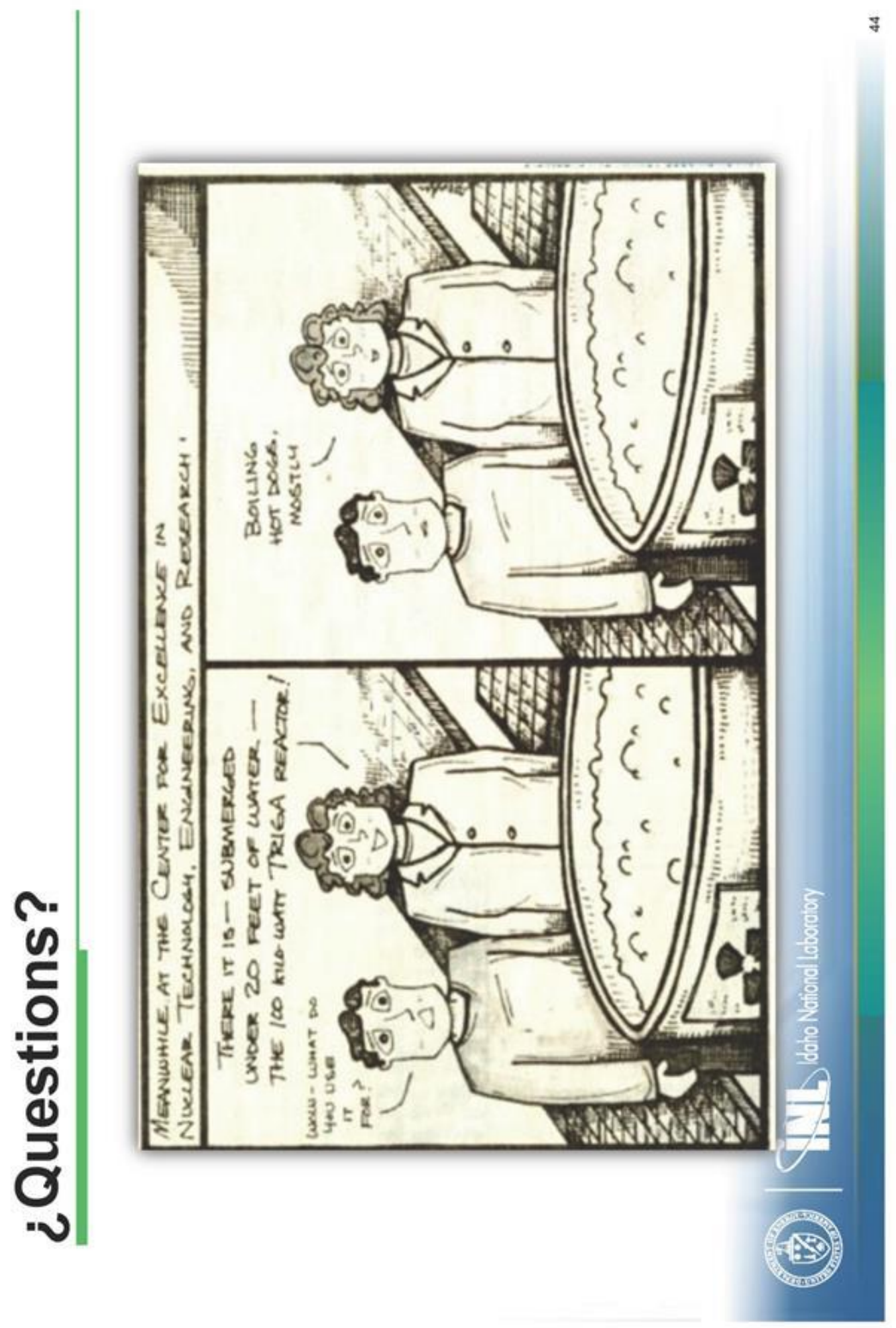




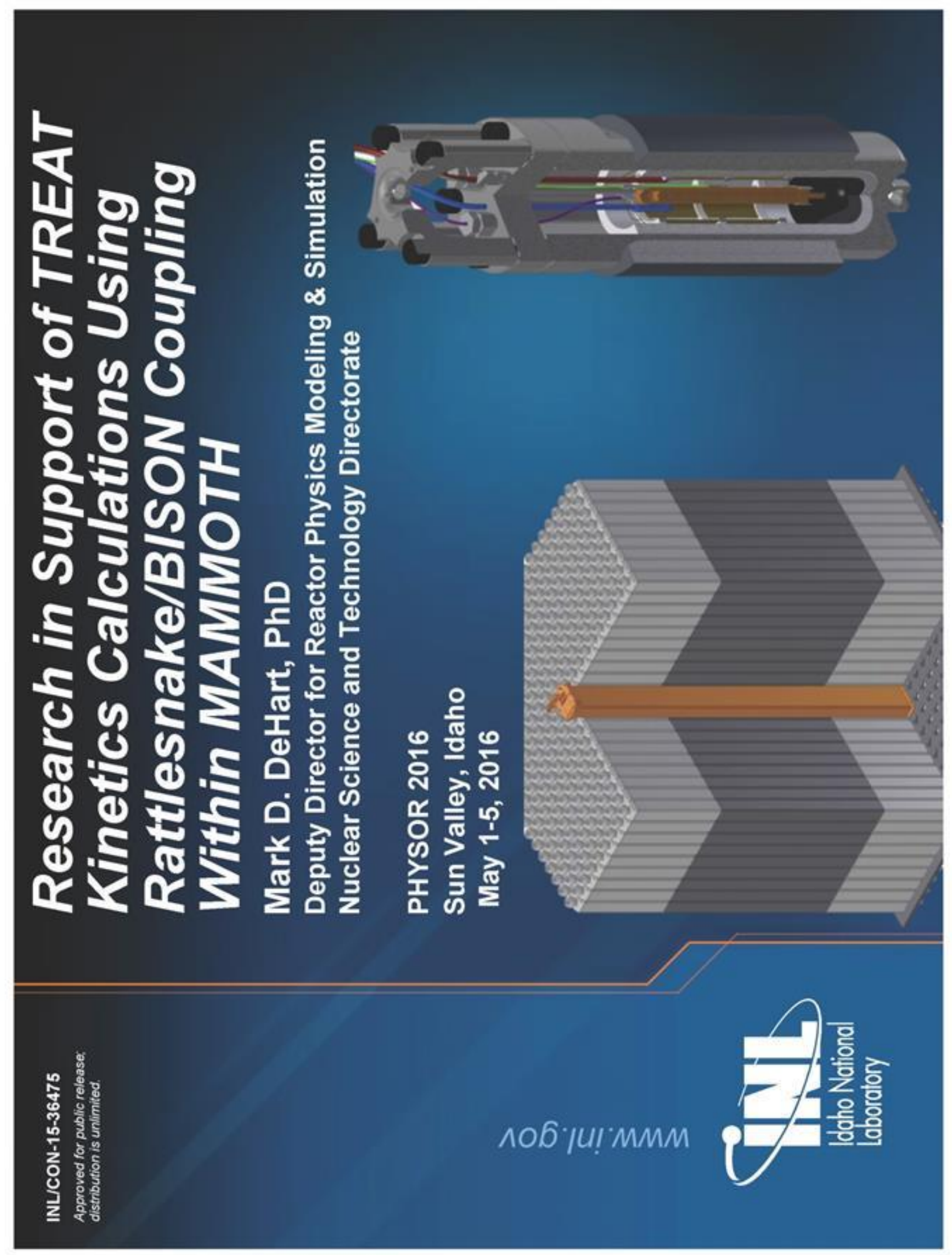




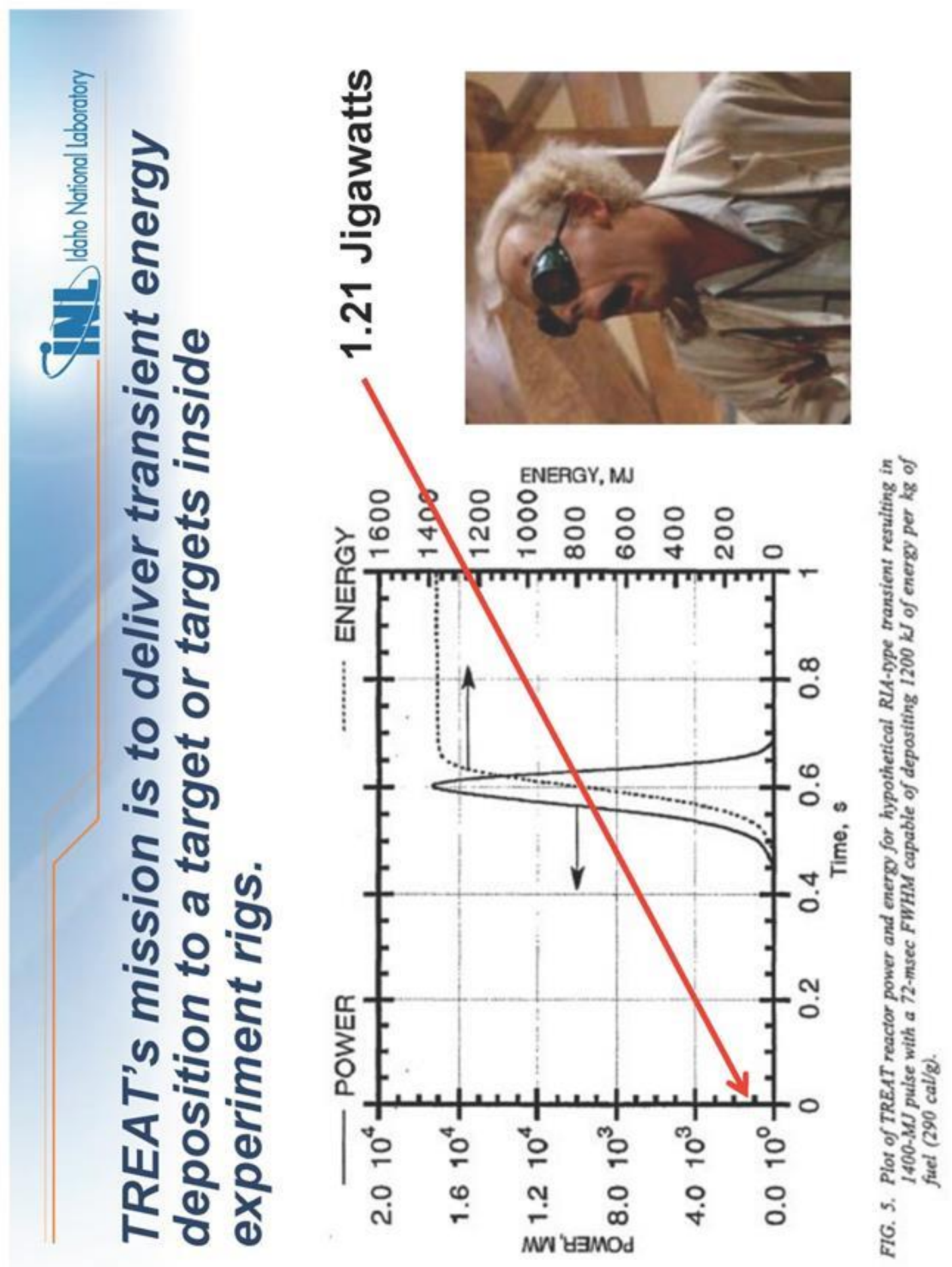




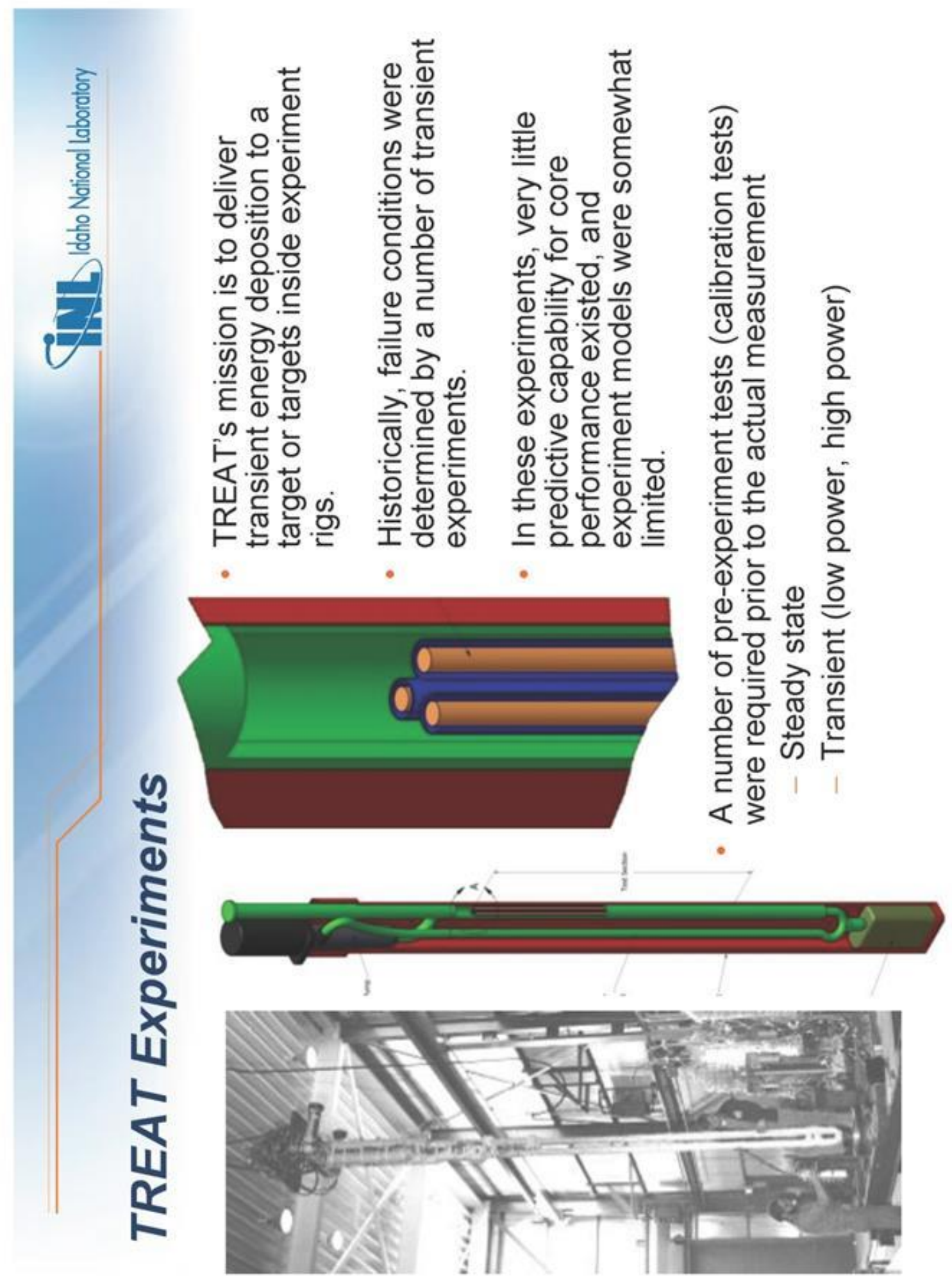




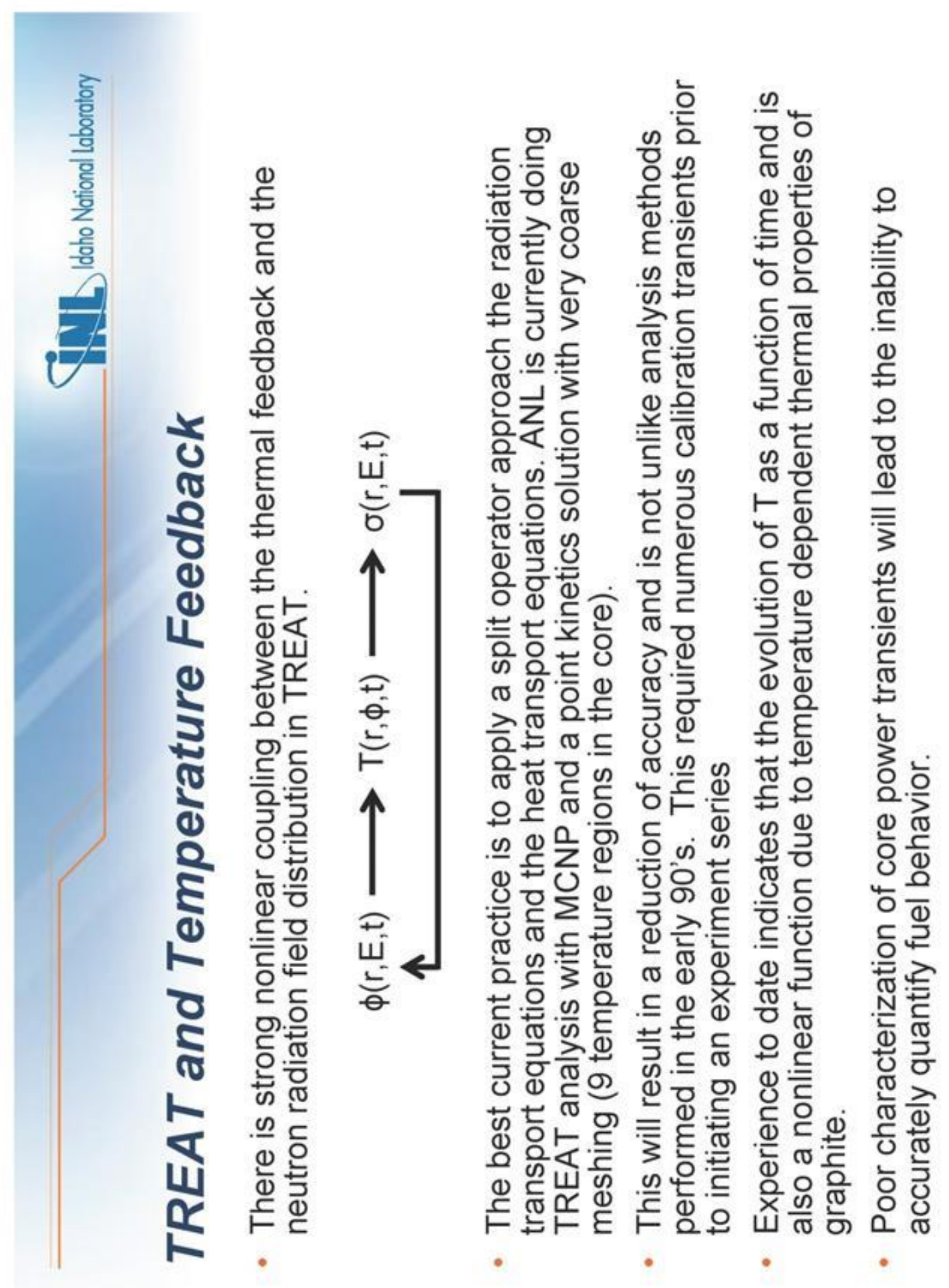




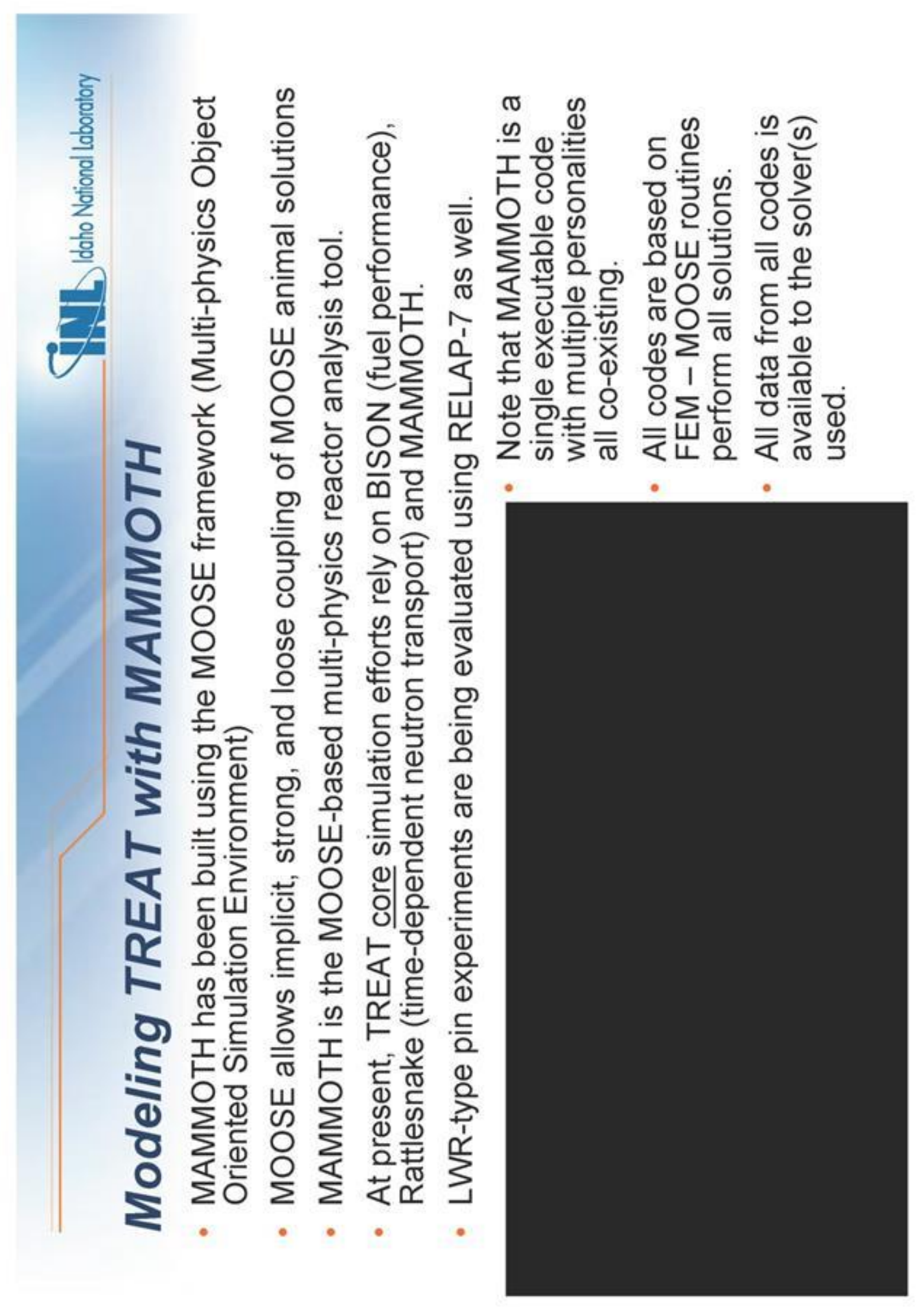




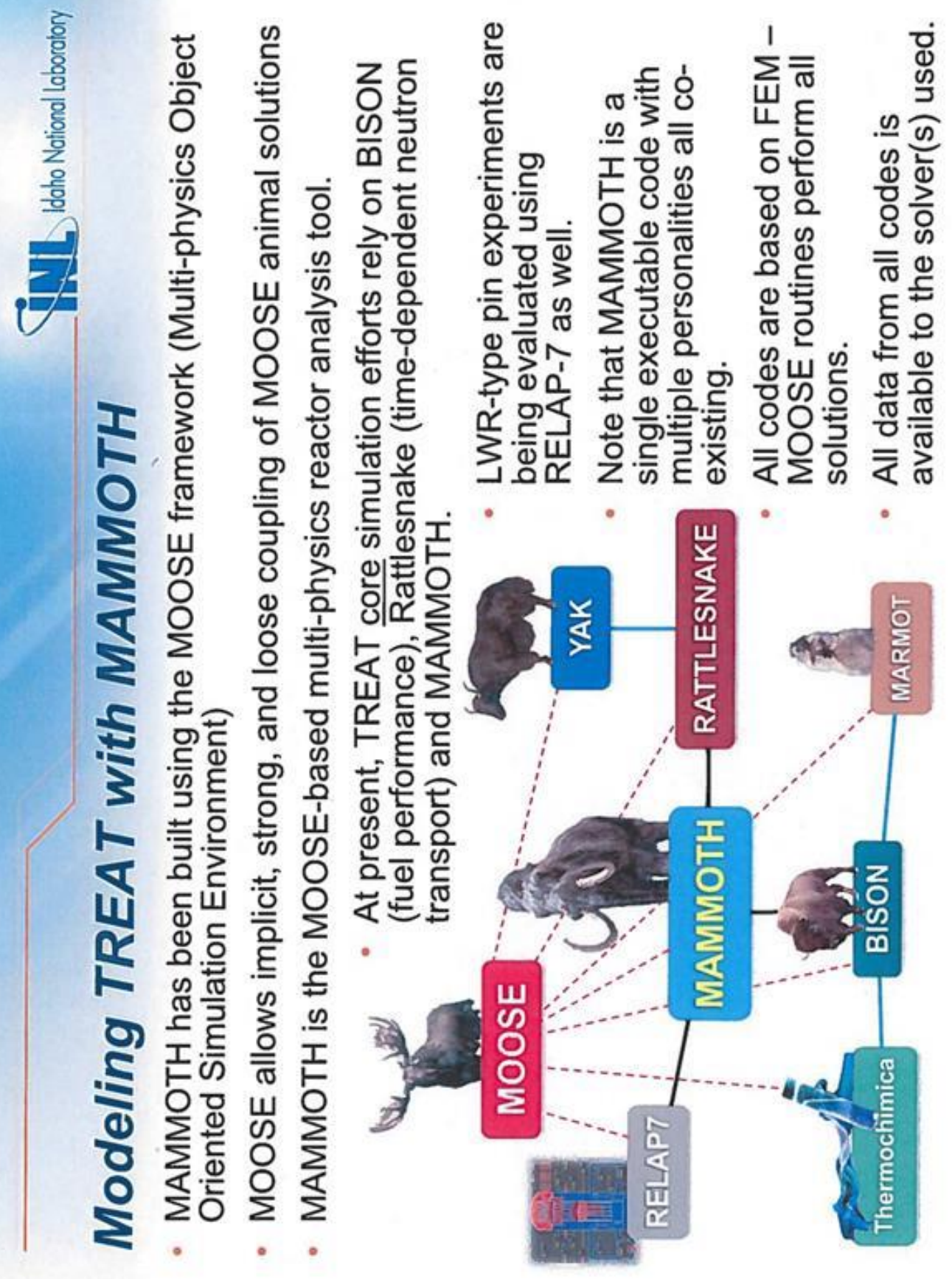




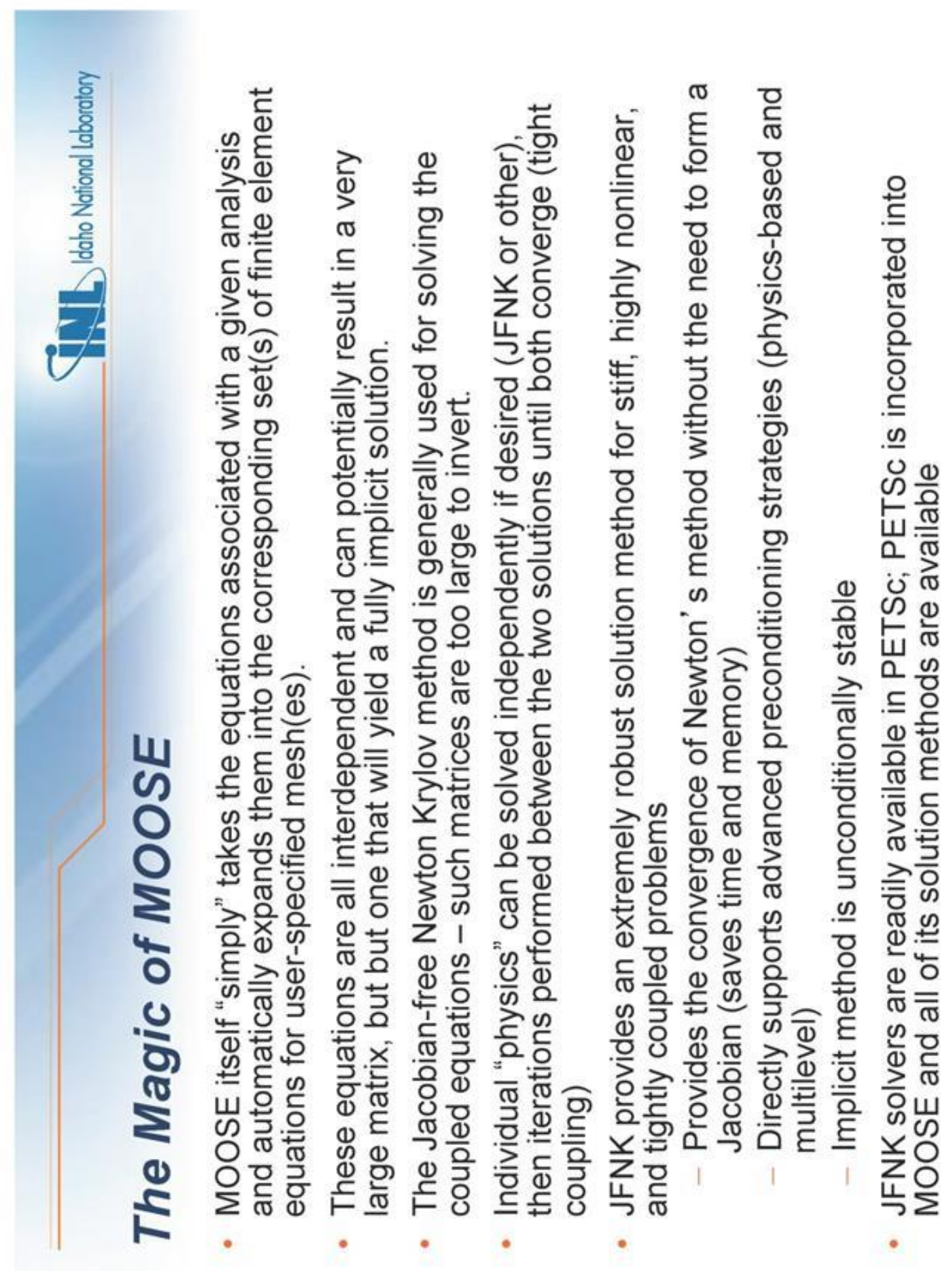




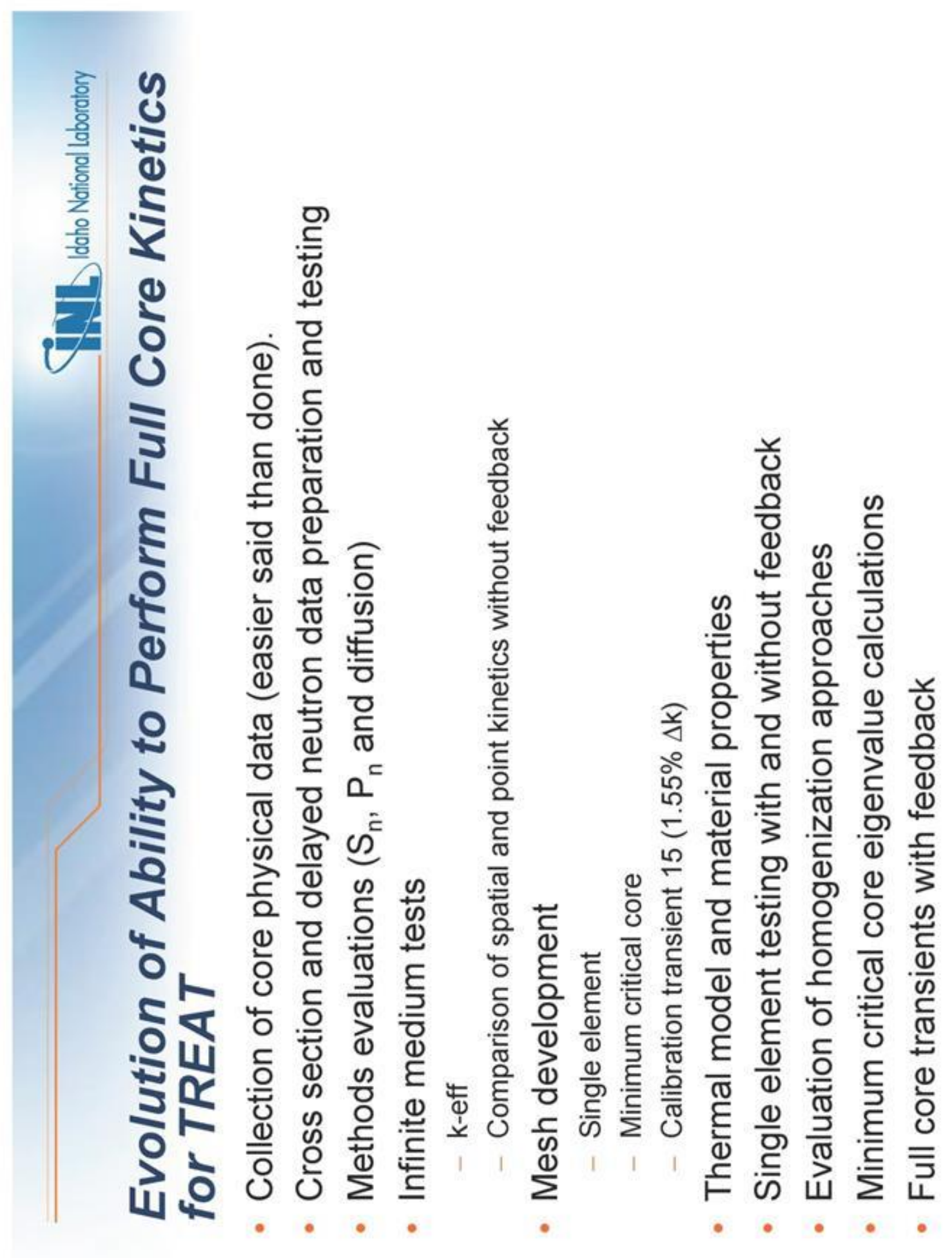




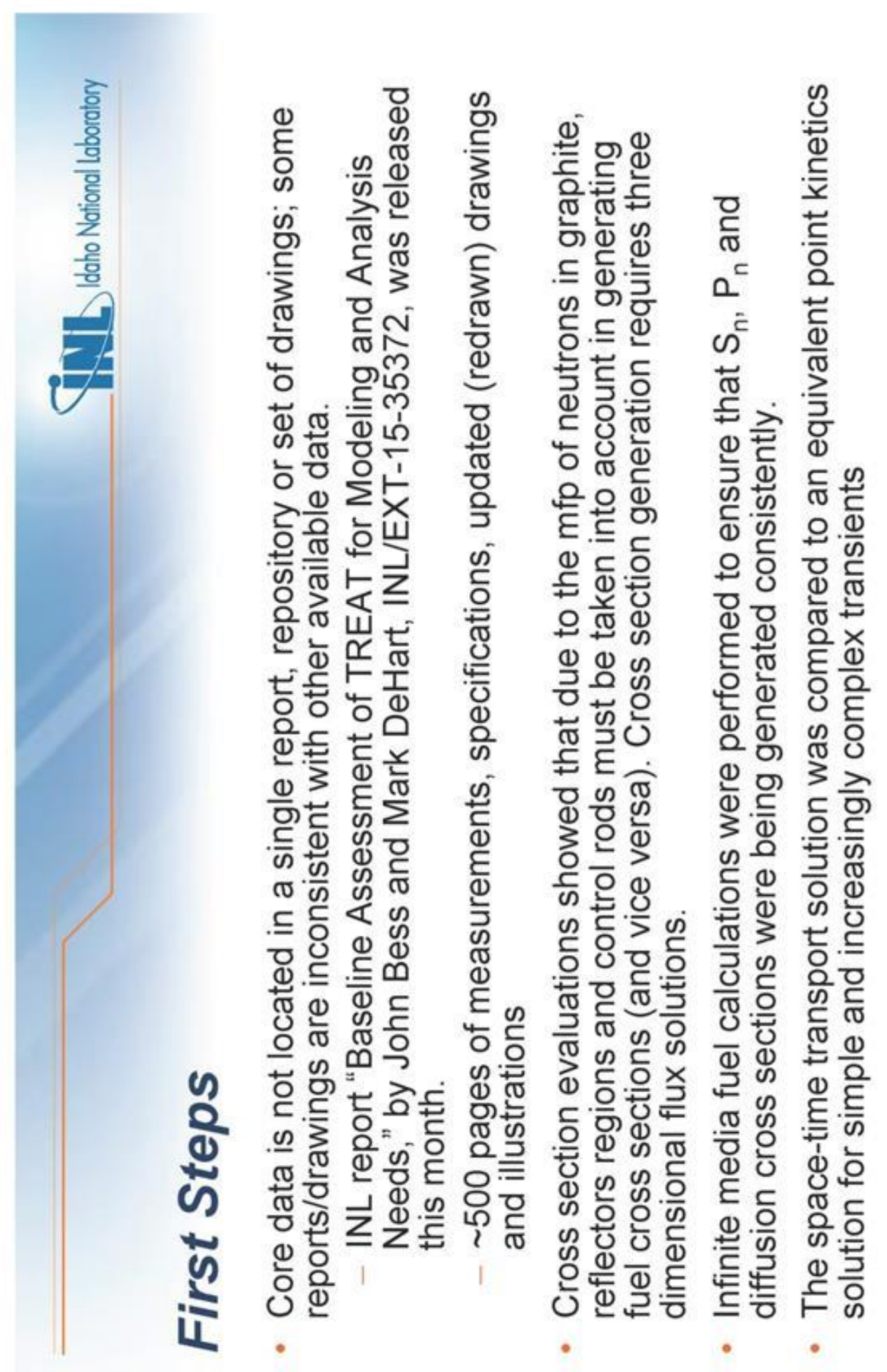




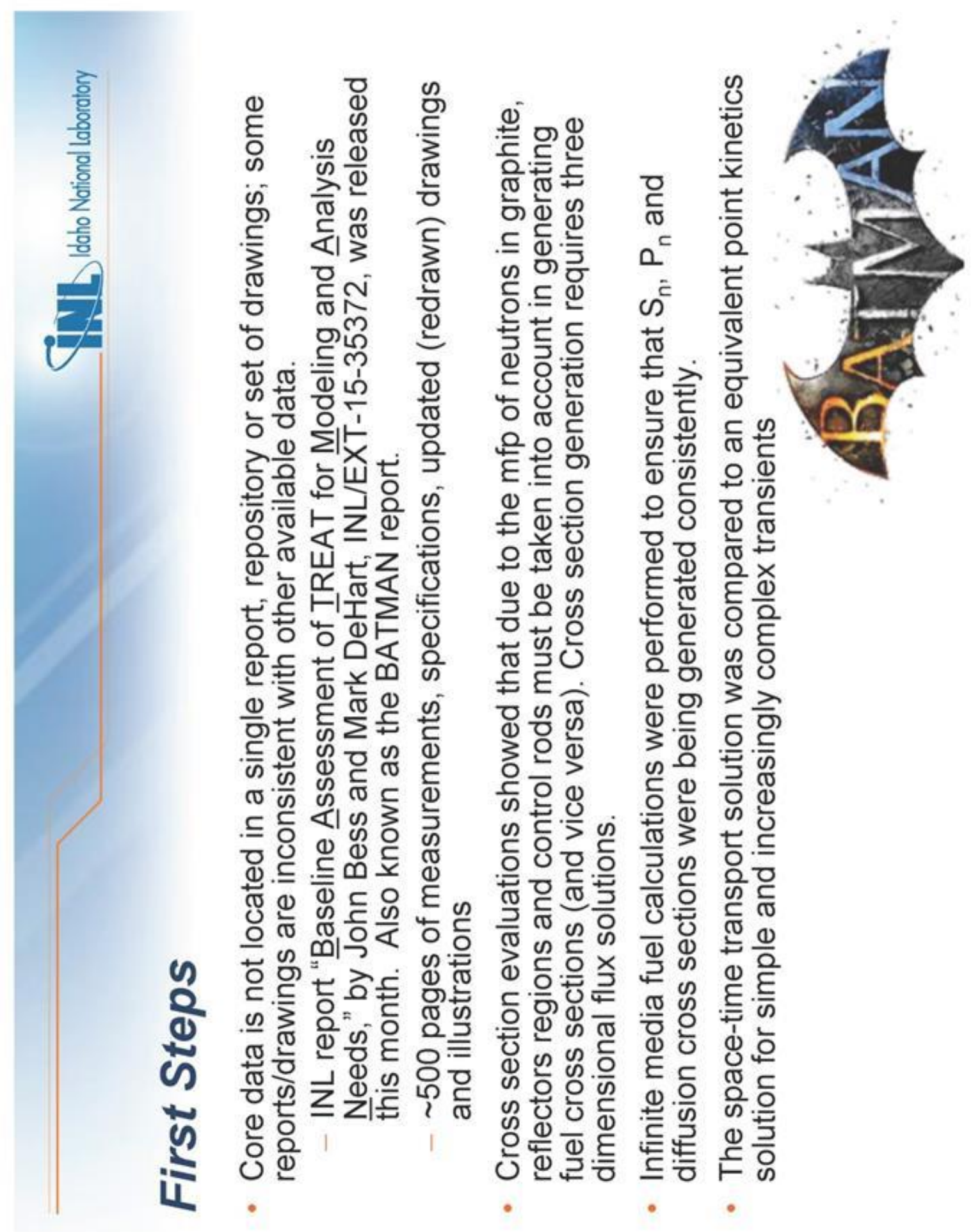




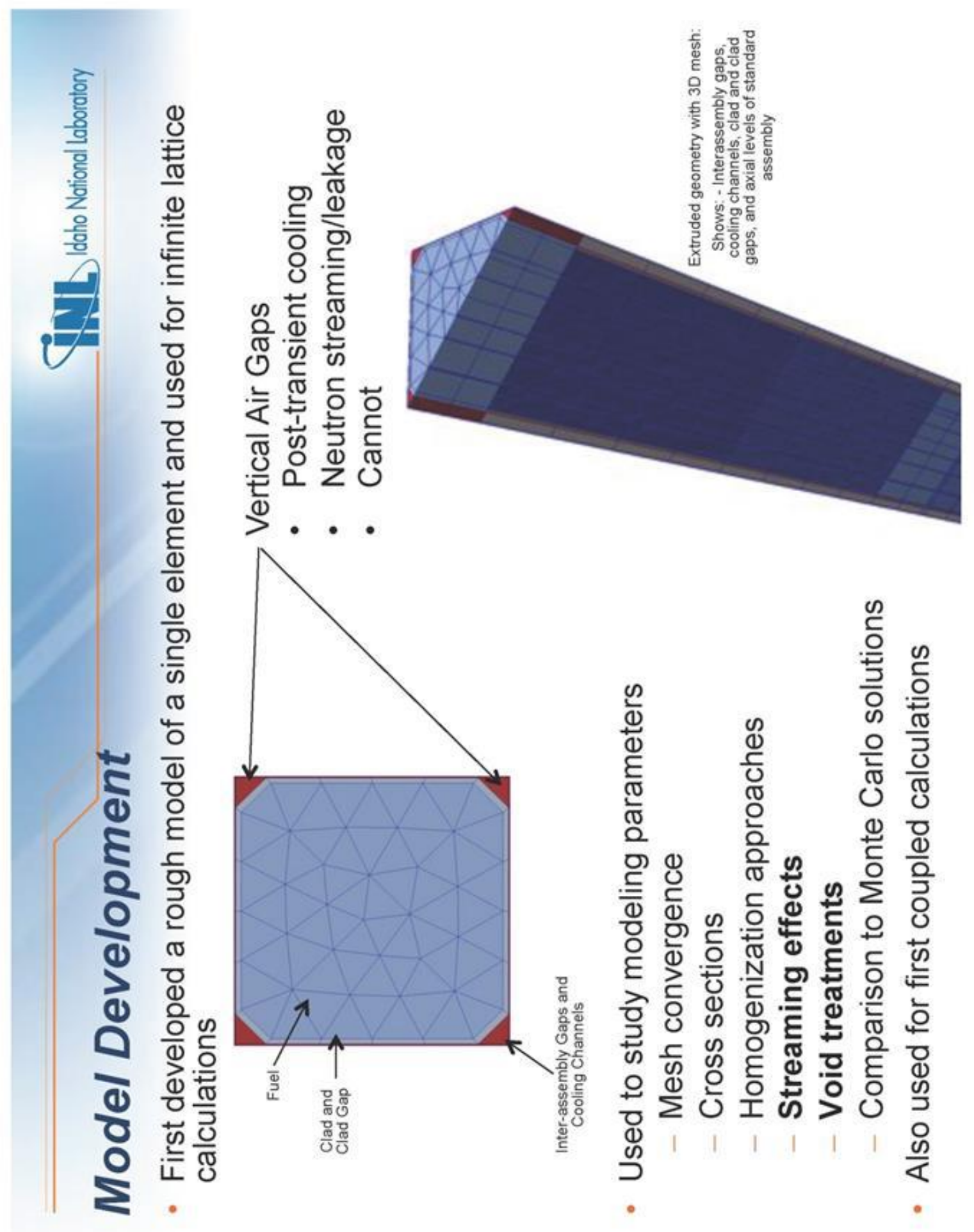




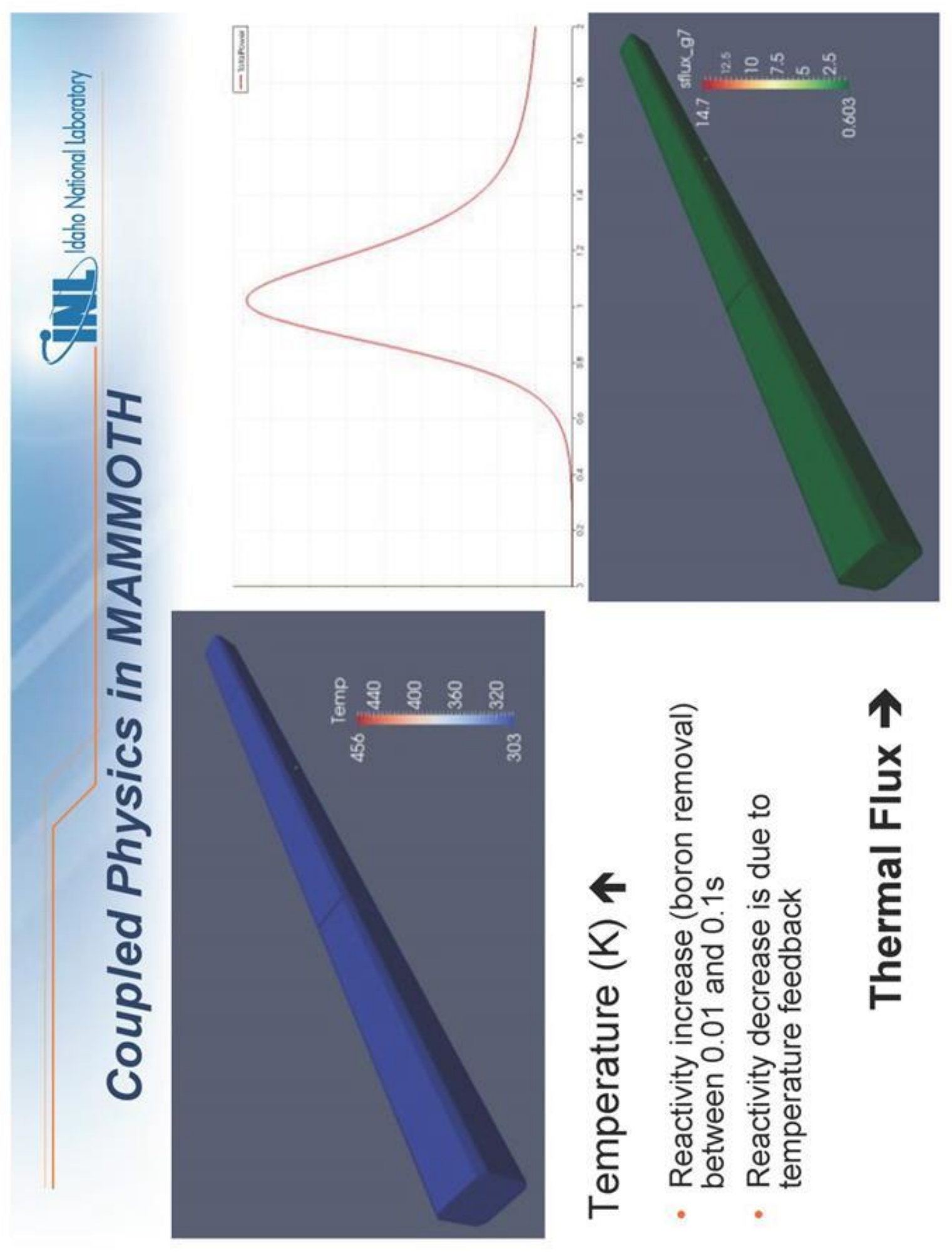




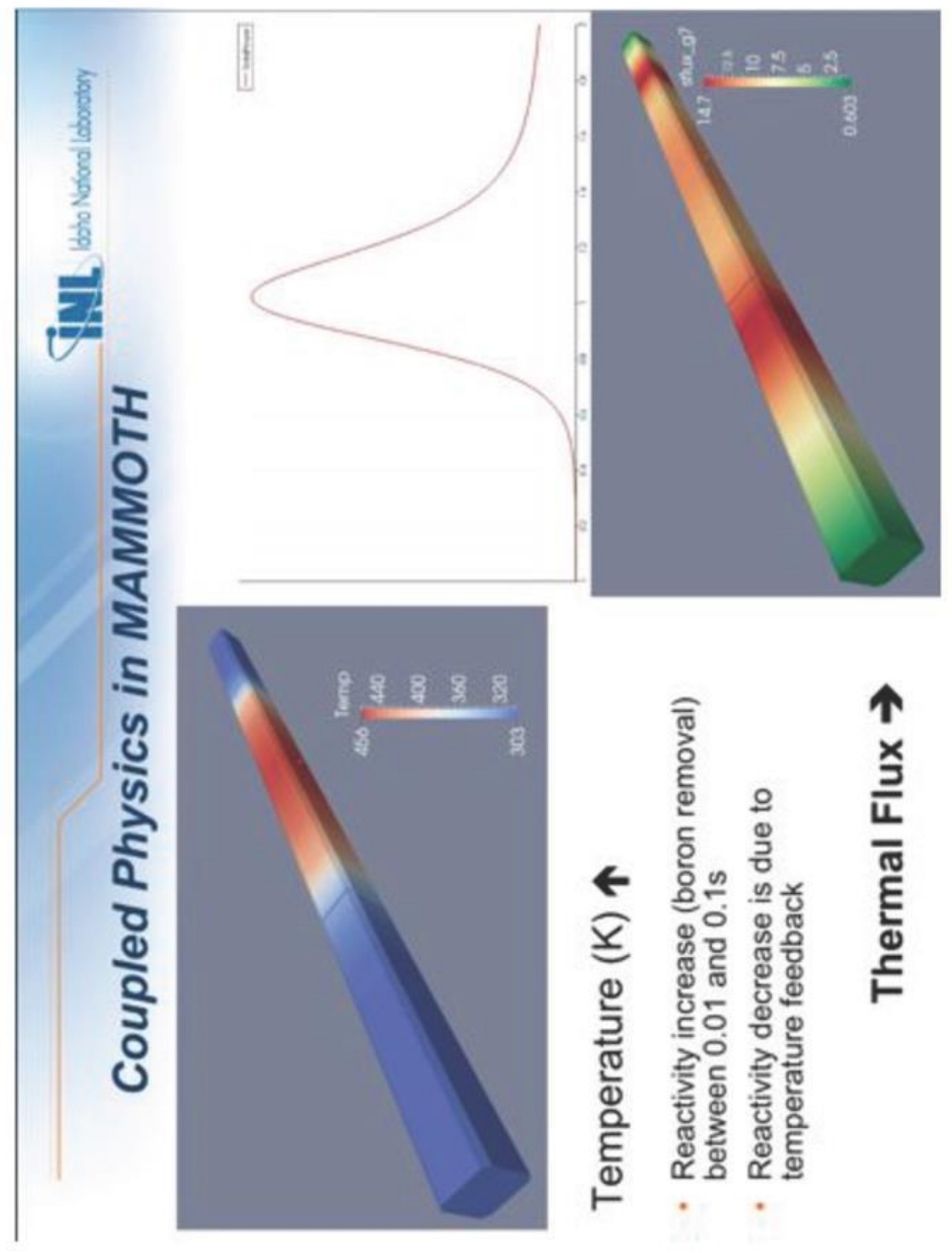




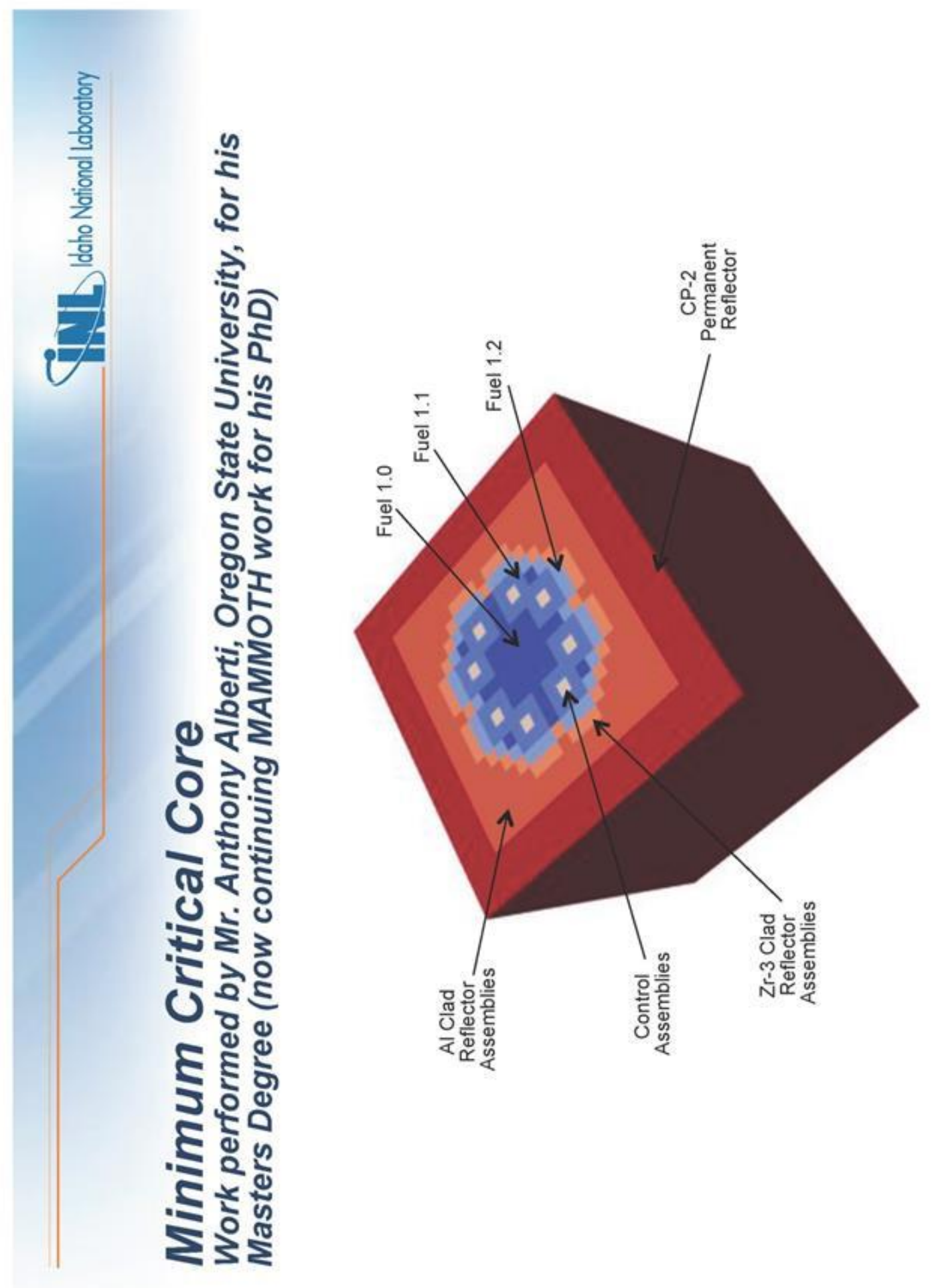




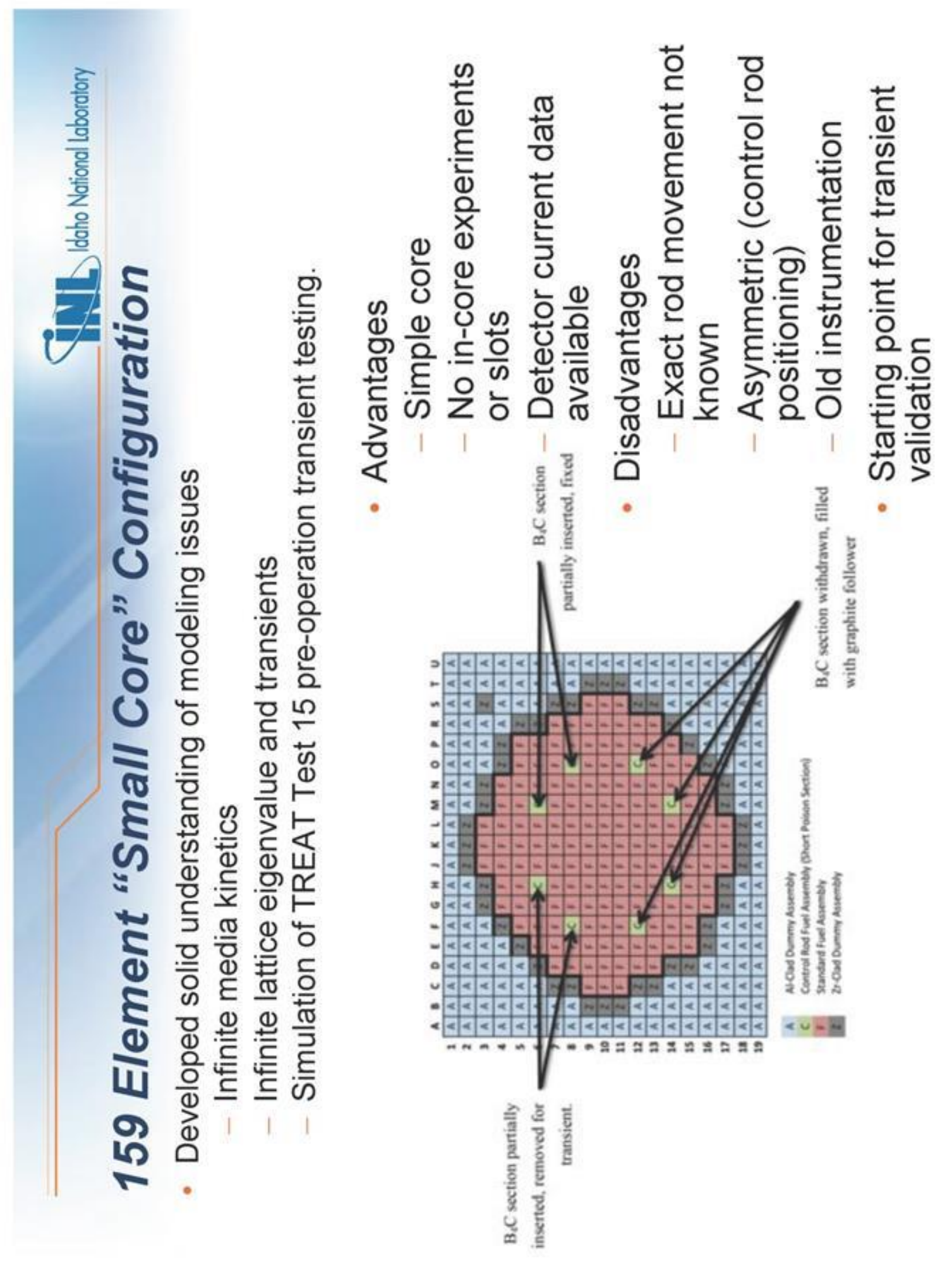




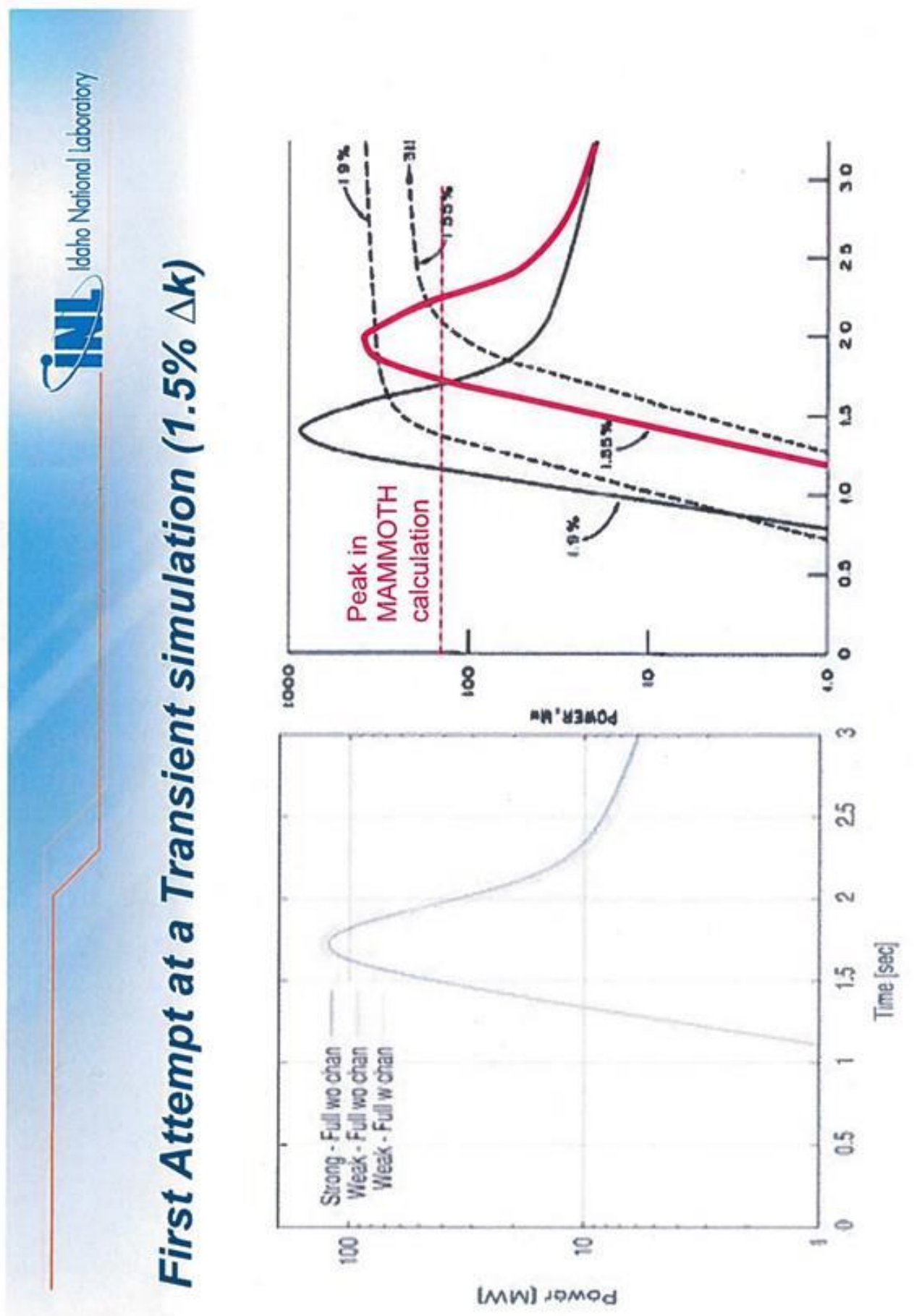



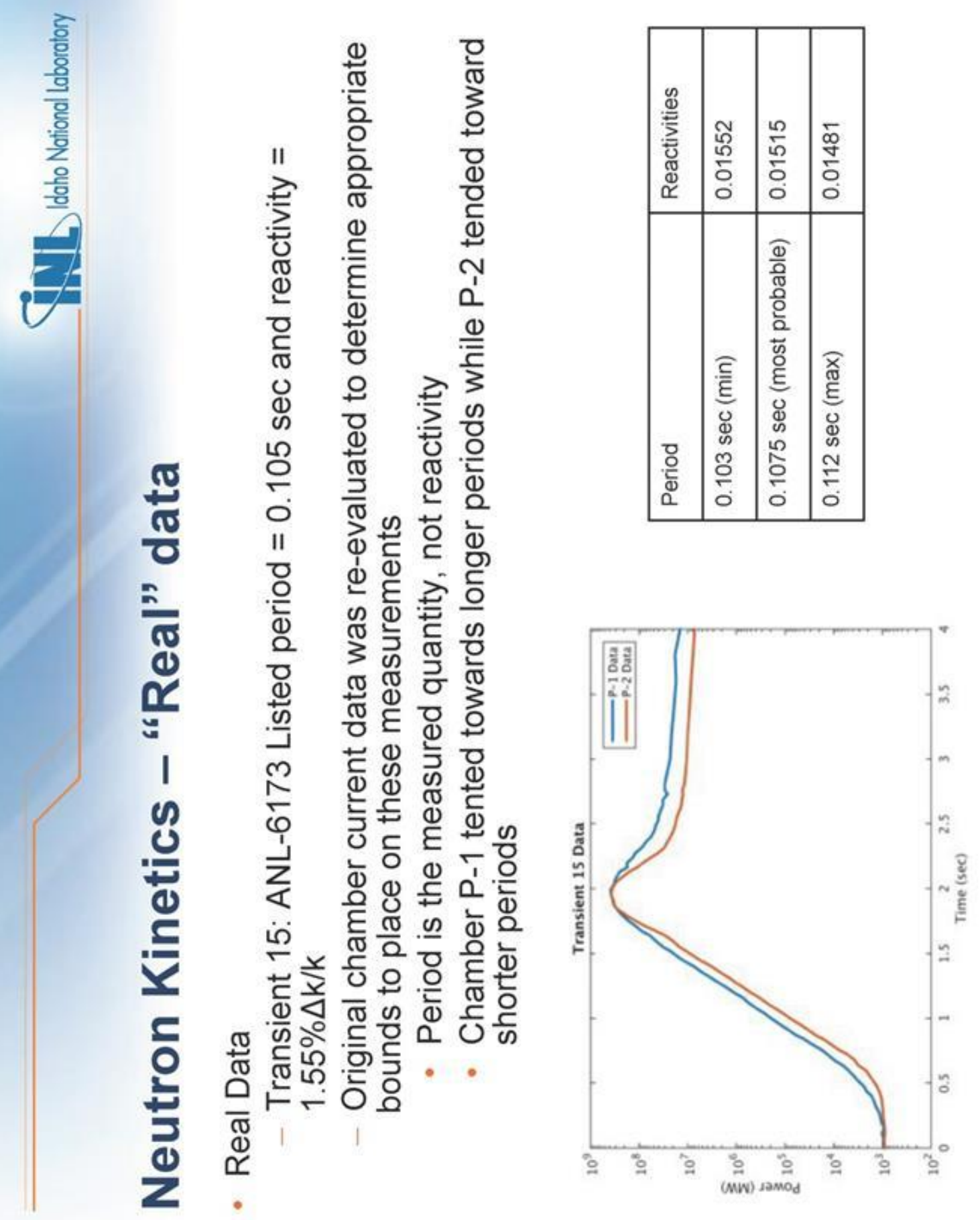


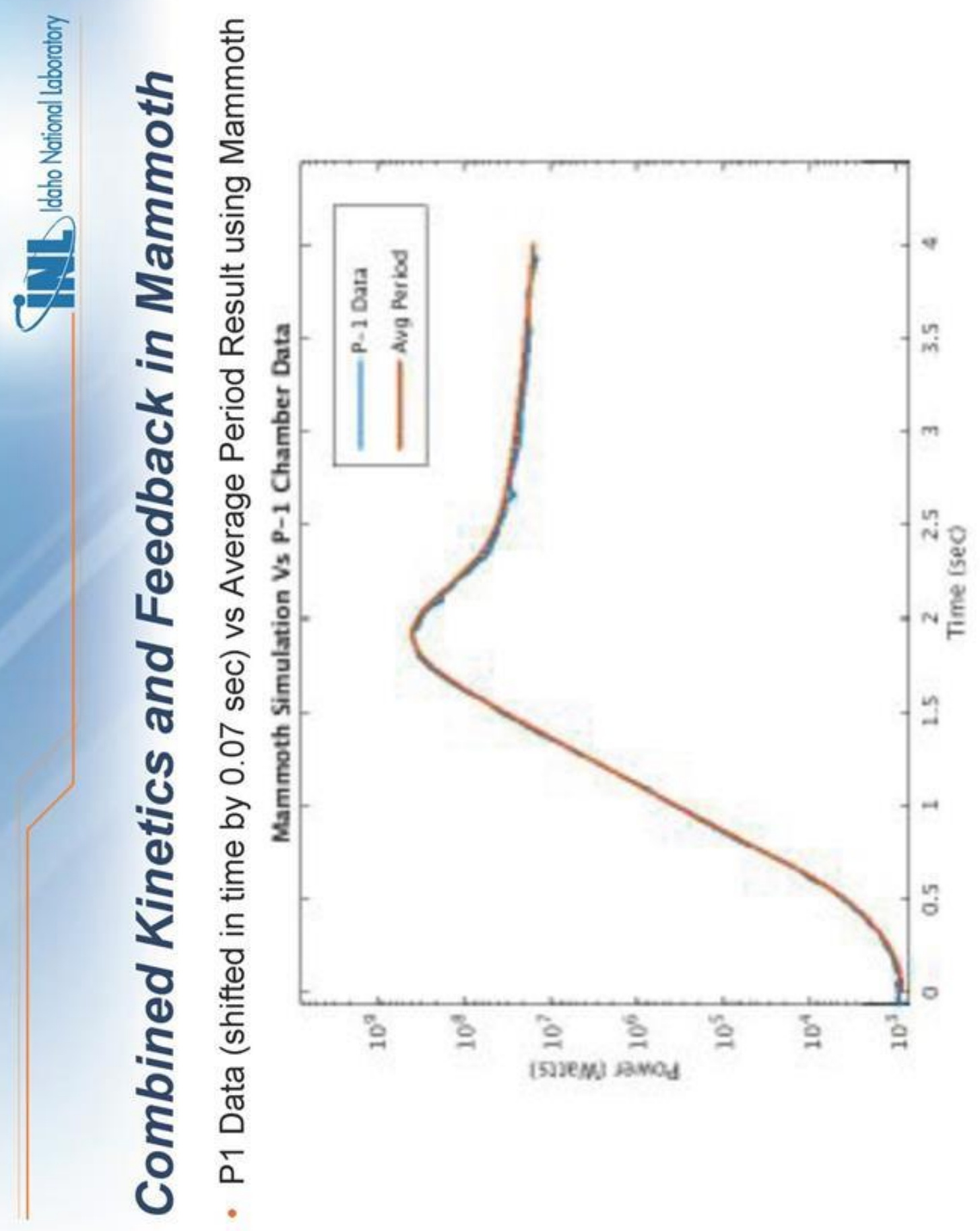




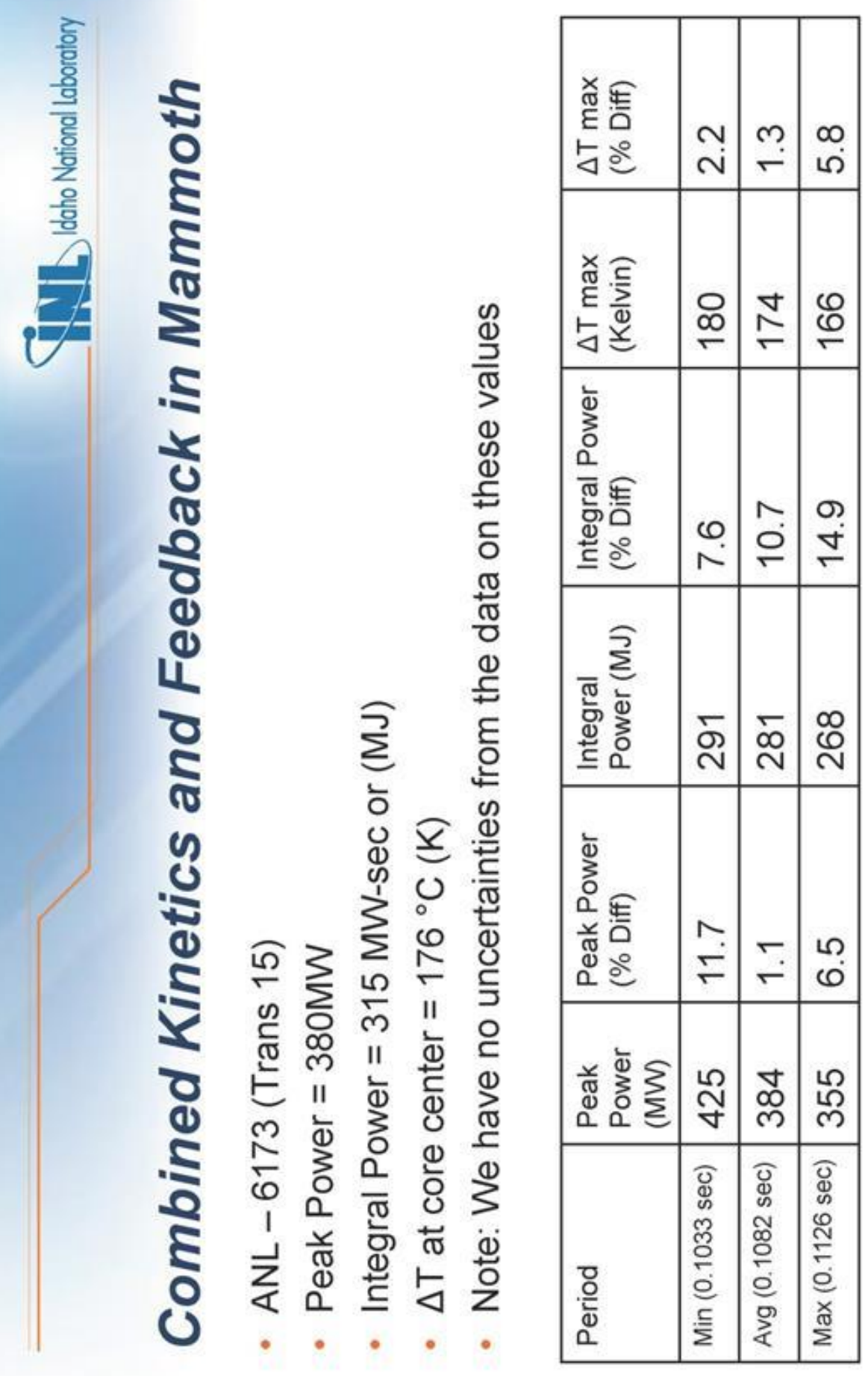




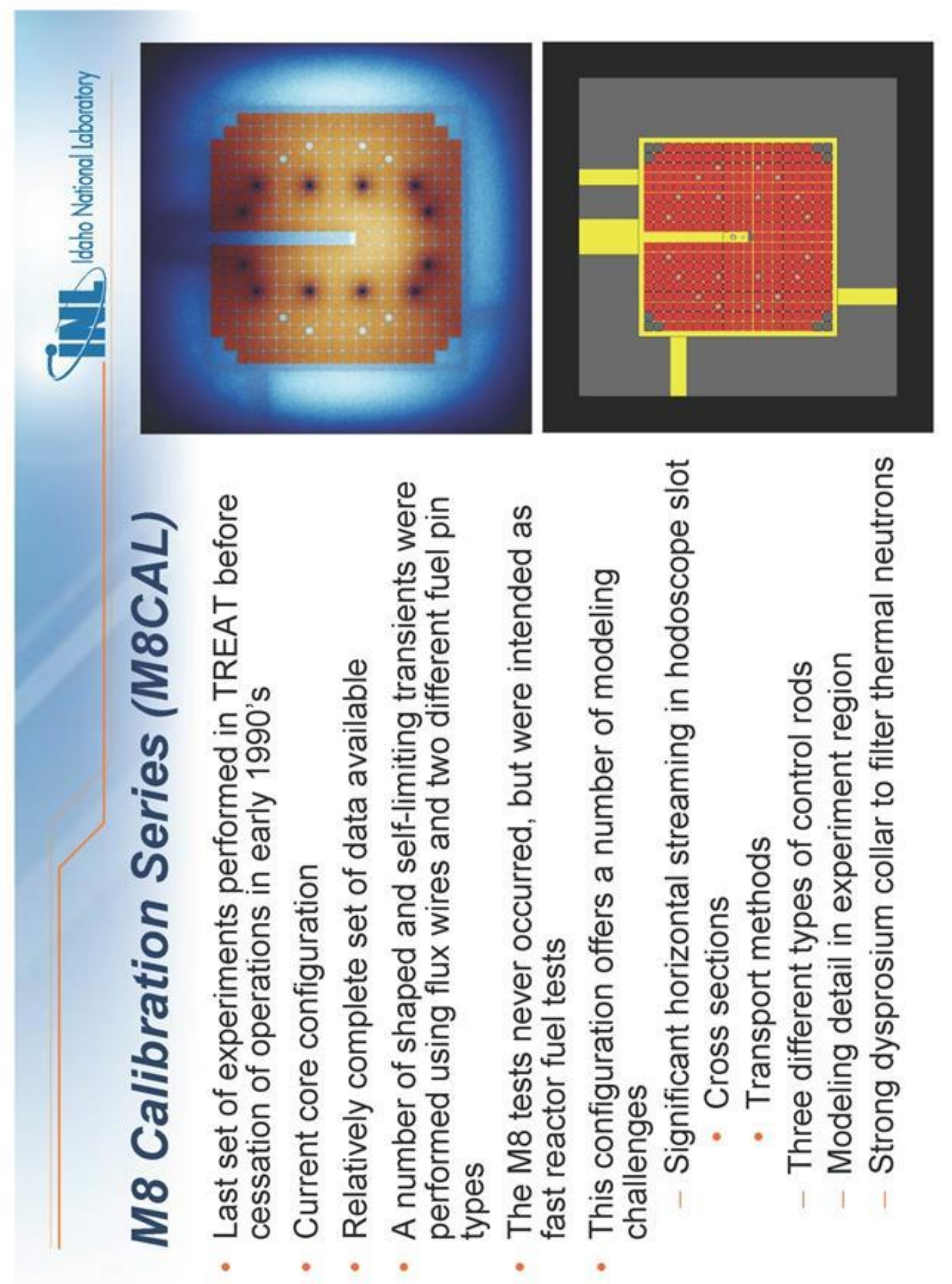




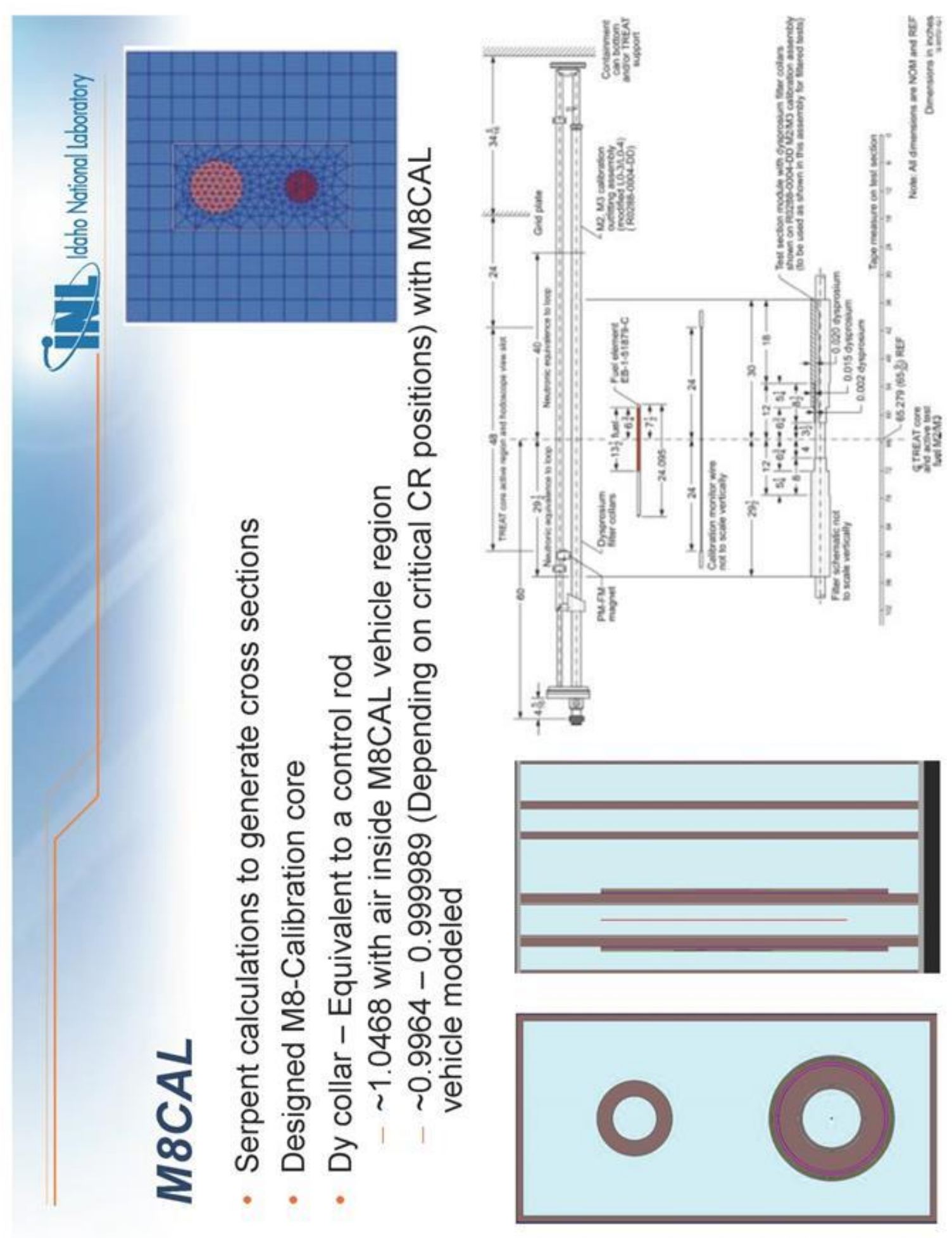




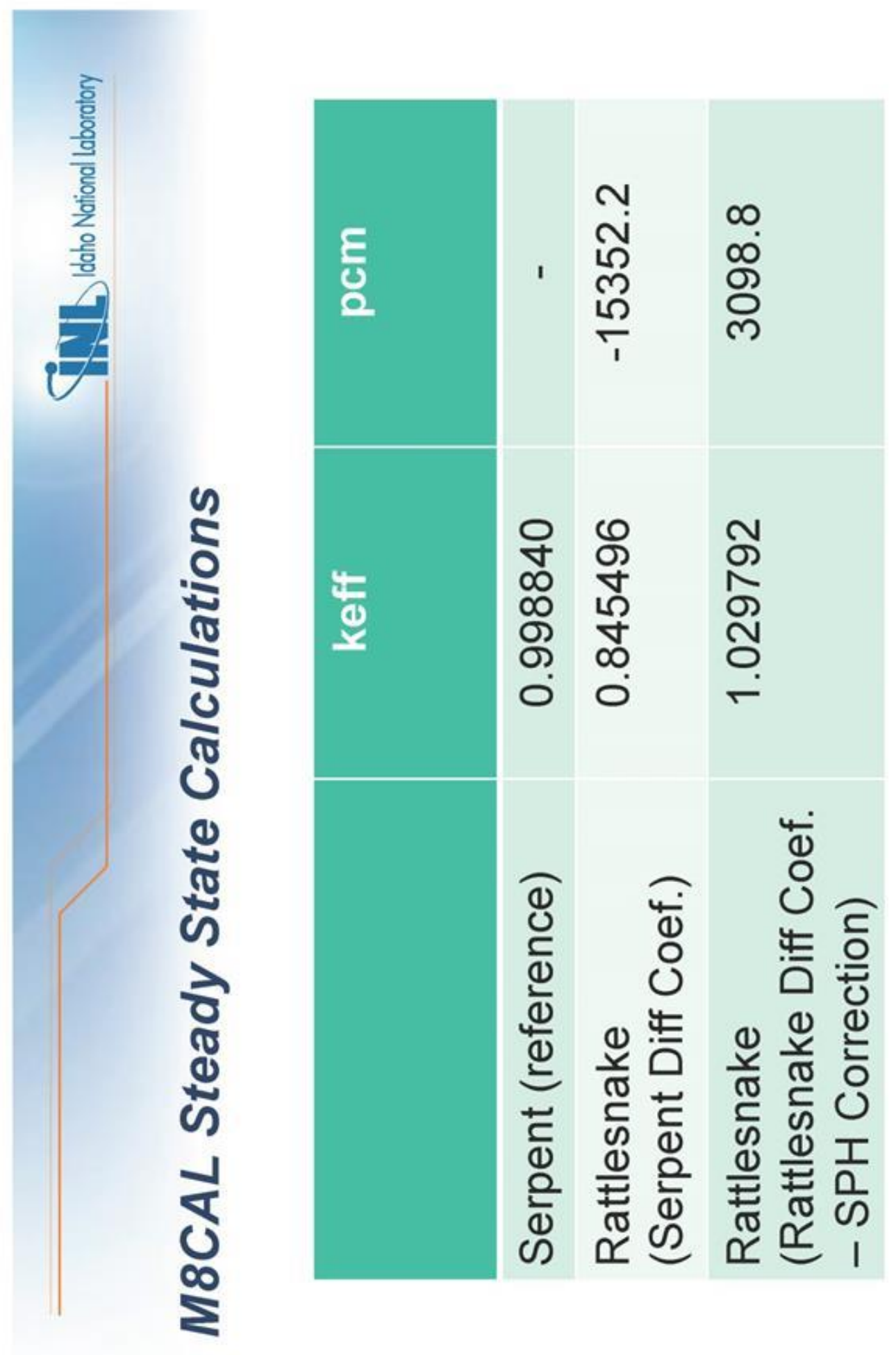



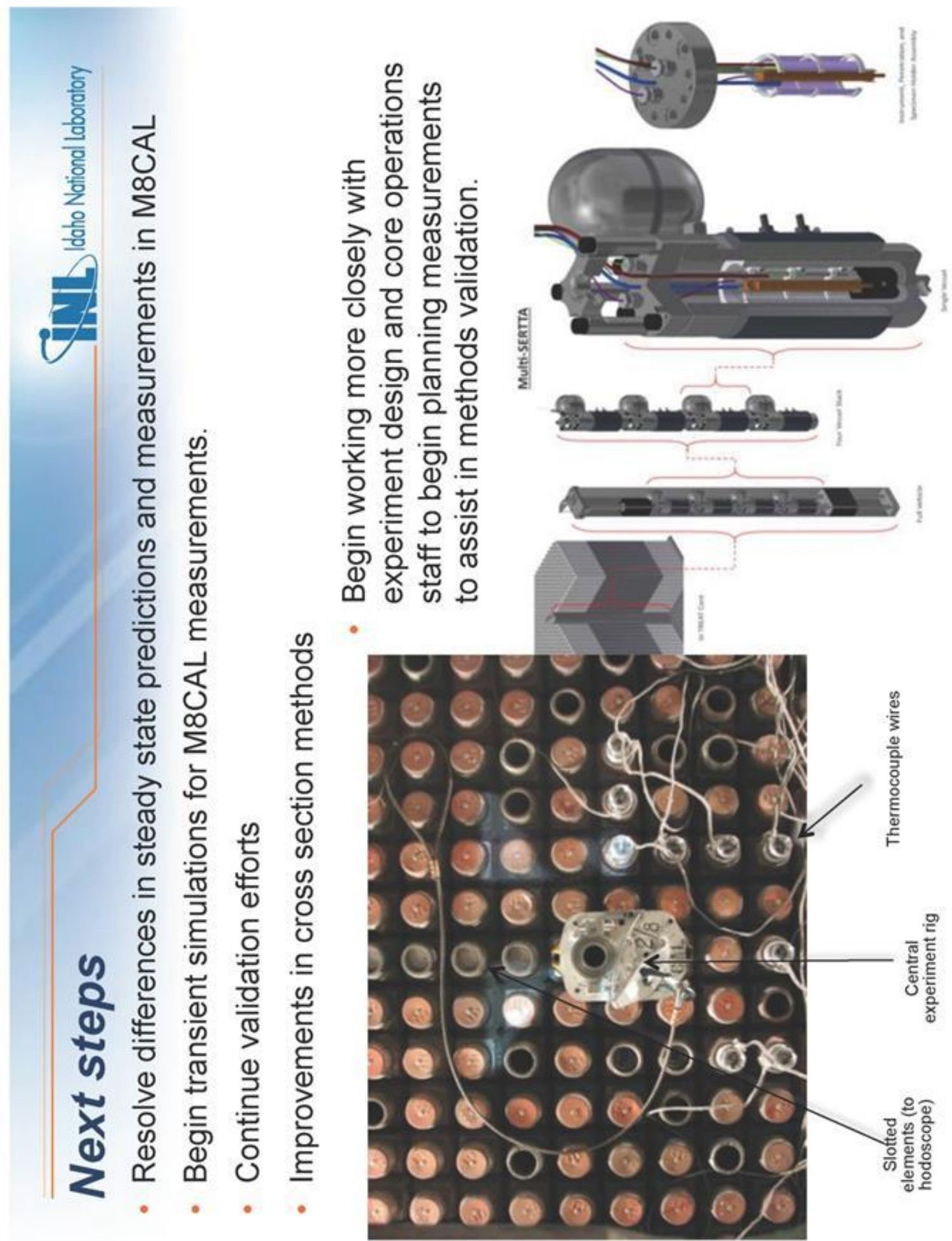


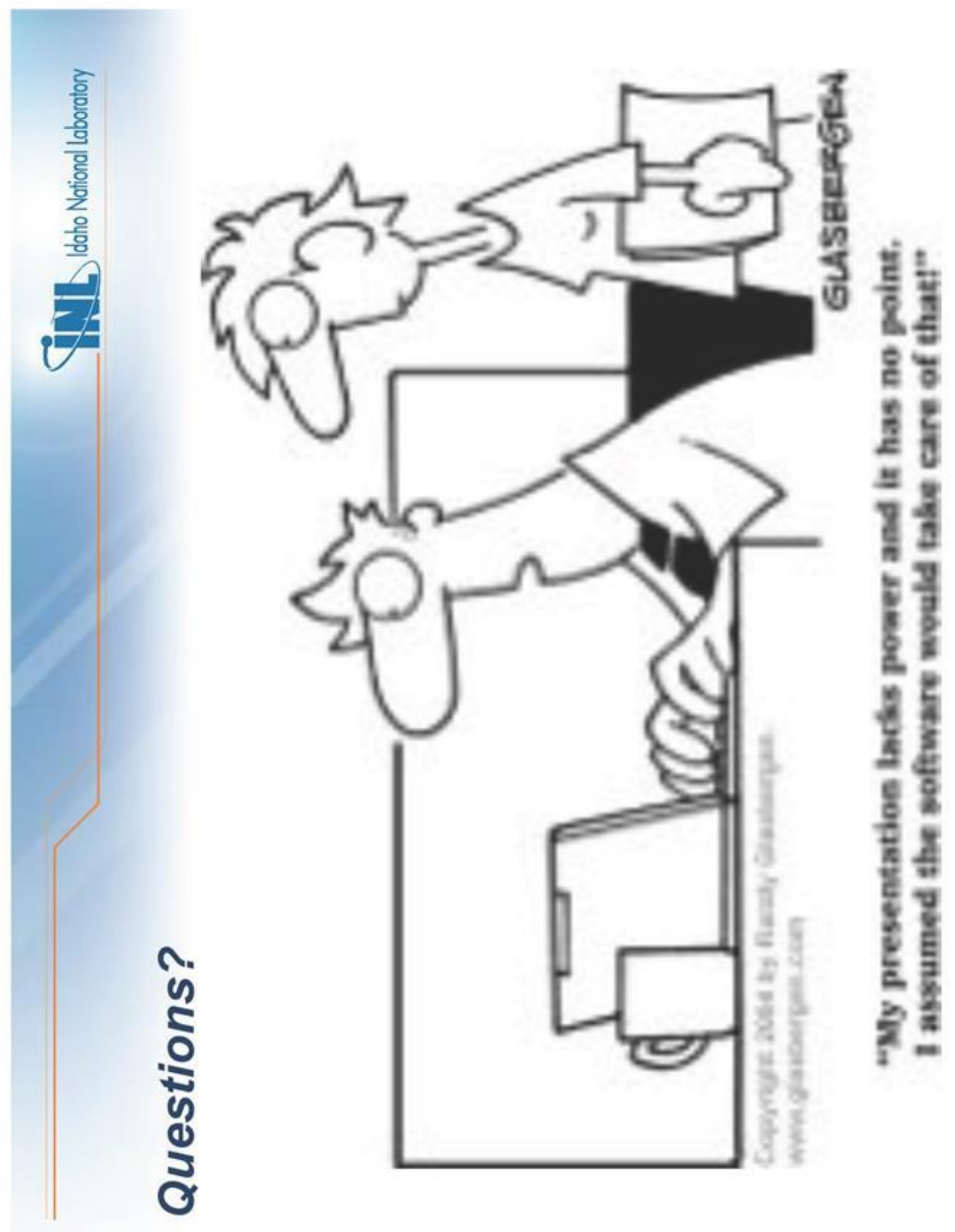



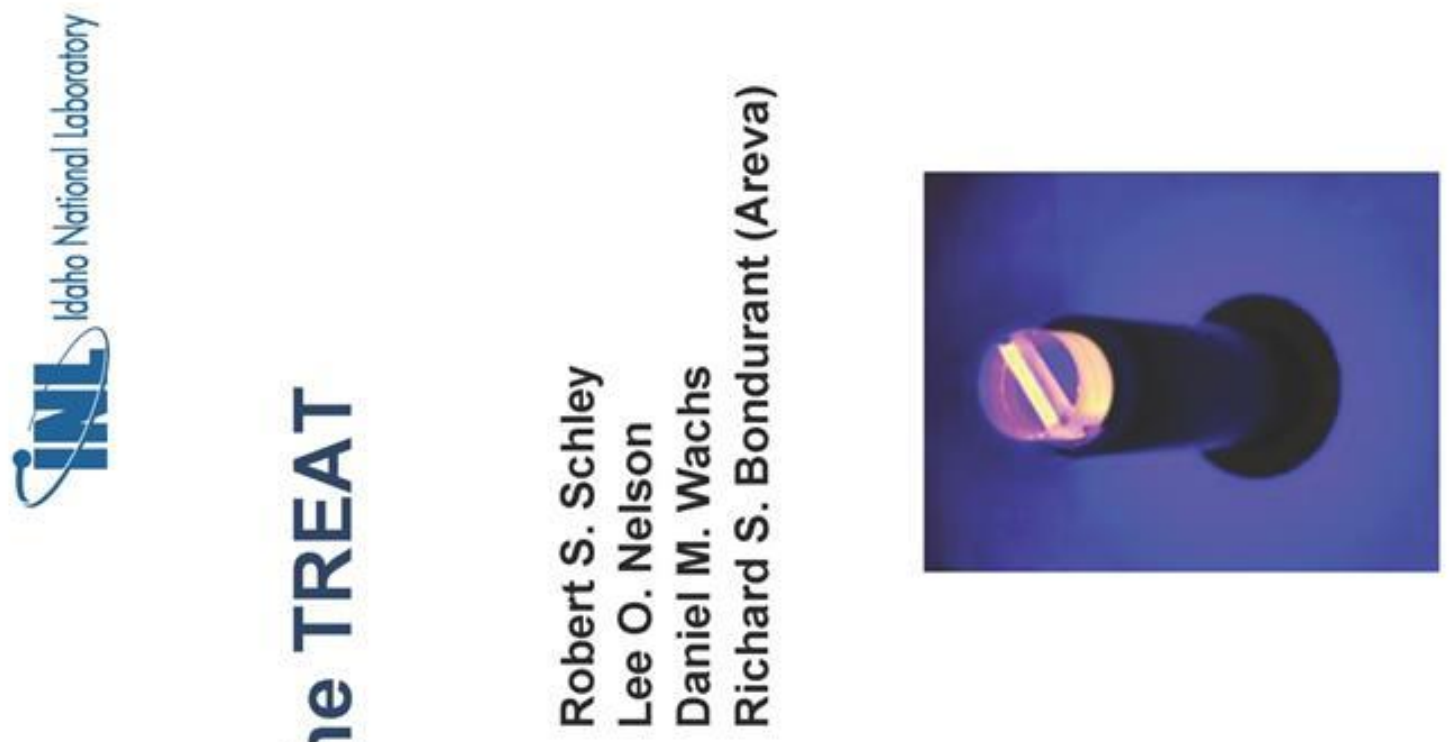

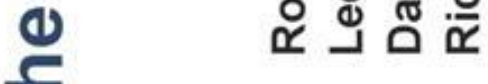

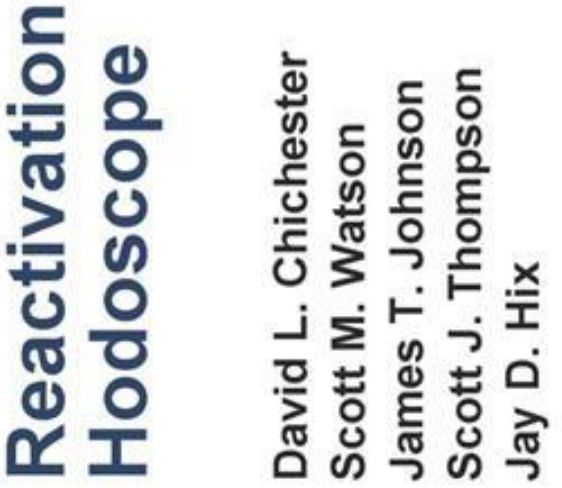

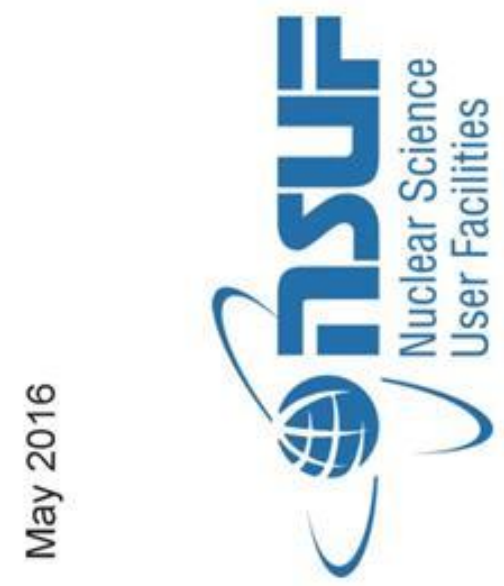

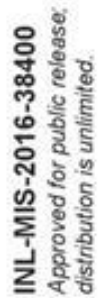

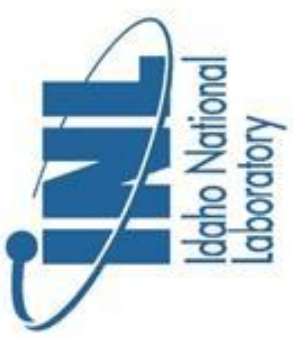



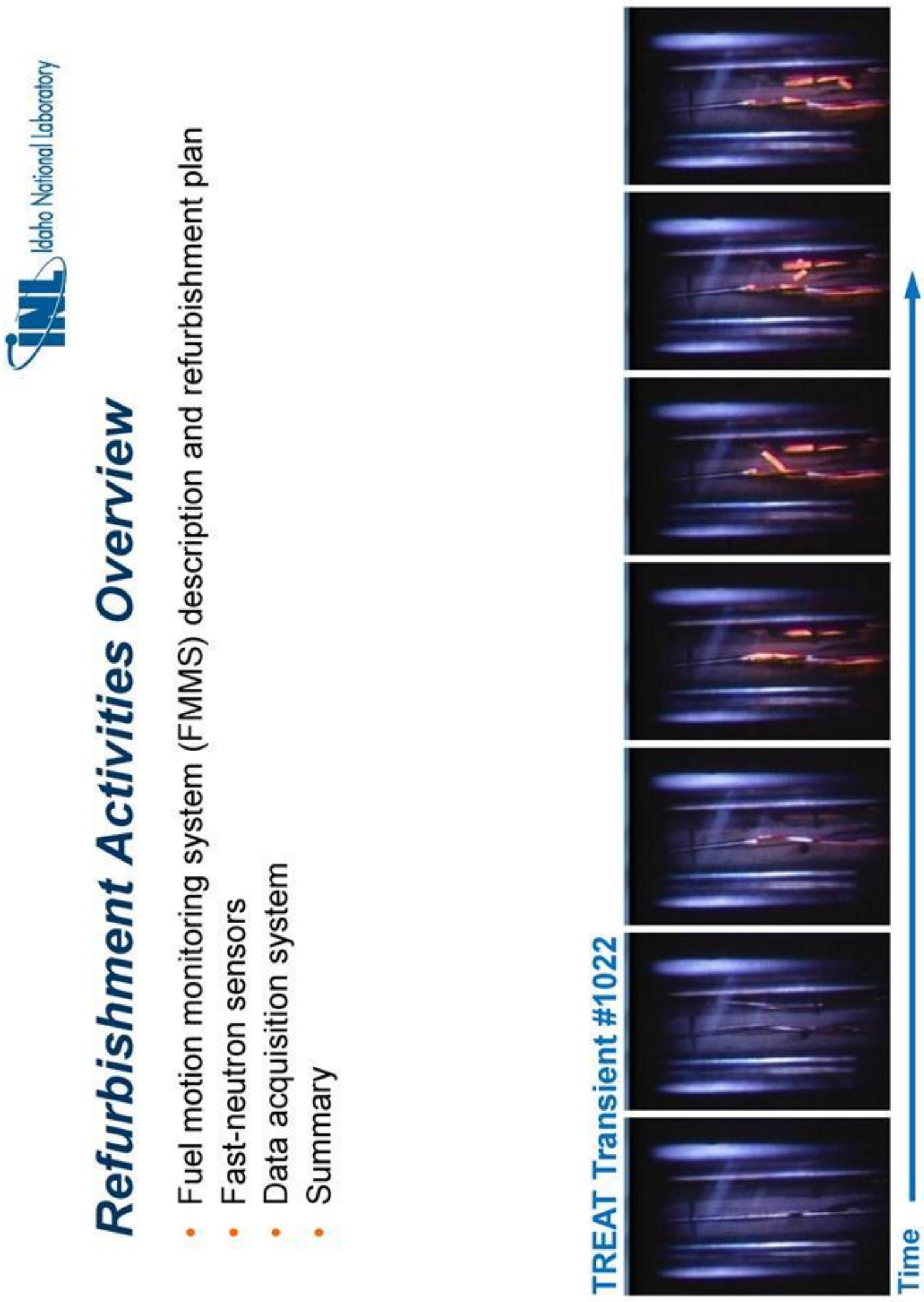


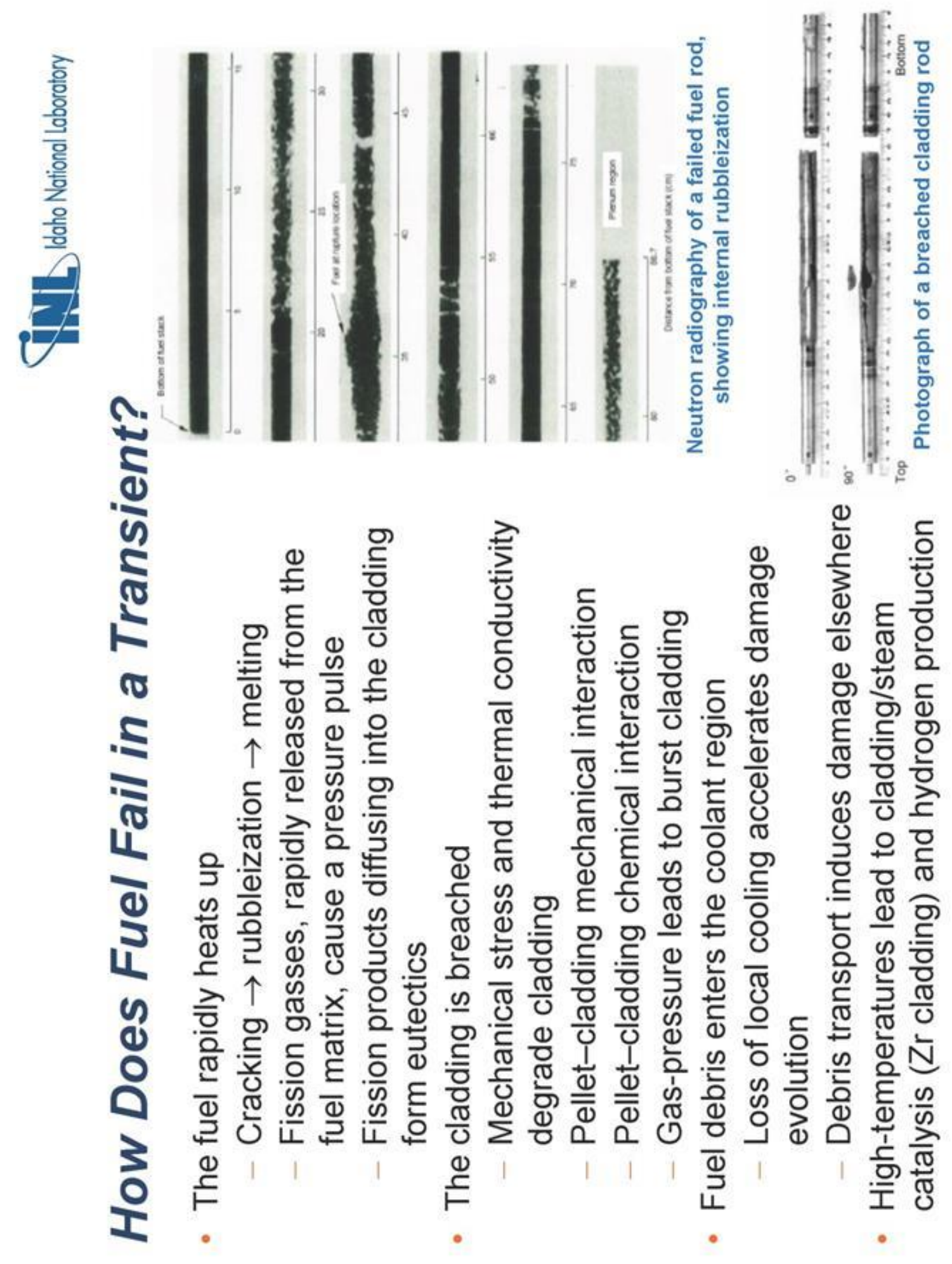



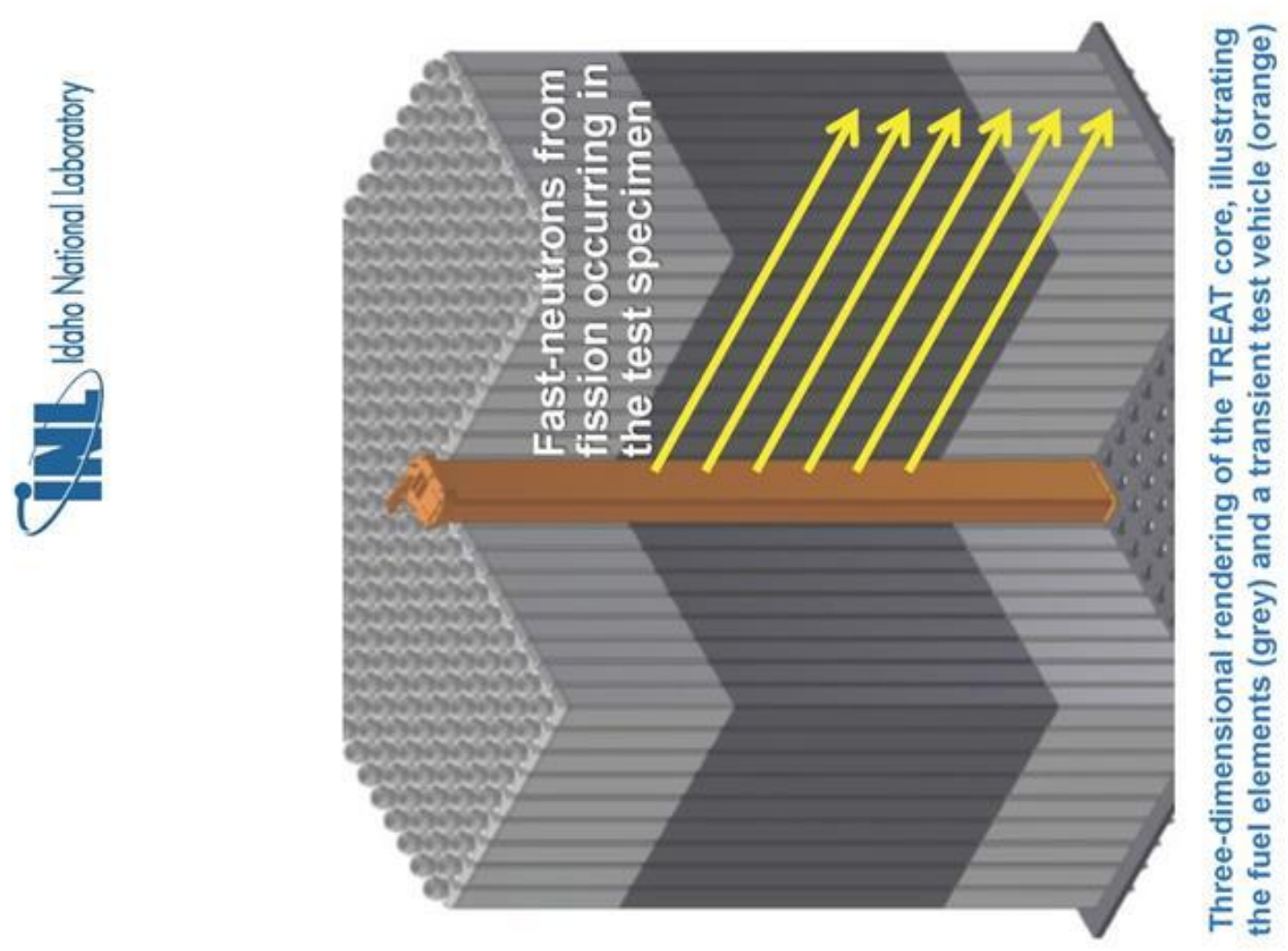

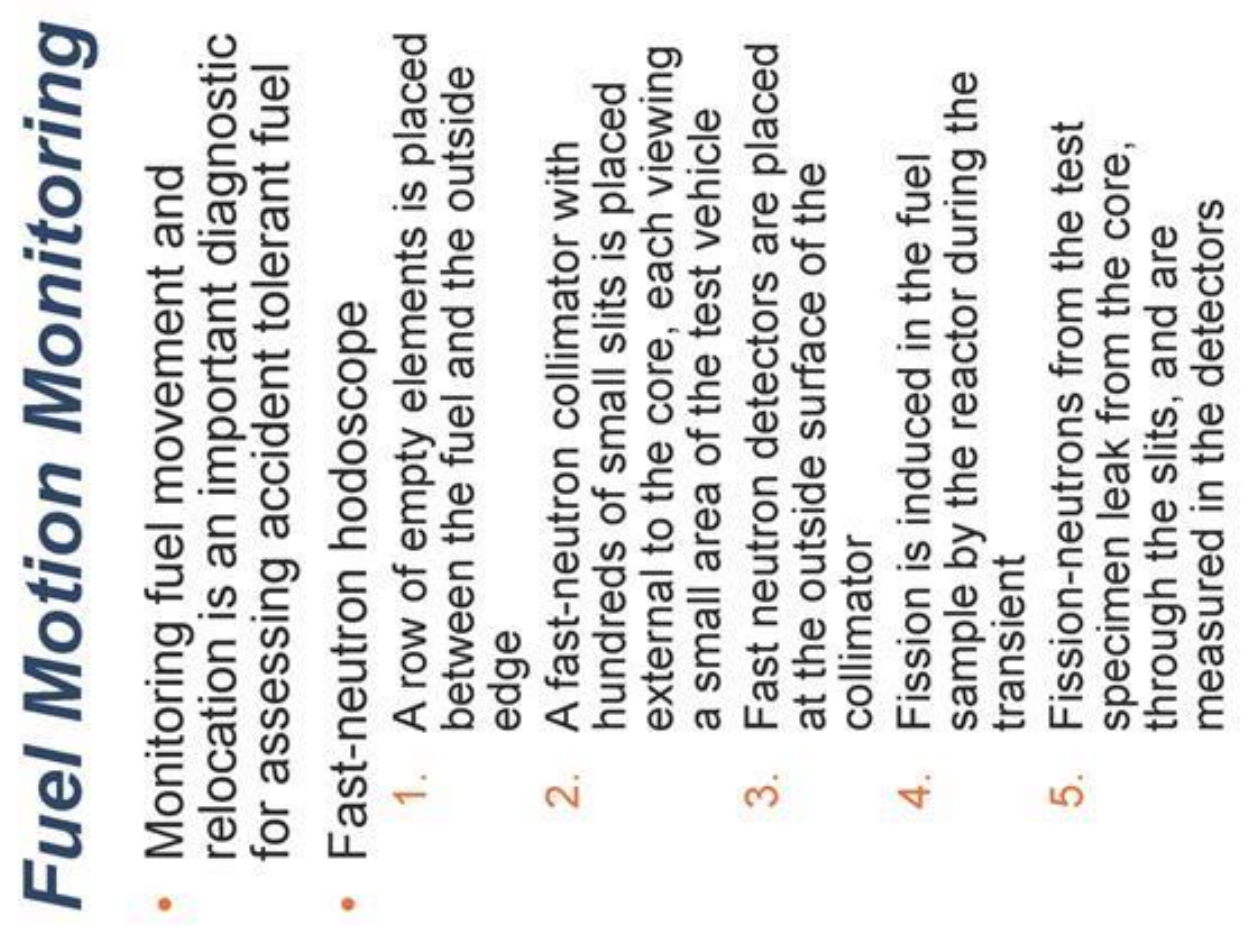




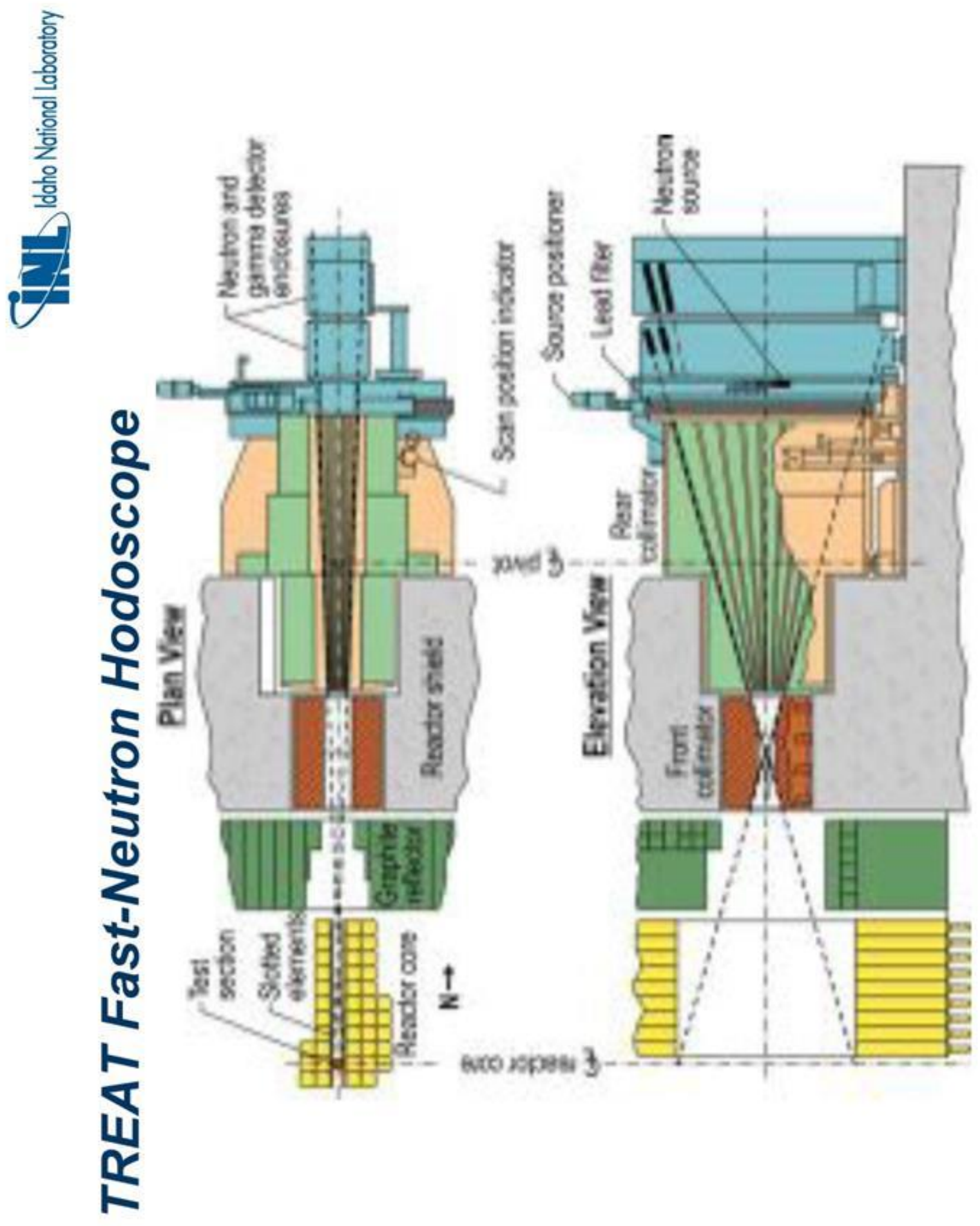



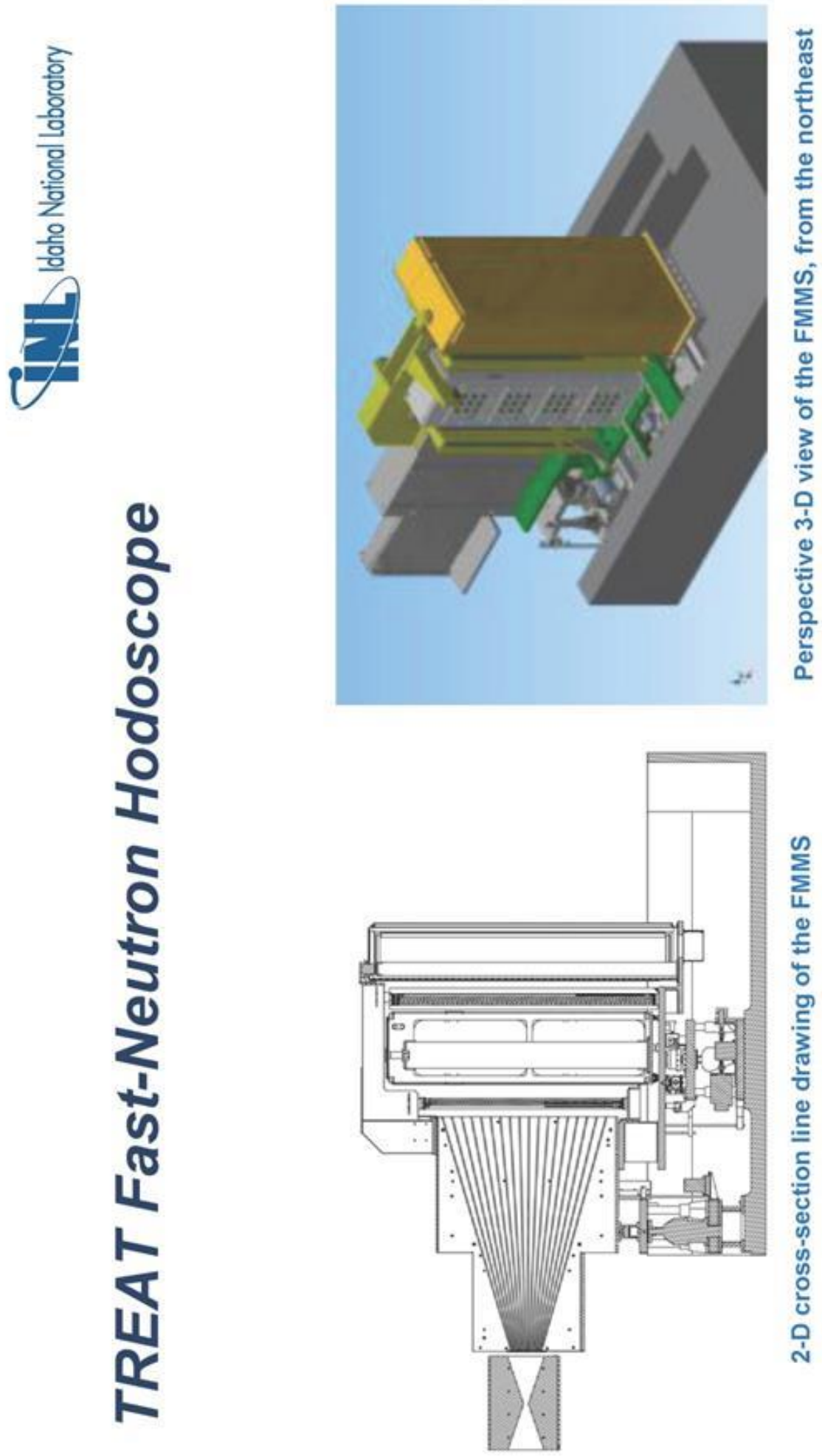

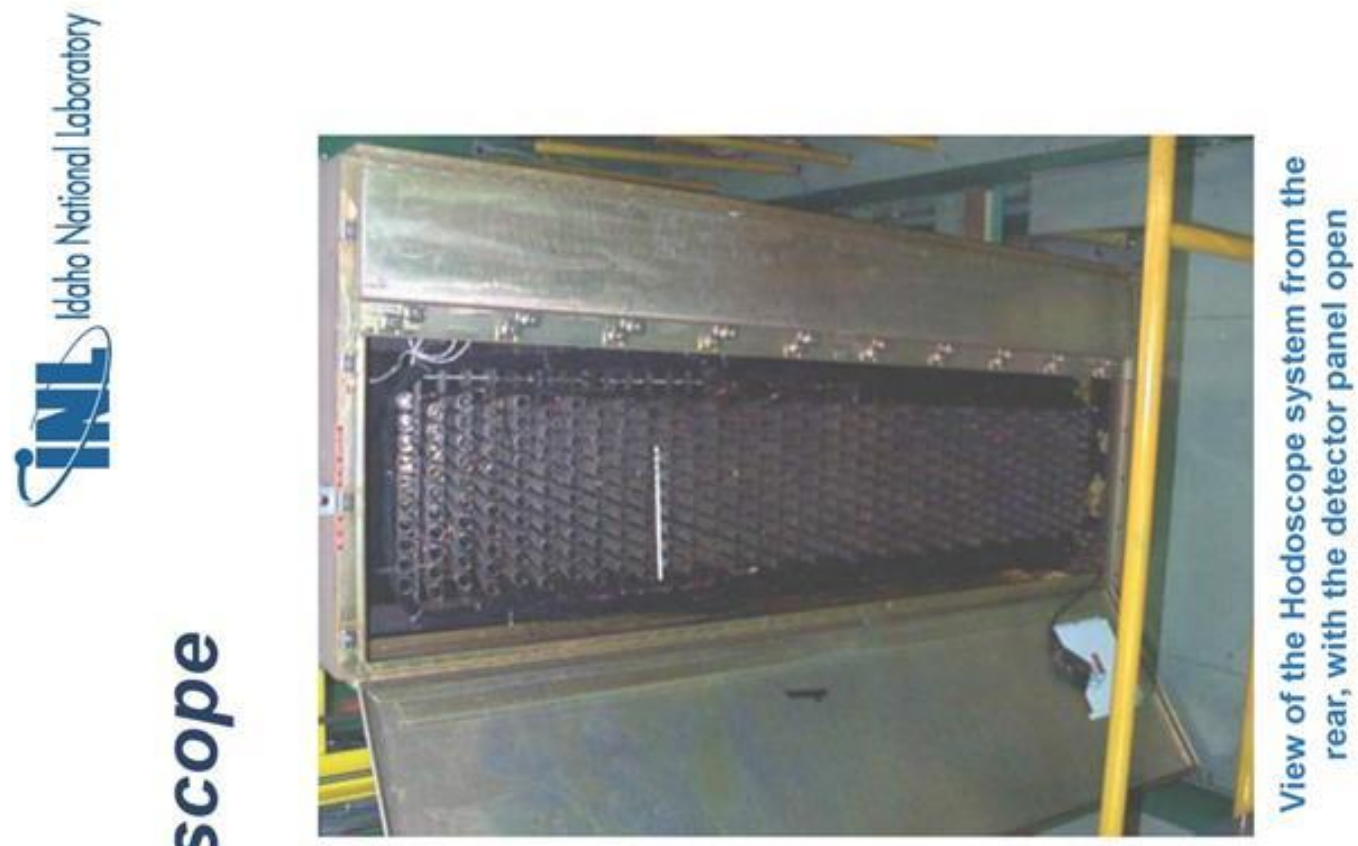

응

(1)

ร

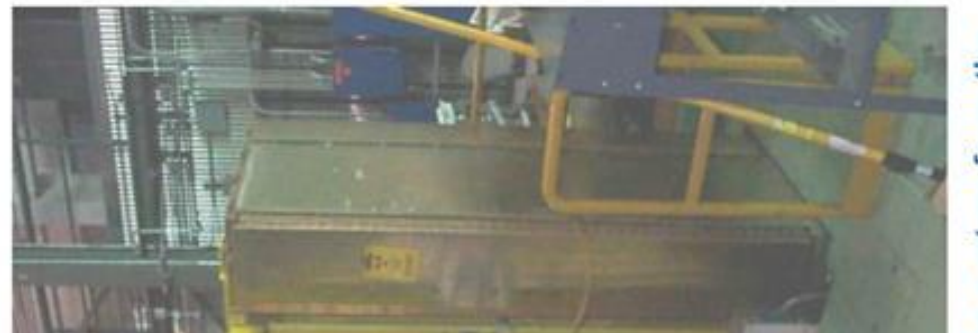

है

(1)

1

()

ชิ

上

II

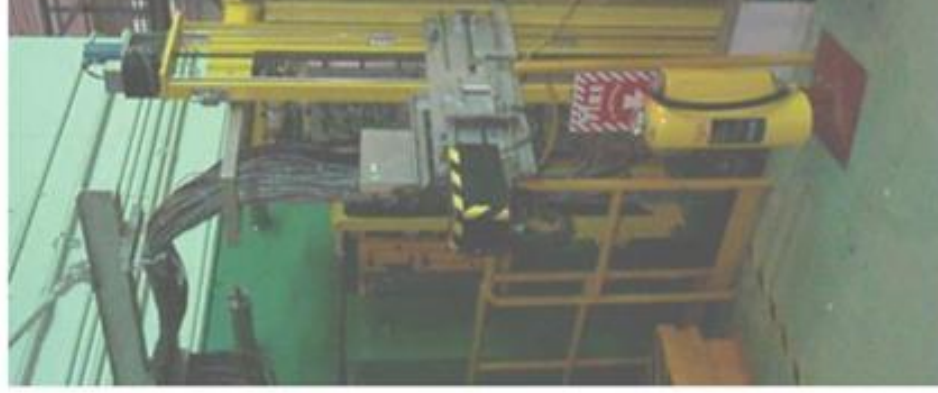

응 은

。

오

든

O

3 

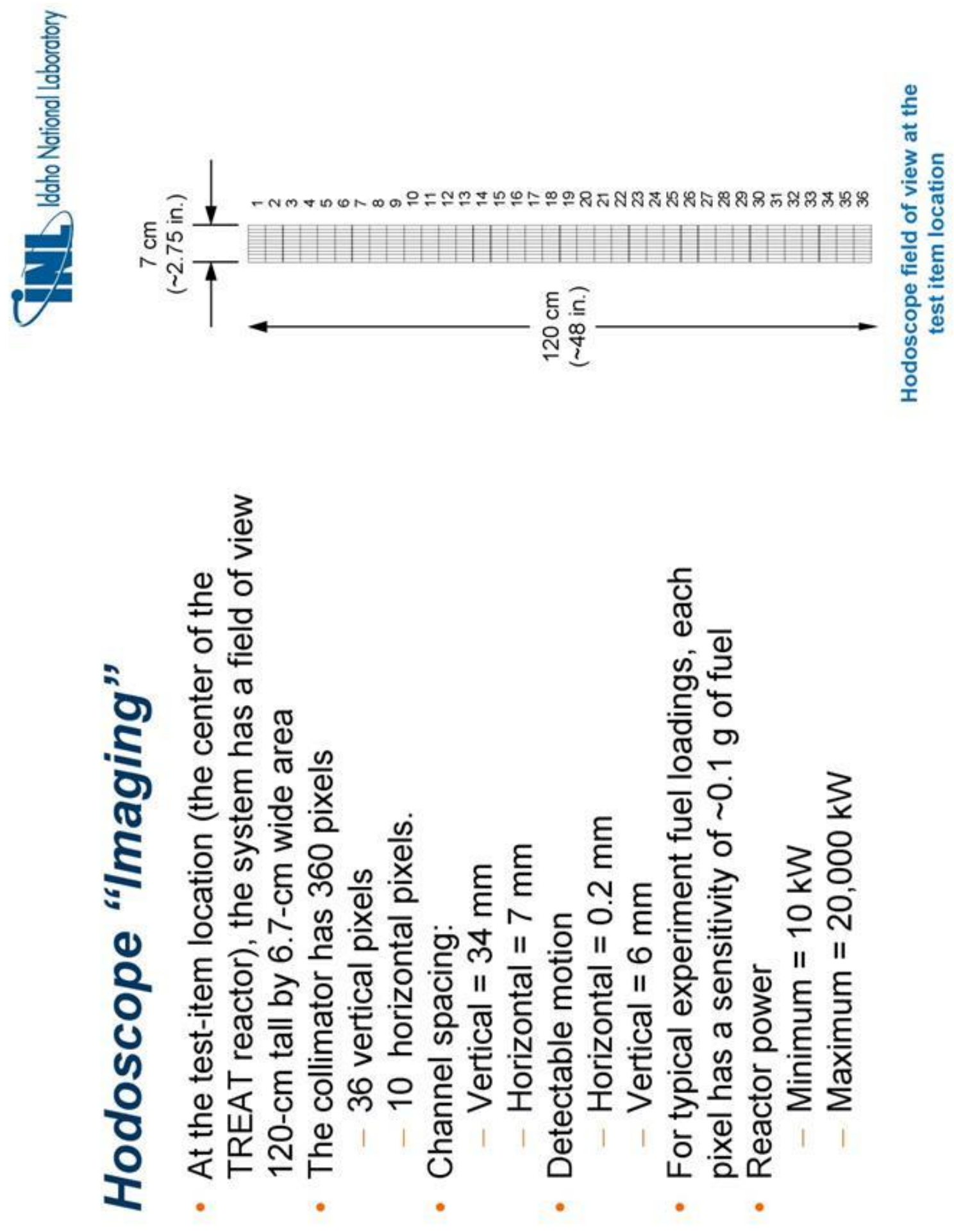

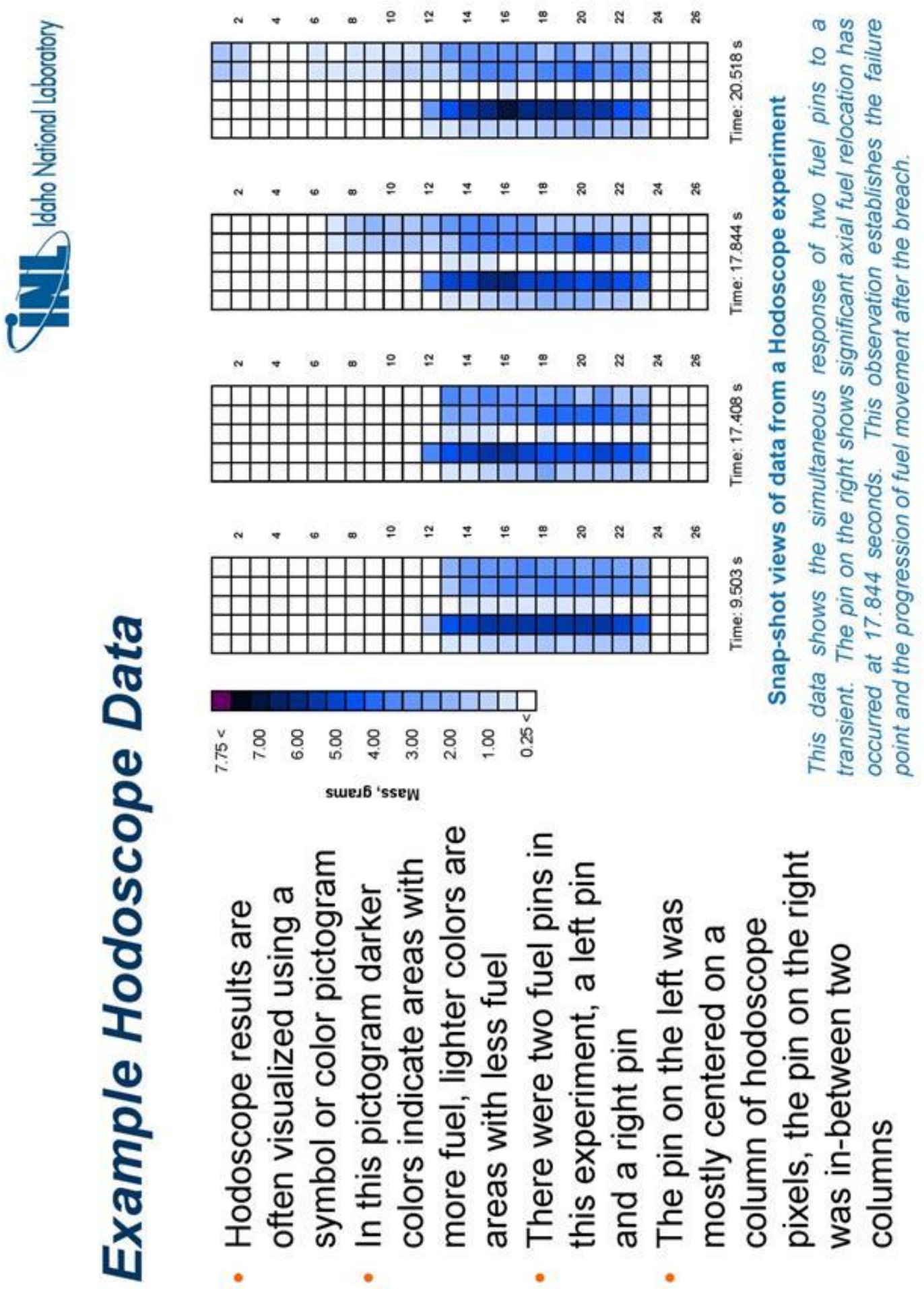

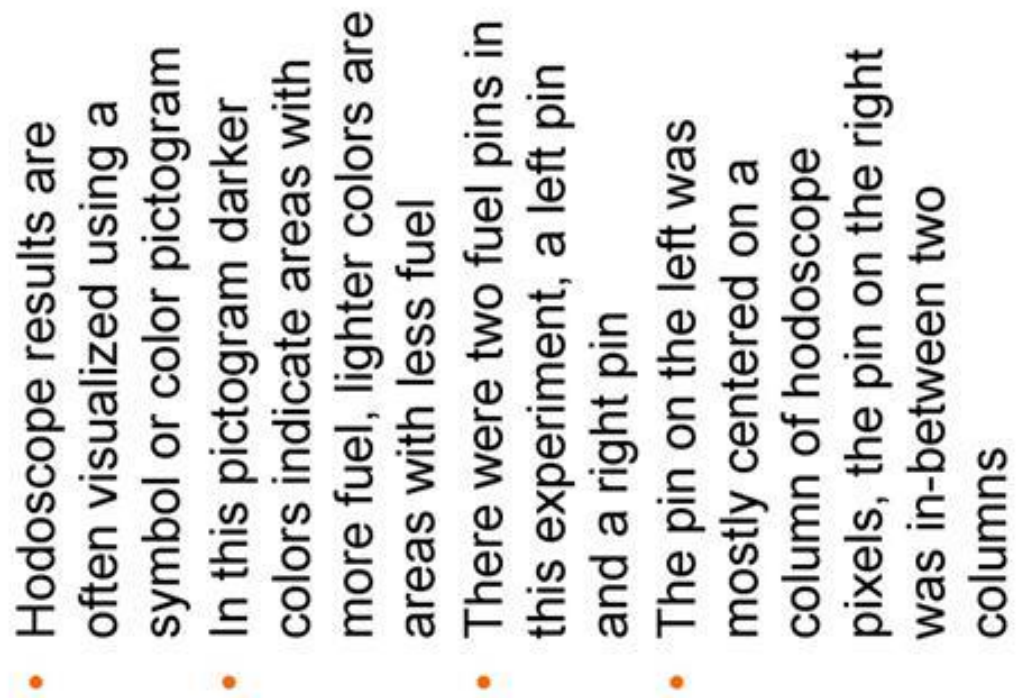




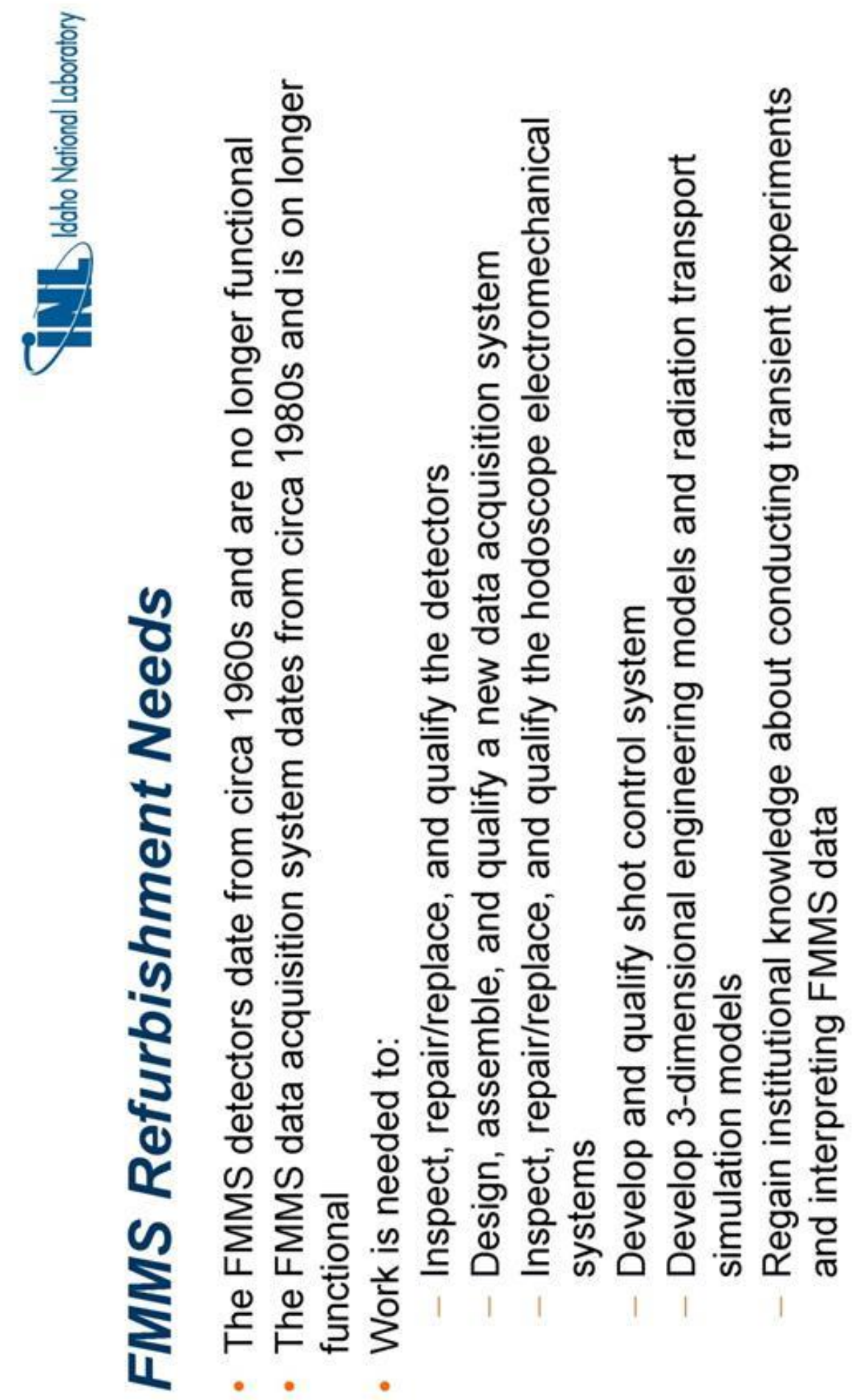



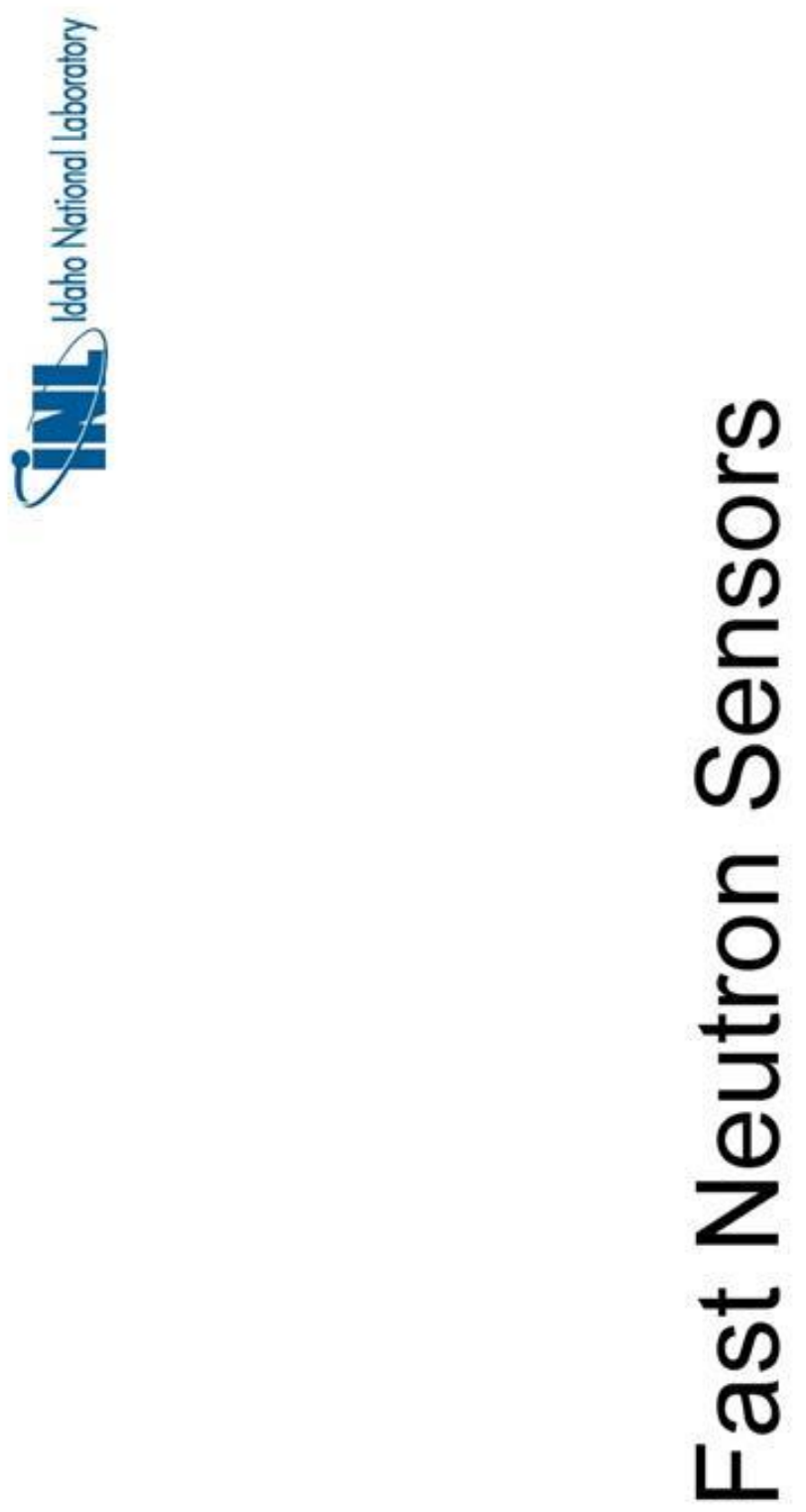


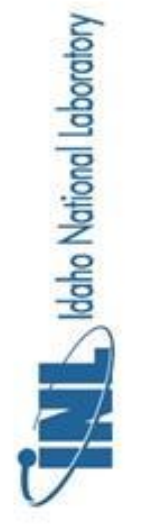

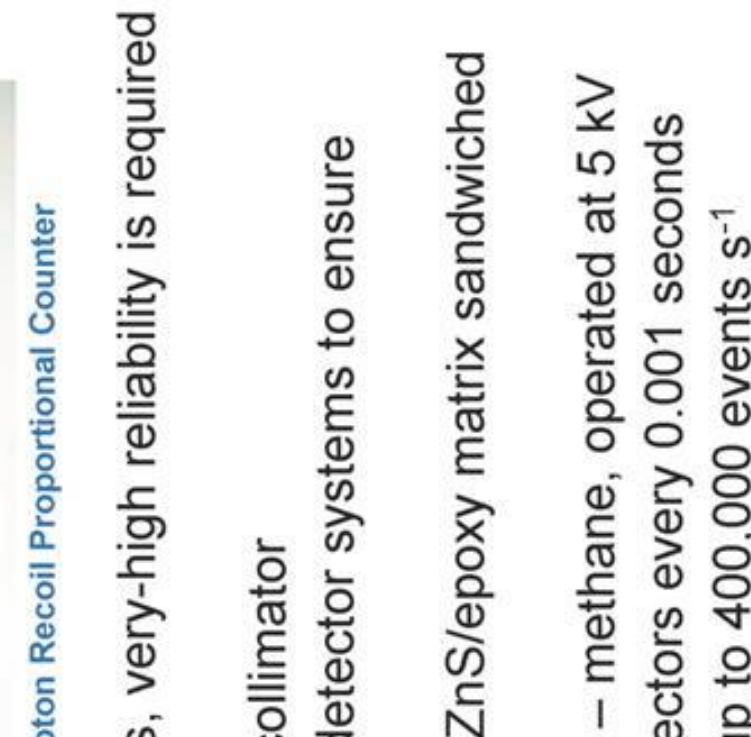

ज़

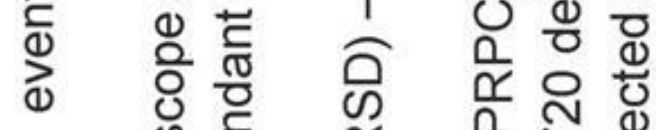

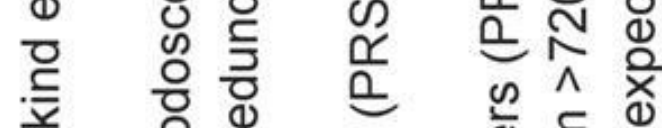

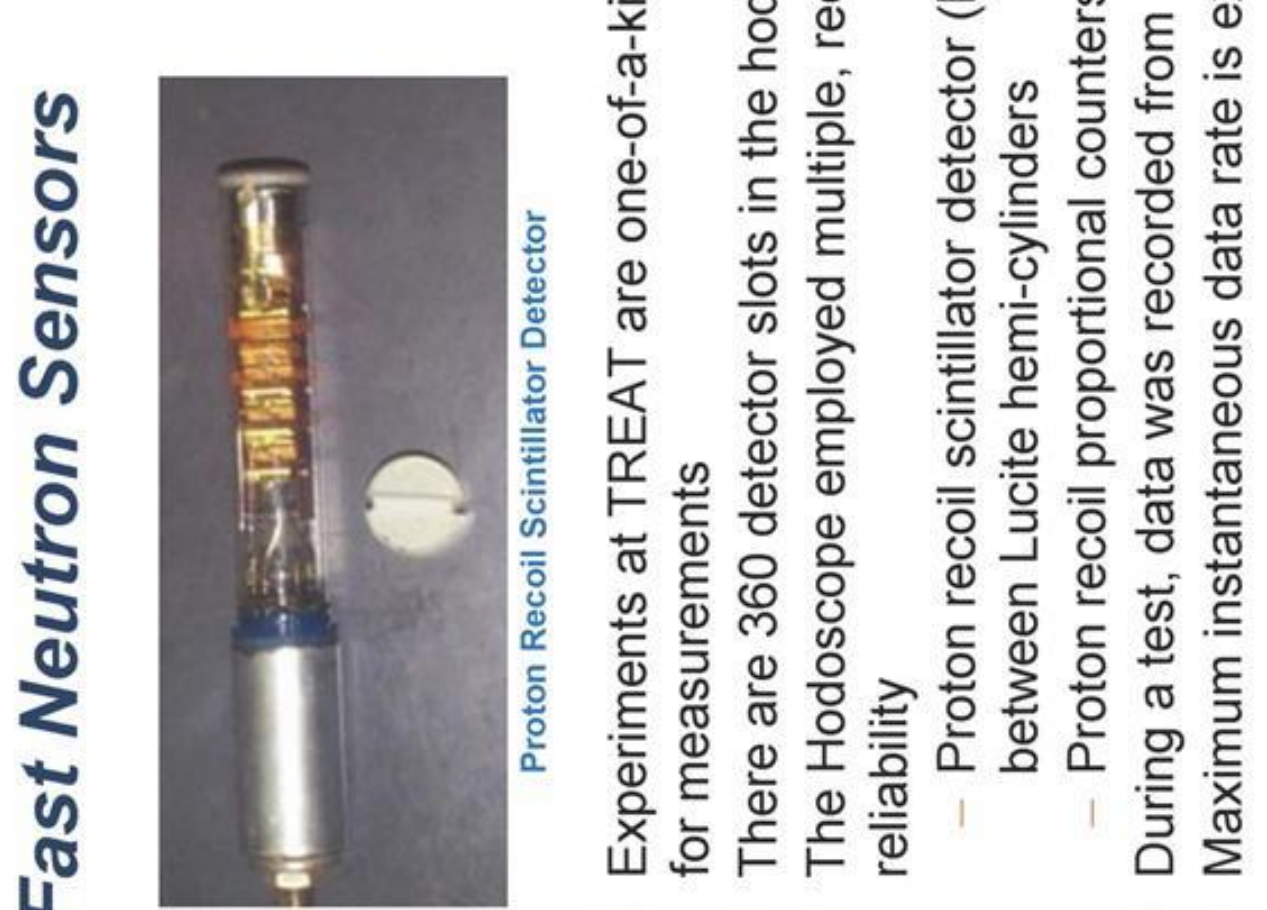




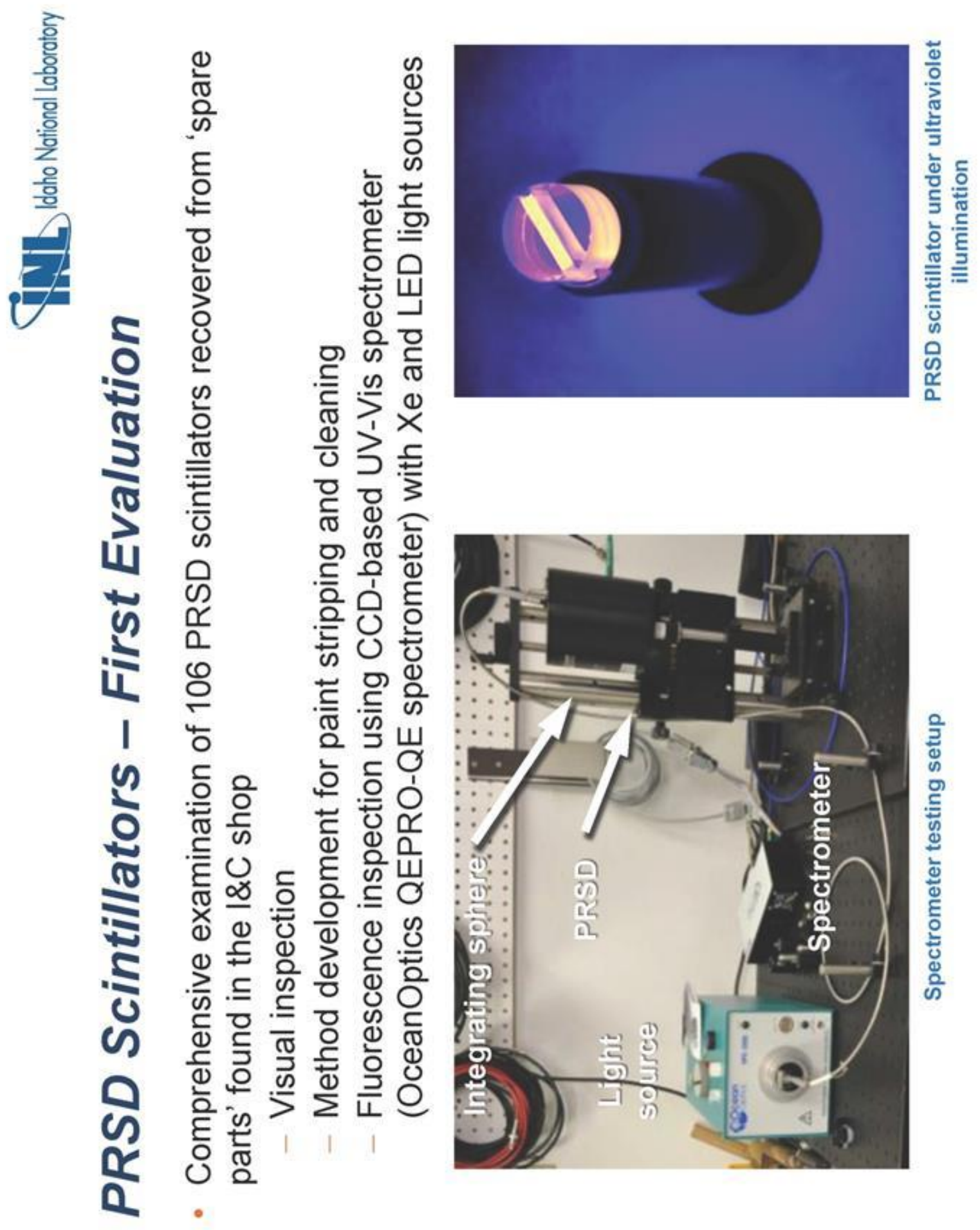




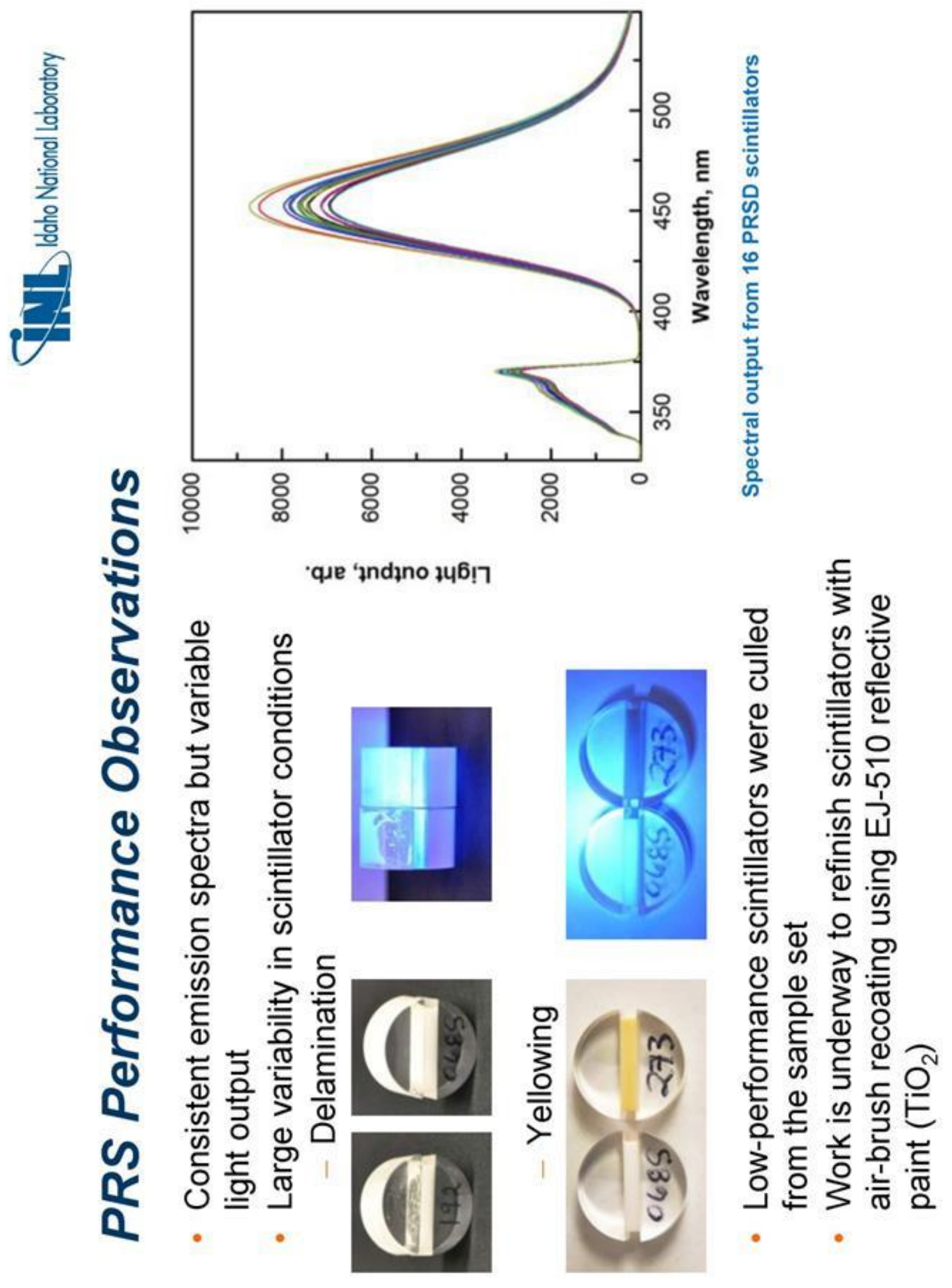



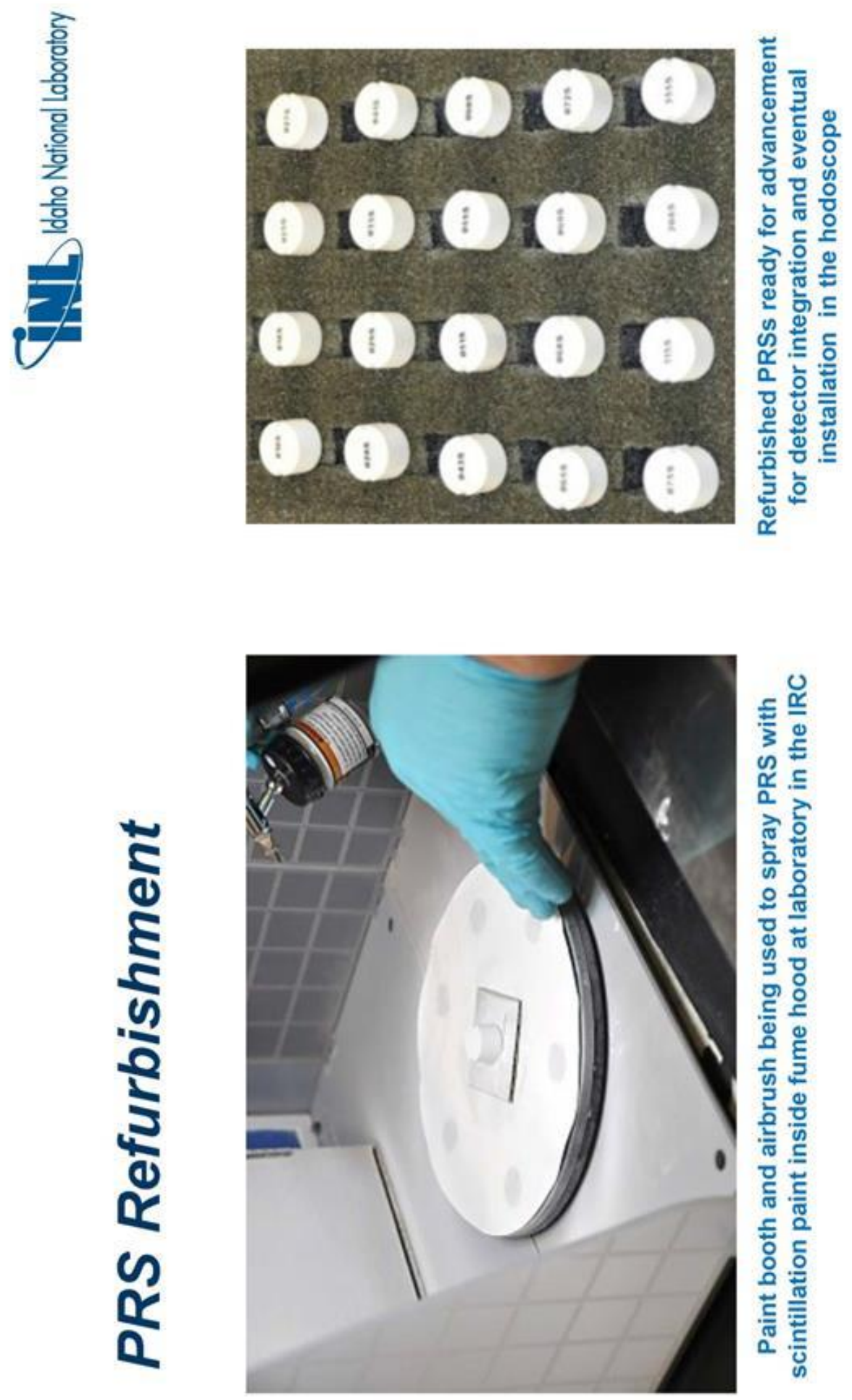

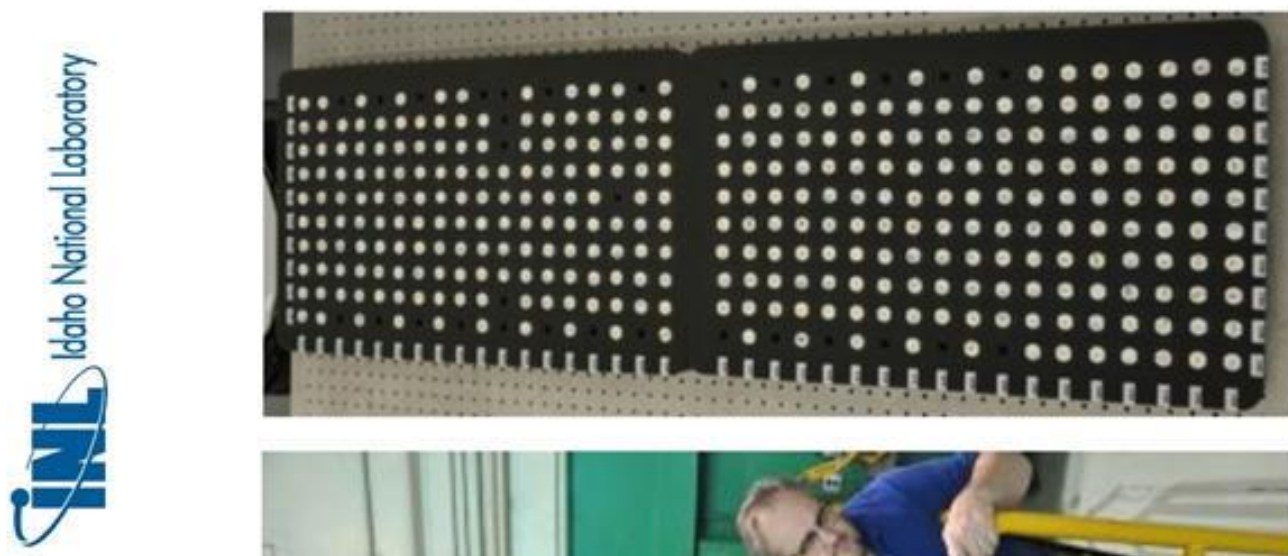

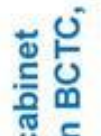

잉.

혼 뜸

U⿺ 증

융 통 흥

해은

is 은

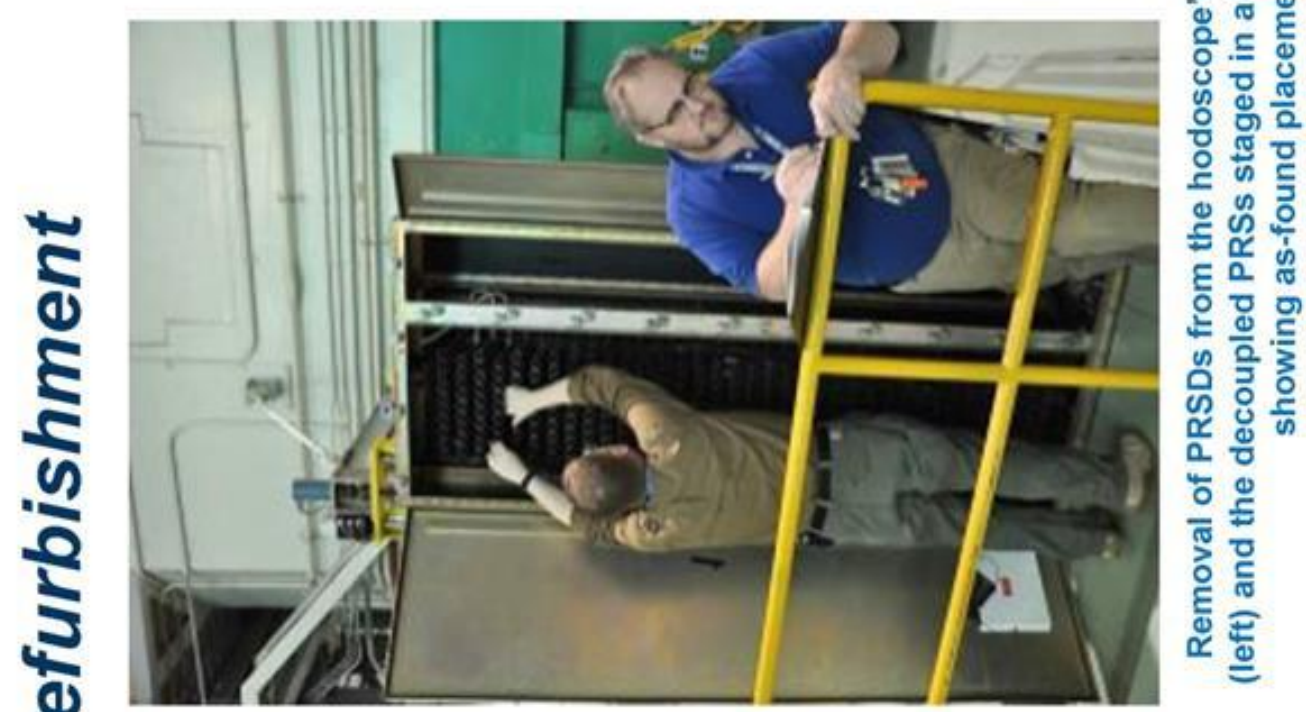

당

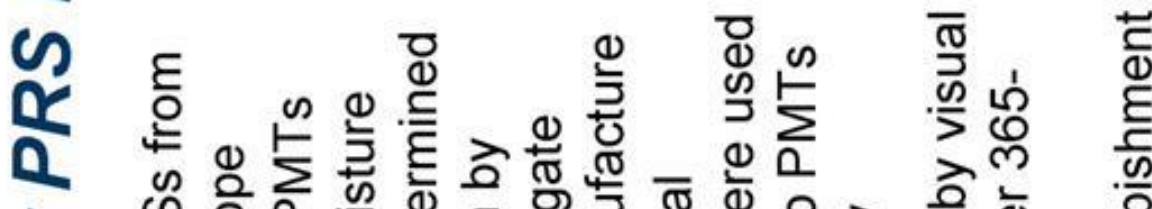

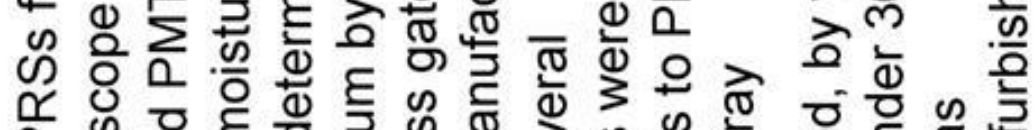

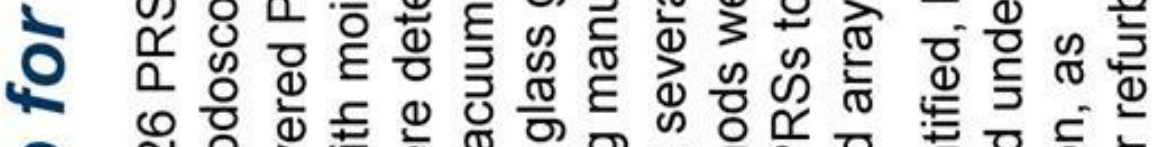

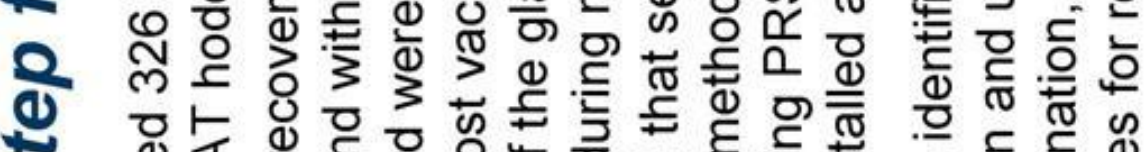

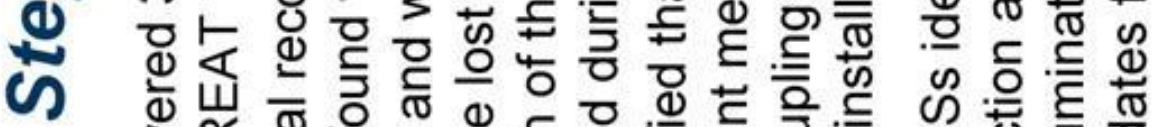
* (1) শ્丶 


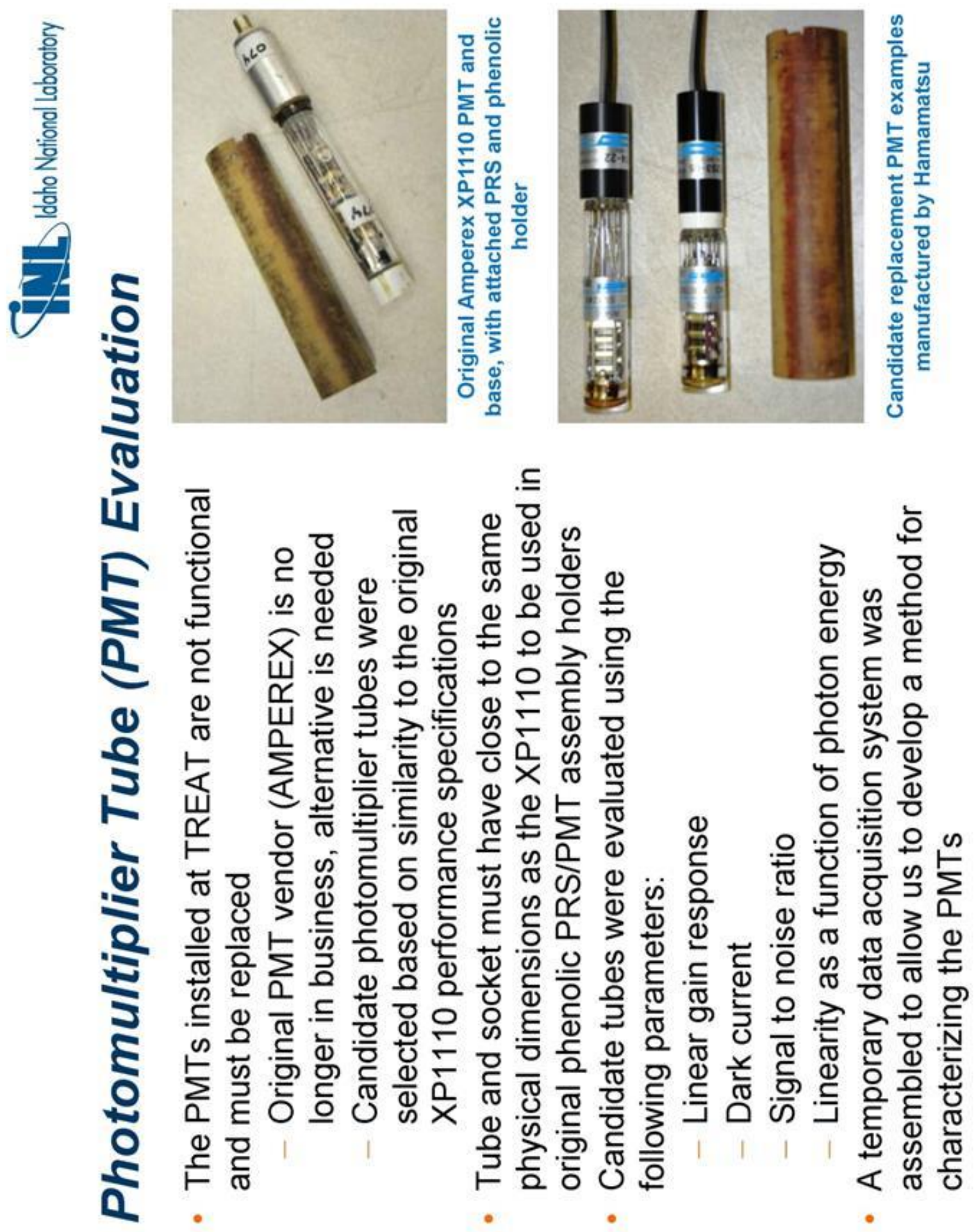



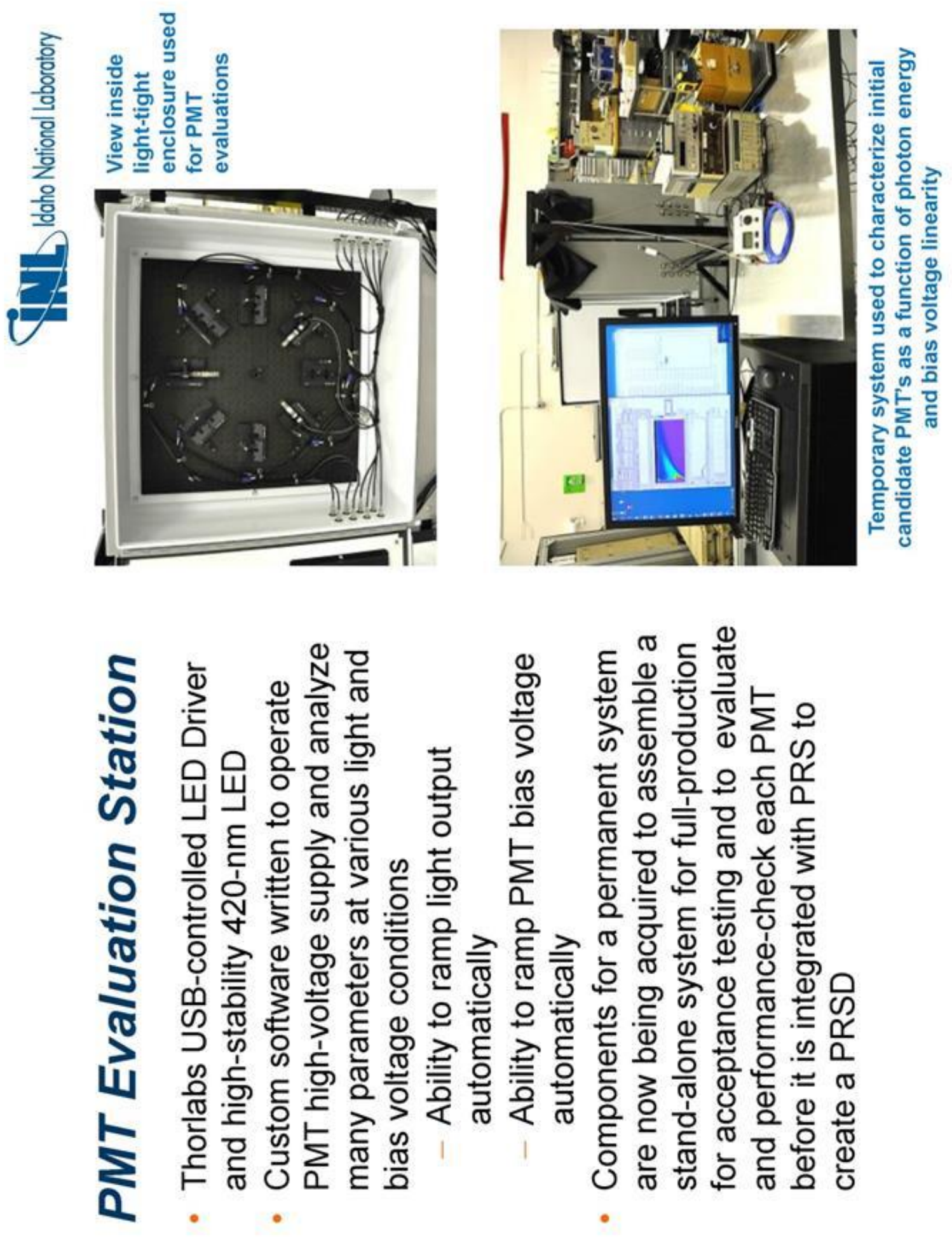


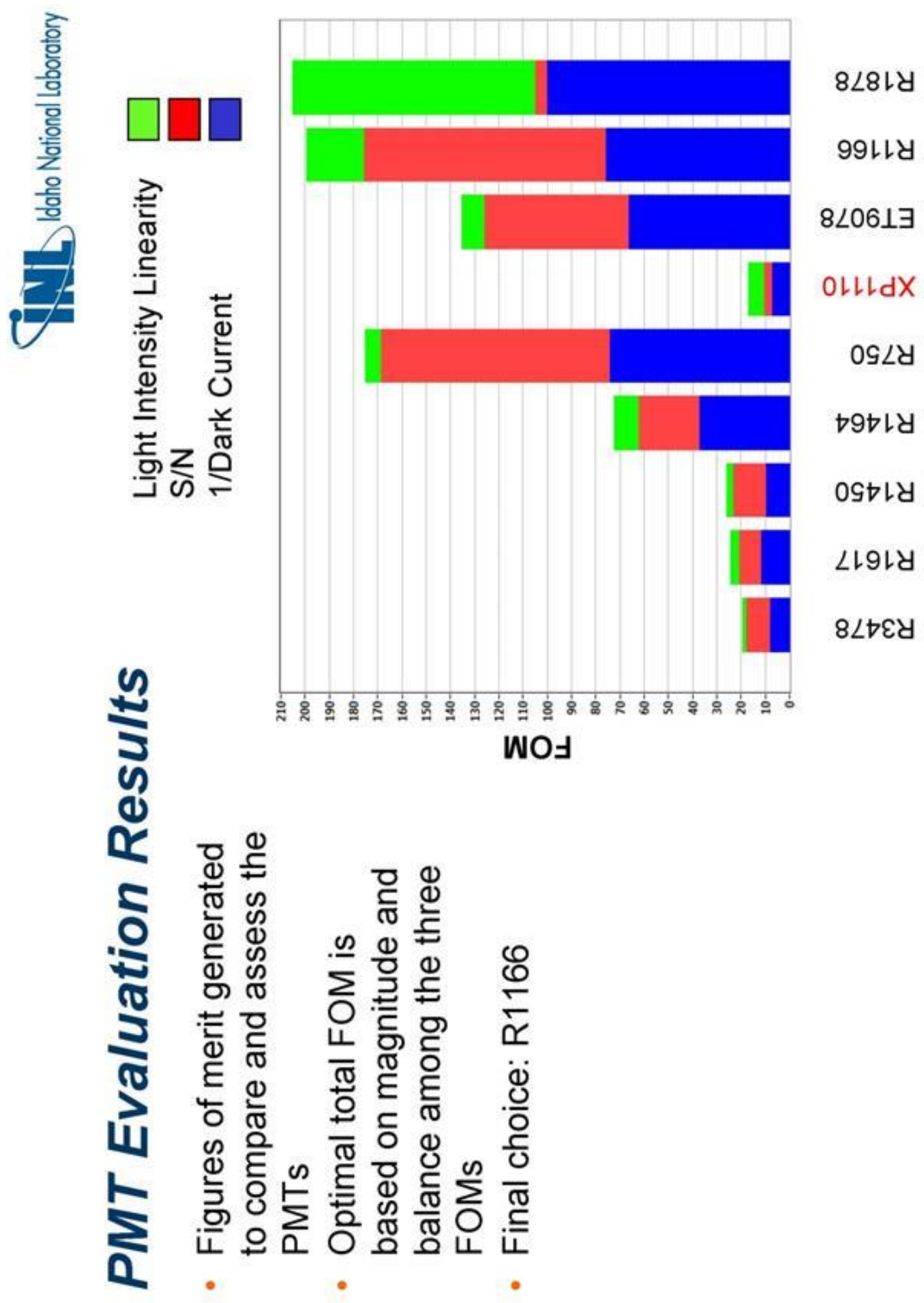



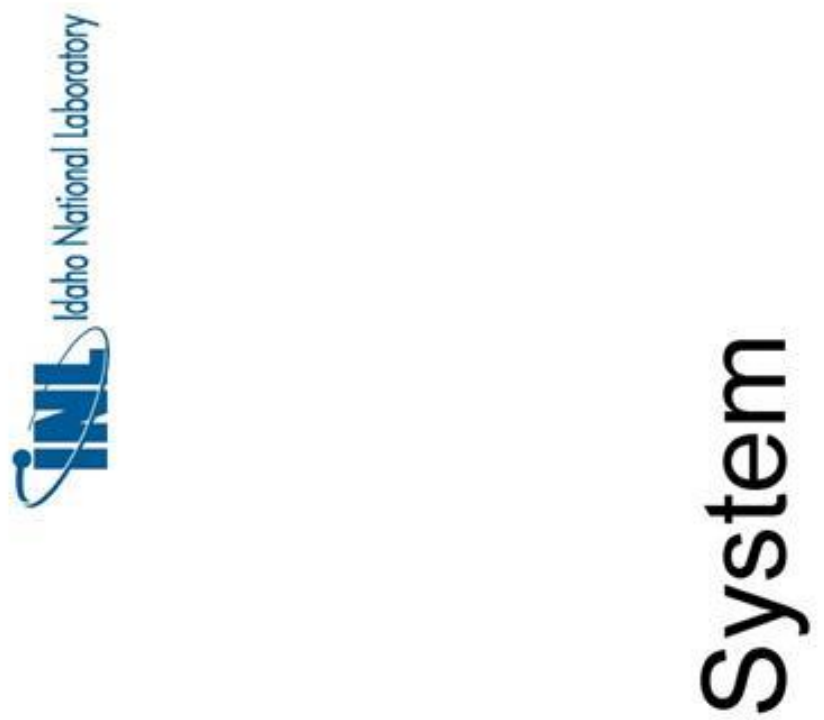

음

5

0

0

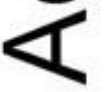

CD

ธช

$\square$ 

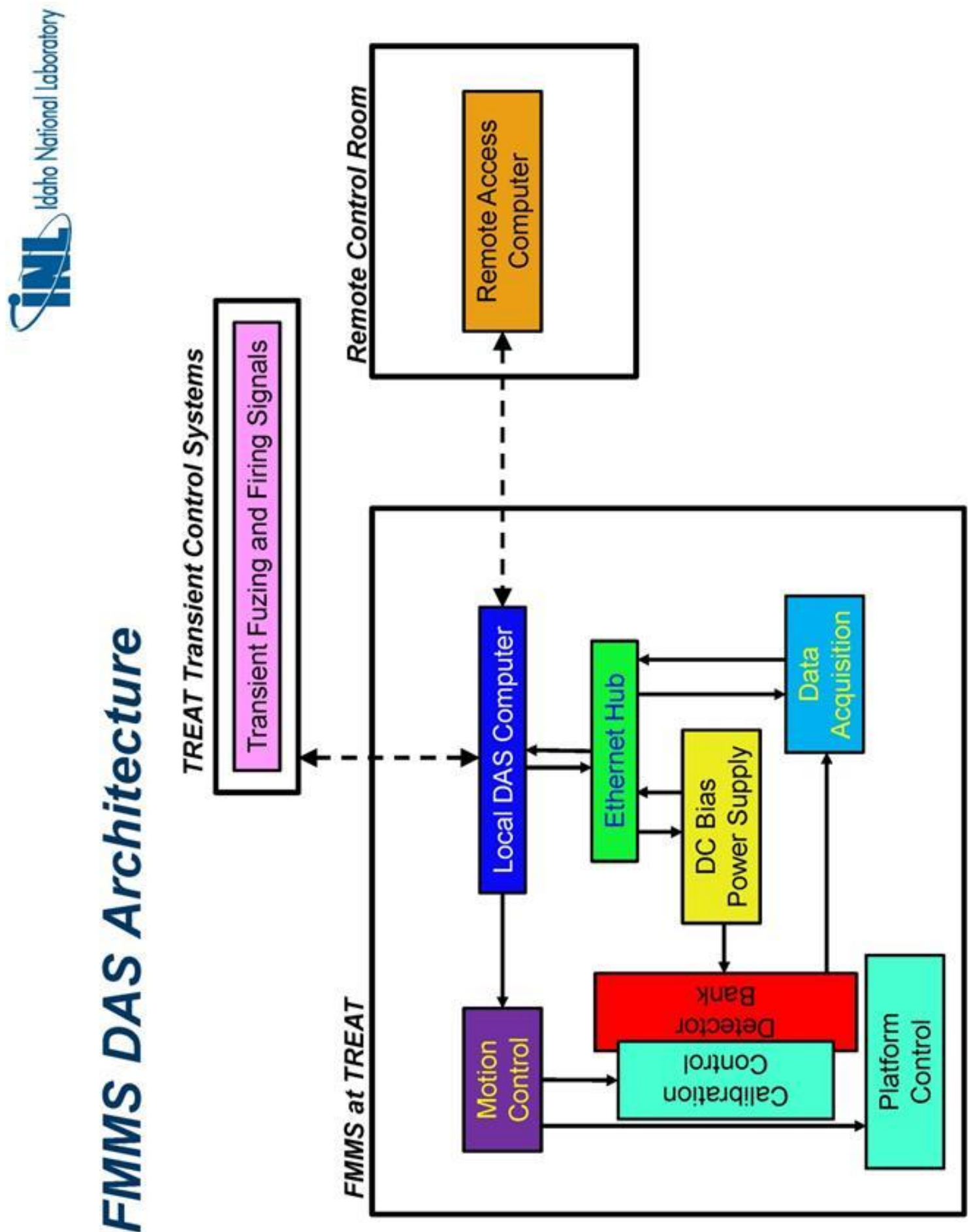


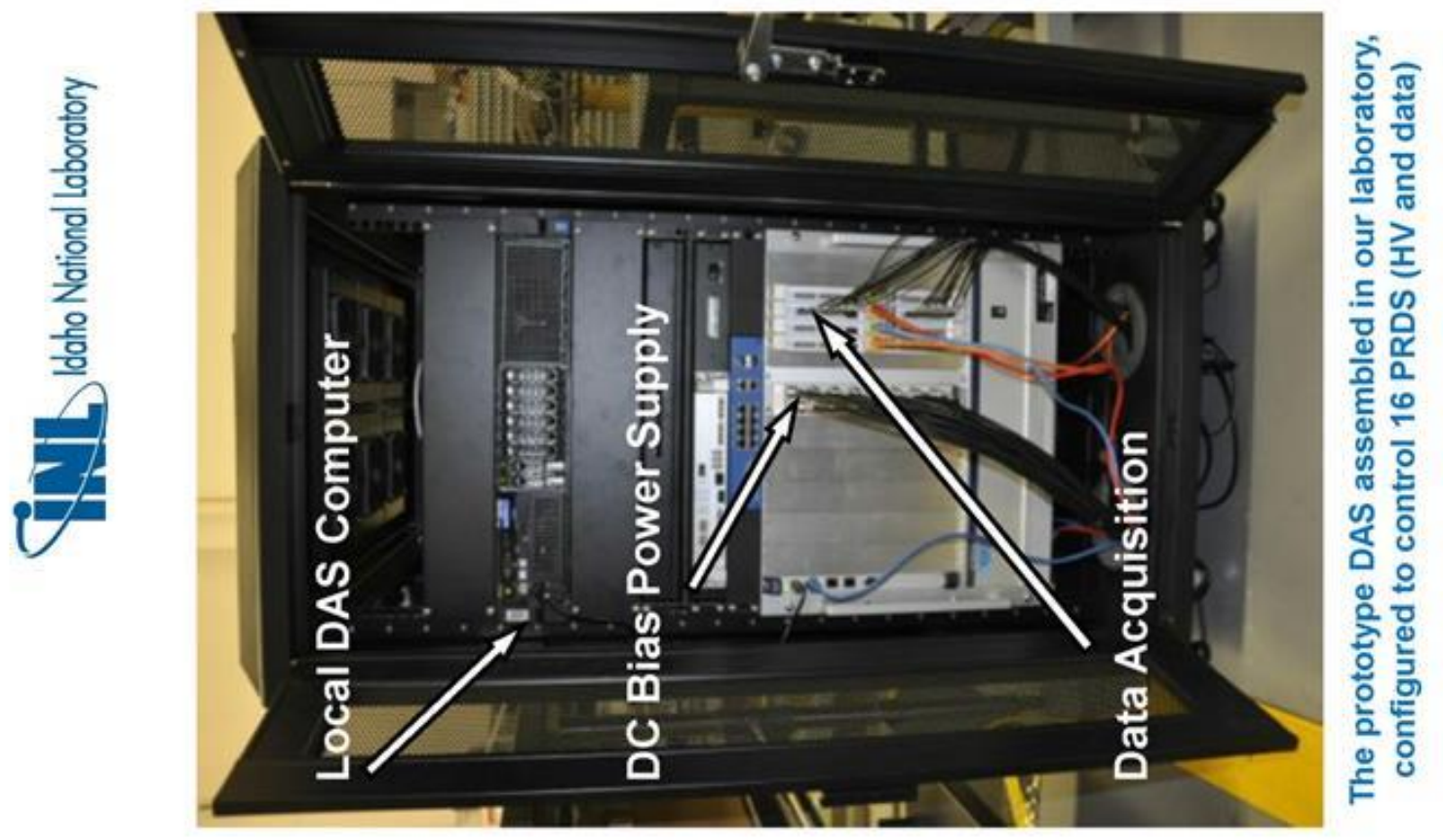

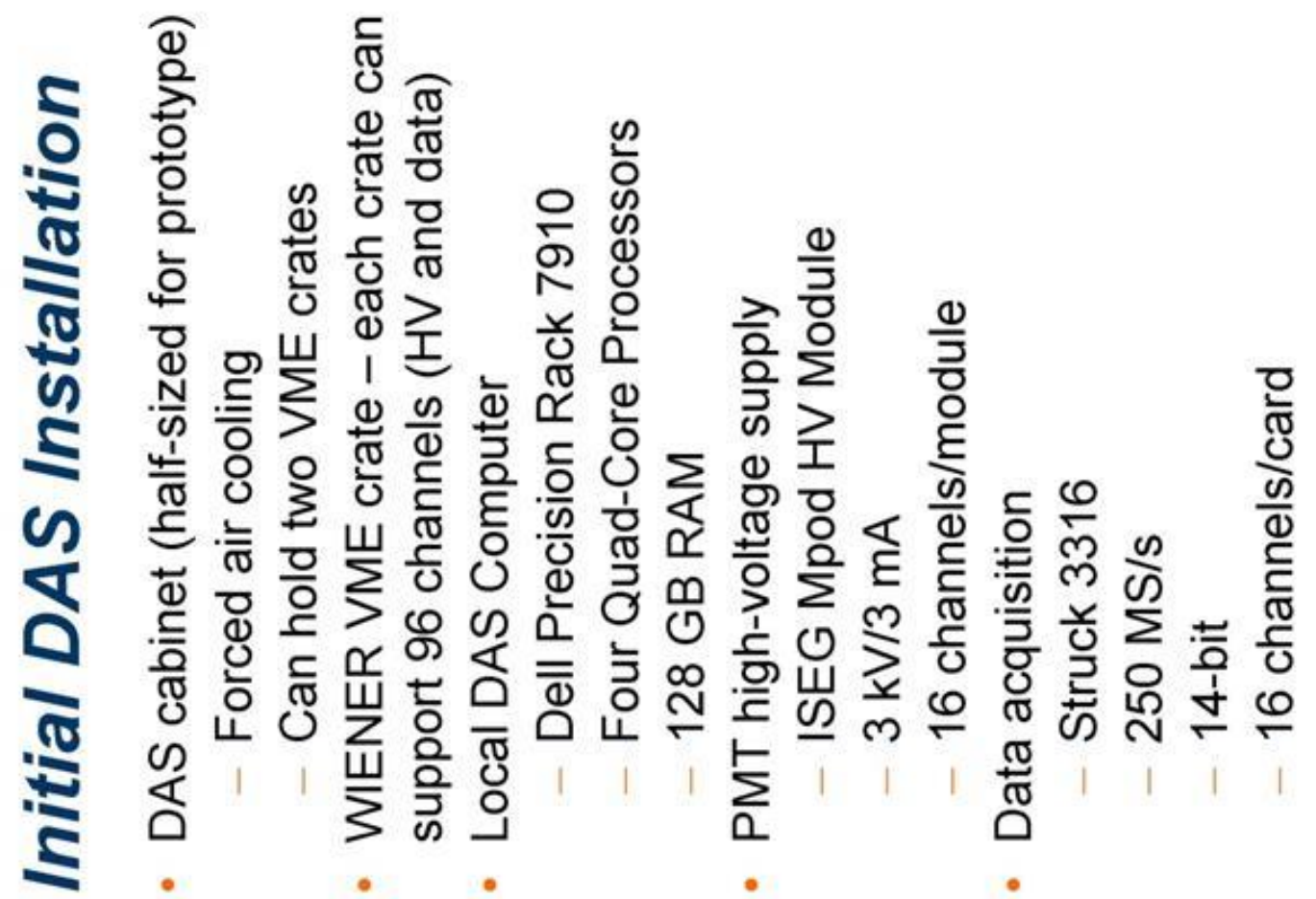




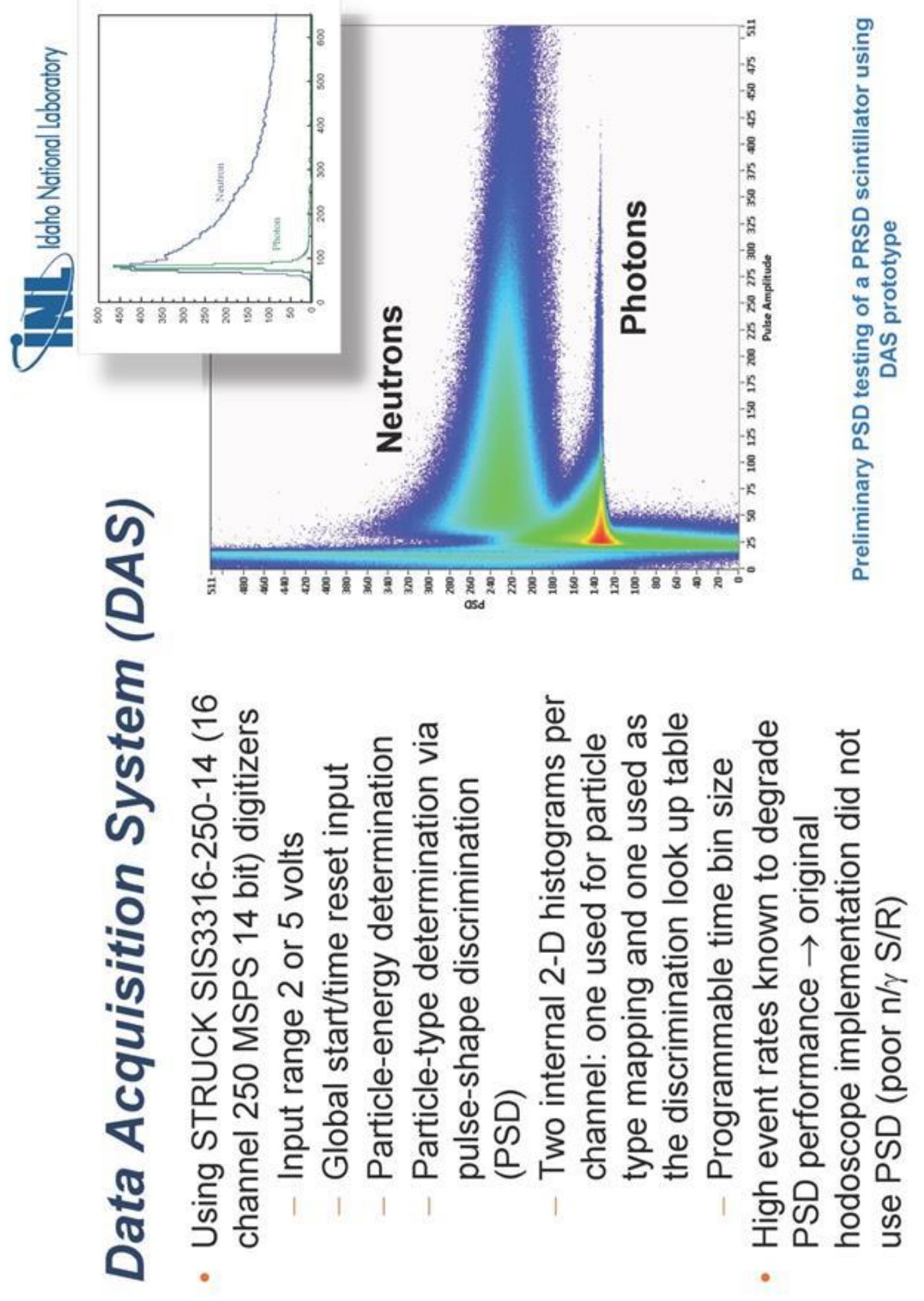



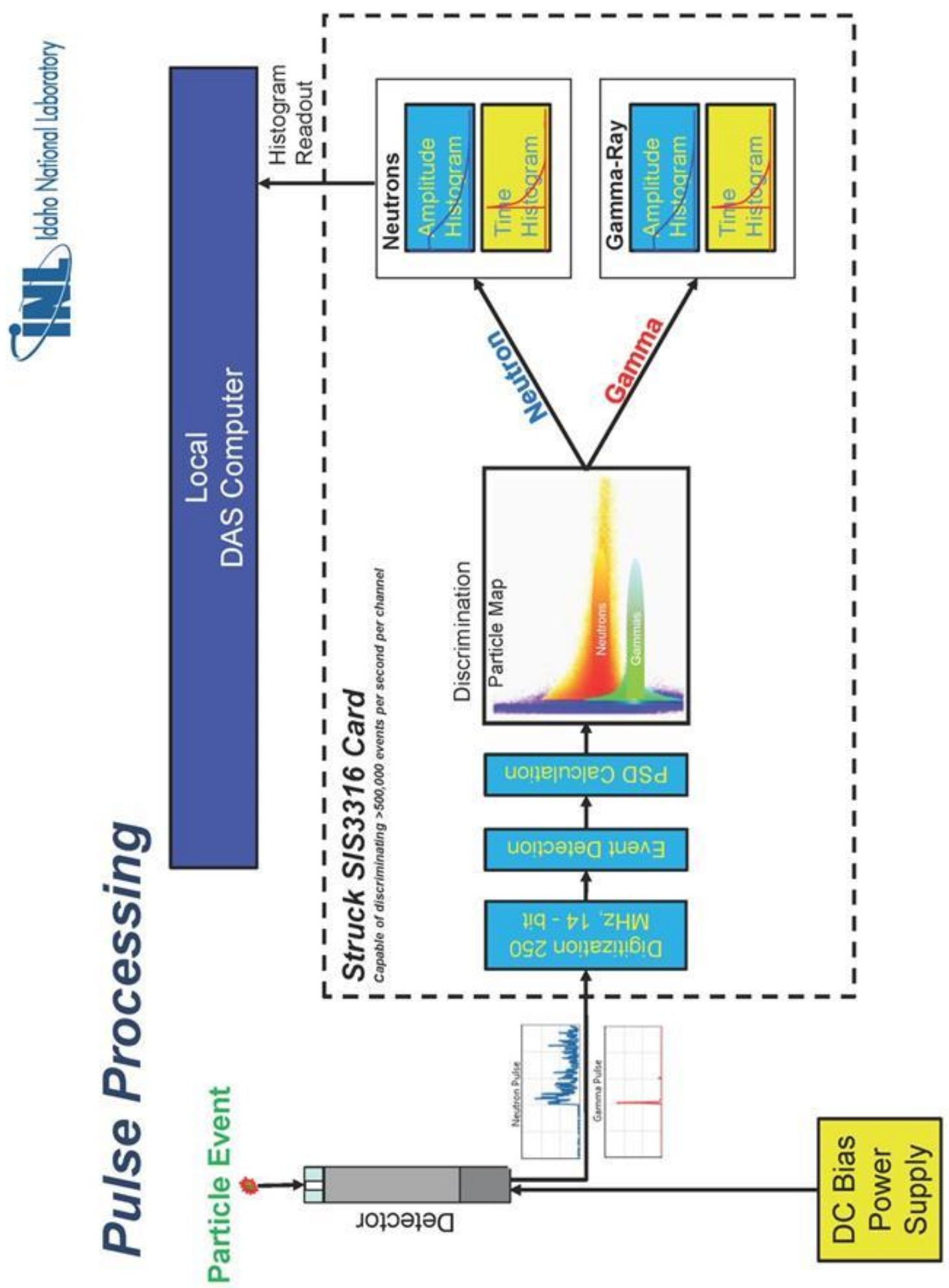


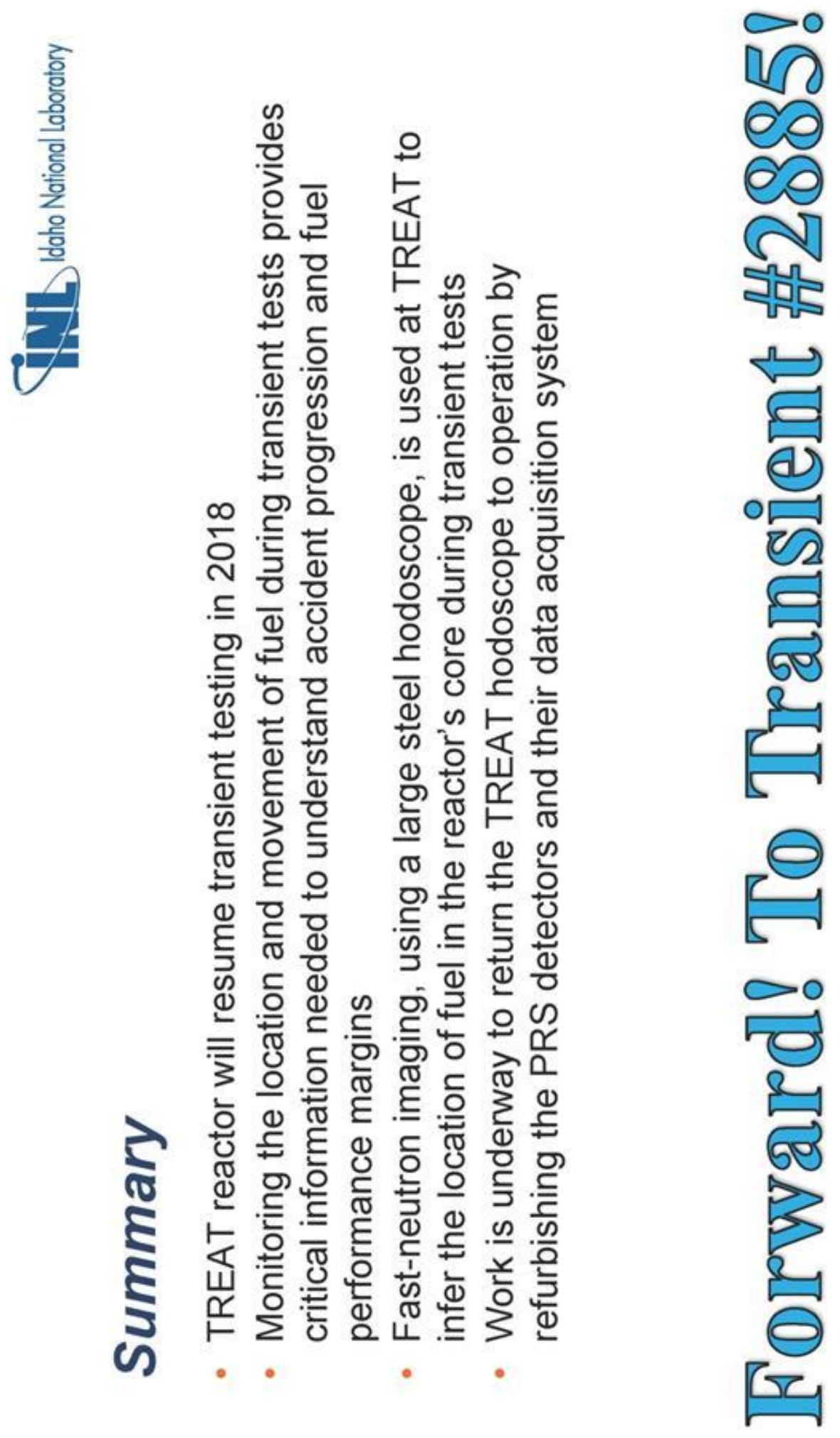




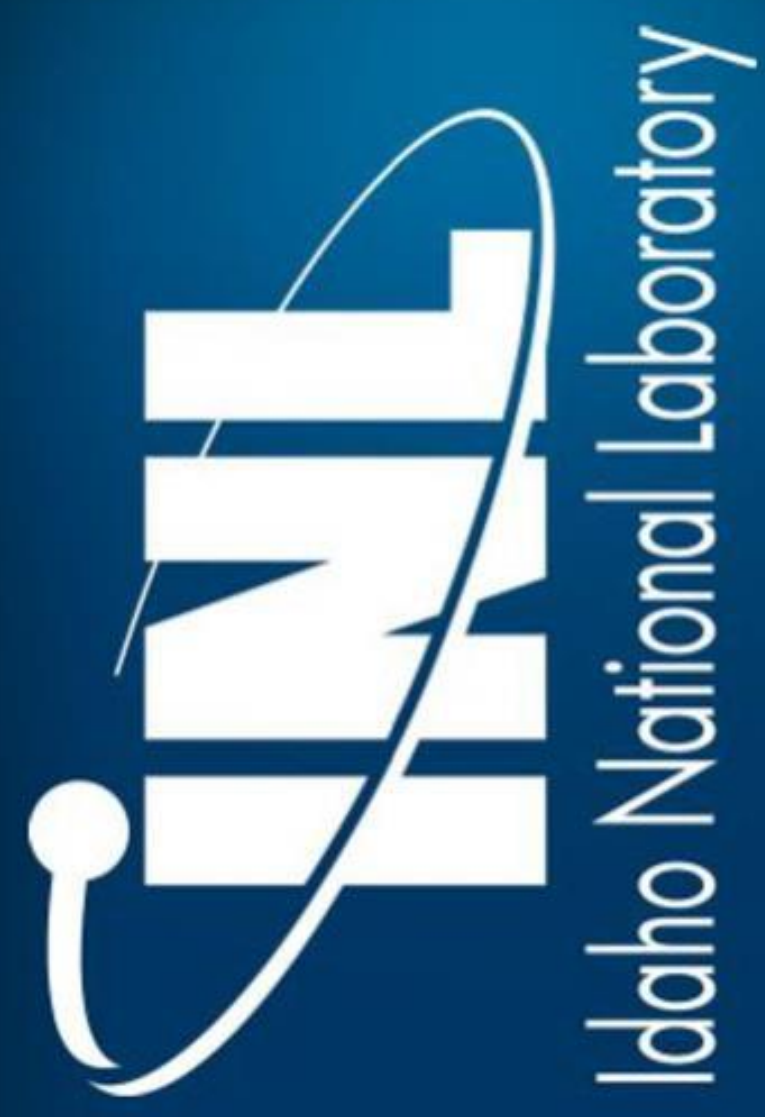



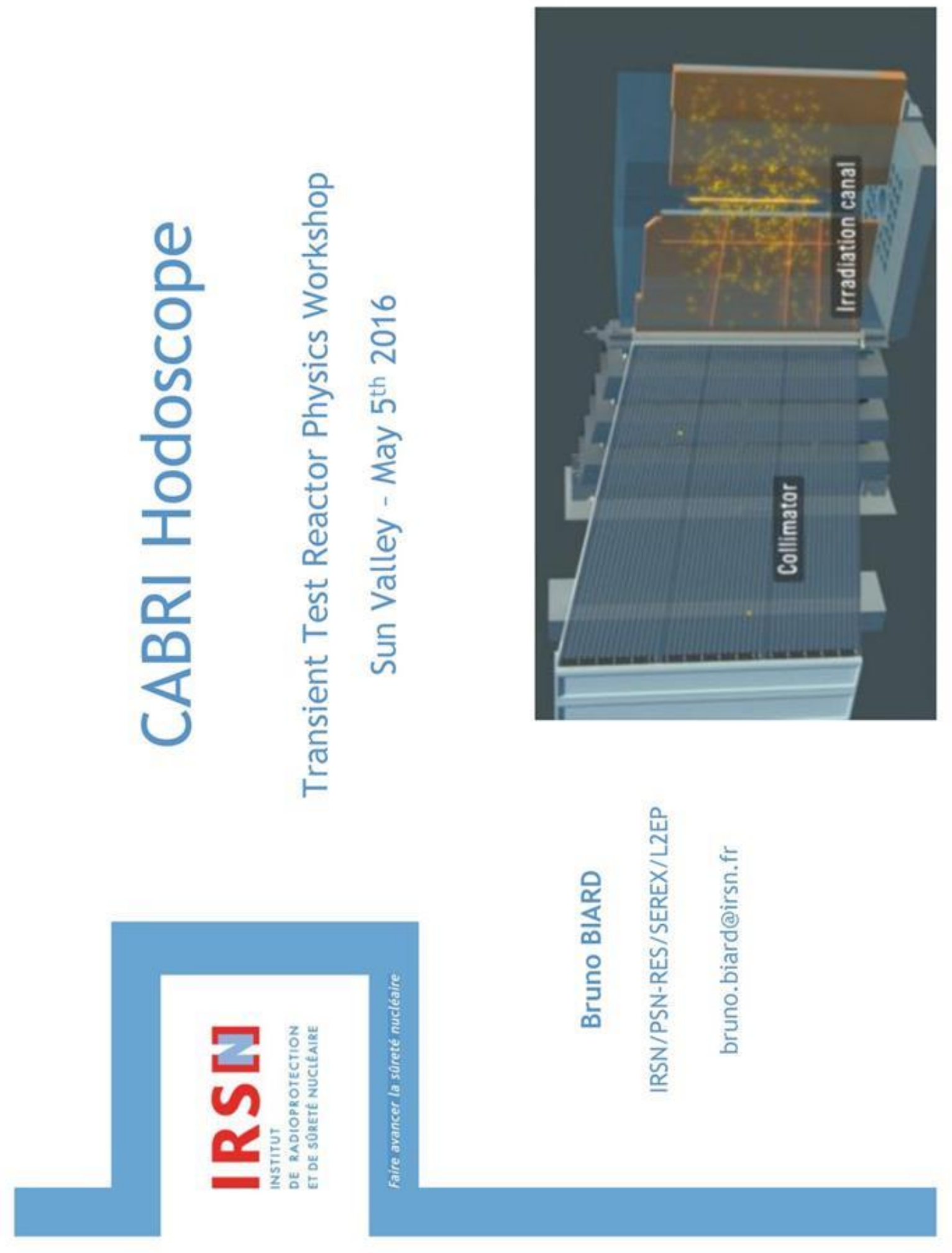


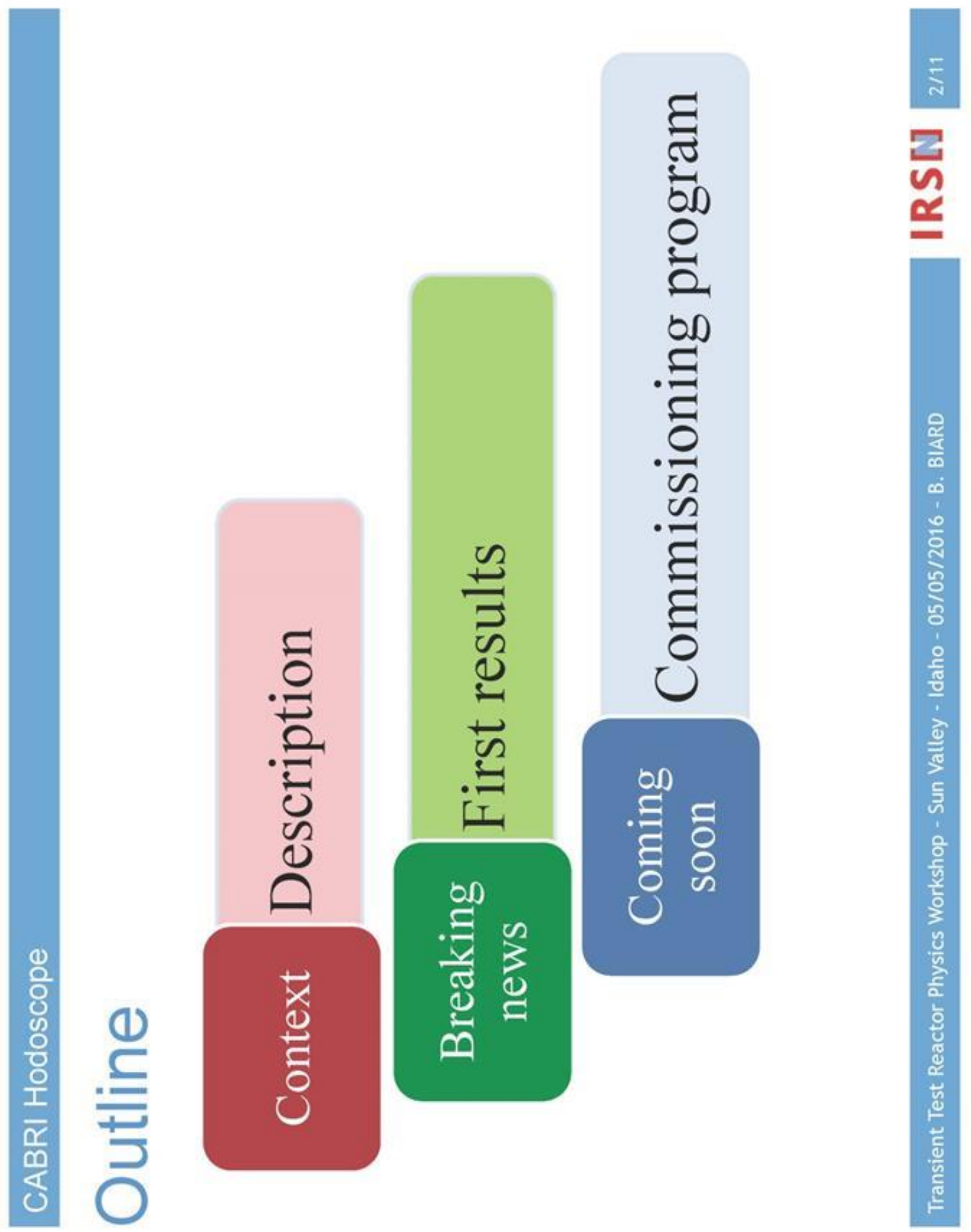




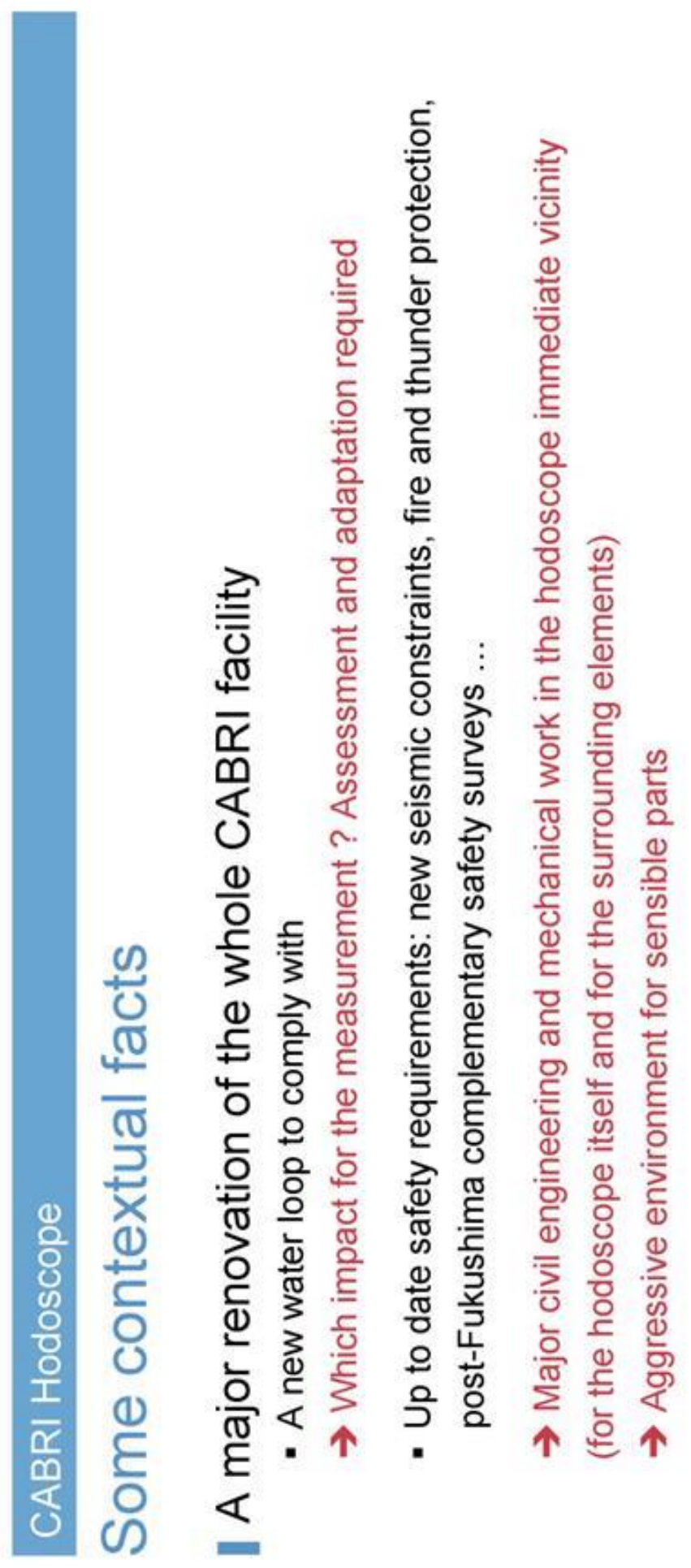

రి

.

을

응

요

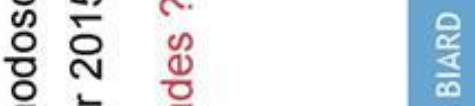

도 전

든 은 윽

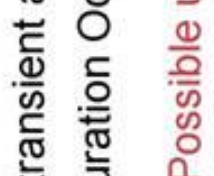

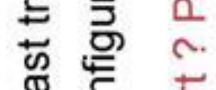

뜽 능

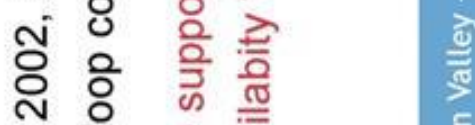

论 느 임

유 \}

(1) 3 त

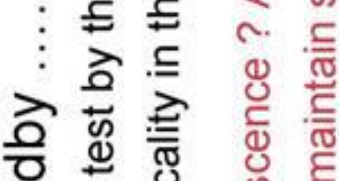

它 壬 :

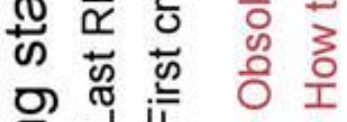

응. 모 


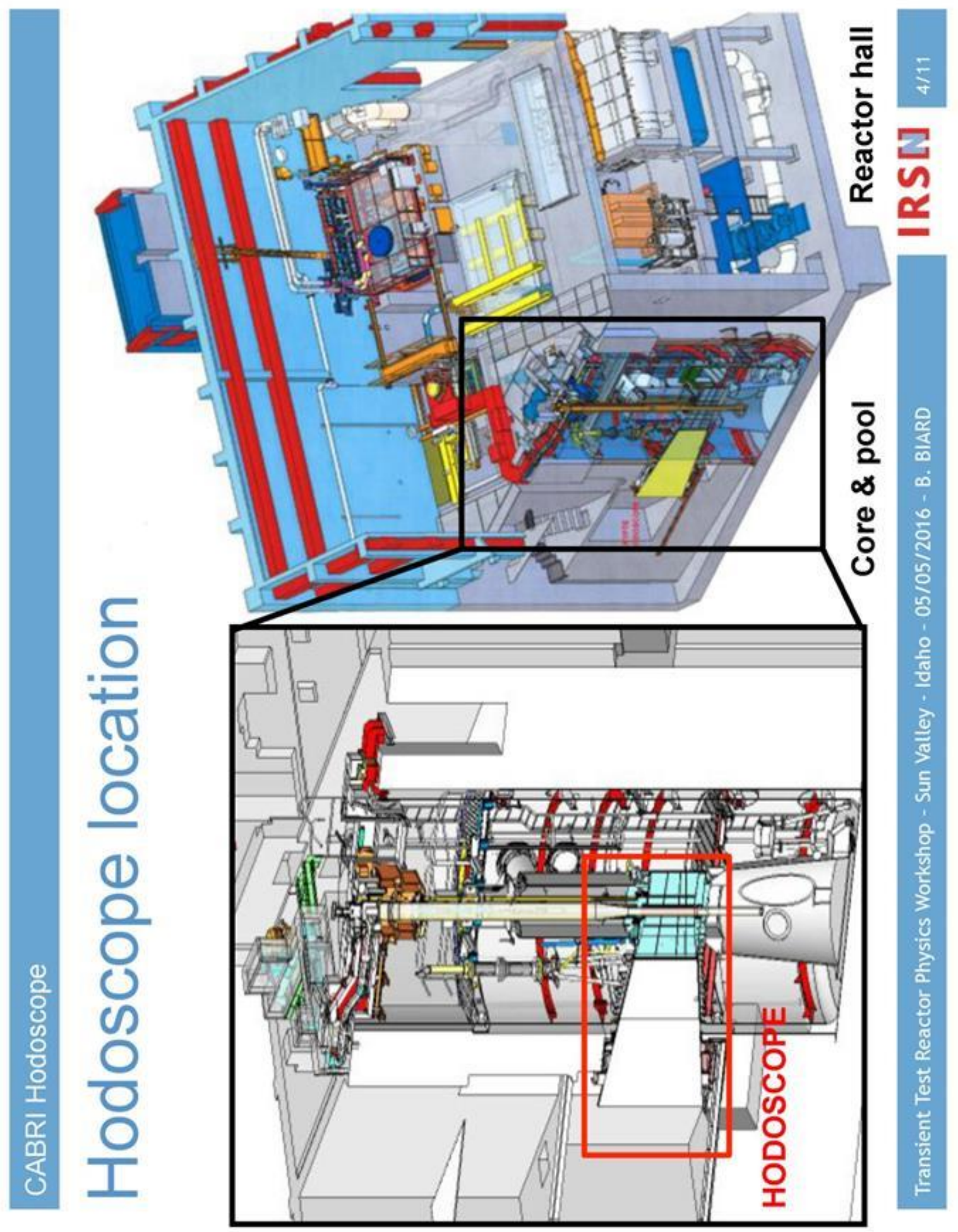




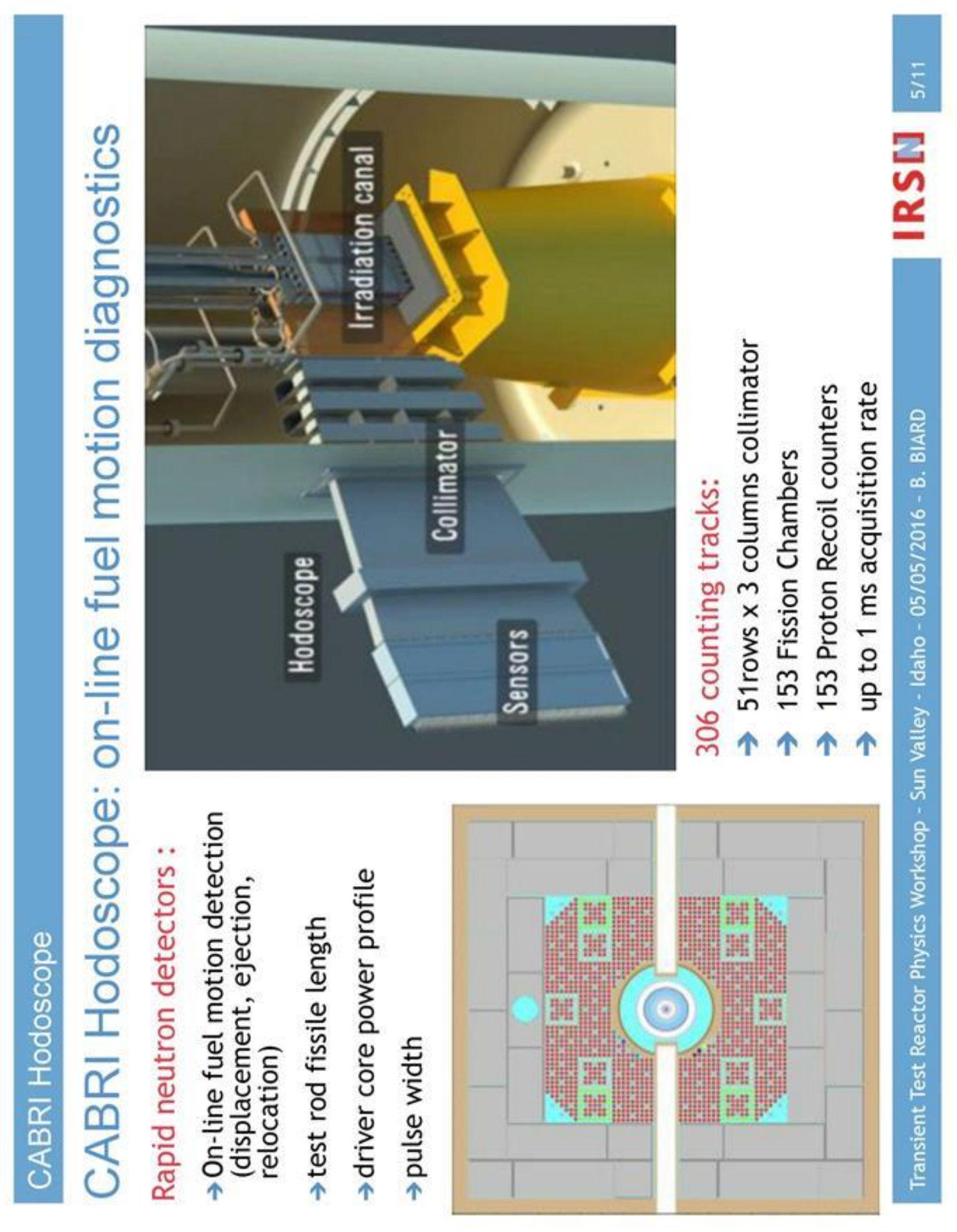




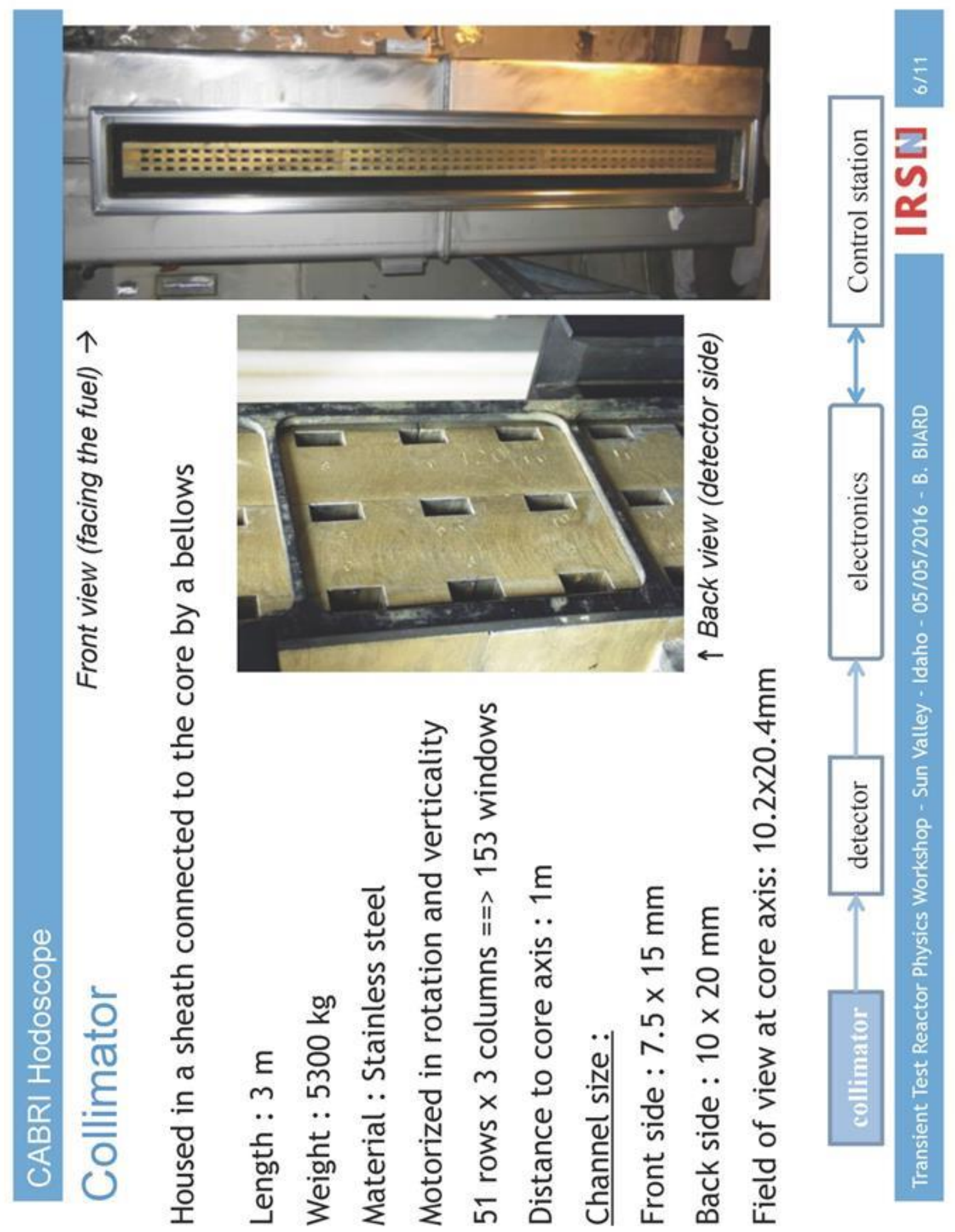




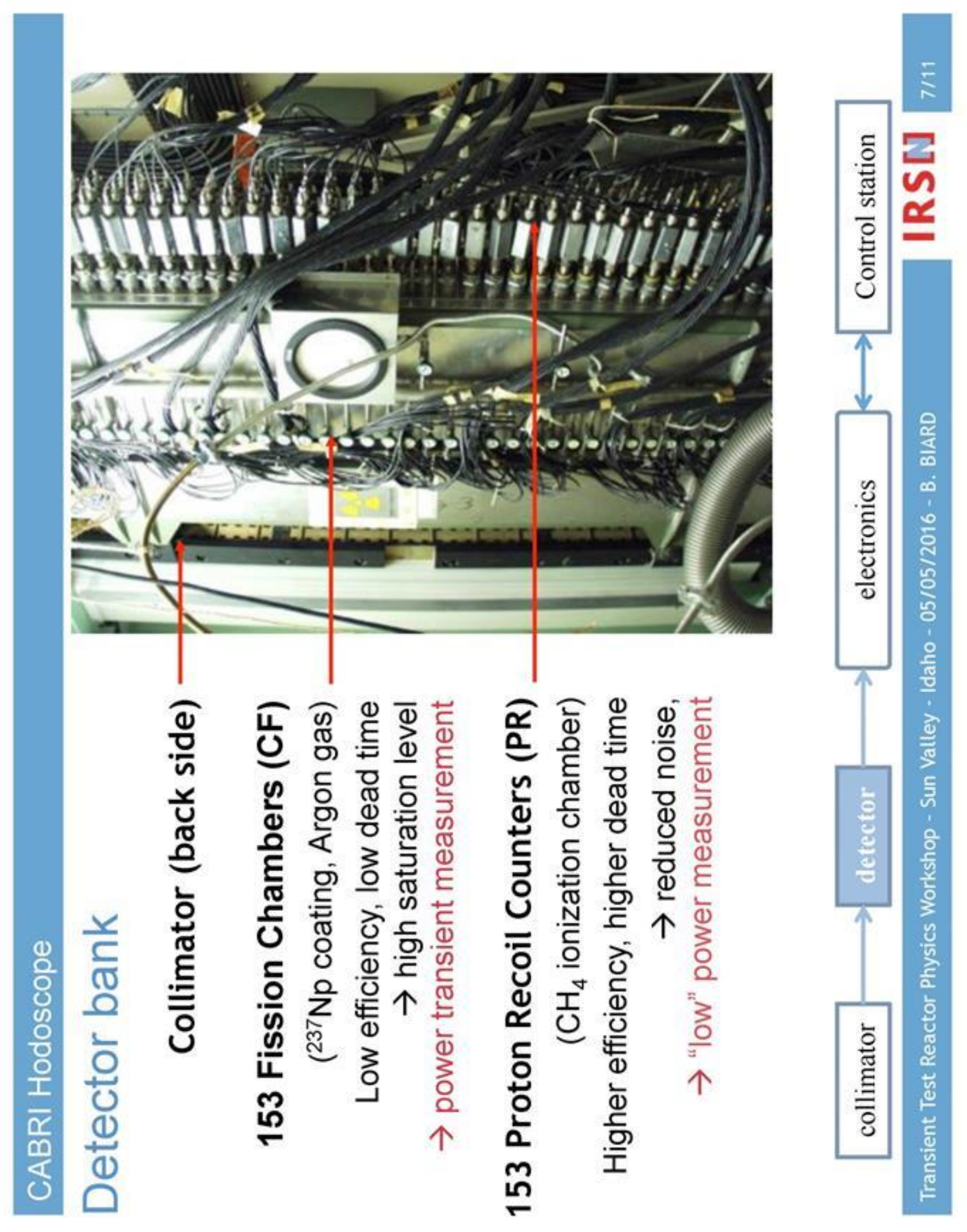




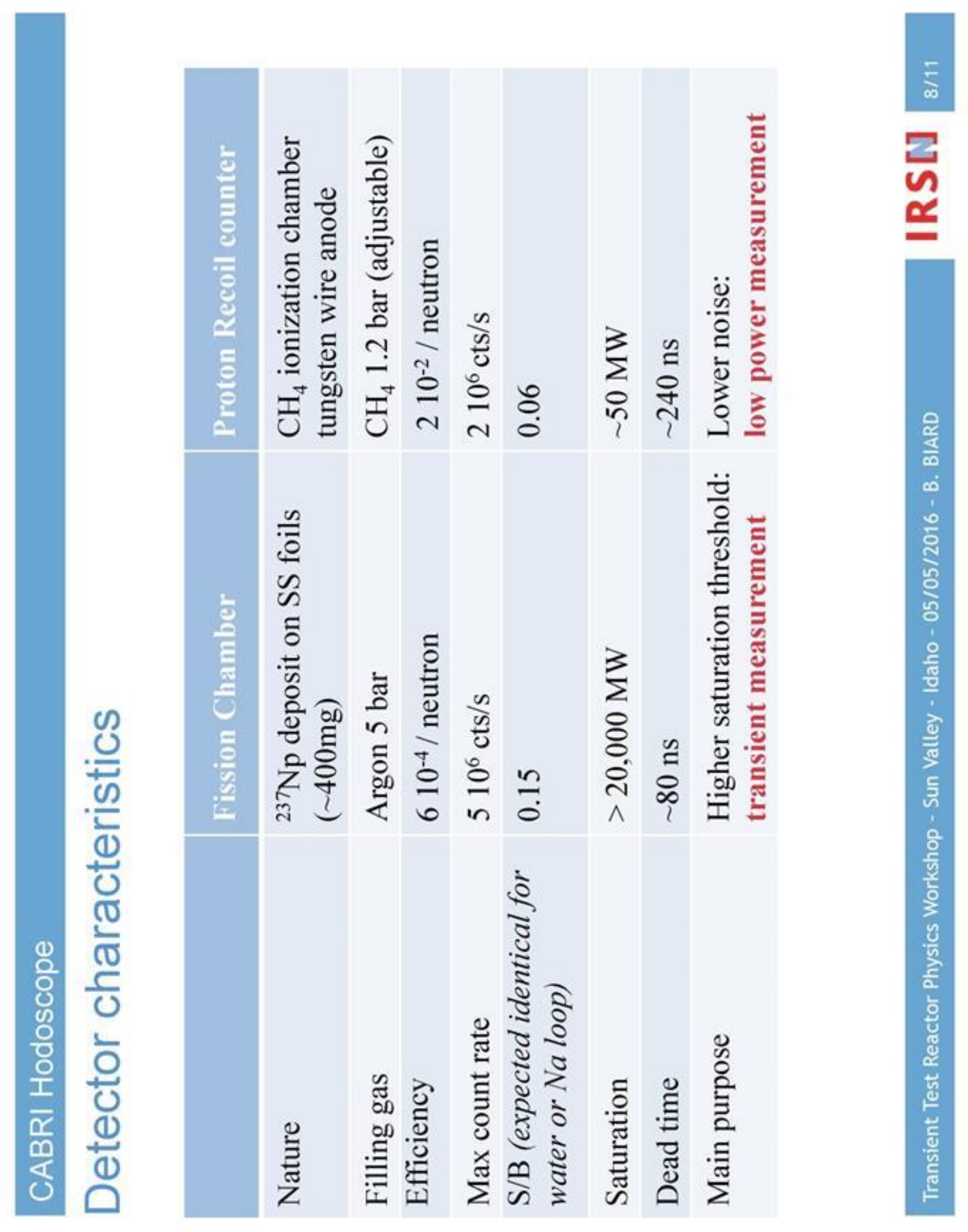




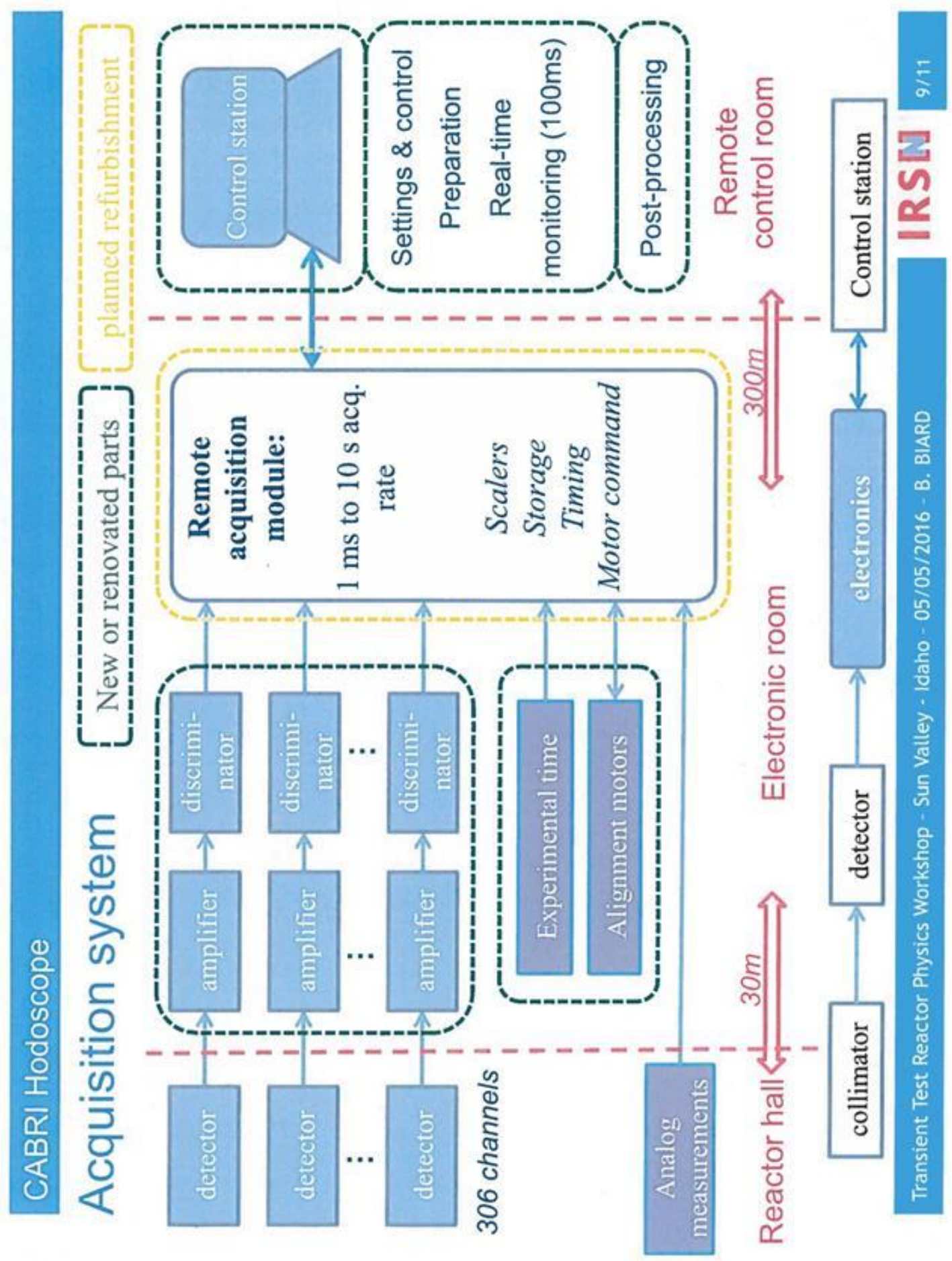




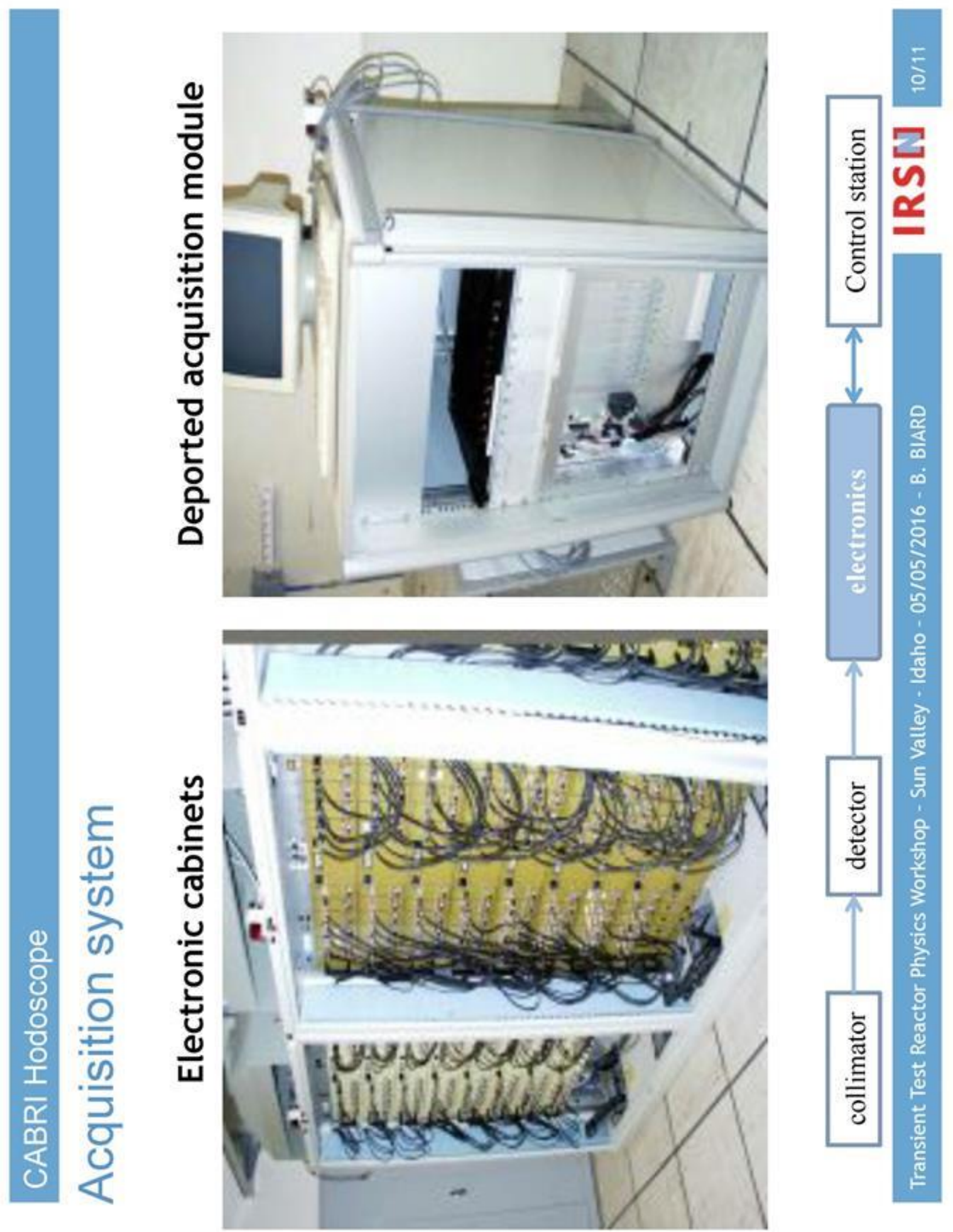



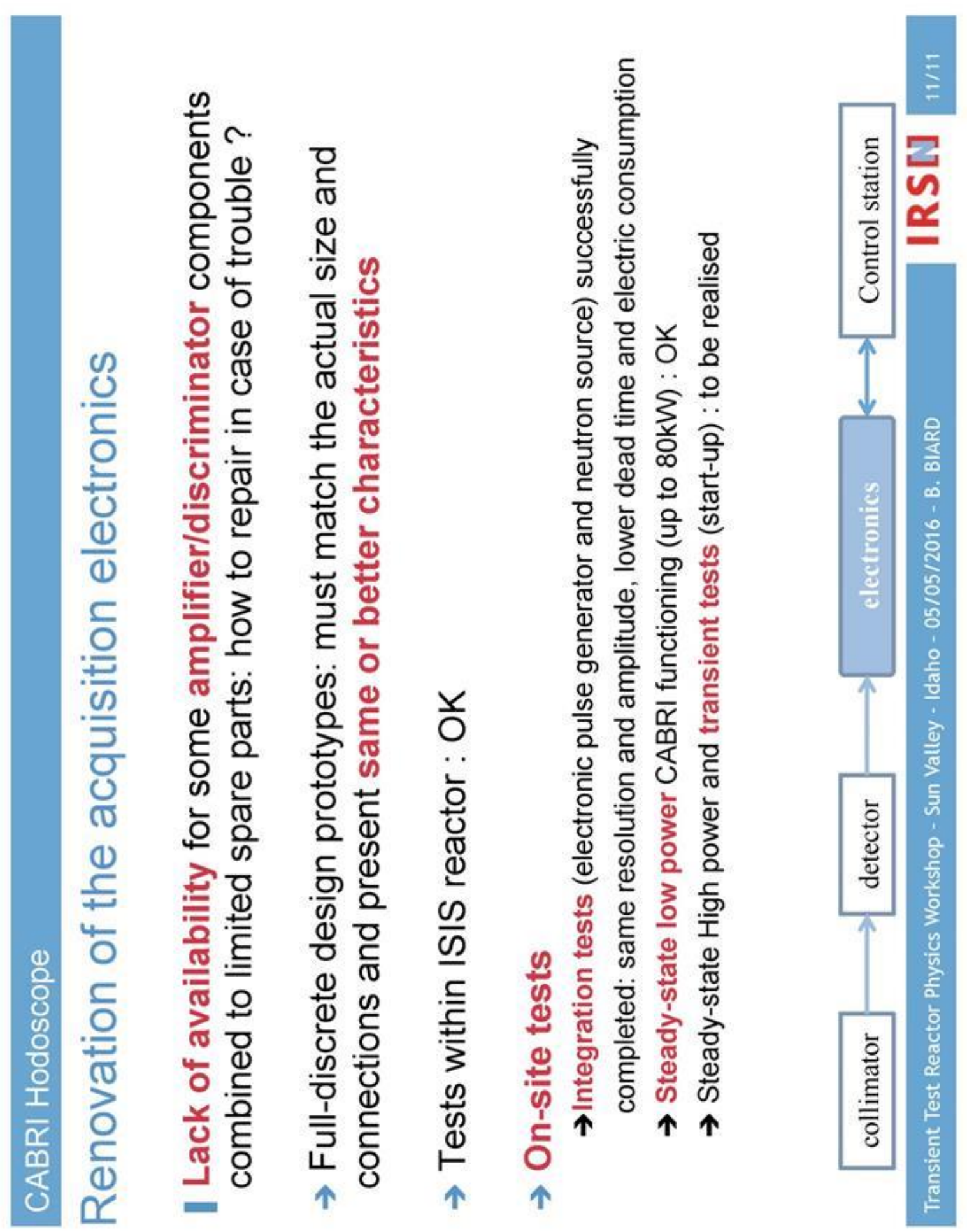


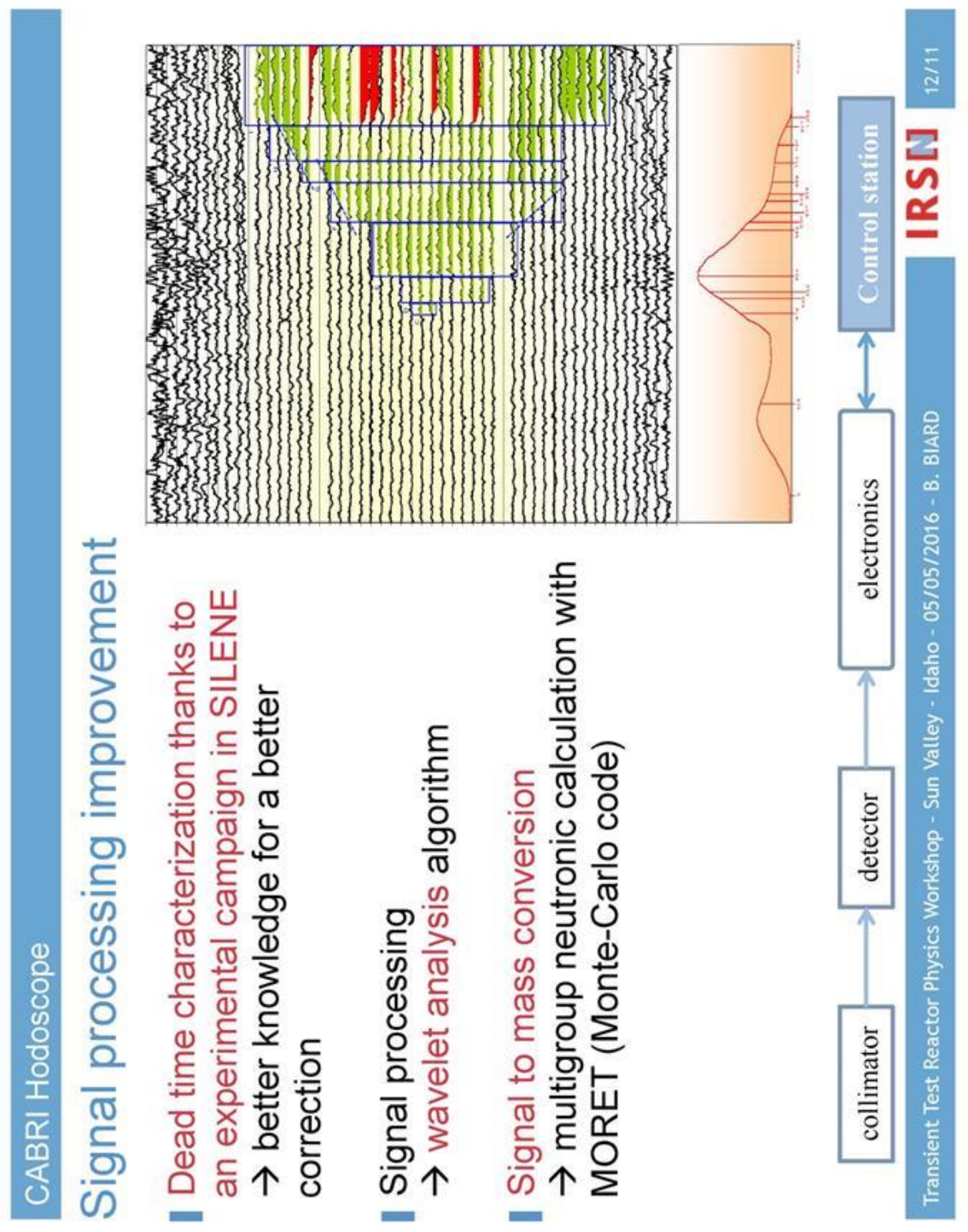




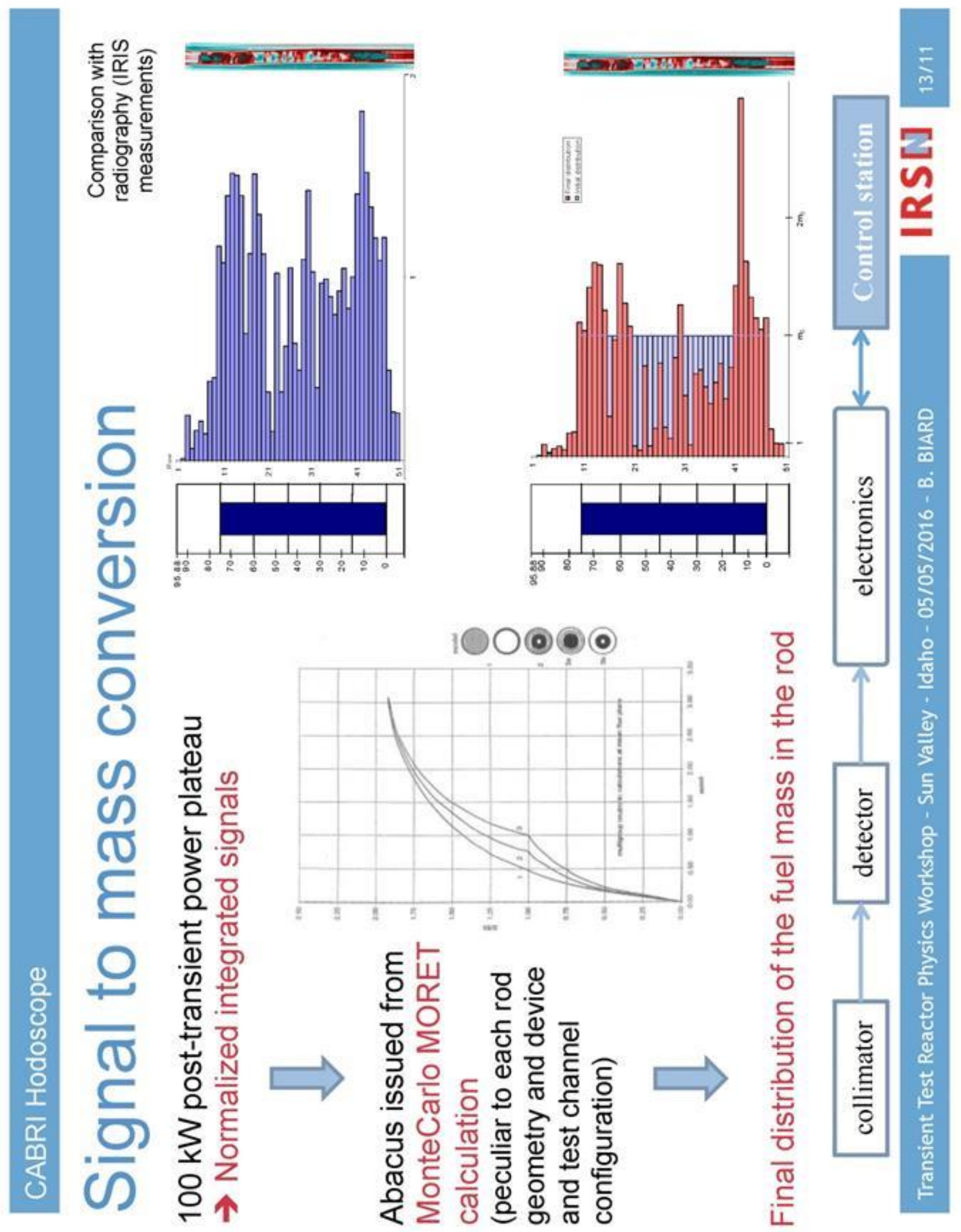




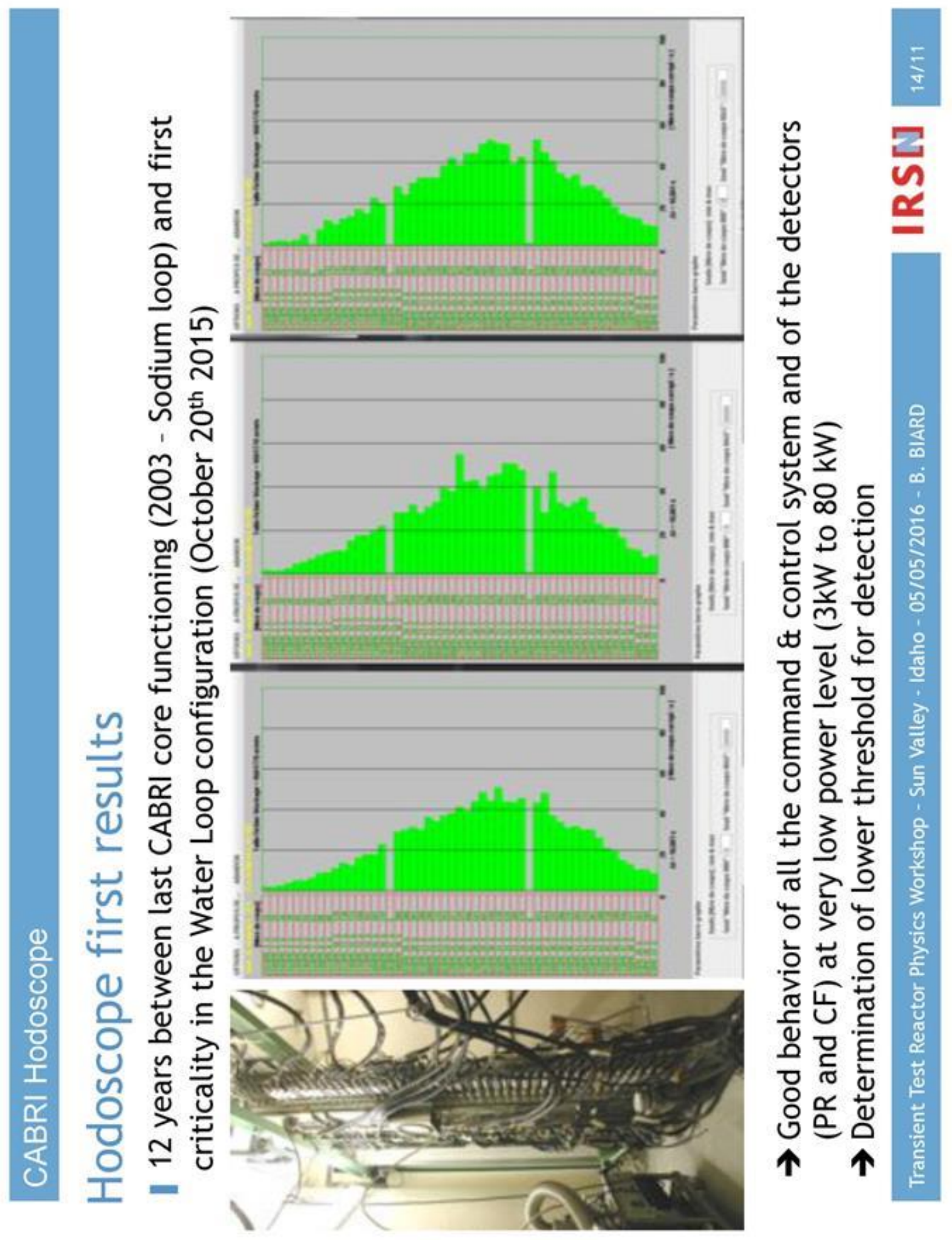



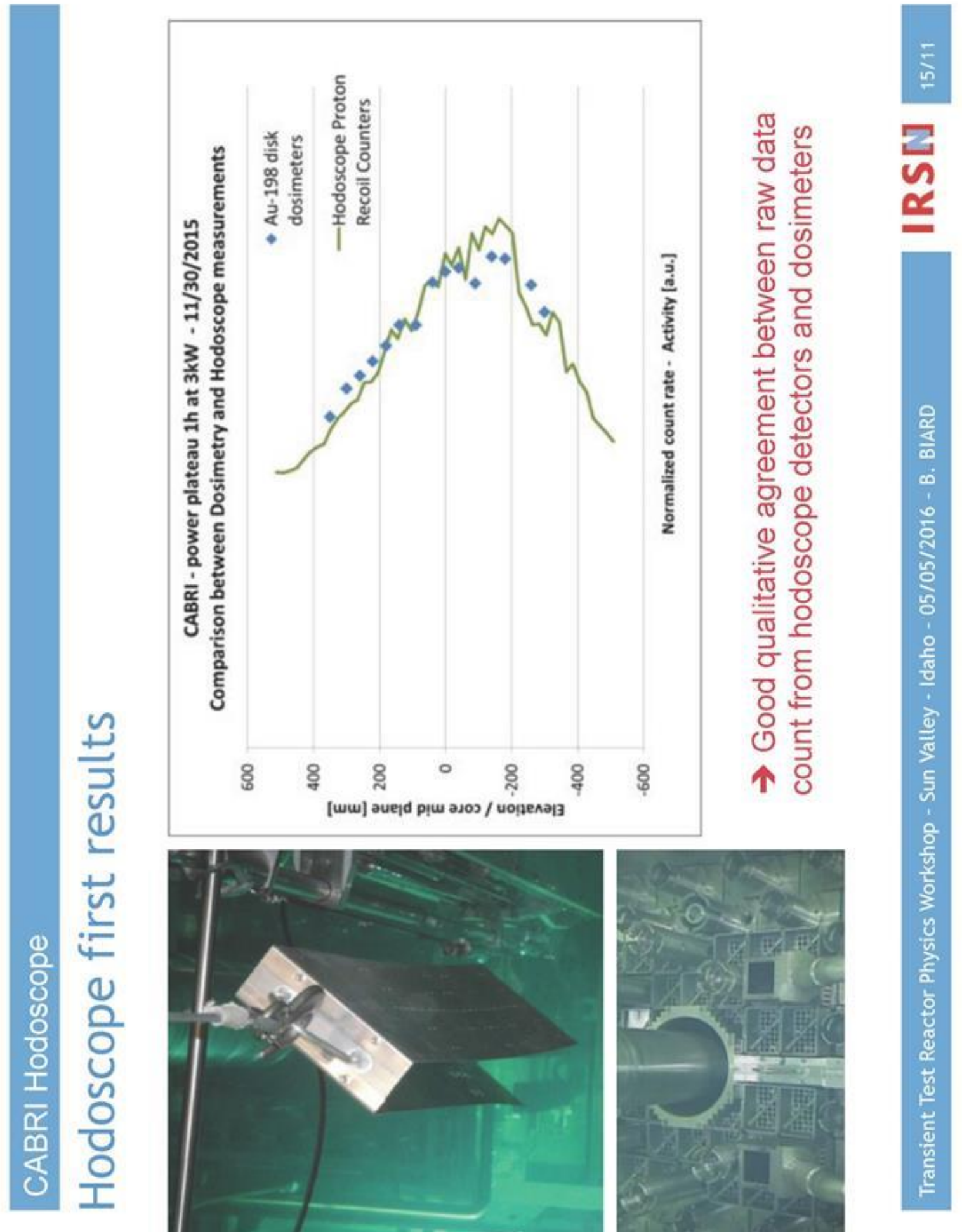


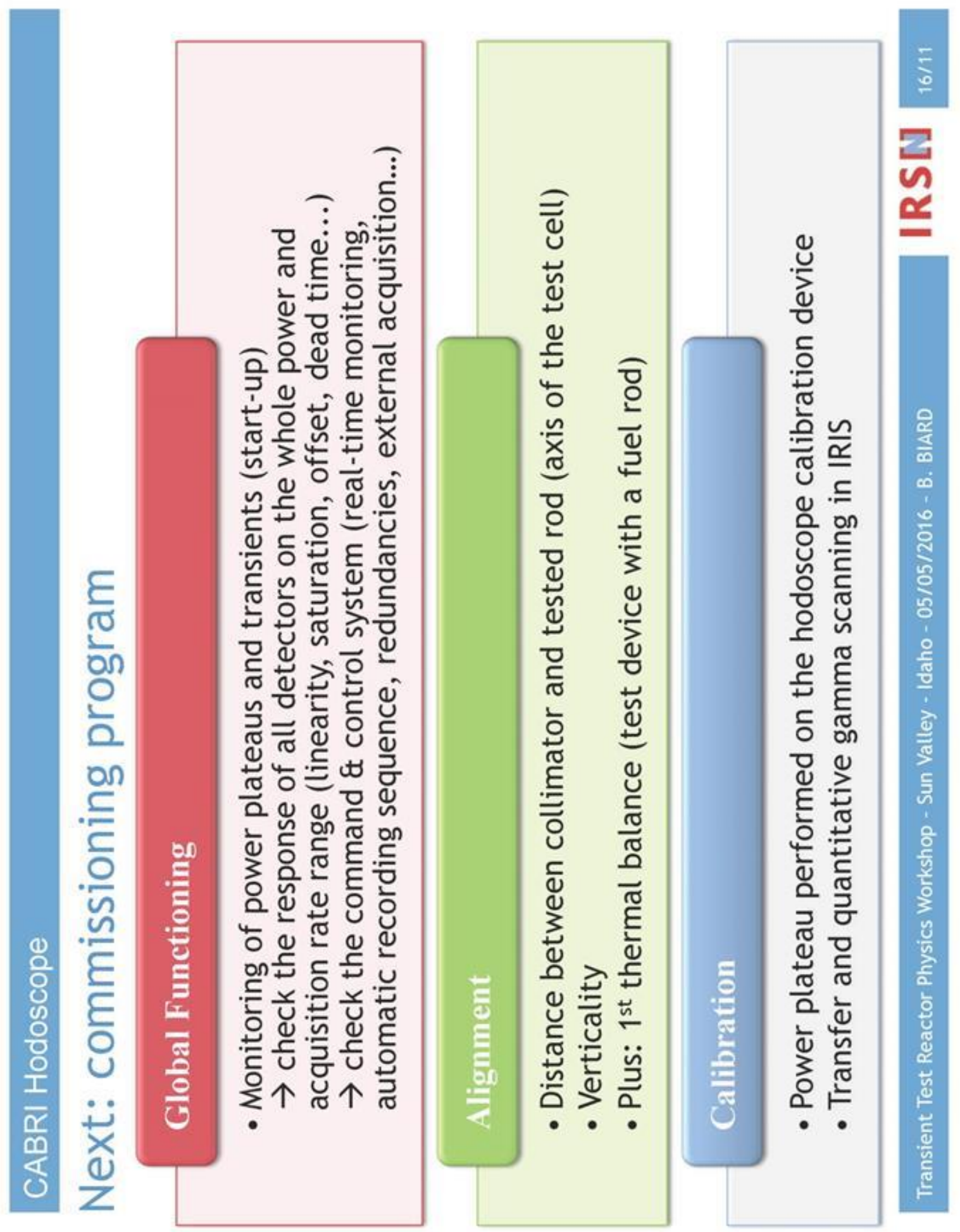




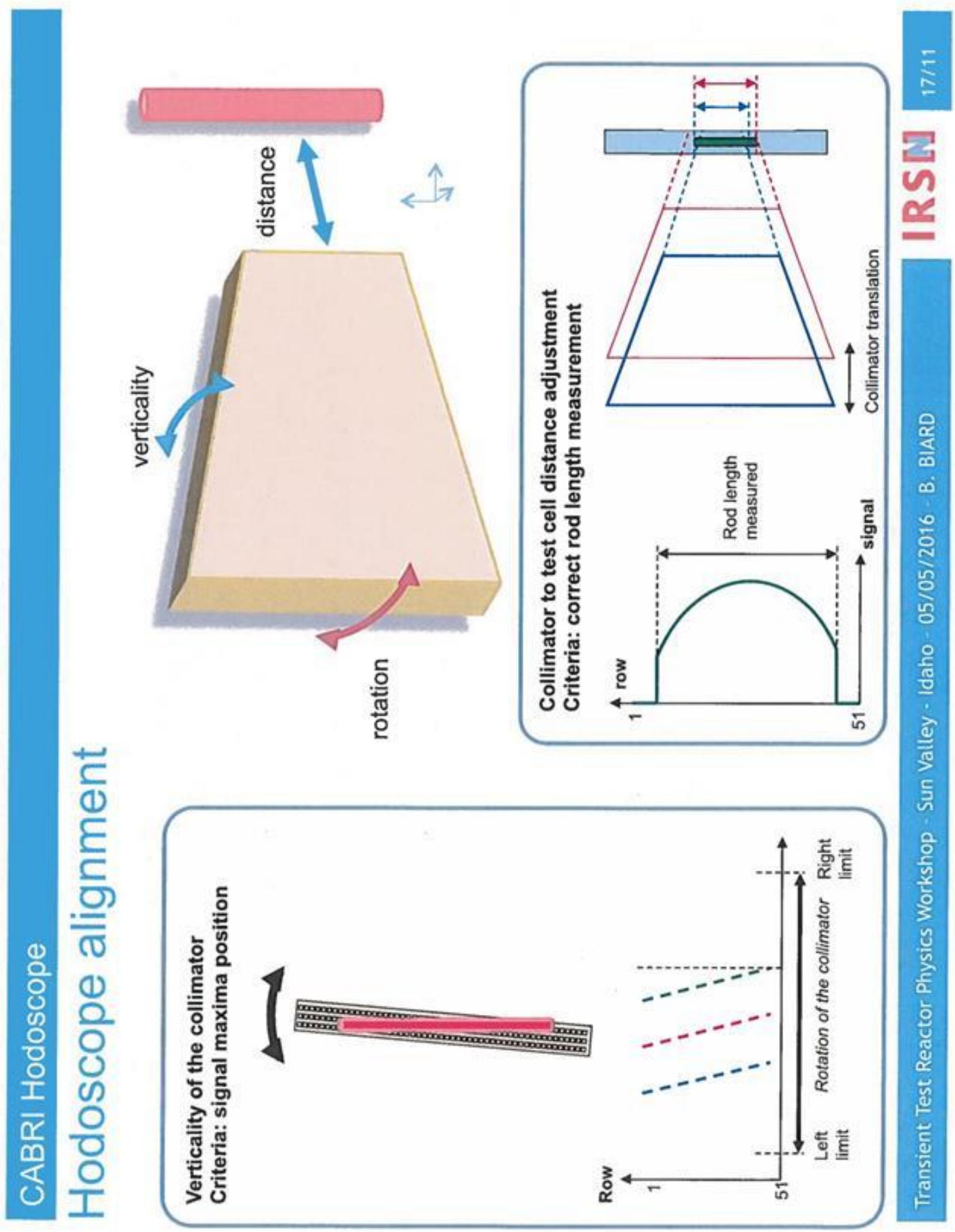




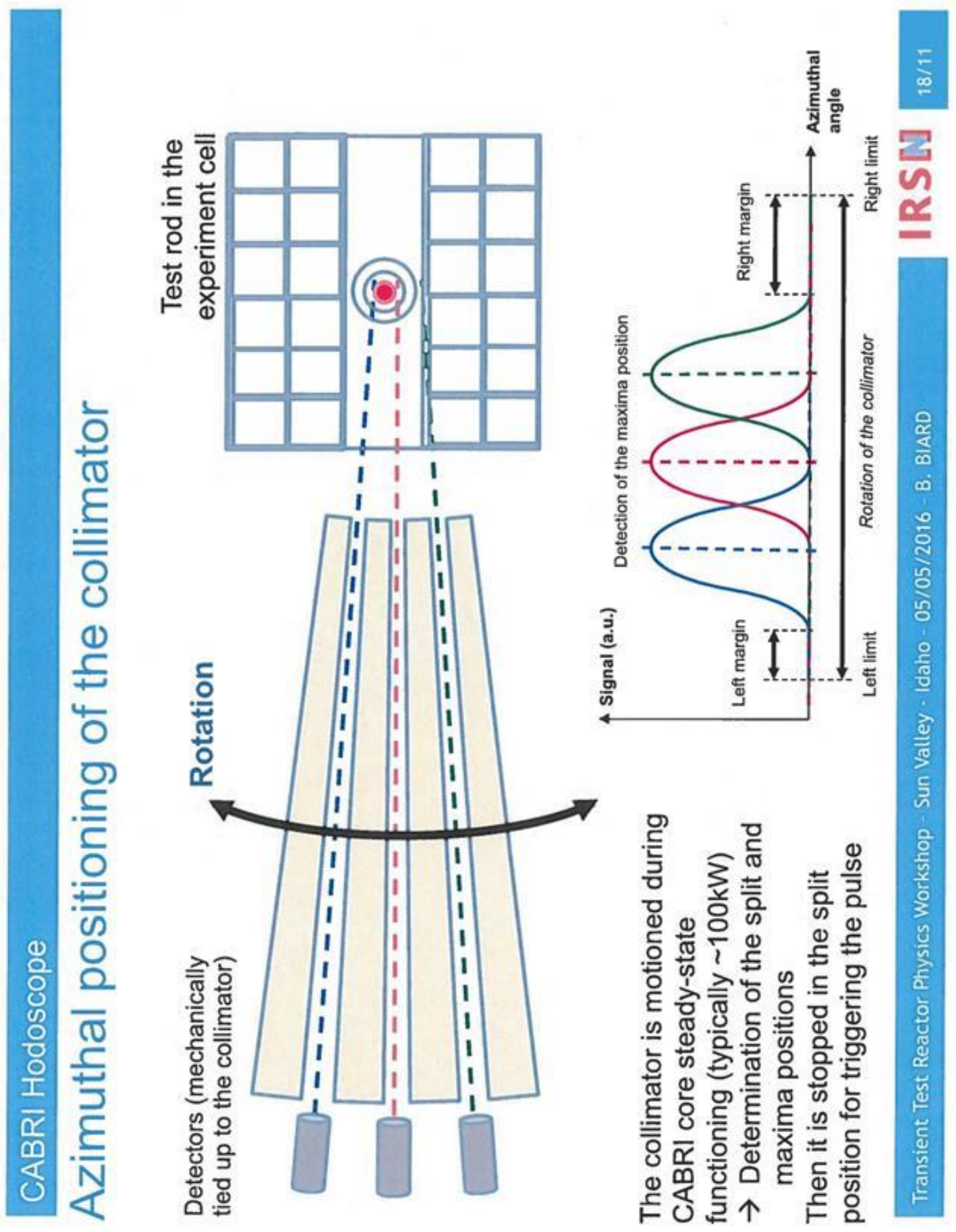



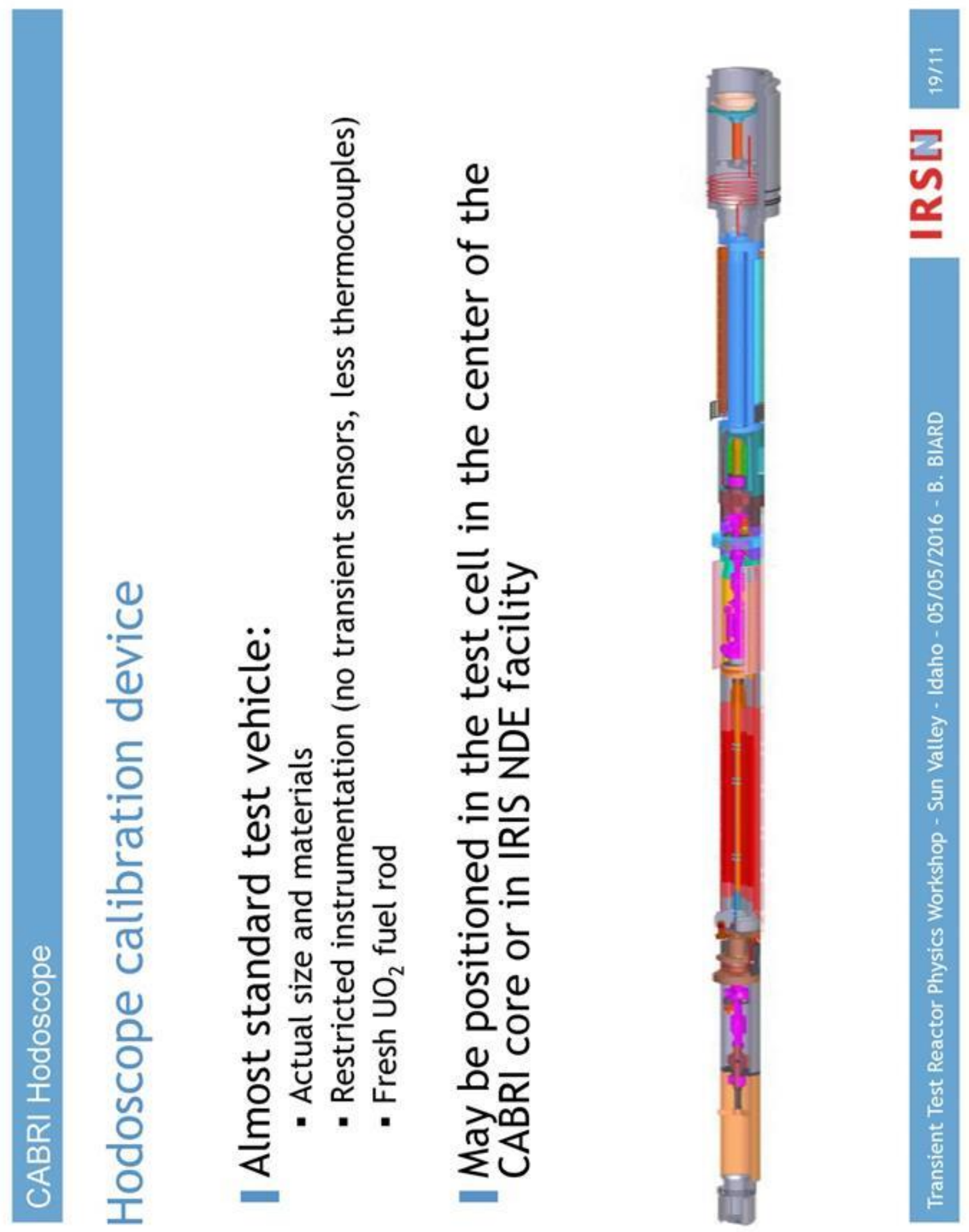


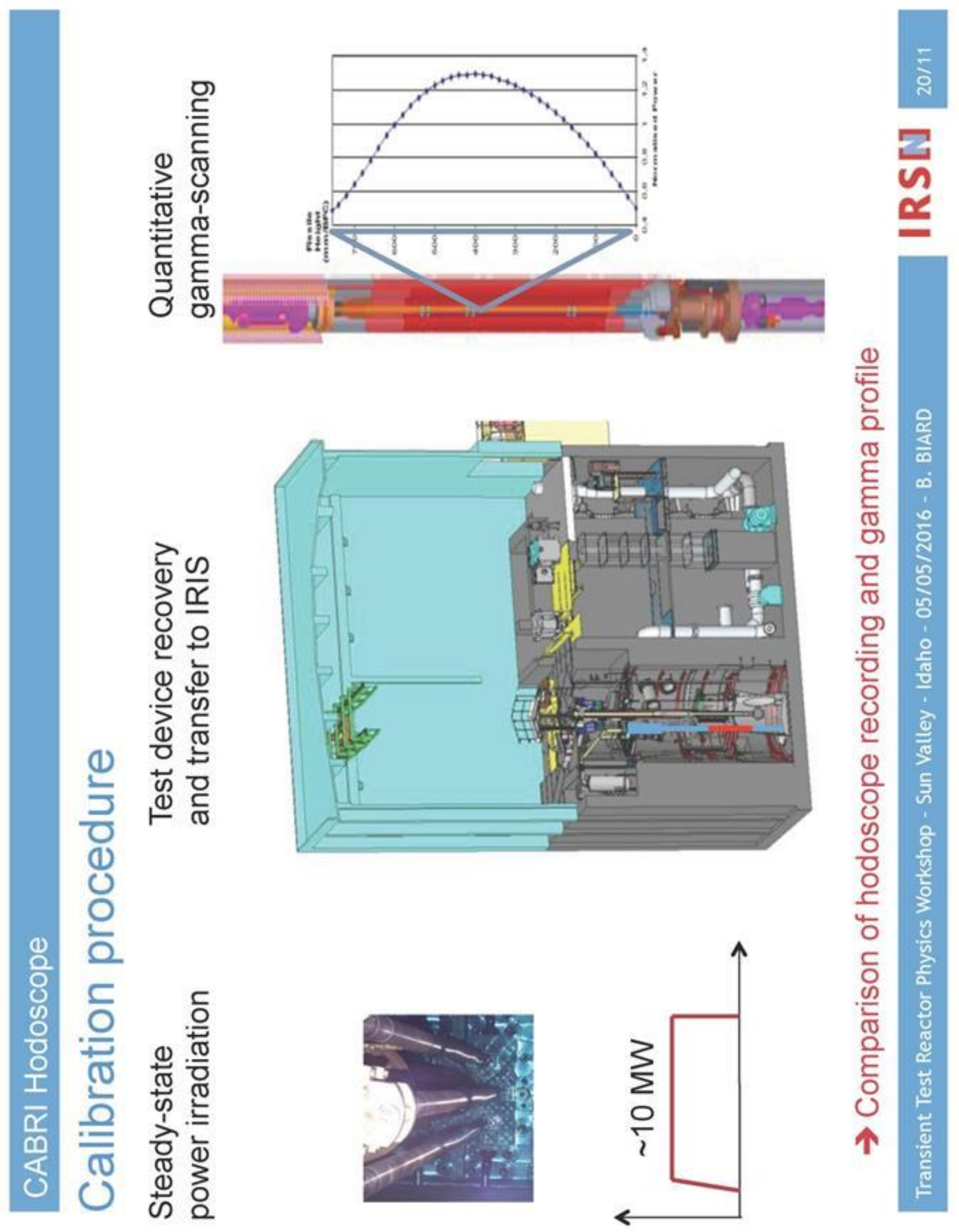



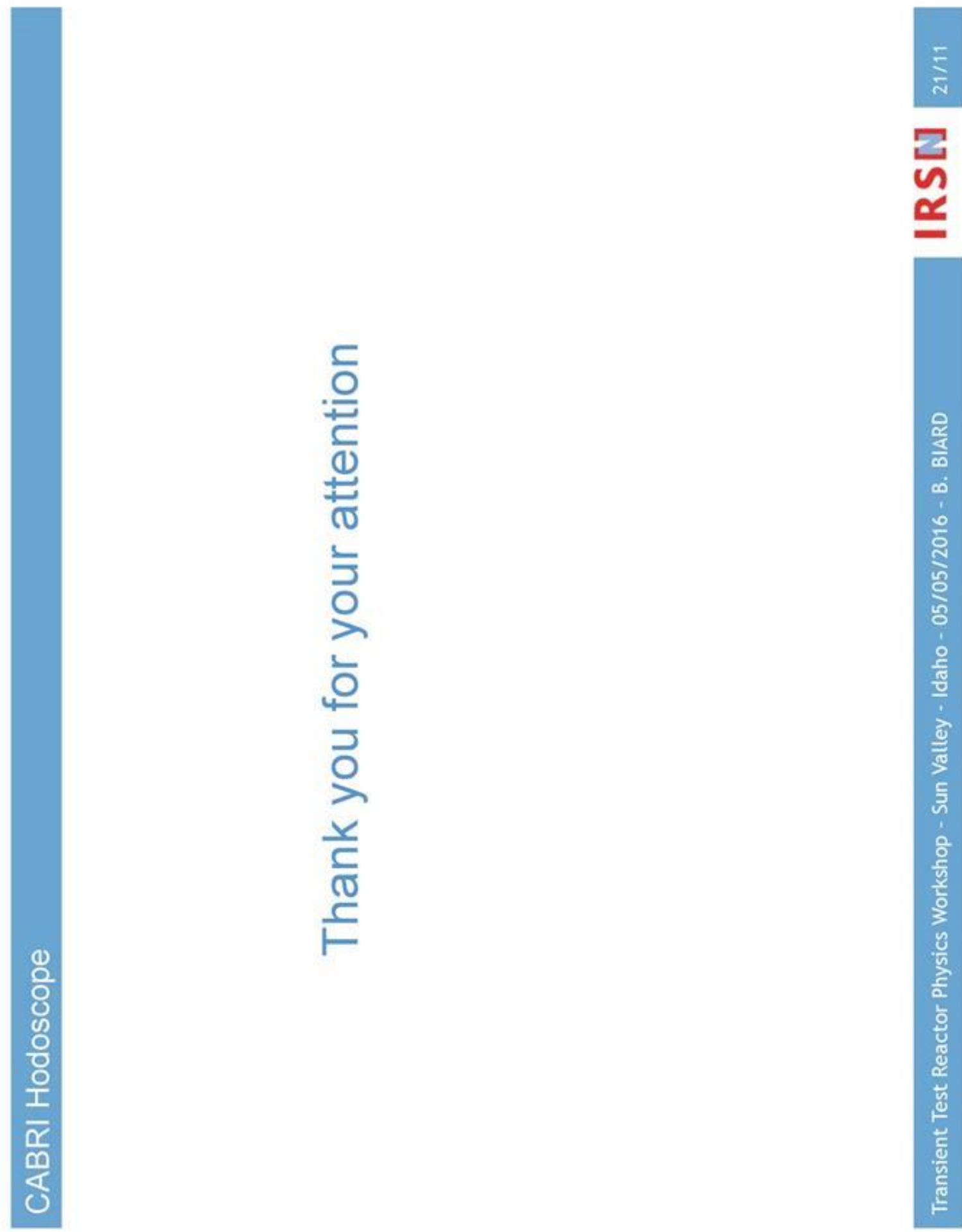

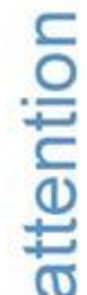

ํํㄱ

는

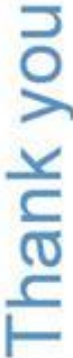

